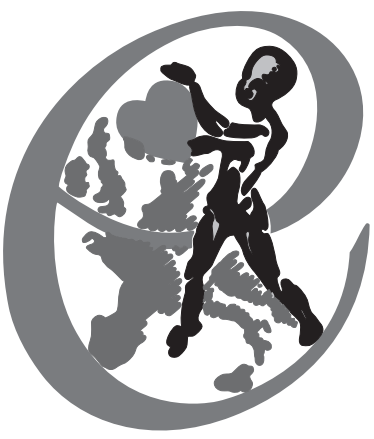

Volume 28 • Supplement 1 • Pages S1-S176

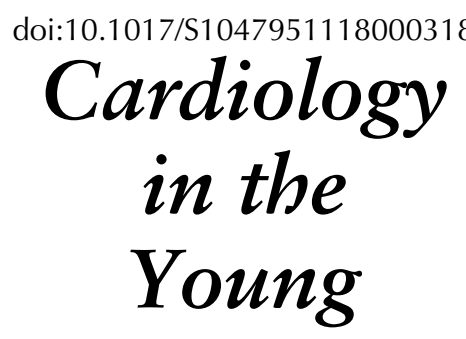

(C) 2018 Cambridge University Press

\title{
52nd Annual Meeting of the Association for European Paediatric and Congenital Cardiology (AEPC) \\ Megaron Athens International Conference Centre, Athens, Greece I May 9-12, 2018
}

O1-1

Predictors of ECG failure for subcutaneous ICD in children: a prospective multicenter study

Sreeram N. (3), Campbell M. (1), Moore J.P. (2), von Alvensleben J.C. (4), Shah A. (5), Batra A. (6), Law I. (7), Sanatani S. (8), Thomas V. (9), Nik-Ahd F. (2), Williams S. (5), Nosavan N. (6), Maldonado J. (7), Hart A. (8), Nguyen T. (1), Balaji S. (1) Oregon health and Science University, Portland, USA (1); University of California at Los Angeles Medical Center, Los Angeles, USA (2); University Hospital of Cologne, Cologne, Germany (3); Children's Hospital Colorado, Aurora, USA (4); Children's Heart Center at OU Medical Center, Oklahoma City, USA (5); University of California Irvine, Orange, USA (6); University of Iowa Stead Family Children's Hospital, Iowa City, USA (7); British Columbia Children's Hospital, Vancouver, Canada (8); University of Nebraska Medical Center, Omaha, USA (9).

Introduction: The subcutaneous Implantable Cardioverter Defibrillator (SICD) shows promise for select patients at risk for sudden death. However, patients need to pass an ECG screening (ECG-S) test before they can receive an SICD. Predictors of ECG-S failure in children are unclear.

Methods: Children $\leq 18$ years with a pre-existing ICD underwent SICD ECG-S. Electrocardiographic and demographic data were analyzed for factors predictive of failure.

Results: Seventy-three patients (44 males (60\%), mean age $14.2 \pm 3.3$, range $5-18$ years) with hypertrophic cardiomyopathy $(\mathrm{n}=24)$, long QT syndrome $(\mathrm{n}=18)$, other inherited arrhythmia syndromes $(n=20)$, congenital heart disease $(n=9)$, and miscellaneous $(n=2)$ with an existing transvenous ICD system underwent prospective ECG-S. Nineteen (26\%) failed ECG-S. Failed patients had a longer QTc duration $(457 \mathrm{~ms}$ vs $425 \mathrm{~ms}$, $\mathrm{p}=0.03)$, QRS duration (120 ms vs $98 \mathrm{~ms}, \mathrm{p}=0.04)$, and R:T amplitude ratio in $\mathrm{aVF}(5 \mathrm{v} 4, \mathrm{p}=0.001)$. Multivariable logistic regression identified QTc (OR 4.31; $\mathrm{p}=0.04)$, QRS duration (OR 4.93, $\mathrm{p}=0.03)$, R:T ratio in $\mathrm{avF}(\mathrm{OR} 3.13, \mathrm{p}=0.08)$ as predictive of failure. A risk score with one point each for QTc $>440 \mathrm{~ms}$, QRS $>120 \mathrm{~ms}$, and R:T ratio $<6.5$ in aVF was associated with probabilities of $15.4 \%$ (1 point), $47.4 \%$ ( 2 points) and $88.6 \%$ (3 points) for failure respectively.

Conclusion: SICD ECG-S failure occurred in $26 \%$ of children which is higher than the reported incidence in adults. Factors predicting ECG-S failure included longer QTc, longer QRS duration, and a lower $\mathrm{R}: \mathrm{T}$ ratio in $\mathrm{aVF}$.
O1-2

Cardiac resynchronization therapy for treatment of chronic pulmonary right ventricular dysfunction in congenital heart disease

Kovanda J., Kubuš P., Ložek M., Tomek V., Gebauer R., Janoušek J. Children's Heart Center, 2nd Faculty of Medicine, Charles University in Prague and Motol University Hospital, Prague, Czech Republic (Supported by the grant No 15-28029A, Agency for Medical Research, Ministry of Health of the Czech Republic)

Introduction: Chronic pulmonary right ventricular (RV) dysfunction is common in congenital heart disease (CHD). We sought to evaluate mid-term results of permanent RV cardiac resynchronization therapy (RV-CRT) in this setting.

Methods: Six patients with repaired tetralogy of Fallot (3), pulmonary atresia (1), absent pulmonary valve syndrome (1) and Ebstein's anomaly (1) having complete right bundle branch block and aged median $13.6(1.0-19.2)$ years underwent RV-CRT for symptomatic chronic RV dysfunction/failure. Implantation procedures were associated with other cardiac surgery aimed to improve hemodynamic residua in $3 / 6$ patients. RVCRT was accomplished by atrial-synchronized RV free wall pacing at sites of late RV activation (q-RV interval= mean $83 \pm 7 \%$ of QRS duration) in maximal fusion with intrinsic ventricular activation as checked by regular 24-hour ECG. Patients were followed-up prospectively for median 14.3 (1.3-46.4)

Table.

\begin{tabular}{|c|c|c|c|c|c|}
\hline & $\begin{array}{l}\text { QRS } \\
\text { duration } \\
{[\mathrm{ms}]}\end{array}$ & $\begin{array}{l}\text { NYHA } \\
\text { class }\end{array}$ & $\begin{array}{l}\text { NTproBNP } \\
{[\text { ng/l] }}\end{array}$ & $\begin{array}{l}\text { RV } \\
\text { FAC } \\
{[\%]}\end{array}$ & $\begin{array}{l}\mathrm{RV} \max .+\mathrm{dP} / \\
\mathrm{dt}[\mathrm{mmHg} / \mathrm{s}]\end{array}$ \\
\hline $\begin{array}{l}\text { Before } \\
\text { After } \\
\text { P }\end{array}$ & $\begin{array}{l}158(29) \\
113(20) \\
=0.002\end{array}$ & $\begin{array}{l}2.3(0.8) \\
1.3(0.4) \\
=0.048\end{array}$ & $\begin{array}{l}842(756) \\
229(196) \\
=0.118\end{array}$ & $\begin{array}{l}18(9) \\
34(4) \\
=0.006\end{array}$ & $\begin{array}{l}316(153) \\
444(161) \\
=0.051\end{array}$ \\
\hline & RV MPI & $\begin{array}{l}\text { Septal flash } \\
{[\mathrm{N}]}\end{array}$ & \multicolumn{3}{|c|}{$\begin{array}{l}\mathrm{RV} \text { septal to free wall } \\
\text { mechanical delay }[\mathrm{ms}]\end{array}$} \\
\hline $\begin{array}{l}\text { Before } \\
\text { After } \\
\text { P }\end{array}$ & $\begin{array}{c}0.48(0.44) \\
0.26(0.10) \\
=0.247\end{array}$ & $\begin{aligned} & 5 / 6 \\
& 0 / 6 \\
= & 0.015\end{aligned}$ & $\begin{array}{c}149(72) \\
23(70) \\
=0.044\end{array}$ & & \\
\hline
\end{tabular}

Mean $(\mathrm{SD}) . \mathrm{FAC}=$ fractional area of change; $\mathrm{MPI}=$ myocardial performance index 
months and assessed by conventional echocardiography and speckle tracking imaging.

Results: All patients survived and improved clinically as well as in terms of RV function and synchrony (Table). RV-CRT had to be terminated after 3.2 years in one patient because of exit block on the RV lead. None of the patients experienced worsening of left ventricular function due to $\mathrm{RV}$ pacing.

Conclusions: Permanent pulmonary RV-CRT alone or in association with redo cardiac surgery carried sustained clinical benefit and improvement in RV function and synchrony over mid-term follow-up in patients with CHD and RV dysfunction associated with electromechanical dyssynchrony. RV-CRT may be a useful adjunct to long-term management of RV dysfunction and failure in selected patients.

\section{O1-3 \\ Brugada Syndrome in the paediatric population: experience from a single center \\ Gribaudo E., Giustetto C., Gabbarini F., Biava L., Bordese R., Agnoletti G., Gaita F. \\ Città della salute e della scienza di Torino, Turin, Italy}

Introduction: Brugada syndrome $(\mathrm{BrS})$ is an arrhythmogenic disease typically diagnosed during adulthood. Few studies exist on paediatric population.

Aim of this study was to analyse the clinical, electrocardiographic (ECG) and genetic characteristics in the paediatric patients of the Brugada Registry of the Piedmont Region of Italy.

Methods: 1044 patients were included in the Brugada Registry from 2001 to 2017. Database was analysed looking for patients younger than 18 years old.

Results: 53 patients were included in the study. Mean age at diagnosis was $12,8+/-4,8$ years. 40 patients $(75 \%)$ were male. Most patients $(42,79 \%)$ were asymptomatic at presentation: diagnosis was made during occasional ECG in 26 (62\%), familial screening in 11 (26\%), ECG made during fever in $4(10 \%)$ or ECG during Ic anti-arrhythmic treatment in one case. Among symptomatic patients $(11,21 \%) 3$ died suddenly (SD); sustained ventricular tachycardia (sVT) and syncopal episodes were documented in one and 7 cases respectively. Mean age at first event was $9+/-6$ years. Two of the patients with SD had spontaneous type 1 Br ECG in multiple leads and homozygous SCN5A mutations. Type 1 pattern was identified in 30/53 (57\%), in 10 during fever; supraventricular arrhythmias were documented in 3 patients ( 2 atrial fibrillation and one atrial ectopic tachycardia). Genetic testing was performed in 30 cases (57\%) and SCN5A mutation was identified in 15 (50\%). During follow-up (49+/- 56 months) 6 patients experienced a new syncopal episode. 7 patients received Hydroquinidine therapy, 4 of them were symptomatic (sVT, syncope) and 3 were asymptomatic with spontaneous type 1 ECG. No patient had symptoms during follow-up. Among symptomatic patients, at presentation or during follow-up, 10/14 (71\%) had a spontaneous type $1 \mathrm{Br}$ ECG, while in asymptomatic patients type 1 was identified only in 20/39 (51\%, p < 0.001).

Conclusions: Spontaneous type 1 Brugada ECG seems to be more frequent in symptomatic patients and SCN5A mutation has been found in a higher proportion of patients than in adults.

\section{O1-4}

A comparison of different risk stratification strategies for sudden cardiac death in pediatric hypertrophic cardiomyopathy

Östman-Smith I. (1), Sjöberg G. (2), Rydberg A. (3), Larsson P. (4), Fernlund E. (5)
Department of Pediatric Cardiology, Queen Silvia Childrens Hospital, Sahlgrenska Academy Gothenburg, Sweden (1); Department of Pediatric Cardiology, Astrid Lindgren Childrens Hospital, Karolinska Hospital, Stockholm, Sweden (2); Unit of Pediatrics, Department of Clinical Sciences, Umeå University Hospital, Umea, Sweden (3); Department of Pediatric Cardiology, Uppsala University Childrens Hospital, Uppsala, Sweden (4); Department of Pediatrics, Linköping University Hospital, Linköping, Sweden (5)

Introduction: Studies on pediatric patients with hypertrophic cardiomyopathy (HCM) have failed to confirm that risk-factors for sudden death considered important in American Heart Association (AHA) 2011 risk-stratification guidelines, the only ones recognized for patients $<16$ yrs of age, are significant predictors in childhood-HCM, with the exception of ventricular tachycardia (VT).

Methods: A national Swedish cohort of patients with a diagnosis of hypertrophic cardiomyopathy (HCM) presenting $<19$ years of age contains 140 patients, follow-up of 10.9 (SD \pm 9.0 ) yrs, with both ECG- and wall thickness measurements, data on syncope, and family history of sudden death (FHSCD). 129 have had $24 \mathrm{~h}$ Holter-ECG or equivalent monitoring. There were 27/140 sudden deaths/cardiac arrests (SD/CA) in the total group and 21/ 129 in the latter group. The ability to predict SD/CA for proposed risk predictors was studied with ROC-curve analysis.

Results: Analyzed as continuous function in the ECG-monitored group the number of "major" AHA risk-factors (FHSCD, max wall thickness $\geq 3 \mathrm{~cm}$, unexplained syncope) had area under the curve (AUC) 0.53 [0.39-0.67, p = 0.66]. Using AHA2011 criteria and incorporating presence of $\mathrm{VT}$ and/or abnormal BP-response to exercise if other major risk factor present, improves AUC only to $0.56[p=0.40]$, but expressing max wall thickness as Detroit Z-score with a cut-off at $\geq 6$ instead of $\geq 3 \mathrm{~cm}$ improves AUC to $0.64[0.51-0.77, p=0.042)$. Examining individual AHA-risk factors, neither FHSCD $(p=0.64)$, or pathological BP-response on exercise $(p=0.20)$ were significant, whereas VT on Holter had $\mathrm{AUC}=0.79[0.63-0.86, \mathrm{p}<0.001]$. A pediatric modification of scoring using only max wall thickness Detroit score $\geq 6$, VT on Holter and unexplained syncope as risk factors improved AUC further to 0.80 [0.69-0.91, $\mathrm{p}<0.001]$. In the same population, both ECG-risk score $>5$ points $\mathrm{AUC}=0.87 \quad[0.80-0.93$, $\mathrm{p}<0.001]$, last Detroit Z-score $\geq 4.5 \mathrm{AUC}=0.80 \quad[0.70-0.89$, $\mathrm{p}<0.001]$, and as continuous functions: ECG risk score $\mathrm{AUC}=$ $0.90[0.85-0.95, p<0.001]$ and last Z-score AUC $=0.86[0.78-$ $0.93, \mathrm{p}<0.001$ ], performs better as risk predictors. ROC-curves in total group gave virtually identical values.

Conclusions: "Major" risk-factors stated in AHA 2011-guidelines have too low sensitivity and specificity in pediatric HCM-patients, and risk-stratification based on ECG-phenotype is more effective. There is a need for the development of a pediatric risk calculator based on childhood risk-factors and including ECG-phenotype.

\section{O1-5}

Pacemaker treatment in a national cohort of patients after Fontan surgery.

Alenius Dahlqvist J. (1), Wiklund U. (2), Berggren H (3),

Hanseus K. (4), Nygren A (5), Johansson Ramgren J. (6), Strömvall-Larsson E. (5), Sunnegårdh J. (5), Rydberg A. (1).

(1) Department of Clinical Sciences, Umeå University, Sweden.

(2) Department of Radiation Sciences, Biomedical Engineering, Umeå University, Sweden. (3) Department of Molecular and Clinical Medicine, Children's Heart Center, The Queen Silvia's Children's Hospital, Göteborg, Sweden. (4) Department of Pediatric Cardiology, Skåne University Hospital, Lund, Sweden. (5) Department of Pediatric 
Cardiology, The Queen Silvia Children's Hospital, Sahlgrenska University Hospital, Gothenburg, Sweden. (6) Pediatric Cardiac Surgery Unit, Children's Hospital, University Hospital, Lund, Sweden.

Objective: In patients with univentricular heart defects Fontan type of surgery has been performed in Sweden since the beginning of the 1980 's, and increasingly so during the last 30 years. We aimed to examine the prevalence of, indications for and risk for pacemaker, with regard to type of Fontan surgery.

Methods: We retrospectively reviewed all Swedish patients who underwent complete Fontan surgery from 1982 to 2017 ( $n=581)$. Patients were identified from four different registries and clinical records were reviewed.

Results: Fourteen percent (79/581) of the patients with a completed Fontan circulation had received a pacemaker. The mean follow-up time from complete Fontan was $12.6 \pm 7.2$ years. Four patients had an atriopulmonary connection (whereof 3 had a pacemaker); 230 had a lateral tunnel (LT) and 343 had an extracardiac conduit (EC). In Sweden the surgical method of choice has developed from from LT to EC, thus the follow-up time was shorter in the EC group than in the LT group. A Kaplan-Meier analysis was performed to compare the need for pacemaker in the patients with LT/EC who received their pacemaker after the Fontan completion. The incidence of pacemaker treatment was higher in patients operated with LT (17\%) than in patients with EC (4\%) (Figure).

The most common indication for pacemaker was sinus node dysfunction (66\%) followed by atrioventricular block (30\%). In 4\% of the cases the indication was protection for bradycardia due to need for treatment with anti-arrhythmic drugs for ventricular or supraventricular tachyarrhythmias.

A Kaplan Meier analysis was performed in patients with pacemaker treatment only due to sinus node dysfunction (lateral tunnel $=29$, extra-cardiac conduit $=10$ ) and we still found a significantly higher prevalence in patients with LT compared to EC $(\mathrm{p}<0.001)$.

Conclusions: In the Swedish national cohort the incidence for a pacemaker in patients with Fontan circulation operated between 1982 and 2017 was 14\%. The main indication was sinus node

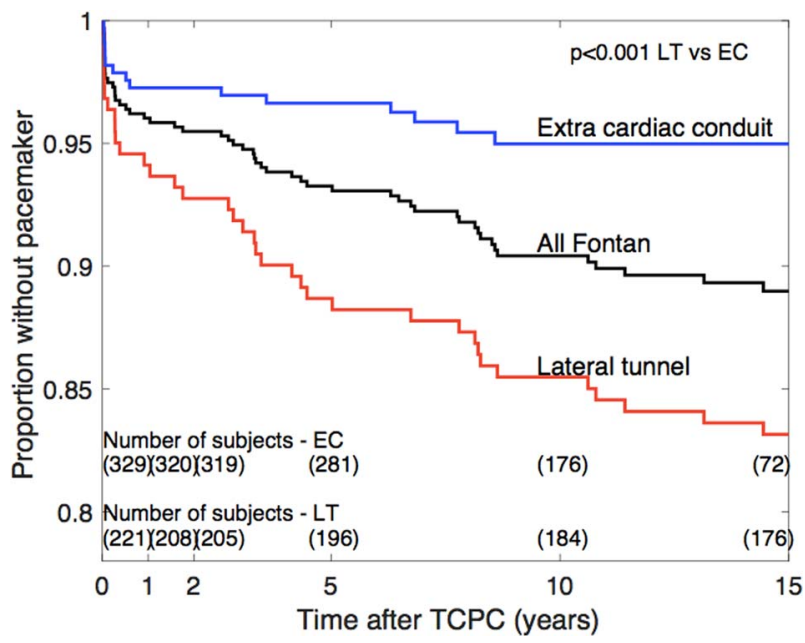

Figure.

Kaplan-Meier curve showing the proportion of patients that were free from pacemaker treatment at different times after TCPC. Numbers in parentheses refers to the remaining number of EC/LT patients at the time points $0,1,2,5,10$ and 15 years affer TCPC. TCPC = Total Cavo Pulmonary Connection, $L T=$ lateral tunnel, $E C=$ extracardiac conduit. dysfunction $(66 \%)$. The incidence of pacemaker treatment was higher in patients operated with lateral tunnel than in patients operated with an extra-cardiac conduit $(\mathrm{p}<0.001)$.

O1-6

Cryoablation for AV Nodal Reentrant Tachycardia in Children and Congenital Heart Disease Patients: Risk Factors for Recurrence

Avramidis D., Bonou P., Tzifa A., Papagiannis J.

Mitera Children's Hospital Athens Greece

Background: AV nodal reentrant tachycardia (AVNRT) is a common arrhythmia in children and patients with congenital heart disease (CHD). Cryoablation is used frequently in pediatric electrophysiology centers, to minimize risk of AV block, but there is significant risk of recurrence. Factors associated with recurrence are not clearly defined.

Purpose: To determine factors related to recurrence in pediatric and congenital patients with AVNRT.

Methods and results: From 17/11/2004-29/06/2015, 101 consecutive patients underwent cryoablation for AVNRT by the same operators. A 3D mapping system (NavX St Jude Medical) was used in all cases. We labeled the first 41 pts as early group and the final 60 as late group. Acute success was achieved in every studied patient. Follow-up ranged from 2.44 to 13.05 years. We compared pts without recurrence (Group A, $n=90$ ) to those with recurrence (Group B, $n=11$ ). There were no differences between groups in patient age $(12.5 \pm 4.7$ vs $12.7 \pm 4$ years $p=0.89)$. Patients in Group A, were more likely to belong in the late era $(p=0.022)$. They tended to have longer procedure time $(193 \pm 55$ vs $165 \pm 35 \mathrm{~min} p=0.11$ ), although the difference was not statistically significant. Fluoroscopy times were similar, although they trended downwards with more experience $(4.59 \pm 4.2$ early group vs $3.3 \pm 4.6$ clate group $p=0.178)$. The number of cryolesions, residual slow pathway conduction and presence of CHD did not show any relationship with recurrence. There were no complications in either group.

Conclusions: Cryoablation is extremely safe and with very high acute success for AVNRT ablation. No risk factors associated with higher recurrence rates were identified, other than less experience. Larger studies are needed to determine methods to increase longterm success.

O2-1

Perioperative cerebral growth is associated with neurodevelopmental outcome in newborns operated for congenital heart disease

Knirsch W. (1,2), Meuwly E. (1,3), Von Rhein M. $(1,3)$, Dave H. $(1,4)$, Tuura R. $(1,5)$, Scheer I. $(1,5)$, Latal B. $(1,3)$, Jakab A. $(1,5)$ Children's Research Center (1), Pediatric Cardiology, Pediatric Heart Center (2), Child Development Center (3), Congenital Cardiovascular Surgery, Pediatric Heart Center (4), Diagnostic Imaging, MR-Center (5), University Children's Hospital, Zurich, Switzerland

Objectives: We aimed to characterise global and local cerebral growth trajectories in neonates suffering from severe congenital heart disease (CHD), and compare against normal development and neurodevelopmental outcome at one year of age.

Methods: 47 neonates with CHD and 44 term controls were included (CHD, gestational age (GA) at the time of first MRI: 40.7 (37.6-51.6) weeks, controls, GA: 42.4 (40.2-45.7)). Diagnostic 3.0 T MRI comprised T2-weighted tri-orthogonal sequences, which were combined and re-sampled to isotropic 
$0.5 \mathrm{~mm}$ voxel space. Global brain volume and lobar volumes were determined by non-linearly aligning a gestational week specific neonatal brain atlas. Growth rate was given as a volumetric change between the second and first MRI scan per week (pre- and postoperative, respectively), while regional growth rate maps were calculated by implementing the deformation-based morphometry approach. We correlated growth rate with the 1-year Bayley Scales of Infant Development using a general linear model approach in the FSL image analysis package, correcting for MRI software version, gender and socio-economic status.

Results: The rate of the global brain volume increase was lower in CHD infants $\left(11 \mathrm{~cm}^{3} /\right.$ week) compared to the normal population $\left(22.3 \mathrm{~cm}^{3} /\right.$ week $)$. The lag behind normal development was most pronounced in the frontal and parietal lobes (2.72 and $1.81 \mathrm{~cm}^{3} /$ week, respectively), where growth rate was greatly under the norm $\left(6.13 \mathrm{~cm}^{3} /\right.$ week and $4.48 \mathrm{~cm} 3 /$ week, respectively). Regional growth rate in the left posterior superior temporal gyrus and left planum temporale correlated significantly ( $p$ corrected $<0.02$ ) with the 1-year language development score in CHD infants.

Conclusion: Our findings not only confirmed the global reduction of cerebral volume in CHD infants, but also imply a furtherreaching impairment consisting of reduced brain growth after corrective surgery and the potential of local brain growth rate to predict neurodevelopmental outcomes.

O2-2

Predictors of neoaortic insufficiency after Ross procedure Klymyshyn Y., Romaniuk O., Lebid I., Khanenova V., Rudenko N., Kalashnikova R., Artemenko Y., Pozniak Y.

Ukrainian Children's Cardiac Center, Kyiv, Ukraine

Objective: To examine predictors of neoaortic insufficiency (neoAI) after Ross operations.

Material and Methods: Ross procedure was performed in 215 consecutive patients from 1996 to 2016 . The mean age at the time of operation was $145 \pm 101$ months (from 1 to 648 months). 159 patients were male - $159(74 \%)$ and $56(26 \%)$ - female - . Patients age was less than 5 years in $48(22,3 \%), 6-10$ years in $39(18,2 \%)-$, $11-17$ years in $66(30,7 \%)$ - and more than 18 years in $62(28,8 \%)$. Aortic stenosis was found in $113(52,5 \%)$ patients, aortic insufficiency in $73(33 \%)$ and complex lesion in $29(14,5 \%)$ patients. Overall mortality was $9 \%, 14(6,5 \%)$ patients died in early postoperative period, $5(2,3 \%)$ - during follow-up. Follow-up was $96 \%$ complete i.e. in 188 patiens. Regression and correlation analysis were used to assess the results.

Results: 11 (5,8\%) patients required neoAV reoperation (in 4 cases - repair of AV and in 7 cases - AVR). Indications for reoperation were severe neoAV insufficiency and AscAo dilatation, $\mathrm{n}=10$ $(5,1 \%)$, reSubAo stenosis $n=1(0,5 \%)$. The mean period before neoAV reoperation was $154,3 \pm 35$ mon (from 27 to 212 mon). Freedom from neoAV reoperation was $98,9 \%, 95,4 \%, 93,7 \%$ after 10,15 and 20 years respectively.

Independent predictors of neoAI more than mild were older age of patients at the time of surgery $(r=0,277, p=0,003)$, acquired AV lesion (rheumatism $(r=0,252, p=0.001)$ and infective endocarditis $(r=0,314, p=0,006), L V$ dilatation before surgery $(r=0,477$, $\mathrm{p}=0.001)$. Surgical technique modification - neoaortic root reinforcement $(r=-0,287, p=0.002)$, congenital nature of aortic valve lesion $(r=-0,405, p=0.001)$, bicuspid aortic valve $(r=-0,351$, $\mathrm{p}=0,001)$ had a positive effect on the long-term outcome.

Conclusions: RP has low risk of autograft reoperation $(5,8 \%)$. Independent predictors of neoAI after RP were: older age of patients at the time of surgery, cause of AV lesion (rheumatism, infective endocarditis), LV dilatation before surgery.
O2-3

Mortality after cardiopulmonary resuscitation events occurring after paediatric cardiac surgery is associated with procedure complexity

Kramer P., Miera O., Photiadis J., Berger F., Schmitt K.R.L. German Heart Center Berlin, Germany

Objectives: Children after cardiac surgery have an increased risk for cardiopulmonary resuscitation (CPR) events while survival after $\mathrm{CPR}$ in these patients is reported to be higher compared to other arrest aetiologies. In the present study, we wanted to assess the institutional incidence and outcomes of CPR events after paediatric cardiac surgery and identify risk factors for mortality.

Methods: In a retrospective review of the past decade from 20072017 , we identified all children $<18$ years of age who experienced $\mathrm{CPR}$ events after cardiac surgery in our institution. Clinical variables and outcomes were analysed.

Results: A total of 232 children (median age 0.31 [IQR 0.05-0.63] years) required CPR after cardiac surgery (incidence $4.9 \%$ ). Univentricular heart malformation was present in 73 patients (31.5\%). In 49 patients $(21.1 \%)$ extracorporeal membrane oxygenation was established during CPR (ECPR) and a total of 79 patients (34.1\%) requires any type of mechanical circulatory support during hospital stay. Surgical procedures were generally complex with median STS-EACTS procedure risk category 4 [IQR 2-4]. Survival to discharge was $71.1 \%(n=165)$ and survival with good neurological outcome was $62.9 \% \quad(n=146)$. Mortality was associated with several resuscitation variables (longer duration of CPR, higher lactate, lower $\mathrm{pH}$, ECPR), patient variables (younger age, univentricular heart malformation) and procedural risk as assessed by STS-EACTS mortality risk score and category (all $\mathrm{p}<0.001)$. In multivariable analysis, STS-EACTS mortality risk category was an independent risk factor for mortality together with CPR duration, higher lactate, lower $\mathrm{pH}$ and requirement of any type of mechanical circulatory support during hospital stay, while age, univentricular heart malformation and ECPR were not associated with increased mortality risk.

Conclusions: Overall survival and neurological outcome in children with CPR events after paediatric cardiac surgery is good. Interestingly, univentricular heart disease and requirement of ECPR were not independent mortality risk factors in multivariable analysis. Procedural risk of preceding cardiac surgery, however, is an independent risk for mortality and consequently, comparison and appraisal of resuscitation outcomes has to take this factor into account.

\section{O2-4}

Aortopulmonary Collaterals in Neonates with d-Transposition of the Great Arteries - Clinical Significance early after Arterial Switch Operation Christmann M. (1,3), Wipf A. (1,3), Navarini-Meury S. (1,3), Dave H. (2,3), Quandt D. (1,3), Knirsch W. (1,3), Kretschmar O. (1,3) University Children's Hospital Zurich, Heart Center, Pediatric Cardiology, Zurich, Switzerland (1); University Children's Hospital Zurich, Heart Center, Division of Congenital Cardiovascular Surgery, Zurich, Switzerland (2); Children's Research Center, University of Zurich, Switzerland (3)

Objectives: Purpose of this study is to evaluate the clinical significance of major aortopulmonary collateral arteries (MAPCAs) during the early postoperative course after arterial switch operation (ASO) in d-transposition of the great arteries (dTGA).

Methods: Clinical data of 98 patients with simple dTGA between January 2007 and December 2016 at the University Children's Hospital Zurich, Switzerland were analyzed retrospectively. 
Results: 37 from 98 patients (38\%) required cardiac catheterization (CC) due to an early complicated postoperative course or difficult coronary transfer due to special coronary anatomy. In 15 (15\%) patients, hemodynamically relevant MAPCAs were found during $\mathrm{CC}$ and coil embolization was performed. Patients with relevant MAPCAs had a significantly longer PICU stay (7 versus 6 days, $\mathrm{p}=0.021)$, longer hospital stay (41 versus 27 days, $\mathrm{p}=0.005$ ), longer mechanical ventilation time ( 5 versus 3 days, $p=0.005)$, longer need for inotropic support ( 5 versus 4 days, $p=0.001)$ and delayed chest closure time ( 3 versus 2 days, $p=0.030$ ) in those in whom it was left open in comparison to all other patients. In patients having CC, pre-surgery oxygen saturation was significantly lower in patients with relevant MAPCAs $58 \%$ vs $70 \%$, $\mathrm{p}$ 0.019). Echocardiography had a sensitivity of 53\% and a specificity of $100 \%$ in detecting relevant MAPCAs, accurately.

Conclusions: MAPCAs are frequently found in dTGA patients and can be associated with lower baseline oxygen saturation and a prolonged postoperative course after ASO. Transthoracic echocardiography cannot replace $\mathrm{CC}$ as diagnostic tool. If significant MAPCAs are suspected, early CC should be performed for diagnostic and therapeutic reasons.

\section{O2-5}

Systemic Atrioventricular Valve Replacement by mechanical prosthesis in Children: Evolution in Practice and Predictors of Long-Term Outcome

Moreau de Bellaing A., Bajolle F., Haydar A., Vouhé P., Bonnet D. Hôpital Necker-Enfants-Malades, Paris, France

Introduction. Systemic atrioventricular mechanical valve replacement (SAVR) in children, especially children aged $<5$ years, has been associated with a high complication rate and long-term outcome is generally perceived as poor because of mandatory anticoagulation and fixed size prosthesis.

Methods. A retrospective review of clinical and surgical records of patients undergoing SAVR in Necker hospital between 2000 and 2017, was performed. 94 children underwent 108 SAVR procedures at a median age of 4.2 years (range, 1 month to 17 years) and a median weight of $15 \mathrm{~kg}$ (range, 2.5 to $68 \mathrm{~kg}$ ). 25 patients (27\%) were $<1$-year-old. Fifteen patients $(16 \%)$ had more than one SAVR. Median follow-up for operative survivors was 5.8 years (range, 1 month to 18 years, 96\% complete).

Results. Thirty-day mortality was $9 \%(\mathrm{n}=8)$. One-year, five-year and ten-year patient survival was respectively $87 \%, 82 \%$ and $79 \%$. By multivariate analysis, high operative mortality in the younger children <1-year-old with Shone's syndrome was the most important contributor to poor long-term survival $(p=0.03)$. Among survivors, the 10 -year freedom from reoperation was $68 \%$. 5 -year survival substantially increases in the second period of the study (72\% before 2008 vs $89 \%$ after $2008, p=0.03$ ) irrespective of age and disease aetiology. From 2008, age-adjusted variable predictors of death that changed were: the median valve size $(23 \mathrm{~mm}$ vs $17 \mathrm{~mm}, \mathrm{p}=0.007)$, the shape of prosthesis $(28 \%$ inversed aortic prosthesis vs $3 \%, \mathrm{p}=0.007)$ and the prosthesis position $(28 \%$ in the supra-annular position vs $54 \%, p=0.017)$. Complications after SAVR included heart block requiring pacemaker (13\%) with a higher risk in case of atrioventricular canal $(p=0.004)$, bleeding $(8 \%)$, prosthesis thrombosis $(3 \%)$, stroke $(3 \%)$ and endocarditis (4\%).

Conclusions. Early mortality after SAVR is low and decreases from 2008 thanks to operative technic modifications. Complications associated with surgery and long-term anticoagulation are rare. This study gives confidence in mechanical prosthesis especially when they are performed in children aged $>1$ year.

\section{O2-6}

Improvement of early postoperative outcome after total cavopulmonary connection by early extubation strategy Kramer P., Nordmeyer S., Cho M.-Y., Photiadis J., Miera O., Berger F., Ovrutskiy S.

German Heart Center Berlin, Germany

Objectives: Short cardiopulmonary bypass time and short duration of mechanical ventilation are important aspects of optimizing the early postoperative course after total cavopulmonary connection. In our study, we investigated the influence of early postoperative extubation strategy in our cohort of Fontan patients during the past two decades.

Methods: We performed a retrospective study including 249 consecutive patients who were selected for total cavopulmonary connection in our institution from 1995-2017. Early postoperative course was evaluated by analysis of early postoperative hemodynamic parameters and early outcome after surgery in terms of mortality and length of hospital stay. For analysis, the patient cohort was sub-divided into a "fast-track" extubation group $(<6$ hours ventilation, $\mathrm{n}=87$ ) and a prolonged ventilation group ( $>6$ hours ventilation, $\mathrm{n}=162)$ and outcome was compared according to preoperative patient's characteristics, duration of cardiopulmonary bypass and duration of mechanical ventilation.

Results: Duration cardiopulmonary bypass did not correlate with duration of mechanical ventilation $(p=0.1)$, was not associated with length of hospital stay in univariate analysis $(p=0.13)$ and did not differ between both groups $(p=0.5)$. Fontan patients in the fast-track group had significantly improved early postoperative hemodynamics with higher arterial pressure and lower pulmonary artery pressure at 6,24 and 48 hours postoperatively $(p=0.02-$ $0.005)$. In multivariable analysis, longer mechanical ventilation was independently associated with length of hospital stay.

Conclusions: Early weaning from mechanical ventilation results in improved early Fontan hemodynamics while early outcome is unrelated to duration of cardiopulmonary bypass indicating that early extubation is an important strategy for improving early results after Fontan.

O3-1

Cardiogenetic Approach in Children Cardiomyopathies. Does It Make Change in Clinical Pathway? Results on The Basis of a Cohort of 250 Children

Baban A. (1), Adorisio R. (1), Magliozzi M. (2), Cali F. (1), Alesi V. (2), Rinaldi S. (3), Cantarutti N. (1), Franceschini A. (1), Iodice F.G. (1), Dionisi-Vici C. (4), Digilio M.C. (5), Silvetti M.S. (1), Parisi F. (1), Amodeo A. (1), Carotti A. (1), Iorio F.S. (1), Dallapiccola B. (6), Novelli A. (2), Drago F. (1)

Pediatric Cardiology and Cardiac Surgery Department. Bambino Gesù Children Hospital and Scientific Institute, Rome - Italy (1); Lab of

Medical Genetics. Bambino Gesù Children Hospital and Scientific Institute, Rome - Italy (2); Health Directorate. Bambino Gesù Children Hospital and Scientific Institute, Rome - Italy (3); Metabolism Unit. Bambino Gesù Children Hospital and Scientific Institute, Rome - Italy (4); Medical Genetics Unit. Bambino Gesù Children Hospital and Scientific Institute, Rome - Italy (5); Scientific Directorate. Bambino Gesù Children Hospital and Scientific Institute, Rome - Italy (6).

Introduction: cardiomyopathies (CMP) are highly heterogeneous in origin. Multiple phenocopies are identified on adult cohorts but less is known in children in whom CMP is a rare disease. Early 90s reports describe $20 \%$ familial origin while late international reports identify up to $50 \%$ of familial cases. Origin varies from genetic to non-genetic background. Again the variability is very high in 
genetic causes including sarcomeric, desmosomal, nuclear, mitochondrial and malformative origins. Different cytogenetic and molecular genetic methods were applied to reach highest detection rate of pathogenic variants. Most of rare PHENOCOPIES in CMP remain undiagnosed, lately diagnosed or misdiagnosed.

Methods: a multidisciplinary transversal and longitudinal approach is applied in this cohort of 252 patients affected by CMP. A multi-organ approach including the investigation of metabolic, hormonal, and genetic factors. The algorithm is not based on the exclusion but the integration of different factors. In other words, we believed in the multifactorial and multigenic model of CMP rather than unifactorial origin of the disease.

Results: we identified 56\% familial background. The majority of genetically positive patients carried sarcomeric mutations. RASopathies represented the majority of early onset HCMP. Rare DCMP phenocopies were recognized: Barth Syndrome (3 families), TMEM70 (2 families), Laminopathies (4 families), EDS (1 family), desmosomal (10 families), desminopathies (1 family), and filaminopathy C (3 families). A comprehensive illustration of the cardiogenetic pathway will be explained. We shall illustrate the systematic "red flag" approach performed in the diagnosis of children CMP.

Conclusions: This cohort represents a preliminary example of personalized medicine approach in children cardiogenetics and how identifying underlying diagnosis can change the clinical course and sometimes the therapeutic approach for the individual and families of children affected by CMP. To the best of our knowledge this approach helps in ameliorating the clinical approach and outcome in rare forms of children CMP.

We acknowledge: 1.Part of this project was awarded Best Investigator Award at 2017 National Congress of the Italian Society of Pediatric Cardiology; 2.This project is part of Good Clinical Practice approach guided by OPBG health directorate; 3.Our Center is HCP participant of the ERN-GUARD-HEART network.

\section{O3-2}

Clinical features and outcomes of Childhood Hypertrophic Cardiomyopathy: a retrospective study in the United Kingdom

Norrish G. (1,2), Field E. (1,2), Mcleod K. (3), Illina M. (3), Stuart G. (4), Bhole V. (5), Uzun O. (6), Brown E. (7), Daubeney P. (8), Linter K. (9), Mathur S. (10), Bharucha T. (11), Adwani S. (12), Jones C. (13), Reinhardt Z. (14), Kaski J.P. (1,2)

Great Ormond Street Hospital, London UK (1); UCL Institute of Cardiovascular science, London, UK (2); Royal Hospital for Children, Glasgow, UK (3); Bristol Royal Children's Hospital, Bristol UK (4); Birmingham Children's Hospital, Birmingham, UK (5); The Noah's Ark Children's Hospital for Wales, Cardiff, UK (6); Leeds Teaching Hospital NHS Trust, Leeds, UK (7); Royal Brompton and Harefield NHS foundation Trust, London UK (8); Glenfield Hospital, Leicester, UK (9); Evelina London Children's Hospital, London, UK (10); University Hospital Southampton NHS Foundation Trust, Southampton, UK (11); Oxford University Hospitals, UK (12); Alder Hey Children's NHS foundation Trust, Liverpool, UK (13); The Freeman Hospital, Newcastle, UK (14)

Objectives: Describe the clinical features and outcomes of Childhood Hypertrophic Cardiomyopathy (HCM) in a national cohort. Methods: A retrospective, multi-centre cohort of children diagnosed with HCM in the United Kingdom (UK) since 1980 was formed from 13 paediatric cardiac centres. Anonymised, non-invasive clinical data were collected.

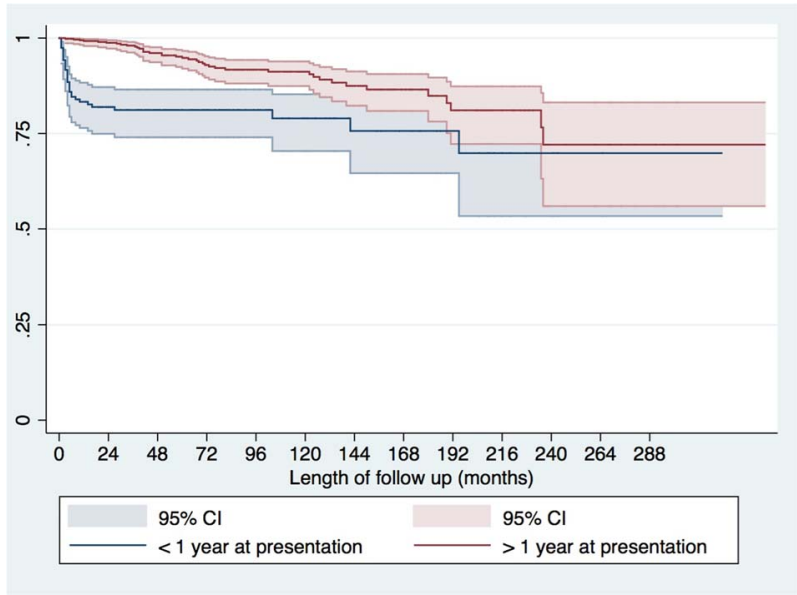

Figure.

Kaplan Meier curve for survival stratified according to age of presentation. Log rank test $p<0.0001$.

Results: 687 patients with childhood HCM were identified with a median age at presentation of 5.2 years (range $0-16$ ). $23 \%$ $(\mathrm{n}=159)$ of patients presented during infancy. The underlying aetiology was varied including idiopathic $(n=433,63 \%)$, Noonan's syndrome (n-121, 17\%), Friedreich's ataxia $(n=58,8 \%)$ and inborn errors of metabolism $(n=64,9 \%)$. Those presenting under 1 year of age were more likely to have a diagnosis of Noonan's syndrome $\left(39 \%\right.$ vs $\left.11 \%, \mathrm{p}=0.000^{\star}\right)$ or inborn error of metabolism (18\% vs $6.4 \% \mathrm{p}=0.000 \star)$.

The majority of patients were asymptomatic, in NYHA/Ross class I at presentation $(n=516,75.4 \%)$, however heart failure symptoms were more common in infants $\left(16 \%\right.$ vs $\left.2.5 \%, \mathrm{p}=0.000^{\star}\right)$. A history of unexplained syncope $(\mathrm{n}=38,5.6 \%)$ or previous aborted cardiac arrest $(n=24,5.6 \%)$ was uncommon.

Overall the prognosis was good, survival without death or transplant was $91.7 \%(89.1-93.7 \%)$ at 5 years. Children diagnosed during infancy or with an inborn error of metabolism had a worse prognosis with a survival of $81 \%$ and $82 \%$ at 1 year respectively. Cause of death was most commonly sudden cardiac death $(\mathrm{n}=20)$ followed by non-cardiovascular causes $(n=17)$ and congestive cardiac failure $(n=12)$. However, sudden cardiac death or an equivalent event (aborted cardiac arrest or appropriate Implantable Cardioverter Defibrillator therapy) was rare, occurring at a rate of 0.012 events/person year follow up.

Conclusions: This national study of childhood HCM describes a heterogeneous cohort whose outcomes are dependent on underlying aetiology and age of presentation. Further studies are needed to systematically investigate risk factors for prognosis in this patient group.

\section{O3-3}

Survival and outcome in pediatric myocarditis: Data of the German multicenter registry "MYKKE"

Degener F. (1,2,3), Opgen-Rhein B. (4), Wagner R. (5), Boehne M. (6), Weigelt A. (7), Müller, G. (8), Racolta A. (9), Rentzsch A. (10), Papakostas K. (11), Reineker K. (12), Kiski D. (13), Wiegand G. (14), Ruf B. (15), Hannes T. (16), Fischer M. (17), Khalil M. (18), Kaestner, M. (19), Freudenthal, N. (20), Steinmetz M. (21), Fischer G. (22), Pickardt T. (23), Messroghli D. (3,24), Schubert S. (1,3) [on behalf of the MYKKE consortium] Deutsches Herzzentrum Berlin, Pediatric Cardiology, Berlin, Germany (1); Charité - Universitätsmedizin Berlin, Institute for Computational and 
Cardiovascular Medicine, Germany (2); DZHK (German Centre for Cardiovascular Research), partner site Berlin, Germany (3); Charité Universitätsmedizin Berlin, Pediatric Cardiology, Berlin, Germany (4); Herzzentrum Leipzig, Pediatric Cardiology, Leipzig, Germany (5); Department of Pediatric Cardiology and Intensive Care Medicine, Hannover Medical School, Hannover, Germany (6); Universitätsklinikum Erlangen, Pediatric Cardiology, Erlangen, Germany (7); Universitäres Herzzentrum Hamburg, Pediatric Cardiology, Hamburg, Germany (8); Herz- und Diabeteszentrum NRW, Pediatric Cardiology, Bad Oeynhausen, Germany (9); Universitätsklinikum des Saarlandes, Pediatric Cardiology, Homburg, Germany (10); Klinikum Links der Weser, Pediatric Cardiology, Bremen, Germany (11); Universitäts-Herzzentrum Freiburg Bad Krozingen, Pediatric Cardiology, Freiburg, Germany (12); Universitätsklinikum Münster, Pediatric Cardiology, Münster, Germany (13); Universitätsklinikum Tübingen, Pediatric Cardiology, Tübingen, Germany (14); Deutsches Herzzentrum München, Technische Universität München, Pediatric Cardiology, München, Germany (15); Uniklinik Köln, Pediatric Cardiology, Köln, Germany (16); Klinikum der Universität München, Pediatric Cardiology, München, Germany (17); Universitätsklinik Gießen, Pediatric Cardiology, Giessen, Germany (18); Universitätsklinikum Ulm, Pediatric Cardiology, Ulm, Germany (19); Universitätsklinikum Bonn, Pediatric Cardiology, Bonn, Germany (20); Universitätsmedizin Göttingen, Kinderherzklinik Göttingen, Pediatric Cardiology, Göttingen, Germany (21); Universitätsklinikum SchleswigHolstein, Pediatric Cardiology, Kiel, Germany (22); Kompetenznetz Angeborene Herzfehler, Berlin, Germany (23); Deutsches Herzzentrum Berlin, Cardiology, Berlin, Germany (24)

Objectives: Myocarditis is a major cause of severe heart failure in childhood. "MYKKE", a multicenter prospective registry for pediatric patients with suspected myocarditis, aims with its ongoing enrollment to gain knowledge on incidence, diagnosis, therapy and outcome.

Methods: Since 2013 twenty centers actively included patients. A sub-analysis on clinical data and a multivariate analysis (MANOVA) of 195 patients enrolled until 2016 was performed according to baseline data and their impact on survival. Patients with typical findings in cardiovascular magnetic resonance (CMR) and/or endomyocardial biopsy (EMB) were defined as myocarditis positive. Results: Until October 2017, 280 patients were enrolled, median (range) age $13.0(0-17)$ years; $66.8 \%$ male. Three age groups were defined according to clinical course and severity of disease: $0-<2$ (24.3\%), 2-12 (20.7\%) and 13-18 years $(55.0 \%) .11 \%$ were listed for heart transplantation (HTx); and 6.8\% subsequently underwent HTx. The overall mortality was $7.5 \%$. Patients $<2$ years had a higher incidence $(59 \%)$ of a severely reduced left ventricular ejection fraction $(\mathrm{EF}<30 \%)$ compared with age groups $2-12 \mathrm{y}$ $(38 \%)$ and $13-18 y(7 \%) ; p<0.001$. They also had the highest rate of mechanical circulatory support (MCS; $\mathrm{p}=0.001)$ and death $(p=0.003)$. A sub-analysis revealed that in $78 \%(n=153 / 195)$, EMB $(n=44)$, CMR $(n=40)$ or both $(n=69)$ were performed with a positive result for myocarditis in $69 \%$. Histology showed: $15 \%$ acute, $43 \%$ subacute/chronic, $12 \%$ status post, $1 \%$ eosinophilic or granulocytic myocarditis, $5 \%$ no infection, $3 \%$ DCM, $1 \%$ HCM, 7\% other. The mortality in the MCS cohort was $21 \%$ $(n=6 / 28)$. The proof of myocarditis had no impact on the possibility of weaning from MCS $(p=0.675)$. Non-MCS patients showed a significantly better survival $(97 \%$ vs. $75 \% ; \mathrm{p}<0.001)$. The MANOVA highlighted age $(p=0.028)$ and EF $(p=0.05)$ as dependent variables in predicting survival. Accordingly, patients $<2$ years and $\mathrm{EF}<30 \%$ carried the highest risk for mortality according to a fulminant course $(\mathrm{p}<0.001)$.

Conclusions: Our data underline that myocarditis stays a lifethreatening disease in the pediatric population, especially in very young children. This first prospective data analysis enabled the definition of a high-risk group. These results emphasize the utility of "MYKKE" as a registry for suspected myocarditis in children and adolescents.

\section{O3-4}

Syk regulates pulmonary vasoconstriction

Tabeling C. (1, 2), Herbert J. (1, 2), Boiarina E. (1, 2),

Hocke A.C. (1), Sewald K. (3); Lamb D.J. (4), Wollin S.L. (4), Fehrenbach H. (5), Kuebler W.M. (6), Braun A. (3), Suttorp N. (1), Weissmann N. (7), Witzenrath $M .(1,2)$

(1) Department of Infectious Diseases and Pulmonary Medicine, Charité - Universitätsmedizin Berlin, Germany; (2) Division of Pulmonary Inflammation, Charité - Universitätsmedizin Berlin, Germany; (3) Department of Airway Immunology, Fraunhofer ITEM, Hannover, Germany; (4) Respiratory Diseases Research, Boehringer Ingelheim Pharma GmbH \& Co. KG, Biberach, Germany; (5) Division of Experimental Pneumology, Priority Area Asthma and Allergy, Research Center Borstel, Airway Research Center North, Member of the German Center for Lung Research, Borstel, Germany; (6) Institute of Physiology, Charité - Universitätsmedizin Berlin, Germany; (7) Excellence Cluster Cardio-Pulmonary System, Justus-Liebig-University, Giessen, Germany

Objectives: Pharmacological inhibition of spleen tyrosine kinase (Syk) is generally well tolerated in humans. In the airways, Syk is known to promote inflammation, smooth muscle cell proliferation and contraction. However, its expression and function in the pulmonary vasculature and its possible involvement in the pathogenesis of pulmonary arterial hypertension $(\mathrm{PAH})$ remain elusive.

Methods: Vascular Syk expression was analyzed in human (PAH versus donor) and murine lung tissue via immunofluorescence and spectral confocal microscopy. The functional role of Syk was studied in human precision-cut lung slices (PCLS) and in isolated perfused and ventilated lungs of wild-type mice or mice deficient in eNOS or protein kinase $\mathrm{C}$ (PKC) $\alpha$ with or without inhibition of Syk, PKC, rho kinase, p38 MAPK and/or NO. Pulmonary vascular hyperresponsiveness was analyzed in isolated lungs following induction of pulmonary Th2 inflammation.

Results: Syk was expressed in smooth muscle cells of remodeled $(\mathrm{PAH})$ and non-remodeled pulmonary arteries (donor). Syk inhibition diminished pulmonary vasoconstriction in human PCLS as well as in isolated mouse lungs independent of eNOS or PKC $\alpha$. The vasopressor response to a broad range of stimuli, namely serotonin, endothelin-1, angiotensin II, thromboxane agonist U46619, sphingosine-1-phosphate and hypoxic ventilation, was shown to be blunted by Syk inhibition (either with BAY 61-3606 or with BI 1002494). In the preconstricted pulmonary vasculature, Syk inhibition rapidly reversed vasoconstriction in a NO-independent manner. Moreover, pulmonary vascular hyperresponsiveness was markedly reduced following Syk inhibition. Inhibition of p38 MAPK reduced pulmonary vasoconstriction to the same extent as Syk inhibition, and simultaneous inhibition of 38 MAPK and Syk did not show any additive effect when compared to Syk inhibition only.

Conclusions: Syk mediates pulmonary vasoconstriction in a NO-independent manner, presumably via p38 MAPK. Our data suggest that Syk inhibition may be a promising therapeutic perspective in $\mathrm{PAH}$.

\section{O3-5}

\section{Experience with safety and efficacy of levosimendan in} pediatric patients

Mulatier C. , Galoin Bertail C., Perouse de Montclos T., Bakloul M., Walton C., Durousset C., Leconte C., Di Filippo S.

Pediatric Cardiology, Cardiovascular Hospital, Lyon, France 
The objective was to describe tolerability and results of levosimendan infusion (LEVO) in critically ill children.

Material and Methods: It is a retrospective analysis of patients aged 0 to 18 years, who received one or more levosimendan infusions, for failure to wean off inotrope and/or ECMO support. Demographic data,clinical, biological, and echocardiographic parameters (LVSF, subaortic VTI and TAPSE) were collected at onset of LEVO (T0), at 2days (T2), 10days (T10), 30days (T30) and 6months (M6) after termination of LEVO.

Results: 71 patients (42males, mean age 3.2y) received 112 levosimendan infusions $(\mathrm{LEVO})=34(48 \%)$ had myocardial dysfunction including 5 acute myocarditis (CMP group), and 37 (52\%) were in postoperative course after surgery for congenital heart disease (CHD). Mean time from surgery to LEVO was 8.3days (median 3), from hospitalisation to LEVO 30.5 days (median 15). NInety-seven percent of the cases were dependent of at least one inotrope support (77\% milrinone) and 32\% of ECMO. Mean dose of LEVO was 0.2 microgrammes $/ \mathrm{kg} / \mathrm{mn}$. Inotrope support was successfully discontinued in 34\% of 32 LEVO $(60 \%$ maintained off support) and $65.6 \%$ of 32 LEVO on ECMO support were weaned off assistance. Mean heart rate decreased from 137 to 131 and 116 bpm at T0, T2 and T30), mean blood pressure increased (64 to 67 and $79 \mathrm{mmHg}$ at $\mathrm{T} 0, \mathrm{~T} 2$ and $\mathrm{T} 30)$, and mean lactates decreased from 2.17 to 1.9 to 1.5 .

Mean LVSF increased from $18.3 \%$ to $21.2 \%, 22.9 \%, 25 \%$ and $31.9 \%$, at T0, T2, T10, T30 and M6. VTI increase was $+1.7 \mathrm{~cm}$, $+3.1 \mathrm{~cm}$ and $+6 \mathrm{~cm}$, at T2, T10, T30 and TAPSE increased $+2.2 \mathrm{~mm}$ and $+2.8 \mathrm{~mm}$ at T10 and T30. LEVO was uncomplicated in 109 of 112 infusions (97.3\%), 3 experienced adverse events (tachycardia and hypotension). Mean CICU time was 66days (median 46) and hospitalisation time was 76days (median 65 ). Fourteen patients underwent heart transplantation and 30 died, i.e. failure occurred in 42 cases (59.2\%). Survival was 50\% at 6th month after termination of LEVO.

Conclusion: Levosimendan is safe and can allow weaning from inotrope and/or ECMO support in hemodynamically compromised pediatric patients with CMP and/or CHD.

\section{O3-6 \\ POTTS shunt as a palliation for patients with idiopathic PAH Bobhate P., Colaco S., Karande T., Kasar P., Kulkarni S. \\ Childrens' heart centre, Kokilaben Dhirubai Ambani Hospital, Mumbai, India}

Introduction: Idiopathic pulmonary artery hypertension ( IAPH) is a progressive disease and may become fatal in many patients. Survival of Eisenmenger's syndrome is much better as compared with patients with IAPH. POTTs shunt has been shown to improve functional class and prolonged survival in patients with IPAH Materials and Methods: This is a prospective study where 10 patients (age $=9$ months-35 years) with PAH on maximal medical therapy, in functional class IV. Clinical symptoms of syncope, functional class, signs and symptoms of right heart failure, upper and lower limb oxygen saturation was looked for in all the patients. PA systolic pressure (PASP), PA acceleration time (PAAT), preejection time (PET) and RV Ejection time (ET) were measured using $2 \mathrm{~d}$ echocardiogram and Doppler. RV mechanics and RV to PA coupling was assessed by RV work (TAPSE X PASP/PAAT) before and after the Potts shunt and at last follow up. 6/10 underwent cardiac catheterization prior to the procedure. NTproBNP and $6 \mathrm{MWT}$ was performed prior to the procedure and at follow up. All patients were on maximal medical therapy before the surgery. 8 underwent surgical POTTS shunt and 2 underwent PDA stenting.
Results: 5 patients in the surgical group and 2 in the PDA stenting survived the procedure. All three who died had severe right ventricular dysfunction. All the survivors were followed up in the $\mathrm{PAH}$ clinic, median follow up was 6 months. $5 / 7$ patients who survived the procedure had significant improvement in functional class (IV vs II, $p=0.02$ ) with decrease in dose and number of pulmonary vasodilators and improvement in $\mathrm{RV}$ function and $\mathrm{RV}$ to PA coupling. 1 patient had no change in the functional status. TAPSE $z$ score improved from $-4.5 \pm 1.5$ to $-1.3 \pm 0.5(\mathrm{p}=0.02)$ and RV to PA coupling improved from $562 \pm 115$ to $883 \pm 113$ $(\mathrm{p}=0.04)$. NT-Pro BNP decreased from 2650 (1126-3400) to 335 (113-446) $\mathrm{p}=0.001$. There were no deaths after discharge. Conclusion: POTTS shunt can be considered as an interim palliative procedure in patients with $\mathrm{PAH}$. Surgery at the earliest indication of clinical deterioration and preoperative stabilization is essential.

\section{O4-1}

Aortic elasticity after aortic coarctation correction: comparison of state of the art therapies by cardiovascular MRI

Pieper T. (1), Latus H. (2), Gummel K. (1), Reich B. (1), Voges I. (3) Paediatric Heart Centre, University Hospital Giessen \& Marburg, Germany (1); Department of Paediatric Cardiology and Congenital Heart Disease, German Heart Centre Munich, Germany (2); Royal Brompton and Harefield NHS Foundation Trust, Cardiovascular Magnetic Resonance Unit and Department of Paediatric Cardiology, London, UK (3)

Background: Previous studies have shown impaired aortic bioelasticity and left ventricular mechanics in patients with aortic coarctation (CoA) despite successful CoA relief. However, rare studies exist comparing patients after surgical and interventional CoA treatment. The aim of this cardiovascular magnetic resonance (CMR) imaging study was to assess aortic bioelasticity and LV properties in CoA patients who underwent either endovascular stent implantation or surgical CoA repair.

Methods: 50 patients $(20.5 \pm 9.5)$ underwent 3-Tesla CMR. Out of them, 18 patients were treated with endovascular stent implantation and 32 patients had surgical CoA repair. We performed volumetric analysis of both ventricles (LV, RV) and left atrium (LA) to assess biventricular end-diastolic and -systolic volumes (LVEDV, LVESV, RVEDV, RVESV) and ejection fraction (LVEF, RVEF) as well as left atrial (LA) volumes and functional parameter $\left(\mathrm{LAEF}_{\text {Passive }}, \mathrm{LAEF}_{\text {Contractile }}, \mathrm{LAEF}_{\text {Reservoir }}\right)$. Furthermore, aortic distensibility at six aortic positions and aortic pulse wave velocity (PWV) at two positions were measured. Native T1 mapping was applied to examine LV myocardial tissue properties. In a subgroup of patients $(n=12)$ additional postcontrast T1 mapping was performed.

Results: CMR measurements of LV, RV and LA parameters did not significantly differ between the surgical and stent group. There was also no significant difference for aortic distensibility, PWV and native T1 relaxation times between both groups. Distensibility of aortic root correlated negatively with age, BMI, BSA and weight $(\mathrm{r}=-0.53$ to $-0.56 ; \mathrm{p}<0.001)$. Native $\mathrm{T} 1$ relaxation times correlated negatively with age, weight, BSA and BMI $(r=-0.58$ to $-0.66 ; \mathrm{p}<0.001)$. The subgroup analysis showed that lower post-contrast $\mathrm{T} 1$ values were associated with lower aortic arch distensibility and higher aortic arch pulse wave velocity $(\mathrm{r}=0.66$ to $0.71 ; \mathrm{p}<0.001)$.

Conclusions: We could not demonstrate a difference in aortic elasticity between patients who underwent surgical CoA repair and patients who were treated with endovascular stent implantation. This suggests that probably other factors such as intrinsic 

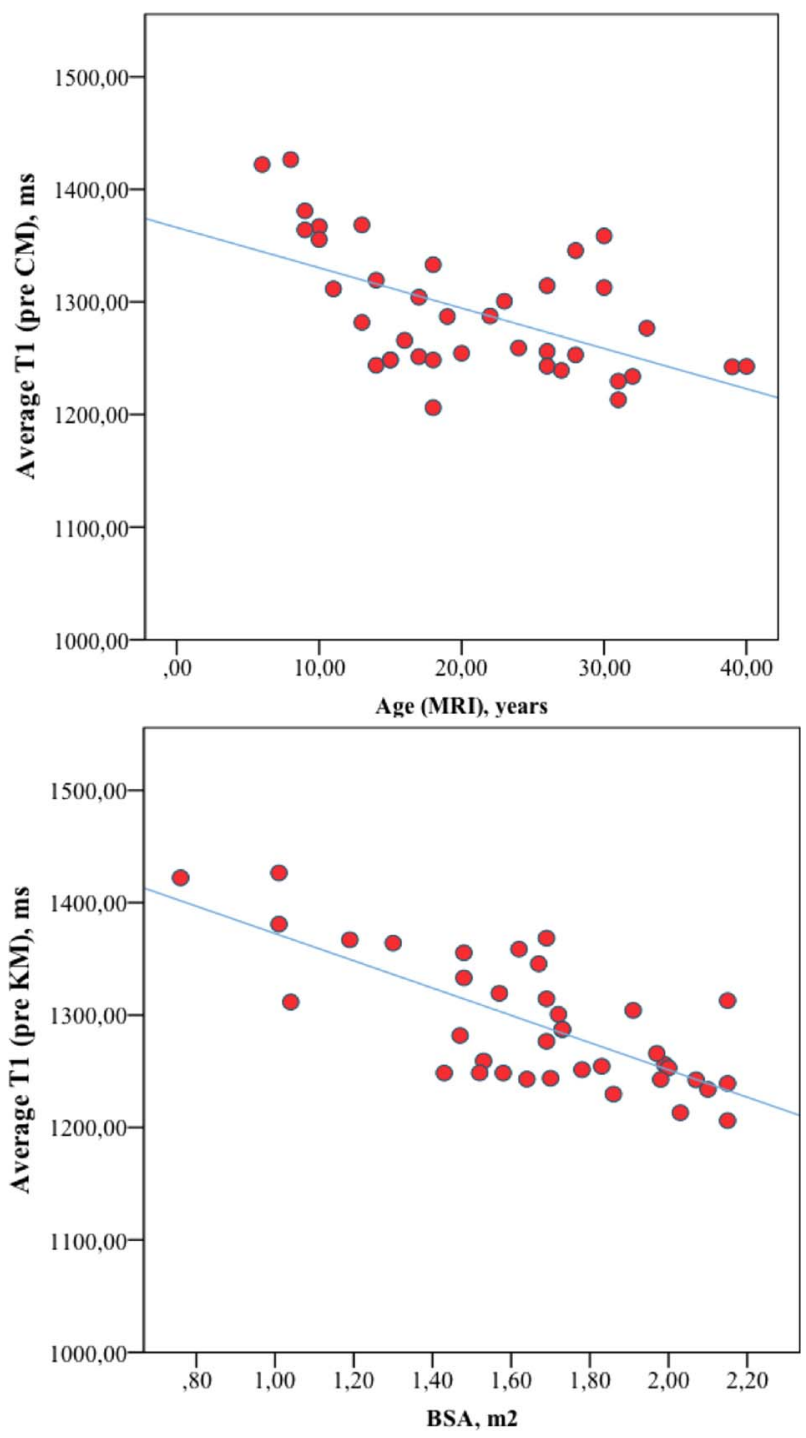

Figure.

aortic wall abnormalities have a greater contribution on aortic wall elasticity. Interestingly, our data suggests that native T1 values are influenced by age, weight, BSA and BMI.

\section{O4-2}

Complimentary coronary artery assessment in Kawasaki Disease with low-dose CT angiography, uncovering vascular pathology missed by echocardiography van Stijn - Bringas Dimitriades D. (1), Planken R.N. (2), Groenink M. (3), Kuijpers T.W.*(1), Kuipers I.M. (4)*

Department of pediatric hematology, immunology and infectious diseases, Emma Childrens Hospital, Academic Medical Centre (AMC), Amsterdam, The Netherlands (1). Department of radiology (cardiovascular imaging, ultrasound, CT, MR), Academic Medical Centre (AMC), Amsterdam, The Netherlands (2). Department of cardiology, Academic Medical Centre (AMC), Amsterdam, The Netherlands (3). Department of pediatric cardiology, Emma Childrens Hospital, Academic Medical Centre (AMC), Amsterdam, The Netherlands (4); * both authors attributed equally

Introduction: Kawasaki Disease (KD) is a vasculitis with formation of coronary artery aneurysms (CAAs) that can lead to myocardial ischemia. To initiate treatment and prevent myocardial infarction, coronary artery assessment is essential. Echocardiography is the primary imaging modality used for coronary assessment in KD despite limited diagnostic accuracy. Coronary angiography $(\mathrm{CAG})$, the gold standard for coronary assessment, is not used as a primary imaging modality due to invasiveness and radiation exposure. Similarly coronary computed tomographic angiography (cCTA) has not been implemented because of high radiation dose. However, state-of-the-art CT-scanners enable low-dose cCTA. The aim of this study was to describe the (I) diagnostic yield of cCTA compared to echocardiography, and (II) to report the radiation dose for the imaging used in KD patients.

Methods: During the past 8 years KD patients underwent cCTA. Low-dose cCTA was available from $2014(n=55)$. Concordant and discordant CCTA and echocardiography findings were compared.

Results: In 70 consecutive KD patients (median age 15.1 yrs [0.48$59.45 \mathrm{yrs}$; $78 \%$ male; $38 \%$ giant CAA in acute KD) a total of 76 CAAs were identified by cCTA in 35 patients. cCTA identified 38 CAAs ( $Z$ score $>3$ ) in 23 patients, not detected by recent echocardiography. In 25 coronary arteries in 15 patients calcifications, plaques and/or thrombi were visualized, undetectable by echocardiography. In 5 patients, the cCTA findings had direct clinical implications for their treatment. The median radiation in doselength-product (DLP) differed between state-of-the-art and older generation CT-scanners (73 [24-283] vs 315 [99-1380]).

Conclusions: Low-dose cCTA enables accurate coronary artery assessment. cCTA is an excellent modality to reveal coronary artery pathology in addition to the echocardiography findings in $\mathrm{KD}$.

O4-3

Approaching routine evaluation of right ventricular performance with advanced echocardiographic techniques in patients with hypoplastic left heart syndrome: a study of feasibility and reproducibility

Ponzoni M. , Federici D., Galletti L., Marcora S.A.

ASST Papa Giovanni XXIII Bergamo, Italy

Introduction: Preserving right ventricle (RV) function is essential for the prognosis of children with hypoplastic left heart syndrome (HLHS). The aim of this study was to assess the feasibility of advanced echocardiographic techniques for routine evaluation of RV sizes and function, speculating the possible impact of the type of Norwood operation.

Methods: 19 children with HLHS treated with Norwood operation (7 with $\mathrm{mBT}$ shunt and 12 with RV-PA conduit; age 0,2-9,9 years) were prospectively recruited in this study. Ventricular volumes and ejection fraction (EF) were obtained by threedimensional echocardiography (3DE), with both semiautomatic border detection and discs summation methods. Global and regional, longitudinal and circumferential Strain and Strain Rate were determined using speckle tracking echocardiography (STE). Images were analyzed offline (Philips Q-lab 10.5). Intra and interobserver reliabilities were estimated using Intraclass Correlation Coefficient (ICC).

Results: Semiautomatic border detection method and discs summation method correlated strongly in defining ventricular volumes and EF. Ventricular volumes didn't differ between the two study groups; mean EF was $52,7 \pm 9,9 \%$ in $\mathrm{mBT}$ shunt group and $45,8 \pm 10,5 \%$ in RV-PA conduit group $(p=0,196)$, using semiautomatic border detection method, and $50,4 \pm 6,7 \%$ and $44,6 \pm 8,4 \% \quad(p=0,161)$ respectively, with discs summation method. Mean global longitudinal strain was $-22,1 \pm 4,2 \%$ in mBT shunt group and $-19,1 \pm 4,5 \%$ in RV-PA conduit group 
$(p=0,200)$, mean global circumferential strain was $-13,1 \pm 3,9 \%$ and $-10,3 \pm 3,0 \%$ respectively $(p=0,138)$. There were no statistical differences in global and regional myocardial deformation indices between the two study groups, except for circumferential Strain Rate in the inferior-lateral segment. ICCs were excellent for almost all 3DE and STE parameters.

Conclusions: 3DE and STE are highly feasible and reproducible for routine evaluation of right ventricular function in children with HLHS. To our knowledge, this is the first study that adopts both three-dimensional echocardiographic methods in this population, proving that they are interchangeable in measuring right ventricular sizes and EF. Further echocardiographic follow up is needed to clearly demonstrate if one variant of the Norwood operation is superior to the other one in our population.

\section{O4-4}

The Three-dimentional Morphometric Change Of Atrial Septum After The Device Closure Of Atrial Septal Defect Along The Growth

Momoki K. (1), Yasukochi S. (2), Takigiku K. (2), Takei K. (2) Saitama Children's Medical Center, Saitama, Japan (1); Nagano Children's Hospital, Nagano, Japan (2)

Introduction: Major risk factor for cardiac erosion after percutaneous device closure of secondary atrial septal defect (ASDII) by Amplatzer Septal Occluder (ASO) is a bald or remnant of aortic rim which was not uncommon in pediatric patients. The study aimed to clarify how each rim of ASDII develops along the sequential growth after implantation of device, using threedimensional echocardiography (3DE).

Methods: Sixty children with ASDII (age: 3-13 years), who underwent device closure by ASO from in January 2007 to December 2013, were enrolled to this study. The device size ranged $9-24 \mathrm{~mm}$ in diameter and $14 \mathrm{~mm}$ as a median) was used for ASO. The three-dimensional distance of each rim was analyzed and measured by off-line software of QLAB from full acquisition of $3 \mathrm{DE}$ data set of apical four-chamber view and its reconstructed data, obtained by iE33 with X7-2 and X5-1 probe (Philips MS). The distance between the edge of device and each rim was compared sequentially at P1 (1-3 days), at P2 (3-11 months), at P3 (1-4 years), and at $\mathrm{P} 4$ ( $>5$ years).

Results: All distance between each rim and the edge of device were significantly increased along the time interval (P1 to $\mathrm{P} 4)$ and the patient's growth; (1) tricuspid valve : 10.3 at $\mathrm{P} 1$ to $13.4 \mathrm{~mm}$ at $\mathrm{P} 4$, (2) mitral valve: 6.9 to $9.0 \mathrm{~mm}$, (3) posterior rim: 5.3 to $7.6 \mathrm{~mm}$, (4) superior vena cava: 14.2 to $17.0 \mathrm{~mm}$, (5) inferior vena

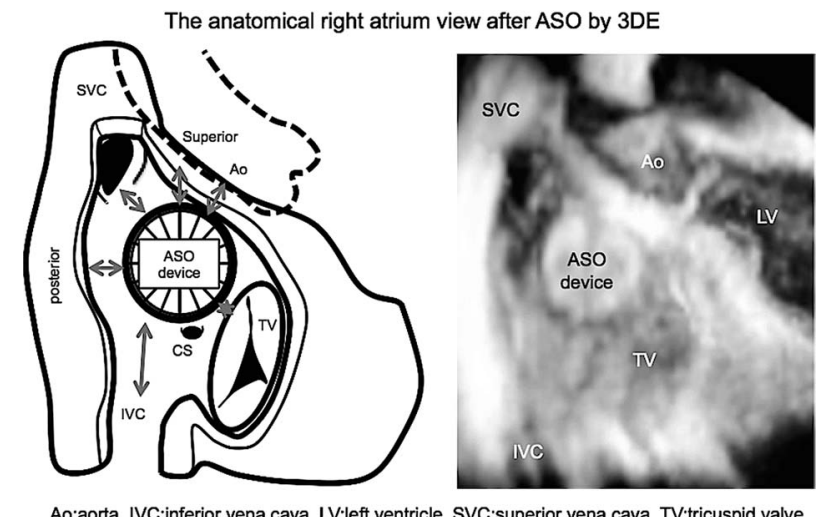

Figure. cava: 14.8 to $18.0 \mathrm{~mm},(6)$ superior rim ( to LA ceiling wall ): 7.9 to $11.2 \mathrm{~mm}$, (7) coronary sinus : 4.1 to $5.7 \mathrm{~mm}$, (7) aortic rim: 1.1 to $1.8 \mathrm{~mm}$. However, cases with absent or bald aortic rim demonstrated no growth of the distance to the edge of devices ( 0 to $0.3 \mathrm{~mm}$ ), regardless how the device edge contact to adjacent rim. Conclusions: The atrial septal rim can grow along patients' growth in all cases but those with absent or bald aortic rim who had a direct contact to device edge. This suggests a potential risk of erosion doesn't reduce along a time in this category of the patients.

\section{O4-5}

Aortic WSS distribution in TGA patients after arterial switch in rest and during dobutamine-stress

Korenhof A. (1), Van der Palen R.L.F. (1), Van den Boogaard P.J. (2). Blom N.A. (1), Lamb H.J. (2). Kroft L.J.M. (2), Westenberg J.J.M. (2), Roest A.A.W. (1)

Department of Pediatrics, division of Pediatric Cardiology, Leiden University Medical Center, Leiden, the Netherlands (1); Department of Radiology, Leiden University Medical Center, Leiden, the Netherlands (2)

Introduction: Aortic root dilatation is an important complication in patients with transposition of the great arteries (TGA) after arterial switch operation (ASO). The interaction between altered geometry (Figure 1A) after ASO and aortic hemodynamics in rest and stress state is unknown. 4D flow enables investigation blood flow and related hemodynamic parameters, including wall shear stress (WSS), known to be associated with vascular remodelling and aortic dilatation. The aim of this study was to assess WSS distribution along the thoracic aorta at rest and stress using 4D flow MRI.

Methods: Sixteen patients (mean age: $15.9 \pm 3.0$ years) after ASO for simple TGA underwent cardiac MRI including 4D flow at rest and during dobutamine-induced stress (dosage $7.5 \mathrm{ug} / \mathrm{kg} / \mathrm{min}$ ) and non-contrast enhanced 3D MR angiography (NCE-MRA) at rest on a Tesla 3.0 scanner (Philips Healthcare). Post-processing of 4D flow data was done with CAAS MR 4Dflow_v1.1 software
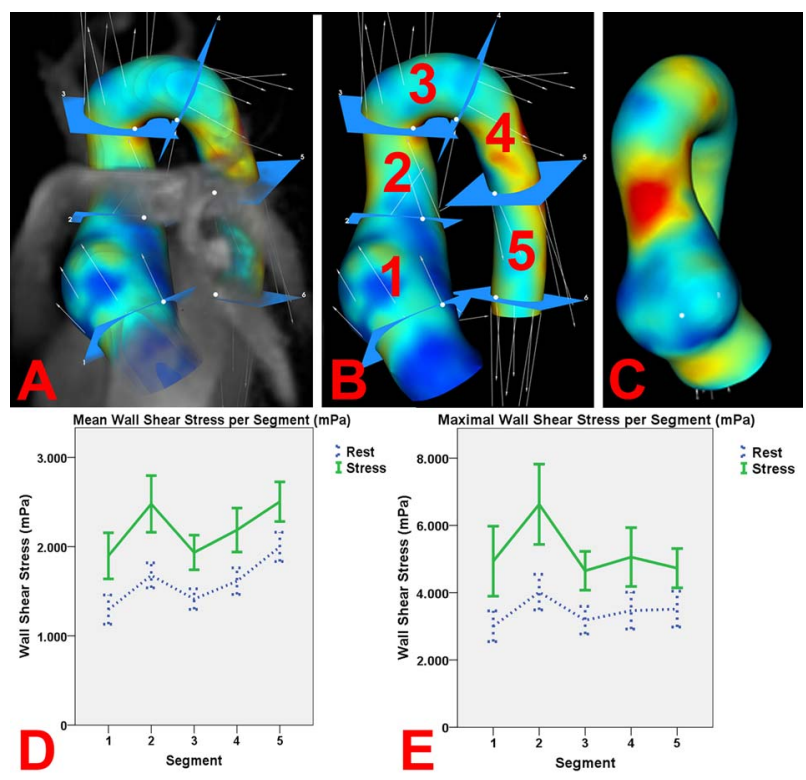

Figure 1.

A. Anatomy after Lecompte. B. Aorta segmentation C. WSSmax distribution along the thoracic aorta with WSS-hotspot in distal AAo. D. WSSmean per aortic segment at rest and dobutamine-stress. $\boldsymbol{E}$. WSSmax per aortic segment at rest and dobutamine-stress. 
(Pie Medical Imaging BV) (Figure 1B). Peak systolic phase was identified for 5 thoracic aortic segments along the cardiac cycle and peak systolic 3D aortic volume was formed and manually adapted where necessary. Mean WSS (WSSmean) and maximal WSS (WSSmax) were calculated for all segments. Furthermore, correlation between aortic diameter differences (ratios) at standardized landmarks and alterations in WSS ( $\Delta$ WSS) were investigated.

Results: A relatively large diameter difference was found between de dilated root and the mid-ascending aorta (AAo) (ratio root/ mid-AAo $=1.62 \pm 0.22$ ). Segmental WSSmean and WSSmax was lowest in the dilated proximal AAo and highest in the distal AAo (Figure 1C) and distal descending aorta in rest and during stress. WSS increased significantly during dobutamine-stress for all aortic segments (WSSmean:506-800 mPa, $\mathrm{P}<0.001$; WSSmax:1215$2610 \mathrm{mPa}, \mathrm{P}<0.000-0.002)$, the most for the distal AAo segment (Figure 1D \& 1E). Moderate correlation was found between $\Delta$ WSSmax in the distal AAo segment and the diameter difference between the root and mid-AAo (ratio) $(r=0.51, \mathrm{P}=0.044)$.

Conclusions: TGA-specific geometry related to the arterial switch operation, evidenced by neo-aortic root dilation and a sudden change in vessel diameter at mid-ascending aorta level, leads to specific wall shear stress distribution along the ascending aorta in TGA patients which aggravates during dobutamine stress.

O4-6

Peripheral venous pressure measurement can replace central venous pressure measurement in pre- and postFontan patients undergoing hemodynamic MRI evaluation Rahkonen O. (1), Ojala T. (1), Martelius L. (2), Happonen J-M. (1) Helsinki University Hospital, Department of Paediatric Cardiology (1) and Department of Radiology (2), Helsinki, Finland

Objectives: Cardiac catheterization has been used to evaluate patients for potential risk factors before and following a Fontan operation. However, catheterization has a low diagnostic yield of outcome in this patient population, and alternative diagnostic strategies have been suggested. Ideal imaging modality should be non-invasive and include high-resolution imaging, flow measurements, and pressure measurements.

Methods: Simultaneous peripheral venous pressure (PVP) and superior caval vein pressure (CVP) were measured in 32 pre- and post-Fontan patients undergoing cardiac catheterization. The measurements were compared using Bland-Altman method. Four patients who underwent both MRI and cardiac catheterization were included in the study. Anatomical and flow measurements in MRI and catheterization were compared. PVRI results derived from combined MRI and catheterization data were compared to the catheterization data alone. Predicted oxygen consumption and Fick principle were used for flow calculations at catheterization. Results: The median (range) PVP was $14 \mathrm{mmHg}(10-23 \mathrm{mmHg})$ and CVP $13 \mathrm{mmHg}(8-21 \mathrm{mmHg})$. Limits of agreement between these two methods were narrow (Figure).

In four patients who underwent combined MRI and catheterization study there was excellent correlation with anatomic measurements. QPi was overestimated in catheterization. In MRI CI and QPi were $3.3 \pm 1.01 / \mathrm{min} / \mathrm{m} 2$ and $2.0 \pm 1.21 / \mathrm{min} / \mathrm{m}^{2}$, whereas in catheterization CI and QPi were $5.0 \pm 2.51 / \mathrm{min} / \mathrm{m}^{2}$ and $2.7 \pm 0.41 / \mathrm{min} / \mathrm{m} 2$, respectively.

Overestimation of QPi was observed in patients with significant collateral flow resulting in underestimation of PVRI $\left(2.1 \pm 1.0 \mathrm{U}^{\star} \mathrm{m}^{2}\right.$ when calculated from catheterization data and $4.7 \pm 4.9 \mathrm{U}^{\star} \mathrm{m}^{2}$ when combined MRI and catheterization data were used). In patients with high collateral flow, overestimation of Qpi was observed if the MRI measurements were done from pulmonary veins rather than from pulmonary arteries.

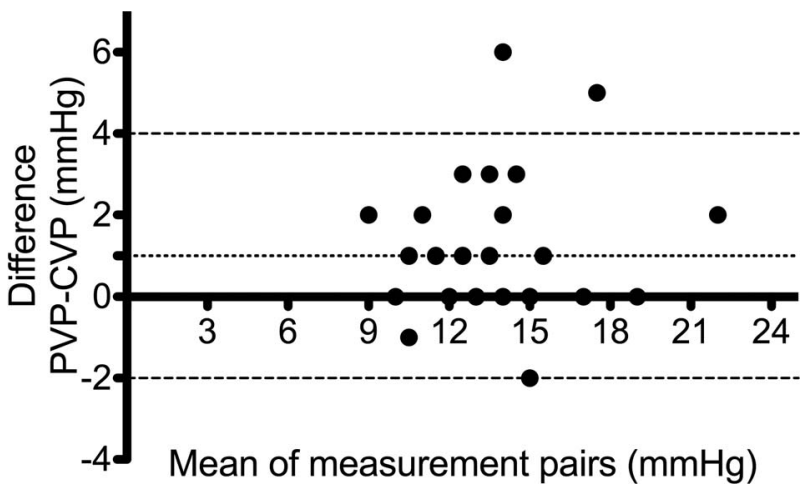

Figure.

Bland-Altman analysis of peripheral venous pressure (PVP) and central venous pressure. $X$-axis: mean of measurement pairs, $y$-axis: difference of PVP and CVP. Bias $1 \mathrm{mmHg}, \mathrm{SD}$ of bias $2 \mathrm{mmHg}$ (95\% limits of agreement from -2 to $4 \mathrm{mmHg}$ ).

Conclusions: 1) PVP can be used instead of CVP for screening of high CVP and PVRI. Use of PVP is non-invasive and can be done without general anesthesia. 2) In patients with significant collateral flow only combined MRI/catheterization may be used to estimate PVRI.

MRI combined with PVP is suitable for initial screening of preand post-Fontan patients. However, we believe that high-risk patients should be evaluated by using combined MRI and catheterization data.

\section{O5-1}

Patient-specific shape modeling to predict response to pulmonary valve replacement in patients with repaired Tetralogy of Fallot

Full P. M. (1), Engelhardt S. (2), Burkhardt B. (1), Tandon A. (1), Velasco Forte M. N. (3), Greil G. F. (1), Wolf I. (4), Lamata P. (5), Hussain T. (1)

Dept. of Pediatrics, UT Southwestern Medical Center, Dallas, US (1); Department of Simulation and Graphics, Otto-von-Guericke-University Magdeburg, Magdeburg, Germany (2); Queen Elizabeth Hospital/King's College London, London, UK (3); Institute for Medical Informatics, Department of Computer Science, Mannheim University of Applied Sciences, Mannheim, Germany (4); School of Biomedical Engineering and Imaging Sciences, King's College of London, London, UK (5)

Introduction: Patients with repaired Tetralogy of Fallot (rTOF) may suffer from pulmonary valve regurgitation and pulmonary valve replacement (PVR) is considered in these patients. We present parameters from statistical shape model (SSM) analysis for the right ventricle $(\mathrm{RV})$ to predict their response to PVR.

Methods: Short axis cine images of $10 \mathrm{rTOF}$ cases before and after PVR, and 10 healthy controls were analyzed. Manual segmentations of the RV at end diastole (ED) were performed for each patient. Three dimensional RV anatomies were automatically reconstructed from the segmentations.

SSM, also referred to as atlas, yield arbitrary many, independent modes of variation of shape by applying principal component analysis (PCA). We considered the first 3 modes covering most variance only. Adverse shape changes in rTOF patients were investigated compared to control cases. If the overall atlas (normal and rTOF cases) showed a shape difference exists between responders (RVEDVolume Index $\left(\mathrm{V}_{\mathrm{i}}\right)=$ after PVR within normal range) and nonresponders, another atlas specifically delineating shape differences within the rTOF group would be created. 


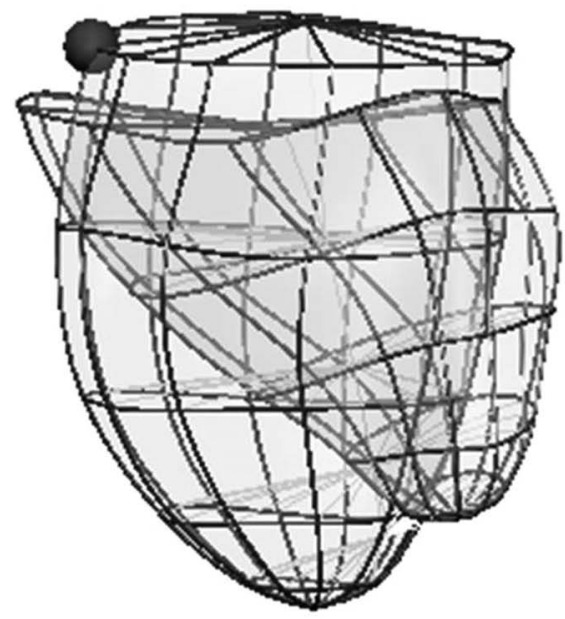

Fig.1a: Normal RV vs rTOF RV

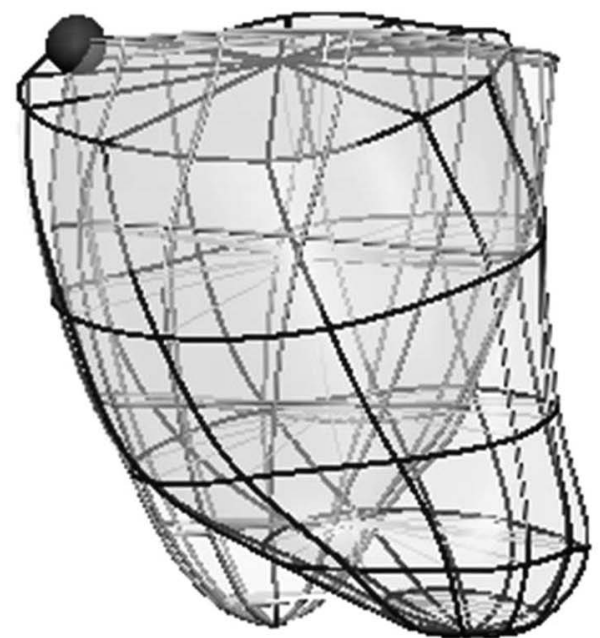

Fig.1b: Responders RV vs non-responders RV

Figure 1.

Two pairs of overlaid meshes are shown. Each pair comprises of two $R V$ meshes and represents the change in shape for the most significant $R V P C A$ mode to distinguish between classes of interest. The observer looks from the $L V$ through the septum on the $R V$. The grey sphere represents the RVOT. All meshes are based on the shape of the $R V$ before PVR.

Fig1a: normal hearts tend towards the gray mesh, pre PVR rTOFs tend towards the black mesh.

Fig1b: pre PVR rTOF with positive response (gray) and no response (black)

Results: The atlas built with pre-PVR rTOF (RVEDV $\left.154.0(+/-22.3) \mathrm{ml} / \mathrm{m}^{2}\right)$ and normal cases $\left(\mathrm{RVEDV}_{\mathrm{i}}=90.7\right.$ (23.3) $\mathrm{ml} / \mathrm{m}^{2}$ ) showed a significant change in size of RV (see Fig.1a, p-value $<0.001$ ), corroborated by $\operatorname{RVEDV}_{\mathrm{i}}$ (p-value $<$ 0.001). Using the same atlas but splitting rTOF cases into responders $\left(\operatorname{RVEDV}_{\mathrm{i}}=148.6(+/-18.6) \mathrm{ml} / \mathrm{m}^{2}\right)$ and nonresponders $\left(\mathrm{RVEDV}_{\mathrm{i}}=159.4(+/-26.6) \mathrm{ml} / \mathrm{m}^{2}\right)$, we observe a significant difference in PCA mode 3 (p-vaule $=0.017$ ), suggesting a novel shape difference exists, although there was no difference in $\mathrm{RVEDV}_{\mathrm{i}}$ between these groups $(\mathrm{p}$-value $=0.477)$. Thus this shape difference adds complementary information to the $\mathrm{RVEDV}_{\mathrm{i}}$ to predict the response to PVR in rTOF. The second atlas using rTOF cases only confirms a significant difference in RV shape $(p$-value $=0.015)$ between responders and non-responders which is manifested by a shifted position of the apex with respect to the base of the heart (see Fig.1b).

Conclusions: In our cohort, the position of the apex relative to the base of the heart is a predictive feature for response to PVR in rTOF. Future work may confirm this and yield shape-dependent biomarkers for clinical decision-making.

\section{O5-2}

Successful Development of a Novel and Rapid Inkjet 3D Printing System for Manufacturing Super Flexible Heart Replicas of Congenital Heart Disease -for Patient Specific Simulation Surgery

Shiraishi I. (1), Kurosaki K. (1), Kanzaki S. (1), Hatanaka K. (2), Hoashi T. (1), Ichikawa I. (1)

National Cerebral and Cardiovascular Center (1), Osaka, Japan; crossEffect Inc. (2), Kyoto, Japan

Introduction: 3D printing technology is now applied for education and training in various clinical fields. To realize rehearsal of surgery of congenital heart disease, we have shown a unique technology that manufactures super flexible polyurethane heart replicas by means of stereolithography followed by vacuum casting. To improve limitations in time and cost of this technique, we here developed a novel and rapid 3D inkjet printing system in collaboration with Japanese chemical and mechanical companies.

Methods: The new machine consisted of 4 inkjet heads with $200 \times 500 \mathrm{~mm}$, which was enough in size for reproducing adult heart and thoracic aorta. Inkjet materials with similar texture to the human heart were also developed. The lamination thickness of the printer was 30 micrometers. We manufactured several different types of congenital heart diseases including ASD, VSD, TOF, DORV, and congenitally corrected TGA. The final products were inspected with industrial CT, where 3D data were compared with the original ones. Results: After inkjet/supporting materials and printing conditions have been carefully optimized, super flexible replicas of congenital heart disease were manufactured with precise external surface and detailed internal structures. The replicas exhibited faithful reproduction in size and shape and similar texture to the real heart, allowing surgeons rehearsal of surgery by cutting and suturing. Whole process takes approximately 48 hours including image processing, 3D printing, and removal of the supporting materials. Conclusions: We have successfully developed a new 3D inkjet printing system based on the industry-academia collaboration. Further improvements in ink materials and printing conditions are necessary to realize more precise surgical simulation.
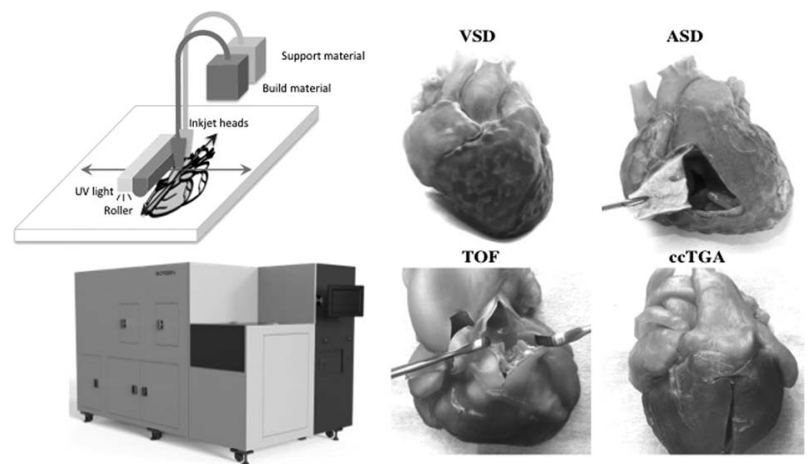

Figure. 


\section{O5-3}

Can we predict the unfavorable coronary features in patients with transposition of the great arteries long-term after an arterial switch operation?

Michalak K.W. (1), Moll M. (2), Sobczak - Budlewska K. (1), Moll J.A. (1), Szymczyk K. (3), Dryżek P. (1), Niwald M. (1), Moll J.J. (2) Department of Cardiology, Polish Mother's Memorial Hospital, Lodz, Poland (1). Department of Cardiac Surgery, Polish Mother's Memorial Hospital, Lodz, Poland (2). Department of Diagnostic Imaging, Medical University, Lodz, Poland (3)

Introduction: Coronary artery complications are the main reason for early mortality after arterial switch operation (ASO), late complications are rather rare and there is no consensus regarding the need and indications for the routine coronary artery evaluation during follow-up, as well as the modality which should be chosen as a first line assessment.

The aim of this study was to present the frequency of coronary abnormalities in asymptomatic patients with transposition of the great arteries (TGA) after an ASO discovered in coronary computed tomography angiography (CCTA) and their potential "red flags" in other examinations.

Patients and methods: Among 750 patients with TGA who had ASO between years 1991-2016, the initial group of 50 consecutive asymptomatic patients, who had routine coronary artery evaluation over 17 years after ASO according to our institutional protocol, were qualified for this study. Additionally all these patients had echocardiographic examination, electrocardiography (ECG), Holter ECG and cardiopulmonary exercise test (CPET), those who had significant abnormalities were also qualified for perfusion scintigraphy or stress MRI.

Results: The frequency of coronary anomalies in this series was $28 \%$. In 30 patients $(60 \%)$ unfavorable coronary features were detected: ostial stenosis (3), muscular bridge (8), coronary fistula (1), interarterial course (4), proximal kinking (13), high ellipticity index (5), proximal acute angulation LCA (<30 degree) of LCA (19), proximal acute angulation of RCA (7). The presence of this features was not correlated significantly with coronary anomalies, surgical technique, associated heart defects or neoaortic root diameters. The presence of acute angulation of coronary arteries was statistically significant correlated with its inter-arterial course $(p=0.037)$; high ellipticity index $(p=0.025)$ and proximal kinking course $(p=0.002)$.

The abnormal CCTA result could not be predicted by reviewing surgical report, ECG, Holter ECG and CPET. The results of perfusion scintigraphy also didn't reveal any significant abnormal findings in those patients who had discovered abnormalities in routine CCTA.

Conclusion: The complex coronary setups with unfavorable, highrisk features are common In patients with TGA after an arterial switch operation. The selection of high risk patients cannot be made indirectly and CCTA provides accurate and useful information for the postoperative management.

\section{O5-4}

The new IQoDS score for fluoroscopy imaging revealed impaired visibility for stents and devices with lower metal portion after Allura Clarity update

Mueller G.C. (1), Stute F. (1), Waschkewitz J. (2), Biermann D. (3), Schoen G. (4), Frenzel T. (5), Kozlik-Feldmann R. (1)

University Heart Center Hamburg, Department of Pediatric Cardiology, Hamburg, Germany (1); University Medical Center HamburgEppendorf, Department for Radiotherapy and Radiation Oncology, Hamburg, Germany (2); University Heart Center Hamburg, Department of Pediatric Cardiac Surgery, Hamburg, Germany (3); University Medical
Center Hamburg-Eppendorf, Institute of Medical Biometry and Epidemiology, Hamburg, Germany (4); University Medical Center Hamburg-Eppendorf, Ambulatory Center, Department of Radiation Oncology, Hamburg, Germany (5)

Introduction: Concerns over the effect of $\mathrm{x}$-ray radiation on patient and staff during cardiac interventions for congenital heart disease have increased in recent years. A wide range of devices and stents made of different materials are available. The study aims at stent and device visibilities after Allura Clarity update under recommendation of the assessability of implants functional safety.

Methods: To evaluate image quality a new score (Image Quality of Devices and Stents) based on device or stent visibility (visualization of endings, wire configuration, integrity and possible fractures) as well as visibility of leading structures like bones, airways and heart shape was developed. Visibility was categorized in four quality classes from clearly identifiable, to blurredly, unsure and not identifiable (score range:7-28points). For analysis of implants a subanalysis of device and stents visibility was performed (subscore range 4-16points). All pediatric patients receiving stents or devices from 2014 to 2016 were included and a representative number of 1119 fluoroscopy series were analyzed before (Allura Xper) and 1038 after the upgrade (Allura Clarity). Implants with higher and lower metal portion were separately evaluated. Diagnoses showed a broad spectrum of congenital heart defects. Patient groups were comparable concerning interventions, investigators, patient's age and weight.

Results: Overall image quality measured by IQoDS score was comparable before and after upgrade to Allura Clarity (IQoDs score: 18.11 vs $17.87 ; \mathrm{p}=0.64)$, whereas visibility of stents and devices with lower metal portion revealed significant lower scores after Allura Clarity update (8.74 vs $7.79 ; p=0.012$ ). Imaging of implants with higher metal portion was comparable before and after Allura Clarity update.

Conclusion: Allura Clarity update is without impact on overall image quality. Nevertheless visibility of devices and stents with lower metal portion becomes more difficult.

\section{O5-5}

Initial data of international study of early vascular aging in children with coarctation of aorta

Sendzikaite S. (1,2), Sudikiene R. (2), Litwin M. (3), Rybak A. (4), Obrycki L. (3), Jankauskiene A. (1)

Paediatric Centre, Institute of Clinical medicine, Vilnius University, Lithuania (1); Centre of Heart and Chest surgery, Institute of Clinical medicine, Vilnius University, Lithuania (2); Department of Nephrology and Arterial hypertension, The Children's Memorial Health Institute, Warsaw, Poland (3); Department of Cardiology, The Children's Memorial Health Institute, Warsaw, Poland (4)

Introduction: Despite the improved survival continued morbidity related to hypertension persists in $42-70 \%$ of patients after repair of coarctation of aorta $(\mathrm{CoA})$. According to the recent literature CoA is classified as one of the highest cardiovascular risk condition. However data of early vascular aging development in this population remains scarce.

Methods: 48 patients ( $64.6 \%$ boys) aged $12.7 \pm 3.7$ years (from 6 to 18 years old) and $4.7 \pm 4.2$ years after $\mathrm{CoA}$ repair were included into the study. 24 hour ABPM, non-invasive oscillometric central blood pressure (CBP) measurement, left ventricular mass index (LVMi), carotid (cIMT) and femoral intima-media thickness (fIMT) were assessed in all patients. Endothelial function was gauged by right brachial flow mediated vasodilatation (FMD), with value less than $10 \%$ considered abnormal. 
Results: Arterial hypertension (AH) was diagnosed in 35 patients (72.9\%). 28 (58.3\%) patients had a previous diagnosis of AH and received treatment with one, two or three antihypertensive agents, however all of them were still hypertensive on follow-up based on the 24 hour ABPM criteria. 7 patients (14.5\%) were newly diagnosed with $\mathrm{AH}$ according to 24 hour ABPM results. Out of all hypertensive patients systolic central BP above 95'th percentile was observed in 22 patients. Left ventricular hypertrophy was diagnosed in 22 patients (46.0\%). Average right cIMT was $0.50 \mathrm{~mm}$, CI $(0.48 ; 0.52)$, while mean of normative cIMT values was $0.45 \mathrm{~mm}$. Right cIMT SDS was $2.53 \pm 1.3$. Average right flMT was $0.27 \mathrm{~mm}$, CI $(0.26 ; 0.29)$, while average of normative fIMT values was $0.32 \mathrm{~mm}$, fIMT SDS $-1.6 \pm 1.48$. Right brachial artery FMD value was $6.3 \% \pm 6.0$ and 31 patients $(64.6 \%)$ had FMD less than $10.0 \%$.

Conclusions: Our results indicate high prevalence of non properly controlled $\mathrm{AH}$ in children after correction of CoA in spite of antihypertensive therapy with more than one antihypertensive agent. Children after CoA repair present also signs of subclinical arterial injury and disturbed endothelial function. Lower fIMT in femoral arteries might suggest disturbed pattern of blood flow in aorta despite CoA repair.

\section{O5-6}

Three-Dimensional Image Fusion to Facilitate Guidance of Complex Cardiac Catheterizations in Congenital Heart Defects - Results from an International Multi-center Registry

Goreczny S. (1), Schubert S. (2), Dryzek P. (1), Kim S. (3), Sandoval J.P. (4), Morgan G.J. (5), von der Wettern J.M. (2), Moszura T. (1) Department of Cardiology, Polish Mother's Memorial Hospital, Research Institute, Lodz, Poland (1); Department of Congenital Heart Disease/ Pediatric Cardiology, Deutsches Herzzentrum Berlin, Germany (2); Department of Pediatrics, Sejong General Hospital, South Korea (3); Department of Pediatric Cardiology / Congenital Heart Disease, Ignacio Chavez National Institute of Cardiology, Mexico City, Mexico (4); Department of Cardiology, Colorado Children's Hospital \& Department of Adult Congenital Cardiology, University of Colorado Hospital, USA (5)

Introduction: Developments in fusion imaging software have facilitated the use of three-dimensional (3D) roadmaps based on preregistered Computed Tomography (CT) or Magnetic Resonance Imaging (MRI) datasets for live guidance of trans-catheter interventions. VesselNavigator (Philips Healthcare) allows direct fusion of fluoroscopic two-dimensional (2D) images with CT or MRI derived 3D roadmaps: 2D-3D registration.

Methods: International multi-centre, prospective study to evaluate fusion of CT and MRI datasets for guidance of cardiac catheterizations in congenital heart disease. Consecutive patients with pre-existing cross sectional imaging deemed clinically suitable by the operator for VesselNavigator assisted cardiac catheterization were included in the study.

Results: From 10/2016 until 11/2017, VesselNavigator was applied in a total of 141 cardiac catheterizations: in 126 patients for interventional guidance (89\%) and in 15 for procedural planning $(11 \%)$. In one hundred patients $(71 \%)$, the fusion imaging was derived from contrast CT scans and the remaining 41 patients (29\%) underwent MRI. Ninety-six interventions were performed in 92 patients with 34 patients having diagnostic catheterization. Stenting of right ventricular outflow tract $(n=24)$, aortic coarctation $(n=22)$ or pulmonary artery $(n=17)$ was the most common intervention followed by balloon dilation of aortic coarctation $(n=11)$ or pulmonary artery $(n=10)$, collateral closure $(n=5)$ and other $(n=7)$. Successful $2 D-3 D$ registration was achieved on $96 \%$ of occasions $(n=121)$. In the remaining 5 patients 3D-3D registration was used. Internal markers were used for registration in 102 patients (80\%) and angiography was used in 25 patients $(20 \%)$. In 42 patients $(33 \%)$ catheterization was performed solely with 3D guidance with the remaining 85 patients $(67 \%)$ requiring additional angiography. Overall, VesselNavigator guidance was judged useful or very useful in 107 patients (85\%) and essential in 17 patients (13\%). In three patients (2\%) VesselNavigator guidance was not deemed useful.

Conclusions: Fusion of 2D fluoroscopic images with CT or MRI datasets is safe and effective method of guidance of cardiac catheterizations in various congenital heart defects. In selected patients 3D guidance allows percutaneous treatment without initial contrast application.

\section{O6-1 \\ Ion channel TRPM4 modulates RV remodeling under right ventricular pressure load in rat \\ Uhl S. (1,2), Frede W. (1,2), Medert R. (2), Freichel M. (2), \\ Gorenflo M. (1) \\ Department Pediatric and Congenital Cardiology, University Medical \\ Center, Heidelberg, Germany (1); Institute of Pharmacology, University \\ Medical Center, Heidelberg, Germany (2)}

Objectives: The survival of children with congenital heart defects including increased right ventricular pressure load (i.e. Tetralogy of Fallot, TOF) or pulmonary arterial hypertension (PAH) is dependent on the function of the right ventricle (RV). RV remodeling under pressure load has several adaptive and maladaptive effects with progressive transition from compensated status to heart failure. Transient receptor potential melastatin 4 (TRPM4) forms calcium-activated nonselective cation channels expressed in myocardium of human, rat and mouse, which was shown to modulate left ventricular hypertrophy in mice. Aim of this study was to identify the cardiac role of TRPM4 regarding right ventricle in healthy rats and under Monocrotaline (MCT)induced pressure load.

Methods: We performed experiments with untreated adult rats and under MCT-induced pressure load. RV function was characterized by echocardiographic measurements and contractility measurements of isolated papillary muscles. Hypertrophy was investigated by echocardiography and by organ weight measurements. Expression of TRPM4 in human and rat RV was detected by western blot. Brain natriuretic peptide (BNP) level was evaluated in rat by enzyme-linked immune assay.

Results: TRPM4 was detected in RV of a child with TOF and in rat. In healthy rats, no difference was seen between wild type (WT) and TRPM4-deficient (TRPM4-/-) rats concerning cardiac function or RV diameter. In rats with MCT-induced RV pressure load (42 days after MCT-injection) TRPM4 expression was reduced by $67 \%$ (TRPM4/Calnexin, p 0.002). Both genotypes showed increased RV hypertrophy and subsequently improved basal contractility in isolated papillary muscles $([\mathrm{mN} \pm \mathrm{SEM}]: \mathrm{WT}$ untreated $10.1 \pm 0.3$, treated $13.8 \pm 1.0$, p0.04; TRPM4 $-/-$ untreated $10.0 \pm 0.5$, treated $16.2 \pm 1.4$, $\mathrm{p}<0.001)$, but TRPM $4-/-$ rats showed significantly increased hypertrophy in comparison to WT (echocardiography: RVAWd $[\mathrm{mm}]$ TRPM4 $-/-0.89 \pm 0.06$, WT $0.63 \pm 0.05$, p0.002; RV weight/ tibia length $[\mathrm{mg} / \mathrm{mm}]:$ TRPM $4-/-11.6 \pm 0.5$; WT $8.6 \pm 0.7, \mathrm{p}<0.001$ ). Cardiac contractility (echocardiography RVFAC [\%]: TRPM4 -/- 33 \pm 2 , WT $37 \pm 1$; papillary muscle contractility after application of $100 \mu \mathrm{m}$ Isoprenaline [mN]: WT $19.3 \pm 1.5$, TRPM $4-/-20.3 \pm 1.9)$ as well as survival rate and BNP level were comparable in TRPM4 $-/-$ and WT rats. 
Conclusion: TRPM4 modulates right ventricular pressure-loadmediated hypertrophy in rats representing a potential candidate for pharmacological approach of right ventricular remodeling.

O6-2

Symptomatic hypertrophic cardiomyopathy in 2 infants with Noonan-syndrome and RIT1-mutation: Successful treatment with the MEK inhibitor trametinib

Hofbeck M. (1), Marquis C. (2), Raboisson M.J. (3), Karalis A. (4), Waldmüller S. (5), Wiegand G. (1), Gelb B.D. (6), Zenker M. (7), Delrue M.A. (4), Andelfinger G. (8)

Department of Pediatric Cardiology, Pulmology and Paediatric Intensive Care Medicine, University Children's Hospital Tübingen, Germany (1); Department of Pharmacy, CHU Sainte Justine, Department of Pediatrics, Université de Montréal, Montréal, QC, Canada (2); Service of Cardiology, CHU Sainte Justine, Department of Pediatrics, Université de Montréal, Montréal, QC, Canada (3); Service of Genetics, CHU Sainte Justine, Department of Pediatrics, Université de Montréal, Montréal, QC, Canada (4); Institute of Medical Genetics, University Hospital of Tübingen, Germany (5); Mindich Child Health and Development Institute and Departments of Pediatrics and Genetics and Genomics Sciences, Icahn School of Medicine at Mount Sinai, New York, NY (6); Institute of Human Genetics, University Hospital Magdeburg, Germany (7); Cardiovascular Genetics, CHU Sainte Justine, Department of Pediatrics, Université de Montréal, Montréal, QC, Canada (8)

Introduction: Noonan syndrome (NS) belongs to the spectrum of RASopathies caused by germline mutations of genes involving the RAS/MAPK pathway. NS is frequently associated with cardiovascular involvement including hypertrophic cardiomyopathy (HCM) in $20 \%$ of the patients; some mutations are associated with a higher risk of HCM, such as RIT1 mutations. Symptomatic HCM in infants with NS carries a poor prognosis (31\% first year survival). So far therapeutic options are limited. We report successful treatment of severely symptomatic infants with trametinib, a MAPK-pathway inhibitor.

Patients: Pregnancy in patient 1 was complicated by polyhydramnios requiring repeat amnioreduction procedures. Following delivery at 36 weeks the girl required mechanical ventilation due to respiratory insufficiency. Genetic testing confirmed the diagnosis of NS with RIT1 F82L de novo mutation. Echocardiography showed biventricular HCM and pulmonary stenosis. HCM progressed with increasing subvalvular obstruction despite treatment with propranolol up to $10 \mathrm{mg} / \mathrm{kg} /$ day. Clinical deterioration at the age of 3 months required resuscitation, mechanical ventilation and chest tubes for bilateral chylothoraces. In patient 2 fetal echocardiogaphy showed mild hydramnios, cardiac valve dysplasia and hypertrophic cardiomyopathy. Neonatal echocardiography confirmed prenatal findings. Genetic testing revealed NS with de novo RIT1 S35T mutation. Despite propranolol treatment up to $10 \mathrm{mg} / \mathrm{kg} /$ day the patient showed rapid aggravation of HCM with significant increase of intracavitary gradients.

Due to the dismal prognosis and in the absence of further alternatives we opted for treatment with a MEK inhibitor. Following discussion with our clinical ethics boards and informed parental consent we started trametinib with $0.025 \mathrm{mg} / \mathrm{kg} / \mathrm{d}$ at the age of 13 and 14 weeks respectively. Treatment resulted in dramatic clinical and cardiac improvement in both patients with regression of hypertrophy and intraventricular gradients. Side effects were limited to transitory acneiform skin rash in patient 1 and accentuation of physiological alopecia in patient 2. Clinical improvement persists in both patients at the age of 12 months.

Conclusions: Treatment with trametinib in 2 infants with RIT1associated severe progressive hypertrophic cardiomyopathy resulted in dramatic cardiac and clinical improvement persisting to the end of the first year of life. According to this experience MEKinhibition might represent a completely new treatment option for these patients.

\section{O6-3}

Analysis of Notch pathway activity in cardiac progenitor cells of the patients with congenital heart defects Kozyrev I.A., Ignatieva E.V., Golovkin A.S., Pervunina T.M., Grekhov E.V., Gordeev M.L., Vasichkina E.S., Kostareva A.A., Malashicheva A.B.

Almazov National Medical Research Centre, St. Petersburg, Russia

Introduction: Recently fine-tuned sequential activation of Notch genes has been shown to be responsible for the proper heart chamber development. In addition, mutations in several genes of the Notch pathway have been shown to be associated with Tetralogy of Fallot (TOF) and hypoplastic left heart syndrome (HLHS). To elucidate more precisely the role of Notch pathway in these congenital defects we aimed to estimate expression of Notch signaling related genes in cardiac progenitor cells (CPC) in the patients with TOF, HLHS and ventricular septal defect (VSD).

Methods: Cardiac progenitor cells were isolated from leftovers after surgical intervention (VSD - 17, HLHS - 10, TOF- 40 patients) by collagenase digestion and were cultured for several passages in vitro. The cells were immunophenotyped by flow cytometry using CD31, CD146, CD117, CD34, CD90, CD166. Gene expression of Notch family genes (NOTCH1, NOTCH2, NOTCH3, NOTCH4, HEY1, HEY2, JAG1, DLL4, BMP2, BMP4) was assessed by qPCR. Notch receptors and ligands surface expression were also estimated by flow cytometry.

Results: In cardiac progenitor cells derived from patients with hypoplastic left heart syndrome, NOTCH4 and DLL4, as well as BMP4, was significantly lower than in cells from patients with a ventricular septal defect. In the cells from patients with tetralogy of Fallot, the expression of Notch family genes had large variability ranging from low to high expression level by both flow cytometry and qPCR.

Conclusion: Our data on cardiac progenitor cells derived from VSD, HLHS and TOF patients suggest direct involvement of Notch pathway dysregulation in the pathogenesis of the HLHS. Regarding tetralogy of Fallot, the role of Notch pathway remains questionable and requires further research.

\section{O6-4 \\ Next-Generation Sequencing with a 482 Gene Panel on 208 Cases of Congenital Heart Defects, Heterotaxy, Primary Ciliary Dyskinesia and Aortopathies \\ Bouvagnet P. (1,9), Simonet T. (2), Lambert L. (3), Morice Picard F. (4), Scheidecker S. (5), Willemin M.C. (6), Alice Maltret A. (7), Reix P. (8), Bonnet D. (7), Perouse de Monclot T. (9) \\ Laboratory of Cardiogenetics, Hospices Civils de Lyon, Lyon, France (1); CBC, Hospices Civils de Lyon, Lyon, France (2); Service de Génétique, CHU Nancy, Nancy, France (3); Service de Génétique, CHU \\ Bordeaux, Bordeaux, France (4), Service de Génétique, CHU Strasbourg, Strasbourg, France (5); Service de Pneumologie, CHRU Lille, Lille, France (6); Service de Cardiologie, Hôpital Necker-Enfants Malades, Paris, France (7); Service de Pneumologie, Hôpital Femme- Mère-Enfant, Hospices Civils de Lyon, Lyon, France (8); Service de Cardiologie Pédiatrique, Hospices Civils de Lyon, Lyon, France (9)}

Introduction: We set up a panel of 482 genes that had been involved through published studies in congenital heart defects, heterotaxy, 
primary ciliary dyskinesia (PCD) and aortopathies because these pathologies are largely overlapping. A series of 208 cases falling into one or several of the selected pathological groups were sequenced by Next-Generation Sequencing.

Method: DNA was enriched by hybridization on a customized set of probes (Nimblegen, Roche). Eight DNA samples by run were paired-end sequenced on a NextSeq500 (Illumina) with a mid-size flow cell. Sequences were analyzed through a pipeline designed locally. CNV were detected with DeCovA. Putative causative variants were confirmed by Sanger sequencing or qPCR and the segregation of selected variants was performed on family members. Results: the causal mutation was found in $71 \%$ of PCD patients, in $21 \%$ of aortopathies, $47 \%$ of heterotaxy patients (and 30\% of heterotaxy patients without PCD), in 39\% of congenital heart defects patients and in $23 \%$ of fetuses with complex cardiac malformations. Overall, the causal mutation was found in $44 \%$ of the cohort.

Conclusion: a large panel of genes is very efficient to find the causal mutation in nearly a half of the cohort of 208 cases. These mutations fulfilled stringent criteria for pathogenicity and a positive familial segregation. These data were sufficiently strong to provide a genetic counseling including in fetus cases.

\section{O6-5 \\ Novel disease genes for childhood-onset cardiomyopathy: TMOD1 and NRAP}

Vasilescu C. (1), Ojala T.H. (2), Hinterding H. (1), Brilhante V. (1), Ojanen S. (1), Lohi J. (3), Carroll C.J. (1), Suomalainen A. (1,4,5) Research Programs Unit, Molecular Neurology, Biomedicum-Helsinki, University of Helsinki, Helsinki, Finland (1). Department of Paediatric Cardiology, Helsinki University Hospital and University of Helsinki, Helsinki, Finland (2). Department of Pathology, Helsinki University Hospital and University of Helsinki, Helsinki, Finland (3). Department of Neurology, Helsinki University Hospital and Clinical Neurosciences, University of Helsinki, Helsinki, Finland (4). Neuroscience Center, University of Helsinki, Helsinki, Finland (5)

Introduction: The discovery of molecular underpinnings of childhood cardiomyopathies raises expectations for new, more tailored forms of treatment. Only 35\% of the children suspected of genetic cardiomyopathy receive a molecular diagnosis with current genetic approaches, suggesting that many new disease genes are yet to be discovered. From the single center in Finland performing cardiac transplants, we have collected the KidCMP cohort of 74 paediatric patients, cohort that offers unique opportunities to discover novel disease genes for severe childhood cardiomyopathies.

Methods: The patients' DNA underwent whole-exome sequencing. The subsequent screening of family members involved Sanger sequencing. The patient's heart and/or skeletal muscle biopsies enabled the evaluation of functional consequences of mutations at mRNA and/or protein level. Similar experiments were performed in myotubes differentiated from patient myoblasts, supporting the findings from tissue samples. Moreover, we used molecular modeling with Discovery Studio 4.5 to assess the possible functional implications of the TMOD1 variant.

Results: We uncovered the homozygous missense variant $\mathrm{p}$. R189W in TMOD1 (Tropomodulin-1) as the genetic cause of a teenage-onset dilated cardiomyopathy with short episodes of ventricular tachycardia. Ventricular tachycardia responded to betablocker treatment, and cardiac function stabilized to the level of mild dysfunction. TMOD1 localizes to the pointed end of actin filaments, regulating their dynamics and stability. In the three dimensional structure of TMOD1, the patient's variant determines the exposure of a hydrophobic amino acid at the protein surface. This is the first report of TMOD1 dysfunction causing human disease.

Exome analysis of a second patient, presenting with dilated cardiomyopathy at 3.5 years of age, led to the identification of the homozygous nonsense variant p.Y448* in NRAP (Nebulinrelated-anchoring protein). NRAP anchors the terminal actin filaments to the membrane. Our results showed that the patient's NRAP mRNA is present in cells, escaping at least partially the nonsense-mediated decay. Recently, another homozygous nonsense variant in NRAP has been described in adult-onset dilated cardiomyopathy with incomplete penetrance.

Conclusions: Whole-exome sequencing enabled the identification of two novel disease genes in childhood dilated cardiomyopathy: TMOD1 and NRAP. The molecular diagnoses facilitate the causative understanding of the diseases, crucial for future development of individualized treatment choices.

\section{O6-6 \\ Unraveling the causes of arrhythmias in very-long chain acyl-CoA (VLCAD) deficiency through the combined use of non-targeted lipidomic and molecular analyses in a mouse model}

Lefort B. (1,2), Gélinas R. (1), Forest A. (1), Bouchard B. (1),

Daneault C. (1), Robillard-Frayne I. (1), Ruiz M. (1), Des Rosiers C. (1) Institut de Cardiologie de Montréal, Montréal, Canada (1); Unité de cardiologie pédiatrique et congénitale adulte, CHU Tours, Tours, France (2)

Introduction: VLCAD catalyses the first reaction of mitochondrial fatty acids (FA) $\beta$-oxidation. Inherited VLCAD deficiency predisposes to severe neonatal arrhythmias whose pathophysiology is not fully understood. We hypothesized that VLCAD deficiency disrupts cardiac lipid homeostasis, which could contribute to arrhythmia.

Methods: Our study model is the VLCAD null mouse (VLCAD-/-), which is known to develop a prolonged QT interval and ventricular arrhythmias. Mice were fed a chow or high fat (HF) diet, the latter being known to worsen the cardiac phenotype in VLCAD deficiency. Non-targeted lipidomic profiling of heart tissues was achieved using liquid chromatography-mass spectrometry (LC-QTOF). The expression of genes or proteins involved in polyunsaturated FA synthesis, phospholipid (PL) remodeling, endoplasmic reticulum (ER) stress and calcium handling were assessed using RT-qPCR or Western blotting.

Results: Compared to their littermate counterparts, VLCAD-/mouse hearts displayed alterations in the FA composition of PL characterized by an increase in arachidonic acid and a decrease in docosahexaenoic acid, a profile that was worsened with the HF diet. These changes in PL composition were associated with dietdependent increased expression of gene or protein markers for (1) PL remodeling, especially the calcium-dependent phospholipase A2 and the lysophosphatidylcholine-acyltransferase 2, (2) ER stress, namely CHOP and GRP78, and (3) calcium handling, namely SERCA2a and phospholamban (PLB). In contrast, the phosphorylated form of PLB (Ser16) and RyR2 (Ser2808) was decreased.

Conclusions: Altogether, results from this study highlight perturbations in PL remodeling in VLCAD-/- mouse hearts, which are associated with ER stress and alterations in proteins involved in calcium handling and exacerbated by a HF diet. These perturbations represent novel potential mechanisms, which could contribute to the pathogenesis of arrhythmias in VLCAD-/- mice as well as in humans. 
O7-1

Decades of increasingly elevated risk of Pulmonary Hypertension following premature birth

Naumburg E. (1,2), Söderström L. (2)

Department of Clinical Science, Paediatrics, Umeå University, Umeå, Sweden (1); Unit of Research, Education and Development, Östersund Hospital, Östersund, Sweden (2)

Objectives: Pulmonary hypertension (PH) among children and adults has been linked to premature birth even after adjustments for known factors such as congenital heart disease (CHD) and lung disease. The aim of this population-based registry study was to assess the risk of $\mathrm{PH}$ following exposure to premature birth over decades when modern neonatal care was introduced and the survival rates increased.

Methods: Information on $\mathrm{PH}$ and perinatal factors was retrieved from the Swedish Pulmonary Hypertension Registry (SPAHR), the Swedish Registry of Congenital Heart Disease (SWEDCON) and the Medical Birth Registry in Sweden. Cases were adults and children over five years of age with PH born in Sweden between 1973 and 2010. Six controls were randomly selected from the Swedish Medical Birth Registry, matched by birth year and delivery hospital to each case. Information on risk factors was retrieved through the registries. Conditional multiple logistic regression was performed adjusting for $\mathrm{CHD}$, gender, chromosomal abnormalities, congenital diaphragm hernia and acute lung disease, for the total study population and by birth grouped in five-year intervals.

Results: The overall adjusted risk of $\mathrm{PH}$ was associated with premature birth, $\mathrm{OR}=4.46(95 \% \mathrm{CI} ; 2.46--8.11)$. The risk of $\mathrm{PH}$ following premature birth for each five-year period increase several times for children born in 2000's and later, $\mathrm{OR}=14.44$ (95\% CI 4.45-45.88).

Conclusion: $\mathrm{PH}$ following premature birth has increased over the last decades. Our study indicates that new factors may play a role in the risk of developing $\mathrm{PH}$ among preterm born infants.

O7-2

Role of MiRNA in Pulmonary Hypertensive Premature with Bronchopulmonary Dysplasia

Pamukcu O., Narin N. (1), Bayat E. (1), Taheri S. (2), Sunkak S. (1), Tasci O. (1), Baykan A. (1), Uzum K. (1)

Erciyes University, Pediatric Cardiology Department, Kayseri, Turkey (1); Erciyes University, Medical Biology Department, Kayseri, Turkey (2)

Aim: Microsomal RNAs' contribution was established in pulmonary hypertension $(\mathrm{PH})$ process by smooth muscle proliferation in pulmonary artery, resistance to apoptosis, endothelial damage and vascular remodeling.

In this study, it was aimed to determine role of miRNAs in pulmonary hypertensive infants with BPD and to investigate whether miRNAs are appropriate markers for risk stratification.

Material method: The study included $21 \mathrm{BPD}$ patients with $\mathrm{PH}$ (group 1), 17 without $\mathrm{PH}$ (group 2) and 21 healthy preterm (group 3). All participants were aged between 28 days and 3 months. Echocardiography was performed by using Vivid 7 Ultrasound system (GE, Hortens, Norway). Tricuspid velocity, mean pulmonary artery pressure (PAP), pulmonary acceleration time, RV end diastolic and end systolic diameter, RV Tei, RV FS, TAPSE, IVC index, LV eccentricity index parameters were measured by echocardiography.

For miRNA assays, peripheral blood samples were drawn into EDTA tubes at time of diagnosis and expression of 25 miRNAs were analyzed.
Results: Mean weight of patients: 1000 (850-1340)gr, 1100 (8321345)gr, 1360 (1100-1512)gr for Group 1,2,3 respectively. Mean $\mathrm{PAP}$ and PVR was found significantly high in group 1 . Nineteen types of miRNA were associated to pulmonary hypertension. Expression of the 15 miRNAs of these 19; was found increased in group 1 (miR 23a-3p, miR 23b-3p, miR 21-5p, miR 17-5p, miR 199a-3p, miR 451a, miR 191-5p, mir 206, miR 145-5p, miR $20 a-5 p, \operatorname{miR} 26 b-5 p, \operatorname{miR} 29 c-3 p, \operatorname{miR} 126-3 p$, miR 130a-3p, miR 208b-3p). It was also found that expression was significantly higher in 3 miRNAs in group 1 when compared to group 3 (miR 221-3p, miR 221-5p, miR 223-3p). miR 150-3p expression level was significantly lower in group 2 than group 3 .

Conclusion: Pulmonary hypertension is a disorder developing due to environmental and genetic reasons, in which underlying mechanism isn't fully understood. The genes controlled by miRNAs found to be related to $\mathrm{PH}$ in our study may have a mechanistic role in $\mathrm{PH}$. In the future, it could be possible to establish novel approaches that may contribute early diagnosis and treatment of pulmonary hypertension by focusing target genes of miRNA found to be related in this study.

\section{O7-3}

Right Ventricular End-Systolic Remodeling Index in the Assessment of Pediatric Pulmonary Arterial Hypertension - Data from the European Pediatric Pulmonary Vascular Disease Network

Koestenberger M. (1), Avian A. (2), Gamillscheg A. (1), Grangl G. (1), Burmas A. (1), Schweintzger S. (1), Baumgartner D. (1), Cvirn G. (3), Kurath-Koller S. (1), Hansmann G. (4)

Division of Pediatric Cardiology, Department of Pediatrics, Medical University Graz, Austria (1); Institute for Medical Informatics, Statistics and Documentation, Medical University Graz, Austria (2); Institute of Physiological Chemistry, Centre of Physiological Medicine, Medical University Graz, Austria (3); Department of Pediatric Cardiology and Critical Care, Hannover Medical School, Hannover, Germany (4)

Objectives: In adults with pulmonary hypertension (PH) determination of the RV end-systolic remodeling index (RVES RI) with echocardiography was found to be of clinical value for the assessment of disease severity. We aimed to determine if increased RVES RI values will also occur in pediatric $\mathrm{PH}$. We further aimed to correlate these RVES RI values with conventionally used echocardiographic variables such as the tricuspid annular plane systolic excursion (TAPSE), the ( $\left.\mathrm{S}^{\prime}\right)$, the left ventricular eccentricity index (LVEI), the pulmonary artery acceleration time (PAAT), and hemodynamic values such as the mean pulmonary artery pressure (mPAP) and the pulmonary vascular resistance index (PVRi).

Methods: We conducted an echocardiographic study in 49 children with $\mathrm{PH}$, and in 123 healthy children.

Results: The RVES RI was increased in children with $\mathrm{PH}$ compared to 49 matched healthy children $(1.45 \pm 0.16$ vs. $1.16 \pm 0.06$; $\mathrm{p}<0.01)$. The RVES RI negatively correlated with RV systolic function parameters (TAPSE, $\mathrm{r}=-0.487, \mathrm{p}<0.001 ; \mathrm{S}^{\prime}, \mathrm{r}=-0.471$, $\mathrm{p}=0.001)$. RVES RI positively correlated with the magnitude of RV-LV interaction (LVEI, $r=0.633, p<0.001$ ) and the PAAT $(\mathrm{r}=-0.343, \mathrm{p}=0.016)$. The RVES RI was positively associated with the invasive hemodynamic variables mPAP $(r=0.464$, $\mathrm{p}=0.004)$ and PVRi $(r=0.579, \mathrm{p}<0.001)$, demonstrating the RVES RI to reflect disease severity in our pediatric $\mathrm{PH}$ patient cohort.

Conclusions: The RVES RI is increased in children with $\mathrm{PH}$ compared to age-matched healthy subjects and correlates with conventional echocardiographic and invasive hemodynamic 
variables of RV function, PAP and PVR. The RVES RI is a simple, reproducible, and clinically relevant echocardiographic measure for the assessment of $\mathrm{PH}$ in children.

\section{O7-4 \\ Ventricular assist devices in pediatric cardiomyopathy and congenital heart disease patients: an analysis from the German National Register for Congenital Heart Defects Lammers A. (1), Sprenger K. (1), Diller G.P. (2), Helm P.C. (3), Asfour B. (4), Abdul-Khaliq H. (5), Uebing A. (1), Ewert P. (6), Bauer U.M.M. (3), Tutarel O. (6) \\ Department of Paediatric Cardiology, University Hospital Münster, Münster/Germany (1); Division of Adult Congenital and Valvular Heart Disease, Department of Cardiovascular Medicine, University Hospital Münster, Münster/Germany (2); National Register for Congenital Heart Defects and Competence Network for Congenital Heart Defects, Berlin/ Germany, DZHK (German Centre for Cardiovascular Research), Berlin/ Germany (3); Asklepios Klinik Sankt Augustin GmbH, German Pediatric Heart Center, Sankt Augustin/Germany (4); Department of Pediatric Cardiology, Saarland University Medical Center, Homburg/Germany (5); Department of Paediatric Cardiology and Congenital Heart Disease, German Heart Centre Munich, Technical University of Munich, Munich/Germany (6)}

Introduction: Heart failure is a major contributor to morbidity and mortality in pediatric cardiomyopathy and congenital heart disease (CHD) patients. Advanced therapies for heart failure like ventricular assist devices (VAD) are increasingly used in patients with acquired heart disease. Limited data exists, however, on the use and outcome of this technology in pediatric cardiomyopathy and CHD patients.

Methods: Patients who were supported with a VAD were identified from the German National Register for Congenital Heart Defects. We report patient demographics, underlying cardiac defect, status of surgical repair/palliation, associated conditions, complications, and 30-day and overall mortality in this population. Univariate regression analysis was performed to identify predictors of an adverse outcome.

Results: Overall, 91 patients (median age 4.29 years [IQR, 0.44$15,96], 45.1 \%$ female) received a VAD. 45 pat. (49.5\%) had a congenital heart defect, while $46(50.5 \%)$ were reported as cardiomyopathy. The type of VAD implanted was Berlin Heart in 31 pat. (34.1\%), Heartware in 16 (17.6\%), Levitronix in 9 (9.9\%), unknown in $28(30.8 \%)$. In 67 pat. (73.6\%) the systemic ventricle was supported, in 4 (4.4\%) the subpulmonary ventricle, and in $20(22.0 \%)$ both. Reasons for implantation were heart failure in $46(50.5 \%)$, post-surgery in $24(26.4 \%)$, transfer from ECMO in $16(17.6 \%)$, and miscellaneous in $5(5.5 \%)$. At least one complication occurred in 54 pat. $(59.3 \%)$ such as bleeding $(\mathrm{n}=37)$, thrombosis $(n=15)$, embolic $(n=19), 20$ of which involved cerebral complications.

Cause for explantation was clinical improvement in 22 (24.2\%), death in $22(24.2 \%)$, heart transplantation in $39(42.9 \%)$, and change to ECMO in 3 (3.3\%). Overall, 30-day mortality rate was $19.8 \%$. Longer term all-cause mortality rate was $39.6 \%$. On unvariate regression analysis, no significant predictor could be identified for 30-day mortality. For all-cause mortality, age at implantation (OR:1,12; 95\%CI 1.06 to $1.18, \mathrm{p}<0.0001$ ) and the presence of a congenital heart defect (OR: 4.92; 95\%CI 1.97 to $12.32, \mathrm{p}<0.001)$ were the only univariate predictors.

Conclusions: The use of VAD for heart failure in pediatric cardiomyopathy and CHD patients is increasing. Even though, mortality remains high in this cohort, reflecting the complexity of this particular patient population presenting with heart failure.

\section{O7-5}

Outcome of rescue heart transplantation in paediatric congenital heart disease patients - Single centre results Cardoso B. (1), Reinhardt Z. (2), Simpson E. (1), Hasan A. (3), De Rita F. (3), Crossland D. (2)

Department of Paediatric Intensive Care, Freeman Hospital, Newcastle upon Tyne, UK (1); Department of Paediatric Cardiology, Freeman Hospital, Newcastle upon Tyne, UK (2); Department of Cardiothoracic Surgery, Freeman Hospital, Newcastle upon Tyne, UK (3)

Introduction: A subset of children undergoing surgery or intervention for CHD remain unwell despite optimal conventional management and cannot be discharged from hospital. Some of these can be considered for cardiac transplant. We define transplant in this group of patients as 'rescue transplant' (RT), describe the outcome of RT for CHD and compare this to other CHD patients undergoing transplant (nRT group).

Methods: Retrospective analysis of the records of children with CHD who underwent cardiac transplantation in our centre between January 2012 and November 2017.

Results: Rescue transplant accounted for 19 out of 49 CHD transplants (39\%). Fourteen had undergone palliative or redo surgery (8 single ventricle palliations, 2 coronary procedures; 2 LVOTO relief; 2 other surgeries) and 5 had undergone interventional procedures. RT recipients tended to be younger than $\mathrm{nRT}$ patients (median 1.5 vs 6 years, $\mathrm{p}=0.074$ ) and of lower weight (median 7.6 vs $16.5 \mathrm{~kg}, \mathrm{p}=0.005$ ), with no significant difference in gender or anatomical classification. At the time of transplant, $47 \%$ of the RT group were on VAD/ECMO vs $13 \%$ of the nRT group $(\mathrm{p}=0.009)$ and more were ventilated $(79 \%$ vs $27 \%, \mathrm{p}<0.005)$. Post-transplant ECMO was required in $47 \%$ of the RT group vs $23 \%$ of the nRT group ( $\mathrm{p}=0.08)$.

RT patients stayed longer in PICU (median 55 vs 24 days, $\mathrm{p}=0.001)$ and more required renal replacement therapy (58\% vs $30 \%, \mathrm{p}=0.05)$ and tracheostomy (53\% vs $23 \% ; \mathrm{p}=0.036)$. Neurological injury (26\% vs $3 \%, \mathrm{p}=0.017)$, parenteral nutrition (47\% vs $0 \%, p<0.005)$, chylothorax $(32 \%$ vs $3.3 \%$; $=0.006)$ and abdominal surgery $(16 \%$ vs $0 \% ; \mathrm{p}<0.005)$ were all more frequent in the RT group. No significant difference in overall survival was observed (RT $88.9 \%$ vs nRT $93.1 \%$ at 1 month, $77 \%$ v. $89.5 \%$ at 1 year, $\mathrm{p}=0.106)$.

Conclusions: Despite requirement for high levels of support going into transplant, RT can be carried out with acceptable survival in patients with CHD. However, this group of patients accumulate considerable morbidity which should be taken into account when counseling parents and planning resource allocation.

\section{O7-6}

\section{Outcome After Transplantation in Patients With} Eisenmenger Syndrome

Hjortshøj C.S. (1), Gilljam T. (2), Dellgren G. (2), Pentikäinen M. (3), Møller T. (4), Jensen A.S. (1), Holmstrom H. (5), Turanlahti N. (4), Thilén U. (6), Gustafsson F. (1), Sondergaard L. (1) Department of Cardiology, Rigshospitalet, Copenhagen, Denmark (1); Department of Medicine, Sahlgrenska University Hospital/Östra and Sahlgrenska Academy (2); Department of Paediatric Cardiology, Helsinki University Hospital, Helsinki, Finland (3); Department of Cardiology, Rikshospitalet, Oslo, Norway (4); Department of Paediatric Cardiology, Rikshospitalet, Oslo, Norway (5); Department of Cardiology, Lund University Hospital, Lund, Sweden (6)

Introduction: Eisenmenger syndrome (ES) is the gravest expression of any unrepaired congenital cardiac defect with a systemic-topulmonary communication leading to pulmonary arterial hypertension $(\mathrm{PAH})$, bidirectional or reversed flow, and cyanosis. 
Novel therapy with pulmonary arterial hypertension (PAH)targeted therapies offers long-term benefits for exercise capacity, clinical symptoms and possibly survival, however transplantation (Tx), either as combined heart-lung-transplantation (HLTx) and single/double-lung transplantations (LTx) with concomitant cardiac repair, is still the only curative treatment option available.

The aim of the present study was to evaluate Nordic patients with Eisenmenger syndrome who have undergone Tx from 1985-2012 and to describe morbidity-free survival along with overall survival. Methods: This was a retrospective, descriptive multicentre study on patients transplanted due to ES.

Results: From 1985-2012 63 of 714 patients with ES (8.8\%) in the Nordic region received a HLTx $(90.5 \%)$ or LTx with concomitant repair of the cardiac defect $(9.5 \%)$. Transplanted patients were 31.9 years (21.1-42.3 years) at the time of transplantation, ranging from 10 to 67 years. Paediatric transplantations accounted for $12.7 \%$.

A total of 39 deaths $(61.9 \%)$ occurred during the study of which 7 (11\%) were peri-operative deaths within 30 days of transplantation. Overall median survival was 12.0 years (95\%CI 7.6-16.4 years) and the overall 1-, 5-, 10-, and 15-year post-Tx survival rates were $84.1 \%, 69.7 \%, 55.8 \%$, and $40.6 \%$, respectively. Survival conditioned by surviving the first year after Tx was 13.9 years (95\%CI 7.0-20.8 years) with 1-, 5-, 10-, and 15-year survival rates of $94.4 \%, 77.4 \%$, $63.0 \%$, and $47.3 \%$, respectively. There was no difference in median survival after HLTx and LTx (14.9 years vs. 10.6 years, $p=0.718$ ). Median survival free of cardiac allograft vasculopathy, bronchiolitis obliterans syndrome (BOS), and dialysis/kidney transplant were 11.2 years (95\% CI 7.8-14.6), 6.9 years (95\% CI 2.6-11.1), $11.2(95 \%$ CI 8.8-13.7), respectively.

The leading cause of death after the perioperative period was infections $(36.7 \%)$, followed by BOS (23.3\%) and heart failure $(13.3 \%)$.

Conclusions: This study shows that lengthy post-transplant survival without severe comorbidities is achievable in patients with Eisenmenger syndrome. Cause-specific mortality after the perioperative period is dominated by causes not directly related to transplantation.

\section{O8-1 \\ Long-term survival after univentricular heart surgery Sunnegardh J. (1), Synnergren M. (1), Dalén M. (2) \\ The Queen Silvia Children's Hospital, Sahlgrenska University Hospital, Institution of Clinical Sciences, Gothenburg University, Sweden (1); Department of Molecular Medicine and Surgery, Karolinska Institutet, Department of Cardiac Surgery, Karolinska University Hospital, Stockholm Sweden (2)}

Objective: To examine long-term survival after surgery for univentricular heart defects.

Methods. All children operated due to univentricular heart defects in our institution before the age of 18 years from Jan 1st 1994 to May 31st 2017 were included. Patient files were analyzed and cross-checked as of May 31st 2017 against the National Population Registry allowing for reliable and complete data on survival. Primary outcome was all-cause mortality. Additionally, riskfactors of death were analyzed.

Results: 431 patients (41\% female), median age and weight at first surgery of 10 days (quartile [Q]1, Q3: 6, 21) and $2.6 \mathrm{~kg}(\mathrm{Q} 1, \mathrm{Q} 3$ : $3.1,4.1)$ were included. Mean follow-up time was $10.1 \pm 8.9$ years, with loss to follow-up of one patient. 108 deaths (25\%) occurred with a median age at death of 88 days. Right ventricular dominance was found in 228 (52.9\%) patients and left or biventricular dominance in $199(46.2 \%)$, with a survival at 10 years of age of $63.3 \%$ and $89.9 \%$, respectively $(p<0.001)$. No mortality difference between these two groups was found in conditional survival in patients surviving the first two years after surgery. In multivariate analysis, risk factors for death were severe AV-valve regurgitation before first stage palliation (hazard ratio [HR] 2.21, $95 \%$ confidence interval $[\mathrm{CI}] 1.00-4.85, \mathrm{p}=0.049)$, hypoplastic left heart syndrome (HR 1.79, 95\% CI 1.10-2.92, p =0.019) and right ventricular dominance (HR 2.32, 95\% CI 1.31-4.10, $\mathrm{p}=0.004)$. No significant difference was found in mortality at three years of age between patients born 1994-2004 ( $\mathrm{n}=57 / 200$, $28.5 \%)$ as compared to those born 2005-2017 ( $\mathrm{n}=40 / 182,22 \%)$. Heart transplantation was performed in 20 patients (4.6\%) with four late deaths. As of May 31st 201734 patients were awaiting a stage 2 or 3 operation and 251 were alive with a complete Fontan circulation.

Conclusion: This study gives prognostic information of survival for patients undergoing surgical palliation due to univentricular heart defects. Patients with a systemic ventricle of left ventricular dominance have a significantly better long-term survival compared with patients with right ventricular dominance. However, this was due to a significantly higher mortality only during the first two years after the initial surgery.

\section{O8-2}

\section{Neonatal Ross-Konno Procedure after Fetal Aortic Valve} Dilatation

Sames-Dolzer E. , Kreuzer M., Benedikt P., Mair Ro., Wickenhauser E., Tulzer A., Tulzer G., Mair R.

Children's Heart Center Linz, Linz, Austria

Objectives: Fetal aortic valve intervention can induce left ventricular functional improvement and growth of left sided cardiac structures in patients with critical aortic stenosis. A neonatal Ross-Konno procedure might be necessary to offer relentless relief of left ventricular outflow tract obstruction and enable a biventricular pathway.

Methods: Our single center retrospective study includes 15 patients who underwent a neonatal Ross-Konno operation after fetal aortic valve intervention between 2005 and 2017. Intervention was performed between the 26th and 34th week of gestation under the following criteria: dilated left ventricle with severely impaired function $(\mathrm{FS}<15 \%)$ and signs of EFE; LV long-axis z-score $>-1$ or $>0$ depending on gestational age; left to right shunt across the foramen ovale and a reverse flow in the aortic arch. Neonatal Ross-Konno was done enlarging the LVOT with a pericardial patch and doing a concomitant aortic arch repair in 5 patients. Age at operation was between 5 and 19 days, weight ranged between 2,18 and $3,9 \mathrm{~kg}$.

Results: Mitral valve diameter $\mathrm{z}$-score ranged between $-0,62$ and $-3,85$, mitral valve abnormalities were described in 9 pts., 8 children had EFE grade III, 6 underwent an EFE resection. 8 pts. had a preoperative $\mathrm{FS} \leq 20 \%$. The median discriminant score was $-2,43$. Three pts. needed postoperative ECMO. In hospital mortality was $6,7 \%$ (1 pt. due to NEC on pod 37). One patient underwent a take-down procedure at the age of 5 months due to chronic CHF and died 5 months later after BDG.

Left ventricular function is considered good or excellent in 11 pts. and moderately reduced without PHT in 2 pts. at last FU. Autograft function is good in all pts. without reoperation during the FU of up to 12,3 a. 2 children needed reoperations for mitral valve surgery, one required a pacemaker.

Conclusions: The neonatal Ross-Konno procedure can offer successful biventricular repair even in children with severe long lasting LVOT obstruction, who probably would not have become 
biventricular without fetal aortic valve dilatation. The absence of LVOT reoperation indicates a long lasting form of repair.

O8-3

Single centre long-term results of Contegra grafts with 1700 patient years of follow-up

Schmiady M. (1,2), Podonyi A. (1,2), Balmer C. (1),Weber R. (1), Cannizzaro V. (1), Greutmann M. (2),Hübler M. $(1,2)$, Dave H. $(1,2)$ (1) Children's Heart Centre, Children's Research Centre, University Children's Hospital Zürich, Switzerland. (2) Heart Centre, University Hospital Zürich, Switzerland.

Objectives: To report the long-term outcome of 170 Contegra grafts implanted in Right Ventricular Outflow tract at a single institution from 2001-2007.

Methods: 170 consecutive Contegra grafts implanted were followed up. Median age and weight were $9(0.01-73.5)$ years and $23(1.9-96.4) \mathrm{kg}$ respectively. Indications for implantation were pulmonary valve dysfunction late after primary repair (91), Repair of TOF, Pulmonary atresia, Truncus arteriosus and Rastelli (43), Ross Procedure (29) and Miscellaneous (8). 57 grafts were $<16 \mathrm{~mm}$ and 114 were $\geq 16 \mathrm{~mm}$. $Z$ value of the grafts was $1.6(-3.1$ to 7.3). Pulmonary arborization associated with chronic regurgitation was called dilatative, that in Ross Procedure was called normal and that associated with stenotic/restrictive substrate was called obstructive. Follow-up duration was 9.6 (0.6-15.3) years and was $95 \%$ complete.

Results: Kaplan Meier patient survival was $90 \pm 2.4 \%$ at 14 years (No. remaining 54), none of the death was related to Contegra. Freedom from replacement was $55.3 \pm 4.3 \%$ at 14 years (No. remaining 22). Contegra survival was significantly better in older patients, bigger grafts, in orthotopic position, in patients coming late after primary repair (Fig 1) as well in dilated pulmonary vascular tree (Table 1). Contegra survival stratified according to diagnostic class is detailed in Figure 1. 50\% of the Contegra $<16 \mathrm{~mm}$ were replaced at 4.5 years. $80 \%$ of all the surviving Contegra were free of intervention and $57 \%$ free of composite dysfunction (Mean gradient $\geq 20$ and/or Regurgitation $\geq$ Moderate) at 14 years (Table 1 ).

Table 1.

\begin{tabular}{|c|c|c|c|c|c|}
\hline $\begin{array}{l}\text { Freedom from } \\
\text { Replacement }\end{array}$ & $\begin{array}{l}\text { Survival } \\
\%\end{array}$ & $\begin{array}{l}\text { a in } \\
\text { years }\end{array}$ & $\begin{array}{l}\text { No. At } \\
\text { Risk }\end{array}$ & $\begin{array}{l}\mathbf{p} \\
\text { Value }\end{array}$ & $\begin{array}{l}\text { Mean } \\
\text { Survival }\end{array}$ \\
\hline \multicolumn{6}{|l|}{ By Age group } \\
\hline$<2$ years & $24 \pm 6$ & 10 & 10 & \multirow[t]{3}{*}{$<0.001$} & 7 (5-8) yrs. \\
\hline $2-10$ years & $54 \pm 10$ & 10 & 13 & & 11 (9-13) yrs. \\
\hline$>10$ years & $82 \pm 5$ & 10 & 51 & & 14 (13-14) yrs. \\
\hline \multicolumn{6}{|l|}{ By Contegra size } \\
\hline$<16 \mathrm{~mm}$ & $26 \pm 6$ & 10 & 12 & \multirow[t]{2}{*}{$<0.001$} & $6(5-7)$ yrs. \\
\hline$\geq 16 \mathrm{~mm}$ & $78 \pm 4$ & 10 & 65 & & 13 (13-14) yrs. \\
\hline \multicolumn{6}{|l|}{$\begin{array}{l}\text { Pulmonary } \\
\text { arborization }\end{array}$} \\
\hline Obstructive & $31 \pm 7$ & 10 & 14 & \multirow[t]{3}{*}{$<0.001$} & 7 (6-8) yrs. \\
\hline Normal & $52 \pm 11$ & 10 & 10 & & 11 (10-13) yrs. \\
\hline Dilatative & $86 \pm 4$ & 10 & 53 & & 14 (13-14) yrs. \\
\hline \multicolumn{6}{|l|}{ Implant Position } \\
\hline Orthotopic & $68 \pm 5$ & 10 & 59 & \multirow[t]{2}{*}{$<0.001$} & 12 (11-13) yrs. \\
\hline Heterotopic & $47 \pm 7$ & 10 & 18 & & 9 (8-11) yrs. \\
\hline $\begin{array}{l}\text { Freedom from } \\
\text { Endocarditis }\end{array}$ & $93 \pm 2.4$ & 14 & 42 & \multicolumn{2}{|c|}{$\begin{array}{l}\text { Incidence of endocarditis } \\
0.5 \% \text { per patient year }\end{array}$} \\
\hline $\begin{array}{l}\text { Freedom from Cath } \\
\text { Interv. }\end{array}$ & $80 \pm 3.5$ & 14 & 25 & & \\
\hline $\begin{array}{l}\text { Freedom from comp. } \\
\text { dysfun. } \\
\text { (PS } \geq 20 \mathrm{mmHg} \text {. } \\
\text { \&/or PR } \geq \text { mod.) }\end{array}$ & $57 \pm 7$ & 14 & 23 & & \\
\hline
\end{tabular}

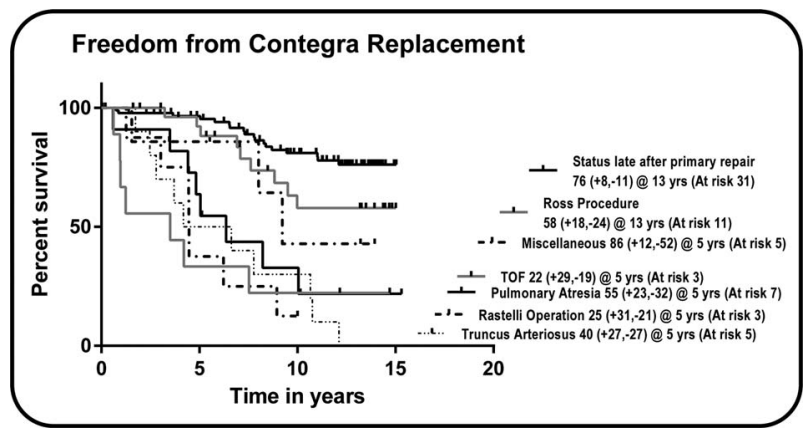

Figure 1.

Conclusions: The search for longer lasting alternatives to smaller Contegra including conduit-less reconstruction techniques continues. Despite this, their off-the-shelf availability and tubular morphology render Contegra a place in every congenital cardiac centre. The results of larger Contegra justify their place as a pulmonary valve substitute typically in teenage patients presenting late after primary repair.

\section{O8-4}

Long-Term Transplant-Free Survival of Patients with Single Ventricle Congenital Heart Disease: Does Ventricular Morphology Matter?

Oster M., Knight J., Suthar D., Kochilas L.

Emory University, Atlanta, GA, USChildren's Healthcare of Atlanta, Atlanta, GA, US

Background: There have been conflicting studies regarding the association of ventricular morphology with long-term outcomes for children with single ventricle (SV). We used data from the Pediatric Cardiac Care Consortium (PCCC), a large multi-center US-based surgical registry, to examine the association of ventricular morphology with long-term transplant-free survival for children with SV.

Methods: This is a retrospective cohort study of individuals who had surgery for SV at $<1$ year of age at 45 centers in the PCCC from 1982-2003 and were linked with records from the US National Death Index (NDI) and Organ Procurement Transplantation Network (OPTN) registry through 2014. To examine overall survival and survival among those who had successful Fontan palliation, we constructed separate Kaplan-Meier transplant-free survival curves and mortality/transplant hazard ratios, adjusting for sex, syndrome, age at Fontan, type of Fontan (aortopulmonary, lateral tunnel or extracardiac), creation of fenestration at the time of Fontan, center, and birth era (early:1982-1992, middle: 1993-1997, and late: (1998-2003).

Results: Of the 3903 patients identified, the dominant singleventricle morphology was right ventricle (RV) in $43.4 \%$, left ventricle (LV) in 36.3\%, and non-classifiable (NC) in $20.3 \%$. Primary heart transplant or in-hospital death following first surgery occurred in $15.2 \%$ of LV, $17.6 \%$ of NC and $45.6 \%$ of RV. Among those who survived to discharge following the first surgery, overall 20 -year transplant-free survival was $71.6 \%$ for LV, $63.2 \%$ for NC and $52.7 \%$ for RV. Compared to those with dominant LV, adjusted 20-year hazard ratio for mortality/transplant was 2.1 (95\% CI 1.8-2.4) for RV and 1.4 (95\% CI 1.2-1.7) for NC. Among the 1498 patients who survived Fontan hospitalization, 15-year transplant-free survival was $84.5 \%$ for RV, $92.8 \%$ for $\mathrm{LV}$, and $88.8 \%$ for NC. Compared to those with dominant LV, adjusted 


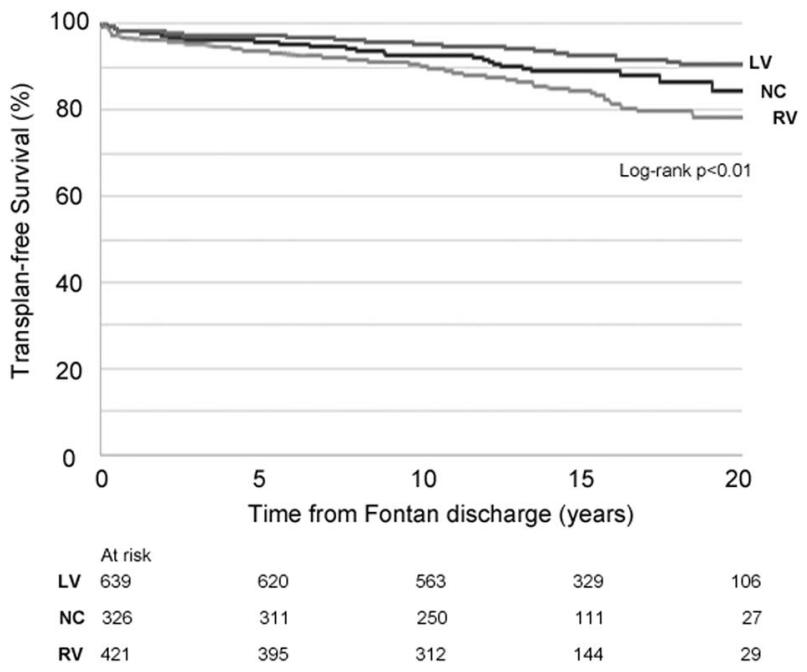

Figure.

15-year hazard ratio for mortality/transplant following Fontan was 2.4 (95\% CI 1.6-3.7) for RV and 1.6 (95\% CI 1.2-3.0) for NC. Conclusion: In this study, long-term transplant-free survival for patients with SV was greater for those with LV morphology. These findings have important prognostic value for providers, patients, and families.

\section{O8-5}

Aortic Valve Replacement by mechanical prosthesis in Children: a positive Long-Term Outcome including for patients with an infantile Marfan syndrome

Moreau de Bellaing A., Raisky O., Haydar A., Bonnet D., Bajolle F. Hôpital Necker-Enfants-Malades, Paris, France

Introduction: Aortic mechanical valve replacement (AVR) in children, especially children with infantile Marfan syndrome (IMS), has been associated with a high complication rate. Long-term prognosis of IMS, a rare congenital inheritable connective tissue disorder, is generally perceived as poor.

Methods: A retrospective review of clinical and surgical records of patients undergoing AVR in Necker hospital between 2000 and 2017, was performed. 55 children (41 boys and 14 girls) underwent 57 AVR procedures at a median age of 12.4 years (range, 1.2 to 17.4 years) and a median weight of $40 \mathrm{~kg}$ (range, 7.5 to $86 \mathrm{~kg}$ ). 11 patients (20\%) had a MS diagnosed in early childhood. Median follow-up for operative survivors was 4.5 years (range, 1 month to 16 years, $95 \%$ complete).

Results. Thirty-day mortality was $4 \%(\mathrm{n}=2)$. One-year, five-year and ten-year patient survival was respectively $96 \%, 93 \%$ and $93 \%$. Among survivors, the 10 -year freedom from reoperation was $87 \%$. Complications after AVR included heart block requiring pacemaker $(5 \%)$, bleeding (4\%), stroke (11\%) and endocarditis (4\%). There was no prosthesis thrombosis. All the patients with an IMS had an aortic root dilatation and underwent a Bentall procedure at a median age younger than children without connective tissue disorder $(9.5$ years, $\mathrm{p}=0.008)$. Eight of these $(73 \%)$ had previous aortic root replacement with valve sparing technique. Two children had an associated severe mitral prolapse and underwent concomitant mitral valve replacement. All patients with IMS survived.

Conclusions: Long-term results after AVR are excellent. Complications associated with surgery and long-term anticoagulation are rare. This study gives confidence in mechanical prosthesis including for children with an infantile MS.
O8-6

Causes of Late Mortality of Children Surviving Initial Congenital Heart Surgery: A Report from the Pediatric Cardiac Care Consortium

McCracken C., Spector L., Knight J., Bradshaw J., Menk J., Moller J., Kochilas L.

Emory University, Atlanta, GA, USAUniversity of Minnesota, Minneapolis, MN, USA

Background: Children living with operated congenital heart disease (CHD) continue to be at risk for causes of death that are different from the general population. We used the Pediatric Cardiac Care Consortium (PCCC), a large US-based registry for interventions for $\mathrm{CHD}$, to examine the underlying causes of death (COD) of children operated at $<21 \mathrm{y}$ of age for a CHD.

Methods: This is a retrospective cohort study of children who were operated for CHD at 45 centers in the PCCC between 1982 and 2003 and were linked with the US-National Death Index (NDI) through 2014. Data provided by NDI-Plus included International Classification of Disease codes for underlying COD and associated morbidities/events contributing to death. Cause-specific mortality for individuals with operated CHD was compared to the general population with adjustment for age and sex. Patients with known chromosomal abnormalities or inadequate identifiers were excluded from the analysis.

Results: Among 31,132 patients surviving their initial congenital heart surgery there were 2,527 deaths $(8.1 \%)$ over a follow-up period of 33 years (median 18.1, IQR:14.5 -22.2). Median age at death was 1.8 year (IQR: $0.5-12.8$ ). COD varied by severity and type of CHD but, overall, $83.2 \%$ of late mortality was associated with CHD or another cardiovascular condition (Figure 1A). As patients aged, the proportion of deaths caused by external causes and neoplasms increased (Figure 1B). The overall standardized mortality (SMR) for patients with operated CHD compared to the general population was 22.6 (95\% CI: 19.5-25.7) from external causes and 5.8 (95\% CI: 4.2-7.3) from neoplasms.

Conclusion: Patients operated for even the mildest forms of CHD are at high risk for premature cardiovascular death directly or indirectly associated to their underlying CHD warranting continued monitoring to address associated morbidities.

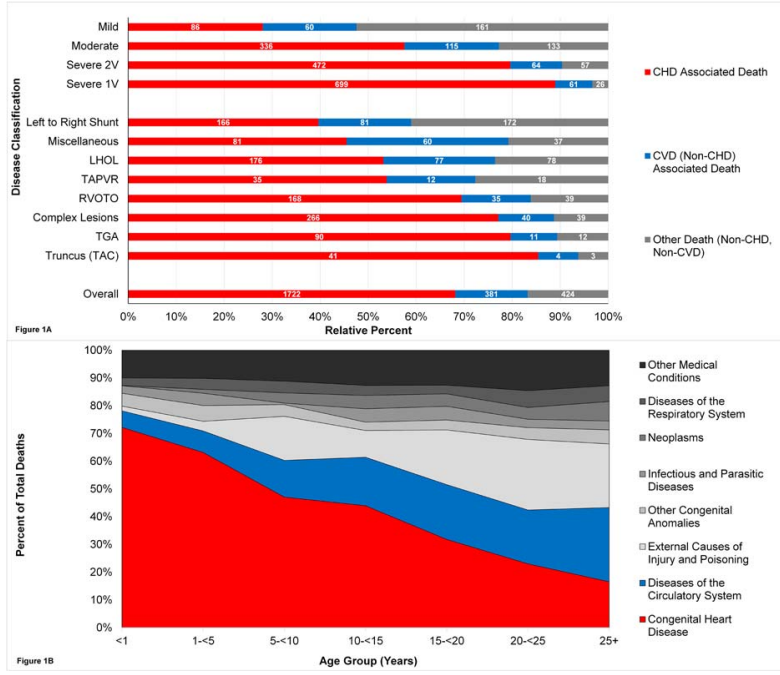

Figure 1. 
09-1

Implantation of the transcatheter heart valve for pulmonary position in native large right ventricular outflow tract with severe pulmonary regurgitation Guzeltas A. (1), Tanidir I.C. (1), Sahin M. (1), Gokalp S. (1), Kasar T. (1), Topkarci M.A. (2)

Istanbul Saglik Bilimleri University, Mehmet Akif Ersoy Thoracic and Cardiovascular Surgery Center, Department of Pediatric Cardiology, Turkey (1); İstanbul Saglik Bilimleri University, Mehmet Akif Ersoy Thoracic and Cardiovascular Surgery Center, Department Anesthesiology, Turkey (2)

Introduction: Transcatheter Pulmonary Valve Replacement (tPVR) into right ventricular to pulmonary artery conduits is being increasingly performed in many centers. There are few options for patients with native and large right ventricular outflow tracts (RVOT). An option for these patients is off label use of Edwards valve. We aimed to review the outcomes for the SAPIEN-XT and S3 valves for pulmonary position in patients with large and native RVOT. Between November 2014 and November 2017, 42 procedures were performed. Among them 32 (\%76) had native RVOT.

Method: We evaluated; drop in blood pressure, clear waist, balloon stability and residual leak to the PA after contrast injection into the RV during a 30-mm TyShak balloon inflated across the RVOT. If there were no contraindications tPVR procedure was initiated. Andra XXL stents with Z-Med-II balloons preferred for prestenting. If RVOT is very large we prefer to wait $6-8$ weeks after presenting procedure before $\mathrm{PVR}$ procedure.

Results: Thirty-two patients underwent RVOT stenting before tPVR. Four of them are still waiting for valvulation. Procedure was unsuccessful in 2 of them. Median age and weight of the remaining patients were 19 (12-46 years) and 54 (33-89) kg, respectively. Primary underlying diagnosis was tetralogy of Fallot $(n=20 / 26)$. Stenting was performed simultaneously with tPVR in 5 (\%19) cases whereas pre-stenting was performed 6 to 14 weeks earlier in 21 patients. Before tPVR median antero-posterior and lateral sizes of the stents were $27(23-30) \mathrm{mm}$ and 28 (24-31) $\mathrm{mm}$, respectively. Valve sizes were XT-26 mm $(\mathrm{n}=4), \mathrm{XT}-29 \mathrm{~mm}$ $(\mathrm{n}=18)$ and $\mathrm{S} 3-29 \mathrm{~mm}(\mathrm{n}=4)$. In 16 patients additional volume (median $2.5 \mathrm{ml}(1-4 \mathrm{ml})$ ) was added to the valves' balloon for stabilization. One of the patients underwent hybrid procedure due to the occlusion of the both femoral and jugular veins.

Complications: One patient had moderate tricuspid regurgitation related with the procedure. There were no deaths during a median follow-up of 9 (1-34) months. Valve function was preserved in all patients during follow-up.

Conclusion: The Edwards SAPIEN-XT and S3 valves may be an alternative for patients with native and large RVOT.

\section{O9-2}

Incidence of arterial hypertension long-term after stenting for native and recurrent coarctation of the aorta

Hosny Fayed M. (1,2), Tanase D. (1), Georgiev S. (1), Genz T. (1), Hager A. (1), Ewert P. (1), Abdellatef A. (2), Ahmed A. (2), Bambul Heck P. (1), Eicken A. (1)

Department of Pediatric Cardiology and Congenital Heart Diseases, German Heart Centre Munich, Technische Universität München, Germany (1); Department of Pediatrics, Faculty of Medicine, Zagazig University, Egypt (2)

Objectives: Today, stent implantation is the first line treatment for coarctation of the aorta $(\mathrm{CoA})$ in children, adolescents and adults in many cardiac Centers. However, there is limited information
Table.

\begin{tabular}{lllll}
\hline & $\begin{array}{l}\text { Hypertension and } \\
\text { Hentihypertensive } \\
\text { medication }\end{array}$ & $\begin{array}{l}\text { without } \\
\text { antihyperten- } \\
\text { sive } \\
\text { medication }\end{array}$ & $\begin{array}{l}\text { Normoten- } \\
\text { sive and } \\
\text { antihyperten- } \\
\text { sive } \\
\text { medication }\end{array}$ & $\begin{array}{l}\text { Normoten- } \\
\text { sive without } \\
\text { antihyperten- } \\
\text { sive } \\
\text { medication }\end{array}$ \\
\hline $\begin{array}{c}\text { Before CoA } \\
\text { Stenting } \\
(\mathrm{n}=220)\end{array}$ & $75(33 \%)$ & $104(48 \%)$ & $9(3 \%)$ & $32(16 \%)$ \\
$\begin{array}{c}\text { After CoA } \\
\text { Stenting } \\
(\mathrm{n}=176)\end{array}$ & $26(15 \%)$ & $11(6 \%)$ & $84(48 \%)$ & $55(31 \%)$ \\
\hline
\end{tabular}

about the fate of hypertension \& cardiovascular changes following stenting on the long term. This retrospective, single-centre study reported the long term results in children, adolscents, and adult patients who underwent endovascular stent placement for both native COA and recurrent (Re-COA).

Methods: Between February 1999 and October 2017, a total of 220 patients (145 males) underwent aortic stent implantation in our hospital. From these patients 101 patients were diagnosed as native CoA and 119 patients with Re-CoA. Median age at intervention was 17 years old (range 6 - 62 years); median weight was $63 \mathrm{~kg}$ (16 $-130 \mathrm{~kg}$ ). Only dilatable stents to adult size were implanted. Covered stents were used in 77 patients (35\%), bare metal stents in 143 patients $(65 \%)$.

Results: The median minimal diameter of the stenosed aortic segment increased from $8 \mathrm{~mm}(0-19)$ to $14 \mathrm{~mm}(6.2-24)(\mathrm{p}<0.001)$. The median invasive peak-to-peak systolic pressure gradient declined from $23 \mathrm{mmHg}(5-109)$ to $0 \mathrm{mmHg}(0-50)$ ( $\mathrm{p}<0.001)$. Two adults experienced an aortic rupture: one patient died and one was rescued by covered stent implantation. Median follow-up was 50 months (1-216 months). Re-catheterization was performed in 135 patients $(62 \%)$. In 82 patients $(38 \%)$ redilatation and in 18 patients (8\%) second stent implantation were necessary. Data on arterial hypertension are shown in the table.

Conclusion: CoA stenting is an effective means for treatment of native CoA and Re-CoA. However, up to $46 \%$ of these patients need repeated catheterization with balloon angioplasty or a second stent implantation. Although CoA stenting did not reduce the need for antihypertensive medications, it improved the response of patients to these medications, with better control of hypertension.

\section{O9-3}

Efficacy and safety of catheter closure of atrial septal defects using the Amplatzer versus the Cocoon septal occluder. A Multicenter Randomized Study

Thanopoulos B.D. (1,4), Dardas P. (1), Ninios V. (1), Giannakoulas G. (2), Deleanou D. (3), Lancovici S. (3)

Agios" Lukas Clinic, Thessaloniki, Greece (1); Ahepa University Hospital, Thessaloniki, Greece (2); Ares Cardiology Center, Bucharest, Romania (3); Iatrikon Medical Center, Athens, Greece (4)

Introduction: Transcatheter closure of atrial septal defect (ASD) using the Amplatzer Septal occluder (ASO) has become the procedure of choice in most cardiac centers. However, despite its technical simplicity the procedure is still associated with complications which, although very rare, are potentially life-threatening. The Cocoon septal occluder (CSO) is an improved ASO with certain design features (Nanoplatinum coating, softness) that may potentially reduce the risk of device related erosions and nickel allergic reactions. We design a randomized controlled study to prospectively compare the efficacy and safety of these two devices. 
Methods: 636 patients (median age 14.5 years) from 4 major centers in Greece and Romania were randomly assigned in a 1:1 ratio to catheter ASD closure using the ASO (group 1) and CSO (group 2 ), respectively. The procedure was guided by fluoroscopy and $2 \mathrm{D}$ and 3D transesophageal echocardiography.

Results: Mean echocardiographic ASD diameter was $21 \pm 7 \mathrm{~mm}$ (range $14-35 \mathrm{~mm}$ ), and $22 \pm 6 \mathrm{~mm}$ (range $12-34 \mathrm{~mm}$ ) in group 1 and group 2, respectively. Mean device diameter was $24 \pm 9 \mathrm{~mm}$ (range $17-40 \mathrm{~mm}$ ) and $22 \pm 8 \mathrm{~mm}$ (range $14-38 \mathrm{~mm}$ in group 1 and group 2 , respectively. The device was permanently implanted in $310(98 \%)$ and 309 (97\%) patients of the group 1 and group 2, respectively. Follow-up (FU) ranged from 6- 48 months. Complete ASD occlusion at 1 month FU was $99 \%$ in both groups of patients. One device erosion (required surgical removal of the device) and 3 severe skin allergic reaction (AR) that required chronic treatment with cortisone were observed in one and 3 patients, respectively, of group 1. No device erosions or ARs were observed in group 2. Device embolization due to insufficient septal rims was observed in 3 and 4 patients of group 1 and group 2 , respectively. No other major complication occurred in both groups of patients.

Conclusions: The ASO is a safe and effective in the great majority of cases. The CSO is a safe and effective device that adds to our armamentarium for as much safer catheter ASD closure. Further studies with a larger patient population are needed to confirm our results.

\section{O9-4}

\section{Interventional closure of Sinus Venosus ASD}

Velasco Forte M.N. (1,2,3), Riahi M. (4), Byrne N. (5), Hermuzi A. (4), Jones M. (4), Baruteau A.E. (4), Israel Valverde (1,2,4), Qureshi S. (1,2), Rosenthal E. $(1,2)$

1. Division of Imaging Sciences and Biomedical Engineering, King's College London, London, United Kingdom; 2. Cardiovascular Pathology Unit, Institute of Biomedicine of Seville, IBIS, Virgen del Rocio University Hospital/CSIC/University of Seville, Spain; 3. Queen Elizabeth University Hospital, Glasgow; 4. Department of Paediatric and Adult Congenital Heart Disease, Evelina London Children's Hospital, Guy's and St Thomas NHS Foundation Trust, London, United KingdomDepartment of Medical Physics, Guy's and St. Thomas' NHS Foundation Trust, London, United Kingdom; 5. Department of Medical Physics, Guy's and St. Thomas' NHS Foundation Trust, London, United Kingdom

Introduction: Superior sinus venosus atrial septal defect (SVASD) is commonly associated with partial anomalous pulmonary venous drainage (PAPVD). In selected patients, it is possible to correct this anomaly by deploying a covered stent at the level of the superior vena cava (SVC) and right atrial (RA) junction. Objectives: We describe the first series of percutaneous SVASD and PAPVD correction using a two-step simulation for procedural planning. Methods: Patients with SVASD and right PAPVD with a clinical indication for correction were selected. They underwent a twostep evaluation consisting in an ex-vivo procedural simulation on a 3D-printed model followed by an in-vivo simulation using balloon inflation in the targeted stent landing zone. The percutaneous procedure consisted in deploying a $10 \mathrm{Zig}$ custom-made covered stent in the SVC-RA junction.

Results: Five patients were referred for pre-procedural anatomical evaluation and were deemed suitable for percutaneous correction. The procedure was successful in all patients with no residual interatrial shunt and successful redirection of the pulmonary venous drainage to the left atrium. At a median clinical follow-up of 8.1 months (2.6-19.8), no adverse events were noted and all

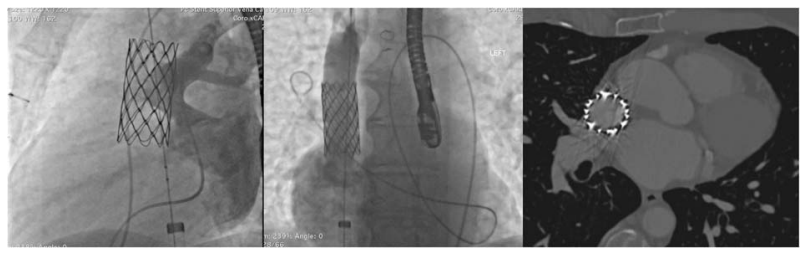

Figure.

patients showed clinical improvement. During follow-up, transthoracic echocardiography and MDCT in 4 patients or invasive angiography in one patient demonstrated a patent SVC stent, no residual SVASD and unobstructed PV drainage in all patients.

Conclusions: In carefully selected patients using a two-staged simulation strategy, percutaneous correction of SVASD with PAPVD is feasible, safe and led to favourable midterm outcomes.

\section{O9-5}

The impact of improved percutaneous pulmonary valve design on patient characteristics: Smaller, younger and more complex

Lehner A. (1), Flaig M. (1), Fernandez Rodriguez S. (1), Ulrich S. (1), Kanaan M. (2), Dalla-Pozza R. (1), Haas N.A. (1)

(1) Ludwig-Maximilians University, Department of Pediatric Cardiology and Pediatric Intensive Care, München, Germany, (2) Heart and Diabetes Center NRW, Department of Pediatric Cardiology, Bad Oeynhausen, Germany

Objectives: The SAPIEN 3 (S3; Edwards Lifescience, Irvine, CA, USA) is a new design of a transcatheter heart valve (THV) with sizes from 23 to $29 \mathrm{~mm}$. Our objective was to compare the patient characteristics receiving earlier Edwards THV types (Sapien valves and Sapien XT valves) to the current patient population eligible for percutaneous pulmonary valve implantation (PPVI).

Methods: During 2011 and 2017, 74 patients were treated with PPVI in two centers. Patient data and valve types were evaluated retrospectively.

Results: From 2011 to 2013 the Sapien valve was implanted in 24 cases, Sapien XT from 2013 to 2016 in $n=24$. Since 2015 PPVI was increasingly performed with the $\mathrm{S} 3$ valve $(\mathrm{n}=26)$. In the S3 group, patients showed a tendency to be younger $(p>0,05)$ and were significantly smaller $(\mathrm{p}<0,05)$ when compared to earlier THV types:

Sapien $3(\mathrm{n}=26)$ : Median age 13,5 ; range $5,8-52$ years; Median weight 51,5 kg; range 15,0-93,0 kg; $(\mathrm{n}=4<20 \mathrm{~kg})$

Sapien XT $(n=24)$ : Median age 17,7; range 7,8-44,2 years; Median weight $64 \mathrm{~kg}$; range $21,5-106 \mathrm{~kg}$.

Sapien $(n=24)$ : Median age 17,9; range 6,6 - 42 years; Median weight $60 \mathrm{~kg}$; range $20,0-135 \mathrm{~kg}$;

Valve sizes used were mostly 23 and $26 \mathrm{~mm}$ (Sapien $\mathrm{n}=23$; XT $\mathrm{n}=16$; S3 $\mathrm{n}=20$ ). The largest $29 \mathrm{~mm}$ model is increasingly used during the last years (XT $n=3, S 3 n=6)$.

Compared to the initial Sapien group, the S3 group showed a broader variety of underlying congenital heart diseases (CHD) and increased procedural complexity (Sapien-in-Melody implantation, PPVI in a single PA, PPVI in the native RVOT).

Conclusion: Compared to the Edwards Sapien and XT valves, patients who received the latest Edwards Sapien 3 valve tended to be smaller (even below $20 \mathrm{~kg}$ ) and younger. Right now PPVI is applicable in a broader variety and more complex cohort of CHD. A THV size of $29 \mathrm{~mm}$ is increasingly utilized. 
O9-7

Hybrid Procedure of Right Ventricle Outflow Tract Stenting in Small Infants with Pulmonary Atresia and Ventricular Septal Defect: Early and Mid-term Results from a Single Center

Bondanza S., Calevo M.G., Derchi M.E., Santoro F., Marasini M. Gaslini Institute, Genoa, Italy

Introduction: Pulmonary Atresia, Ventricular Septal Defect (PA-VSD) with Major Aorto-pulmonary Collateral Arteries (MAPCAs) and Hypoplasia of Pulmonary Arteries is a rare and complex congenital defect which requires early interventions to relieve cyanosis and enhance the growth of native pulmonary arteries. The treatment of these patients is still controversial. Surgical techniques require cardiopulmonary bypass which can be poorly tolerated by small infants. Percutaneous techniques such as radiofrequency perforation can be very challenging. The hybrid technique consists of perventricular stenting of the Right Ventricle Outflow Tract (RVOT) through medial sternotomy, to restore native pulmonary flow.

Methods: We retrospectively reviewed the cardiovascular database of our Institute in order to analyze our experience in hybrid procedure. We detected 6 patients with PA-VSD, MAPCAs and Hypoplasia of native Pulmonary Arteries, who underwent hybrid first approach between November 2007 and December 2015. We report our early results and mid- term outcomes.

Results: Median age at the procedure was 26 days, median weight was $3150 \mathrm{~g}$, median Nakata index was $52 \mathrm{~mm} 2 / \mathrm{m} 2$. All procedures were successful except for one: this patient underwent a surgical shunt. No immediate and early deaths or major complications occurred and Oxygen saturation levels increased in all the patients. Patients were followed up for a period of 12 to 103 months: 4 of them underwent a second procedure of unifocalization at the mean age of 12.5 months. One of these had a late conduit thrombosis and died 2 years later.

Conclusions: We reported data from the largest series of patients who underwent this hybrid procedure. Our experience demonstrated encouraging results to expand the use of this approach to bridge high risk patients with diminutive pulmonary arteries to final surgical repair. Hybrid RVOT perventricular stenting is a safe and feasible approach in selected patients. Mid and longer term outcomes of this cardiopathy depend mainly on native pulmonary arteries size and growth.

\section{O10-1}

A Novel Non-invasive Quantification of Myocardial Work of the Left Ventricle in Patients after Repairing Tetralogy of Fallot

Yasukochi S., Saikawa Y., Takigiku K., Takei K.

Nagano Children's Hospital, Azumino, Japan

We investigated an efficacy of regional myocardial work index (MWI) of the left ventricle (LV) in children after repairing tetralogy of Fallot (TOF) by using a novel non-invasive quantification echocardiographic imaging. MWI at 18 segments of LV was calculated by pre-installed program of E95 and EchoPac (GE HMS) from regional LV pressure (LVP)-strain loop areas which was constructed from assumed LVP curve in combination with strain by speckle tracking from apical three images of LV as previously described (Eur H J 2012:33:724-733). The sixteen patients of TOF (14.9y) and 16 normal control (N) (13.6y) were enrolled.

Results: The global strain and MWI were significantly higher in $\mathrm{N}$ $(-19,2064 \% / s)$ than in $\operatorname{TOF}(-17.3,1682)$ as same as Myocardial work waste $(\mathrm{MWW})$ in $\mathrm{N}(92 \% / \mathrm{s})$ and in TOF $(74.9 \% / \mathrm{s})$. This

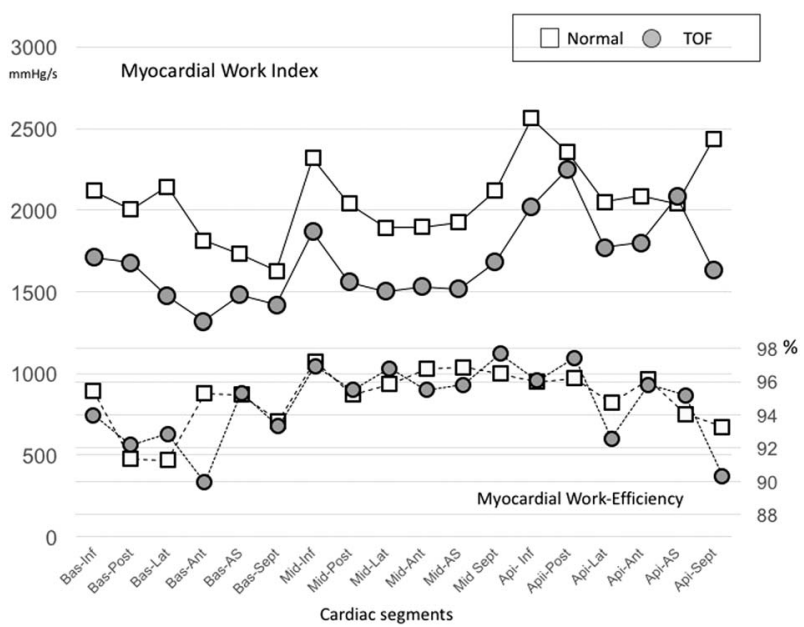

Figure 1.

results suggested $\mathrm{MW}$-efficiency (MWE), defined as $\{(\mathrm{MWI}-$ MWW)/MWI $\}$ was reserved in TOF as Fig.1. In TOF, MWW tended to demonstrate weak positive correlation with the ratio of $\mathrm{RVp} / \mathrm{LVp}$ and negative one with the ratio of RVEDV/LVEDV, however, MWE was reserved in patients not related to LV ejection fraction if not with heart failure. This means the regional myocardial energetic efficiency remains reserved until LV collapsed.

In conclusion, this non-invasive quantification of MWI could provide a new insight of regional myocardial energetics beyond deformation.

O10-2

Daily heart rate dynamics reflects global Fontan pathophysiology

Suzuki D., Ohuchi H., Morimoto Y., Nigishi J., Sakaguchi H., Shiraishi I., Kurosaki K.

National Cerebral Cardiovascular Center, Suita, Japan

Introduction: A large body of evidence shows that increased resting heart rate (HR) and arrhythmia are associated with poor prognosis in patients with chronic heart failure. Although Fontan pathophysiology shares common pathophysiology of chronic heart failure, precise association of daily HR dynamics with Fontan pathophysiology has not been elucidated.

Methods: We prospectively evaluated daily HR dynamics (bpm) with Holter ECG monitoring in consecutive 223 patients after the Fontan operation (age 17.1 \pm 8.3 years). We obtained maximum heart rate (Max), minimum heart rate (Min), mean heart rate (Mean), and total number of premature atrial and ventricular contraction (PAC, PVC, respectively) as their daily HR dynamics. We compared these HR-dynamics with central venous pressure (CVP), cardiac index $(\mathrm{CI})$, arterial oxygen saturation $(\mathrm{SaO} 2)$, ejection fraction of the systemic ventricle (EF), and peak oxygen uptake (VO2) during exercise testing. We excluded Fontan patients with pacemaker implantation and/or those with beta blocker.

Results: Max, Min, Mean was $123 \pm 20,62 \pm 13,84 \pm 13$, respectively. PAC and PVC was found in $74.9 \%$ and $67.3 \%$ in all patients. Max, Min, and Mean were inversely correlated with age $(\mathrm{p}<0.001$ for all). Min was lower in male patients $(\mathrm{p}=0.0435)$ and positively correlated with CI $(\mathrm{p}<0.0001)$. PAC was more frequent in patients with atrio-pulmonary Fontan connection $(\mathrm{p}=0.0015)$ and the total number of PAC was inversely correlated with CI $(\mathrm{p}<0.0001)$. Increased PVC was inversely correlated with 
EF $(p=0.0587)$, arterial oxygen saturation $(p=0.014)$, and peak $\mathrm{VO} 2(\mathrm{p}<0.0001)$. Max and increased number of PVC were independently correlated with lower exercise tolerance $(\mathrm{p}<0.05-0.01)$. Conclusions: Daily HR-dynamics, including arrhythmia, based on Holter ECG monitoring reflects global Fontan pathophysiology. Periodic HR-monitoring with Holter monitoring is a useful noninvasive tool to monitor Fontan pathophysiology.

\section{O10-3}

Ambulatory Intravenous Inotropic Support and or Levosimendan in Failing Pediatric and Congenital Heart Disease: Safety, Survival, Improvement or Transplantation Apostolopoulou S.C., Vagenakis G.A., Tsoutsinos A., Kakava F., Rammos $S$.

Onassis Cardiac Surgery Centre, Athens, Greece

Introduction: End-stage heart failure (HF) frequently needs continuous inotropic support in hospital and has high morbidity and mortality. It is often treated with mechanical circulatory support or transplantation, both options associated with significant adverse events and not readily available in many countries.

Methods: This is a retrospective analysis of our experience using continuous ambulatory inotropes (AI) and/or periodic levosimendan (LS) infusions in pediatric end-stage HF patients in a tertiary care center focusing on outcome, efficacy and safety of AI and/or LS infusions in HF patients.

Results: The study included 27 patients aged 9.3 7.4 (0.1-26.1) years with severe HF (6 myocarditis, 13 dilated cardiomyopathy, 2 restrictive cardiomyopathy, 6 repaired congenital heart disease) needing continuous inotropic support. Overall, 21 patients received dobutamine and milrinone $\mathrm{AI}$ through a permanent central catheter for $1.1 \pm 0.9$ (0.3-3.7) years. Additionally, 14 AI patients and the remaining 6 study patients received periodic LS infusions for $1.4 \pm 1.0(0.1-4.2)$ years. Inotropes were used for $1.4-0.4$ years as bridge to recovery in 6 improved myocarditis patients, who remained stable after discontinuation on follow-up. AI and or LS infusions were used as bridge to transplantation in 6 patients with only 3 survivors, in 2 of which inotropes preoperatively reversed severe combined pre and postcapillary pulmonary hypertension allowing successful heart only transplantation. Finally, inotropes were used as mainstay therapy in 15 patients for 0.3-4.2 years, mostly with good quality of life and family dynamics. Four patients died of worsening HF after 0.8-2.1 years of therapy. During 3.6 \pm 5.3 (0.321.3) years of follow-up, we observed 4 central line infections treated with antibiotics and 4 catheter reinsertions due to dislodgement. Parenteral inotropes were also discontinued in 1 cardiomyopathy patient who received a left ventricular assist device and is still waiting for transplant 2.5 years later.

Conclusions: AI and/or LS infusions in HF is safe and beneficial for long periods even in small infants and children, allowing stabilization, discharge from hospital and cost reduction, good quality of life. It may provide precious time for heart transplantation or myocardial remodeling, improvement and possible discontinuation even after long periods of support.

\section{O10-4}

Early cardiac evaluation in Duchenne or Becker muscular dystrophy using quantitative assessment of late gadolinium-enhanced magnetic resonance imaging

Takeda A. (1), Aikawa T. (2), Oyama-Manabe N. (3), Yamazawa H. (1), Izumi G. (1), Sasaki O. (1), Abe J. (1), Fujimoto T. (1) Department of Pediatrics, Hokkaido University Hospital, Sapporo, Japan (1); Department of Cardiovascular Medicine, Faculty of Medicine and
Graduate School of Medicine, Hokkaido University, Sapporo, Japan (2); Department of Diagnostic and Interventional Radiology, Hokkaido University Hospital, Sapporo, Japan (3)

Introduction: Cardiac involvement in Duchenne or Becker muscular dystrophy (DMD/BMD) has been postulated to be often overlooked. Recently, cardiac magnetic resonance imaging with late gadolinium enhancement (CMR-LGE) has enabled visualization of the fibrotic area in the myocardium, making it a reliable modality for early diagnosis of cardiomyopathy in DMD/BMD. However, quantitative assessment of CMR-LGE in DMD/BMD has not been established.

Methods: Forty-six consecutive CMR-LGE images in 26 patients with DMD/BMD (2.9-29.0 years old, median 11.0 years old) were studied retrospectively. \%tLGE (ratio of LGE area to LVmass), LGEn (number of segmental area with positive LGE), Indexed left ventricular end-diastolic volume (LVEDVI) and left ventricular ejection fraction (LVEF) were obtained at later analysis. 1) The relationship between \%tLGE and LGEn, and 2)The relationship between LVEDVI, LVEF and \%tLGE, and the cut-off value of $\%$ LGE for LVEDVI $>92 \mathrm{ml} / \mathrm{BSA}$ and LVEF $<50 \%$ were studied.

Using 26 CMR-LGE data at first scan of each patient, 3) The relationship between LVEDVI, LVEF, \%tLGE and age, and the cut-off age for LVEDVI $>92 \mathrm{ml} / \mathrm{BSA}$ and LVEF $<50 \%$ were studied. 4) The comparison of LVEDVI, LVEF, \%tLGE between two groups divided by an age (Group A $(n=11),<10$ years old, GroupB $(n=16),>=10$ years old) were studied.

Results: 1) \%tLGE were strongly correlated with LGEn ( $\mathrm{r}=0.98)$. 2) Both LVEDVI and LVEF were strongly correlated with \%tLGE $(\mathrm{r}=0.63,0.73$ respectively). Cut-off value of $\% \mathrm{tLGE}$ for $\mathrm{LVEF}<$ $50 \%$ and LVEDVI $>92 \mathrm{ml} / \mathrm{BSA}$ were $5 \%$ and $28 \%$, respectively. 3) With advancing years with age, \%LGE increased $(r=0.27)$, LVEF decreased $(\mathrm{r}=0.33)$, and LVEDVI mildly increased $(r=0.42)$. Ten years old was a cut-off point of both $\mathrm{LVEF}<50 \%$ and LVEDVI $>92 \mathrm{ml} / \mathrm{BSA}$.

4) \%tLGE and LVEDVI were significantly higher in Group B. LVEF was significantly lower in Group B.

Discussion: \%tLGE reflected the extension of fibrosis in LV myocardium as well as LV pump function. We concluded that 10 years old was a turning point of progressing LV fibrosis and reducing LV pump function. Therefore, cardioprotection should be considered before 10 years old.

Conclusions: Quantitative assessment of CMR-LGE could be a gold standard method for cardiac evaluation in patients with DMD/BMD.

\section{O10-5}

Surgical Therapy of Obstructive Airway Disease in Patients with Intrinsic Tracheobronchial Malformations or Airway Stenosis Associated with Vascular Ring - Long Term Results

Loukanov T. (1), Fonseca E. (1), Springer W. (2), Rauch H. (3), Uhl S. (2), Ziesenitz V. (2), Gorenflo M. (1) Congenital Cardiac Surgery, University Hospital Heidelberg, Germany (1); Paediatric \& Congenital Cardiology, University Hospital Heidelberg, Germany (2); Anesthesiology, University Hospital Heidelberg, Germany (3)

Introduction: We analyzed the outcome of surgical airway reconstruction using cardiopulmonary bypass (CPB) as additional technique during more extensive reconstruction procedures which would have been limited by conventional ventilation.

Methods: In this retrospective single center analysis, we included all patients presenting in the period between 2008 and 2017 with 
severe respiratory failure due to airway obstruction/compression caused by (1) intrinsic malformations of the trachea leading to respiratory failure or (2) vascular and/or congenital heart defects associated with respiratory failure. All patients underwent bronchoscopy, additional imaging was performed by CT or MRI, and in selected cases by cardiac catheterization. Follow-up data were obtained from follow-up examinations.

Results: 114 patients, aged 0.8 years [10 days -49 years] (Median [range]) presenting with vascular ring (78 out of 114), intrinsic tracheal malformations $(n=24 / 114)$, pulmonary sling $(n=12$ / 114) were included. $33 / 114$ patients had additional cardiac malformations including VSD $(n=15 / 33)$, Fallot/DORV 4/33, aortic arch hypoplasia (1/33) or complex CHD (13/33). Surgical techniques included a) decompression of airway by transection of ligaments or vascular remnants $(n=82), b)$ partial resection of stenotic segments of the trachea $(n=19)$, c) slide tracheoplasty $(n=9)$. In selected cases, external re-expansion of trachea was used $(\mathrm{n}=4)$. CPB was used in $75 / 114$ patients and was a pre-requisite for all patients in whom tracheal procedures were needed or whenever additional intracardiac repair was performed. During the follow-up period, 10 syndromic patients died: 3 patients (Trisomy $21 \mathrm{n}=1$, VACTERL $\mathrm{n}=2$ ) died due to neurological complications. Seven patients died due to multi-organ failure (one due to sepsis after leakage of tracheal anastomosis). The surviving 104 patients showed clinical improvement or - if previously ventilator dependent - could be weaned successfully.

Conclusions: Relief of vascular compression and/ or reconstruction of the trachea and main bronchi can be performed safely in patients with severe airway malformations by using $\mathrm{CPB}$ and modern reconstructive techniques. However, pre-existing syndromatic disease and co-existing multimorbidity must be taken into account in decision making.

\section{O10-6}

Neurodevelopment outcomes following fetal diagnosis of Ebstein Anomaly or Tricuspid Dysplasia

Marini D. (1), Lim J.M. (1), Saini B. (1), Roy C. (1), Mawad W. (1), Ly L. (1), Chau V. (1), Grosse-Wortmann L. (1), Yoo S.J. (1),

Jaeggi E. (1), Macgowan C. (1), Miller S. (1), Seed M. (1)

The SickKids Hospital, Toronto, Canada

Background: Data concerning neurodevelopmental outcome in subjects with Ebstein Anomaly (EA) or severe Tricuspid Valve Dysplasia (TVD) are lacking. New cardiovascular magnetic resonance (CMR) technology allowing hemodynamic assessment in the fetus could provide insights into the mechanisms of brain injury and dysmaturation as well as prognostic information. We aimed to investigate the clinical and neurodevelopment outcome of patients following fetal diagnosis of EA/TVD.

Population and methods: From 2013 to 2017, we identified 15 fetuses with EA and 6 fetuses with TVD at our institution at a median gestational age of 23 weeks. Of this cohort, 12 had fetal $\mathrm{CMR}$ at a median gestational age of 36 weeks. Fetal biometry and hemodynamics were compared with those in 30 normal fetuses. Results: Two fetuses underwent early termination of pregnancy. Three fetuses died in utero, four developed hydrops but survived to birth, five had circular shunts, 11 had functional or anatomical pulmonary atresia, and five had pericardial or pleural effusions. The tricuspid regurgitation was severe in 17 , moderate in three and mild in one. CMRI revealed significantly lower combined ventricular output $(p<0.001)$, superior vena caval flow $(p<0.01)$ and fetal oxygen delivery $(\mathrm{p}<0.01)$ in EA/TVD fetuses compared to controls. EA/TVD fetuses had increased oxygen extraction fraction $(p<0.01)$, but body and brain weight $z$-scores were still

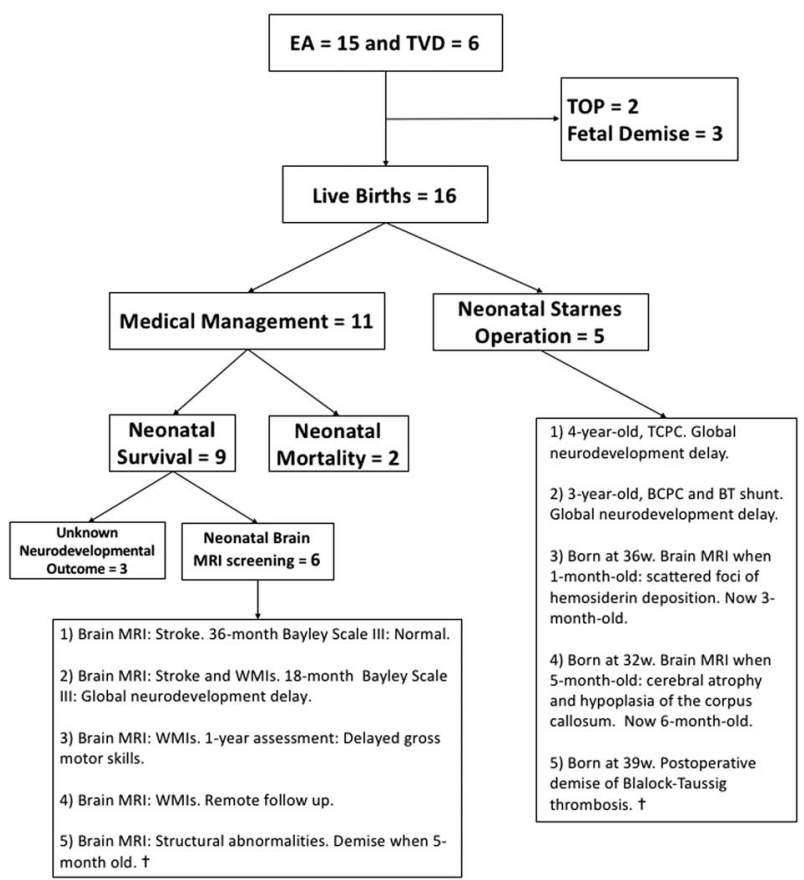

Figure 1.

BCPC: bidirectional cavopulmonary connection; EA: Ebstein anomaly; TCPC: total cavopulmonary connection; TVD: Tricuspid Valve Dysplasia; TOP: termination of pregnancy.

reduced $(\mathrm{p}<0.01$ and $\mathrm{p}<0.001)$. Of the 12 fetuses having had fetal CMR, 9 survived and 8 had neurodevelopment assessment. Brain MRI, neurodevelopmental and clinical outcomes are summarized in Figure 1.

Conclusions: Neurodevelopmental delay appears to be very common in all severe forms of EA and TVD regardless of the mode of management. This might be explained by the severity of cerebral hypoxia ischemia in this form of congenital heart disease, which is already present in fetal life, and which persists postnatally, both in subjects undergoing palliative surgery and in subjects managed conservatively. Cerebral lesions are generally present on brain MR at birth, even when patients are born at term and managed conservatively. These preliminary data might be of help in prenatal counselling and strongly support the need for early neurodevelopmental assessment and life-long follow-up, irrespective of the surgical approach.

O11-1

From neonates to adults in Marfan syndrome- Diagnosis, progress and transition to adult care

Stark V.C. (1), Olfe J. (1), Doering K. (1), Kozlik-Feldmann R. (1), Mueller G. C. (1), Seggewies F. (1), von Kodolitsch Y. (2), Mir T.S. (1) Clinic for Pediatric Cardiology, University Heart Center, Hamburg,

Germany (1), Clinic for Cardiology, University Heart Center, Hamburg, Germany (2)

Objectives: Medical care for chronic diseases like Marfan syndrome (MFS) is a big challenge. Thereby long-term outcome and quality of life (QoL) are essential and highest aim for patients. Early diagnosis, due to improvement of genetics and clinical judgment, allows insights of disease development and possible preventive strategies in childhood. In consequence, patients require lifelong follow-up and need an effective transition to adult medicine. We hereby present more than 10 years of follow-up with a distinguish insight of all aspects of diagnosis and prophylaxis in MFS. 
Table 1. MFS Pathologies in children/ young adults.

\begin{tabular}{llll}
\hline Pathology & $\begin{array}{l}\text { Prevalence } \\
(<\mathbf{1 8 y})\end{array}$ & $\begin{array}{l}\text { Prevalence } \\
(>\mathbf{1 8 y})\end{array}$ & $\mathbf{P}$ \\
\hline Sinus valsalvae & $73 / 115(63 \%)$ & $24 / 34(70.6 \%)$ & $\mathrm{ns}$ \\
MVP & $59 / 115(51 \%)$ & $19 / 34(55.9 \%)$ & $\mathrm{ns}$ \\
Systemic & $54 / 115(46 \%)$ & $18 / 34(52.9 \%)$ & $\mathrm{ns}$ \\
$\quad$ manifestation & & & \\
Ectopia lentis & $21 / 115(18 \%)$ & $8 / 34(23.5 \%)$ & $\mathrm{ns}$ \\
Dura ectasia & $37 / 69(53 \%)$ & $19 / 31(61.2 \%)$ & $\mathrm{ns}$ \\
\hline
\end{tabular}

Methods: Since 2008 we investigated 424 patients in our specialized pediatric Marfan clinic whereas in 149 MFS was diagnosed $(9.5 \pm 5.8 \mathrm{y} ; \mathrm{m}: 62 \%)$ according Ghent Criteria (GC). We attended 978.5 patient years. We analyzed criterions of GC, requirement of prophylaxis and QoL in children and adults and evaluated effectiveness of transition to adult care.

Results: Analysis of GC showed age dependent increase of prevalence (Table 1). Medical prophylaxis was initialized in 80 patients (<18y 52\%; >18y 62\%; 10.2 \pm 5.1 y). Significant z-score reduction could be achieved with sartan and/or betablockers (BB). Seven patients required aortic root replacement $(11.5 \pm 5.6 \mathrm{y})$ in childhood. There was no dissection or mortality. Compared to adults QoL did not show significant impairment in children. From 34 adults $59 \%$ were accompanied to adult care, 35\% are still in pretransition section, $5 \%$ are lost in follow-up.

Conclusions: Age dependent onset of symptoms remains a challenge in MFS. Especially onset of cardiovascular abnormalities in young adults is very dynamic. To prevent complications early medical prophylaxis is effective and essential in childhood (Sartan and/or BB). Lifelong follow-up examinations need to be standardized and a safe cooperation of pediatric cardiologists and adult cardiology is important. Patients in transition progress require special attention, assistance and a close contact to the patient and family. Finally, success of transition needs to be reevaluated before pediatric patient care ends.

\section{O11-2}

Functional Outcomes in children with Transposition of the Great Arteries after Arterial Switch Operation

Häcker A.-L. (1,2), Reiner B. (2), Hager A. (1), Oberhoffer R. (1,2), Ewert P. (1), Müller J. (1,2)

Department of Pediatric Cardiology and Congenital Heart Disease, Deutsches Herzzentrum München, Technische Universität München, Germany (1); Institute of Preventive Pediatrics, Technische Universität München, Germany (2)

Objective: Despite decreasing mortality and morbidity in children with transposition of the great arteries (TGA) after arterial switch operation (ASO), recent studies still report functional impairments. This study analyzes intima-media-thickness (IMT), healthrelated physical fitness (HRPF) and health-related quality of life (HRQoL) in children after ASO.

Methods: In total, 68 children after ASO (12.9 \pm 3.7 years, 19.1\% female) were investigated between August 2014 and October 2017. The IMT was measured by ultrasound, HRPF was assessed by five tests of the FITNESSGRAM $®$ and HRQoL was analyzed with a self-report questionnaire (KINDL-R). All test results were compared to a recent healthy reference cohort (RC: $n=2116$, $49.1 \%$ female) and adjusted for sex and age differences.

Results: Children after ASO had a significantly increase in IMT (TGA: $0.489 \mathrm{~mm}$, SE: 0.006, RC: $0.465 \mathrm{~mm}$, SE: 0.001, p <.001) and impairments in HRPF (TGA z-score: $-0.58 \pm 0.81, \mathrm{p}<.001$ ) compared to the RC whereas HRQoL did not differ between these groups (HRQoL score: TGA: 75.2, SE: 1.280, RC: 76.5, SE: $0.360, p=0.315)$. Neither the classification into simple and complex TGA, nor the statistical analysis regarding the coronary pattern, resulted in a significant difference in-between the groups for all health-related outcomes.

Conclusions: Children after ASO exhibit detrimental IMT and impaired HRPF. Long-term consequences of a thickened IMT remain unclear and demand further physiological analysis. But the need for long-term follow-ups is emphasized by this analysis and special promotion might be necessary to reduce impairments concerning health-related outcomes in children after ASO.

\section{O11-3}

Challenges of clinic surveillance following coarctation of the aorta repair

Dumitrascu-Biris I., Theocharis P., Prabhu N., Charakida M., Simpson J.M. Department of Fetal and Paediatric Congenital Heart Disease, Evelina London Children's Hospital,UK

Introduction: Systemic hypertension following coarctation of the aorta (COA)repair is well described in adulthood. However, data in childhood are limited and blood pressure monitoring in clinical setting remains challenging.

Aims: The aim of this study was to assess the incidence of high blood pressure in COA patients following surgical or interventional repair and assess its determinants.

Methods: Our study population consisted of 123 patients who attended the cardiac outpatient clinic between January 2016January 2017 and had surgical or interventional COA repair. Patients with isolated COA, associated atrial or ventricular septal defects or in associations with complex lesion were included.

Study design: Retrospective data collection from electronic cardiac outpatient clinic letters. Systolic and diastolic blood pressure (BP) was measured using automated device in a sitting position. BP measurements were converted to $\mathrm{z}$ scores using Cardio $\mathrm{Z}$ for patients over 1 year and New Zeeland nephrology guidelines for $<1$ year.

Results: From the 123 children only 105 had reliable observations recorded. Systolic BP was $>95$ th gentile in $27 \%$ of children. $18 \%$ of patients were on antihypertensive medication (ACE inhibitor or beta-blocker). $62 \%$ of the patients had isolated COA, $16 \%$ had COA associated with VSD /ASD and 20\% had COA in association with complex lesions. Median age at intervention was 15 days (1-5581) and a metal suspicion was raised in $35 \%$. There was poor correlation between LWPW $z$ scores and SBP $z$ scores $(r=0.330, p=0.746)$. The SBP was significantly associated with the Doppler velocity in the descending aorta $(r=0.319, p=0,012)$ and age at operation and hypertension had a positive correlation $(\mathrm{r}=0.301, \mathrm{p}=0.016)$. Conclusion: The data from this retrospective study demonstrate that increase BP is of concern in childhood. Although one third of children were hypertensive in the cardiac outpatient clinic, half of those were on antihypertensive therapy. Further studies are needed to demonstrate whether aggressive BP medication in childhood will translate in long term cardiovascular benefit.

\section{O11-4}

Transgenerational effects of maternal obesity and gestational diabetes on child body composition and left ventricle mass - RADIEL study $\mathbf{6}$ years follow-up Litwin L. (1,2), Sundholm J.K.M. (1), Rönö K. (3), Koivusalo S.B. (3), Eriksson J.G. (4,5), Sarkola T. (1)

Children's Hospital, University of Helsinki and Helsinki University Hospital, Helsinki, Finland (1); Department of Congenital Heart Defects 
and Pediatric Cardiology, SMDZ in Zabrze, SUM, Katowice, Poland (2); Women's Hospital, University of Helsinki and Helsinki University Hospital, Helsinki, Finland (3); University of Helsinki and Helsinki University Hospital, Helsinki, Finland (4); Folkhälsan Research Center, Helsinki, Finland (5)

Objectives: Maternal obesity and gestational diabetes (GDM) are related to child's early weight gain and long-term cardiovascular risk. Fetal programming has been hypothesized to be the underlying mechanism. We aimed to investigate the influence of maternal adiposity and GDM on child body composition and left ventricle mass (LVM) at 6 years of age.

Methods: The observational follow-up study included 201 motherchild pairs (boys, $n=111$ ), a subcohort from the Finnish Gestational Diabetes Prevention Study. GDM was diagnosed in 96 mothers, 36 of whom required medication. Mother and child's anthropometrics, body composition, blood pressure, and offspring echocardiography were performed at the mean of 6,1 years (SD 0,5) from delivery.

Results: Maternal pre-pregnancy BMI $\left(30,5 \mathrm{~kg} / \mathrm{m}^{2}\right.$; SD 5,6$)$ correlated with child body fat percentage $(\mathrm{BF} \% ; \mathrm{r}=0,2 ; \mathrm{p}=0,01)$ and was reflected in increased child BMI (mean z-score $0,45 \mathrm{~kg} / \mathrm{m}^{2}$; SD 0,93). LVM correlated with child and maternal anthropometrics, lean body mass (LBM), fat mass, child's age, sex, and systolic blood pressure (Table 1). In a stepwise linear regression model, LVM was independently predicted only by child LBM $\left(\mathrm{r}^{2}=0,434\right)$. LVM, left ventricle mass index (LVMI, $\mathrm{g} / \mathrm{m}^{2.09}$ ) and LVM $\mathrm{z}$-score were not associated with pre-pregnancy BMI, GDM exposure, or child BF\%. LVMI and LBM were higher ( $<<0,001 ; \mathrm{p}=0,002)$, but BF\% lower $(\mathrm{p}<0,001)$, in boys compared with girls.

Conclusions: LVM at six years of age depends predominantly on child LBM, which is correlated with height, weight, and sex, and influenced by maternal LBM. Maternal pre-gestational adiposity is reflected in child adiposity, increasing future cardiovascular risk, but no association between LVM and child adiposity or evidence of fetal programming related to GDM was found in early childhood.

Table 1. Determinants of LVM.

\begin{tabular}{lcr}
\hline & r & p-value \\
\hline Age & 0,241 & 0,001 \\
Height & 0,629 & $<0,001$ \\
Weight & 0,605 & $<0,001$ \\
BMI Z-score & 0,308 & $<0,001$ \\
Waist-hip ratio & $-0,06$ & 0,41 \\
Lean body mass & 0,659 & $<0,001$ \\
Fat mass & 0,435 & $<0,001$ \\
Body fat \% & 0,096 & 0,174 \\
Systolic blood pressure & 0,265 & $<0,001$ \\
Maternal height & 0,294 & $<0,001$ \\
Maternal weight & 0,199 & 0,005 \\
Maternal lean body mass & 0,313 & $<0,001$ \\
Maternal fat mass & 0,147 & 0,04 \\
Maternal body fat \% & 0,044 & 0,54 \\
Pre-pregnancy BMI & 0,126 & 0,08 \\
\hline
\end{tabular}

\section{O11-5}

CHIP-Family to improve psychosocial wellbeing of young children with congenital heart disease and their families van der Mheen M. (1), McCusker C.G. (2), van Beynum I.M. (1), Dulfer K. (1), van Galen E. (3), Bogers A.J.J.C. (1), Hillegers M.H.J. (1), Helbing W.A. (1), Utens E.M.W.J. $(1,4,5)$

Erasmus MC - Sophia Children's Hospital, Rotterdam, The Netherlands (1); School of Applied Psychology, University College Cork, Cork,
Ireland (2); Dutch Patient Association for Congenital Heart Disease, Maarssen, The Netherlands (3); Research Institute of Child Development and Education, University of Amsterdam, Amsterdam, The Netherlands (4); Academic Centre for Child and Adolescent Psychiatry the Bascule/ Department of Child and Adolescent Psychiatry, Academic Medical Centre, Amsterdam, The Netherlands (5)

Introduction: Children with congenital heart disease (CHD) are at increased risk for behavioral, emotional, and cognitive problems. They often have reduced stamina and participate less in sports. Moreover, parents of children with CHD are at risk for psychosocial problems. Therefore, a family-centered psychosocial intervention for CHD-children is needed. The previously developed 'Congenital Heart Disease Intervention Program (CHIP) School' targeted parents only and resulted in non-significant, though positive, effects as to child psychosocial wellbeing. We expanded CHIP by adding a specific child module and including siblings, creating the multidisciplinary CHIP-Family intervention. Methods: Ninety-three families of children with CHD (4-7 years old) participated in this single-blinded randomized controlled trial. Participants were randomized into CHIP-Family or care as usual. CHIP-Family consisted of a separate one-day parent and child workshop. The child workshop was provided by two psychologists and a physiotherapist and consisted of cognitive behavioral exercises and sports exercises. The parent workshop was provided by two psychologists and a pediatric cardiologist and focused on problem prevention therapy, psychoeducation, parenting skills, and medical issues. Approximately four weeks after the workshop, parents received an individual follow-up session. Baseline (T1) and follow-up assessments (T2 $=6$ months after T1) consisted of questionnaires filled out by the child, parents, and teacher.

Results: The acceptability of the CHIP-Family program to the parents in the intervention group was assessed approximately 2 weeks after completing the program. On average, overall program satisfaction was rated with an 8 (on a scale of 1 to 10). Parents rated the explanation of the cardiologist (86.4\%) and meeting other families of children with CHD (77.3\%) as most helpful components of the program. Preliminary results with regards to the effect of CHIP-Family on child emotional and behavioral problems will be presented.

Conclusions: Multidisciplinary psychosocial interventions for families of children with CHD are needed. The CHIP-Family intervention has been positively evaluated by participating parents.

O11-6

Economic Evaluation of Outreach Clinics in Pediatric Cardiology in the Province of Québec

Fournier A. (1), Ndao E.M. (2), Borgès Da Silva R. (2)

CHU Sainte-Justine (1), Public Health School, University of Montreal

(2); Montreal, Quebec, Canada

Introduction and Objectives: In the west of the Quebec province, the CHU Sainte-Justine (CHUSJ), a university pediatric hospital located in Montreal, offers pediatric cardiology services for the diagnosis and management of all cardiac conditions. To minimize the cost of family travel, pediatric cardiologists set up outreach clinics in the local hospitals of the network. The objective of this study was to conduct an economic evaluation of pediatric cardiology outreach clinics from patient and health care system perspectives.

Methods: The sample of this economic evaluation consisted of all patients who had a pediatric cardiology consultation or follow-up in the outreach clinics for the year 2015 (5292 visits). Two alternatives were evaluated: identification and evaluation of the costs (direct and indirect) associated with a visit in an outreach clinic 
with an equivalent visit at the CHUSJ. Both alternatives were compared using the cost differential method.

Results: The direct medical costs for the patients was zero because the medical visits are completely reimbursed by the state. The loss of productivity associated with the visit if the patient had to come to the CHUSJ ranged from 0 to 231 Canadian dollars (\$) with a statistically significant average of $29 \$$ per patient per visit, $p<0.001$. This average increased with remoteness. The indirect costs associated with a visit at CHUSJ were higher, on average $84 \$$ per patient. The visits in the outreach clinics were statistically less expensive than the visits at the CHUSJ, $\mathrm{p}<0.0001$. The visit in an outreach clinic would bring an average total gain of $113 \$$ per patient compared to a visit at CHUSJ, a total of almost $600000 \$$ for the year 2015. Outreach clinics cost an average surplus of $21 \$$ per patient to the health care system. As with the cost paid directly by the patients, the costs for the health care system increased with the distance between the patient's home and the place of consultation, but with smaller differences. Conclusion: Outreach clinics not only increase access to specialized health care in the vicinity of the home of the patients but also reduce associated direct and indirect costs.

\section{O12-1}

Long-term results after percutaneous pulmonary valve implantation (PPVI) - Single centre experience

Eicken A., Tanase D., Georgiev S., Hager A., Meierhofer C., Ewert P. Department of Paediatric Cardiology and Congenital Heart Disease, Deutsches Herzzentrum München, Technische Universität München, Germany

Percutaneous pulmonary valve implantation (PPVI) is the first line treatment option for suitable patients with right ventricular outflow tract (RVOT) dysfunction in many centres. We report on our long-term experience with PPVI.

Since $12 / 2006$ a total of 240 patients (female 84 ) were treated with PPVI. In 225 patients a Medtronic Melody valve was used and in 15 patients an Edwards Sapien valve was implanted $(23 \mathrm{~mm} \mathrm{n}=3$, $26 \mathrm{~mm} \mathrm{n}=8,29 \mathrm{~mm} \mathrm{n}=4)$. Median patient age was 18.4 years (range 4.1-78.9y), weight was $59 \mathrm{~kg}$ (19-176 kg). Indication for treatment was leading RVOT stenosis in 102, regurgitation 38 . both 100. Diagnoses: TOF/PA + VSD 121, common arterial trunc 40, TGA after Rastelli 20, AoS after Ross 27, and miscellaneous 32. The valves were placed in a bioprosthesis in 215 patients, a "native" RVOT was present in 25 patiens. Nearly all patients (97\%) had prestenting of the RVOT with a variety of stents. Periprocedural mortality was $2 / 240 \quad(0.8 \%$, coronary occlusion 1, fatal conduit rupture 1). The gradient in the RVOT was lowered from 38 to $10 \mathrm{mmHg}(\mathrm{p}<0.001)$ and the RV:aortic pressure ratio fell from $67 \%$ to $36 \%$ respectively $(p<0.001)$. During a follow-up of 922 patient years $91 \%$ of all patients still live with the percutaneously implanted pulmonic valve; 15 valves had to be explanted due to endocarditis (8), outgrowth (7) and in six patients a valve in valve procedure was performed.

In conclusion PPVI can be performed after careful patient selection with a low periprocedural morbidity and mortality. Coronary compression and conduit rupture are the procedural hazards. Longterm results are promising but lifelong surveillance is necessary.

\section{O12-2}

Are comorbidities an issue in adults with congenital heart disease? An analysis of the German National Register for Congenital Heart Defects

Tutarel O. (1), Helm P. (2), Diller G.P. (3), Ewert P. (1), Bauer U. (2) Paediatric Cardiology and Congenital Heart Disease, German Heart Centre Munich, Munich/Germany (1); National Register for Congenital Heart Defects and Competence Network for Congenital Heart Defects,
Berlin/Germany, DZHK (German Centre for Cardiovascular Research), Berlin/Germany (2); Division of Adult Congenital and Valvular Heart Disease, Department of Cardiovascular Medicine, University Hospital Muenster, Muenster/Germany (3)

Objectives: Due to the great achievements in the field of congenital heart disease, the number of adults with congenital heart disease (ACHD) is increasing. As congenital heart disease patients are getting older, comorbidities are more frequently encountered. Therefore, the contribution of these comorbidities to patient outcome is gaining importance. An analysis of the prevalence of comorbidities from a contemporary nationwide cohort is lacking. Methods: ACHD (defined as age $>18$ years) with a medical record within the last five years were identified from the German National Register for Congenital Heart Defects. We report data on patient demographics, underlying cardiac defect, disease complexity, associated conditions, and type of procedures.

Results: In total, 4054 patients (mean age $32.95 \pm 11.86$ years, female $n=1948(48.1 \%)$ ) were included. $1142(28.2 \%)$ pat. had a simple defect, 1909 (47.1\%) a defect of moderate complexity, and $886(21.9 \%)$ a complex defect. In 117 pat. (2.9\%) miscellaneous defects were present. At least one comorbidity was present in 2145 pat. (52.9\%). 716 pat. (17.7\%), had two or more comorbidities. Endocrinological (16.9\%) and neurological disorders (16.2\%) were the most common. Comorbidities affecting the musculoskeletal system were also frequently encountered $(10.3 \%)$, as well as those affecting the gastrointestinal system (9.6\%). 907 patients were older than 40 years $(22.4 \%)$. At least one comorbidity was present in $67.3 \%$ of these patients compared to $48.8 \%$ of patients with an age between 18 and 40 years $(\mathrm{p}<0.0001)$. At least two comorbidities were present in $47.0 \%$ of the patients older than 40 years compared to $25.0 \%$ in the younger age group ( $<<0.0001$ ).

Conclusions: Comorbidities were found in a significant proportion of ACHD. As our patients are getting older, this burden further increases. Future research should focus on the impact of these comorbidities on the outcome of ACHD patients.

O12-3

Pulmonary atresia and intact ventricular septum (PA-IVS) - A population based follow-up in Sweden between 1980 and 2016

Ekman-Joelsson B-M. (1), Söderberg B. (1), Hanséus K. (2) Queen Silvia Childrens Hospital, Gothenburg, Sweden (1); Lund University Hospital, Lund, Sweden (2)

Introduction: Pulmonary atresia and intact ventricular septum is a rare and heterogeneous congenital heart defect that still represent a challenge to pediatric cardiac surgeons and cardiologists.

Objective: To study the outcome of all children born with pulmonary atresia and intact ventricular septum in Sweden between January 1980 and August 2016.

Methods: A retrospective, descriptive study based on medical reports and the initial echocardiographic examination.

Results: The cohort consists of 171 subjects, giving an incidence of 4.6 per 100000 live births. 74/171 (43\%) had ventriculo coronary arterial communications (VCAC). 164 subject were offered treatment, for 147 subjects the initial treatment was based on surgery and for 17 it was based on percutaneous treatment. At the end of the study period 130 subjects were alive, 71 had biventricular repair, five one- and an half ventricular repair and 55 univentricular palliation with completed total cavopulmonary connection in 42 cases. Follow-up time ranged from 2.5 months to 35 years (median 14 years). The overall mortality was $37 \%$ (34/ 91) between 1980 and 1999, and $8 \%(7 / 80)$ between 2000 and 
2016. Of the 41 that died, $34(83 \%)$ died before the age of one year, no death due to the heart defect after the age of nine years. In 26 subjects the heart defect was diagnosed prenatally, of whom 10 ended up with a biventricular circulation. Statistical analysis of incremental risk factors for death showed statistical significance for low birth weight, having systemic-to-pulmonary shunt as the only surgical procedure. The survival after surgery is statistically ameliorated during the time period.

Conclusions: The incidence of 4.6 per 100000 live births remain unchanged in spite of developed prenatal imaging. The majority $71 / 130(55 \%)$ of the survivors has biventricular repair. The mortality rate has declined over time, and there was no cardiac death after the age of nine years.

\section{O12-4}

Fontan operation: comparison of intra-atrial lateral tunnel and extra-cardiac conduit

van den Bosch E. (1, 2, 3), Bossers S.S.M. (1,2), Bogers A.J. J.C. (4), Robbers-Visser D. (5), van Dijk A.P.J. (6), Roos- Hesselink J.W. (7), Breur J.M.P.J. (8), Haas F. (9), Kapusta L. (10, 11),

Helbing W.A. $(1,2,11)$

Erasmus University Medical Center, Sophia Children's Hospital, Department of Paediatric Cardiology, Rotterdam, The Netherlands (1); Erasmus University Medical Center, Department of Radiology,

Rotterdam, The Netherlands (2); Netherlands Heart Institute, Utrecht, The Netherlands (3); Erasmus University Medical Center, Department of Cardiothoracic Surgery, Rotterdam, The Netherlands (4); Academic Medical Center, Amsterdam, Department of Cardiology, Amsterdam, The Netherlands (5); Radboud University Medical Center, Department of Cardiology, Nijmegen, The Netherlands (6); Erasmus University Medical Center, Department of Cardiology, Rotterdam, The Netherlands (7); University Medical Center Utrecht, Wilhelmina Children's Hospital, Department of Paediatric Cardiology, Utrecht, The Netherlands (8); University Medical Center Utrecht, Wilhelmina Children's Hospital, Department of Pediatric Cardiac Surgery, Utrecht, The Netherlands (9); Pediatric Cardiology, Dana-Dwek Children's Hospital, Sourasky Medical Center, Tel Aviv University, Tel Aviv, Israel (10); Radboud University Medical Center, Amalia Children's Hospital, Department of Pediatric Cardiology, Nijmegen, The Netherlands (11)

Objectives: To compare the outcome of current modifications of staged total cavopulmonary connection (TCPC): The extracardiac conduit (ECC) and intra-atrial lateral tunnel (ILT) technique. To compare the modern prosthetic ILT technique with the ECC technique.

Methods: We included patients that underwent staged TCPC between 1988 and 2008. Records were reviewed for patients demographics, operative details and events (arrhythmias, surgical and catheter-based reinterventions and death) during follow-up. Late complications were defined as complications after 30 days of the TCPC or after hospital discharge.

Results: 208 patients were included, 103 ILT (51 Baffle and 52 Prosthetic) and 105 ECC patients. Median follow-up duration was 14.0 years (interquartile range 10.7-17.2). At 15-year post TCPC a) overall survival was $81 \%$ for ILT, $90 \%$ for ECC patients $(p=0.122), b)$ freedom from late surgical and catheter-based reintervention was $63 \%$ for ILT and $45 \%$ for ECC ( $p=0.024)$, c) freedom from late arrhythmia was $72 \%$ for ILT, $85 \%$ for ECC $(p=0.020)$. There were no differences in freedom from late arrhythmias between prosthetic ILT and ECC technique (82\% vs $85 \%, \mathrm{p}=0.567$ (Figure 1)).

Conclusions: Outcome after staged ILT and ECC Fontan are good, with comparable overall survival. Reinterventions occurred more frequently in the ECC group. Late arrhythmias were more

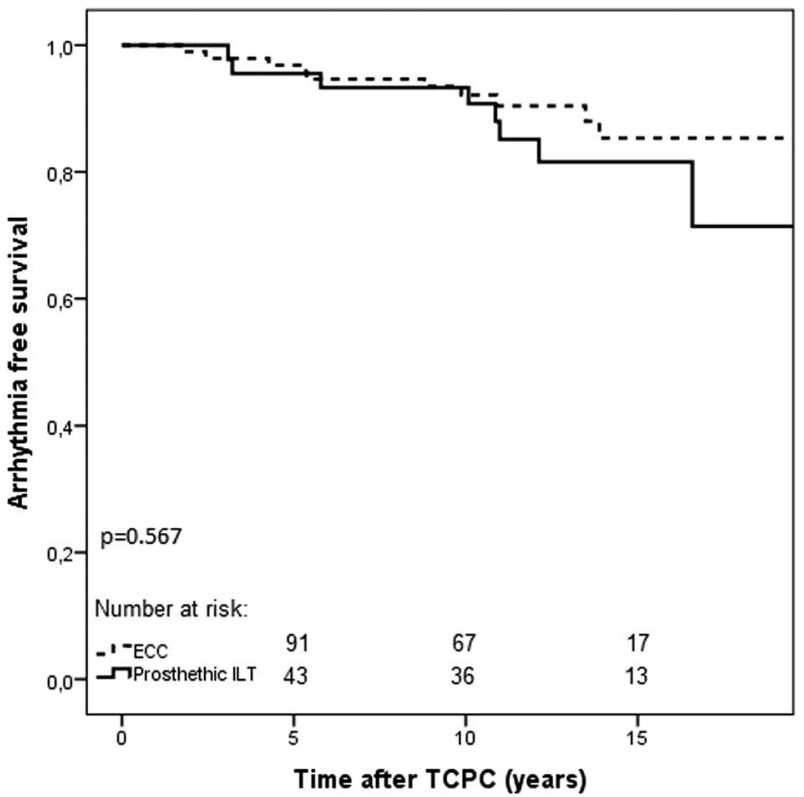

Figure 1.

Late arrhythmia free survival, ECC vs Prosthetic ILT

common in the ILT-group, but not in comparison between recent ILT modification and ECC.

O12-5

Inspiratory Muscle Training did not improve Physical Capacity in adult Patients with Fontan Circulation Fritz C. (1), Müller J. (1,2), Oberhoffer R. (1,2), Ewert P. (1), Hager A . (1) German Heart Centre Munich, Technical University of Munich, Munich, Germany (1); Institute of Preventive Pediatrics, Technical University of Munich, Munich, Germany (2)

Introduction: Impairments in respiratory musculature strength are associated with a reduction in physical capacity. Furthermore, intervention studies have shown a beneficial effect of an inspiratory muscle training (InMT) on physical capacity measures.

This study investigates the effect of a daily six-month InMT on physical capacity in adult patients with Fontan circulation.

Methods: After receiving a symptom limited cardiopulmonary exercise and lung function test, 42 patients (50\% female; $30.8 \pm 8.2$ years) with Fontan circulation were randomized into either an intervention (IG) or control group (CG). The IG performed a telephone supervised InMT of 3 sets with 10-30 repetitions with the device POWERbreathe Medic (POWERbreathe International Ltd., Southam, UK). Since four patients dropped out, retesting was performed in 18 patients of the IG and 20 of the CG six months after baseline.

Results: After six month of InMT, peak oxygen uptake (\%predicted) did not increase in the IG in comparison to the CG (IG: $-0.3 \pm 2.3 \mathrm{ml} / \mathrm{min} / \mathrm{kg}$ vs. CG: $-0.2 \pm 3.0 \mathrm{ml} / \mathrm{min} / \mathrm{kg}$; $\mathrm{p}=0.952)$. There was also no difference in ventilatory efficiency (IG: $-0.5 \pm 3.1 \mathrm{ml} / \mathrm{min} / \mathrm{kg}$ vs. $\mathrm{CG}:-0.3 \pm 2.5 \mathrm{ml} / \mathrm{min} / \mathrm{kg}$; $\mathrm{p}=0.844)$ nor in peak workload (IG: $-3.6 \pm 11.1 \mathrm{Watt} / \mathrm{kg}$ vs. CG: $-3.4 \pm 9.5 \mathrm{Watt} / \mathrm{kg} ; \mathrm{p}=0.938)$ after Intervention.

Neither improved forced vital capacity (IG: $1.6 \pm 7.1 \%$ vs. CG: $-0.4 \pm 7.8 \% ; \mathrm{p}=0.402)$ nor forced expiratory volume in the first second (IG: $0.2 \pm 6.2 \%$ vs. CG: $-2.0 \pm 7.0 \% ; \mathrm{p}=0.315$ ). 
Conclusion: A daily six-month InMT did not improve physical capacity measures in patients with Fontan circulation. Advanced studies should investigate further training forms suitable for patients with Fontan circulation enhancing physical capacity including a reduced mortality and morbidity.

012-6

Predicting heart transplantation outcome in the failing Fontan patient: A challenging problem

Polyviou S. (1), O'Sullivan J. (1,2), Hasan A. (1), Coats L. (1,2) Adult Congenital and Paediatric Heart Unit, Freeman Hospital, Newcastle upon Tyne, UK (1); Cardiovascular Research Centre, Institute of Genetic Medicine, Newcastle University, Newcastle upon Tyne, UK (2)

Introduction: High-risk heart transplantation (HT) currently offers the only sustainable approach to modify the natural history of the failing Fontan circulation. However, it remains unclear at assessment which patients will gain most benefit. The aim of this study was to assess the validity of factors (lower age, shorter Fontan-transplantation interval, lower ejection fraction, pretransplantation cardiac/renal mechanical support, moderate/ severe systemic atrioventricular valve regurgitation, higher MELD-XI score) identified to predict poor post-transplantation outcome [Berg CJ et al AmJCardiol 2017;119 (10):1675-1679].

Methods: We reviewed pre-transplantation data for all paediatric and adult patients who underwent HT for failing Fontan between January 2009 and October 2017 at our Institute. The association of all-cause mortality with individual demographic, echocardiographic and clinical variables was assessed using the Cox proportional hazards model.

Results: Thirty-seven patients (median age 21.3 years, range 3.4-43.5) underwent HT for failing Fontan circulation. Median follow-up was 2.8 years (range $0-8.1$ ). Survival was $73 \%$ at 1 year and $60.1 \%$ at 5 years. Most deaths $(10 / 13)$ were early and preceded hospital discharge. Factors predictive of higher posttransplantation mortality in our cohort were MELD-XI score, bridging to transplantation with renal replacement therapy and Fontan failure with preserved systolic ventricular function (Table). Transplantation age below 18 years $(n=14,5$ deaths) was not a statistically significant predictor of mortality compared to adult age ( $n=23,8$ deaths) and neither were gender, age at Fontan, Fontanto-transplantation interval, degree of pre-transplantation systemic AVVR and use of ECMO as a bridge to transplantation.

Conclusion: In this mixed population of children and adults with failing Fontan circulations, higher MELD-XI score, renal replacement therapy and preserved ventricular function predicted

Table.

\begin{tabular}{lll}
\hline Variable & $\begin{array}{l}\text { Hazard Ratio } \\
\mathbf{( 9 5 \%} \mathbf{C I})\end{array}$ & $\begin{array}{l}\mathbf{P} \\
\text { value }\end{array}$ \\
\hline Male gender & $0.697(0.23-2.12)$ & 0.524 \\
Age at Fontan & $1.001(0.993-1.009)$ & 0.824 \\
Moderate/severe systemic AVVR & $0.88(0.3-2.63)$ & 0.822 \\
Preserved systemic ventricular & $4.53(1.5-13.66)$ & $\mathbf{0 . 0 0 7}$ \\
$\quad$ function & $1.14(1.02-1.27)$ & $\mathbf{0 . 0 2 5}$ \\
MELD-XI & $4.9(1.32-18.22)$ & $\mathbf{0 . 0 1 8}$ \\
Renal replacement therapy & $0.046(0-14107)$ & 0.634 \\
Pre-transplantation ECMO & $0.999(0.994-1.004)$ & 0.696 \\
Fontan-transplantation interval & $0.995(0.95-1.04)$ & 0.825 \\
Transplantation age & & \\
\hline
\end{tabular}

post-transplantation mortality. This contrasts with others' findings and underlines the need for multi-centre cooperation to identify robust risk factors that can be utilized in this rare and challenging patient group.

O13-1

Changes in right ventricular function and growth after inutero pulmonary valvuloplasty in fetuses with pulmonary atresia with intact septum or critical pulmonary stenosis Tulzer A. (1), Arzt W. (2), Gitter R. (1), Prandstetter C. (1), Grohmann E. (1), Mair R. (3), Tulzer G. (1)

Children's Heart Center Linz, Department of Pediatric Cardiology, Kepler University Hospital, Linz, Austria (1); Institute of Prenatal Medicine, Kepler University Hospital, Linz, Austria (2); Children's Heart Center Linz, Department of Pediatric Cardiac Surgery, Kepler University Hospital, Linz, Austria (3)

Objectives: to assess the immediate effects of fetal pulmonary valvuloplasty on right ventricular (RV) size and function as well as in-utero RV growth and postnatal outcome.

Methods: Thirty-five fetal pulmonary valvuloplasties were performed in 23 fetuses with pulmonary atresia with intact ventricular septum (PAIVS) $(\mathrm{n}=15)$ or critical pulmonary stenosis (CPS) $(\mathrm{n}=8)$ at a median gestational age of $28+4$ weeks $(23+6$ to $32+1)$. RV morphologic and functional parameters were obtained before and 1-2 days after the procedure and analyzed retrospectively. Longitudinal data was collected only from fetuses who were followed in our center. Outcome was assessed using a prediction- score for a non-biventricular outcome.

Results: There were no fetal deaths. Immediately after successful intervention $\mathrm{RV} / \mathrm{LV}$ length ratio, TV/MV ratio, $\mathrm{RV}$ filling time, $\mathrm{RV}$ TVI ${ }^{\star} \mathrm{HR}$ increased and tricuspid regurgitation velocity decreased significantly. In fetuses followed longitudinally to delivery $(n=5)$ ratios of $\mathrm{RV} / \mathrm{LV}$ and $\mathrm{TV} / \mathrm{MV}$ remained constant or improved further. Fetuses with an unsuccessful intervention $(\mathrm{n}=2)$ became univentricular all others had either a biventricular $(\mathrm{n}=15)$, one and a half ventricular $(\mathrm{n}=2)$, or a still undetermined $(n=4)$ outcome. Five of nine fetuses with a predicted nonbiventricular outcome became biventricular while 3 of nine still have an undetermined circulation.

Conclusion: In selected fetuses with PAIVS or CPS in-utero pulmonary valvuloplasty leads to immediately larger RVs caused by reduced afterload and increased filling. This is followed by continued RV growth improving the chances for a biventricular outcome even fetuses with a predicted non-biventricular circulation.

O13-2

Semilunar valve growth in fetal transposition of the great arteries:Semilunar valve size is predictive of postoperative aortic root dilatation

Clur S.A. (1), van der Palen R.L.F. (2), van der Zee C.W. (1),

Vink A.S. (1), Knobbe I. (3), du Marchie Sarvaas G.J. (4), Jurgens S.J. (1), Bax C.J. (3), Pajkrt E. (2), Blom N.A. (1,2,3), Haak M. (3),

Bilardo C.M. (4)

Academic Medical Center, Amsterdam (1); University Medical Center, Leiden (2); Free University Medical Center, Amsterdam (3); University Medical Center, Groningen (4)

Introduction: Transposition of the great arteries (TGA) is treated with the arterial switch operation (ASO). Despite excellent longterm survival, important residual lesions can be identified from 

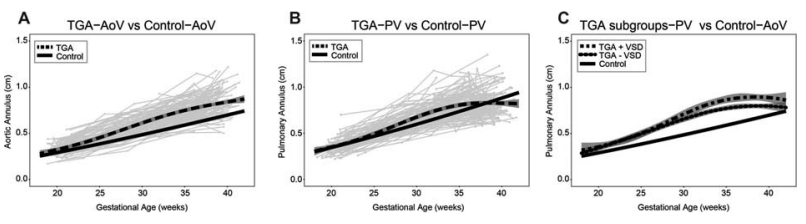

Figure.

early childhood, which may require re-interventions, such as neo-aortic root (NAoR)-dilatation.

The aim was to document semilunar valve growth in fetal-TGA and to determine if NAoR-dilatation can be predicted prenatally. Methods: All fetuses diagnosed with TGA suitable for ASO in four academic institutions, where stored data was available, were included (2000-2017). Measurements included diameters of semilunar valve annuli prenatally and postnatally; and NAoR at 1 year and last check-up. Annulus growth was analysed using a linear mixed-effect model and compared to normal values (Schneider et al. 2005). Semilunar valve and NAoR Z-scores were correlated.

Results: 138 fetuses were included of which 88 (63,7\%) were male. $51(37 \%)$ had a significant ventricular septal defect (VSD) requiring surgical closure (12 with Taussig-Bing anatomy). 17 (12\%) died (1 intrauterine death, 5 terminations of pregnancy, 5 preASO due to comorbidity and $6(4,3 \%)$ postASO. 123 underwent an ASO (3 are still in utero). 382 fetal echocardiograms were performed. A postnatal echocardiogram was available in 118 giving preASO data from 500 echocardiograms. The median number of repeated measurements was $5(1-10)$ per patient. The median postnatal follow-up was 2.9 years ( 4 months-13.3yrs) $(n=119)$. TGA-aortic valve (AoV) annuli were significantly larger than controls (A in Figure), but matched the control pulmonary valves (PV). TGA-PV diameters were comparable with control PV annuli but were significantly larger than control AoV annuli, especially in the sub-group with a VSD (B and C in Figure). Semilunar valve Z-scores correlated significantly with NAoR-dilatation, $(\mathrm{p}=<0.001$ PV 26-36 weeks'; $\mathrm{p}=0.004$ AoV 26-30 weeks'). The best cut-off value to predict a postASO NAoR $\geq Z+2$ at last followup was a PV Z-score $\geq 0.08$ at $26-30$ weeks' (sensitivity $70 \%$; specificity $80 \%)$.

Conclusion: Fetal-TGA semilunar valve annuli show accelerated growth, especially when there is a significant VSD. Significant correlations between fetal-TGA semilunar Z-scores and postASO NAoR-dilatation were found.Factors besides postoperative hemodynamics such as prenatal flow, quality and quantity of connective tissue and genetic factors may influence semilunar valve growth and NAoR-dilatation.

\section{O13-3}

Antenatal diagnosis of Double Aortic arch: Associated chromosomal and extra cardiac abnormalities, differential growth of arches during gestation and post-natal outcome Bartsota M. $(1,3,4)$, Jowett V. $(1,3)$, Carvalho J.S. $(1,2)$

Royal Brompton \& Harefield NHS Trust, London, UK (1); St George's hospital, London, UK (2); Centre for Fetal Care, Queen Charlotte's \& Chelsea hospital, London, UK (3); Chelsea \& Westminster hospital, London, UK (4)

Introduction: Double aortic arch (DAO) is a rare congenital malformation that results from failure of regression of both arches during embryogenesis creating complete vascular ring that causes variable degree of compression of the trachea and/or oesophagus. In the absence of large antenatal series, counselling regarding risk of associated chromosomal and extra-cardiac abnormalities (ECA) and postnatal need for surgery remains challenging. From personal observations we also hypothesised that there is differential growth of the arches during pregnancy and initially, a patent left arch may present as atretic postnatally.

Methods: Retrospective, case series review. From computerised databases, we identified all fetuses with DAO seen in four tertiary centres between $01 / 2014$ and 11/2017. We reviewed prenatal findings to assess patency of both arches, associated ECA and chromosomal abnormalities and reviewed postnatal outcome data. Results: Twenty cases of DAO were identified; three of them diagnosed antenatally as right aortic arch (RAO) and postnatally as DAO. In the majority $(60 \%)$, indication for cardiac scan was suspected RAO. Mean gestational age at diagnosis was $21+3$ weeks (range $16+0$ to $28+4)$. One $(10 \%)$ of 10 cases that had genetic testing had 22q11 deletion. In the majority (84\%) right arch was dominant, in all cases arterial duct was left-sided and in all antenatally diagnosed as DAO, the left arch was patent. In three cases $(15 \%)$ there were minor additional cardiac abnormalities and one $(5 \%)$ had ECA. Postnatally, computerised tomography scan (CT) showed atretic left segment in six patients $(54 \%)$. In the majority $(73 \%)$ of cases that have been assessed by $\mathrm{CT}$, surgical or catheter intervention has been either performed (mean age $=5.1$ months) or planned.

Conclusions: Antenatal diagnosis of DAO is feasible and essential for prompt postnatal management. From our study it appears that risk of chromosomal abnormalities is similar and risk of ECA less when compared with $\mathrm{RAO}$ and majority of patients require intervention during infancy. These provisional data also support the theory that during gestation there is preferential growth of the dominant right arch as postnatally some of the patients presented as DAO with atretic left segment.

\section{O13-4}

Home Fetal Heart Rate Monitoring for Surveillance of Fetal Arrhythmias: A Cohort Analysis

Freire G.A. (1), Hanson J. (1,2), Morelli D. (2), Wilhm M. (1), Amankwah E. (3,4), Goldenberg N. $(2,5)$

Johns Hopkins All Children's Heart Institute, Johns Hopkins All Children's Hospital, St. Petersburg, FL, USA (1); Johns Hopkins All Children's Clinical and Translational Research Organization, Johns Hopkins All Children's Hospital, St. Petersburg, FL, USA (2); Johns Hopkins All Children's Health Informatics Core, Johns Hopkins All Children's Hospital, St. Petersburg, FL, USA (3); Department of Oncology, Johns Hopkins University School of Medicine, Baltimore, MD, USA (4); Departments of Pediatrics and Medicine, Johns Hopkins University School of Medicine, Baltimore, MD, USA (5)

Background: Sustained fetal arrhythmias (SFAs) can result in fetal demise. Traditionally, monitoring at-risk fetuses is performed episodically via serial in-office assessments. However this monitoring approach may be impractical, costly, and suboptimally sensitive to the development of clinically significant arrhythmias. In recent years, hand-held Doppler monitors have been introduced into clinical practice at some centers as an alternative for fetal heart rate (FHR) monitoring. The overall objective of this work was to conduct an analysis of a single-institutional mixed retrospective-prospective cohort of pregnant women undergoing FHR monitoring at Johns Hopkins All Children's Hospital, comparing traditional monitoring versus home-based monitoring. Methods: The study included a contemporary cohort of pregnant women carrying fetuses at increased risk of SFA, using FHR home monitoring ( 3 times/day) in addition to regularly-scheduled clinic visits, and a historical cohort monitored with clinic visits. At risk: non-sustained tachycardia (HR $>180 \mathrm{bpm}$ for $<12 \mathrm{~h} /$ day), complex ectopy or bradycardia $(\mathrm{HR}<100 \mathrm{bpm})$ were included. SFA 
was defined as arrhythmia present $>$ than 12 hours/day. Compliance with documentation was evaluated using FHR home Measurement logs. Agreement in FHR between Doppler ultrasound-based home monitors (HDUM) and in-office Doppler ultrasound (ODU) was measured using Bland-Altman analysis. The time to detection (TTD) to SFA was compared between cohorts by independent t-test and frequencies of clinical outcomes (hydrops and mortality) with Fisher's exactor Chi-squared testing. Results: There were 44 subjects in the retrospective cohort (traditional FHR monitoring) and 21 in the prospective cohort (home-based FHR monitoring). Agreement in FHR measurement (HDUM vs ODU) was very strong, with ICC $=0.986(95 \%$ CI, 0.98-1.0). The median TTD to a SFA was longer in the retrospective cohort than the prospective cohort (11, range 5-48 vs. 9 , range 9-14 days; $p=0.49)$. There were 3 cases of hydrops $(7 \%)$ in the retrospective cohort and none $(0 \%)$ in the prospective $(\mathrm{P}=0.49)$.

Conclusions: Home FHR monitoring appears to be feasible in atrisk fetuses and has the potential to lead to earlier identification of (and intervention for) SFAs. Larger cooperative studies are needed to further substantiate a potential benefit of home FHR monitoring on clinical outcomes in at-risk patients.

\section{O13-5}

Short- and long-term outcomes of fetal balloon aortic valvuloplasty - single-center experience

Grzyb A. $(1,4)$, Kolesnik A. $(2,4)$, Kowalczyk-Domagala M. (1), Zubrzycka M. (2), Brzezinska-Rajszys G. (1,2), Kansy A. (3), Maruszewski B. (3), Debska M. (5), Dangel J. (4) Cardiology Department (1), Heart Catheterization Laboratory (2), Cardiac Surgery Department (3), The Children's Memorial Health Institute, Warsaw, Poland; Perinatal Cardiology and Congenital Anomalies Department (4), Obstetrics and Gynecology Department (5), Centre of Postgraduate Medical Education, Warsaw, Poland

Introduction: Fetal balloon aortic valvuloplasty (FBAV) for critical aortic stenosis is performed to prevent the progression to hypoplastic left heart syndrome (HLHS). Multiple selection criteria for postnatal biventricular (BV) outcome were developed, however, the management of borderline-LV patients remains highly variable and center-dependent. The aim of this study was to summarize the results of FBAV performed in a single fetal cardiology center.

Methods: We evaluated the data of 72 fetuses in whom 78 FBAV was performed between 2011-2017. Searching for the predictors of BV outcome we analyzed the time of FBAV, threshold score, and selected left-heart measurements (aortic valve, ascending aorta, mitral valve, LV length, width and indexed volume) pre-, post-intervention, and in the newborn. The follow-up data were collected from target cardiology units.

Results: From the study group, 59 children (89\%) were live-born, at median 39 weeks, $40 \%$ via cesarean section. 3 neonates died before any treatment. From the remaining group, 30 patients (54\%) were qualified for single-ventricle (SV) palliation, 17 (30\%) for BV treatment, and in $9(16 \%)$ the hybrid procedure was performed. From the last group, 1 patient followed SV palliation strategy, and 2 were converted to BV circulation. In the BV group, 13 newborns had BAV performed (1 followed by Ross-Konno operation), and 1- surgical valuloplasty; 3 required no treatment. 3 children, with very poor function of LV died post-intervention. Overall, out of 53 treated children with known follow-up, $14(26.4 \%)$ have BV circulation and 23 (41\%) SV palliation. All results are summarized below:

Out of prenatal echocardiographic parameters, the bigger AAO diameter, higher LV pressure and later performed FBAV

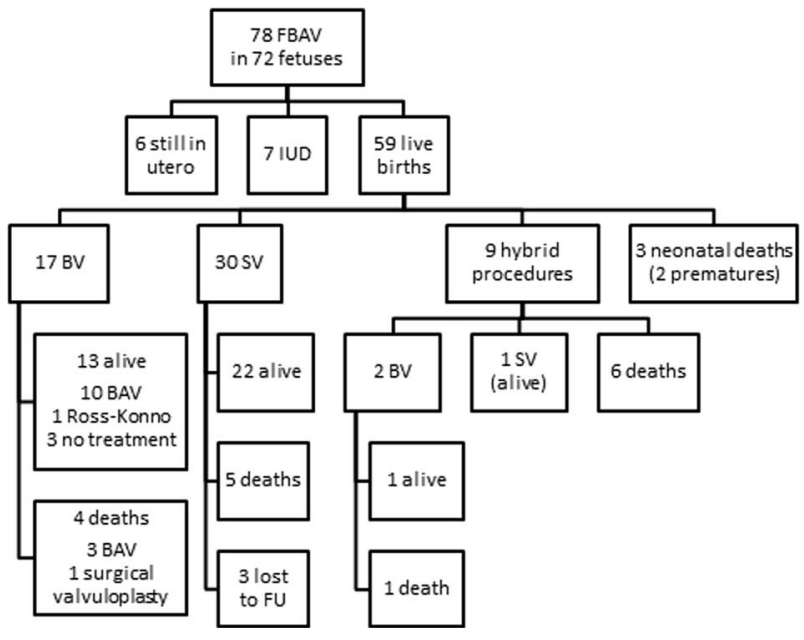

Figure.

(26 vs. 24 weeks) significantly correlated with BV postnatal treatment. The threshold score $\geq 4$ also occurred predictive for BV outcome (sensitivity 94\%, specificity $41 \%, \mathrm{p}<0.05$ ). In the newborns' echocardiographic examinations, all left-heart structures diameters and LV indexed volume were significantly larger in the BV group. Conclusions: FBAV allows for postnatal BV treatment in a subset of patients with critical aortic stenosis. Nevertheless, the postintervention course is highly unpredictable, and the treatment method depends on the newborn's condition and left-heart structures development, as well as center experience.

O13-6

Outcome with Routine vs Random Transplacental Antiinflammatory Treatment for Anti-Ro Antibody-Mediated Fetal Heart Disease

Mawad W. (1), Hornberger L.K. (2), Diab K. (3), Cuneo B. (4), Moon-Grady A (5), Silverman E. (1), Jaeggi E. (1)

The Hospital for Sick Children, Toronto, Canada (1); Stollery Children's Hospital, Edmonton, Canada (2); Rush University Medical Center, Chicago, USA (3); Children's Hospital Colorado, Colorado, USA (4); Benioff Children's Hospital, San Francisco, USA (5)

Background: Cardiac manifestations of neonatal lupus (NLE), including heart block (AVB), endocardial fibroelastosis (EFE) and dilated cardiomyopathy (DCM,), are associated with significant mortality.

Transplacental fetal treatment (TFT) with dexamethasone from the time of NLE diagnosis to birth, $\beta$-stimulation for bradycardia $<50$ beats/minute, and intravenous immune globulin (IVIG) for EFE has been routinely used at our institutions to improve outcomes. The optimal management including the use of dexamethasone is still debated.

Methods: We reviewed 95 consecutive fetuses with cardiac NLE that received TFT as per our institutional guidelines (1996-2016) and compared the findings with inconsistently treated TFT (Lopes 2008; Eliasson 2011; Levesque 2015).

Results: The table compares our ("current") with the predominantly untreated cohorts of the above studies. Neonatal ( $93 \%$ vs $84 \%$ and $77 \%$ ) as well as long-term survival ( $89 \%$ vs $87 \%, 69 \%$ and $69 \%$ ) were better in our series including cardiac, non-cardiac and unknown causes of death. The incidence of late onset DCM was also lower in our series ( $2 \%$ vs $19 \%, 7 \%$ ). 
Table.

\begin{tabular}{|c|c|c|c|c|}
\hline & Current & $\begin{array}{l}\text { Levesque } \\
(n=198)\end{array}$ & $\begin{array}{l}\text { Eliasson } \\
(n=175)\end{array}$ & $\begin{array}{l}\text { Lopes } \\
(n=57)\end{array}$ \\
\hline $\begin{array}{l}\text { Anti-Ro antibody- } \\
\text { mediated }\end{array}$ & $95(100 \%)$ & $100 \%$ & $140(80 \%)$ & 41 (72\%) \\
\hline Complete AVB & $78 \%$ & $100 \%$ & $83 \%$ & $75 \%$ \\
\hline Other Diagnosis & $22 \%$ & $0 \%$ & $17 \%$ & $25 \%$ \\
\hline $\begin{array}{l}\text { Age at diagnosis } \\
\text { (weeks) }\end{array}$ & 23 & 23 & 24 & 30 \\
\hline Fetal hydrops & $14 \%$ & $11 \%$ & $9 \%$ & $11 \%$ \\
\hline $\begin{array}{l}\text { Endocardial } \\
\text { fibroelastosis }\end{array}$ & $36 \%$ & $21 \%$ & - & $5 \%$ \\
\hline Prenatal therapy & $95(100 \%)$ & $75(39 \%)^{\star}$ & $67(38 \%)^{\star}$ & $11(19 \%)^{\star}$ \\
\hline $\begin{array}{l}\text { Fluorinated } \\
\text { steroids }\end{array}$ & $100 \%$ & $39 \%$ & $38 \%$ & $11 \%$ \\
\hline Duration (weeks) & $11(1-18)$ & $8(1-18)$ & $10(1-21)$ & - \\
\hline Beta-mimetic & $36 \%$ & - & $23 \%+$ & $16 \% \dagger$ \\
\hline IVIG & $27 \%$ & $2 \% \star$ & $0.6 \%$ * & $0 \% \star$ \\
\hline $\begin{array}{l}\text { Cases with known } \\
\text { outcome }\end{array}$ & 95 & 198 & 164 & 57 \\
\hline Fetal survival & 93 (98\%) & $174(93 \%)$ & 149 (91\%) & - \\
\hline Neonatal survival & $88(93 \%)$ & - & $138(84 \%) \dagger$ & $44(77 \%) \dagger$ \\
\hline 10-year survival & $85(89 \%)$ & & & $39(69 \%)^{\star}$ \\
\hline Late onset DCM & $2 \%$ & $19 \% \star \star$ & $7 \% \dagger$ & $5 \%$ \\
\hline
\end{tabular}

$\star_{p}<0.0001$ compared to current; $\dagger p<0.05$ compared to current

Conclusions: In our cohort, we have found good fetal survival, and lower neonatal and long-term mortality as well as lower incidence of late-onset DCM compared to published series. These findings support routine treatment in this condition to alter neonatal and long-term outcomes.

014-1

Anomalous Aortic Origin of a Coronary Artery: Outcomes of Surgical and Non-surgical Treatment in a Single-Center Bibevski S., Ruzmetov M., Chan K.-C., Latson L., Perryman R., Scholl F.G.

The Pediatric Heart Institute, Joe DiMaggio Children's Hospital at Memorial Healthcare, Hollywood, FL, USA

Background: Anomalous aortic origin of the coronary artery (AAOCA) has been associated with coronary ischemia, myocardial infarction, and sudden death. Risk stratification and management remain controversial, especially for the asymptomatic children. The aim of this study was to analyze all patients with AAOCA managed surgically or non-surgically at our Center over a 10-year period.

Methods: Retrospective chart review of patients with isolated diagnosis of AAOCA from 2007 to 2017. Follow-up obtained by chart review or contact with cardiologists. Treatment algorithm based on surgical intervention for patients who are symptomatic with right AAOCA and asymptomatic older than 10 years with left AAOCA.

Results: Fifty patients were managed for AAOCA. Mean age was $20.7 \pm 15$ years (range, $5-63$ years). Thirty patients $(60 \%)$ had right AAOCA and twenty patients (40\%) had left AAOCA. There is no significant difference in age between left and right AAOCA patients $(20 \pm 18$ vs $21 \pm 13 ; p=0.88)$. Age of surgically treated patients and non-surgical patients at last follow-up are shown in Figure. Twenty-eight underwent surgical intervention (14 with right AAOCA and 14 with left AAOCA). Surgical intervention included unroofing in $23(82 \%)$, reimplantation in $2(7 \%)$, and coronary artery bypass grafting in $3(11 \%)$ patients. Median followup was 2 years ( $0-10$ years) and follow-up was completed in $82 \%$ of patients. There has been only 1 death that was perioperative in an adult patient. All patients have been free of any cardiac symptoms postoperatively. At follow-up $74 \%$ of surgical patients

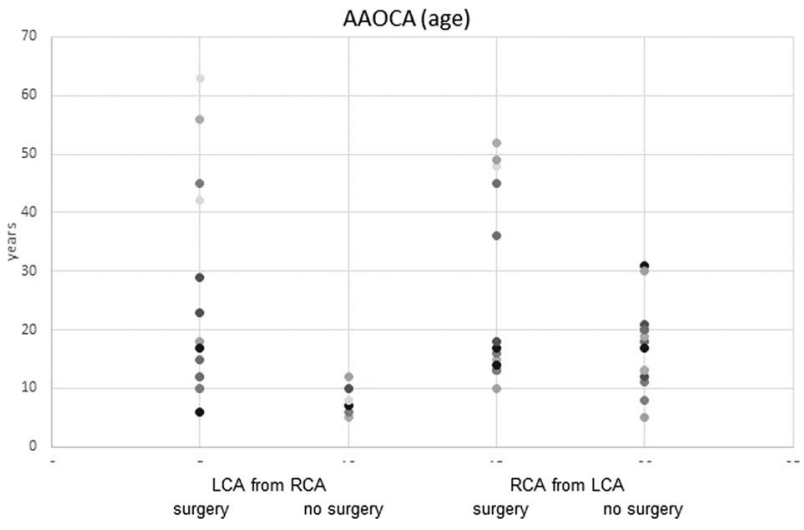

Figure.

$(\mathrm{n}=20)$ underwent functional testing and had no evidence of ischemia. In the non-surgical group $(n=22)$, surgery was not recommended yet in 20 (91\%), and $2(9 \%)$ are undergoing further testing.

Conclusions: Our program uses a treatment algorithm to select patients for surgical intervention. Only a half subset of AAOCA patients (symptomatic with right AAOCA and asymptomatic older than 10 years with left AAOCA) requires an operation, and we favor unroofing and translocation techniques. With this paradigm, outcomes are excellent, as validated with anatomic- and function-based testing. Further follow up and subtype classification will be important for risk stratification.

O14-2

Comparison of right coronary artery with left coronary artery arising from opposite sinus: clinical presentation and risk of sudden death

Courand P.Y., Bozio A., Ninet J., Bakloul M., Walton C., Perouse de Montclos T., Galoin-Bertail C., Boussel L., Metton O., Henaine R., Di Filippo $S$.

Congenital Cardiology, Cardiovascular Hospital, Lyon, France

The objective of this study was to assess wether right coronary artery from opposite sinus (RCAOS) was less severe than left coronary artery (LCAOS).

Material and Methods: Patients diagnosed with RCOS and LVOS were compared regarding clinical presentation, outcomes and surgical mnagement.

Results: 35 patients were diagnosed with anomalous aortic origin of a coronary artery, including 16 LCAOS (31\% males), 19 RCAOS (42\% males). Age at diagnosis was $24.1 \pm 21.4 \mathrm{y}$ in LCAOS and $18.5 \pm 17.1 \mathrm{y}$ in RCAOS. Patients were asymptomatic in $12.5 \%$ of LCAOS and $26.3 \%$ of RCAOS $(p=0.24)$. Chest pain occurred in $56.2 \%$ vs $36.8 \%(p=0.38)$, syncope or sudden death in $37.5 \%$ vs $42.1 \%(p=0.03)$ and dyspnea in $12.5 \%$ vs $10.5 \%(p=0.2)$ of patients with respectively LCAOS and RCAOS. Patients with an inter-arterial course between the great arteries presented more frequently with syncope/sudden death compared with patients without (43.3 vs. $5.9 \%, \mathrm{p}=0.007)$. Diagnosis was reached by first-line transthoracic echocardiography in half of the cases. Surgical repair was performed in 24 patients (68.5\%): $64.7 \%$ of RCAOS and $76.2 \%$ of LCAOS. All operated patients (23 direct implantation, 1 un-roofing) had been diagnosed with an inter-arterial course between the great arteries, and were asymptomatic at a median postoperative follow-up of 6.5 years (range from 3.1 to 14.6). 
Conclusion: The frequency of severe clinical symptoms was similar in RCAOS with inter-arterial course than LCAOS. Outcome as well as incidence of surgical repair did not differ between both groups.

\section{O14-3 \\ Late coronary complications in patients with Bland- White-Garland syndrome (ALCAPA) \\ Lammers A.E. (1), Stegger J. (1), Kubitza P. (1), Tutarel O. (2), \\ Bauer U. (3), Uebing A. (1) \\ Paediatric Cardiology, Münster University Hospital, Germany (1), German Heart Centre Munich, Germany (2), Competence Network Congenital Heart Defects, Berlin (3)}

Objectives: Anomalous origin of the left coronary artery from pulmonary artery (ALCAPA) is rare and accounts for $0,5 \%$ of all congenital heart disease. Age and mode of presentation vary. Surgical treatment aims to establish a normal coronary blood supply to the left coronary artery (LCA) and includes reimplantation of the LCA into the aortic root, ligation of the origin of the LCA, bypass grafting and the Takeuchi-procedure.

Methods: Registry Search of the German Competence Network for Congenital Heart Defects with the aim to assess morbidity in this patient cohort.

Results: 107 patients with ALCAPA were identified within the registry. In $9 / 107$ patients $(8.4 \%)$ coronary artery problems occurred after a median follow-up of 4.3 years from initial repair, performed at a median age of 6.8 years (LCA reimplantation $n=3$, Tacheuchi-Operation $n=2$, bypass grafting $n=1$, other $n=3$ ).

Coronary problems reported were myocardial infarction $(\mathrm{n}=2)$, coronary artery occlusion $(n=1)$, stenosis $(n=5)$ and aneurysmatic ectasia of the LCA $(n=1)$. Two patients underwent percutaneous transluminal coronary angioplasty (PTCA) with stent insertion, one underwent surgical augmentation of the stenosed coronary artery and two patients received an coronary artery bypass graft. One patient with initial PTCA and stent placement received a bypass graft following in-stent stenosis. Four patients had no specific revascularization procedures. $8 / 9$ patients are still alive at 24 years (median; 3.6-47.6 years) of follow-up. 5/8 survivors had a normal left ventricular function. In $3 / 8$ patients contractility was impaired. Associated risk factors for coronary artery disease were not reported in all but one patient (arterial hypertension and obesity). Four patients had additional valvar procedures (mitral valve replacement $\mathrm{n}=2$, aortic valve replacement $\mathrm{n}=1$, Contegra graft for supravalvar pulmonary stenosis after reconstruction of the pulmonary root). One patient required ICD implantation for episodes of sustained ventricular tachycardia. Conclusions: Although rare after surgical treatment of ALCAPA, late coronary artery problems occur and account for significant morbidity and need for subsequent intervention or surgery. Valve disease (mainly left sided) also contribute to morbidity. Thorough life-long follow-up for and awareness of these complications are warranted to improve the long-term outcome for patients with ALCAPA.

\section{4-4}

Coronary artery complications following arterial switch operation: A Registry analysis of the German Competence Network Congenital Heart Defects

Lammers A.E. (1), Orwat S. (2), Stegger J. (1), Lebherz C. (3), Bauer U. (4), Uebing A. (1)

Paediatric Cardiology (1), Clinic for Adult Congenital Heart Disease (2), Münster University Hospital, Münster, Germany (1,2); Clinic for Adult Congenital Heart Disease, RWTH University Hospital Aachen, Germany (3); Competence Network Congenital Heart Disease, Berlin (4)
Objectives: The introduction of the arterial switch operation (ASO) for repair of transposition of the great arteries (TGA) has transformed survival with this condition. However, coronary complications resulting from coronary transfer with reimplantation into the neo-aortic root remain a concern. We aimed to evaluate coronary complications in this national cohort of TGA patients.

Methods: Registry search of the German Network for Congenital Heart Disease.

Results: At time of analysis, 1737 TGA patients were included in the networks registry, of whom 871 had undergone the ASO. CA complications were reported on in only $13(1.5 \%)$ patients: two presented with myocardial infarction; five were diagnosed with CA occlusion and another 6 with coronary stenosis. Of those, four patients underwent CAVB surgery, in 3 the CA was re-implanted and another 3 underwent coronary angioplasty with stent placement. In 3 patients no revascularization was undertaken. It is noteworthy, that 4 of the 13 patients with CA complications had an unusual coronary anatomy (common origin, double ostium, intramural course of the CA, right CA from left sinus and hypoplastic left CA from right sinus), 2 of whom died 2.2 and 14.4 years after ASO. These patients did not undergo any revascularization procedure. 2 of the 11 patients who were still alive after 19.8 years of follow-up developed ventricular tachycardia resulting in ICD implantation 16 and 19 years following their ASO. Conclusions: Albeit the technical challenge of reimplantation of the CA during the ASO, late CA complications appear to be rare. The true incidence, however, remains unclear as this complication might be underdiagnosed and underreported. Survival with this complication seems guarded. Particular caution at follow-up should be taken in patients with an unusual CA anatomy pattern. Coronary complications are associated with adverse outcomes and routine coronary angiography after the ASO could be considered, particularly in patients with unusual coronary anatomy.

O14-5

Glenn procedure with additional flow enlarges pulmonary size without extra cardiac-stress

Sonota K., Hamamichi Y., Nukaga S., Komiya E., Ishii T., Kishiki. K., Inage A., Ueda T., Yazaki S., Yoshikawa T.

Sakakibara Heart Institute, Tokyo, Japan

Introduction: We often leave additional flow to pulmonary arteries (PA) in Glenn procedure to grow PA larger. However, additional flow (Add. flow) is suggestive of imposing over-loads against mono- ventricular circulation. We investigated cardiac function and pulmonary circulation in Glenn patients with Add. flow.

Methods: The medical records of 73 Glenn patients were reviewed who were confirmed Add. Flow on cardiac catheterization. Control was 53 Glenn patients who were not confirmed Add. flow. They all underwent cardiac catheterization between 2010 and 2017. We compared cardiac performances and pulmonary circulation indexes between two groups.

Results: Additional flow was reserved by following methods, such as antegrade flow from ventricular outflow tract (30), conduit from right ventricle (29), and shunting from aortic branch (14). All additional flows were lessened by narrowing their tracts. Study ages were almost same between Add. flow group and non-Add. flow group. Indexes of PA were larger in Add. flow group (276 vs. $228 \mathrm{~mm} 2 / \mathrm{m} 2: \mathrm{p}=0.023$ ); left PA diameters were larger (18.3 vs. $16.3 \mathrm{~mm} 2 / \mathrm{m} 2: \mathrm{p}=0.034)$; right PA diameters were larger $(20.9$ vs. $18.2 \mathrm{~mm} 2 / \mathrm{m} 2: \mathrm{p}=0.0025)$. Pulmonary flow was more in Add. flow (3.8 vs. $2.8 \mathrm{~L} / \mathrm{min} / \mathrm{m} 2: \mathrm{p}<0.0001)$; pulmonary resistance was lower in Add. flow (1.6 vs. $2.0 \mathrm{U}^{\star} \mathrm{m} 2$ : $\left.\mathrm{p}=0.014\right)$; pressure of 
superior vena cava was higher in Add. flow (13.9 vs. $12.3 \mathrm{mmHg}$ : $\mathrm{p}=0.039)$. As for ventricular performances, there were no significant differences in following indexes between two groups: end-diastolic volume, end-systolic volume, and ejectuin fraction. Pressure indexes were not significantly different between two groups: pulmonary capillary wedge, end-diastolic ventricle, and end-systolic ventricle. Values of NT-proBNP were not significantly different between two groups.

Conclusion: Additional flow grew pulmonary artery diameters larger in Glenn patients. Contrary to our expectation, cardiac functions were not depressed in Glenn patients with additional flow. One explanation for not increasing cardiac loads is that we make shunting narrower on leaving additional flow. Our study showed that additional flow to PA leaves in Glenn patients could turn out a good strategy to grow PA without excess cardiac loads. We could make additional flow coexisting in Glenn patients without depressing cardiac functions.

\section{O14-6}

Coarctation of the aorta repair in neonates under three months of age: mortality and risk factors for reintervention Lehnert A., Villemain O., Gaudin R., Raisky O., Bonnet D. M3C-Necker Enfants malades, AP-HP, Université Paris Descartes, Sorbonne Paris Cité, Paris, France

Objectives: The main challenge of aortic coarctation repair in neonates is to get durable results without morbidity. We aimed to describe the pronostic factors of mortality and secondary intervention procedure in order to adapt the diagnosis announcement, the surgical strategy and the long-term follow-up.

Methods: We performed a retrospective study on neonates under three months of age who underwent primary aortic coarctation repair without associated congenital heart disease (except VSD), between January 2000 and March 2014.

Results: We reviewed 530 patients. Three hundred and eight (58\%) patients had isolated coarctation and $222(42 \%)$ had a coarctation with ventricular septal defect (CoA + VSD). Three hundred and eighty five patients (72.6\%) underwent an isolated coarctation repair, 51 patients $(9.6 \%)$ had coarctation repair with closure ventricular septal defect (VSD), and 94 patients (17.8\%) had coarctation repair and banding pulmonary artery (PA). Mean follow-up was 4.87 years $+/-4.5$ years $(95 \%)$. Freedom from event were $80 \%$ at one year and $70 \%$ at five years for the whole population, and $85 \%$ at one year and $80 \%$ at five years for isolated coarctation. Overall mortality was 3.6\% (19 patients) without significant difference between surgical strategies. In multivariate analysis, the risks factors of death were mitral stenosis $(p=0.0074)$, presence of VSD $(p=0.037)$ and low weight at surgery $(p=0.001)$. The risk factors of reintervention were postnatal diagnosis $(p=0.0081)$, presence of VSD $(p=0.0001)$, prostaglandin treatment before surgery $(p=0.0171)$ and low weight at surgery $(\mathrm{p}=0.0294)$.

Conclusion: This study underlines the necessity to improve the antenatal diagnosis and the use of prostaglandin to decrease the reintervention risk in neonates with aortic coarctation. Weight at surgery is one of the mortality and reintervention risk factors, therefore surgery must be delayed if possible.

\section{ON-1}

The effects of Transition Care Models in adolescents with Congenital Heart Disease: preliminary results from a quasiexperimental study

Flocco S.F. (1), Dellafiore F. (2), Caruso R. (2), Pittella F. (2),

Giamberti A. (1), Carminati M (1), Chessa M. (1)
(1) Pediatric and Adult Congenital Heart Disease Center, IRCCS Policlinico San Donato, San Donato Milanese (Milan, Italy); (2) Health Professions Research and Development Unit, IRCCS Policlinico San Donato, San Donato Milanese (Milan, Italy)

Introduction: The number of adolescents with Congenital Heart Disease (CHD) continues to grow, evend considering the improving of surgical and medical techniques. CHD represents a new challenge for the health care systems due to their specific peculiarities, such as the need of a specific care planning and a lifetime clinical follow-up. The literature shows how transition care models from adolescence to adulthood are strategic to ensure the continuity of the care and to improve adolescents' knowledge and self-managing skills, even considering the psychological issues related to their condition.

Currently, the literature describe "Transition Clinic models (TCm)" as a standard set of educational and support interventions, with a multidisciplinary approach to patients and their families. However, there is a gap in knowledge regarding the clinical relevance of TCm. TCm could improve patient outcomes such as knowledge, adherence and lifestyles. However, there is a gap of empirical evidences in the literature and - in order to fill it - a team of researchers and clinicians started to adopt the TCm at IRCCS Policlinico San Donato (Milan, Italy). This article presents the preliminary results of a study aimed at describing the effects of the TCm on CHD adolescents' outcomes.

Methods: A quasi-experimental study designs was conduct after approval from the San Raffaele Hospital (Milan, Italy) Ethics Committee (number: 36/INT/2015). The data were collected from September 2015 to 2017, using four questionnaires on the subject of knowledge, health condition and quality of life and submitted before the implementation of the TCm (T0) and one year after the enrolment (T1)

Results: Was enrolled 250 CHD patients with 12-18 years old, but this study is related to 100 patients enrolled (59\% boys with $16.71 \pm 5.2$ years). The overall results showed a good impact of TCm on CHD adolescents' outcomes, describing in T1 a reduction of pain and anxiety and improvement of life satisfaction, health and quality of life.

Conclusion: The TCm seems to provide high-quality care to the patient, by a multidisciplinary team. The TCm needs to be standardized and then shared with all the other Italian centers that are mainly focused on CHD patients.

\section{ON-2}

Ready for the transfer to the adult care - Factors associated with transition readiness in adolescents with congenital heart disease

Burström Å. (1,2), Acuña Mora M. (3,4), Öjmyr-Joelsson M. (1,5), Sparud-Lundin C. (3), Rydberg A. (6), Hanseus K. (7), Frenckner B. (1,5), Nisell M, (1,8), Moons P. (3,4), Bratt E.-L. $(3,9)$

Institution for Women's and Children's Health, Karolinska Institutet, Stockholm, Sweden (1); Department of Paediatric Cardiology, Astrid Lindgren Children's Hospital, Stockholm (2); Institute of Health and Care Sciences, University of Gothenburg, Gothenburg, Sweden (3); KU Leuven Department of Public Health and Primary Care, Leuven, Belgium (4); Department of Paediatric Surgery, Astrid Lindgren Children's Hospital, Stockholm, Sweden (5); Department of Clinical Sciences, Pediatrics, Umeå University, Umeå, Sweden (6); Department of Pediatric Cardiology, Skane University Hospital, Lund, Sweden (7), The Red Cross University College, Stockholm, Sweden (8); Department of Pediatric Cardiology, The Queen Silvia Children's Hospital, Sweden (9)

Introduction: Transfer to adult care for adolescents with Congenital Heart Disease (CHD) is predominantly determined by age even 
though international guidelines recommend taking selfmanagement skills, maturity and transition readiness into consideration. To improve preparation for transfer to adult care transition readiness is essential to scrutinize. The aim of this study was to (i) describe the level of readiness for transition in adolescents with CHD, (ii) to compare the level of readiness for transition assessed by the adolescents with parental assessments, and (iii) to study potential correlates of transition readiness in adolescents with CHD.

Methods: A cross-sectional questionnaire study was conducted. Adolescents aged 14-18y with a CHD and their parents were invited. Data collected: Sociodemographic information, CHD-complexity, Readiness for Transition (RTQ), Empowerment (GYPES), Knowledge (KnoComh), Quality of life (LAS), Illness Perception (IPQ), Health status (PedsQL) and Health Behavior (HBS). Parents filled out RTQ-proxy version.

Results: Data from 157 triads (adolescents, mothers and fathers) were included. Mean age was $15.7 \mathrm{y}$, and $45.9 \%$ were females. Adolescent overall readiness (A-OR) and adolescent responsibility $(\mathrm{A}-\mathrm{AR})$ increased with age (A-OR $\mathrm{p}=0.03, \mathrm{~A}-\mathrm{AR} \mathrm{p}=0.002)$. Parental involvement assessed by adolescents (A-PI) decreased with increasing age $(p=0.045)$. Adolescent scored their A-OR higher than parents $(p=0.001)$. Fathers scored PI lower than the mothers and adolescents (father-PI mean $3.2 \pm 0.8$, mother-PI mean3.6 \pm 0.5 , adolescent-PI mean3.6 $\pm 0.6 ; \mathrm{p}<0.001)$. A-OR was associated with mother-OR $(p=0.002)$ and father-OR $(p=0.001)$. A higher level of empowerment $(p=0.03)$ and lower IPQ-score $(p=0.047)$ predicted a higher level of A-OR. A higher level of A-AR was associated with increased age $(p=0.04)$, mother-AR $(p=0.003)$ and father-AR $(p=0.02)$. The A-PI was predicted by the mothers-PI $(p=0.023)$ and adolescents age $(\mathrm{p}=0.025)$.

Conclusion: Age was associated with transition readiness and increased adolescent responsibility as well decreased parental involvement. Age in itself does not solely determine when adolescents are ready for transfer. Empowerment and illness perception also are related to transition readiness. Therefore, emphasis should be given to empowering adolescents, increase awareness about their condition to promote self-management during transition to adulthood and transfer to adult care.

ON-3

It's like balancing on a slackline - A description from adults living with congenital heart disease

Bay A. (1), Lämås K. (1), Berghammer M. (2,3), Sandberg C. (1), Johansson B. (1)

Umea University Sweden (1); University West Trollhattan Sweden (2); Sahlgrenska University Hospital Gothenburg Sweden (3)

Introduction: Several studies have shown that adults with congenital heart disease have reduced exercise capacity and do not reach the recommended daily level of physical activity. With this in view, it is of great importance to investigate how this population experiences physical activity. The aim of the study is to illuminate how adults with congenital heart disease describes themselves in relation to physical activity.

Methods: Semi-structured interviews with fourteen adults with complex congenital heart disease were performed. Patients were recruited from the clinic waiting list, based on their scheduled follow up and diagnosis. Interviews were analysed by qualitative content analysis.

Results: The overall theme It's like balancing on a slackline illustrates how adults with congenital heart disease described themselves in relation to physical activity. The overall theme consists of four themes: Being an adventurer- enjoying the challenges of physical activity, Being a realist- adapting to physical ability, Being a non-doer- lacking prerequisites for physical activity and Being an outsider- feeling excluded depending on physical ability.

Conclusions: The descriptions on themselves as a physically active were not constant or one-dimensional and the descriptions varied during the interviews, related to different time periods in life. It meant that they could described themselves as being an adventurer liking tough challenges, but at the same time describing themselves as being a non-doer with uncertainty over their physical strength. The findings point out specific factors for adults with CHD that might constitute as obstacles, but also possibilities for being physically active.

\section{ON-4}

Patient and parent feedback in relation to a dedicated cardiomyopathy transition clinic

Green L., Mackay R., Casey C., Daubeney P., Prasad S.

The Royal Brompton Hospital London England

Introduction: The Inherited Cardiac Conditions Service offers transitional care to young people and their families transitioning from children's to adult services. Incorporated in this is a dedicated cardiomyopathy clinic for young people between the ages of 16 to 20 years. Transitional care aims to provide the necessary skills for self-management and participation in care (Acuna Mora, 2017). The National Institute for Clinical Excellence (2016) recommend that young people should be involved in transition service design, delivery and evaluation.

Method: 'Quick feedbacks sheets' (QFS) were provided to young people and their carers during their attendance to the cardiomyopathy transition clinic in order to obtain feedback and suggestions for practice development. An 'emotional touchpoint' (ETP) was performed to explore a patient's experiences of transition.

Results: The QFS was completed by 14 patients and their parents who attended the transition clinic. Patients commented on members of the multi-disciplinary team as 'helpful' thus contributing to them having a 'positive experience'. The ETP sourced data in relation to experiences of transition. Feelings were expressed including; feeling "nervous" "It's a new stage in life, things may be a little different from before" and a general theme of support was conveyed, "everyone wants the best for me". Themes of inclusion and communication were also present; 'contact with nurse excellent' and 'I feel included in decisions'. Service development areas were identified, patients responded that they would like the opportunity to meet other young people; a 'way to let patients talk with the same condition to offer support and advice' would be appreciated. Another recommendation was that a patient stated 'I would have liked a transition talk earlier before I turned 16'.

Conclusions: Feedback was obtained from patients, and their parents, attending a cardiomyopathy transition clinic. Using the specified tools, themes were identified, thus reinforcing the need for communication and supportive practice during the transition process. It was recognised that transition consultations are required earlier than at the age of 16 years. Service development should be considered to incorporate the enabling of young people to meet others with the same condition to facilitate peer discussion and support.

YIA-1

$\mathrm{X}$ gene variant identified in childhood pulmonary arterial hypertension induces activation of p53 signaling pathway and a decrease in pulmonary arterial smooth muscle cell apoptosis Chida A. (1), Furutani Y. (1), Inai K. (1), Furukawa T. (2,3), Nakayama T. (4), Ono Y. (5), Nakanishi T. (1) 
Department of Pediatric Cardiology, Tokyo Women's Medical University, Tokyo, Japan (1); Institute for Integrated Medical Sciences, Tokyo Women's Medical University, Tokyo, Japan (2); Department of Histopathology, Tohoku University Graduate School of Medicine, Sendai, Japan (3); Department of Pediatrics, Toho University Omori Medical Center, Tokyo, Japan (4); Department of Pediatric Cardiology, Shizuoka Children's Hospital, Shizuoka, Japan (5)

Background: Mutations in the bone morphogenetic protein receptor type 2 (BMPR2) gene and other several genes have been reported in heritable pulmonary arterial hypertension (HPAH) and idiopathic pulmonary arterial hypertension (IPAH). However, 60 to $90 \%$ of IPAH cases have no mutations in these genes. This study was conducted to identify a novel cause of PAH and clarify the pathogenesis of PAH.

Methods: In order to find disease causing variants, we performed direct sequencing and multiplex ligation dependent probe amplification to analyze 18 families with multiple affected family members with PAH. In one of the 18 families with PAH, there were no disease causing variants in BMPR2, ACVRL1, ENG, SMAD1/4/8, BMPR1B, NOTCH3, CAV1, or KCNK3. In this family, a female proband and her paternal aunt developed $\mathrm{PAH}$ in their childhood. To identify novel disease causing variants efficiently, whole exome next generation sequencing was performed in the $2 \mathrm{PAH}$ patients and the proband's healthy mother. Based on the result, we performed functional analysis using human pulmonary arterial smooth muscle cells (hPASMCs), candidate gene small interfering RNA, and candidate gene constructs.

Results: We identified a X gene variant, R554L, in the 2 family members with $\mathrm{PAH}$, but not in the proband's mother without $\mathrm{PAH}$. Knockdown of the $\mathrm{X}$ in hPASMCs induced p53 signaling pathway activation and decreased cell viability. Western blotting showed R554L X in hPASMCs induced lower expression of p53 and $\mathrm{p} 21$, and lower phosphorylation of $\mathrm{p} 53$ in nucleus compared to wild type X. Furthermore, hPASMCs proliferation and viability were higher for cells transfected the R554L X than for them transfected wild type X. In addition, R554L X induced a decrease in apoptotic cells in hPASMCs compared to wild type X.

Conclusions: We identified a novel variant in $\mathrm{X}$ gene in a Japanese family with PAH. The variant, R554L X, revealed gain of function and induced decreasing nucleus p53 and p21, lower phosphorylation of nucleus p53, decreasing apoptotic hPASMCs, and increasing hPASMCs proliferation and cell viability compared to wild type X. This study can contribute to elucidate mechanism of $\mathrm{PAH}$ pathogenesis.

\section{YIA-2}

\section{Hypobaric hypoxia during air travel in patients after the Fontan operation}

Morimoto Y., Ohuchi H., Suzuki D., Iwasa T., Negishi J.,

Kurosaki K.

National Cerebral and Cardiovascular Center, Osaka, Japan

Introduction: Pulmonary circulation has unique characteristics in patients after the Fontan operation, which includes lack of pulmonary ventricle, non-pulsatile flow, leading to mild hypoxia mainly due to ventilation/perfusion mismatch in the lung. Although Fontan patients have increasing opportunity of air travel, there has been no evidence-based recommendation for air travel because of no available data on an effect of hypobaric hypoxia $(\mathrm{HH})$ during air travel in these patients.

Methods: We prospectively checked changes in percutaneous oxygen saturation $(\mathrm{SpO} ; \%$ ) and heart rate (HR;bpm) (PULSOXMe300) during commercial air travel in 7 clinically stable Fontan patients (male $=3$, aged 15 to 33 years with the postoperative follow-up of 14-23 years). These patients were asked to list a departure point, an arrival point, and any major adverse event (s) or symptoms during air travel, including headache and palpitations. We compared the dynamics of $\mathrm{SpO} 2$ and HR during air travel with recent hemodynamics, pulmonary function test and cardiopulmonary variables during exercise testing (CPX).

Results: Decrease in $\mathrm{SpO} 2$ from baseline $(92.4 \pm 3.7 \%)$ to 1 st stabilized $\mathrm{HH}(87.9 \pm 5.3 \%)$ during air travel was $4.5 \pm 2.3 \%$ $(0.8-7.3 \%)$ and was correlated with decrease in SpO2 during CPX $(\mathrm{p}=0.0499) . \mathrm{SpO} 2$ at 1 st stabilized $\mathrm{HH}$ correlate with the lowest $\mathrm{SpO} 2$ at CPX $(70-94 \%)(p=0.0192)$. The peak VO2 was correlated with the lower $\mathrm{SpO} 2$ at 1st stabilized $\mathrm{HH}(\mathrm{r}=0.9132$, $\mathrm{p}=0.0041)$. Any hemodynamic data during catheterization did not predict the $\mathrm{SpO} 2$ dynamics during corresponding flight. The flight duration was inversely correlated with the magnitude of $\mathrm{HH}$ $(\mathrm{r}=-0.9613, \mathrm{p}=0.0387)$. One patient with the lowest $\mathrm{SpO} 2$ of $78 \%$ at 1 st stabilized $\mathrm{HH}$ experienced headache and malaise during his flight.

Conclusions: Fontan patients showed significant $\mathrm{HH}$ during air travel with a wide individual variety. Exercise-induced hypoxia strongly predicted the hypobaric-induced hypoxia and CPX with $\mathrm{SpO} 2$ monitoring may help clinicians to guide the air travel in these patients.

\section{YIA-3}

Fever within $48 \mathrm{~h}$ after Melody implantation is a risk factor of late infective endocarditis

Villemain O. (1), Ben Moussa N. (2), Malekzadeh-Milani S. (1), Meot M. (1), Mostefa-Kara Ma. (1), Bonnet D. (1), Boudjemline Y. (1) M3C-Necker Enfants malades, AP-HP, Université Paris Descartes, Sorbonne Paris Cité, Paris, France (1); Cardiology Department, Hopital Européen Georges Pompidou, Paris, France (2)

Objectives: Fever following pulmonary valve implantation is known but it's frequency and impact have been poorly described. The aim of this study was to analyze clinical and biological parameters of inflammation just after the Melody valve implantation, and to see if early onset of inflammation can predict the risk of late infective endocarditis (IE).

Methods: We performed a retrospective study on patients with Melody valve implanted for dysfunctional RVOT in our unit between January 2008 and December 2016. All clinical and biological inflammatory parameters following Melody implantation were recorded.

Results: 198 patients were included (median age $=22.8$ [Q1-Q3: 15.4-31.5] years). Before the procedure, the RVOT statute of patients was: $74(37.4 \%)$ pulmonary valved conduits, 57 (28.8\%) natives (with or without valve), 50 (25.2\%) homografts, and 17 $(8.6 \%)$ valveless conduits. Within $48 \mathrm{~h}$ post procedure, 58 patients $(29.3 \%)$ had fever $\left[\mathrm{T}>37.9^{\circ} \mathrm{C}\right]$ and 140 patients $(70.7 \%)$ had no fever, 98 patients $(13.6 \%)$ had a CRP $>5 \mathrm{mg} / \mathrm{L}$, and 61 patients $(30.8 \%)$ had white blood cells (WBC) $>10 \mathrm{G} / \mathrm{L} .55$ patients received large spectral antibiotic regimen waiting for results of blood culture. No patients were found to have early IE and antibiotics were stopped after a median duration of 2 [1-2] days. Length of stay was longer in patients with fever compared to those without fever $(\mathrm{p}<0.001)$.

The median follow-up was 3.3 [1.3-5.3] years. Twenty-one patients developed IE (10.6\%), with a median at 1.8 [0.3-3.5] years. At 5 years, freedom from IE was $87.2 \%$. Fever within $48 \mathrm{~h}$ post procedure was a significative risk factor $(\mathrm{HR}=6.1, \mathrm{IC} 95 \%$ [2.5-15.1], p < 0.001). At 5 years, freedom from IE was $88.5 \%$ for patients without fever and $67.7 \%$ patients with fever within $48 \mathrm{~h}$ 
post procedure $(p<0.001)$. Elevated post-procedural WBC $(p=0.62)$, CRP level $(p=0.90)$, and initial RVOT statute of patients $(p=0.70)$ were not a risk factor for IE.

Conclusion: Patients with fever within 48 hours after Melody valve implantation are at particular risk of IE in their life. The reasons might be related to a specific genetic profile. Further studies are needed to understand the causes behind.

\section{YIA-4}

A validation study of the paediatric guidelines of the 2014 European Society of Cardiology risk stratification algorithm for sudden cardiac death in hypertrophic cardiomyopathy

Norrish G. (1,2), Field E. (1,2), Mcleod K. (3), Illina M. (3), Stuart G. (4), Bhole V. (5), Uzun O. (6), Brown E (7), Daubeney P. (8), Linter K. (9), Mathur S. (10), Bharucha T. (11), Adwani S. (12), Jones C. (13), Reinhardt Z. (14), Kaski J.P. (1,2)

Great Ormond Street Hospital, London UK (1); UCL Institute of Cardiovascular science, London, UK (2); Royal Hospital for Children, Glasgow, UK (3); Bristol Royal Children's Hospital, Bristol UK (4); Birmingham Children's Hospital, Birmingham, UK (5); The Noah's Ark Children's Hospital for Wales, Cardiff, UK (6); Leeds Teaching Hospital NHS Trust, Leeds, UK (7); Royal Brompton and Harefield NHS foundation Trust, London UK (8); Glenfield Hospital, Leicester, UK (9); Evelina London Children's Hospital, London, UK (10); University Hospital Southampton NHS Foundation Trust, Southampton, UK (11); Oxford University Hospitals, UK (12); Alder Hey Children's NHS foundation Trust, Liverpool, UK (13); The Freeman Hospital, Newcastle, UK (14)

Introduction (or Basis or Objectives): Sudden cardiac death (SCD) is the most common cause of death in children with hypertrophic cardiomyopathy (HCM). However, identifying which patients are at high risk and may benefit from an implantable Cardioverter Defibrillator (ICD) is challenging. A personalised risk score for adults with HCM has recently been incorporated into the European Society of Cardiology (ESC) guidelines, but cannot be used in children. Instead, current ESC recommendations suggest consideration of an ICD in children with HCM if two or more clinical risk factors are present. However, this approach to risk stratification in childhood HCM has not been formally validated. Methods: 687 paediatric HCM patients derived from a retrospective, national cohort study in United Kingdom were assessed for 4 clinical risk factors (RF) for SCD: severe left ventricular hypertrophy (maximal wall thickness $>30 \mathrm{~mm}$ or $\mathrm{Z}$ score $>6$ ); unexplained syncope; non-sustained ventricular tachycardia; and family history of SCD. The primary end point was a composite of SCD, appropriate ICD therapy or sustained ventricular tachycardia.

Results: Over a follow up period of 4596 patient years (median follow up 5.2 years), SCD or equivalent event occurred in 33 of $531(6.2 \%)$ patients with no RF, 20 of $138(14.5 \%)$ patients with 1 RF, 4 of $16(25 \%)$ patients with 2 RF and 1 of 2 patients $(50 \%)$ of patients with 3 RF. Annual rate of SCD was $0.01 \%, 0.02 \%, 0.04 \%$ and $0.08 \%$ respectively. The risk of SCD increased with additional clinical risk factors (1 RF hazard ratio 1.84, $\mathrm{p}=0.032 ; 2 \mathrm{RF}$ hazard ratio $3.58 \mathrm{p}=0.016 ; 3 \mathrm{RF}$ hazard ratio $9.00 \mathrm{p}=0.08$ ). The positive predictive value for SCD if 2 or more risk factors were present is $27.8 \%$. The concordance statistic for the ability to correctly identify a patient at high risk based on the number of risk factors was 0.62 at 1 year and 0.65 at 5 years.

Conclusions: The risk of SCD is higher for patients with increasing numbers of clinical risk factors. However, the current ESC guidelines have a low ability to discriminate between high and low

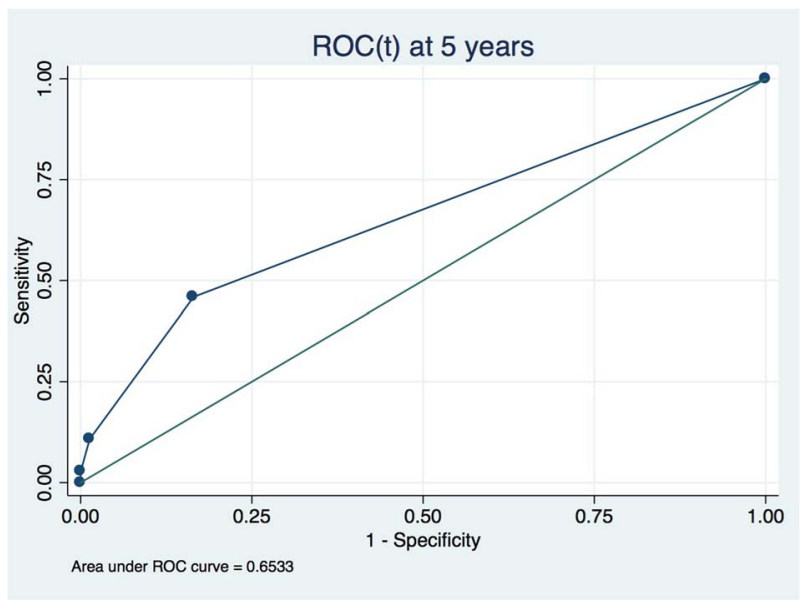

Figure.

risk individuals. Future studies are required to develop and evaluate risk stratification strategies in childhood HCM.

Legend: Receiver operating characteristic $(\mathrm{ROC}(\mathrm{t}))$ Curves for the European Society of Cardiology (ESC 2014) guideline at 5 years.

\section{YIA-5}

An innovative cell therapy using mitochondrial drug delivery system for doxorubicin cardiomyopathy Abe J. (1), Yamada Y. (2), Takeda A. (1), Harashima H. (2) Department of Pediatrics, Faculty of Medicine, Hokkaido University, Sapporo, Japan (1); Laboratory for molecular design of pharmaceutics, Faculty of Pharmacentical Sciences, Hokkaido University, Sapporo, Japan (2)

Objectives: Recent molecular researches have revealed that doxorubicin (DOX)-induced cardiomyopathy happens mainly due to poor mitochondrial biogenesis and exaggerated oxidative stress. Resveratrol has a potent of anti-oxidative ability mediated through multiple functions in mitochondria. Here, we investigated mitochondrial therapeutic effect in stem cell antigen-1 positive cardiac progenitor cells (Sca1-CPCs) after mitochondrial delivery of resveratrol.

Methods: A MITO-Porter system, mitochondrial drug delivery system, was used to deliver resveratrol into mitochondria in Sca1-CPCs. We encapsulated resveratrol in MITO-Porter, and observed the intracellular trafficking after transfection into Sca1-CPCs. We next investigated the therapeutic effects using DOX-exposure cells by measuring the mitochondrial enzymatic activity and membrane potential. Moreover, we validated the therapeutic effects using DOX-induced cardiomyopathy model mice to assess survival rate and tissue pathology. We conducted a randomized study wherein 24 mice received an intramyocardial injection of placebo-medium, naked Sca1-CPCs, or Sca1-CPCs with MITO-Porter before DOX treatment. The survival rate was assessed by Kaplan-Meier curve, and the assessment of the left ventricular shortening fraction (LVFS) was followed 4 weeks after injection. To investigate mitochondrial function in the host heart, the mitochondrial protein expression levels, and mitochondria related gene expression levels were measured 3 days after the DOX treatment. Results: Intracellular observation showed that MITO-Porter could accumulate in mitochondria of Sca1-CPCs. We confirmed that mitochondrial delivery of resveratrol protected Sca1-CPCs from DOX-induced toxicity due to the preservation of the mitochondrial enzymatic activity and membrane potential in vitro. In Sca1-CPCs with MITO-Porter-injected mice at 4 weeks, the 
values of LVFS were kept within the normal range, and these were associated with the better survival rate (log-rank test; $\mathrm{P}<0.05)$. In the myocardium of Sca1-CPCs with MITO-Porter-injected mice, mitochondrial electron transport complex proteins were partially preserved $(\mathrm{P}<0.05)$, and the OXPHOS and mitochondrial biogenesis related genes expression levels were also higher than naked Sca1-CPCs $(\mathrm{P}<0.05)$.

Conclusions: The transplant of Sca1-CPCs with MITO-Porter loading resveratrol, which possess activated mitochondria and reduce oxidative stress, would have more efficiency rather than the conventional CPCs transplant in doxorubicin-induced cardiomyopathy.

\section{YIA-6}

Longitudinal hemodynamic assessment of fetuses with TGA to predict the perinatal course - The pilot study Grzyb A. (1,3), Kolesnik A. (2,3), Duliban J. (3), KowalczykDomagala M. (1), Zubrzycka M. (2), Brzezinska-Rajszys G. (1,2), Bokiniec R. (4), Dangel J. (3) Cardiology Department, The Children's Memorial Health Institute, Warsaw, Poland (1); Heart Catheterization Laboratory, The Children's Memorial Health Institute, Warsaw, Poland (2); Perinatal Cardiology and Congenital Anomalies Department, Centre of Postgraduate Medical Education, Warsaw, Poland (3); Neonatology and Neonatal Intensive Care Department, Medical University of Warsaw, Warsaw, Poland (4)

Introduction: Transposition of the great arteries (TGA) is one of the most common congenital heart diseases, well-tolerated prenatally, however life-threatening for the newborn. The main concerns are: the foramen ovale (FO) restriction, sometimes coexisting with persistent pulmonary hypertension (PPHN), which may preclude efficient intracardiac mixing. Numerous parameters have been described to predict the need for urgent balloon atrioseptostomy (BAS) and the occurrence of PPHN after birth, with often conflicting results. The aim of this study is to develop and test a different method, based on a thorough, longitudinal observation of intracardiac blood flows, to predict the hemodynamic status of the newborn with TGA.

Methods: Retrospective-prospective analysis of echocardiographic examinations of 66 fetuses diagnosed with simple TGA (small VSDs included) in a reference fetal cardiology center between 2011-2017. Results: Based on our observations we developed a flowchart of fetal TGA assessment presented below. Its usefulness in predicting the newborn's condition is shown in the table.

1. FO flow $\mathrm{R} \rightarrow \mathrm{L}$ or bidirectional, blood mixing assessed by Color Doppler:

a. mixing clearly visible $\rightarrow$ NO RESTRICTION.

b. mixing limited by interatrial septum [IAS] $\rightarrow$ go to point $2 / 3$.

2. Short, thickened, usually hypermobile $\mathrm{FO}$ valve, $\mathrm{R} \rightarrow \mathrm{L}$ unrestrictive DA flow, systolic velocity usually $\mathrm{PT}=\mathrm{Ao}$ or $\mathrm{PT}>\mathrm{Ao} \rightarrow$ FO RESTRICTION.

3. Long FO valve bulging deeply into the left atrium:

a. DA L $\rightarrow \mathrm{R}$ diastolic flow, systolic velocity $\mathrm{PT}<\mathrm{Ao} \rightarrow \mathrm{FO}$ RESTRICTION, possibly technically difficult BAS.

b. If in subsequent examinations the atrial septum excursion decreases or septum becomes hypermobile; end-systolic and/or diastolic $\mathrm{L} \rightarrow \mathrm{R}$ DA flow $\rightarrow$ increased pulmonary flow $\rightarrow$ RISK OF PPHN.

4. DA restriction/narrowing OR long lasting ( $\geq 5$ weeks) limited interatrial mixing $\rightarrow$ HIGH RISK OF PPHN.

5. Obligatory assessment every 1-2 week after 35 week of pregnancy.
Table.

\begin{tabular}{lllllllll}
\hline & Outcome & \multicolumn{1}{c}{} & & & & & \\
\cline { 2 - 5 } Prediction & $\begin{array}{l}\text { No } \\
\text { restriction }\end{array}$ & $\begin{array}{l}\text { Urgent } \\
\text { BAS }\end{array}$ & $\begin{array}{l}\text { BAS + } \\
\text { PPHN }\end{array}$ & Sensitivity & Specificity & PPV & NPV \\
\hline No restriction & 25 & 1 & 0 & $83,3 \%$ & $97,2 \%$ & $96,2 \%$ & $87,5 \%$ \\
Urgent BAS & 3 & 11 & 5 & $78,6 \%$ & $84,6 \%$ & $57,9 \%$ & $93,6 \%$ \\
BAS + PPHN & 2 & 2 & 17 & $77,3 \%$ & $90,9 \%$ & $81,0 \%$ & $88,9 \%$ \\
\hline
\end{tabular}

Conclusions: Longitudinal assessment and interpretation of fetal TGA hemodynamics seems to predict the newborn's condition with high accuracy and specificity, which is important in planning the perinatal period, especially in cases with suspected PPHN.

MP1-1

Changes of gene expressions in pulmonary arterial hypertension rat models after ambrisentan treatment Hong Y.M., Kim K.C.

Departments of Pediatrics (1); Thoracic and Cardiovascular Surgery (2); Ewha Womans University School of Medicine, Seoul, Korea

Introduction: Pulmonary arterial hypertension (PAH) causes right ventricular failure due to a gradual increase in pulmonary vascular resistance. Ambrisentan is an oral, once-daily, endothelin (ET) A -selective endothelin receptor antagonist (ERA) for the treatment of $\mathrm{PAH}$. The purpose of this study was to investigate the effect of ambrisentan on right ventricular (RV) pressure, lung pathology and gene expressions.

Methods: The rats were grouped as follows: the control (C) group; the $\mathrm{M}$ group, monocrotaline (MCT) $60 \mathrm{mg} / \mathrm{kg}$, sc); the A group (MCT + ambrisentan $3 \mathrm{mg} / \mathrm{kg} /$ day by gavage feeding). Hemodynamic study was performed by catheterization into the external jugular vein and pathological changes were investigated by Victoria blue staining in the lung tissues. Changes of protein expression levels of ET-1, ERA, endothelial nitric oxide (eNOS) and NADPH oxidase (NOX) 4 were confirmed by western blot analysis.

Results: The mean RV pressure was significantly reduced in the A group at weeks 2 (Mvs. A, $27.00 \pm 1.47 \mathrm{mmHg}$ vs. $16.60 \pm$ $0.40 \mathrm{mmHg})$ and 4 (M vs. A, $32.40 \pm 0.76 \mathrm{mmHg}$ vs. $14.67 \pm 0.80 \mathrm{mmHg}) . \mathrm{LV}+$ septum $/ \mathrm{RV}$ ratio was significantly reduced in the A group at week 4. Reduced medial wall thickness in the pulmonary arteriole was noted in the A group at week 4 . Reduction in number of intra-acinar muscular pulmonary arteries was observed in the A group at week 4. Protein expression level of ET-1 significantly decreased in the A group at week 2 . Protein expression level of ERA significantly decreased in the A group at weeks 2 and 4. Protein expression level of NOX4 significantly increased in the $\mathrm{M}$ group compared with the $\mathrm{C}$ group, but there was no difference between the $M$ group and the A group. Protein expression level of eNOS significantly increased in the A group at weeks 2 and 4.

Conclusion: There was significant improvement of mean RV pressure, RV hypertrophy and pulmonary pathology after ambrisentans treatment. Significant reduction in ET-1 and ERA protein expressions and an increase of eNOS protein expressions were also detected.

\section{MP1-2}

Acute Rheumatic Fever - Review of the Previous 40 Years and a Critical Appraisal of the Jones Criteria: A SingleCenter Experience

Akca Dinc G. (1), Kucuk M. (2) , Baysal K. (3) , Inan Aydemir N. (4), Tanyeri B. (5), Uzun N. (6) 
Ondokuz Mayıs University Department of Pediatrics Samsun Turkey (1); Samsun Obstetrics and Pediatrics Hospital Pediatric Cardiology Samsun Turkey (2); Ondokuz Mayıs University Department of Pediatric Cardiology Samsun Turkey (3); Samsun Obstetrics and Pediatrics Hospital Department of Pediatrics Samsun Turkey (4); Bezmialem University Department of Neonatology Istanbul Turkey (5); Afyonkarahisar Public Hospital Department of Pediatrics Afyon Turkey (6)

Background: Acute Rheumatic Fever (ARF) develops following group-A $\beta$-hemolytic streptococcus infections, and may result with severe complications including rheumatic heard disease (RHD). The aim of this study is to evaluate the general demographic and clinical features of patients that followed-up with a diagnosis of ARF during the last four decades at our department, and establishing an epidemiological database for further studies.

Methods: Patients that diagnosed and followed-up with a diagnosis of ARF at Ondokuz Mayis University Faculty of Medicine, Department of Pediatric Cardiology, between 19782016 were retrospectively evaluated. Assessments were separated into 4 time periods as 1978-1988, 1990-2001, 2001-2011 and 2011-2016 to reflect the temporal trends. Diagnostic criteria have been updated according to the revisions by American Heart Association in 1992 and 2015 and by World Health Organization in 2003.

Results: A total of 885 ARF cases were evaluated in the study. The incidence of ARF was found to be in a decreasing trend and most recent incidence rate was $0.164 \%$, nd RHD incidence was $0.15 \%$. there was a tendency towards female gender, and age distribution between genders were similar. Recent data suggests that isolated major findings according to Jones criteria were distributed as $58.3 \%$ for carditis, and $8.4 \%$ for arthritis. Most frequent combined major findings were carditis + arthritis $(18.7 \%)$, carditis + chorea $(12.5 \%)$, and carditis + erythema marginatum (2.1\%). The frequency of isolated chorea, erythema marginatum and subcutaneous nodules were decreased over time, and subcutaneous nodules have not been observed recently. Recent treatments resulted with cure in $55.7 \%$ of cases, and $3.4 \%$ of cases had additional valve lesions.

Conclusion: According to our findings, major complications and mortality have been controlled substantially over time, but incidence rates are still high due to shortcomings in secondary prophylaxis. Particularly subcutaneous nodules are decreased to undetectable rates, and may be excluded from the major criteria of ARF in the next revision.

Keywords: Acute rheumatic fever, rheumatic heart disease, carditis, Jones criteria, subcutaneous nodules, Turkey, epidemiology

MP1-3

Circulating endothelial cells and endothelial progenitor cells in relation to the severity of primary hypertension in children

Shi L., Zhang Y., Lin Y., Liu Y., Cui X., Li J.

Capital Institute of Pediatric, Beijing, China

Introduction: Endothelial dysfunction contributes to the progression of primary hypertension. Circulating endothelial cells (CECs) and endothelial progenitor cells (EPCs) have been used as indicators of endothelial dysfunction and related to the degree of hypertension in adults. Little is known about the changes of CECs and EPCs in children with primary hypertension. This study is to examine CECs and EPCs in children with different degrees of primary hypertension and their changes after the treatments.
Methods: We enrolled three age- and sex-matched groups of children (age $11.5 \pm 2.93 \mathrm{yrs}$ ), including 64 with primary hypertension (PH), 30 with pre-hypertension (PHP) and 30 healthy children. In $\mathrm{PH}$ group, patients were divided into two groups with target organ damage (TOD, $n=30$ ) and without (non-TOD, no $=34$ ) based on clinical tests. CECs and EPCs were measured by flow cytometry. Non-TOD group received 6-month treatment with recommended diet and exercise regime; TOD group had additional antihypertensive agents (angiotensin-converting enzyme inhibitor and calcium antagonist). Data were collected before and after the treatment period.

Results: CECs in PHP and HP groups were higher than those in healthy group $(41.43 \pm 11.52 \mathrm{cell} / \mathrm{ul}, 45.59 \pm 25.72 \mathrm{cell} / \mathrm{ul}$, and $21.23 \pm 8.91$ cell/ ul, $\mathrm{P}<0.05)$, while EPCs lower $(12.90 \pm$ $7.73 \mathrm{cell} / \mathrm{ul}, 12.70 \pm 7.98 \mathrm{cell} / \mathrm{ul}$, and $22.47 \pm 15.32 \mathrm{cell} / \mathrm{ul}$, $\mathrm{P}<0.05)$. CECs in TOD group $(48.35 \pm 19.48$ cell/ul $)$ were higher than those in non-TOD group $(43.35 \pm 19.64 \mathrm{cell} / \mathrm{ul}$, $\mathrm{P}<0.05)$, while EPCs lower $(12.60 \pm 7.98 \mathrm{cell} / \mathrm{ul}, 13.85 \pm 8.15$ cell/ul, $\mathrm{P}<0.05)$. After 6-month treatment of diet and exercise in non-TOD group, CECs reduced to $30.88 \pm 29.64$ cell $/ \mathrm{ul}$ $(p<0.05)$.EPCs did not change significantly. No significant changes were found in TOD group, attributable to different organ damages and antihypertensive agents.

Conclusions: Children with pre-hypertension and primary hypertension have endothelial dysfunction as indicated by CECs and EPCs, particularly those with TOD. Healthy diet and exercise regime may help to improve endothelial function.

\section{MP1-4}

Impact of national prenatal screening program on prenatal detection of Transposition of the Great Arteries over a 10-Year Period: Improved but not enough

Hautala J. (1), Helle E. (2), Gissler M. (3,4), Ritvanen A. (5), Pitkänen O. (2), Tekay A. (1), Stefanovic V. (1), Sarkola T. (2), Pihkala J. (2), Pätilä T. (6), Mattila I. (6), Jokinen E. (2),

Räsänen J. (1), Ojala T. (2)

1. Obstetrics, and Gynecology, Women's Hospital, University of Helsinki and Helsinki University Hospital, Finland; 2. Pediatric Cardiology, Children's Hospital, University of Helsinki and Helsinki University Hospital, Finland; 3. National Institute for Health and Welfare Finland, Helsinki, Finland; 4. Department of Neurobiology, Care Sciences and Society, Division of Family Medicine, Karolinska Institute, Stockholm, Sweden; 5. Retired, Finnish Register of Congenital Malformations, Information Services Department, National Institute for Health and Welfare, Helsinki, Finland; 6. Pediatric Cardiac Surgery, Children's Hospital, University of Helsinki and Helsinki University Hospital, Finland

Introduction: Transposition of the great arteries (TGA) is one of the most common cyanotic congenital heart defects requiring early neonatal surgery. Earlier research has demonstrated an increase in prenatal detection of simple TGA, improved survival, and quality of life in the current era.

We aimed to evaluate the impact of the introduction of a systematic national prenatal screening program on prenatal detection of simple TGA (+/- ventricular septal defect) and to evaluate the regional differences in total prevalence in the five university districts in an 11 -year national cohort from Finland.

Methods: Systematic national prenatal screening was introduced in January 2007 and has been fully implemented from January 2010 onwards. We compared prenatal detection rates of TGA in three time periods: Before the introduction of systematic ultrasound screening 2004-2006, transition period 2007-2009 and screening period 2010-2014. Data on births (all births $n=651$ 970) and 
pregnancy terminations with the diagnosis of simple TGA during 2004-2014 were assessed from five national registers (Register of Pediatric Cardiac Surgery, Register of Congenital Malformations, Register of Induced Abortions, Medical Birth Register and Cause of Death Register).

Results: There were 145 cases with simple TGA (132 live births, one stillbirth and 12 terminations of pregnancy). National total prevalence of simple TGA was 2.24/10 000 births (western part of Finland 1.78/10 000, southern 2.30/10 000, northern $2.28 / 10000$ and eastern 3.14/10 000). There was a significant difference in the total prevalence between eastern and western part of the country $(p=0.020)$.

The overall prenatal detection rate was $29 \%(42 / 145)$. The proportion of prenatally diagnosed cases increased significantly from $14.0 \%$ (2004-2006), 22.5\% (2007-2010), to 43.5\% (20102014) $(\mathrm{p}=0.001)$. However, the impact of the screening program had significant regional variation $(\mathrm{p}<0.05)$.

Conclusions: Introduction of the national prenatal screening program has significantly increased the prenatal detection of TGA $(14.0 \%-43.5 \%)$, but the impact of the screening program varied inside the country. Interestingly, even genetic isolate country such as Finland has significant regional variation in prevalence of TGA warranting for future studies.

\section{MP1-5}

Accuracy of prenatal diagnosis of coarctation of the aorta using z-scores

Vigneswaran T.V. (1), Chivers S. (2), Charakida M. (1,2), Allan L.D. (1), Zidere V. (1,2), Simpson J.M. (1,2), Akolekar R. (1)

King's College Hospital, London, UK (1)Evelina London Children's

Hospital, London, UK (2)

Aim: To assess the utility of new $\mathrm{z}$-scores and prospective measurements of the outflow tracts in the prenatal diagnosis of coarctation of the aorta (COA).

Methods: All continuing cases of COA (including with ventricular septal defect or persistent left superior caval vein) from 1998 to 2016. The period was divided into two eras, era 1 between 1998-2007 where the outflow tract measurements were made retrospectively and era 2 between 2008 - 2016 where the measurements were made prospectively. Diameters of the aortic valve, pulmonary valve, distal transverse aortic arch (DTAA) and arterial duct $(\mathrm{AD})$ were taken from the echocardiogram closest to 20 weeks. New z-scores created from prospectively made measurements from 7000 normal fetuses were used to calculate z-scores for the measured parameters.

Results: There were 193 fetuses with prenatal suspicion of COA. COA was confirmed after birth in 56/107 (52\%) in era 1 and in $42 / 86$ (49\%) in era 2 . There was no significant difference between the true COA and false positive groups in the incidence of nuchal translucency measurement $(p=0.3)$, presence of left superior vena cava $(\mathrm{p}=0.3)$ or VSD $(\mathrm{p}=0.7)$. Bicuspid aortic valve was seen in $22 / 37(59 \%)$ of the true coarctation group and 13/46 (28\%) of the false positive group $(p=0.04)$.

The $\mathrm{z}$-scores of the cardiac measurements are shown in Table 1. The aortic valve and DTAA $z$-scores were significantly smaller in the true COA cases compared to the false positive cases, but there was considerable overlap. Multiple regression analysis demonstrated that DTAA z-score was the only significant predictor $(p=0.004)$ with an area under the receiver operator curve of 0.712 $(\mathrm{p}<0.001)$. DTAA $z$-score $<0$ confers a sensitivity of $100 \%$, but with a false positive rate of $90 \%$. To achieve a false positive rate of $50 \%$ the DTAA z-score cut-off would be -2.65 and a sensitivity of $83 \%$ would need to be accepted.
Table 1.

\begin{tabular}{|c|c|c|c|}
\hline & $\begin{array}{l}\text { Era 1: } 1998-2007 \\
\text { True coarctation } \\
(\mathrm{n}=56)\end{array}$ & $\begin{array}{l}\text { Era 2: 2008-2016 } \\
\text { False positives } \\
(\mathrm{n}=51)\end{array}$ & $\begin{array}{l}P \\
\text { value }\end{array}$ \\
\hline $\begin{array}{l}\text { Gestation at scan } \\
\text { (range) in weeks }\end{array}$ & $21(19-32)$ & $22(16-36)$ & 0.59 \\
\hline Pulmonary valve $Z$ score & $1.5(-1.5$ to 3.3$)$ & $0.8(-2.2$ to 4.1$)$ & 0.15 \\
\hline Aortic valve $\mathrm{Z}$ score & $-1.5(-6.8$ to 2.0$)$ & $-1.3(-5.4$ to 3.8$)$ & 0.04 \\
\hline $\begin{array}{l}\text { Aortic:pulmonary valve } \\
\text { diameter }\end{array}$ & $0.62(0.39-0.85)$ & $0.68(0.49-1.02)$ & 0.001 \\
\hline Arterial duct $Z$ score & $0.9(-4.4$ to 3.4$)$ & $0.4(-3.3$ to 3.8$)$ & 0.11 \\
\hline DTAA diameter & $1.8(1.1-3.2)$ & $2.3(1.3-4.3)$ & $<0.001$ \\
\hline DTAA Z score & $-3.7(-9.3$ to -0.2$)$ & $-2.1(-7.1$ to +2.5$)$ & $<0.001$ \\
\hline DTAA: arterial duct & $0.66(0.26-1.18)$ & $0.81(0.53-1.50)$ & $<0.001$ \\
\hline \multirow{2}{*}{$\begin{array}{l}\text { No. of cases of bilateral } \\
\text { superior vena cava }\end{array}$} & $10 / 55$ & $9 / 52$ & 0.91 \\
\hline & $\begin{array}{l}\text { True coarctation } \\
(\mathrm{n}=42)\end{array}$ & $\begin{array}{l}\text { False positives } \\
(n=46)\end{array}$ & $\begin{array}{l}P \\
\text { value }\end{array}$ \\
\hline $\begin{array}{l}\text { Gestation at scan } \\
\text { (range) in weeks }\end{array}$ & $22(15-32)$ & $23(19-33)$ & 0.57 \\
\hline Pulmonary valve $\mathrm{Z}$ score & $1.5(-1.4$ to 2.5$)$ & $1.0(-6.9$ to 3.4$)$ & 0.44 \\
\hline Aortic valve $\mathrm{Z}$ score & $-2.0(-6.2$ to 2.7$)$ & $-1.4(-5.7$ to 4.8$)$ & 0.06 \\
\hline $\begin{array}{l}\text { Aortic:pulmonary valve } \\
\text { diameter }\end{array}$ & $0.62(0.40-1.03)$ & $0.70(0.45-0.91)$ & 0.01 \\
\hline Arterial duct $Z$ score & $0.8(-4.0$ to 2.7$)$ & $0.4(-3.5$ to 2.7$)$ & 0.3 \\
\hline DTAA diameter & $2.0(0.8-3.3)$ & $2.3(1.3-3.5)$ & 0.007 \\
\hline DTAA Z score & $-3.8(-10.9$ to -0.3$)$ & $-2.7(-6.2$ to +1.2$)$ & 0.005 \\
\hline DTAA: arterial duct & $0.66(0.3-1.09)$ & $0.75(0.26-1.45)$ & 0.02 \\
\hline $\begin{array}{l}\text { No. of cases of bilateral } \\
\text { superior vena cava }\end{array}$ & $9 / 42$ & $11 / 44$ & 0.70 \\
\hline
\end{tabular}

Conclusion: Bicuspid aortic valve is overrepresented in the false positive group compared to the normal population. Despite the acquisition of prospectively acquired measurement of the outflow tracts, biometry alone is not sufficient to reduce the false positive rate, this suggests that further factors need to be identified to improve the prenatal diagnostic accuracy.

\section{MP1-6}

Fetal Aortic Arch variants: Left or Right matters!

Germanakis I. (1), Pepes S. (1), Sifakis S. (1), Nikoletakis G. (2), Malathrakis D. (2), Chlapoutakis K. (2), Mantas N. (2), Mousourakis S. (2), Giakoumakis I. (2), Mataliotakis I. (1), Makrigianakis A. (1)

Faculty of Medicine, University of Crete, Heraklion, Greece (1); Private Praxis of Obstetrics/Obstetric Ultrasound, Heraklion/Rethymnon/ Chania, Greece (2)

Introduction: Following routine anomaly ultrasound improved heart imaging, a greater number of aortic arch (AA) abnormalities is expected to be detected. The clinical impact of this remains to be determined.

Methods: Retrospective study of 3,414 fetal echocardiogram (FE) records of a tertiary academic referral centre (2008-2107) regarding: 1) antenatal diagnosis of AA variants, including combinations of right aortic arch (RAO) with/or without aberrant left subclavian artery (ALSCA), retroesophageal ductus arteriosus (rPDA), double aortic arch (DA) and left aortic arch (LAO) with aberrant right subclavian artery (ARSCA) 2) AA diagnosis prior or following FE, 3) isolated (ISO) or associated with congenital heart disease (CHD) 4) karyotype abnormalities 5) NT findings, 6) postnatal outcome.

Results: 52 pregnancies, median GW: 23 wk (18-35), maternal age: $30,4 y r s(20-44)$, IVF $(n=6)$ were included. 
Table.

\begin{tabular}{lllllll}
\hline $\begin{array}{l}\text { AA } \\
\text { variant }\end{array}$ & $\begin{array}{l}\text { Abnormal } \\
\text { Karyotype }\end{array}$ & $\begin{array}{l}\text { Abnormal } \\
\text { NT }\end{array}$ & $\begin{array}{l}\text { Fetal } \\
\text { Malformation }\end{array}$ & $\begin{array}{l}\text { Feeding } \\
\text { Problems }\end{array}$ & $\begin{array}{l}\text { Feeding- } \\
\text { operation }\end{array}$ & $\begin{array}{l}\text { CHD- } \\
\text { operation }\end{array}$ \\
\hline $\begin{array}{c}\text { ISO- RAO } \\
(\mathrm{n}=19)\end{array}$ & $2 / 16(12 \%)$ & $4 / 14(22 \%)$ & $1 / 18(6 \%)$ & $2 / 8(25 \%)$ & $1 / 8(12 \%)$ & \\
$\begin{array}{c}\text { ISO- } \\
\text { ARSCA } \\
(\mathrm{n}=15)\end{array}$ & $0 / 13(0 \%)$ & $1 / 13(7 \%)$ & $1 / 15(7 \%)$ & $1 / 9(11 \%)$ & $0 / 9(0 \%)$ & \\
$\begin{array}{c}\text { CHD-RAO } \\
(\mathrm{n}=12)\end{array}$ & $2 / 9(22 \%)$ & $1 / 10(10 \%)$ & $3 / 11(27 \%)$ & $0 / 7(0 \%)$ & $0 / 7(0 \%)$ & $4 / 7(57 \%)$ \\
$\begin{array}{c}\text { CHD- } \\
\text { ARSCA } \\
(\mathrm{n}=6)\end{array}$ & $1 / 5(20 \%)$ & $1 / 6(14 \%)$ & $0 / 7(0 \%)$ & $1 / 3(33 \%)$ & $0 / 3(0 \%)$ & $0 / 3(0 \%)$ \\
\hline
\end{tabular}

In $20(38 \%)$ the AA variant was first detected during FE (ISO $\mathrm{n}=15)$, corresponding to isolated AA variant as new finding $0.44 \%$ ( 1 in 227$)$.

$34(65 \%)$ were isolated defects: ARSCA-LAO $(n=15)$, RAO $(\mathrm{n}=1)$, DA $(\mathrm{n}=1), \mathrm{RAO}-\mathrm{ALSCA}(\mathrm{n}=2)$, RAO-ALSCA-rPDA $(\mathrm{n}=8)$, RAO-rPDA $(\mathrm{n}=7) .18(35 \%)$ were CHD-associated: ARSCA-LAO $(n=6)$, RAO $(n=1)$, RAO-ALSCA, RAOALSCA-rPDA, RAO-rPDA, a single case each. Associated CHD $(\mathrm{n}=18,35 \%)$ included VSD $(\mathrm{n}=6), \operatorname{ToF}(\mathrm{n}=5)$, suspected CoA $(\mathrm{n}=4)$, TrA, PAtr.-VSD, PS, a single case each. Abnormal NT-values (14\%), fetal malformations (10\%), abnormal Karyotype $(12 \%$, Di George $n=2)$ were documented.

The side of aortic arch (RAO vs LAO-ARSCA) and the presence of CHD (ISO vs CHD) had a weak (non-significant) association with the probability of abnormal karyotype, abnormal NT, fetal malformation and postnatal outcome (Table).

Conclusions: Isolated left aortic arch variants (ARSA) might have a weaker association with fetal karyotype abnormalities and better outcome compared to right arch variants or those associated with CHD.

\section{MP1-7}

Microalbuminuria, proteinuria and renal function in children with cyanotic congenital heart disease

Roginska N. (1), Kawalec W. (1)., Żuk M. (1)., Litwin M. (2)., Brzezińska-Rajszys G. (1)

Children's Memorial Health Institute Warsawa 1 - Dept. of Cardiology; 2 - Dept. of Nephrology, Kidney Transplantation and Arterial Hypertension

Introduction: Cyanotic congenital heart diseases (CCHDs) are recognized as a potential cause of cyanotic nephropathy. There have been few reports on changes in urinalysis (proteinuria, albuminuria) and renal function in patients with CCHD due to reduced concentration of oxygen in systemic arteries and tissues.

The aim of this study was to estimate the albuminuria, proteinuria and glomerular filtration rate (GFR) in patients with cyanotic congenital heart disease (CCHD) in comparison to patients with non-cyanotic congenital heart disease (non-CCHD).

Methods: 126 children with congenital heart disease hospitalized in Cardiology Clinic were examined. The exclusion criteria were any known kidney disease and urinary tract pathology. Patients were divided into non-CCHD ( $\mathrm{SaO} 2$ above 90\%, $\mathrm{N}=67$, aged $5.3+/-4.8$ yo) group and CCHD ( $\mathrm{Sa} 02$ below $90 \%, \mathrm{~N}=59$, $3.9+/-2.9$ yo) group. Total protein/creatinine ratio (PCR) and albumin/creatinine ratio (ACR) in morning spot urine was evaluated.

GFR (calculated using the Schwartz formula GFR = height $\mathrm{x}$ $\mathrm{k} / \mathrm{Cr}$ ) was estimated for each patient.
Table.

\begin{tabular}{|c|c|c|c|c|c|c|c|c|}
\hline & Group & $\mathbf{n}$ & Me & IQR & $\begin{array}{l}\text { Mean } \\
\text { Rang }\end{array}$ & $\mathbf{U}$ & $\mathbf{Z}$ & $\mathbf{p}$ \\
\hline \multirow[t]{2}{*}{ ACR } & $\mathrm{CCHD}$ & 59 & 11.61 & 15.1 & 70.86 & 1598.5 & -1.98479 & 0,047 \\
\hline & Non-CCHD & 67 & 7.73 & 7.2 & 57.86 & & & \\
\hline \multirow[t]{2}{*}{ PCR } & $\mathrm{CCHD}$ & 59 & 0.28 & 0.36 & 76.28 & 1273.5 & -3.554 & 0,00038 \\
\hline & Non-CCHD & 67 & 0.17 & 0.17 & 53.01 & & & \\
\hline \multirow[t]{2}{*}{ GFR } & $\begin{array}{r}\text { Group II } \\
\text { CCHD }\end{array}$ & 59 & 102.34 & 24.1 & 57.82 & 1639.5 & 1.786 & 0,073 \\
\hline & Non-CCHD & 67 & 108.79 & 26.6 & 64 & & & \\
\hline
\end{tabular}

The statistical significance of the differences in ARC, PCR and GFR was assessed by the U-Mann Whitney test. The frequency of significant proteinuria, albuminuria and GFR were evaluated by Chi-square test.

Results: In non-CCHD group 11 children had PCR above the norm (16.4\%), and in CCHD group 29 children had PCR above the norm $(45.7 \%)(p<0.05)$. Median ACR and PCR values were greater in CCHD group in comparison with non-CCHD group (both $\mathrm{p}<0.05)$.

Reduced GFR (<90 ml/min/1/73 m2) was observed with similar frequency in both groups: in 7 children without CCHD, and 9 children with CCHD. However, patients with CCHD had significantly lower GFR values in comparison with non-CCHD patients $(\mathrm{p}<0.05)$.

Conclusion:

1. Patients with CCHD had significantly greater ACR and $\mathrm{PCR}$ in comparison with non-CCHD patients

2. $45 \%$ of CCHD patients had pathological levels of albuminuria and proteinuria in comparison with $16 \%$ among nonCCHD patients.

3. Although still in the normal range, GFR was significantly lower in CCHD than non-CCHD.

MP1-8

Adverse Lipid and Inflammatory Changes in Young Nondiabetic First-Degree Relatives of Type 1-Diabetic Patients

Englund E., Maxedius A., Odermarsky M., Liuba P.

Pediatric Cardiology, Pediatric Heart Center, Skåne University Hospital, Lund, Sweden

Background: Previous studies from our and other centers on young patients with type 1 diabetes (T1D) have indicated pro-atherosclerotic changes in the carotid arteries and in the lipid and systemic inflammatory profiles without a clear relationship between these changes and the hyperglycemic control. We have also earlier found in these patients a certain HLA-related genetic susceptibility to adverse vascular changes.

Objective: To assess whether pro-atherosclerotic changes are present in apparently healthy first-degree relatives of patients with T1D.

Methods: Plasma lipids, C-peptide (index of insulin secretion), C-reactive protein (CRP), and the carotid artery intima-media thickness (CA-IMT), compliance (CAC) and stiffness index (SI) were assessed in up to 116 non-diabetic first-degree relatives (FDR; mean age: 12.6 years; 56 female) of patients with T1D and in up to 43 age-matched control individuals (mean age: 13.3 years; 23 female) without heredity for cardiovascular risk factors. The number and type of clinically manifest acute infections during 


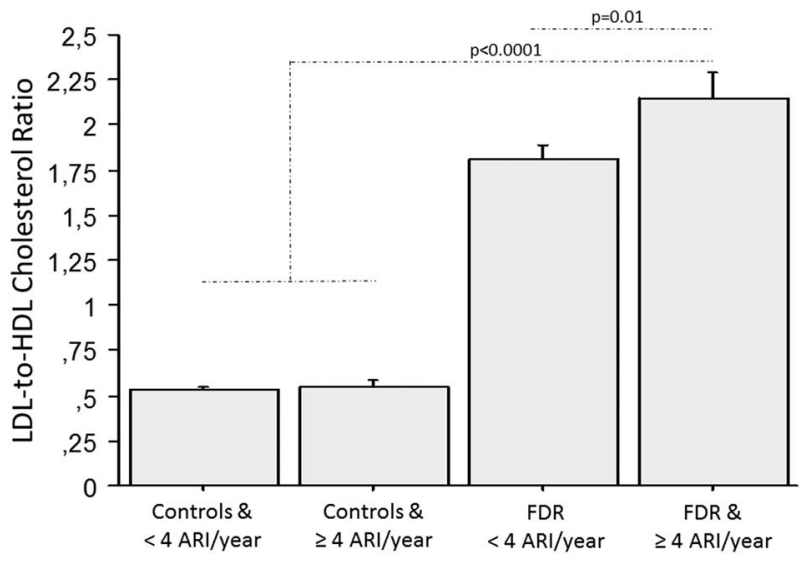

Figure.

the past year were obtained via a questionnaire validated in our previous studies.

Results: There was no difference in age, gender, body mass index, arterial blood pressure and C-peptide levels between the FDR and control groups $(p>0.2)$. Also, there was no significant difference in SI $(p=0.2)$, CAC $(p=0.1)$ and CA-IMT $(p=0.9)$ between the groups. In the FDR group, plasma CRP and LDL- cholesterol (LDL-C) were increased ( $\mathrm{p}<0.05$ for both) and HDL-C was decreased $(p<0.0001)$ as compared to the control group. The changes in LDL-C and HDL-C were most marked in FDR individuals with $\geq 4$ acute respiratory infections during the past year (Figure).

Conclusion: Adverse lipidemic and inflammatory changes are present in healthy non-diabetic first-degree relatives of T1D patients, probably related to their genetic susceptibility to this disease. Further analyses of additional indices of peripheral arterial function in these groups are under way.

MP1-9

Subtle cardiomyopathic changes in childhood cancers survivors on 10 year-follow-up

Wolf C.M. (1), Kuehn A. (1), Hager A. (1), Müller J. (1,2),

Reiner B. (1,2), Meierhofer C. (1), Oberhoffer R. (1,2), Ewert P. (1), Schmid I. (3), Weil J. (1)

(1) Department of Pediatric Cardiology and Congenital Heart Disease, German Heart Center Munich, Technical University Munich, Germany; (2) Institute of Preventive Pediatrics, Faculty of Sport and Health Sciences, Technical University Munich, Germany; (3) Department of Hematology and Oncology, Dr. von Hauner University Children's Hospital, LudwigMaximilians-University Munich, Germany

Introduction: Survivors of childhood cancer are at risk for anthracycline-induced cardiomyopathy. The aim of this study was to assess long-term cardiac natural history.

Methods: Patients underwent cardiopulmonary exercise test (CPET), transthoracic echocardiography (TTE) with tissue doppler imaging (TDI) and speckle tracking, and cardiovascular magnetic resonance (CMR) with contrast. Findings were correlated to anthracycline and radiation dose, and compared to data from 27 gender- and age-matched patients with childhood onset hypertrophic cardiomyopathy (HCM).

Results: Cardiac evaluation was performed in 55 patients ( 27 males) at $11.3 \pm 4.4$ years after cancer treatment. Oncologic diagnosis at a mean age of $10.3 \pm 4.9$ years was Hodgkin lymphoma in 13 , sarcoma in 17 , acute lymphoblastic or relapse leukemia in 18 ,

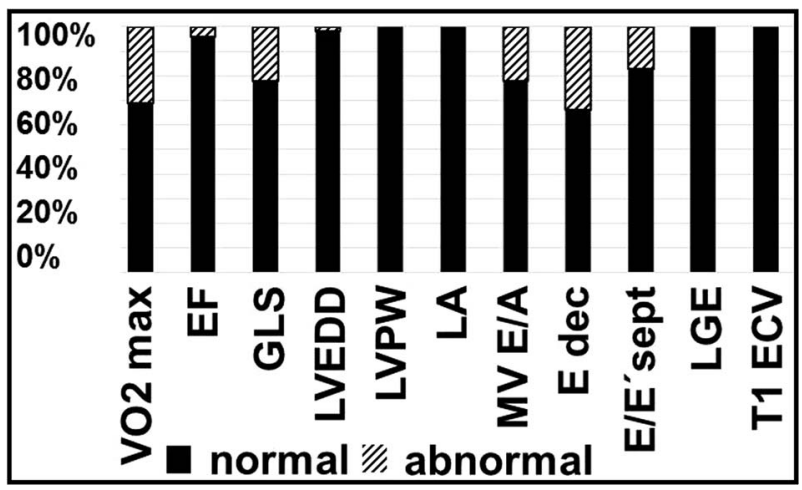

Figure.

and others in 7 patients. Cumulative anthracycline dose was more than $300 \mathrm{mg} / \mathrm{m}^{2}$ in 24 patients. 25 patients also received radiation therapy. Decreased peak maximum respiratory oxygen uptake in $\%$ predicted (VO2 max) on CPET, increased global longitudinal strain (GLS) on TTE speckle tracking, and diastolic dysfunction on TDI were the most prominent findings (Figure: percentage of patients with normal (black bars) and abnormal (striped bars) values). Decreased CPET exercise capacity and increased septal $\mathrm{E} / \mathrm{E}^{\prime}$ ratio, respectively, correlated significantly with increased anthracycline dosing $(\mathrm{p}<0.001$, Pearson correlation). There was no correlation between radiation dose and abnormal cardiac findings.

In contrast to HCM patients, childhood cancer survivors did not show left ventricular hypertrophy (LVPW z-score $0.5 \pm 0.6$ vs. $2.8 \pm 2, \mathrm{p}<0.001)$, hyperdynamic systolic function on TTE (Ejection fraction (EF) $62 \pm 8 \%$ vs. $71 \pm 12 \%, p=0.001$ ), or fibrotic myocardial changes on CMR (Late gadolinium (LGE) positive $0 / 13$ vs. $12 / 20, p=0.001$; extracellular volume fraction (ECV) $22 \pm 2 \%$ vs. $28 \pm 4 \%$, p < 0.001) at time of follow-up.

Conclusions: Long-term childhood cancer survivors exposed to cardiotoxic therapy show subtle cardiomyopathic changes best detected by CPET and TTE including TDI and speckle tracking. This underlines the importance of follow-up in specialty outpatient clinics for this patient population.

MP1-10

Early detection of sudden infant death syndrome: ECG, genetic study and familial cascade screening

Sarquella-Brugada G. (1), Zambrano M.D. (1), Cesar S. (1), Fiol V. (1), Fernandez-Falgueras A. (2), Brugada R. (2), Brugada J. (1), Campuzano O. (2)

(1) Pediatric Arrhythmia and Sudden Death unit, hospital Sant Joan de Déu, University of Barcelona, Spain; (2) Cardiovascular Genetic Center, IDIBGI, Univerisity of Girona, Spain

Arrhythmogenic diseases have been proven to cause infant sudden death. Some of them can be diagnosed performing a 12-lead ECG. We have performed a prospective analysis of ECG performed at 24 to 72 hours of life to new-borns of a single centre. Of those which QT interval was above normal, saliva was collected for DNA genetic analysis using a gene panel related to sudden death using ultrasequencing technology. Clinical and genetic analysis of the family members has been performed accordingly.

During the first year of the project we have performed 600 ECG in new-borns (51\% males). We have identified 14 cases of prolongation of the QTc above $470 \mathrm{~ms}$ and ECGs of the first-degree family members has been performed. In 7 of them (QTc below 490ms) 
normalization of the ECG has been observed along the first 6 months of life and no family memebrs have been detected abnormal. In the remaining 7 cases with persistent QTc interval above 490 ms., a genetic analysis has been performed. In 6, family members ECG were abnormal. In 5, a rare genetic variant has been described (one of them, de novo).

Neonatal ECG screening allows early detection of potentially lethal cardiac diseases. Genetic study helps identifying the underlying mechanism of the disease. Family cascade study is mandatory in order to detect other disease carriers.

\section{MP1-11}

\section{Comparison of echocardiographic findings and insuline resistance in obese children}

Giannisi F. (1), Keivanidou A. (1), Sakellari I. (2), Hassapidou M. (2), Makedou A. (1), Giannopoulos A. (1)

2nd Pediatric Department, Aristotle University of Thessaloniki, AHEPA General Hospital, Thessaloniki, Greece (1); Alexander Technological Educational Institute of Thessaloniki, Department of Nutrition and Dietetics, Thessaloniki, Greece (2)

Introduction: Obesity in children and adolescents has increased over the past decades and is considered a strong risk factor for future cardiovascular morbidity and mortality. Obesity has been associated with myocardial structural alterations that may influence cardiac mechanics. The aim of this study was to detect early changes in LV structure and function in obese normotensive children and whether they are associated with anthropometric measurements and insuline resistance.

Methods: A total of 51 children aged 7-12 years (34 obese and 17 normal-weight (NW) children) were studied. The obese children were divided into two groups according to BMI z-score (Overweight $(\mathrm{OW}):>+1 \mathrm{SD}(\mathrm{N}=13)$, obese $(\mathrm{OB}):>+2 \mathrm{SD}(\mathrm{N}=21)$ ). All children had normal resting blood pressure. Homeostasis model of insulin resistance was calculated by the formula [fasting glucose $(\mathrm{mg} / \mathrm{dl}) X$ fasting insulin $(\mu \mathrm{IU} / \mathrm{ml})] / 405$. Twodimensional ultrasound was performed to assess the LV mass index (LVMI), calculated as LV mass/height (2.7) and pulsedwave doppler to assess transmitral early and late diastolic inflow peak velocities (E \& A ). Tissue Doppler imaging was used to record the mitral annular velocities (e' \& a').

Results: The three children groups (NW,OW,OB) didn't differ significantly regarding age and sex.

Waste-to-hip ratio $(0,91 \pm 0,13$ and $0,95 \pm 0,03$ versus $0,845 \pm$ $1,33, \mathrm{p}=0,012)$, HOMA-IR index $(2,76 \pm 1,8$ and $2,9 \pm 1,2$ versus $1,67 \pm 0,63 \mathrm{p}=0,025$ and LVMI $(37,6 \mathrm{~g} / \mathrm{m} 2.7 \pm 7,6$ and $41,96 \mathrm{~g} / \mathrm{m} 2.7 \pm 10,8$ versus $27,4 \mathrm{~g} / \mathrm{m} 2.7 \pm 5,5, \mathrm{p}<0,001)$ were significantly higher in $\mathrm{OW}$ and $\mathrm{OB}$ children compared to controls. Mitral valve E/A ratio was significantly higher in NW compared to $\mathrm{OB}(2,21 \pm 0,84$ versus $1,68 \pm 0,32 \mathrm{p}=0,016)$ reflecting better diastolic function, but in all the patient groups, the mitral valve E/A ratio was $>1$, within the normal range. Tissue Doppler Imaging measurements of $\mathrm{e}^{\prime}$ and $\mathrm{a}^{\prime}$ velocities as well as E/e' ratio didn't differ between cases and controls.

Conclusions: In paediatric obesity, myocardial size and diastolic function seem to be affected early so cardiac ultrasound should always be considered when assessing obese children.

\section{MP1-12}

Criteria for aortic dilatation diagnosis in adolescents with Bicuspid Aortic valve

Siurana J.M. (1), Sabate A. (2), Ayerza A. (3), Jimenez L. (4), Escriba S. (5), Gonzalez M.A. (6), Sorli M. (7), Rodriguez M. (8), Dominguez O. (9), Rellan S. (10), Portillo M. (11), Albert L. (12),
Alvarez R. (13), Figueras M. (14), Teodoro S. (15), Collell R. (16), Lopez B. (17), Manso B. (18), Solana R. (19), Riaño B. (20), Rezola E. (21), Rojo H. (22), Cantero T. (23), Isla R. (1), Jimenez M. (24), Tejero M.A. (25), Perez A.M. (26), Moriano A. (27), Plata B. (28), Salido C. (29) Hospital Hospital de Nens de Barcelona-Spain (1); Hospital Universitari Vall d'Hebron Pediatric Cardiology Barcelona-Spain (2); Hospital Lozano Blesa Zaragoza-Spain (3); Hospital Miguel Servet ZaragozaSpain (4); Hospital Son Espases Mallorca-Spain (5); Hospital de Ciudad Real-Spain (6); Hospital de Cartagena-Spain (7); Hospital Monteprincipe Madrid-Spain (8); Hospital Virgen de la Salud ToledoSpain (9); Hospital Clinico Valladolid-Spain (10); Hospital de Merida Badajoz-Spain (11); Hospital 12 de Octubre Madrid-Spain (12); Hospital Sant Pau Barcelona-Spain (13); Hospital Josep Trueta GironaSpain (14); Hospital Parc Tauli Sabadell-Spain (15); Hospital Sant Joan Reus-Spain (16); Hospital de Santiago de Compostela-Spain (17); Hospital Virgen del Rocio Sevilla-Spain (18); Hospital Infanta Leonor Madrid-Spain (19); Hospital San Pedro Logroño-Spain (20); Hospital de Donosti-Spain (21); Hospital Príncipe de Asturias Madrid-Spain (22); Hospital de Palencia-Spain (23); Hospital General Segovia-Spain (24); Hospital Reina Sofia Cordoba-Spain (25); Hospital General de Catalunya Sant Cugat del Valles-Spain (26); Hospital Lluis Alcanyis Xativa-Spain (27); Hospital de Salamanca-Spain (28); Hospital de Jerez-Spain (29).

Objectives: Establish the threshold to define aortic root and ascending aorta dilatation in adolescents with bicuspid aortic valve. Specifically in those adolescents with adult body surface area (BSA).

Methods: From the Spanish registry for children ( $\leq 18$ years) with bicuspid aortic valve (REVAB), under the Spanish Society of Pediatric Cardiology and Congenital Heart Diseases (SECPCC), patients $\geq 10$ years were analyzed $(\mathrm{N}=464)$. The aortic root and the ascending aortic measures were compared in absolute value, value indexed by BSA and $\mathrm{z}$-score, with the threshold of $40 \mathrm{~mm}$, $27.5 \mathrm{~mm} / \mathrm{m}^{2}$ and $+2 /+3$ respectively according to AHA guidelines. A second analysis was performed comparing patients $\geq 10$ years with BSA $<1.5 \mathrm{~m}^{2}$ and BSA $\geq 1.5 \mathrm{~m}^{2}$. Results: Overall, adolescents had a higher $\mathrm{z}$-score of the ascending aorta than $<10$ years: $1.82 \pm 1.8 \mathrm{SD}$ vs $0.92 \pm 2.1 \mathrm{SD}, \mathrm{p} 0.02$ (see figure); while there was no difference for the aortic root: $0.40 \pm 1.3 \mathrm{SD}$ vs

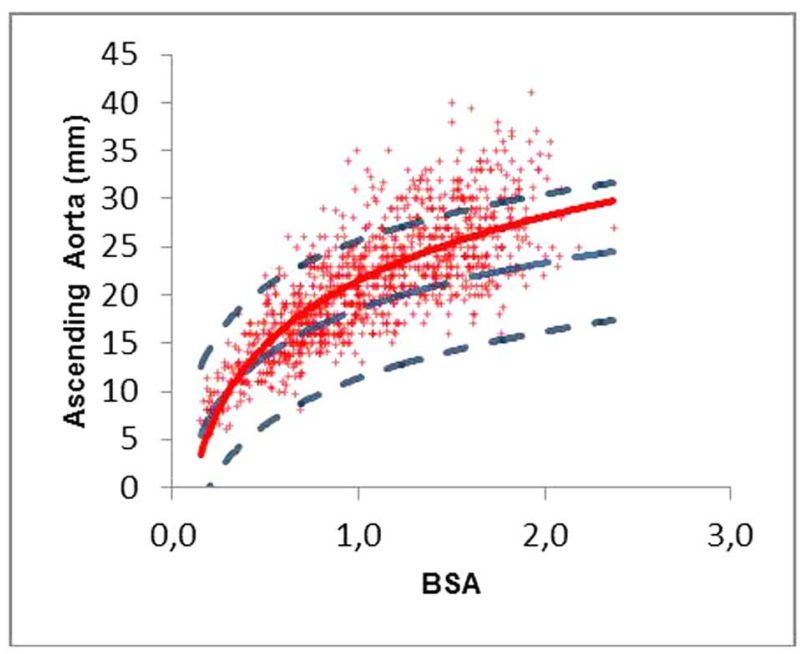

Figure.

Relationship between ascending aorta diameter and BSA. Dashed lines represent mean \pm 2 standard deviation of expected size. Continuous line represent mean of our series size. 
$0.16 \pm 1.4$ SD. When analyzing patients $\geq 10$ years, a total of 214 patients $(46.1 \%)$ had a $z$-score of the ascending aorta $\geq 2$ and 126 patients $(27.1 \%)$ had a $z$-score $\geq 3$ while only $7(1.5 \%)$ had an indexed value $\geq 27.5 \mathrm{~mm} / \mathrm{m}^{2}(\mathrm{p}=0.003$ and $\mathrm{p}=0.002$ respectively) and 2 had an absolute value $\geq 40 \mathrm{~mm}$ ( $\mathrm{p}=0.195$ and $\mathrm{p}=0.067$ respectively). For the aortic root, 51 patients $(10.9 \%)$ had a $\mathrm{z}$-score $\geq 2$ and $13(2.8 \%)$ a $\mathrm{z}$-score $\geq 3$ while 1 patient had an indexed value $\geq 27.5 \mathrm{~mm} / \mathrm{m}^{2}$ ( $\mathrm{p}=1$ for both) and 1 patient had an absolute value $\geq 40 \mathrm{~mm}$ ( $\mathrm{p}=0.105$ and $\mathrm{p}=0.027$ respectively). Almost half of the adolescents had a BSA $\geq 1.5 \mathrm{~m}^{2}$ (231, 47.7\%), of those, 109 (47.2\%) had an ascending aorta $z$-score $\geq 2$, 67 (29\%) had a $\mathrm{z}$-score $\geq 3$, none had an indexed value $\geq 27.5 \mathrm{~mm} / \mathrm{m}^{2}$, and 2 had an absolute value $\geq 40 \mathrm{~mm}(0.9 \%)$. For the aortic root, 20 $(8.7 \%)$ had a $z$-score $\geq 2,4(1.7 \%)$ had a $z$-score $\geq 3$, none had an indexed value $\geq 27.5 \mathrm{~mm} / \mathrm{m}^{2}$, and 1 had an absolute value $\geq 40 \mathrm{~mm}(0.4 \%)$.

Conclusions: There are a considerable number of patients who, depending on the dilatation criteria used, can be considered patients with a normal or dilated aorta, especially regarding the ascending aorta. This discrepancy is clinically significant, especially in the adolescent population when recommendations for lifestyle, follow-up and prognosis must be made.

\section{MP2-1}

The subcutaneous ICD (S-ICD) in congenital heart disease - what lessons have we learnt so far?

Ghaeni L., Danne F., Yigitbasi M., Berger F., Peters B. German Heart Center Berlin, Germany

Introduction: The S-ICD offers a considerable alternative to conventional systems in patients with congenital heart disease (CHD), as it avoids vascular access and thoracotomy.

Methods: S-ICDs were implanted in a heterogeneous group of 15 patients with structural CHD (minimum age $8.9 \mathrm{y}$, height $131.0 \mathrm{~cm}$, weight $28.0 \mathrm{~kg}$ ). The majority presented with challenging hemodynamic and anatomical features, such as missing anatomical vascular access (e.g. univentricular anatomy and Glenn anastomosis), tricuspid valve dysplasia, existing ventricular assist device (VAD), or chronic left-to-right shunt. In 4 patients S-ICD was combined with epicardial pacing. Lead and device positioning had to be modified according to anatomic variations such as dextrocardia. 14 devices were implanted intramuscular or submuscular.

Results: Follow-up was up to 7 years. Cosmetic results and clinical tolerance was good even in subjects below $30 \mathrm{~kg} .7$ patients received appropriate shocks. 4 patients with $\mathrm{RBBB}$ presented with $\mathrm{t}$-wave-oversensing (TWOS) due to altered t-wave morphology under higher heart rate. 2 patients consequently received inappropriate shocks in sinus tachycardia under exercise. The other 2 patients received "pseudo-appropriate" shocks (treated episodes that were misinterpreted by device resulting from TWOS, though patient had clinical benefit from shock); one with hemodynamically relevant SVT and the other with slow VT. Subsequently, TWOS could be successfully addressed by modified programming and firmware updating. Combined epicardial pacing was feasible and safe although potential interactions may occur, as experienced in one patient with false (unipolar) programming and unreasonable template acquisition. One patient with appropriate shocks due to monomorphic VTs developed subsequently slow VTs below the programmable shock zone (170 $\mathrm{bpm}$ ) and drug-induced chronotropic incompetence. Another patient with subcutaneous implantation and progressive obesity suffered from late device migration. The two latter patients had to be switched to transvenous systems. The VAD patient died of unknown reason.

Conclusion: S-ICD therapy is feasible even in patients with complex CHD without transvenous access or systemic intracardiac shunting. Thorough pre-implantation screening combined with modifications in lead or device placement and adapted programming strategies are indispensable to allow proper function even in severely distorted CHD anatomy. Post-implantation exercise testing is mandatory to address TWOS, especially in RBBB. Cosmetic and functional results are excellent if protective implantation techniques are applied; nevertheless, the bulky size of the current device generation precludes its use below abodyweight of approximately $25-30 \mathrm{~kg}$. Despite successful application with combined (epicardial) pacing, the lack of antibradycardia and antitachycardia pacing represents the other major limitation towards use in CHD.

\author{
MP2-2 \\ Cardiac Arrhythmias in Hypoplastic Left Heart Syndrome: \\ Incidence, Type, And Course \\ Papagiannis J., Yimer M.A., Tisma-Dupanovic S., Malloy-Walton L., \\ Connelly D., Noel-Macdonnell J., O’ Brien J., Papagiannis J. \\ Children's Mercy Hospital, Kansas City, USA
}

Introduction: Despite significant advances, the prognosis of patients with hypoplastic left heart syndrome (HLHS) remains guarded. Arrhythmias are common in the postoperative course, but their characteristics and impact on outcome have not been studied adequately.

Objective: To determine the types, incidence, and risk factors of arrhythmias, and their impact on morbidity and mortality in HLHS patients.

Methods: We performed a retrospective study of 120 consecutive patients with HLHS and variants (82\% HLHS, 11\% double outlet right ventricle and $8 \%$ right dominant $\mathrm{AV}$ canal) who underwent surgical palliation in our center from $1 / 2006$ to $12 / 2016$. Demographic, anatomic, surgical and clinical variables were reviewed. Statistical analysis was performed using Fisher's exact test or student's t-test as indicated. Survival analysis was performed using Kaplan-Meier method with log-rank test.

Results: Group 1 consisted of 31 (25.8\%) patients with arrhythmia (supraventricular arrhythmia in 25 and other arrhythmias in 10). Some patients had more than one arrhythmia. Group 2 consisted of $89(74.2 \%)$ patients without arrhythmias. Arrhythmia diagnoses were ectopic atrial tachycardia (EAT) in $11(35.5 \%)$, paroxysmal supraventricular tachycardia (PSVT) in 7 (22.6\%), junctional ectopic tachycardia in $5(16.1 \%)$, sinus node dysfunction in 5 $(16.1 \%)$, 2nd or 3rd degree heart block in $3(9.7 \%)$, atrial flutter in $2(6.5 \%)$ and ventricular tachycardia in $2(6.5 \%)$. Most arrhythmias occurred between stage 1 and 2 [21 (67.7\%)] with a median age at arrhythmia of 9 days (IQR: 7, 132). Thirty (96.8\%) of the patients with arrhythmia required therapy but only 15 (48.4\%) were discharged with medication. Arrhythmia post stage 1 was more likely to resolve compared to post stage 2 or $3(\mathrm{P}=0.0034)$. There were no differences in anatomical, surgical or clinical variables, except that Group 1 patients were younger at stage 1 (7 vs 9 days, $\mathrm{P}=0.015)$. Arrhythmia was associated with longer hospital stay (66 vs 41 days) after stage $1(\mathrm{P}=0.025)$ but not with mortality (25.5\% vs $24.7 \%, \mathrm{P}=0.95)$.

Conclusion: Arrhythmias, especially supraventricular, are common in HLHS patients, with EAT and PSVT being the most common types. Most arrhythmias resolved spontaneously. Although arrhythmias were associated with longer hospital stay, they did not affect mortality. 
MP2-3

How to Determine the QT-interval in the Paediatric Population: a Comprehensive Analysis of a Large Cohort of Long QT-Syndrome Patients and Controls

Vink A.S. (1), Neumann B. (2), Lieve K.V.V. (1), Hofman N. (1), el Kadi S. (1), Schoenmaker M.H.A. (1), Slaghekke H.M.J. (1),

Clur S.A.B. (1), Blom N.A. (1,3), Kaab S. $(2,4)$, Sinner M.F. $(2,4)$, Wilde A.A.M. (1), Postema P.G. (1)

Academic Medical Center, Amsterdam, The Netherlands (1); University

Hospital Munich, Ludwig-Maximilians-University, Munich, Germany (2); Leiden Medical Center, Leiden, The Netherlands (3); German Centre for Cardiovascular Research, Munich, Germany (4);

Introduction: Long QT-syndrome (LQTS) is associated with potentially fatal arrhythmias at young age. Treatment is very effective but its diagnosis may not be simple. Importantly, different methods are used to assess the QT-interval, which troubles its recognition. QT-experts advocate manual measurements with the tangent or threshold method. However, differences between these methods and their performance in LQTS diagnosis have not been established. We aimed to assess differences between these two methods for QT-analysis.

Methods: Confirmed pathogenic mutation carriers in KCNQ1 (LQT1), KCNH2 (LQT2) and SCN5A (LQT3) genes were included as LQTS cases and their genotype-negative family members as controls when they were under the age of 18 years. Consecutive complexes from baseline ECGs were analyzed with both methods in two separate sessions by three readers. One reader additionally remeasured $10 \%$ of the ECGs.

Results: We included 451 individuals (subgroups: 120 LQT1, 137 LQT2, 45 LQT3 and 127 controls) from 176 families, aged $8 \pm 5$ years and $50 \%$ females. In the total cohort, QT-tangent was $10.5 \mathrm{~ms}$ shorter ( $\mathrm{p}<0.0001)$ compared to QT-threshold $(95 \%$ limits of agreement \pm $17.2 \mathrm{~ms})$. In all the subgroups the QT-tangent was shorter $(\mathrm{p}<0.0001$ all groups) compared to QT-threshold, but this was less pronounced in LQT2. Both methods had a high inter- and intra-observer validity (Intraclass correlation coefficient $>0.96$ ), and a high distinctiveness between LQTS patients and controls (area under the curve $>0.86$ ). Using the current guideline cut-off value (QTc $480 \mathrm{~ms}$ ), both methods had similar specificity ( $99 \%$ versus $98 \%$ ) but the QT-threshold yielded a higher sensitivity (20\% versus $27 \%$ ). Optimal cut-off value for boys was QTc-tangent $417 \mathrm{~ms}$ (78\% sensitivity, 85\% specificity) and QTcthreshold $428 \mathrm{~ms}$ ( $80 \%$ sensitivity, 79\% specificity). For girls QTctangent $418 \mathrm{~ms}$ (83\% sensitivity, 80\% specificity) and QTc-threshold $446 \mathrm{~ms}$ (64\% sensitivity, 93\% specificity).

Conclusion: The length of the QT-interval is different depending on the method used for determination. Both methods have a high validity. However, for diagnostic purposes current guideline cutoff values yield different results for these two methods, and could result in inappropriate reassurance or treatment. Adjusted cut-off values are suggested to diagnose LQTS.

\section{MP2-4}

Electroanatomic mapping-guided catheter ablation of supraventricular tachycardia in children with Ebstein's anomaly

Ergül Y. (1), Koca S. (2), Akdeniz C. (2), Тuzcu V. (2)

(1)Saglik Bilimleri University, Mehmet Akif Ersoy Thoracic and Cardiovascular Surgery Center, Department of Pediatric Cardiology / Electrophysiology, Istanbul, Turkey; (2)Istanbul Medipol University Hospital, Department of Pediatric Cardiology/Electrophysiology, Istanbul, Turkey

Background: In Ebstein's anomaly (EA), arrhythmias are common, tachycardia substrates are complex, and accessory pathway (AP) ablations are often challenging. Recent advances in electroanatomical mapping technologies have decreased or eliminated fluoroscopic exposure during catheter ablation procedures.

Objective: This study demonstrates the utility of the EnSite Velocity cardiac mapping system (St. Jude Medical, St Paul, MN) in the catheter ablation of supraventricular tachycardia (SVT) in children with EA.

Methods: Twenty patients (Female/Male $=8 / 12$ ) with EA who underwent catheter ablation guided by the EnSite-Velocity system between December 2011 and December 2016 were retrospectively evaluated.

Results: Patient median age was 11.5 years (range: 2 years 9 months -18 years), and the median weight was $41 \mathrm{~kg}$ (range $11-73 \mathrm{~kg}$ ). Five patients had severe EA (two in the univentricular repair pathway - fontan palliation), two had moderate EA, and thirteen had mild EA. Fourteen patients (70\%) presented with Wolff-Parkinson-White (WPW) syndrome-related SVT, four (20\%) with wide QRS tachycardia, and two with narrow QRS tachycardia. The most common indications for ablations were palpitations/syncope and treatment-resistant arrhythmias. Thirty-one tachycardia substrate foci (21 manifest WPW non-decremental-AP, 2 concealed-AP, 4 Mahaim-AP, 3 focal atrial tachycardias, and 1 typical atrioventricular nodal reentrant tachycardia) were detected in twenty patients in the electrophysiological study. There were multiple tachycardia substrates in eleven patients (55\%). All WPW patients had right-sided APs (most commonly right posterior-posteroseptal), and six (6/14, $43 \%)$ of these patients were high risk. The patient-based acute procedure success rate was 19/20 (95\%), and the tachycardia-based success rate was 30/31 (97\%). The mean procedure time was $170 \pm 43 \mathrm{~min}$ (range: 90-265), and fluoroscopy was not used in 15 $(75 \%)$ patients. The mean fluoroscopy time in the remaining five patients was $3.6 \pm 2.9 \mathrm{~min}$ (range:0.7-7.8). During a mean followup of $35.1 \pm 20.3$ months (range:6-60), tachycardia recurred in four patients $(4 / 19,21 \%)$, two of whom underwent a second successful procedure. No complications were seen.

Conclusion: Catheter ablation of arrhythmias can be performed effectively and safely in pediatric EA patients by using a limited fluoroscopic approach with the help of electroanatomical mapping systems. However, the rate of tachycardia recurrence at follow-up remains high.

\section{MP2-5}

Therapeutic Use of Transplacental Nonsteroidal Antiinflammatory Drugs (NSAIDS) for Fetal Right Heart Failure with Circulatory Shunt

Jaeggi E. (1), Torigoe T. (1), Van Mieghem T. (2), Golding F. (1), Seed M (1), Honjo O (1), Ryan G. (2), Mawad W. (1)

The Hospital for Sick Children (1); Mount Sinai Hospital (2), Toronto, Canada

Background: Circular shunt (CS) describes the disastrous pathophysiology resulting from a widely patent arterial duct (DA), severe pulmonary and tricuspid regurgitation and a failing right ventricle. In this instance, a significant amount of blood recirculates ineffectively between the ventricles, contributing to poor systemic perfusion, hydrops and death.

Methods: In three hydropic fetuses with Ebstein anomaly (EA) and CS, pharmacological DA constriction to birth, followed by immediate surgical pulmonary artery ligation and tricuspid valve closure was attempted. Treatment response to transplacental indomethacin (IND; loading: $100 \mathrm{mg}$; maintenance: 100-300 mg/d) was defined as DA constriction significant enough to resolve fetal hydrops. 
Table.

\begin{tabular}{llccc}
\hline & & Case 1 & Case 2 & Case 3 \\
\hline Gestational age (weeks) & & 30 & 26 & 30 \\
DA diameter (mm) & Baseline & 2.8 & 3.8 & 3.1 \\
& IND & 3 & 1.8 & 1.3 \\
UA end-diastolic flow & Baseline & reversed & absent & reversed \\
& IND & reversed & anterograde & anterograde \\
MCA pulsatility index & Baseline & 2.44 & 2.81 & 3.1 \\
& IND & 1.9 & 1.9 & 1.85 \\
DA max/min flow (m/s) & Baseline & $0.82 / 0.12$ & $1.1 / 0.1$ & $1.6 / 0.35$ \\
& IND & $1.16 / 0.14$ & $3.2 / 0.8$ & $2.8 / 1.4$ \\
Treatment (days) & Total & 12 & 49 & 42 \\
Outcome & & Died & Alive & Alive \\
\hline
\end{tabular}

Results: The table summarizes the findings at baseline and with indomethacin. Prior to IND, all three fetuses had normal sized DA, continuous retrograde DA flow with low flow velocities, and absent or reversed end-diastolic umbilical artery (UA) and middle cerebral artery (MCA) flows. Case 1 showed no signs of improvement with IND for 9 days and died from postoperative complications. Cases 2-3 responded instantly to IND with DA constriction, normalized UA and MCA flows and resolution of hydrops. Case 2 was electively delivered at 32 weeks when hydrops recurred while on maximal IND. Sustained DA constriction to birth was achieved in case 3 . Both survivors required postoperative ECMO and hemofiltration for transient respiratory and renal failure. They are currently well and awaiting secondstage single ventricle surgery at home.

Conclusions: This is the first report of the successful use of IND to manage the circulatory failure and delay the delivery and surgery of two fetuses with EA/CS. The long-term risks of a significant CS on the neurodevelopment and the use of IND on renal function should be further addressed in a prospective longitudinal study.

MP2-6

Impaired lung function in children and adolescents with Fontan circulation may improve after endurance training Hedlund E.R., Ljungberg H., Söderström L., Sjöberg G., Lundell B. Department of Women's and Children's Health, Karolinska Institutet, Stockholm, Sweden.

Objectives: To study lung function and physical capacity in children and adolescents after Fontan palliation compared with matched healthy controls. We also studied the effect of endurance training on lung function.

Methods: Fontan patients $(\mathrm{n}=30)$ and healthy age- and gendermatched control subjects $(n=25)$ performed dynamic and static spirometry, pulmonary diffusing capacity and maximal oxygen uptake before and after a 12 -week endurance training program and at follow-up after one year.

Results: Fontan patients have a restrictive lung pattern, a reduced pulmonary diffusing capacity $(4.27 \pm 1.16 \mathrm{mmol} \cdot \mathrm{kPa}-1 \cdot \mathrm{min}-1 \mathrm{vs}$. $6.61 \pm 1.88 \mathrm{mmol} \cdot \mathrm{kPa}-1 \cdot \mathrm{min}-1, \quad \mathrm{p}<0.001)$ and a reduced maximal oxygen uptake $(35.0 \pm 5.1 \mathrm{ml} \cdot \mathrm{min}-1 \cdot \mathrm{kg}-1$ vs. $43.7 \pm$ $8.4 \mathrm{ml} \cdot \mathrm{min}-1 \cdot \mathrm{kg}-1, \quad \mathrm{p}<0.001)$ when compared to controls. Patients had signs of air trapping with a higher portion of residual volume of total lung capacity than controls ( $26 \pm 6 \%$ vs. $22 \pm 5 \%$, $\mathrm{p}<0.05$ ). Vital capacity increased for patients (from $2.80 \pm 0.97$ liters to $2.91 \pm 0.95$ liters, $\mathrm{p}<0.05)$ but not for controls after an endurance training program.

Conclusions: Fontan patients have a restrictive lung pattern, reduced pulmonary diffusing capacity and a reduced maximal oxygen uptake when compared to healthy controls. Fontan patients may also have air trapping. Endurance training seems to improve vital capacity in this patient group, possibly because of an improvement of chest musculature and movement. Lung function and exercise tests provide important information when managing this patient group. Apart from general beneficiary effects, exercise may improve lung function in young Fontan patients and should be encouraged.

\section{MP2-7}

Establishing a UK and Ireland wide model for collating cardiac catheter lab morbidity to improve risk assessment and case planning: A retrospective review of 5 major congenital cardiac centres

Shepherd E. (1,2), Bentham J. (3), Kenny D. (4), Smith B. (5), Taliotis D. (1), Tulloh R. (1,2), Morgan G. $(6,7)$ Bristol Royal Hospital for Children, Bristol, UK (1); University of Bristol, Bristol, UK (2) Leeds Congenital Heart Unit, The Leeds Teaching Hospitals NHS Trust, Leeds, UK (3); Our Lady's Children's Hospital, Crumlin, Dublin, Ireland (4); The Queen Elizabeth University Hospital, Glasgow, UK (5); The Evelina Children's Hospital, London, UK (6); Children's Hospital of Colorado and University Hospital Colorado, Denver, USA (7)

Introduction: Analysis of mortality and morbidity data has been focused on surgery, with no effective system national system scrutinising congenital catheterisation. In the USA two quality assessment tools focused on congenital catheterisation have been developed; the C3PO database and the CRISP RISk Score. Both are modelled on retrospective data from several thousand of congenital catheterisations, mainly within the USA.

Method: We retrospectively analysed morbidity and mortality data from 1500 consecutive catheterisations from five major congenital heart disease centres across the UK and Ireland, between February 2015 and October 2016. We excluded electrophysiology cases. One independent observer retrospectively calculated CRISP RISk scores and categories, using 8 parameters, for each individual case. We then compared the risk score with observed catheterisation complications, classified by NICOR definitions.

Results: 1500 procedures were analysed from 5 congenital cardiac centres. Centres 1,3 and 5 provided paediatric and adult congenital catheters and centres 2 and 4 paediatric only. We excluded 17 incomplete data sets leaving $n=1483$; evenly split between each centre. 1130 were paediatric and 353 adult ( $\geq 18$ years). Table 1 displays the CRISP quoted adverse effect risk in comparison to the observed adverse effect rate across all centres. There were 5 catastrophic complications; 1 in RISk Category 3, 2 in RISk Category 4, and 2 in RISk Category 5. There was an

Table 1. CRISP RISk compared to the observed adverse effect rate. Mild complications (e.g. small haematoma) as classified by NICOR's cardiac catheterisation complication severity definitions have been excluded as not applicable to CRISP RISk score.

\begin{tabular}{|c|c|c|c|c|}
\hline $\begin{array}{l}\text { CRISP } \\
\text { RISk } \\
\text { Score } \\
\text { Category }\end{array}$ & $\begin{array}{l}\text { CRISP } \\
\text { Quoted } \\
\text { Adverse } \\
\text { Effect Rate } \\
(\%)\end{array}$ & $\begin{array}{l}\text { Observed } \\
\text { Adverse Effect } \\
\text { Rate: } \\
\text { Paediatrics \& } \\
\text { Adults (\%) }\end{array}$ & $\begin{array}{l}\text { Observed } \\
\text { Adverse Effect } \\
\text { Rate: } \\
\text { Paediatrics } \\
\text { Only (\%) }\end{array}$ & $\begin{array}{l}\text { Observed } \\
\text { Adverse } \\
\text { Effect Rate: } \\
\text { Adults Only } \\
(\%)\end{array}$ \\
\hline CRISP 1 & 1.0 & $3 / 202$ (1.5) & 2/132 (1.5) & 1/70 (1.4) \\
\hline CRISP 2 & 2.6 & 19/612 (3.1) & $16 / 440(\mathbf{3 . 6})$ & 3/172 (1.7) \\
\hline CRISP 3 & 6.2 & $34 / 478$ (7.1) & $25 / 373$ (6.7) & 9/105 (8.6) \\
\hline CRISP 4 & 14.4 & 28/171 (16.4) & $25 / 165$ (15.2) & $3 / 6(\mathbf{5 0 . 0})$ \\
\hline CRISP 5 & 36.8 & $7 / 20$ (35.0) & $7 / 20$ (35.0) & $0 / 0(\mathbf{0})$ \\
\hline
\end{tabular}


additional 1 death during anaesthetic induction before the procedure.

Conclusion: Although designed for use in paediatrics in the USA, quantitative data has shown important links between the CRISP Catheterization RISk Score and cardiac catheterisation complication rates and severity within paediatric and adult congenital cardiac catheterisation practice, within the UK and Ireland. As well as providing a much needed structure for recording data, the CRISP Catheterization RISk Score may facilitate meaningful morbidity data essential for individualised risk stratification and case planning.

\section{MP2-8}

The optimal transcatheter method to achieve long term patency of atrial septal defects

Sideris E. (1), Sideris C. (1), Toumanides S. (2), Moulopoulos S. (2) Athenian Institute of Pediatric Cardiology (1); Department of Clinical

Therapeutics, University of Athens, Greece (2)

Introduction: Long term patency of transcatheter atrial septal defects (TASDs) is required for both paliative L-R shunts (i.e. Fontan Operation, Isolated Left Heart Failure) or paliative R-L shunts (i.e. Right Heart Failure, Eisenmenger syndrome). Atrial balloon seprostomy and static pressure balloon septostomy (SPBS) have only temporary results and standard stents have been found problematic.

Methods: We developed and tested in piglets, 2 new methods for longer patency of TASDs. Alcohol septal defect angioplasty and special flat stent placement after static pressure balloon angioplasty. A $9 \mathrm{~mm}$ TASD was created in 10 one month old piglets by SPBS. In 5 of them alcohol septal angioplasty was performed using an alcohol infiltrated polyurethane foam sleeve on an angioplasty balloon. In the rest of them, a specially made flat nitinol stent was used. The Stent had a $15 \mathrm{~mm}$ proximal and distal ring and a $9 \mathrm{~mm}$ septostomy central ring. All rings were polyurethane covered for endothelialization/thrombogenicity purposes. The animals were followed for 4 months and then had autopsies.

Results: All alcohol septostomies had good acute results and no acute complications; however none of the defects were patent at 4 months. All flat stents had successful creation of TASDs. At 4 months, there was excellent endothelialization, no thrombogenicity and full patency of the ASDs.

Conclusions: The application of the flat septal stent as made, was the optimal method for long term patency of experimental TASDs. Clinical trials are justified.

\section{MP2-9}

Up to 11 years of experience with the Melody valved stent Cools B., Boshoff D., Heying R., Eyskens B., Roggen M., Suys B., Troost E., Budts W., Louw J., Frerich S., Gewillig M.

Leuven University, Belgium.

Objective: To report up to11 years follow up after Melody ${ }^{\circledR}$ valve implantation in pulmonary position.

Methods: Single institution non randomized prospective observational study. All patients discharged with a Melody valve in pulmonary position from 2006 until the end of $2017(n=186)$ were included. Follow-up consisted of 1,3, 6, 12 month followed by annual visits. Clinical evaluations, chest radiography and standard echocardiography were performed.

Results: 190 Melody valved stents were implanted in 186 patients. Mean age 19.4 y (std 13.1), 66.7\% male. Indication : stenosis $(45 \%)$, regurgitation $(33 \%)$ and mixed $(22 \%)$. Basic diagnosis: tetralogy of Fallot (54\%), Ross (18\%), truncus arteriosus (6\%), DORV (9\%), pulmonary stenosis (13\%). Pre-stenting was performed in all except the initial four patients. In stenotic lesions there was an immediate reduction of the gradient mean $66>23 \mathrm{mmHg}$ PIG with slow increase to $36 \pm 12 \mathrm{mmHg}$ PIG after 11 years. In dominant regurgitation there was $t$ reduction from median $4 / 4$ to $0 / 4$, progressing to maximal $2 / 4$ after 11 years. Stent fractures were observed in $8.4 \%$ of patients mean $4.2 \pm 2.6$ years after implantation; only 1 had a grade III fracture where the clinician decided for redo PPVI. Re-interventions included surgical removal in $8(4.3 \%)$, redo PPVI in $5(2.7 \%)$ and balloon angioplasty to accommodate for somatic growth in 8 (4.3\%). Re-interventions were performed mean $4 \pm 2.5$ years after implantation. Endocarditis was diagnosed in $19(10.2 \%)$ patients a median of 2.3 years (range: $0.7-8.8$ ) after Melody implantation. Nine got sterilized (47,4\%), 3 got redo PPVI (15.8\%) and 7 were surgically removed (36.8\%). Three patients showed thickened wall of the valved stent with inceased gradient but without signs of endocarditis. There were 3 deaths, none were procedure or valve related.

Conclusions: The Melody valve shows a good preserved leaflet function up to 11 years after implantation as well in stenotic as in regurgitant lesions. The main reason for redo PPVI or surgical removal is endocarditis, although in half of the patients with endocarditis no re-intervention was needed. Stent fractures are observed but lead very exceptionally to intervention.

\section{MP2-10 \\ Does arterial duct morphology predict success and/or failure of stent treatment ? \\ Gewillig M., Cools B., Heying R., Roggen M., Eyskens B., BoshoffD. Leuven University, Belgium}

Introduction: Stenting the arterial duct appears an elegant palliation for patients with decreased pulmonary flow. However results vary from good lasting palliation to catastrophic procedure.

Methods: From 2001 till 2017, 117 patients with duct dependent pulmonary flow. 22pts were not referred for duct stenting (clinician preference 10, too tortuous 12). In straight ducts Prostin IV was tailored to have moderate constriction at time of stent deployment. The duct was crossed with a Progreat ${ }^{\mathrm{TM}}$ microcatheter, and exchanged for a stiff 0.014 " IronMan ${ }^{\mathrm{TM}}$ coronary catheter. A coronary stent was positioned through a $4 \mathrm{~F}$ sheath. Acetyl-salicylic acid $1-2 \mathrm{mg} / \mathrm{kg} / \mathrm{d}$. When prolonged ductal flow was required, elective restenting was performed, and later redilation up to $5.5 \mathrm{~m}$ as indicated.

Results were retrospectively assessed on anatomic features: A/ straight duct as in PA-IVS; B/ duct in severe PS single ventricle; $\mathrm{C}$ / duct in $\mathrm{PA}-\mathrm{VSD}$, angle $>120^{\circ}$ very tortuous; $\mathrm{D} / \mathrm{duct}$ from subclavian artery to single lung; E/ duct from subclavian artery to both lungs.

Results: Duct stenting was attempted in 95 patients. At initial procedure stenting failed in $14 \mathrm{pts}$ (no wire crossing 11, no positioning stent 2, too large 1) and was delayed in 13 (duct too large 7 in type $\mathrm{A}$, need for other access 3 in type C, other). Good stenting was obtained in 81 pts. Type A-B-D were "easy", type C was demanding. Early re-intervention was required in 8 (incomplete covering 3, exclusion left PA 3, abundant flow requiring flow reductor 1 , kink 1 ).

8 patients died at interval; 2 possibly stented duct related (acute collaps at home). The duct was abandoned in 15 after 17 (1.5-91) months, actively closed in 3 . In 32 patients elective restenting was performed at $7(1.5-21)$ months. In 13 pts re-redilation was performed at $16(7-90)$ months to allow repair at bigger size. 
Pts evolved to Glenn 19, PA-VSD repair 10, Nikaidoh 3, RV overhaul 2, unifocalisation 2.

Conclusions: Ductal stenting in selected patients can give good lasting palliation until successful repair. The vertical tortuous duct (D) and very long type (E) require experience and adapted techniques.

\section{MP2-11}

Patient Radiation Dose during Transcatheter Atrial Septal Defect Occlusion

Kollaros N. (1), Tsapaki V. (2), Plemmenos C. (1), Mastorakou I. (1), Apostolopoulou S.C. (1)

Onassis Cardiac Surgery Center, Athens, Greece (1), Konstantopoulio General Hospital, Athens, Greece (2)

Introduction: Transcatheter atrial septal defect (ASD) occlusion is the procedure of choice for eligible patients with ASD compared to surgical occlusion. Despite the worldwide use of the procedure, international literature has extremely limited data on related radiation dose levels. The purpose of this study was to determine radiation dose, fluoroscopy time (FT) and Number of images (I) in transcatheter ASD occlusion in both paediatric and adult patients. Methods: Patients were divided according to age in groups, 5-10y, $10-15 y, 15-18 y$ and $>18$ y. Various clinical (weight, BMI, BSA etc) and radiation related data, Kerma Area Product (KAP), fluoroscopy time (FT) and number of images $(\mathrm{N})$ ) were recorded. Results: A total of 161 patients were included in the study. The median values obtained for Fluoroscopy Time (FT), Number of Frames (N), and Kerma Area Product (KAP) analyzed by age group are depicted in the Table.

Conclusions: Radiation dose varies greatly during transcatheter ASD occlusion according to age. Fluoroscopy time and number of frames are not adequate measures for radiation exposure monitoring, and only fluoroscopy time (except in the $10-15$ y group) significantly correlated with radiation dose. Given the radiosensitivity of paediatric patients, optimisation of radiation protection measures and establishment of diagnostic reference levels should be encouraged.

Table.

\begin{tabular}{lllll}
\hline $\begin{array}{l}\text { Age at } \\
\text { occlusion }\end{array}$ & $\begin{array}{l}\text { Fluoroscopy } \\
\text { Time (FT) }\end{array}$ & $\begin{array}{l}\text { Frames } \\
\text { (N) }\end{array}$ & $\begin{array}{l}\text { Kerma Area } \\
\text { Product (KAP) }\end{array}$ & $\begin{array}{l}\text { Correlation } \\
\text { KAP-FT }\end{array}$ \\
\hline $\begin{array}{c}5-10 \text { year } \\
\text { old }\end{array}$ & $6.6 \mathrm{~min}$ & 639 & $8.0 \mathrm{~Gy} . \mathrm{cm}^{2}$ & 0.71 \\
$\begin{array}{c}10-15 \text { year } \\
\text { old }\end{array}$ & $4.6 \mathrm{~min}$ & 409 & $11.0 \mathrm{~Gy} \cdot \mathrm{cm}^{2}$ & -0.04 \\
$\begin{array}{c}15-18 \text { year } \\
\text { old }\end{array}$ & $5.7 \mathrm{~min}$ & 575 & $14.0 \mathrm{~Gy} . \mathrm{cm}^{2}$ & 0.87 \\
$\begin{array}{c}>18 \text { year } \\
\text { old }\end{array}$ & $3.2 \mathrm{~min}$ & 305 & $7.0 \mathrm{~Gy} . \mathrm{cm}^{2}$ & 0.41 \\
\hline
\end{tabular}

\section{MP2-12}

Long-term survival and need of reintervention after treatment of critical valvular aortic stenosis in the neonate; a complete national study in Sweden

Kjellberg Olofsson C. (1,2), Berggren H. (2), Johansson Ramgren J. (3), Hanseus K. (3), Sunnegårdh J. (2)

Department of Paediatrics, Sundsvall Hospital, Sweden (1); The Queen Silvia Children's Hospital, Sahlgrenska University Hospital, Gothenburg, Sweden (2); Children's Heart Center, Skåne University Hospital, Lund, Sweden (3)
Objective: To examine long-term survival after treatment of critical valvular aortic stenosis during the neonatal period in a complete national cohort with surgical valvotomy being the preferred method of initial treatment.

Methods: All children in Sweden treated due to critical aortic stenosis during the neonatal period from Jan 1st 1994 to Dec 31st 2016 were included. Patient files were analyzed and cross-checked against the Swedish National Population Registry as of Nov 21st 2017 allowing for reliable and complete data on survival. The diagnosis was confirmed by reviewing echo-studies and echo-reports. Critical aortic stenosis in the neonate (younger than 30 days of age) was defined as a valvular stenosis with duct-dependent systemic circulation or depressed left ventricular function (fractional shortening $\leq 27 \%$ ). Primary outcome was all cause mortality and secondary outcomes were the need of re-intervention and aortic valve replacement.

Results: Sixty-one patients were identified (50 boys, 11 girls) with gestational age median 39 weeks (range 26-42). All medical files were retrieved with no patient lost to follow-up. Initial treatment was surgical valvotomy $(n=52)$, balloon valvotomy $(n=6)$, closed transventricular valvotomy $(n=2)$ and Ross procedure $(n=1)$. Median age at initial treatment was five days (range 0-26). There were no 30 day mortality but four late deaths at five and 10 months and at two and 21 years of age. Re-intervention was needed in 34 patients at median 3.4 months (range 4 days- 17.3 years). Freedom from re-intervention was $64,59,52,48,44 \%$ at $1,5,10,15,20$ years respectively. Aortic valve replacement was needed in 22 patients $(36 \%)$ : Ross procedure $(n=11)$, mechanical prosthesis $(n=6)$, homograft $(n=3)$, biological prosthesis $(n=2)$. In two patients conversion to a single-ventricle palliation was performed and two further patients had a heart transplantation.

Conclusion: Surgical valvotomy is a safe and reliable initial treatment method in these critically ill neonates, but the need of reintervention is high with a freedom from re-intervention of $52 \%$ at 10 years of age. In 10 out of 11 patients who reached the age of 18 years during the study the native aortic valve had been replaced.

MP3-1

Cardiomyopathy versus congenital heart diseases - 10 years in pediatric heart transplantation

Ablonczy L., Vilmányi Cs., Prodán Zs., Nagy Zs., Sápi E. Szatmári $A$.

Hungarian Institute of Cardiology, Pediatric Cardiac Center, Budapest, Hungary

Introduction: Pediatric heart transplant (htx) program was established 10 years ago in Hungary. The results of htx were analyzed regarding the underlying diseases (cardiomyopathy - CM versus congenital heart diseases - CHD).

Methods: 32 pediatric htx were performed between 2007 and 2016 in our center (m/f: 16/16, CM/CHD:21/11). Mean age was 9 yrs in CM pts (group 1.) and 8.4 yrs in CHD pts (group 2.) at time of htx. Need for prae-htx ventricle assist device (VAD), short and long-term mortality, complications, post-htx rejection, transplantrelated morbidities were examined.

Results: Almost every children had previous surgery in the CHD group (10/11). Need for praetransplant VAD was higher in the CM group $(8 / 21$ versus $1 / 11$ cases, $p<0.01)$. All transplants were successfully performed, no early mortality ( $<6$ months post-htx) was observed. Mean follow-up time was shorter in the CHD group (52 versus 32 months, $\mathrm{p}=0.04) .5$ children died in the CM group during the follow-up, compared to only 1 child in the CHD group $(5 / 21$ versus $1 / 11, \mathrm{p}=0.63)$. There was no significant difference between the 2 groups in rejection $(4 / 21$ vs $3 / 11$, 
$\mathrm{p}=0.66$ ) required anti-rejection therapy (ISHLT grade $=>2 \mathrm{R}$ or/and antibody-mediated rejection - AMR). From the CM group 2 children died due to complications of severe rejection requiring VAD. Chronic graft failure developed in a highly sensitized patient from the CHD group due to recurrent episode of AMR. There were no significant differences in cytomegalovirus (CMV) and Ebstein-Barr virus (EBV) replication/infection required specific therapy between the 2 groups (CMV $3 / 21$ versus $1 / 11-p=0.67$, EBV 4/21 versus $2 / 11-p=0.95)$. CMV was fatal in 1 case, one futher patient died because of EBV-associated posttransplant lymphoproliferative disease (PTLD). Incidence of diabetes mellitus (DM) and autoimmune diseases (AD) were similar in both groups (group 1.: $2 \mathrm{DM}, 2 \mathrm{AD}$, group 2.: 1 DM, 1 AD).

Conclusions: Htx is a good and durable option for children with end-stage heart failure. Higher late mortality rate was observed in the CM group. Longer follow-up time and more frequent need for praetransplant VAD in the $\mathrm{CM}$ group can explain the differences.

MP3-2

Pulmonary Vasodilator Therapy in Borderline Pulmonary Arterial Hypertension due to Congenital Heart Disease: Treat and Repair or Repair and Treat?

Apostolopoulou S.C., Moutsou A., Tsoutsinos A., Vagenakis G.A., Kakava F., Kyriakoulis, K., Rammos S.

Onassis Cardiac Surgery Center, Athens, Greece

Introduction: Pulmonary arterial hypertension (PAH) associated with congenital heart disease (CHD) is an important issue both in the adult and pediatric population that may influence outcome and even preclude repair. Pulmonary vasodilators (PV) in borderline cases, before or after surgery, may improve PAH and possibly change the operability criteria for these patients.

Methods: This study reports our experience using PV in 2 groups of CHD patients with borderline PAH: 1) patients with initially prohibiting $\mathrm{PAH}$, where PV improved $\mathrm{PAH}$ enough to permit intervention (treat and repair), 2) patients initially deemed operable but developing significant postoperative PAH (repair and treat).

Results: The 1st group included 12 patients aged $13.0 \pm 14.2$ (0.9-43) years, 4 with single ventricle (SV) physiology. Pulmonary vascular resistance index (PVRI) was $6.4 \pm 2.6$ (4.4-10.1) Wood units (WU) in the nonSV and $2.5 \pm 0.6 \mathrm{WU}$ in the SV patients, not permitting further intervention. PV therapy for $2.0 \pm 1.3$ years improved $(\mathrm{p}<0.01)$ systolic and mean pulmonary pressure, PVRI and PVRI/SVRI and permitted repair in the nonSV patients, Fontan in 2 and fenestration occlusion in 2 SV patients. PV were discontinued in 4 patients $0.9 \pm 0.3$ years after intervention without problems for $5.6 \pm 1.4$ years subsequent follow up.

The 2nd group included 12 patients aged $13.1 \pm 21.1(0.4-72.7)$ years, 6 with SV physiology. PV were instituted postoperatively before discharge in 3 nonSV patients due to increasing RV estimated pressures and $6 \mathrm{SV}$ patients due to increasing central venous pressures and pleural effusions. Therapy was discontinued after $2.6 \pm 1.9$ years after invasively measured improvement in 5 patients without problems for $4.2 \pm 3.2$ years subsequent follow up. PV were instituted 2 months to 2.8 years postoperatively in 3 patients with septal defects due to invasively measured increased pulmonary pressures and resistances and patients remain on PV during 2.0-8.0 years.

Conclusions: $\mathrm{PV}$ in borderline $\mathrm{PAH}$ due to $\mathrm{CHD}$ may help to manipulate pulmonary vascular resistance pre and postoperatively, permit repair or intervention in otherwise prohibiting resistances and possibly shift the operability criteria in these patients. Further research with larger populations and longer follow up is needed before recommendations for pre and/or postoperative PV therapy can be issued and followed.

MP3-3

Subclinical atherosclerosis in cyanotic congenital heart disease

Tarp J.B. (1), Sorgaard M. (1), Christoffersen C. (1), Jensen A . (1), Sillesen H. (1), Erikson P. (2), Celermajer D. (3), Estensen ME. (4), Holstein-Rathlou NH. (5), Engstrom T. (1), Sondergaard L. (1).

(1) Rigshospitalet, Copenhagen, Denmark; (2) University of Göteborg, Göteborg, Sweden; (3) Royal Prince Alfred Hospital, Sydney, Australia; (4) Rikshospitalet, Oslo, Norway; (5) Copenhagen University,

Copenhagen, Denmark

Introduction: The survival of patients with cyanotic congenital heart disease (CCHD) has improved dramatically due to better care and treatment options, which has resulted in an ageing population with risk of acquired heart disease. Previously uncontrolled studies have suggested that these patients are protected against the development of atherosclerosis.

The aim of the present study was to evaluate the prevalence of subclinical atherosclerosis in patients with CCHD and a matched control population.

Method: The prevalence of atherosclerosis was investigated in adults with CCHD and in controls matched for age, sex, body-mass-index, and smoking status. The patients were included from centers in Denmark, Norway, Sweden and Australia. Coronary artery atherosclerosis was assessed by computer tomography with measurement of coronary artery calcification (CAC) score with subclinical atherosclerosis defined as a CAC $>0$. Carotid artery atherosclerosis was evaluated using ultrasound by measuring plaque burden and carotid intima media thickness (CIMT). Lipid status was evaluated as additional atherosclerotic risk factor.

Results: Data on 63 patients with CCHD (mean age 48.5 years $( \pm 12.7)$ and 59 matched controls 48.6 years $( \pm 13,0)$ showed no difference in CAC score (CAC >0: $21 \%$ of patients vs. $17 \%$ of controls $(\mathrm{CI} ;-10 \% ; 18 \%, \mathrm{p}=0.57)$. Similar, no differences were found in the present of carotid plaque burden (23\% in patients vs. $12 \%$ in controls, $\mathrm{p}=0,11)$, CIMT $(0.62 \mathrm{~mm}( \pm 0.13)$ in patients vs. $0.61 \mathrm{~mm}( \pm 0,14)$ in controls, $p=0,48)$. Lipid status on ultracentrifugation did not differ between the groups (total cholesterol: $4.76 \mathrm{mmol} / 1( \pm 0.97)$ in patients vs. $5.14 \mathrm{mmol} / 1( \pm 0.95)$ in controls, $\mathrm{p}=0,08$; low-density-cholesterol: $2.59 \mathrm{mmol} / 1( \pm 0.64)$ in patients vs. $2.85 \mathrm{mmol} / \mathrm{l}( \pm 0.84)$ in controls, $\mathrm{p}=0.11)$.

Conclusion: In this controlled study we did not find an increased burden of subclinical atherosclerosis in patients with CCHD by combining a variety of assessment methods.

We could not confirm the results of previous studies, which by analysing smaller samples have indicated a decreased risk. Thus, the present study indicates that patients with CCHD should apply to the same prophylaxis precautions including life-style modifications as recommended for the general population in order to avoid atherosclerotic diseases.

MP3-4

Incidence of late arterial hypertension after aortic coarctation repair: differences between surgical correction and stent implantation

Yammine M.L., Chinali M., Cafiero G., Giannico S., Giordano U. Bambino Gesù Children's Hospital, IRCCS, Rome, Italy

Objectives: We sought to evaluate long-term outcomes in terms of blood pressure (BP) and echocardiographic parameters in children 
and adults with aortic coarctation corrected by surgical repair or stent implantation.

Methods: We identify 64 patients with isolated coarctation of the aorta with mean follow-up of 13,93 years after successful aortic repair. Patients were divided into two groups based on the type of aortic correction: 38 with surgical repair (end-to-end anastomosis, patch angioplasty or subclavian flap) and 26 with stent implantation (either with native aortic coarctation or recurrent coarctation after previous surgical correction). All subjects underwent 24-hour ambulatory blood pressure monitoring (ABPM) and trans-thoracic echocardiography to assess left ventricular mass.

Results: Antihypertensive treatment was recorded in 18/26 (69.2\%) patients with aortic stenting compared to $21 / 38$ (55.3\%) $(\mathrm{p}=$ n.s.) of those with surgical repair of the aorta. ABPM revealed arterial hypertension with higher prevalence in the stented group $(19.2 \%$ vs $13.2 \% ; p=$ n.s.), with $11.5 \%$ of subjects having elevated values in the day-time compared to $5 \%$ of those in the surgical Group $(\mathrm{p}=$ n.s.). Pulse pressure (PP) mean and median values were higher in patients with aortic stenting, and $\mathrm{PP}$ values exceeding $50 \mathrm{mmHg}$ have been recorded in $21 / 26(80.8 \%)$ compared to $22 / 38(57.9 \%)(p=0.03)$ in the surgical group. Finally, in patients with stenting of the aorta, echocardiogram showed a higher proportion of patients $(46.2 \%)$ with significant late residual gradient across aortic site repair (defined as $>20 \mathrm{mmHg}$ ), compared to $28.9 \%$ in the surgical Group $(\mathrm{p}=0.07)$. Also, left ventricular mass index (LVMi) demonstrated a higher prevalence of left ventricular hypertrophy in patients corrected with aortic stenting $(30.1 \%$ vs $13.2 \%)(\mathrm{p}=0.059)$.

Conclusions: Although comparable successful outcomes in the early time-period, patients after stenting for aortic coarctation seem to have worse long-term outcomes compared to patients with surgical correction. Stenting management showed a higher prevalence of resting arterial hypertension, higher need of antihypertensive therapy and higher incidence of elevated left ventricular mass index.

MP3-5

Long-Term Transplant-Free Survival of Patients after Lateral Tunnel vs Extracardiac Fontan for Single Ventricle Lesions: Does type of Fontan procedure matter?

Kochilas L., Knight J., Suthar D., Oster M.

Emory University, Atlanta, GA, USChildren's Healthcare of Atlanta, Atlanta, GA, US

Background: Despite its multiple modifications, Fontan palliation for single ventricle (SV) lesions is associated with significant morbidity leading to premature mortality or need for transplant. After 1990, the two predominant types of Fontan are the lateral tunnel (LT) and the extracardiac conduit (EC). Advantages and disadvantages for each procedure have been reported from previous studies; however, data from direct comparison of long-term outcomes between each type of procedure are sparse. We used data from the Pediatric Cardiac Care Consortium (PCCC), a large multi-center US-based surgical registry, to compare the long-term transplant-free survival for children with LT vs EC Fontan procedure.

Methods: This is a retrospective cohort study of SV patients born between 1990 and 2001 and underwent Fontan palliation in the PCCC. We used the PCCC dataset and linkage data from the US National Death Index and the Organ Procurement Transplantation Network registry to examine long-term survival and transplant status for LT and EC procedure through 2014. To address differences in characteristics of patients we compared survival after application of propensity score matching for potential confounders
Table. Characteristics and outcomes of patients after lateral tunnel or extracardiac Fontan

\begin{tabular}{|c|c|c|c|c|}
\hline & Total & Lateral Tunnel & Extracardiac & p-value \\
\hline Whole cohort & $\mathrm{N}=1,223$ & $\mathrm{~N}=432(35.3 \%)$ & $\mathrm{N}=791(64.7 \%)$ & \\
\hline Male sex & $760(62.1)$ & $276(63.9)$ & $484(61.2)$ & 0.3520 \\
\hline \multicolumn{5}{|l|}{ Year of birth } \\
\hline 1990-1995 & $463(37.9)$ & $263(60.9)$ & $200(25.3)$ & $<0.0001$ \\
\hline $1996-2000$ & $760(62.1)$ & $169(39.1)$ & $591(74.7)$ & \\
\hline $\begin{array}{c}\text { Chromosomal } \\
\text { abnormality }\end{array}$ & $83(6.8)$ & $28(6.5)$ & $55(7.0)$ & 0.754 \\
\hline \multicolumn{5}{|c|}{ Dominant systemic ventricle } \\
\hline Right ventricle & $386(31.6)$ & $140(32.4)$ & $246(31.1)$ & 0.0015 \\
\hline Left ventricle & $528(43.2)$ & $208(48.2)$ & $320(40.5)$ & \\
\hline Not classifiable & $309(25.3)$ & $84(19.4)$ & $225(28.5)$ & \\
\hline Fenestrated Fontan & $780(58.0)$ & $322(78.4)$ & $458(49.1)$ & $<0.0001$ \\
\hline $\begin{array}{l}\text { Median age in yrs } \\
\text { (IQR) }\end{array}$ & $2.9(2.3-3.9)$ & $2.6(2.0-3.3)$ & $3.2(2.5-4.2)$ & $<0.0001$ \\
\hline In-hospital survival & 1, $145(93.6)$ & $389(90.1)$ & $756(95.6)$ & 0.0002 \\
\hline $\begin{array}{l}\text { Median follow up in } \\
\text { yrs (IQR) }\end{array}$ & $13.5(11.3-16.6)$ & $16.5(13.3-18.6)$ & $12.5(10.6-15.3)$ & $<0.0001$ \\
\hline $\begin{array}{l}\text { 10-year post Fontan } \\
\text { survival }\end{array}$ & 93.4 & 94.0 & 93.2 & 0.325 \\
\hline $\begin{array}{l}\text { Propensity score } \\
\text { matched }\end{array}$ & 482 & $N=241$ & $N=241$ & \\
\hline In-hospital survival & $447(91.7)$ & $224(93.0)$ & $223(92.5)$ & 0.861 \\
\hline $\begin{array}{l}\text { 10-year post Fontan } \\
\text { survival }\end{array}$ & $417(93.2)$ & $208(92.8)$ & $209(93.7)$ & 0.859 \\
\hline
\end{tabular}

such as type of systemic ventricle, sex, presence of genetic syndrome, creation of fenestration at the time of Fontan, year of birth and age at Fontan.

Results: Of the 1223 patients identified, 432 (35.3\%) underwent LT and 791 (64.7\%) EC. LT was performed in younger children (2.4 vs 3.2 yrs of age, $p<0.0001)$ and more frequently in the early years of the study period (Table). In-hospital survival was higher for EC $(95.6 \%$ vs $90.1 \%, p=0.0002)$; however, 10-year survival after discharge was similar for LT and EC (94\% vs. 93.2\% respectively, $\mathrm{p}=0.325)$. Multivariable logistic regression comparing transplant-free survival for 482 propensity score matched patients within this cohort did not reveal any in-hospital (OR 0.72 for LT vs EC; 95\% CI: 0.16-3.26) or long-term (OR 1.14 for LV vs EC; 95\% CI: 0.61-2.11) benefit for either type of procedure.

Conclusion: LT and EC types of Fontan have similar long-term transplant-free survival outcomes. Additional studies comparing differences in morbidity will be needed to evaluate for potential long-term benefits of the one vs the other procedure.

MP3-6

Smaller regional brain volumes are correlated with adverse neurodevelopmental outcome at one year of age in complex congenital heart disease

Knirsch W. (1,2), Meuwly E. (1,3), Feldmann M. (1,3), Von Rhein M. (1,3), Dave H. (1,4), Tuura R. (1,5), Jakab A. $(1,5)$, Latal B. $(1,3)$ Children's Research Center (1), Pediatric Cardiology, Pediatric Heart Center (2), Child Development Center (3), Congenital Cardiovascular Surgery, Pediatric Heart Center (4), Diagnostic Imaging, MR-Center (5), University Children's Hospital, Zurich, Switzerland

Objectives: The purpose of this ongoing prospective cohort study is to analyse, if pre- and postoperatively measured brain volumes in neonates suffering from severe congenital heart disease (CHD) predict one- year- neurodevelopmental outcome.

Methods: Infants with severe CHD undergoing neonatal cardiopulmonary bypass surgery were examined with a pre- and postoperative cerebral MRI. Neurodevelopmental testing at 1 year was performed using the Bayley III.

Results: 78 neonates with CHD and 43 term controls were included (mean gestational age at birth was 39.2 weeks for the CHD children and 39.6 weeks in the control group). The CHD 
Table 1.

\begin{tabular}{|c|c|c|c|c|c|c|c|c|c|}
\hline & \multicolumn{3}{|c|}{ Cognitive } & \multicolumn{3}{|c|}{ Language } & \multicolumn{3}{|c|}{ Motor } \\
\hline & Beta & $\mathbf{P}$ & $\mathbf{R}^{2}$ & Beta & $\mathbf{P}$ & $\mathbf{R}^{2}$ & Beta & $\mathrm{p}$ & $\mathbf{R}^{2}$ \\
\hline Whole brain & 0.145 & $0.025^{\star}$ & 0.125 & 0.130 & $0.024 \star$ & 0.132 & 0.111 & 0.051 & 0.192 \\
\hline Cortex & 0.206 & $0.017^{\star}$ & 0.137 & 0.173 & $0.025^{\star}$ & 0.131 & 0.159 & $0.037 \star$ & 0.200 \\
\hline White matter & 0.275 & $0.022^{\star}$ & 0.129 & 0.238 & $0.025^{\star}$ & 0.131 & 0.218 & $0.039^{\star}$ & 0.199 \\
\hline Frontal lobe & 0.498 & $0.019^{\star}$ & 0.133 & 0.447 & $0.018^{\star}$ & 0.142 & 0.381 & $0.042^{\star}$ & 0.196 \\
\hline Parietal lobe & 0.624 & $0.03 \star$ & 0.120 & 0.433 & 0.092 & 0.089 & 0.515 & $0.041^{\star}$ & 0.197 \\
\hline Occipital lobe & 1.202 & $0.039 \star$ & 0.112 & 1.133 & $0.028^{\star}$ & 0.127 & 1.035 & $0.042^{\star}$ & 0.197 \\
\hline Temporal lobe & 0.5 & 0.123 & 0.08 & 0.499 & 0.102 & 0.115 & 0.076 & 0.799 & 0.132 \\
\hline Cerebellum & 1.247 & $0.019 \star$ & 0.133 & 0.825 & 0.086 & 0.091 & 0.889 & 0.059 & 0.187 \\
\hline
\end{tabular}

$\star=p<0.05$

infants had lower total and regional brain volumes in all regions compared to healthy controls (mean whole brain volume CHD infants postoperatively $395.2 \mathrm{~cm}^{3}$, controls $434.7 \mathrm{~cm}^{3}, \mathrm{p}<0.001$ ). Female CHD infants had lower total brain volumes compared to males $(\mathrm{p}<0.05)$. The Bayley-III composite standard scores were lower in CHD infants compared to healthy controls in all three domains: cognition: $104(55-140)$ versus $117(85-135)(\mathrm{p}<0.01)$, language; $92(56-132)$ versus 97 (79-124) $(\mathrm{p}<0.05)$, motor: 92 $(46-135)$ versus 103 (73-121) ( $\mathrm{p}<0.01)$. Logistic regression analyses correcting for gender, postmenstrual age at time of MRI and protocol group showed that postoperative brain volumes (whole brain, cortex, white matter, lobes (except temporal lobe), cerebellum) predicted cognitive outcome in CHD (see Table 1) but not in control children.

Conclusion: Our findings confirm that there is a global reduction of cerebral volume in newborns with severe CHD. Further, the data shows that postoperative brain volumes are a predictor for the cognitive outcome in CHD infants. Children with low cerebral volume could be detected early on which would allow for early therapeutic interventions.

\section{MP3-7}

\section{Aortic Valve Repair in Congenital Heart Surgery using} CardioCel ${ }^{\circledR}$ patch material

Kretzschmar J. (2), Nordmeyer S. (1,3), Murin P. (2), Schulz A. (2), Danne F. (1), Nordmeyer J. (1), Sumbadze D. (1), Schmitt K. R. L. (1), Miera O. (1), Cho M.-Y. (2), Sinzobahamvya N. (2), Berger F. (1), Sigler M. (4), Ovroutski S. (1), Photiadis J. (2)

German Heart Center Berlin, Department of Congenital Heart Disease and Paediatric Cardiology, Berlin, Germany (1); German Heart Center Berlin, Department of Surgery for Congenital Heart Disease and Paediatric Cardiology, Berlin, Germany (2); Charité-

Universitätsmedizin Berlin, Institute for Cardiovascular Computerassisted Medicine (3); Georg-August University, Department of Paediatric Cardiology, Göttingen, Germany (4)

Objectives: The optimal material for aortic valve repair (AVR) has yet to be found. In this study we report our experience of AVR in congenital heart surgery (CHS) using CardioCel ${ }^{\circledR}$ patch material. Methods: In this retrospective study we reviewed data of 40 consecutive patients who underwent AVR using CardioCel $^{\circledR}$ patch (Admedus Regen Pty Ltd, Perth, WA, Australia) between February 2014 and August 2016. Median age at operation was 9 (2-34 years), 26 patients presented initially with aortic valve insufficiency (AI) and 14 with stenosis (AS). For a median postoperative follow-up (FU) of 22 (6-42) months until August 2017, clinical and echocardiographic data was included. In case of reoperation, explanted CardioCel ${ }^{\circledR}$ patch material was examined histologically. Besides standard histological staining, Kossa stain (for identification of calcifications) and

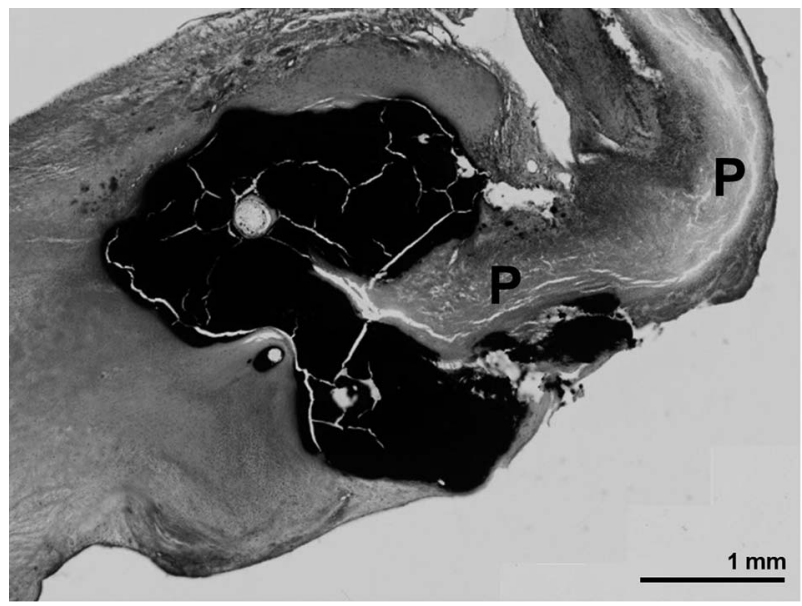

Figure.

immunohistochemical staining was applied with antibodies specific for muscular, inflammatory, and connective tissue component antigens. Results: 9/40 patients (23\%) suffered from an adverse event during FU (death: $\mathrm{n}=1,2.5 \%$; re-operation: $\mathrm{n}=8,20 \%)$. Overall, the probability of freedom from re-operation/death was $100 \%, 89 \% \pm 6 \%$ and $56 \% \pm 12 \%$ at median 10, 20 and 30 months FU. Reason for re-operation was insufficiency in 4 patients (10\%), stenosis in 3 patients $(8 \%)$ and 1 patient $(2.5 \%)$ was diagnosed with aortic valve endocarditis. 2 out of the remaining 31 patients are scheduled for re-operation (AS: $n=1, A I: n=1$ ) and 9 patients show decrease of aortic valve function with moderate AI. Freedom from developing combined endpoint (death/re-operation/moderate degree of aortic valve dysfunction (AS, AI)) after AVR was $97 \% \pm 2.5 \%, 73 \% \pm 8 \%$ and $33 \% \pm 9 \%$ at 10,20 and 30 months, respectively. Histologically, all specimen $(8 / 8=100 \%)$ showed tissue proliferation and $50 \%$ showed inflammatory cell infiltration. 6 of 8 samples with implant times of 23 to 29 months revealed low-grade calcifications (Figure) and only the two specimen with "short" implant times of 8 and 11 months showed no calcifications. Conclusions: AVR using $\mathrm{CardioCel}^{\circledR}$ pericardial patch material in patients with congenital aortic valve disease shows unsatisfactory results at 30 months FU. Explanted CardioCel ${ }^{(\mathbb{R}}$ patches demonstrated proliferations and calcifications as possible reasons for patch performance failure.

MP3-8

What determines quality of life in pre-school aged children with single ventricle congenital heart disease - Surgery, Hemodynamics, Bayley Scales or Brain Volumes?

Knirsch W. (1,2), Heye K.N. (1,2,3), Tuura R. (1,4), Beck I. (3), Wetterling K. (5), Schranz D. (6), Hahn A. (7), Hofmann K. (6), Latal B. (1,3), Landolt M. $(1,8,9)$, Reich B. (6)

Children's Research Center (1), Pediatric Cardiology, Pediatric Heart Center (2), Child Development Center (3), Diagnostic Imaging, MR-Center (4), University Children's Hospital, Zurich, Switzerland; Child Development Center (5), Frankfurt/Main, Germany; Pediatric Heart Center (6), Pediatric Neurology (7), University Hospital Giessen, JustusLiebig-University, Giessen, Germany; Department of Psychosomatics and Psychiatry (8), University Children's Hospital, Zurich, Switzerland; Division of Child and Adolescent Health Psychology (9), Department of Psychology, University of Zurich, Switzerland 
Objectives: Little is known about health-related quality of life (HRQoL) in pre-school age in children with congenital heart disease (CHD) undergoing Fontan palliation. We aimed on HRQoL in preschoolers with single ventricle (SV) CHD by determining patient-related, clinical variables, and their impact of HRQoL with various dimensions of daily functioning.

Methods: Prospective two-center cohort study in children without genetic comorbidity undergoing Fontan palliation for SV CHD at $26.3 \pm 3.4$ (mean $\pm \mathrm{SD}$ ) years of age. HRQoL was assessed by parental report of the Preschool Pediatric Cardiac Quality of Life Inventory (P-PCQLI) one year later at 3-4 years, compared to normative data of children with mixed types of CHD using linear multivariable regression analysis. The influence of hemodynamics determined by catheter measurements, cerebral findings by magnetic resonance imaging, and neurodevelopmental outcome by the Bayley scales III, all assessed before Fontan palliation on later HRQoL were also determined.

Results: 46 children were evaluated with the HRQoL at an age of $38.9 \pm 3.6$ years. Total HRQoL score was comparable to children with mixed $\mathrm{CHD}$ at $41.7 \pm 3.3$ years. Perioperative variables (summarized in a surgery severity score) were significant determinants of HRQoL at that age, explaining a variability for the total score of $24 \%$, for the physical score of $28 \%$ and for the social score of $18 \%$. The surgery severity score and preterm birth predicted the therapy HRQoL (adjusted R2 $=0.22$ ), whereas preterm birth was the only predicting variable for functional HRQoL $(\mathrm{R} 2=0.15)$. Pre Fontan hemodynamics and brain volumes showed mild univariate correlations with HRQoL subscores, but were not significant in multivariable analysis (i.e. determining $<10 \%$ variability for physical score). The Bayley scales III as well as the presence and type of cerebral lesions on MRI were not predictive for HRQoL at pre-school age.

Conclusions: Despite substantial health-related burden, preschoolers with SV CHD are reported to have a good HRQoL. Perioperative risk factors have a stronger impact on HRQoL at pre-school age compared to pre Fontan hemodynamics, brain volumes and neurodevelopmental outcome assessments. In future, reports on patient-individual self-rated HRQoL at school age are needed to determine the impact of the analyzed factors on follow up at school age.

\section{MP3-9 \\ Telemetrically adjustable pulmonary artery banding with the FloWatch device: the Greek experience \\ Kalavrouziotis G., Kallikourdis A., Giannakopoulou E., Andreou N., Eleftherakis N., Karanasios E., Konstantopoulou G., Kourtesis A., Paphitis C. \\ "Aghia Sophia" Children's Hospital, Athens, Greece}

Introduction: Pulmonary blood flow control in congenital heart diseases (CHDs) has been a "dream" for decades which have come true. We present the first series in Greece of patients with CHDs and pulmonary hypertension, who had telemetrically adjustable pulmonary artery banding (PAB) with the FloWatch ${ }^{\circledR}$ device.

Methods: There were 10 infants (4 male). Median age at the time of surgery was 38 (range, 9-290) days, and median body weight was 3.6 (range, 3.0-6.2) kg. CHDs were: ventricular septal defect $(n=6)$, complete atrioventricular septal defect $(n=4)$, patent ductus arteriosus $(n=4)$, secundum atrial septal defect $(n=2)$, aortic coarctation $(n=1)$. All patients were in heart failure under maximal pharmaceutic support.

Results: The implantation of the device was easy. Mortality was nill. Three patients had had septic complications. In one patient, an infected pericardial effusion was revealed intraoperatively. Drainage of the effusion and the appropriate antibiotic treatment led to a smooth postoperative course. A second patient developed fever during surgery, and 24 hours later, septic shock. She was resuscitated successfully, and her course was uneventful thereafter. A third patient had prolonged fever of unknown origin and fluid collection in the mediastinum. An exploratory repeat sternotomy was done and the fluid was drained. Postoperative course was uneventful thereafter. In no case the device was explanted. The device was gradually adjusted with the guidance of bedside echocardiography within the next 2-3 days till the desired pressure gradient across PAB was achieved. Median ICU and hospital stay were 2 and 12 days, respectively. All patients undergone total repair of their CHD 18 to 35 months after FloWatch implantation. The explantation of the device was easy. No pulmonary artery reconstruction was required. In two cases with a spontaneously closed or insignificant VSD, the redo procedure included only FloWatch ${ }^{\circledR}$ removal, without the need of CPB in one case.

Conclusions: 1. The FloWatch ${ }^{\circledR}$ device can precisely control pulmonary blood flow in both directions (decrease - increase). 2. Surgical mortality, and surgical morbidity for PAB adjustment is nill. 3. The device demonstrated resistance to an infected/septic environment without the need for explantation.

MP3-10

Accuracy of Echocardiography in diagnosing Coronary Anatomy in Transposition of the Great Arteries - Helping or confusing the surgeon?

Amini K. (1,3), Christmann M. (1,3), Wipf A . (1,3), Weber R. (1,3), Balmer C. (1,3), Quandt D. (1,3), Kretschmar O. $(1,3)$, Hübler M.

(2,3), Knirsch W. (1,3)

University Children's Hospital Zurich, Heart Center, Pediatric

Cardiology, Zurich, Switzerland (1); University Children's Hospital

Zurich, Heart Center, Division of Congenital Cardiovascular Surgery,

Zurich, Switzerland (2); Children's Research Center, University of

Zurich, Switzerland (3)

Objective: To determine the accuracy of echocardiography in diagnosing the coronary anatomy in neonates with d-transposition of the great arteries (dTGA) before arterial switch operation (ASO) and to evaluate the impact of a correct or incorrect echocardiographic diagnosis regarding the intra- and postoperative course after ASO.

Methods: In this single center observational study 131 neonates $(72.5 \%$ male) with simple dTGA, diagnosed from 2004 to 2017 were enrolled. We compared coronary patterns found in preoperative echocardiography with intraoperative findings and evaluated the findings with parameters of the intra- and postoperative clinical course.

Results: 90 (68.7\%) patients had a usual coronary anatomy (1LCx2R) and $41(31.3 \%)$ patients showed coronary variants intraoperatively $(1 \mathrm{~L} 2 \mathrm{RCx} \mathrm{n}=18$, single coronary artery $\mathrm{n}=7$, intramural course $n=5$, others $n=11$ ). In children with usual coronary anatomy echocardiographic diagnosis was correct in $92.2 \%$ in contrast to $19.5 \%$ with coronary variants. Overall, echocardiographic diagnosis was correct in $68.7 \%(n=90)$. The incorrectly diagnosed patients were coronary variants in $83 \%$ $(\mathrm{n}=34)$. Incorrect diagnosed patients showed significantly longer duration of surgery (261 vs. $276 \mathrm{~min}, \mathrm{p}=0.042$ ) and extracorporal circulation time (ECC, 165 vs $178 \mathrm{~min}, \mathrm{p}=0.026$ ), whereas common parameters of the postoperative course (inotropic support, ventilator support, ICU and total hospital stay) were not significantly different in both groups. Of note, coronary variants itself showed a significantly longer duration of surgery 
(261 vs $295 \mathrm{~min}, \mathrm{p}=0.025)$, aortic cross clamp time (102.5 vs $122 \mathrm{~min}, \mathrm{p}=0.004)$ and ECC time (166.5 vs $188 \mathrm{~min}, \mathrm{p}=0.016)$ without differences in parameters of the postoperative course. Conclusion: Accuracy of echocardiographic diagnosis of coronary anatomy is high in usual coronary pattern (1LCx $2 \mathrm{R}, 92.2 \%)$ but low in coronary variants (19.5\%). Incorrect diagnosis is associated with a longer intraoperative but not with a more complicated or longer postoperative course. Nevertheless, biasing the impact of a wrong echo diagnosis, a more difficult coronary transfer in coronary variants influences these factors as well. Surgical strategy was not fundamentally influenced by coronary anatomy as ASO could be performed in all children (100\%) regardless of assumption of coronary variants or wrong diagnosis during preoperative echocardiography.

\section{MP3-11}

Arterial Switch Operation for Transposition of the Great Arteries with Intact Ventricular Septum in Infants Older than 21 days

Yıldız O., Ozturk E., Onan S., Tanıdır C., Ergul Y., Güzeltaş A., Haydin $S$.

Department of Pediatric Cardiovascular Surgery and Pediatric Cardiology Istanbul SBU Mehmet Akif Ersoy Thoracic and Cardiovascular Surgery Center Istanbul, Turkey

Introduction: We have previously published that the primary arterial switch operation (ASO) is a feasible strategy for patients with transposition of the great arteries and intact ventricular septum (TGA-IVS) beyond age 21 days. We wanted to share our increasing experience in this study.

Methods: Between 2010 and 2017, 22 patients with the diagnosis of TGA/IVS whom ASO was performed after the postnatal 3rd week were evaluated retrospectively from the hospital data base. The demographic and echocardiographic features, cardiac catheterization findings were recorded. The timing and course of the operations, postoperative problems in the intensive care unit were evaluated.

Table. The demographic and clinical data

\begin{tabular}{|c|c|c|c|c|c|c|c|c|c|c|}
\hline no & $\begin{array}{l}\text { Age } \\
\text { day }\end{array}$ & Sex & $\begin{array}{l}\text { Weight } \\
\text { (kg) }\end{array}$ & $\begin{array}{l}\text { BSA } \\
(\mathrm{m} 2)\end{array}$ & BAS & ASD & Cath & PDA & $\begin{array}{l}\text { VSD } \\
\text { type }\end{array}$ & SO2 $\%$ \\
\hline 1 & 25 & $\mathrm{M}$ & 4,2 & 0,26 & $\mathrm{Y}$ & Non-R & Y & 0 & Type 3 & $>70$ \\
\hline 2 & 25 & M & 4,0 & 0,22 & $\mathrm{~N}$ & $\mathrm{R}$ & $\mathrm{N}$ & 0 & Type 2 & $>70$ \\
\hline 3 & 45 & F & 3,1 & 0,2 & $\mathrm{Y}$ & Non-R & $\mathrm{N}$ & 0 & Type 1 & $>70$ \\
\hline 4 & 43 & F & 4,0 & 0,23 & $\mathrm{~N}$ & Non-R & Y & 0 & Type 3 & $<70$ \\
\hline 5 & 125 & M & 5,4 & 0,29 & $\mathrm{~N}$ & $\mathrm{R}$ & Y & 0 & Type 2 & $<70$ \\
\hline 6 & 43 & M & 3,2 & 0,21 & $\mathrm{~N}$ & $\mathrm{R}$ & $\mathrm{N}$ & large & Type 1 & $>70$ \\
\hline 7 & 24 & $\mathrm{~F}$ & 3,3 & 0,2 & $\mathrm{~N}$ & $\mathrm{R}$ & $\mathrm{N}$ & 0 & Type 1 & $<70$ \\
\hline 8 & 23 & M & 3,2 & 0,2 & $\mathrm{~N}$ & Non-R & $\mathrm{N}$ & large & Type 2 & $>70$ \\
\hline 9 & 60 & $\mathrm{~F}$ & 4,6 & 0,24 & $\mathrm{~N}$ & Non-R & Y & 0 & Type 3 & $<70$ \\
\hline 10 & 40 & M & 4,5 & 0,25 & $\mathrm{~N}$ & Non-R & $\mathrm{N}$ & 0 & Type 1 & $>70$ \\
\hline 11 & 22 & M & 2,3 & 0,16 & $\mathrm{~N}$ & Non-R & $\mathrm{N}$ & large & Type 1 & $>70$ \\
\hline 12 & 43 & $\mathrm{~F}$ & 3,4 & 0,2 & $\mathrm{~N}$ & $\mathrm{R}$ & $\mathrm{N}$ & large & Type 1 & $<70$ \\
\hline 13 & 26 & F & 3,6 & 0,22 & $\mathrm{~N}$ & $\mathrm{R}$ & $\mathrm{N}$ & 0 & Type 1 & $<70$ \\
\hline 14 & 55 & M & 4,4 & 0,23 & Y & $\mathrm{R}$ & $\mathrm{N}$ & 0 & Type 2 & $<70$ \\
\hline 15 & 50 & M & 3,2 & 0,2 & $\mathrm{Y}$ & $\mathrm{R}$ & Y & 0 & Type 2 & $>70$ \\
\hline 16 & 120 & M & 5,5 & 0,29 & $\mathrm{Y}$ & $\mathrm{R}$ & Y & 0 & Type 3 & $<70$ \\
\hline 17 & 80 & $\mathrm{~F}$ & 4,6 & 0,25 & $\mathrm{~N}$ & Non-R & $\mathrm{N}$ & large & Type 2 & $>70$ \\
\hline 18 & 900 & $\mathrm{M}$ & 11 & 0,52 & $\mathrm{~N}$ & Non-R & $\mathrm{N}$ & 0 & ${ }_{3}^{\text {Type } 2-}$ & $>70$ \\
\hline 19 & 48 & $\mathrm{~F}$ & 3,3 & 0,2 & $\mathrm{~N}$ & Non-R & $\mathrm{N}$ & 0 & Type 1 & $>70$ \\
\hline 20 & 28 & $\mathrm{~F}$ & 3,2 & 0,2 & $\mathrm{~N}$ & $\mathrm{R}$ & $\mathrm{N}$ & 0 & Type 1 & $<70$ \\
\hline 21 & 55 & M & 4,4 & 0,23 & $\mathrm{Y}$ & $\mathrm{R}$ & Y & 0 & Type 2 & $<70$ \\
\hline 22 & 58 & $\mathrm{M}$ & 4 & 0,25 & $\mathrm{~N}$ & Non-R & $\mathrm{Y}$ & med & ${ }_{3}^{\text {Type } 2-}$ & $>70$ \\
\hline
\end{tabular}

F:Female, M:Male, BSA:Body surface area, BAS:balloon atrial septostomy, ASD:Atrial septal defect, VSD: Ventricular Septal defect Y:Yes, N:No.
Results: The median age of operation was 44 days (range 22900days), median weight was $4 \mathrm{~kg}$ (range $2.3-11 \mathrm{~kg}$ ). 11 patients had severe cyanosis $(\mathrm{SO} 2 \leq 70 \%), 16$ patients were receiving PGE1 infusion, and 7 patients were on mechanical ventilation at baseline. Balloon atrial septostomy was on 7 patients previously. Preoperative catheterization was performed on 10 patients. 15 patients were transferred to the intensive care unit with opened chest postoperatively. The median duration of mechanical ventilation, intensive care unit and hospital stay were 5 (range 2-33) days, 9.5 (range 3-48) days and 16 (range 7-57) days, respectively. 3 patients needed postoperative and one patient needed preoperative extracorporeal life support. There was no mortality.

Patients were grouped into 3 types according to the interventricular septum-left ventricular geometry on preoperative echocardiography. Type I: Interventricular septum (IVS) bulging into the right ventricle ( 9 patients), Type II: straightened IVS (7 patients), type III: IVS bulging in to the left ventricle (bananashaped) (6 patients).

Conclusions: Late arterial switch operation can be performed safely in patients with transposition of the great arteries and intact ventricular septum after a detailed evaluation and with an efficient, advanced, and suitable postoperative intensive care unit monitorization.

\section{MP3-12}

The "Heart-and-Brain Interaction" in Hypoplastic Left Heart Syndrome - The Impact of Hemodynamics on Neurodevelopmental Outcome before Fontan Procedure

Knirsch W. (1,2), Heye K.N. (1,2,3), Tuura R. (1,4), Beck I. (3), Dave H. (1,5), Wetterling K. (6), Akintürk H. (7); Schranz D. (7), Hahn A. (8), Jux C. (7), Kretschmar O. (1,2), Latal B. (1,3), Reich B. (7) Children's Research Center (1), Pediatric Cardiology, Pediatric Heart Center (2), Child Development Center (3), Diagnostic Imaging, MRCenter (4), Congenital Cardiovascular Surgery, Pediatric Heart Center (5), University Children's Hospital, Zurich, Switzerland; Child Development Center, Frankfurt/Main, Germany (6); Pediatric Heart Center (7), Pediatric Neurology (8), University Hospital Giessen, Justus-Liebig-University, Giessen, Germany

Objectives: The long-term impact of altered hemodynamics after stage II in children with hypoplastic left heart syndrome (HLHS) on cerebral growth and neurodevelopmental outcome is unknown. Elevated vascular pressures before Fontan procedure may be associated with smaller brain volumes and poorer neurodevelopmental outcome. Methods: Semi-automated segmentation of cerebral MRI scans and neurodevelopmental assessment with Bayley-ScalesIII (BS) and neurological examination were conducted in 29 children with HLHS (18 male, 25 Hybrid) before Fontan procedure at mean age of $27.4 \pm 4.1$ months, and in 8 controls. Hemodynamic measures before Fontan were assessed with cardiac catheterization. Results: Superior caval vein (SVC) pressure and atrial pressure were median (range) 10 (5-18) and 6 (4-14) $\mathrm{mmHg}$, respectively. BS were favourable with mean $( \pm \mathrm{SD})$ Cognitive Composite Scale (CCS) $97 \pm 12$, Language Composite Score (LCS) $96 \pm 14$, and Motor Composite Scale (MCS) $96 \pm 16$. Brain volumes were significantly reduced compared to controls, while cerebrospinal fluid (CSF) volumes were elevated. White and deep gray matter volumes were significantly lower after Norwood procedure. (Table 1). Atrial pressure correlated with reduced brain volumes (total brain volume (gray, deep grey and white matter) $r=-0.46, p=0.012$, white matter 
Table 1. Data are given as $\mathrm{n}(\%)$, mean and SD or median and interquartile-range (Q1, Q3) P-values by Kruskal Wallis and post hoc Mann-Whitney $U$ test.

\begin{tabular}{|c|c|c|c|}
\hline & Hybrid $(n=24)$ & $\begin{array}{l}\text { Norwood } \\
(\mathbf{n}=5)\end{array}$ & Control $(n=8)$ \\
\hline Age at MRI [months] & $27.6 \pm 4.3$ & $26.4 \pm 3.3$ & $29.7 \pm 9.5$ \\
\hline $\begin{array}{l}\text { Total grey matter } \\
\text { volume }[\mathrm{ml}]\end{array}$ & $622.6 \pm 60.7$ & $577.6 \pm 42.1$ & $685.2 \pm 86.2$ \\
\hline $\begin{array}{l}\text { Subcortical grey matter } \\
\text { volume }[\mathrm{ml}]\end{array}$ & $44.4 \pm 3.9$ & $38.4 \pm 4.1$ & $49.6 \pm 6.7$ \\
\hline $\begin{array}{l}\text { White matter volume } \\
{[\mathrm{ml}]}\end{array}$ & $284.9 \pm 30.8$ & $255.2 \pm 18.6$ & $331.1 \pm 64.3$ \\
\hline \multirow{2}{*}{$\begin{array}{l}\text { Cerebrospinal fluid } \\
\text { volume [ml] }\end{array}$} & $17.6(13.1,22.5)$ & $17.9(13.1,23.6)$ & $11.9(9.5,13.8)$ \\
\hline & $\begin{array}{l}\text { P-value Hybrid vs. } \\
\text { Norwood }\end{array}$ & $\begin{array}{l}\text { P-value Hybrid } \\
\text { vs. Control }\end{array}$ & $\begin{array}{l}\text { P-value Norwood } \\
\text { vs. Control }\end{array}$ \\
\hline Age at MRI [months] & 0.63 & 0.45 & 0.28 \\
\hline $\begin{array}{l}\text { Total grey matter } \\
\text { volume }[\mathrm{ml}]\end{array}$ & 0.101 & 0.051 & 0.030 \\
\hline $\begin{array}{l}\text { Subcortical grey matter } \\
\text { volume }[\mathrm{ml}]\end{array}$ & 0.005 & 0.051 & 0.019 \\
\hline $\begin{array}{l}\text { White matter volume } \\
{[\mathrm{ml}]}\end{array}$ & 0.032 & 0.041 & 0.045 \\
\hline $\begin{array}{l}\text { Cerebrospinal fluid } \\
\text { volume [ml] }\end{array}$ & 0.933 & 0.009 & 0.045 \\
\hline
\end{tabular}
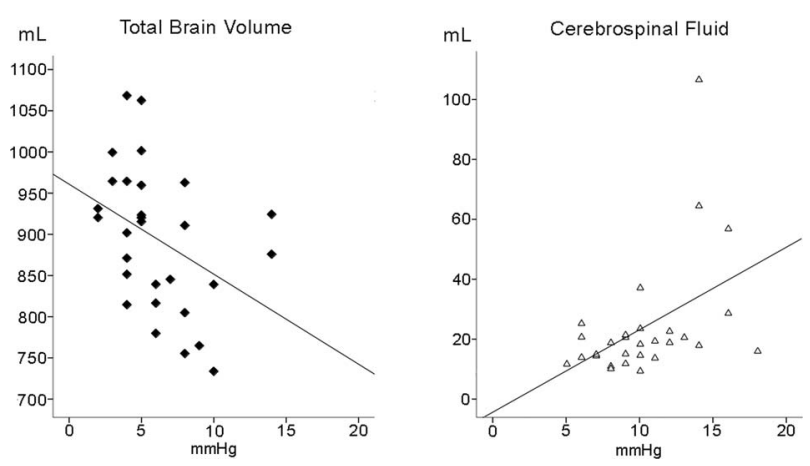

Figure.

$r=-0.47, p=0.010$; gray matter $r=-0.46, p=0.013$ ), while SVC pressure did not correlate with brain volumes, but with increased CSF volume $(r=0.45, p=0.014)$. The later finding correlated neurodevelopmental outcome for all BS with CCS $r=-0.40$ $(p=0.028), \operatorname{LCS} r=-0.59(p=0.003)$, MCS $r=-0.51(p=0.004)$. SVC pressure greater than $12.5 \mathrm{mmHg}$ was associated with poorer performance for all BS (CCS $p=0.025$, LCS $p=0.001$, MCS $\mathrm{p}=0.012$ ).

Conclusions: Impaired hemodynamics before Fontan procedure in HLHS patients may affect the "Heart-and-Brain" interaction: On one hand, cerebral growth (total, white, gray matter) may be affected by impaired myocardial performance (determined by increased atrial pressures) resulting in an impaired somatic and cerebral perfusion. On the other hand, impaired cerebral venous downstream (determined by increased SVC pressure) results in increased CSF volume and impaired neurodevelopmental outcome. Future studies have to evaluate other potentially contributing factors such as CSF flow dynamics, altered aortic arch vascular compliance after Norwood surgery, use of systemic medication reducing systemic and pulmonary vascular resistance for a better understanding of this "Heart-and-Brain" interaction.
MP4-1

Profile of resistance to IVIG treatment in patients with Kawasaki disease and concomitant infection

Poupart S. (1), Dionne A. (1,2), Le C.K. (3), Meloche-Dumas L. (1), Turgeon J. (4), Autmizguine J. (5), Dahdah N. (1)

Division of Pediatric Cardiology, CHU Ste-Justine, Montreal, Canada (1); Division of Pediatric Cardiology, Boston Children's Hospital, Boston, United States (2); Department of Family Medicine, St-Mary's Hospital, McGill University, Montreal, Canada (3); Division of Pediatrics, CHU Ste-Justine, Montreal, Canada (4); Division of Pediatric Infectious Disease, CHU Ste-Justine, Montreal, Canada (5)

Introduction (or Basis or Objectives): Kawasaki disease (KD) is a pediatric systemic vasculitis that can be associated with concomitant viral or bacterial infections. Patients with persistent or recurrent fever 36 hours after the end of intravenous immunoglobulin (IVIG) are considered to be resistant to treatment and are at increased risk for coronary complications. However, it is unknown how concomitant infection influences the response to IVIG treatment. The aim of this study was to determine the impact of concurrent infection on the prevalence of IVIG resistance and coronary outcome.

Methods: Retrospective study of 154 children (mean age at diagnosis: $3.4 \pm 2.8$ years) diagnosed with KD, between 2008 and 2016 in a tertiary pediatric university hospital, of which $59(38 \%)$ had concomitant infection.

Results: Delay in diagnosis ( $>10$ days of fever) was similar between patients with and without concomitant infection (7\% vs $7 \%$, $\mathrm{p}=0.89)$. Patients with concomitant infection were more likely to have fever 48 hours after initial treatment ( $36 \%$ vs $20 \%, p=0.05$ ) and to be treated with a second dose of IVIG (33\% vs $18 \%$, $\mathrm{p}=0.04)$. Patients with infection had higher C-reactive protein at the time of diagnosis (148 vs $112 \mathrm{mg} / \mathrm{L}, \mathrm{p}=0.04$ ), which persisted after IVIG administration ( $111 \mathrm{vs} 59 \mathrm{mg} / \mathrm{L}$ at 48 hours, $\mathrm{p}=0.003$ ). However, there was no statistically significant difference in the prevalence of coronary artery (CA) complications (coronary artery Z-score $>2.5$ ) between patients with and without concomitant infection $(36 \%$ vs $39 \%, \mathrm{p}=0.68)$. Conclusions: Children with KD and concomitant infection are more likely to have persistent fever and elevated inflammatory markers after treatment requiring a second dose of IVIG. Nevertheless, this is not associated with an increased risk of CA complications. Larger scale studies are needed to help distinguish IVIG resistance from infection in children with persistent fever and guide management of this population.
A

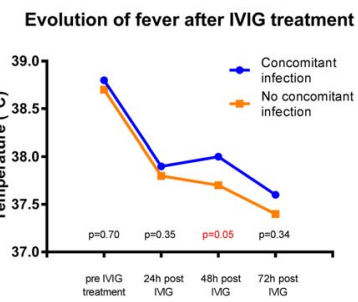

B

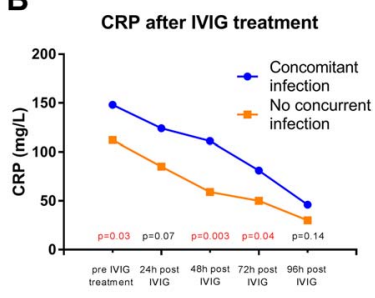

Figure.

MP4-2

Normal values for left and right ventricular speckletracking systolic strain in healthy Italian children Cantinotti M. (1), Franchi E. (1), Assanta N. (1), Viacava C. (1), Koestenberger M. (3), Santoro G. (1).

Fondazione CNR-Regionae toscana G. Monasterio, Massa and Pisa, Italy (1) Institute of Clinical Physiology, CNR, Pisa, Italy, (2); Division of Pediatric Cardiology, Department of Pediatrics, Medical University Graz, Austria (3) 
Background: There is an increasing interest in echocardiographic strain measurements for the assessment of ventricular myocardial function in children, however pediatric nomograms remain limited. Nomograms for the left ventricle (LV) strain values are all numerically limited (with all the studies inferior to 300 subjects and many less than 80 subjects), while data for the right ventricle $(\mathrm{RV})$ are very limited (all less than to 50 subjects) and heterogenous. Our aim was to establish pediatric nomograms LV and the RV strain measured by two-dimensional speckle tracking echocardiography (2D-STE) in a wide cohort of healthy children prospectively enrolled.

Methods: Echocardiographic measurements included: STE LV longitudinal and circumferential and RV longitudinal global endsystolic strain. Age/weight/height/heart rate (HR), and body surface area (BSA) were used as independent variables in different analyses to predict the mean values of each measurement. Echocardiograms were performed by Philips iE33 systems (Philips, Bothell, USA) and offline measurements on Philips Qlab 9.

Results: In all, 723 subjects (age 31 days-17 years; $47.5 \%$ female) were studied. Feasibility varied from $97.0 \%$ for LV global longitudinal strain (GLS), to $87.0 \%$ for RV GLS, up to $71.6 \%$ for LV global circumferential strain. Low coefficients of determination (R 2) were noted among all of the strain parameters evaluated and adjusted for age/weight/height/BSA/HR (i.e. R 2 all $<0.10$, range $0.02-0.086$ ). This hampered the possibility to perform $z$ scores with a sufficient reliability. In fact the $\mathrm{z}$ scores by using similar R2, generated a difference of more than 20 points among lower and upper limits of normality. We also evaluated the possibility to perform percentiles, but they suffered for the same limitations. Thus, we limited to present data as mean values (plus or minus SD) stratified for age groups (Figure 1).

Age variation of strain values were limited. LV longitudinal strain values decreased with age ( $\mathrm{p}$ inferior to 0.001 ), while no significant age-related variations were noted for RV longitudinal strain. Furthermore, no significant effects were found for most measurements.

Conclusions: We calculated echocardiographic normal values for 2D STE for the LV and RV strain in the greatest cohort of healthy children reported so far, by using vendor specific software. Our results tend to confirm previous observations showing only limited variations of strain parameters with age and gender.

\begin{tabular}{|c|c|c|c|c|c|c|}
\hline Measurements & $\begin{array}{l}31 \text { days- } 24 \\
\text { months (1) }\end{array}$ & $\begin{array}{c}2-5 \\
\text { vears (2) }\end{array}$ & $\begin{array}{c}5-11 \\
\text { vears (3) }\end{array}$ & \begin{tabular}{|c|}
$11-18$ \\
years (4)
\end{tabular} & $\mathbf{p}$ & post hoc* \\
\hline \multicolumn{7}{|l|}{ Left ventricle } \\
\hline Longitudinal Strain $4 \mathrm{C}$ & $26.1 \pm 2.4$ & $25.7 \pm 2.6$ & $25.1 \pm 2.6$ & $24.8 \pm 2.4$ & $<0.001$ & 1 vs 3,$4 ; 2$ vs 4 \\
\hline Longitudinal Strain 2c & $26.7 \pm 2.8$ & $25.6 \pm 2.7$ & $25.4 \pm 2.7$ & $24.3 \pm 2.8$ & $<0.001$ & 1 vs $2,3,4 ; 2,3$ vs \\
\hline Longitudinal Strain $3 c$ & $25.4 \pm 3.3$ & $24.1 \pm 3.1$ & $23.8 \pm 2.9$ & $23.2+2.9$ & $<0.001$ & 1 vs $2,3,4$ \\
\hline Global Longitudinal Strain & $26.0 \pm 2.3$ & $25.0 \pm 2.2$ & $24.7 \pm 2.3$ & $24.0 \pm 2.3$ & $<0.001$ & 1 vs $2,3,4 ; 2,3$ vs \\
\hline Circumferential strain basal & $22.1 \pm 4.8$ & $21.3 \pm 4.4$ & $22.0 \pm 4.6$ & $22.6 \pm 4.2$ & 0.243 & - \\
\hline Circumferential strain medial & $23.4 \pm 6.3$ & $23.5 \pm 4.7$ & $24.8 \pm 4.8$ & $25.9 \pm 4.8$ & 0.003 & 2 vs 4 \\
\hline Circumferential strain apical & $28.0 \pm 8.5$ & $25.7 \pm 5.9$ & $26.9 \pm 6.7$ & $27.5 \pm 5.7$ & 0.238 & -.. \\
\hline Global Circumferential strain & $24.6 \pm 4.2$ & $23.3 \pm 4.3$ & $24.5 \pm 4.5$ & $25.4 \pm 4.2$ & 0.020 & 2 vs 4 \\
\hline \multicolumn{7}{|l|}{ Right ve } \\
\hline Global long itudinal strain & $25.4 \pm 3.9$ & $25.9 \pm 4.0$ & $25.8 \pm 4.7$ & $25.0 \pm 4.1$ & 0.305 & -- \\
\hline
\end{tabular}

Figure.

\section{MP4-4}

Fontan-associated liver disease in patients after Fontan palliation

Schleiger A. (1), Salzmann M. (1), Kramer P. (1), Schmitt K.B. (1), Miera O. (1), Bassir C. (2), Müller H.P. (3), Berger F. (1), Ovroutski S. (1) Department of Congenital Heart Disease- Pediatric Cardiology (1); Department of Pediatric Radiology, Charité- Universitätsmedizin Berlin (2); Department of Hepatology and Gastroenterology, CharitéUniversitätsmedizin Berlin (3)
Objectives: Despite improved long-term survival Fontan palliated patients are prone to the development of Fontan-associated liver disease (FALD) including liver fibrosis, liver cirrhosis and hepatocellular carcinoma. In this study we aimed to develop a diagnostic algorithm for early detection of FALD and identify patients at risk. Methods: In the last 30 years 350 patients received Fontan palliation for single ventricle anatomy in our institution. To identify patients at risk for FALD we extended our routinely performed follow-up program with a detailed hepatic assessment: In the last 12 months 53 patients of the total cohort (median age 19.4 years [7; 49]) underwent a detailed age-adjusted liver examination including laboratory analysis, liver ultrasound $(\mathrm{n}=24)$, transient elastography $(n=30)$, invasive measurement of Fontan pressure $(n=29)$ and liver vein wedge pressure $(n=10)$. In 12 patients a biomarker test (Fibrotest) was performed to estimate the level of liver fibrosis.

Results: Liver ultrasound revealed hepatic parenchymal changes in 18 of 24 patients. Most common findings were heterogeneous parenchymal echotexture, segmental atrophy/hypertrophy or destroyed intrahepatic vascular architecture. In 6 of 24 patients (median age 33.7 years $[19 ; 47]$ ) severe liver cirrhosis was detectable. The presence of hepatic parenchymal changes was associated with the duration of the Fontan circulation (median duration13.3 years $[1 ; 25] ; p=0.04)$ and elevated liver vein wedge pressure $(p=0.05)$. Fontan duration also showed a strong correlation with hepatic stiffness measured by transient elastography $(p=0.003)$, the liver enzymes aspartat aminotransferase $(p=0.034)$ and $\gamma$-glutamyl transferase $(p=0.011)$ and the presence of hepatomegaly $(p=0.001)$. Fibrotest correlated with transient elastography $(p=0.019)$, but not with the duration of the Fontan circulation $(p=0.672)$. Fontan pressure was significantly higher in patients with a failing Fontan circulation $(p=0.001)$ and correlated with liver stiffness measured by transient elastography $(p=0.024)$.

Conclusions: In $75 \%$ of Fontan patients FALD was present. Liver damage detected by ultrasound, laboratory analysis and transient elastography strongly correlated with the duration of the Fontan circulation. A detailed hepatic assessment is indispensable for a long-term follow-up program for Fontan palliated patients.

\section{MP4-5}

Atrial volume and function in univentricular patients after Fontan palliation

Burkhardt B.E.U. (1,2), Michael J.T. (2), Tran A. (2), Tandon A. (2), Hussain T. (2)

Pediatric Heart Center, University Children's Hospital Zurich, Switzerland (1); Department of Pediatrics, University of Texas Southwestern Medical Center, Dallas, Texas, United States of America (2)

Introduction: Left atrial volume can be derived from cardiac magnetic resonance (CMR) cine images. Unlike biplane area-length (AL) calculations, the disc summation (DS) method does not require a specific atrial geometry. We hypothesized that both atria are deformed in patients with functionally univentricular hearts and area-length calculations are inaccurate even for that atrium which corresponds to the functional ventricle, and that atrial emptying may be impaired in Fontan patients.

Methods: Fontan patients and controls with normal cardiac anatomy were prospectively imaged by CMR. Right and left atrial areas and lengths as well as volumes from short axis stacks were obtained at atrial end-diastole, before atrial contraction, and at atrial end-systole. Volumes were indexed to body surface area. Atrial volumes by AL and DS and emptying fractions were compared between Fontan and normal groups, as well as within Fontan patients by ventricular morphology and type of cavopulmonary anastomosis. 
Results: 29 Fontan patients with sinus/atrial rhythm during CMR (age $16.5 \pm 4$ years, $83 \%$ male) and 19 controls (age 14.5 \pm 3.5 years, $37 \%$ male) were included (age $\mathrm{p}=0.91$ ).

Left atrial AL mildly overestimated end-diastolic DS measurements in Fontans $\left(29.2 \pm 14.2 \mathrm{ml} / \mathrm{m}^{2}\right.$ vs. $25.1 \pm 10.2 \mathrm{ml} / \mathrm{m}^{2} ; 95 \%-$ CI of the difference $0.01-8.91 \mathrm{ml} / \mathrm{m}^{2}$ ), but not in controls $\left(40 \pm 7.8 \mathrm{ml} / \mathrm{m}^{2}\right.$ vs. $\left.37 \pm 7.4 \mathrm{ml} / \mathrm{m} 2 ; 95 \%-C I-0.41-6.25 \mathrm{ml} / \mathrm{m}^{2}\right)$. Fontan patients with functional right ventricles had larger right atria at atrial end-diastole than those with functional left ventricles by both methods (AL: $33.8 \pm 18 \mathrm{ml} / \mathrm{m}^{2}$ vs. $7.9 \pm 4.2 \mathrm{ml} / \mathrm{m}^{2}$; $\mathrm{p}=0.001)$ and smaller left atria by $\mathrm{AL}\left(22.3 \pm 8.6 \mathrm{ml} / \mathrm{m}^{2}\right.$ vs. $36.6 \pm 15.5 \mathrm{ml} / \mathrm{m}^{2} ; \mathrm{p}=0.044$. Not by DS: $\left.\mathrm{p}=0.573\right)$. In Fontan patients, ejection fractions of both atria combined did not differ by ventricular morphology or type of cavopulmonary anastomosis. Fontans showed lower atrial active emptying fractions than controls (left: $15.3 \pm 9.5 \%$ vs. $32.3 \pm 7.6 \% ; \mathrm{p}<0.001$; right: $16.8 \pm 11.6 \%$ vs. $27.2 \pm 10.3 \% ; \mathrm{p}=0.03)$ and lower total emptying fractions (left: $33.1 \pm 15.6 \%$ vs. $61.7 \pm 7.2 \%$; $<<0.001$; right: $34.6 \pm 12.3 \%$ vs. $52.7 \pm 7.5 \% ; \mathrm{p}<0.001)$.

Conclusions: Atrial volumes should be measured by DS in Fontan patients, rather than by calculations based on geometrical assumptions. Atrial volume distribution in Fontan patients depends on ventricular morphology. Biatrial active and total emptying fractions are lower in Fontans compared to controls.

\section{MP4-6}

4D flow magnetic resonance postoperative pulmonary stenosis assessment in patients with transposition of the great arteries repaired by arterial switch

Belhadjer Z. (1), Soulat G. (1,2), Azarine A. (2), Legendre A. (1,2), Ladouceur M. (1,2,3), Iserin L. (2), Bonnet D. (1,3), Mousseaux E. (1,2) Paris Descartes University, Paris, France (1); European Hospital Georges Pompidou, APHP, Paris, France (2);Necker Enfant Malades hospital, APHP, Paris, France (3)

Background: Right ventricular outflow tract obstruction (RVOTO) is a common complication in adult patients who had arterial switch (ASO) for transposition of the great arteries (TGA). Reoperation for RVOTO is recommended in symptomatic patients with right ventricular systolic pressure greater than $60 \mathrm{mmHg}$ (velocity of tricuspid regurgitation (IT) $>3.5 \mathrm{~m} / \mathrm{sec}$ ). However, pulmonary flow measurement with transthoracic echocardiography Doppler (TTE) is hardly reliable.

$4 \mathrm{D}$ flow in magnetic resonance is a new imaging method, allowing analysis of blood velocity and flow in an entire volume, permitting detection, quantification and location of vascular stenosis.

Objective: to compare this 4D flow imaging to TTE for the diagnosis of pulmonary stenosis in adult patients after ASO for TGA. Methods and results: 33 patients (19 men, 14 women, mean age 25.5 years old) were prospectively included with a TTE and a MRI 4D flow examination on the same day.

In 16 patients (48.5\%), RVOTO was not correctly evaluated by TTE vs. in $0(0 \%)$ with 4 D flow. TTE detected $11(33.3 \%)$ patients with mild RVOTO (max; velocity $2 \mathrm{~m} / \mathrm{s}$ ) and $2(6 \%)$ patients with moderate RVOTO (max; velocity $3.5 \mathrm{~m} / \mathrm{s}$ ) vs. $14(42 \%)$ and $4(12.1 \%)$ respectively with $4 \mathrm{D}$ flow. The peak flow velocities measurements in Doppler and 4D flow were highly correlated $(\mathrm{r}=0.79 ; \mathrm{p}<0.0001)$.

In addition, peak velocity and the right ventricle mass index correlated in 4D flow better than with TTE $(r=0.74 ; p<0.001$ vs. $r=0.55 ; p=0.02$ )

$4 \mathrm{D}$ flow was also more precise to locate the site of RVOTO on the distal pulmonary trunk in 19 patients and on the pulmonary artery branches in 14 patients.
Conclusion: 4D flow MRI is a reliable tool for assessment of RVOTO after the ASO for TGA in adult patients and should potentially be part of routine evaluation in the monitoring of these patients.

\author{
MP4-7 \\ Long-term follow-up of congenital aortic valvular stenosis \\ by echocardiography \\ Eroglu A.G., Atik S.U., Cinar B., Bakar M. T., Saltik I.L. \\ Istanbul University, Cerrahpasa Medical Faculty, Istanbul, Turkey
}

Introduction: In this study, we evaluated the natural history and the factors effecting the progression of aortic valvular stenosis (AVS) and aortic regurgitation (AR) in 388 patients during long term follow-up by echocardiography.

Methods: Very mild aortic stenosis was defined as a transvalvular Doppler gradient less than $25 \mathrm{mmHg}$, mild stenosis as $25-49 \mathrm{mmHg}$, moderate stenosis as $50-75 \mathrm{mmHg}$, and severe stenosis as more than $75 \mathrm{mmHg}$.

Results: The patients were followed from 1 month to 20.6 years. The degree of AVS was very mild in 138 (35.6\%), mild in 133 $(34.3 \%)$, moderate in $68(17.5 \%)$ and severe in $49(12.6 \%)$ patients, at the time of initial echocardiographic examination. Morphologic characteristic of the aortic valve was unicuspid in $4(1 \%)$, bicuspid in $249(64 \%)$ and tricuspid in $135(34 \%)$ patients. Kaplan-Maier analysis demonstrated that, the patients with very mild and mild AVS are more likely to survive without AVS deterioration then the patients with moderate AVS (13.6 years, CI 12-15.1; 15.3 years, CI 14-16.7; 9.8years, CI 8.2-11.5, respectively). Of 388 patients, 148 (38.1\%) had AR (50 trivial, 74 mild, 20 moderate, and 4 severe) at initial echocardiographic examination and there was no AR in $240(61.9 \%)$ patients. No AR developed in 158 $(65.8 \%)$ of these 240 patients. In $82(34.2 \%)$ AR developed (40 (16.7\%) trivial, 32 (13.3\% mild, and 10 (4.2\%) moderate) after a median 3.2 years, (range 1 month to 16 years) follow-up. During follow up of 148 patients who had AR at initial echocardiography, AR did not deteriorated in 102 (68.9\%) patients and the degree of AR progressed in $46(31.1 \%)$ during a median 4 years (range: 1 month to 14.2 years) follow up. The bicuspid valve morphology increased the risk of AR development/deterioration after adjustment for sex (OR 1.73, CI 1-2.7, p=0.022).

Conclussion: Patients with very mild stenosis may be followed with a noninvasive approach every 1 or 2 years, and an annual followup is suggested for patients with mild stenosis. We recommend that patients with moderate stenosis undergo noninvasive evaluation every 6 months.

\author{
MP4-8 \\ Cardiac Rhabdomyomas, Association With Tuberous \\ Sclerosis Complex and Everolimus Treatment: Single \\ Center Experience \\ Aypar E., Kurucu N., Varan A., Aydın G.B., Yalçn B., Konuşkan B., \\ Anlar B., Ertugrul I., Aykan H.H., Karagöz T., Alehan D, Kutluk T., \\ Akyüz C. \\ Hacettepe University Children's Hospital, Ankara, Turkey
}

Introduction: Cardiac rhabdomyoma (CR) is the most common pediatric heart tumor,have a natural history of spontaneous regression, surgery is only necessary when hemodynamically significant obstruction is present.Rhabdomyomas are closely associated with tuberous sclerosis complex (TSC), an autosomal dominant neurocutaneous disorder where TSC mutation results in abnormal cellular proliferation and differentiation. Recent reports 
on everolimus (mammalian target of rapamycin (mTOR) inhibitor), have shown favorable results for treatment of CRs. We aimed to evaluate clinical presentation, outcome of patients diagnosed as CRs, association with TSC, present our results with everolimus treatment.

Methods: Medical data of children diagnosed as CRs between 2013-2017 were retrospectively reviewed.

Results: 30 patients (median age 7 months (1 day-16 years 8 months), 13 male, 17 female) were diagnosed as CR. 13 patients $(43 \%)$ were diagnosed prenatally.21 patients (\%70) had multiple CRs, 9 patients (30\%) had solitary CR by echocardiography. During the follow-up, 21 patients (70\%) had TSC and epilepsy. Rhabdomyomas were complicated by arrythmias (7 patients, $23 \%)$, left (4 patients, 13\%) and right (1patient, 3\%) ventricular inflow obstruction, left (5 patients, 17\%) and right (1 patient, $3 \%)$ ventricular outflow tract obstruction. 4 patients had ventricular tachycardia, one patient required implantable cardiac defibrillator, 1 patient had supraventricular tachycardia. $7 / 30$ patients (23\%), median age 18 days (7 days-12 years) were given everolimus (optimal dose, $0.25 \mathrm{mg}$, twice per day, 2 days per week). Indications for everolimus were inoperable outflow tract obstruction in 4 patients, inflow obstruction in 3 patients. Everolimus were effective (reduced tumor size by at least $50 \%$, decreased tumor numbers) in $6 / 7$ patients $(86 \%)$. Surgery was performed in 3 patients (10\%). Follow period was median 14 months.

Conclusions: In our series, multiple CRs were more closely associated (95\%) with TSC than previously reported in literature (75\%). Although CRs are benign in nature, 18/30 (60\%) of our patients had complications.Patients with multiple CRs must be under careful follow-up for development of TSC, renal angiolipomas,subependymal astrocytomas. To our knowledge, this is the largest series reported so far with everolimus in treatment of CRs. Rapid tumor regression was observed in $86 \%$ of patients even in one month which were unlikely from a spontaneous regression. Everolimus and other mTOR inhibitors may be possible novel therapies for patients with hemodynamically significant CRs.

\section{MP4-9}

The Use of Speckle Tracking Echocardiography to Assess Myocardial Performance in Monochorionic Diamniotic Twins with and without Twin to Twin Transfusion Syndrome

Breatnach C.R. (2), Bussman N. (1), Vincent D.F. (1), James A.T. (3), Lynch A. (2), Malone F. (1), McCallion N. (1), Franklin O. (2), El-Khuffash A. (1).

The Rotunda Hospital, Dublin, Ireland (1); Our Lady's Children's Hospital, Dublin, Ireland (2); The Hospital for Sick Children, Toronto, Canada (3)

Introduction: Data on myocardial performance in monochorionic diamniotic (MCDA) twins during the early neonatal period is lacking. These infants are at risk of developing twin to twin transfusion syndrome (TTTS). We aimed to assess myocardial function using speckle tracking echocardiography (STE) in MCDA twins with and without TTTS. We hypothesise that infants exposed to TTTS would exhibit lower values for strain and strain rate measured using (STE) during the early neonatal period. Methods: We performed a prospective observational study of 4 twin groups: Uncomplicated MCDA, MCDA twins with selective IUGR, MCDA with TTTS in receipt of SLPCV (MCDA \& LASER) and MCDA twins with TTTS not receiving SLPCV (MCDA no LASER). Serial echocardiography was performed on day one, day two and between days $5-7$ of life. Assessment of myocardial performance included the use of STE.
Results: Forty seven twin pairs were enrolled in the study: 21 uncomplicated MCDA; 14 selective IUGR; 6 TTTS no LASER, and 6 TTTS \& LASER. Recipient TTTS no LASER infants had lower LV and RV strain (which persisted throughout the first week. Function measurements in the TTTS no LASER donor group were significantly higher than the recipient counterparts.

Conclusion: This is the first study using STE to highlight the poor myocardial performance in MCDA twins exposed to TTTS who do not undergo SLPCV. This highlights the need for close monitoring of their haemodynamic status during the early neonatal period. Further study is warranted to explore this condition further.

\author{
MP4-10 \\ Aortic angle predicts neo-aortic root dilatation and \\ regurgitation following arterial switch operation \\ Martins D. (1,2), Legendre A. (1), Boddaert N. (1), Raisky O. (1), \\ Bonnet D. (1), Raimondi F. (1) \\ (1) Hôpital Necker Enfants Malades, APHP. Paris, France; (2) Hospital \\ de Santa Cruz, CHLO. Lisbon, Portugal
}

Introduction: Neo-aortic root dilatation and regurgitation are common progressive long-term complications of the arterial switch operation (ASO) for transposition of the great arteries (TGA) with increasing clinical burden. While several risk factors have been identified, most are constitutional. The acute aortic angle commonly seen after ASO might alter aortic dynamics and facilitate progression of the neo-aortic root dilatation and aortic regurgitation, but insufficient data is available.

Methods: Retrospective analysis of TGA patients undergoing CMR after ASO at a single tertiary center from November 2010 to July 2017.

Results: 180 patients were analyzed, 157 of which having adequate imaging of the aortic arch and root. Neo-aortic root Z score was normally distributed with $73 \%$ of patients having a $Z$ score $>2$. The aortic angle had a significant $(p<0,001)$ inverse relationship with the neo-aortic root $\mathrm{Z}$ score both in univariate and multivariate linear regression. Other significant predictors were male gender and the concomitant presence of a VSD or a dysplastic neo-aortic valve. The presence of neo-aortic regurgitation was also inversely correlated with the aortic angle in both univariate and multivariate logistic regression, with other significant predictors being the presence of a VSD or a bicuspid neo-aortic valve. Conclusions: Acute aortic angles predict more extensive neo-aortic root dilatation and higher incidence of regurgitation. We believe a surgical technique promoting less acute aortic angles has potential for ameliorating the long-term outcomes of TGA.

\author{
MP4-11 \\ The use of 3D Printing in the Evaluation of Complex \\ Congenital Heart Disease (CHD): Registry data from the \\ European Congenital Heart Surgeons Association \\ (ECHSA) \\ Protopapas E. (1,2), Hakim N. (1,2), Zografos P. (1,2), \\ Vazaios C. (5), Bilalis L. (3), Zavaropoulos P. $(1,2)$, \\ Alexopoulos C. (4), Matej N. (5), Polimenakos A. (6), Sarris G. (1,2) \\ Athens Heart Surgery Institute (1); Department of Congenital Cardiac \\ Surgery at Iaso Children's Hospital (2); 3D-Life (3); Pediatric Cardiac \\ Intensive Care Unit at Iaso Children's Hospital (4), Athens, Greece; \\ Childrens Heart Centre (5) Bratislava, Slovakia ; The Children's Hospital \\ of Georgia Heart Center (6) Augusta, USA
}

Introduction: Three-dimensional printing (3D printing) of physical cardiac models is a new tool poised to help surgeons appreciate 
complex cardiac anatomic features and their interrelationships with surrounding tissues, as shown in various published case reports, but, objective evidence of the usefulness of this technology is lacking. We report preliminary analysis of data from an ECHSA multicenter retrospective registry, aiming to establish the usefulness of preoperative 3D Printing models in facilitating clinical decision making, as assessed by each contributing cardiac team. Methods: In each participating center, accurate 3D printed models of the relevant cardiac anatomy were created based on contrastenhanced computer tomography (CT) or magnetic resonance (MR) images in selected cases, as decided by the local cardiac team. The surgeons subsequently evaluated (on a scale 0-3) and reported the usefulness of the 3D printed models in 4 categories: a) Family education and counseling, b) Surgical or percutaneous interventional planning, c) Surgical teaching or simulation, and d) Evaluation of surgical/interventional result.

Results: From June of 2014 till December of 2017, 39 patients with anatomically challenging congenital heart defects, whose preoperative assessment included creation of 3D printed cardiovascular models, were enrolled from 3 different cardiac centers. Issues explored included feasibility of biventricular repair in complex $\operatorname{DORV}(\mathrm{n}=6)$, approach to coronary anomalies (fistula $(\mathrm{n}=1)$, AAOCA $(n=2))$, technical demands of complex aortic $(n=4)$ or pulmonary $(n=3)$ reoperations, and PA-VSD-MAPCAS $(n=5)$, technical demands of complex aortic operations $(n=8)$, TGA's $(n=3)$, single ventricle $(n=1)$, PAPVC $(n=1)$, Truncus $(n=2)$, TOF $(n=2)$, ASD $(n=1)$. Each patient's care team retrospectively evaluated the usefulness of the model. In all cases, the 3D model facilitated diagnostic understanding (median score 3), surgical planning and patient/family education (median score 2), but in only a few cases $(n=7)$ was it used for surgical teaching or simulation.

Conclusions: This study provides preliminary initial evidence favoring the clinical usefulness of $3 \mathrm{D}$ printed models in the management of complex CHD. Printing of 3D cardiac models appears to be a useful tool for the diagnostic assessment and preoperative preparations for patients with CHD's, as judged by the user physician teams. Enlargement of the database and incorporation of more objective assessment methods is warranted and is in evolution.

\section{MP4-12}

Echocardiographic predictors of unfavourable outcome in children with hypertrophic cardiomyopathy

Ziolkowska L., Boruc A., Kowalczyk-Domagala M., Turska-Kmiec A., Paszkowska A., Brzezinska-Rajszys G.

Department of Pediatric Cardiology, The Children's Memorial Health Institute, Warsaw, Poland

Background: Echocardiographic parameters-the maximal wall thickness (MWT), left atrial dimension (LAD) and left ventricular outflow tract gradient (LVOTO) was reported to predict the cardiovascular events in adult patients with hypertrophic cardiomyopathy (HCM). The aim of our study was to evaluate the progression of the disease expressed by these parameters and to determine whether they are predictive of adverse clinical outcome also in the pediatric population with HCM.

Methods: A total of 100 children with HCM aged 0-18 years (mean 9.4), examined since 2002 to 2017 were enrolled and prospectively followed with respect to echocardiography results and to clinical endpoints. All children were classified as group I (gI, reached endpoint) or II (gII, not). The echocardiographic parameters were collected from the first (H1) and last (H2) examination (time of observation 0.2-11.3 years, mean 3.1), and compared between groups I and II. The clinical endpoints were defined as cardiovascular events: sudden cardiac death (SCD), heart failure cardiac death (HFCD), aborted cardiac arrest (CA), appropriate ICD discharges (ICDdx), heart transplantation ( $\mathrm{HTx}$ ).

Results: During a follow-up, mean 7.3 years, 18 (18\%) of children reached the clinical endpoints (gI), while $82(82 \%)$ did not (gII). The following endpoints occurred in gI: SCD $(n=3)$, HFCD $(n=3), \operatorname{CA}(n=2), \operatorname{ICDdx}(n=6), \operatorname{HTx}(n=4)$.

The difference in MWT progression was not significant between the two groups ( $\mathrm{z}$-score $2.9 \pm 4.6$ vs $1.0 \pm 4.7 ; \mathrm{p}=0.11$ ), but significant progression of MWT (mean z-score from $10.8[15.4 \mathrm{~mm}]$ to 13.8 $[19.9 \mathrm{~mm}] ; \mathrm{p}=0.015)$ was observed in gI, while not in gII (mean $z$-score from $9.1[14.9 \mathrm{~mm}]$ to $10.1[17.4 \mathrm{~mm}] ; \mathrm{p}=0.07$ ).

LAD progression was significantly different between groups (z-score $1.1 \pm 3.0$ vs $-1.3 \pm 2.4$ respectively; $p=0.001$ ). LAD remained relatively stable in gI (z-score $4.1[35.4 \mathrm{~mm}]$ to 5.2 $[39.7 \mathrm{~mm}] ; \mathrm{p}=0.156)$ and decreased significantly in gII (z-score 3.6 [35.8 mm] to $2.4[24.8 \mathrm{~mm}] ; \mathrm{p}<0.001)$. The progression of the LAD increased the odds of reaching the endpoint by $3.3(\mathrm{p}=0.04)$.

LVOTO changes were insignificant in both groups, it increased from $21.4 \mathrm{mmHg}$ to $23.5 \mathrm{mmHg}$ and from $18.7 \mathrm{mmHg}$ to $21.7 \mathrm{mmHg}$, respectively.

Conclusions:

(1) Increase of MWT is a significant predictor associated with disease progression and unfavorable clinical outcome in pediatric population with HCM.

(2) LAD progression is the most sensitive indicator of cardiovascular events in children with HCM, therefore should be measured regularly during follow-up.

\section{MPN-1}

Can Nurses master Pediatric Cardiac Auscultation following appropriate Teaching and Practice? Germanakis I. (1), Archontoulaki S. (2), Giusi J. (2), Burianova Bagaki A. (1), Rikos N. (2)

Faculty of Medicine, University of Crete, Heraklion, Greece (1); Nursing Dpt, School of Health and Welfare Services, TEI Crete, Heraklion, Greece (2)

Introduction: Pediatric cardiac auscultation (PCA) proficiency is a key clinical skill, fundamental for the efficiency of any pediatric cardiovascular disease (CVD) screening program and for a costeffective use of pediatric cardiology services.

We aimed to test the hypothesis that non-medical personnel (nurse students) can also become masters of PCA following structured teaching

Methods: Pilot study, including two volunteer nurse students full attending a 3-month teaching program of PCA including attendance of 1) relevant e-learning material (videolectures) 2) interactive, multimedia-based PCA lectures and workshops for medical students, 3) weekly attendance of an academic pediatric cardiology outpatient clinic. Their PCA performance was assessed by 1) both students validating a series of recorded digital phonocardiograms representing innocent and abnormal murmurs, additional sounds (clicks, pericardial friction) and heart tone abnormalities (wide S2 split) 2) one student recording and interpreting digital phonocardiograms of 30 school children participating on a pediatric CVD screening program . Their performance was compared to that of 34 medical students attending the same teaching program (1) and against expert pediatric cardiologist, all validating the same digital phonocardiograms (2).

Results:

1) Both nurse students had $100 \%$ sensitivity $/ 33 \%$ specificity to correctly differentiate abnormal from normal auscultatory 
findings (vs 98\%/65\% average values for medical students). Their auscultation quality scores (composite score of several auscultation variables tested) were 8,7 and 7,8 ( vs 7,78 mean, 7,9 median value for medical students).

2) Comparing the nurse and the expert physician auscultation: Agreement was present in $18 / 30$ validations $(60 \%)$ including normal auscultation $(n=10)$, innocent murmur $(n=6)$, abnormal murmur $(n=1)$, abnormal extra sounds $(n=1)$. Disagreement regarded a) detected only by expert: innocent murmur $(n=4)$, abnormal murmur $(n=1)$, abnormal extra sound $(n=2)$. b) detected only by student: innocent murmur ( $\mathrm{n}=3$, no murmur by expert), abnormal murmur ( $\mathrm{n}=1$, innocent murmur by expert), additional sound ( $\mathrm{n}=2$, abnormal murmur in 1 case by expert).

Conclusions: Pediatric Cardiac Auscultation proficiency can be achieved also by non-physicians, including training nurses, following structured intensive teaching combining e-learning and practical exercise. The role of trained nurses supporting pediatric CVD screening programs, including cardiac auscultation, should be further investigated.

\section{MPN-2}

\section{Coping with new technology - Nursing familiarization with Berlin Heart EXCOR ${ }^{\circledR}$}

Laciakova L., Hadackova I., Krivska D.

Children's Heart Centre, 2nd Faculty of Medicine, Charles University in Prague and Motol University Hospital, Prague, Czech republic

Introduction: Long-term mechanical circulatory support is increasingly used in paediatric patients as bridge to transplant/recovery or candidacy. The aim of the present study was to evaluate the nursing workload imposed on the paediatric cardiac ICU and paediatric cardiology ward during first-time use of a para-corporal left ventricular assist device and to describe the process of familiarization with the system.

Methods: Berlin Heart EXCOR ${ }^{\circledR}$ was used as bridge to transplant in a 19 months old girl with end stage heart failure due to dilated cardiomyopathy until a successful heart transplantation 4 months later. The child spent 124 days in the ICU/cardiology unit while on the device. A questionnaire survey was filled out by the staff nurses $(N=14)$ covering the following topics: familiarization with Berlin Heart $\operatorname{EXCOR}^{\mathbb{B}}$, wound care, physiotherapy and occupational therapy.

Results: The results show how staff nurses were coping with new technology. Newly acquired knowledge was used almost immediately. The results are valuable to focus future education of the nursing staff and further improve nursing care. The process of training nurses for paediatric patients undergoing therapy with the Berlin Heart EXCOR ${ }^{\circledR}$ including photography and video records will be presented.

Conclusions: Long-term mechanical circulatory support by a paracorporal device imposes an additional workload on nursing staff as well as need for training which can be accomplished in a structured and straightforward way.

\section{MPN-3}

CHD Parents Need Questionnaire (CHD-PNQ): development and validation of a protocol study amongst Italian population

Dellafiore F. (1), Flocco S.F. (2), Pittella F. (1), Conte G. (1), Magon A. (1), Giamberti A. (2), Carminati M. (2), Chessa M. (2), Caruso R. (1)

(1) Health Professions Research and Development Unit, IRCCS

Policlinico San Donato, San Donato Milanese (Milan, Italy); (2)

Pediatric and Adult Congenital Heart Disease Center, IRCCS

Policlinico San Donato, San Donato Milanese (Milan, Italy)
Introduction: Parents of Congenital Heart Diseases (CHD) patients play a pivotal role beneath the whole life of their children, both in their cure and growth process. In fact, the CHD parents are extensively involved in care activities, such as accompanying their child to visits, always staying with them for the entire time and administering their medications every day. Moreover, they help as integral members of their child's healthcare team and they give their children support for developing independence and promote their responsibility. Thus, being a parent with those responsibilities is very difficult due to stress, important dilemmas and contradictions. The assessment of those needs could be strategic to establish any shortage in this field. Currently, to the best of our knowledge, there is a lack of information in the literature about experiences and dilemmas of CHD parents, and knowing the needs of CHD parents is important to address a tailored and efficient health-care delivery for the whole family. Therefore, the purpose of this study is to develop and validate a tool (CHD-PNQ), evaluating CHD Parent's Needs, amongst Italian population.

Methods: Three phases methodological study. Phase 1 concerns the drawing up of CHD-PNQ's items, based on literature review. Phase 2 tested content and face validity, aimed to investigate the clearness and the pertinence of the items, when translated in Italian. Content validity was assessed computing content validity ratio (CVR) and index (I-CVIs; S-CVI) from expert panelists' response. Face validity was assessed by the narrative analysis on the answers of three open-ended questions to the expert panelists. Phase 3 involved the data collection from a cross-section of CHD Italian Parents to assess the psychometric proprieties, stability and internal consistency of the CHD-PNQ

Results: This trial has approval from the San Raffaele Hospital (Milan, Italy) Ethics Committee (approval number: 136/INT/ 2017), and it will begin on January 2018, at IRCCS Policlinic San Donato.

Conclusion: The presented protocol study could allow the CHD Parents Need Questionnaire to be developed and validated. Moreover, the evaluation of CHD parents' needs could fill the gap in this area and providing an efficient health-care delivery for the whole family.

\section{MPN-4}

Scrube nurse. Hybrid nurse in the hybrid procedure

Di Iorio F., Pirti C.

Bambino Gesù Children Hospital TaorminaContrada Sirina 98036 Taormina Sicilia, Italy

Introduction: Regionalization is a regulatory approach to rationalization of resource allocation, especially for highly specialized medical services or technologies .For high-risk surgical procedures such as cardiac surgery, regionalization may improve outcomes by consolidating surgical programs and increasing the case volume of surgical centers. Congenital heart surgery is one such highly specialized field. Sicilian Regionalization of a Pediatric Cardiac Department was carried out through a partnership between Regional Health Government and Pediatric Hospital "Bambino Gesù" the Mediterranean Pediatric Cardiology Center is the only cardiologic tertiary care center in Sicily and it serves a population of 6 million inhabitants with about 60000 newborns per year. Method: The hybrid approach has been developed has an alternative strategies for the management hypoplasia left heart syndrome and other forms of complex congenital heart disease. This approach combines both surgical (Pulmonary branch artery banding) and interventional cardiology (stenting of the ductus arteriosus and septic atrial balloon)) techniques and shifts the risk of 
major open-heart surgery and cardiopulmonary bypass to later in infancy (Pediatric Cardiology 2005; 26:190-199) Materials from October 2011 to February 2017, we treat 49 consecutive neonates underwent single stage hybrid procedure for palliation of HLHS and for others specifically cardiac failure.

Results: High level turnover of nurses staff operating in the our department and the high level of this specialty improve the necessity of creating a new nurse role for optimizer human and material resources the "scrub nurse". Scrub nurse is one nurse that have more role, instrumental nurse and hemodynamic nurse, in the same hybrid procedure. We created a specific Job description and a specific map for show position of scrub nurse in the different time, hemodynamic and surgical time, of the hybrid procedure. Our competence are the results of important and specific trial about heart failure, high level know of surgical and hemodynamic treatment for specific congenital cardiac diseases

Conclusion: The clinical results they brought a new concept of work the" team work. The highly complex pediatric patients with congenital heart disease require inter professional teamwork and collaboration to ensure high-quality outcomes with low mortality and morbidity. (Congenital Heart Dis. 2013;8:3-19).

\section{MPN-5 \\ Nursing management in the department of surgical treatment of complex cardiac rhythm disturbances and cardiac pacing \\ Apanovich V., Berzon S., Firsova N. \\ Research Clinical Institute of pediatrics, arrhythmology department, Moscow, Russian Federation}

Background: The range of surgical interventions for the treatment of different types of rhythm disturbances is constantly expanding. Except catheter ablations and pacemaker and ICD implantations we implemented video assisted thoracoscopic (VATS) technology such as sympathectomy and pacemaker implantation in hybrid operation room. Each new method requires correction of the nurse preparation plan and it is important to manage the nursing care in the most efficient way.

Objective: The aim of this work is to describe our basic nurse approach to the preparation of the child for surgical intervention, nurse care during procedure and the algorithm of nurse anesthesia plan.

Methods: We describe the most significant steps which we use for preoperative preparation of the operating room and the preparation of the special surgical instruments and anesthetic equipment. In this topic we focuse on the details of these stages in pediatric patients. We describe the peculiarities of the nursing approach at the main stage of surgical intervention in pediatric patients.

Results: We demonstrated our own approach of nursing in various surgical interventions which we used to cure different heart rhythm disorders including life-threatening arrhythmias in children.

Conclusion: Our approach in preparing for surgery, the features of surgical nursing at the main stage shorten the time of surgical procedure and reduces the risks of postoperative complications.

\section{P-1}

\section{Arrhythmic faces and risk stratifications of hypertrophic cardiomyopathy in children; Single center experience from Turkey}

Ergül Y. (1), Özgür S. (2), Şahin G.T. (2), Kafalı H.C. (2), Şengül F.S. (2), Ayyıldız P. (2), Akıncı O. (3), Güneş M. (4), Haydin S. (4), Güzeltaş A. (2)
Saglik Bilimleri University, Mehmet Akif Ersoy Thoracic and Cardiovascular Surgery Center, Department of Pediatric Cardiology/ Electrophysiology, Istanbul, Turkey (1);Saglik Bilimleri University, Mehmet Akif Ersoy Thoracic and Cardiovascular Surgery Center, Department of Pediatric Cardiology, Istanbul, Turkey (2); Saglik Bilimleri University, Mehmet Akif Ersoy Thoracic and Cardiovascular Surgery Center, Department of Radiology, Istanbul, Turkey (3); Saglik Bilimleri University, Mehmet Akif Ersoy Thoracic and Cardiovascular Surgery Center, Department of Pediatric Cardiac Surgery, Istanbul, Turkey (4)

Background: Hypertrophic cardiomyopathy (HCM) is the most common cause of sudden cardiac death (SCD) in the young adults. Although there are large series about arrhythmic characteristics of HCM in adults, it is limited in childhood. We evaluated pediatric HCM patients followed at our center from different angles.

Patients and Methods: We identified 120 pediatric patients with HCM between January 2010 to August 2017. Demographic characteristics, non invasive and invasive diagnostic tests findings, treatment and follow-up results of the patients were evaluated. Patients with ICD and poor prognosis were scanned backwards and their distinctive and prominent features were searched. The contributor factors, which could help to selection SCD candidate, were tried to be found.

Results: The mean age of patients was $8.2 \pm 5.9$ years $(0.05-21)$ and $64.2 \%$ were males. Electrocardiographically, the most common finding was ventricular preexcitation $(n=12,10 \%)$ except for left ventricular hypertrophy. Echocardiographically, there were concentric hypertrophy in the majority $(51.2 \%)$ and obstruction (LVOTO) in 26 (22\%). Implantable cardioverter-defibrillator (ICD) implantation was performed in 24 patients (14 transvenous, 10 epicardial). Two of them were inserted for secondary prevention; whereas the remaining for primary prevention. ICD dependent complications were seen in 7 (29\%) patients. During follow-up period (mean $23.0 \pm 22.5$ months); 3 (2.5\%) patients has died (2patient with Danon's disease, due to heart failure and uncontrolled VF episodes, 1 patient with Noonan Syndrome in postoperative period) and none of them had an ICD. There was a significant difference between good and poor prognostic groups in terms of QT prolongation, left atrial dimensions, presence of preexcitation, and left ventricular outflow tract obstruction.

Conclusion: HCM is still an important disease, as it is common in the community. In order to avoid both SCD and ICD related complications, the right patient selection for ICD is important. New factors that may contribute to conventional risk factors are being investigated. In our own group, we showed that some of these modifier factors are effective on the prognosis.

\section{P-2}

Alternative Route to Improve Success in Right Sided Supraventricular Tachycardias in Children: Transjugular Approach

Ergül Y. (1), Özgür S. (2), Şahin G.T. (2), Kafalı H.C. (2),

Çelebi S.B. (3), Bay B. (3), Güzeltaş A. (2)

Saglik Bilimleri University, Mehmet Akif Ersoy Thoracic and Cardiovascular Surgery Center, Department of Pediatric Cardiology/ Electrophysiology, Istanbul, Turkey (1); Saglik Bilimleri University, Mehmet Akif Ersoy Thoracic and Cardiovascular Surgery Center, Department of Pediatric Cardiology, Istanbul, Turkey (2); Saglik Bilimleri University, Mehmet Akif Ersoy Thoracic and Cardiovascular Surgery Center, Department of Anesthesiology and Reanimation, Istanbul, Turkey (3)

Background: Catheter ablation via the femoral vein has been widely used in children. However, in certain conditions, an alternative vascular access is required for a successful ablation. Herein we 
reported that, accessory pathways (APs) and ectopic foci which reside right anterior and anterolateral to tricuspid valve orifice can be safely and effectively ablated with transjugular venous approach.

Patients and Methods: Ten procedures performed via the transjugular venous approach were reviewed retrospectively from the 355 electrophysiological procedures performed between March 2016 and November 2017. EnSite 3D electro-anatomic mapping and limited flouroscopy was used in all patients.

Results: The mean age of patients was $15 \pm 2.4$ years (12-18) and $6 / 10(60 \%)$ were males. Six of the patients had previous ablation procedures via the femoral vein approach and due to failure or recurrence the procedures had to be repeated. In these patients mean procedure time shortened from $175 \pm 75$ minutes (105-280) to $112 \pm 21$ minutes (96-143) with the transjugular approach. 9/10 patients had APs, and one patient had focal atrial tachycardia. One of the APs was a Mahaim pathway. Ablation localisations were right anterior and right anterolateral in $7 / 10$ and $3 / 10$ patients respectively. The average procedure time was $145 \pm 50$ (64-220)minutes and the fluoro time was $2.7 \pm 3(0.5-9)$ minutes. All of the RF ablations via transjugular approach were successful $(100 \%, 10 / 10)$. The mean follow-up period was $5 \pm 4.6(2-18)$ months. So far no recurrence has been observed.

Conclusion: Right anterior and anterolateral regions are the most challenging areas in terms of catheter stabilization even when long sheaths are used during femoral route ablation. Ablation attempts in these regions have partial success, frequent recurrence and high complication rates. This study revealed that transjugular approach seems as an effective alternative for those cases where transfemoral approach is unsuccessful for the ablation targets located in the right anterior and anterolateral regions.

\section{P-3}

Ablations in the aortic cusp in children: a single center four years experience

Ergül Y. (1), Özgür S. (2), Şahin G.T. (2), Kafalı H.C. (2), Özcanoğlu D. (3), Güzeltaş A. (2)

Saglik Bilimleri University, Mehmet Akif Ersoy Thoracic and Cardiovascular Surgery Center, Department of Pediatric Cardiology/ Electrophysiology, Istanbul, Turkey (1); Saglik Bilimleri University, Mehmet Akif Ersoy Thoracic and Cardiovascular Surgery Center, Department of Pediatric Cardiology, Istanbul, Turkey (2); Saglik Bilimleri University, Mehmet Akif Ersoy Thoracic and Cardiovascular Surgery Center, Department of Anesthesiology and Reanimation, Istanbul, Turkey (3)

Background: Approximately $10 \%-15 \%$ of idiopathic ventricular tachycardia (VT) originate from the left ventricular outflow tract and can be mapped and ablated from within the aortic root, although it can be challenging owing to the complex anatomic relationships of the aortic valve, coronary arteries, and veins. As we know there are many data on aortic cusp ablation in adults, studies are limited in children. In this study, we aimed to evaluate our patients who underwent ablation in aortic cusps retrospectively. Patients and Methods: Fifteen aortic cusp ablation procedures were performed in 14 patients (seven females; 50\%). First, right ventricular outflow tract was mapped with the EnSite ${ }^{\mathrm{TM}}$ system (St. Jude Medical Inc., St. Paul, MN, USA) in all patients, and in some of them also radiofrequency (RF) ablation test lesions were given there. Finally coronary cusp origine was proved in all patients and left heart was catheterized retrogradely through femoral artery. Results: The mean age was $15.85 \pm 1.63$ years $(10.9-18)$ and the mean body weight was $58.40 \pm 10.83 \mathrm{~kg}(34-80)$. Ablation in the Aortic valve was used for manifest accessory pathway in only one patient. The rest of the patients underwent ablation in aortic cusps to eliminate ventricular tachycardia and/or PVCs. The mean percentage of PVCs on ambulatory ECG was $28 \pm 19 \%(10-75)$. Limited flouroscopy was used in most of the patients $(11 / 14$ patients) during left heart catheterization. The mean procedure time $190.07 \pm 47.68$ minutes $(120-330)$ and in those the mean fluoroscopy time $7.53 \pm 5.62$ minutes $(0-28)$. RF ablation was successful in all patients except one. In this patient the focus was very close to the left main coronary, and we performed RF ablation with low energy-short duration. After that VT recurred and successful cryoablation (with an 8-mm-tip cryocatheter) was performed in the same session.

Conclusion: Arrhythmias originating from the coronary cusps in this series were successfully and safely ablated with RF in children without injury to the coronary arteries or the aortic valve. Coronary cusp origine should be considered when the transition zone of PVC is borderline and/or ablation in RVOT is unsuccessful.

\section{P-4 \\ Cryoablation with an 8-mm Tip Catheter for Typical Atrioventricular Nodal Reentrant Tachycardia in Children: Single Center Seven Years' Experience Ergül Y. (1), Kafal H.C. (2), Özgür S (2), Şahin G.T. (2), Özkilitçi E. (3), Güzeltaş A. (2) \\ Saglik Bilimleri University, Mehmet Akif Ersoy Thoracic and Cardiovascular Surgery Center, Department of Pediatric Cardiology/ Electrophysiology, Istanbul, Turkey (1); Saglik Bilimleri University, Mehmet Akif Ersoy Thoracic and Cardiovascular Surgery Center, Department of Pediatric Cardiology, Istanbul, Turkey (2); Saglik Bilimleri University, Mehmet Akif Ersoy Thoracic and Cardiovascular Surgery Center, Department of Anesthesiology and Reanimation, Istanbul, Turkey (3)}

Background: In children with typical atrioventricular nodal reentrant tachycardia (tAVNRT), 8-mm-tip cryoablation is prefered because of its safety profile and long-term success rates. This study aimed to assess the utility of 8-mm-tip cryoablation catheters for tAVNRT in children.

Patients and Methods: A total of 103 patients (57 females,55\%), who underwent cryoablation using an 8-mm-tip catheter for AVNRT ablation between January 2010 and December 2017 were evaluated retrospectively. Electrophysiological procedures were performed using the EnSite ${ }^{\mathrm{TM}}$ system (St. Jude Medical Inc., St. Paul, MN, USA).

Results: The mean patient age and weight were 14.3 years (6.2$20.7)$ and $54 \mathrm{~kg}$ (30-92), respectively. Ten patients (9.7\%) had structural heart disease (including three Ebstein's anomalies and one single ventricle physiology with bidirectional cavopulmonary anastomosis). Seven patients (6.8\%) had an additional arrhythmia substrate like ventricular tachycardia, Mahaim pathway and focal atrial tachycardia and six patients (5.8\%) also had an atypical AVNRT. The mean procedural time was 157 minutes (90-365). Mean number of effective cryolesion and total duration of cryoapplications were 8 (4-17) and 2529 seconds (240-4920s) respectively. Fluoroscopy was used only in seven patients (\%6.8) for diagnostic purposes in those with structural heart disease, or for vascular access. Mean floroscopy duration and mean radiation dose were 8 minutes (1.9-16) and $1001,6 \mathrm{mGy} \cdot \mathrm{cm}^{2}$ (106-2803) respectively. There were no complications except for a right bundle branch block and a transient atrioventricular block. Acute success rate of cryoablation was 98/103 (95\%). In four of the remaining five patiens, cryoablation failed and the procedure was completed successfully with radiofrequency (RF) ablation in the same session. In the patient with a single ventricule physiology and 
bidirectional cavopulmonary anastomosis, both cryoablation and RF ablation failed and medical therapy was carried on. During the mean follow-up period of 22 months (1-72) one patient with tAVNRT recurred 11 months later, and successfully ablated with $\mathrm{RF}$ in the second session. The resultant long-term success rate of AVNRT ablation was 102/103 (99\%).

Conclusions: Cryoablation of AVNRT with an 8-mm-tip catheter in children appears to be safe and effective by using a limited fluoroscopic approach and can be preferred as a first choice.

\section{P-5}

Electrophysiologic Characteristics and Ablation Results of Tachycardia Substrates Originating from the Coronary Sinus in Children

Ergül Y. (1), Özgür S. (2), Şahin G.T. (2), Kafalı H.C. (2), Güzeltaş $A$. (2)

Saglik Bilimleri University, Mehmet Akif Ersoy Thoracic and

Cardiovascular Surgery Center, Department of Pediatric Cardiology/Electrophysiology, Istanbul, Turkey (1);Saglik Bilimleri

University, Mehmet Akif Ersoy Thoracic and Cardiovascular

Surgery Center, Department of Pediatric Cardiology, Istanbul,

Turkey (2)

Background: Epicardial accessory pathways (APs) and focal atrial tachycardia (FAT) may originate from the coronary sinus system and transcatheter ablation through the coronary sinus is required. However, care must be taken due to the proximity of vital structures like coronary artery branches during ablation procedure. There is only limited data about ablations through coronary sines in children when compared to adults. We aimed to share our six year experience in ablations through coronary sinus.

Patients and Methods: From January 2011 to November 2017, 18 patients (10 female,55.5\%) underwent ablation of arrhythmia substrates through coronary sinus. EnSite 3D-electroanatomic mapping system (St. Jude Medical Inc., St. Paul, MN, USA) was used in all patients with limited fluoroscopic exposure.

Results: The mean age and weight of the patients were $11.13 \pm 3.12$ (4.48-18)years and $39,24 \pm 13,45 \quad(17-83) \mathrm{kg}$, respectively. In four of the patients, FAT was ablated through the proximal portion of the coronary sinus. In the remaining 14 patients, manifest epicardial APs were ablated through the coronary sinus. Interestingly, the APs were evaluated as high-risk pathways for all patients except two. Coronary sinus aneurysm was detected in five $(28 \%)$ patients. In these patients, deep QS pattern was remarkable in lead D-III on surface ECG wherase the same pattern was not found in any of the remaining patients. In 6 patients only cryoablation and another 6 patients only radiofrequency ablation were sufficient for completion of process. But in the remaining patients, combinations were needed. The average procedure time: $196 \pm 48.6(120-320)$ minutes and the fluoroscopy time was $9.66 \pm 9.39$ (0-34.5) minutes. Mean follow-up period after the procedures was $10.8 \pm 7$ (2.6-19.2) months. In only one patient with FAT, the arrhthmia has recurred, which later on successfully ablated with cryoablation again. Acute and long term success rate was 18/18 (100\%).

Conclusion: Coronary sinus itself can be the origine of many different types tachycardia. It can also be used to reach the epicardial foci. Both RF or cryoablation can be used for ablation through it. However, if during RF ablation will be used, one should keep in mind that coronary artery injuries and venous system perforations are more likely and must pay attention to the anatomy of the coronary sinus.

\section{P-6}

Different Arrhythmic Faces of Catecholaminergic Polymorphic Ventricular Tachycardia during Epinephrine Challenge Test in a Six Years Old Child Kafal H.C. (1), Öztürk E. (1), Özgür S. (1), Ergül Y. (2) (1)Saglik Bilimleri University, Mehmet Akif Ersoy Thoracic and Cardiovascular Surgery Center, Department of Pediatric Cardiology, Istanbul, Turkey; (2)Saglik Bilimleri University, Mehmet Akif Ersoy Thoracic and Cardiovascular Surgery Center, Department of Pediatric Cardiology/Electrophysiology, Istanbul, Turkey

Introduction: Catecholaminergic polymorphic ventricular tachycardia (CPVT) is an inherited arrhythmic disorder characterized by exercise-induced ventricular arrhythmias and risk for sudden cardiac death. The heart is structurally normal and the resting electrocardiogram (ECG) is normal. Maximal exercise stress test is a standart method in diagnosis of CPVT, because arrhythmias can be detected only during exercise. In patients, not suitable for exercise stress test due to young age, or where the exercise test is inconclusive, epinehrine challenge test can be used in the diagnosis. Atrial arrhytmias in CPVT patients during exercise testing have been reported already in the literature. But to our best knowledge, this is the first pediatric case, where atrial tachycardias were induced during an epinephrine challenge test.

Case Report: Six years old male patient admitted with syncopy, recuring in the last three years, especially when he was excited. Twelve lead surface ECG, echocardiography and ambulatory ECG were found normal. Treadmill exercise stress test was found inconclusive for CPVT due to non-cooparation for the test. So, adrenalin stress test was performed according to Ackerman protocole. Basal ECG was consistent with normal sinus rhytm (Figure 1a). During $0,25 \mathrm{mcg} / \mathrm{kg} / \mathrm{min}$ infusion first a supraventricular tachycardia with narrow QRS complex was induced (Figure 1b). Next, a bidirectional polymorphic ventricular tachycardia developed (Figure 1c), and at least atrial flutter with varying degrees of AV block was induced. Medical therapy with dideral and flecainid was initiated. The patient is still under control and asymphtomatic with medical therapy.

Conclusion: Not only bidirectional polymorphic ventricular tachycardia, but also several types of atrial tachycardias can be induced during the adrenalin stres test fort he diagnosis of CPVT.

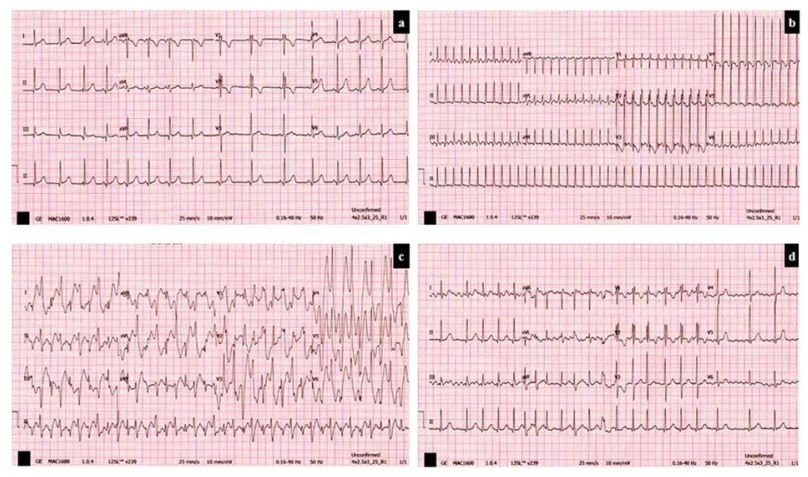

Figure 1.

\section{P-7}

An Unusual Etiology for Acquired Long QT Syndrome

Kasar T. (1), Ayyıldız P. (1), Tuzun B. (1), Ergül Y. (1), Kiyan $G(2)$.

Saglik Bilimleri University, Mehmet Akif Ersoy Thoracic and Cardiovascular Surgery Center, Department of Pediatric Cardiology, Istanbul, Turkey (1); Marmara University, Pediatric Surgery, Istanbul, Turkey (2) 
Introduction: Long QT syndrome (LQTS) is an arrhythmogenic disorder that can be both congenital and acquired. We want to present an interesting, rare cause for acquired LQTS.

Method: An 18 months old female patient admitted to the hospital with tachycardia and cyanosis without a history of syncope or any characteristic feature or an index case in the family. Cardiomegaly was present at chest X-Ray, oxygen saturation was $98 \%$, apical pulse was 91 beats/minute. Cardiac sounds were barely heard at auscultation, along with a 2/6 systolic murmur. The QTc segment was 500$520 \mathrm{msec}$ and there was vectorial suppression at the left precordials indicating both the repolarization and depolarization abnormality (Figure 1a). There was a $40 \mathrm{~mm} \times 40 \mathrm{~mm}$ mass with a circular regular contour neighbouring the left ventricular posterior wall. There wasn't any significant pressure on heart. B-blocker was initiated. Cardiac MRI demonstrated a semi-demarcable solid mass at the inferior left hemithorax extending posteriorly and towards the middle mediastinum neighbouring closely with left ventricular posterior wall anteromedially, inferior lobe of left lung mediastinal pleura posteriorly (Figure 1b). Biopsy of the mass demonstrated a fibrose pseudotumor and surgical excision was planned. The mass was resected partially (about 80-85\%) from its near side to the heart. Cardiac functions were normal at echocardiography on follow-up. At the $10^{\text {th }}$ postoperative day the QTc was regressed to $460 \mathrm{msec}$ on ECG and vectorial supression was normalised (Figure 1c).

Conclusion: LQTS had propensity to cause fatal cardiac events therefore early diagnosis and therapy is vital. Apart from known causes of acquired LQTS like drugs, there are many other causatives reported to lengthen QT interval but to the best of our knowledge there was no reported case of LQTS due to a cardiac mass which is a rare but an important entity.

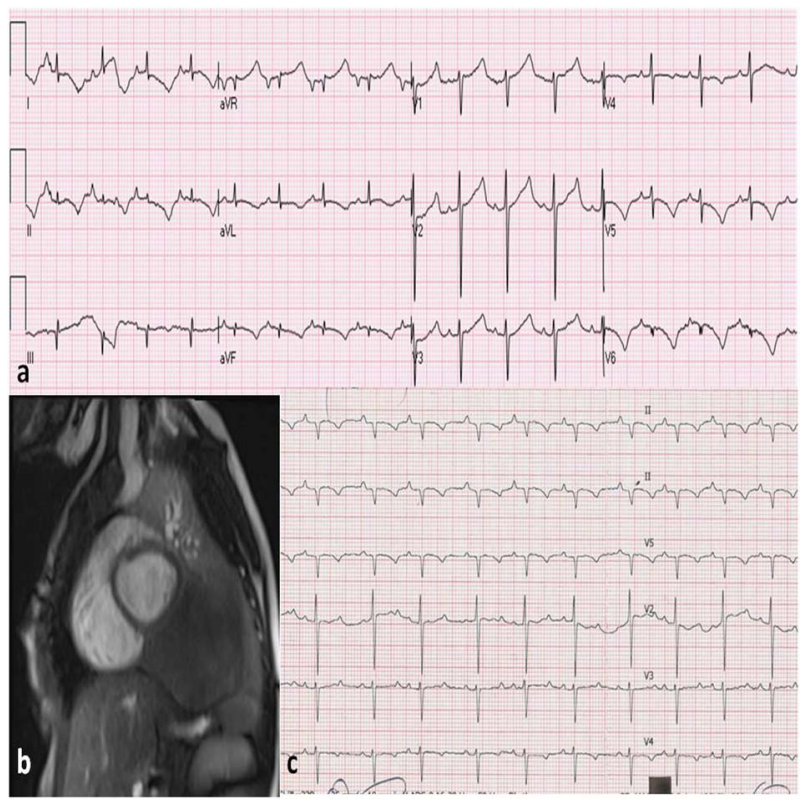

Figure 1.

\section{P-8}

Benign early repolarization pattern: Is there any risk of sudden cardiac death?

Kayali S. (1), Demir F. (2)

Univercity of Health Sciences, Keciören Training and Research Hospital, Pediatric Cardiology, Ankara, Turkey (1);Univercity of Health Sciences, Keciören Training and Research Hospital, Department of Pediatrics, Ankara, Turkey (2)
Introduction: Early repolarization (ER) is a common electrocardiographic (ECG) finding. Although it has been known a benign phenomenon, recent studies have shown that it can be an important marker of cardiac vulnerability and leads sudden cardiac death. Therefore, new studies have been made to distuinguish malignant and benign pattern of ER. However, there are still conflicting datas regarding the prognostic significance of ER in asymptomatic subjects especially in children.

In this study, some spesific ECG markers that reflect ventricular repolarization were investigated in children with benign ER including, QT interval dispersion (QTdis), corrected QT interval dispersion (QTcdis), interval between the peak and the end of $\mathrm{T}$ wave (Tp-e), Tp-e interval dispersion ( $\mathrm{Tp}$-e dis), ratios of $\mathrm{Tp}-\mathrm{e} /$ QT and Tp-e/QTc to predict the risk of sudden death.

Methods: Eighty healthy children (mean age:13,7+/-3,2 years and 71 boy) with benign ER pattern on ECG who have no history of syncope, no history of arrhtymia, no known arrhtymia susceptibility sendromes, no known coronary arter disease, no family history of sudden death were made as the study group and 51 children with normal ECG were included in the study as control group. Benign ER pattern was defined as terminal QRS notching or slurring accompanied by rapidly ascending ST elevation (>1 mV from baseline) in two or more in anterior or inferolateral leads. The following ECG parameters were evaluated at rest by one blinded experienced pediatric cardiologist : QT dis, QTc dis, Tpe dis, Tp-e/QT and Tp-e/QTc.

Results: ER was present in anterior derivations in $52(65 \%)$ and in inferolateral derivations in $28(35 \%)$ children in study group. QRS slurring was observed in $67(83.8 \%)$ and QRS notching in 13 (16.3\%) children with ER.

Study group had higher QT dis, QTc dis, Tpe dis, Tp-e/QT and Tp-e/QTc measurements when compared to controls. ( $\mathrm{p}: 0,05$, p: 0,04, $\mathrm{p}<0,01, \mathrm{p}<0,01, \mathrm{p}<0,01, \mathrm{p}<0,01$ respectively). There were no significant differences among the studied ECG parameters regarding the ER location (anterior, inferolateral), the ER type (slurring or notching).

Conclusion: These findings suggest that benign ER in children may also increase the risk of sudden cardiac death by alterations in ventricular repolarization.

\section{P-9}

\section{Mitral Valve Prolapse Masquerading as Atypical Cardiac} Symptoms

Petropoulos A.C. (1,2,3), Behbudov V. (1), Khudiyeva A. (1), Mustafayeva G. (1), Isayev I. (3)

Deparment of Congenital Cardiology, Merkezi Klinika, Baku, Azerbaijan (1); Department of Congenital Cardiology, XMS

Klinika (2); Department of Pediatrics, Educational and Therapeutically Hospital, Azerbaijan Medical University (3)

Introduction: Mitral valve prolapse $(\mathrm{MvP})$ is a Congenital Heart Disease (CHD), defined as an abnormal displacement of the mitral valve leaflets into the left atrium during systole. It has been thought to be mostly an incidental finding during echocardiography assessment. It can frequently masquerade as an element of syncope or even other atypical cardiac symptoms and signs such as easy fatigability, dizziness, palpitations, atypical chest pain. Occasionally, arrhythmias can be observed, associate with increased parasympathetic tone. Although thought to be a benign condition it needs to be detected and followed-up mostly when mitral regurgitation is present.

Aim: Describing the ever first incidence, clinical presentations and morphology of the defect by echocardiographic assessment of $\mathrm{MvP}$ in Azerbaijan by a retrospective study. 
Population-Method: Since August 2012, in three referral centers for CHD, 2160 patients age one day to 57 years old, (mean age $8+/-6$ years) were referred with suspicion of cardiac symptoms and or signs. From the patients that were diagnosed with MvP, their medical records and additional studies (Holter-ECG) were reviewed. Results: We excluded all syndromes, cardiomyopathies and complex CHD that may involve as an element MvP. Using the echocardiographic criteria of the historical study by Freed et.al [N Engl. J Med. 1999 Jul 1;341 (1)], we found:90patients with MvP (incidence 4.17\%). From these 57 (63\%)were females.51/90 (57\%) presented with symptoms and/or signs. Specifically: a.21/90 (23\%) with palpitations or arrhythmia detected on $24 \mathrm{~h}$ Holter ECG (premature atrial and / or ventricular beats, more than $15 \%$ of regular beats, episodes of Mobitz type I and episodes of nonsustainable junctional rhythm, b. 17/90 (19\%) complained of frequent atypical sharp non- sustainable chest pain, c. 5/90 (5.56\%) had suffered a typical vasovagal syncope and 8/90 (8.9\%) from presyncope. Regarding the morphology of MvP: 63/90 (70\%) involved the anterior leaflet,12/90 (13.4\%) both and 15/90 $(16.7 \%)$ the posterior leaflet. Regarding the amount of regurgitation: $52 / 90(57.8 \%)$ was trivial to mild-vena contracta $<2 \mathrm{~mm}-, 24 / 90(26.7 \%)$ mild- vena contracta $2-3 \mathrm{~mm}$ - and 16/90 (13.5\%) mild to moderate -vena contracta $>3<5 \mathrm{~mm}$ -

Conclusions: $\mathrm{MvP}$ has a high incidence in Azerbaijan, with a unique morphology (mostly anterior leaflet prolapse) and can not only masquerade as atypical cardiac symptoms but also frequently $13.5 \%$ involve significant $\mathrm{MvR}+$.

\section{P-10}

Postinterventional recurrent supraventricular tachycardia due to transient right bundle branch block after a successful high-frequency ablation of a midseptal accessory pathway in a patient with preexcitation sydrome

Postina P., Mitschke T., Follmann P., Lindinger A., Fritsche K., Kriebel T.

Department of pediatrics and pediatric cardiology, Westpfalz-Klinikum GmbH, Kaiserslautern, Germany

Introduction: Right Bundle branch Block (RBBB) may occur by catheter positioning during electrophysiological study (EPS). As this mechanical irritation is normally transient and not associated with significant side effects data of complications are limited.

Case Presentation: Recurrent episodes of apparent life threatening events were noted in a 20 months old girl $(10 \mathrm{~kg})$ with preexcitation sydrome with two episodes already under propanolol therapy. Due to these episodes the patient was transferred to our hospital for EPS.

RBBB occurred during positioning of electrode catheters and was traceable for the rest of the examination. Atrial stimulation showed an antegrade refractory period of the accessory pathway (AP) of $220 \mathrm{~ms}$. A supraventricular tachycardia (SVT) was not inducible. Successful ablation was achieved in the midseptal region with a loss of antegrade (loss of delta wave) and retrograde conduction of the AP.

Two hours later the patient developed a supraventricular tachycardia with RBBB. After administration of adenosine sinus rhythm occurred only for one beat without preexcitation, with subsequent start of the SVT again. Despite propafenon administration sinus rhythm could not be restored. However rate control could be achieved under continuous amiodarone administration with recurrent termination of the SVT and direct onset of the SVT after one sinus beat. The electrophysiological mechanism of the SVT with unusual direct start of SVT after one sinus capture was due to delayed excitation of the right ventricular myocardium and relapse of retrograde conduction of the AP. Due to this time lag the atrial myocardium was inducible again. SVT stopped immediately after loss of RBBB after 80 hours. Despite the relapse of the retrograde conduction the risk of sudden cardiac death through rapid antegrade conduction via the AP was eliminated. In the follow-up examinations after 1, 4 and 8 month no more SVT episodes were detected. Ablation of the retrograde conduction of the AP will be taken into consideration when the patient has gained more weight.

Conclusion: Transient RBBB in combination with an accessory pathway can cause sustained SVT which may be difficult to control.

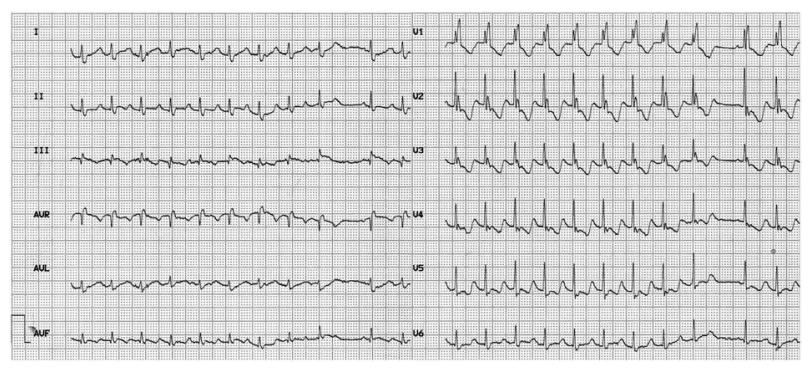

Figure.

\section{P-11}

A Young Patient with mid-septal accessory pathwey accompanied by left ventricular dysfunction Zakaraia I., Paech C., Gebauer R., Wagner R., Dähnert I. Pediatric Cardiology, Leipzig Heart Center, Leipzig, Germany

Objectives: Early septal activation may induce asynchronous ventricular activation in patients with WPW-pattern.

We report the case of a 16-years old patient with WPW-pattern and a highly impaired LV-function (EF 33\%) due to asynchronous ventricular activation because of a midsepteal accessory pathway (AP). The patient is a refugee from Nigeria who presented to our outpatient clinic because of progressive limitation of his physical capacity. X- ray showed cardiomegaly. Neither tachycardia, nor treatment but two times episodes of syncope were reported. Echocardiography revealed an asynchronous septal motion with systolic bulging. A successful resynchronization was achieved by catheter ablation of the AP. Following an improvement of both the dyssynchronia and LV function (EF50\%) could be seen. Despite sustained synchronous ventricular activation in 6 month follow up LV-EF was still slightly reduced. This unexpectedly long persistence of systolic function is presumably explained by the duration of asynchronous ventricular activation of over one decade.

Conclusion: Catheter ablation of septal pathways may result in ventricular dyssynchrony, yet early ablation may be beneficial for complete recuperation of LV function.

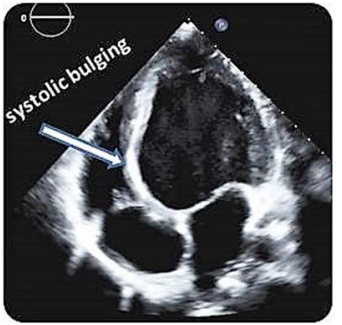

before ablation

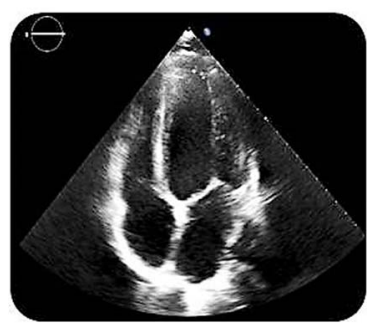

after ablation
Figure. 


\section{P-12}

The outcome of cavotricuspid isthmus-dependent atrial flutter ablation in patients with adult congenital heart disease: A single centre experience

Zaki A., Dua J.S. (1), Jenkins P. (1), Mahadevan V.S. (2), Hoschtitzky A. (1), Shah A. (1) ,Schneider H. (1), Weatherby J. (1), Kirkwood G.J. (1), Muhyaldeen S. (1)

Manchester Royal Infirmary, Manchester, UK (1); UCSF Medical

Center, San Francisco, USA (2)

Background: Although radiofrequency catheter ablation (RFA) is the gold standard treatment for cavotricuspid isthmus (CTI)dependent flutter (AFL) in the general population, its safety and outcome in adult congenital heart disease (ACHD) patients is backed only by published case reports and centre experiences in select ACHD groups. Here we report 4-year data for consecutive ACHD patients undergoing RFA for CTI-AFL in our centre.

Methods: We analysed data of consecutive RFA procedures for treatment of CTI-AFL in patients with ACHD between January 2013 and December 2016. Patients were divided into those with simple and those with moderate/complex ACHD as per the PACES/HRS expert consensus statement in 2015. Acute success was defined as termination of persistent AFL by ablation plus achievement of bidirectional block (BDB) across the CTI, or CTI BDB if ablation was done in sinus rhythm (SR).

Results: 23 patients (mean age:46 years) underwent 25 procedures. Five patients (six procedures) had simple ACHD (one unrepaired; five surgically repaired atrial septal defects) and 18 patients (19 procedures) had previous surgical repair for moderate/complex ACHD (Ebstein:1, tetralogy of Fallot (TOF):5, d-transposition of the great arteries (d-TGA):2, severe pulmonary stenosis (PS):2, Eisenmenger: 1 and combinations:7). Acute ablation success was achieved in $100 \%$ of cases in both groups with no complications. Mean follow up post-procedure was 13.5 months (range:3-54). Freedom from arrhythmias during follow-up was $83 \%$ and $63 \%$ in the simple and moderate/complex ACHD groups respectively $\left(\chi^{2}=0.85 ; p=0.35\right)$. Recurrent paroxysmal or sustained arrhythmias were documented in seven (37\%) moderate/complex patients (TOF:1, d-TGA:1, PS:1, Ebstein repair: 1 and combination: 3 ) and included atrial fibrillation: 2 patients (11\%); typical or atypical AFL: 3 patients (16\%); non-sustained VT:1 patient (5\%); and SVT:1 patient (5\%). Mean time to first recurrence was 153 days (range: 1-395 days). Two patients (one simple; one moderate/ complex ACHD) had recurrent CTI-AFL and underwent successful redo RFA with no recurrence at 3 months follow-up.

Conclusion: RFA of CTI-AFL in patients with simple and moderate/complex ACHD is safe and has high acute success rate. Post procedure freedom from all arrhythmias is more likely to be achieved in simple than moderate/complex ACHD, although the difference was not statistically significant in our cohort.

P-13

Outcome of radiofrequency catheter ablation of supraventricular arrhythmias in patients with adult congenital heart disease: single centre experience Zaki A. (1), Dua J.S. (1), Jenkins P. (1) , Mahadevan V.S. (2), Hoschtitzky A. (1), Shah A. (1) ,Schneider H. (1), Saraf K. (1), Kirkwood G.J. (1), Muhyaldeen S. (1)

Manchester Royal Infirmary, Manchester, UK (1); UCSF Medical Center, San Francisco, USA (2)

Introduction: Data in the literature regarding the safety and outcome of catheter-based radiofrequency ablation (RFA) for the treatment of supraventricular arrhythmias (SVA) in patients with adult congenital heart disease (ACHD) is based on case-reports and centre experiences. Here we report three-year data for consecutive patients with simple and moderate/complex ACHD undergoing RFA for treatment of SVA in our centre.

Methods: Retrospective analysis of consecutive RFA procedures, between January 2014 and December 2016, for treatment of SVA in patients with repaired/unrepaired ACHD. Underlying ACHD was classified as simple (Group A) or moderate/complex (Group B) according to the PACES/HRS expert consensus statement in 2015. SVA was defined as AVNRT, AVRT, intraatrial re-entrant tachycardia (IART), focal atrial tachycardia, atrial fibrillation or cavo-tricuspid/cavo-mitral isthmus atrial flutter. Acute success was defined according to the arrhythmia mechanism as one or more of the following: (1) termination of sustained tachycardia by ablation plus non-inducibility afterwards, or (2) pulmonary vein isolation, or (3) bidirectional block across ablation lines, or (4) non-inducibility of previously inducible arrhythmias. Outcome was defined as equivocal when: (1) substrate modification was performed during sinus/ paced rhythm, plus (2) clinical arrhythmia was non-inducible.

Results: 50 ablation procedures were performed in 41 patients (mean age: $41.8 \mathrm{y})$. Ten patients (12 procedures) had simple ACHD (ASD or VSD) and 31 patients (38 procedures) had complex ACHD (Ebstein, TOF/pulmonary stenosis repair, Fontan repair, arterial switch, Senning repair, Eisenmenger and combinations). Acute success was achieved in 100\% of Group A and $84.2 \%$ of Group B procedures. Outcome was equivocal in 5\% of Group B procedures. There was one non-fatal complication in Group B (asystolic arrest following termination of IART). Postprocedure arrhythmia-free survival was 75\% in Group A and 66\% in Group B during mean follow up of 10 and 14.5 months respectively $(p=0.15)$. Average time to recurrence of the same or different SVA was 1.5 months in Group A and 5.1 months in Group B.

Conclusion: RFA of SVA is safe and has high acute success rate in patients with ACHD. However, long term freedom from arrhythmias appears to be insignificantly lower in patients with moderate/complex compared to simple ACHD.

\section{P-14}

Rhabdomyolysis and cardiac depolarization anomalies: TANGO2 or not?

Tormassian A. (1), Cano A. (2), Koutbi L. (3), Chabrol B. (2), Ovaert C. (1)

Cardiologie pédiatrique et congénitale, Timone enfants, AP-HM,

Marseille, France (1); Centre de reference des maladies métaboliques, Service de neuropédiatrie, Timone enfants, AP-HM, Marseille,

France (2); Rythmologie, CHU La Timone, AP-HM, Marseille, France (3)

Introduction: acute rhabdomyolysis is a rare but potentially severe disease in childhood. Inherited metabolic disorders have to be searched for. Significant repolarisation abnormalities have been described in patients with acute rhabdomyolysis and TANGO2 mutation involved in the Golgi apparatus.

Methods: Between 12/2013 and 01/2017, 4 children (mean age 3,5 years at first crisis) presented in our department with 1 to 3 acute severe rhabdomyolysis crises (CPK > $10000 \mathrm{UI} / \mathrm{l}$ ) associated with significant ECG anomalies. All 4 had neuro-developmental delay (from mild speech delay to spastic tetraparesia, with microcephaly). Rhabdomyolysis occurred after acute viral illnesses or unusual fasting and were associated with neurological deterioration. Cardiac anomalies included significant lengthening of QT and QTc interval in all 4, concomitant bradycardia and life-threatening polymorphic ventricular tachycardia in patient 1 , left bundle 
branch bloc in patient 3 and ventricular arrhythmia and cardiac arrest in patient 4 (figure 1). ECG anomalies were maximal around 10 days after the beginning of crisis and slightly delayed from the peak CPK level. Ventricular dysfunction was associated in patient 2 (mild) and in patient 3 (severe requiring inotrope support). Cardiac anomalies regressed completely in all between the rhabdomyolysis crises. Patient 1 died while admitted for her 3rd rhabdomyolysis crisis, with severe neurological worsening, prolonged QT but no ventricular arrhythmia. TANGO2 mutation was confirmed in patient 1 , suspected but not yet confirmed for the 3 others.

Conclusion: Ventricular conduction and function have to be explored in children with acute rhabdomyolysis in the setting of neurodevelopmental delay. TANGO2 mutations may be responsible. The cardiac disorders are rapidly progressive, potentially lifethreatening but reversible. The mechanism and treatment are poorly defined.

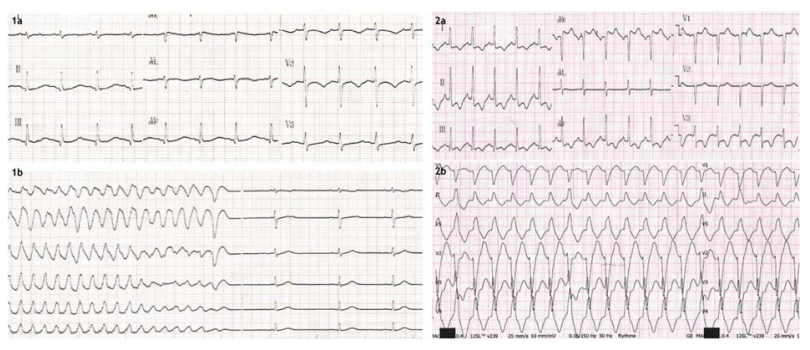

Figure 1.

Abnormal ECGs during acute rhabdomyolysis of patient $1(1 a, 1 b)$ and patient 3 (2a and $2 b)$.

\section{P-15}

Unexplained Syncope in Children

Polyakova E., Trofimova T., Shkolnikova M.

Children's Center for Cardiac Arrhythmias, Moscow, Russian Federation

Basics: Syncopal states are quite frequent in children's population, as $15-20 \%$ of them had at least one episode of it. A syncope of unclear etiology makes up $17-37 \%$ among all syncopes. Unexplained Syncope $(\mathrm{UnS})$ can mask the most life threatening cardiogenic syncope, which can lead to sudden cardiac death. So far, diagnostics of syncope in children have been developed insufficiently.

Methods: We studied 1895 children with recurrent syncope hospitalized at the Children's Center for Cardiac Arrhythmias. Personal and family history, physical examination, including ECG, stress test, holter monitoring, tilt-table and other tests, were made. The average age was $12.1 \pm 4.5$ (from 1 to 17 ).

Results: Out of 1895 hospitalized children with recurrent syncope, $290(15.3 \%)$ had UnS and had implanted loop recorders. Out of 290 children (49\% - boys), monitoring was completed for 259 pts due to the end of a 36-month follow-up, or due to the symptomrhythm correlation or detecting arrhythmia. Clinically positive cases (syncope and/or events) were in 50\% of children. Arrhythmogenic syncope or Arrhythmogenic event were diagnosed in $31 \%$ of pts with UnS and $53.8 \%$ of clinically positive cases. Among the causes for arrhythmic syncope and events we observed both tachyarrhythmias (ventricular fibrillation) and bradyarrhythmias (asystole, AVblock).

Conclusion: The frequency of $\mathrm{UnS}$ in hospitalized children was $15.3 \%$. Implantation of loop recorders in children with UnS was useful in 50\%. Arrhythmogenic syncope or Arrhythmogenic event was diagnosed in $31 \%$ of pts with UnS.
P-16

Thoracoscopic Procedures in Pediatric Patients with Cardiac Arrhythmias

Termosesov S., Khamnagadaev I., Garipov R, Volkova Y., Grishin I., Romantsova Z., Khaspekov D., Shkolnikova $M$.

Research Clinical Institute of Pediatrics, Arrhythmology Department, Moscow, Russian Federation

The Objectives: To evaluate the early outcomes of video $a$ assisted thoracoscopic (VATS) procedures as an adjunctive therapeutic approach in pediatric patients with cardiac arrhythmias.

Methods: In 2016-2017 yrs, 15 pts aged 4 to 15 underwent VATS procedures at our institution. Group I - pts with Long QT syndrome (LQTS) $(\mathrm{n}=7)$ and catecholaminergic polymorphic ventricular tachycardia (CPVT) $(n=5)$. Group II - pts with thirddegree atrioventricular block and body mass less than $15 \mathrm{~kg}(\mathrm{n}=3)$. In Group I all pts underwent left cardiac sympathetic denervation

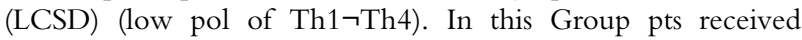
implantable cardioverter defibrillators (ICDs). In all cases beta-blockers therapy were not effective before LCSD. Invasive electrophysiological study (EPS) was performed before and after LCSD. In pts with ICDs EPS was performed via device. Permanent cardiac pacemaker (VVIR mode) was implanted using VATS technology in Group II. Leads were placed to epicardial surface of the left ventricle (screw-in -1 pts).

Results: Follow 7 up period was $4-12$ months. The were no intraoperative and perioperative complications in both groups. The trend to increasing of ventricular effective refractory period was found during EPS just after LCSD. There were no any shocks registered after LCSD in Group I. In 1 pts non sustain ventricular tachycardia was induced during exercise test. Acute and chronic leads thresholds and R-wave amplitude were appropriate in Group II.

Conclusion: VATS procedures can be safely and effectively performed in most patients with cardiac arrhythmias. This minimally invasive procedure can be used in complex therapeutic approach for reducing of life-threatening cardiac arrhythmias episodes.

\section{P-17}

Compound heterozygous or homozygous SCN5A mutation and atrial standstill in paediatric population: two cases report

Raimondo C. (1), Fressart V. (2), Denjoy I. (3), Bonnet D. (1), Maltret $A$. (1)

Hôpital Universitaire Necker-Enfants Malades, Centre de référance Malformations Cardiaques Congénitales Complexes-M3C, Paris, France (1); UF de cardiogénétique et myogénétique moléculaire et cellulaire, CHU Paris-GH La Pitié Salpêtrière-Charles Foix - Hôpital Pitié-Salpêtrière, Paris, France (2); Unité de Rythmologie, Centre de Référence Maladies Cardiaques Héréditaires, CHU Paris Nord-Val de Seine - Hôpital Xavier Bichat-Claude Bernard, Paris, France (3)

Atrial standstill is a rare arrhythmogenic condition characterized by the absence of electrical and mechanical atrial activity. It occurs as idiopathic, familial or sporadic. Recently, studies have linked the familial forms of atrial standstill to the SCN5A gene, which encodes the alfa subunit of the Nav1.5 cardiac sodium channel protein. We describe two pediatric cases of sinus node dysfunction and compound heterozygous SCN5A mutations.

Case1- A 3 years old boy with history of syncopes was implanted with a dual chamber-epicardial pacemaker because of a sinus node dysfunction with severe bradycardia associated with pauses $>3 \mathrm{~s}$. No family history of cardiological disease or sudden cardiac death. The parents are cousins. After implantation, pacemaker parameters 
showed atrial lead dysfunction and elevated ventricular capture. PM was programmed in VVI 40/min. During follow-up visits, PM interrogation showed progressive increasing ventricular stimulation up to $90 \%$, no ventricular arrhythmias and a stable ventricular capture at $1.3 \mathrm{~V}$. PM was then reprogrammed in VVIR $60-130 / \mathrm{min}$. Genetic tests were positive for homozygote SCN5A mutation (c.4380C > A: p.Phe1460Leu). The family genetic test showed the presence of the same heterozygote mutation in both parents.

Case2- A 3 years-old boy hospitalized for a left sylvian ischemic stroke with right hemiparesis and left facial paralysis. The ECG showed a sinus node dysfunction characterized by a bradycardic jonctional rhythm $40 / \mathrm{min}$ with pauses $>2.5 \mathrm{~s}$ and a Brugada pattern in the precordial lead V1. TE echocardiography showed a dilated left atrium with a large thrombus in the left appendage. Anticoagulation therapy was contraindicated a cause of the elevated risk of bleeding. The implantation of an endocardial PM DDD showed the presence of an atrial standstill. The PM was then programmed in VVIR 60-130/min. In the suspicion of a Brugada syndrome, a therapy with hydroquinidine was started. The genetic test found a double mutation of the gene SCN5A: c.559A > C ( $\mathrm{pThr187Pro),} \mathrm{never} \mathrm{described} \mathrm{before,} \mathrm{and} \mathrm{c.4747C}>\mathrm{T}$ (p. Arg1583Cys). The child died of neurological complication.

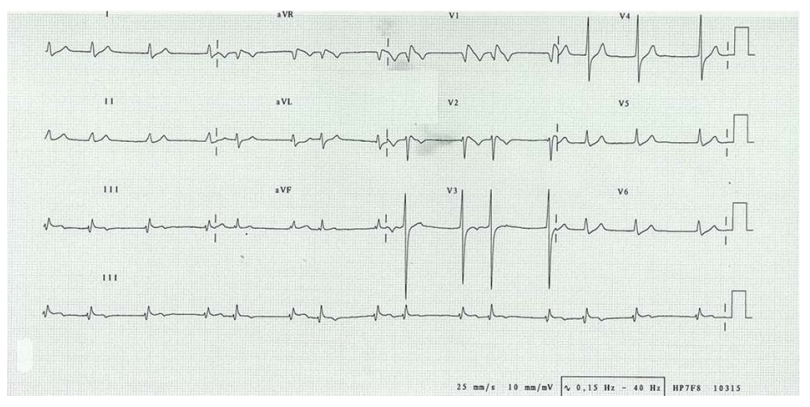

Figure 1.

Admission ECG of patient 2.

\section{P-18}

Predictive factors of cardiogenic shock in children less than one year of age with supraventricular tachycardia

Fournier E., Iriart X., Jalal Z., Thomas J., Mouton J.B., Sarton R., Thambo J.B., Séguéla P.E.

Bordeaux University Hospital, Bordeaux, France

Objectives: Supraventricular tachycardia (SVT) is the most common arrhythmia in children. However, factors leading to cardiac dysfunction are still unknown. We aimed to determine predictive factors of cardiogenic shock in children less than one year of age with SVT.

Methods: All the children under the age of one year and who were referred for SVT to our paediatric cardiologic intensive care unit were included. Cardiogenic shock was defined as a systemic ventricular ejection fraction under $50 \%$ and the need of inotropic drugs. Demographic data, type of SVT, prenatal diagnosis of arrhythmia, clinical characteristics and therapeutic management were reviewed.

Results: 109 children (10 days [1-30]) were included. 17\% of patients (12 days [3.5-17.5]) developed cardiogenic shock. Heart failure was significantly more frequent in case of flutter $(54.5 \%$ vs $13.3 \%$; $<$ < 0.01), when heart rate was high (280 bpm [235-300] vs $230 \mathrm{bpm}[210-280] ; \mathrm{p}=0.04)$ and for sustained tachycardia. $29 \%$ of patients presented pre-natal arrhythmia which was not associated with post-natal heart failure. Neither presence of associated congenital heart disease, nor young age at presentation were at risk of cardiogenic shock.

Conclusions: The incidence of cardiogenic shock is not uncommon in children with SVT. A high heart rate seems to be the main risk factor. $50 \%$ of patients with flutter will develop heart failure in case of prolonged tachycardia. These predictive factors of cardiogenic shock should help to improve the therapeutic management.

\section{P-19}

Closed loop stimulation-guided atrial pacing in young patients with complex congenital heart defects: is it physiological? A case series

Pazzano V. (1), Silvetti M.S. (1), Battipaglia I. (1), Di Mambro C. (1), Saputo F.A. (1), Tamburri I. (1), Carotti A. (2), Torcinaro S. (3),

Drago F. (1)

Paediatric Cardiology and Arrhythmia/Syncope Unit, IRCCS Bambino Gesù Children's Hospital, Rome, Italy (1); Heart Surgery Unit, IRCCS Bambino Gesù Children's Hospital, Rome, Italy (2); Biotronik Italy (3)

Objectives: Young patients with operated complex congenital heart defects (CHD) often develop sinus node dysfunction requiring permanent pacing, with rate-responsive function to respond to physiological stresses. Activity-driven sensors cannot account for non-movement stress and cannot modulate heart rate physiologically. Closed Loop Stimulation (CLS, Biotronik, Berlin, Germany) is a physiological rate-responsive pacemaker based on the indirect measure of ventricular contractility. No data are available on the effects of such pacing strategy in young patients with CHD and single-chamber atrial pacing.

Methods: We report a series of 5 patients with CHD and sinus node dysfunction who underwent CLS atrial pacing with endocardial or epicardial systems. CLS pacing was compared with rate-responsive accelerometer-driven pacing. During the first 15-30 days, the pacemaker was programmed in atrial pacing mode and then was switched to CLS atrial pacing mode. An in-hospital control was scheduled 1-2 months later to evaluate the CLS response to neurovegetative stresses (i.e. non-movement stress - Stroop color test, handgrip - and exercise stress test).

Results: At telemetric interrogation, CLS pacing showed a more physiological pattern of 24-hour heart rate trends than accelerometer sensors. The data obtained during non-movement stress and exercise stress test demonstrated a physiological increase in the pacing rate with CLS. The accelerometer sensor histogram showed a "non-response" behavior, where only lower rate events were documented during neurovegetative stresses.

Conclusions: In young CHD patients, endocardial/epicardial CLS atrial pacing demonstrated a physiological response of heart rate to neurovegetative and physical stresses. This is the first report on the efficacy of CLS-guided single-chamber atrial pacing in ensuring physiological pacing in young patients with complex congenital heart defects and sinus node dysfunction after surgery.

\section{P-20}

Use, effectiveness and safety of Ivabradine in paediatric patients with atrial tachycardia

Loevets T.S., Pervunina T.M., Butish L., Yakovleva E.V., Lebedev D.S., Vasichkina E.S.

Almazov National Medical Research Centre, Saint-Petersburg, Russia

Introduction: Ivabradine is a specific, selective inhibitor of the I (f) channels of the sinus node and the atrioventricular node. Ivabradine decreases heart rate and myocardial oxygen consumption. 
Information about the use in children for the treatment of atrial tachycardia (AT) is limited.

Purpose: To analyze the antiarrhythmic effect of Ivabradine for the treatment of AT in children.

Materials and Methods: We enrolled 20 consecutive patients with atrial tachyarrhythmias (10 girls and 10 boys), who treated with Ivabradine. Patients underwent a complete history, physical examination, laboratory studies (including thyroid function, CK, CK-MB, LDG, Troponin I, proBNP), echocardiography, ECG, Treadmill test and Holter monitoring (HM). Cardiac MRI were performed according to indications and physician's decision.

7 (35\%) had frequently recurrent AT, 9 (45\%) - chronic AT, $2(10 \%)$ - accelerated atrial rhythm, $1(5 \%)$ - frequent premature atrial contractions (burden 45\%). The etiology of AT was idiopathic in 50\% (10/20), post-myocarditis in 30\% (6/20), after surgical treatment of congenital heart disease in $2(10 \%)$, increased automatism of the atrial foci in 2 (10\%). Arrhythmia-induced cardiomyopathy was in 35\% (7/20) cases.

Results: Long-term follow up of patients was 20,38 \pm 19,14 months (range 1 to 60). The mean age of diagnosis of AT was $8,04 \pm 4,82$ year $(0,16-16)$. The Ivabradine dosage after uptitration was $0,15 \pm 0,06(0,08-0,28 \mathrm{mg} / \mathrm{kg} /$ day $)$ twice a day.

All patients received antiarrhythmic therapy prior to treatment with Ivabradine. We use of $\beta$-blockers, class IC and III antiarrhythmic agents and combination therapy. Ivabradine was effective in $70 \%(14 / 20)$ patients: in 6 - the tachycardia was suppressed, in 8 reduced the heart rate and decrease duration of paroxysm of tachycardia (control the ventricular response).

Ivabradine was ineffective in $30 \%(6 / 20)$ patients, in 4 patients with post-myocarditis AT and in 2 patients after surgical treatment of congenital heart disease. Radiofrequency ablation was performed in 6 patients ( 3 of 6 children taking Ivabradine before catheter ablation, 3 of 6 after non-successful RFA).

There were no significant side effects from the use of Ivabradine. Conclusions: Ivabradine is an effective and safe drug for the treatment of idiopathic atrial tachycardias in paediatric patients.

\section{P-21}

Integrated model for the risk assessment of the unfavourable course of binodal pathology in children Vasichkina E.S., Pervunina T.M., Kruchina T.K., Loevets T.S., Egorov D.F. Lebedev D.S.

Almazov National Medical Research Centre, Saint Petersburg, Russia

Aim: This study is aimed to look for the risk factors of an adverse course of binodal pathology and the creation of a comprehensive model of an adverse course of the disease.

Methods: We have enrolled 177 consecutive patients with binodal pathology, $146(82,5 \%)$ male, 31 (17,5\%) female. Inclusion criteria: combined sinus node dysfunction and atrioventricular (AV) conduction disturbance, duration of observation of at least 1 year, at least 2 comprehensive examinations, and the presence of disease dynamics.

All children had carried out examination of the heart at least 2 times: ECG, Holter monitoring (HM), transesophageal electrophysiologic study (TEEPS), and echocardiographic examination, The clinical results obtained during the work were processed using the STATISTICA for Windows software (version 10).

Results: In the analysis of complaints, clinical picture and data of electrocardiographic and electrophysiological parameters, factors that have influenced the course of the binodal pathology were established. Risk factors were: male gender, the age of manifestation of the disease over 14.5 years, the minimum heart rate is less than 52 beats per minute (before the medication), the value of SNRT is more than $1510 \mathrm{~ms}$ (before the test with atropine), the minimum heart rate after the administration of atropine is less than $96 \mathrm{bpm}$. The total risk score is calculated as the sum of the values of these indicators ( 5 risk factors) and can take values from 0 to 5 points. Three levels of total risk score are singled out: 0 points - low probability of negative dynamics; $1-3$ points - an insignificant probability of negative dynamics; $4-5$ points - significant risk of negative dynamics.

Conclusion: We obtained statistically significant $(\mathrm{p}<0.01)$ differences in the frequencies of the negative dynamics of the course of the binodal pathology at different levels of the total risk score.

\section{P-22}

Catheter Ablation for Supra-Ventricular Tachycardias in 15 Patients after Mustard or Senning Procedures

Taniguchi K., Toyohara K., Takeuchi D., Inai K., Sugiyama H., Shoda $M$

Tokyo Women's University, Japan

Introduction: Atrial switch operation (ASO, Mustard or Senning procedure) was performed in patients of d-transposition of great arteries (d-TGA) as palliation and l-transposition of great arteries (1-TGA) as anatomical repair with Jatene or Rastelli procedures. Although patients after ASO often have supra-ventricular tachycardia (SVT), electrophysiological study and catheter ablation (EPS/CA) for them is challenging because of complex postoperative anatomy and multiple damaged substrates.

Methods: We reviewed 24 EPS/CA procedures in 15 patients (d-TGA 9, 1-TGA 6) who underwent ASO (Mustard 8, Senning 7). Results: The mean age at latest EPS/CA was $32.3 \pm 8.2$ years. Isthmus dependent atrial flutter (AFL) was induced in 9 patients. Multiple atrial tachycardias (ATs) were induced in 12 patients. Atrioventricular nodal reentry tachycardia (AVNRT) was induced in 2 patients (common AVNRT 1, uncommon AVNRT 1).

The number of patients who had only one arrhythmia was 3 (AFL 2 and common AVNRT 1 in Senning group), uncommon AVNRT and ATs was 1 (in Senning group), AFL and several ATs was 7 (3 in Senning group, 4 in Mustard group) and several ATs was 4 (in Mustard group).

ATs were often induced in Mustard group (Mustard 8, Senning 4, $\mathrm{p}=0.04$ ). AFL was induced in both (Mustard 4, Senning 5, $\mathrm{p}=0.40)$. Patients of Mustard group tend to have multiple arrhythmias (Mustard 8, Senning 4, p =0.04).

A success rate at first procedure was higher in Senning group (Mustard 0\%, Senning 43\%, $\mathrm{p}=0.04$ ), and the number of procedures per patient was higher in Mustard group (Senning 1.29, Mustard 1.88, $\mathrm{p}=0.03$ ). However, we succeeded in $73 \%$ of patients (11 of 15$)$, and there was no significant difference between two groups.

Although an access to pulmonary vein atrium (PVA) was needed with the exception of two patients of Senning group, there was tendency that Brockenbrough procedure was avoided in Mustard group because of the existence of baffle leak (Mustard 2, Senning 4, $\mathrm{p}=0.06$ ).

Conclusions: Compared to patients of Senning procedure, patients of Mustard procedure tend to have more ATs. Although several CA procedures are needed especially in Mustard group, those are effective in most patients after ASO.

\section{P-23}

The diagnostic accuracy of transesophageal electrophysiology study in adolescents - 6 years follow-up Litwin L. (1,2), Galeczka M. (1,2), Grochowina-Major A. (2), Slezak W. (1), Rojczyk D. (2), Skiba-Zdrzalek A. (2), Bialkowski J. (1,2) 
Department of Congenital Heart Defects and Pediatric Cardiology, SMDZ in Zabrze, SUM, Katowice, Poland (1); Silesian Center for Heart Diseases, Zabrze, Poland (2)

Introduction: Arrhythmia-suggesting symptoms without a documented event are a common clinical scenario in adolescents. Transesophageal electrophysiology study (TES) is a relatively noninvasive diagnostic method with a potential to address this issue, but consensus on TES application in paediatric patients is missing. We evaluate TES diagnostic accuracy among adolescents with different indications for TES.

Methods: TES was performed in 88 consecutive patients between 2011 and 2017 in a single tertiary centre. Examinations were done under local anaesthesia, with a routine protocol, by one experienced paediatric cardiologist. We analysed TES outcomes and medical documentation and performed phone interview at a median 2,6 years (range $0,1-6,8$ ) from TES. Follow-up was available in $58(66 \%)$ patients, aged 15,7 years (SD 1,5). In this cohort, TES was performed due to arrhythmia-suggesting symptoms (collapse, palpitations) in 44, control after radiofrequency ablation (RFA) in 3, risk stratification in asymptomatic preexcitation syndrome in 7 and conduction assessment in 5 patients. Results: Arrhythmia was induced in 10 out of 44 suspected patients. They were referred for invasive electrophysiological study (EP) with RFA in case of a positive result (9 patients, positive predictive value 90\%). Among 34 patients without relevant arrhythmia during TES, 25 patients experienced further symptoms, 26 remained in outpatient observation, and 4 underwent EP with RFA (negative predictive value $88,2 \%$ ). In a subgroup controlled after RFA, no arrhythmia was induced, but one patient needed the second EP with RFA later on. In the pre-excitation subgroup, supraventricular tachycardia episode was induced in one case. This patient and 3 other with effective refraction equal $260 \mathrm{~ms}$ or less were referred for the EP study with subsequent successful RFA. Follow-up in remaining cases was uneventful. In a group referred for conduction assessment, atrioventricular node dysfunction was diagnosed in 3 patients. There was one episode of mild, TES-induced conduction disturbances, and no long-term complications.

Conclusions: TES is an effective and safe procedure with the potential for wide clinical application in adolescents. High positive and negative predictive values in symptomatic adolescents with suspected arrhythmia could improve selection for invasive EP, although no definitive arrhythmia exclusion could be warranted by TES.

\section{P-24}

The contribution of the NGS technology to establish the diagnosis of Marfan or Marfan-like syndrome with congenital heart disease

Tsoutsou E. (1), Kenteroglou O. (1), Marias V. (1), Willems P. (2), Selenti N. (1), Fryssira H. (1)

Choremio Research Laboratory (1), Athens, Greece; GENDIA

(GENetic DIAgnostic Network) (2), Antwrep, Belgium

Introduction: Marfan syndrome (MFS) is an autosomal dominant disorder of connective tissue with prevalence of 1 per 5000 individuals. MFS is characterized by the early development of aortic thoracic dilatations/aneurysms, together with defects of the skeletal and ocular systems. Additionally, its main features are the tall stature, arachnodactyly, pectus excavatum, joint hypermobility and ectopia lentis. Mutations in the Fibrillin-1 gene (FBN1) are the most common cause of the MFS. Nevertheless, mutations in other genes (e.g. TGFBR1, FLNA, SLC2A10, ADAMTSL4,
CBS, SMAD3, TGFB2, MED12, FBN2, PLOD1, BMPR2) have also been associated with this syndrome. New Technologies, such as Next Generation Sequencing, contribute to the identification of novel mutations in these genes.

Materials and Methods: We applied Whole Exome Sequencing (WES) in 7 patients with Marfan or Marfan- like phenotype and heart disease. The WES test sequences and analyses 214.405 exons dispersed throughout the genome. Peripheral blood samples were collected, and genomic DNA was isolated from these patients. Results: We tested 7 patients with Marfan or Marfan - like phenotype with WES. 5 of them were males and 2 of them were females. We found 3 novel mutations in the FBN1 gene, 1 mutation in the BMPR2 gene and 1 mutation in the SLC2A10 gene. In 2 cases no mutation was identified by WES.

Conclusion: We identified 5 novel mutations, all associated with Marfan or Marfan-like syndrome. The use of NGS technologies help to establish the diagnosis of MFS in patients, especially in cases with no FBN1 mutation which until recently remained undiagnosed.

Table.

\begin{tabular}{|c|c|c|}
\hline Patient & Heart disease & Gene mutation \\
\hline Pat. 1 & $\begin{array}{l}\text { Mitral and Tricuspid valve } \\
\text { prolapse } \\
\text { Mild Aortic root dilatation }\end{array}$ & FBN1: c. $3623 \mathrm{G}>\mathrm{A}$ in exon 30 \\
\hline Pat. 2 & Ascending aortic aneurysm & $\begin{array}{l}\text { BMPR2: c.1513dupA in exon } 11 \\
\text { (a premature stop codon) }\end{array}$ \\
\hline Pat. 3 & Mitral valve prolapse & FBN1: c. $299 \mathrm{G}>\mathrm{A}$ in exon 3 \\
\hline Pat. 4 & $\begin{array}{l}\text { Mitral valve prolapse } \\
\text { Mild Pulmonary artery } \\
\text { dilatation }\end{array}$ & No mutation found \\
\hline Pat. 5 & Mitral valve insufficiency & No mutation found \\
\hline Pat. 6 & $\begin{array}{l}\text { Mitral valve prolapse } \\
\text { Aortic root dilatation }\end{array}$ & SLC2A10: c. $859 \mathrm{G}>\mathrm{A}$ \\
\hline Pat. 7 & Aortic dilatation & FBN1: c.724_725ins T in exon 6 \\
\hline
\end{tabular}

P-25

Early disease onset and high penetrance of severe cardiomyopathy in RBM20 p.P638L mutation carriers in toddler's age

Gaertner-Rommel A. (1), Laser K.T. (2), Marga E. (1),

Klauke B. U. (1), Schulz U. (3), Dubowy K.O. (2), Kececioglu D. (2), Gummert J. (3), Milting H. (1)

HDZ NRW, Erich und Hanna Klessmann-Institut für Kardiovaskuläre Forschung und Entwicklung, Bad Oeynhausen, Germany (1); HDZ

$N R W$, Zentrum für Angeborene Herzfehler, Bad Oeynhausen, Germany (2); HDZ NRW, Klinik für Thorax- und

Kardiovaskularchirurgie, Bad Oeynhausen, Germany (3)

Introduction: Mutations in RBM20 are associated with dilated cardiomyopathy (DCM) and aberrant myocardial splicing. A German family with high prevalence of DCM was screened by next generation sequencing resulting in identification of the heterozygous mutation RBM20 p.P638L known for high penetrance and severe cardiomyopathy and/or sudden cardiac death in adults. However, little is known on its molecular pathomechanisms and disease course.

Methods: Aberrant myocardial splicing of TTN and RYR2 was analysed by Real-Time-PCR in 3 patients. To compare the localization of RBM20 wildtype and mutants RBM20-EYFP chimera was analyzed in different cell lines. The heterozygous genotype was modeled in vitro by coexpression of wildtype and mutant. Before genetic testing of 4 pediatric family members $(<1$, $1.5,10,14$ ys.) clinical investigation was done by echo. 
Results: Real-time-PCR revealed in mutation carriers aberrant myocardial splicing of RBM20 target genes - a hallmark of pathogenic RBM20 mutations. In vitro lack of nuclear localization for RBM20 p.P638L suggests a relevant molecular pathomechanism. Interestingly, coexpression of wildtype and mutant RBM20 had no influence on the subcellular localization of the wildtype protein. In both young children aged 2-3 ys. first evidence of subclinical mild DCM was identified by echo. In both adolescents stress echocardiography revealed pathological hemodynamics. The younger of both was later mechanically bridged to heart transplantation (HTx) aged 24. The older received ICDimplantation and is in supervision for HTx.

Conclusions: Detection of myocardial splice defects have impact for the identification of pathogenic RMB20 mutations. RBM20 p.P638L leads to subcellular mislocalisation presumably causing aberrant myocardial splicing, is associated with early onset cardiomyopathy and high penetrance. Echocardiographic evidence was found in early childhood, deterioration under stress in adolescence and terminal heart failure in early adulthood. For RBM20 mutations early onset of cardiac phenotypes and genetic predictive testing for RBM20 in early childhood should be considered. Identification of the molecular pathomechanisms in the affected patients allows insights into the disease mechanisms and may reveal drug targets for preventive or even curative treatment.

\section{P-26 \\ Treatment options of resistent forms of Kawasaki disease, our experience \\ Djukic D., Stanimirovic B., Ljuboja O. \\ University Clinical Center of Republic of Srpska, Banja Luka, Bosnia and Herzegovina}

Introduction: Kawasaki syndrome is an acute vasculitis characterized by fever, bilateral non purulent conjunctivitis, erythema of the mouth, rash and cervical lymphadenopathy. In 15 to $20 \%$ of untreated children, ectasia or coronary artery aneurysms may develop. The most difficult complications are ischemic heart disease and sudden cardiac death.

Objective: The aim of this study is to describe clinical manifestations, laboratory investigations, therapeutic approach, complications and outcome in children with Kawasaki syndrome. Medical records of all children with Kawasaki syndrome during 8 years period (between 2009 and 2017) at the Pediatric Clinic Banja Luka were retrospectively reviewed. 20 children, 12 girls and 8 boys were analyzed. The youngest child was 4 months old, and the oldest child was 7 years old. The most common clinical manifestations were: fever 20/20 (100\%), rush 15/20 (75\%), conjunctivitis $12 / 20(60 \%)$, lips and buccal mucosa hyperaemia 13/20 (65\%), gastrointestinal 9/20 (45\%), cervical lymphadenopathy $8 / 20$ (40\%) and renal involvement $8 / 20(40 \%)$. In $8 / 20(40 \%)$ of patients had coronary involvement. Coronary aneurysm occurred in $5 / 20$ ( $25 \%$ ) of children. 15/20 (75\%) of children had elevated parameters of inflammation. All 20 children were treated with i.v. immunoglobulins (IG) with acetyl salicylic acid. In 2 children, a second dose of IG and methylprednisolone were administered. Two patients required administration of Anti-TNF alpfa monoclonal antybodies-infliximab, given as a single dose, with favourable outcome. During follow-up period 4 children (20\%) had persistent aneurysmal enlargement of the coronary arteries. During the study period no death was reported.

Conclusion: In eight children 8/20 (40\%) who developed coronary involvement, the therapy was started late, after the seventh day of the onset of the disease. The most complicated patient was youngest 4 months old infant who received therapy at the latest from all patients (Day 13). There was no significant difference in clinical manifestations, laboratory results, therapeutic approach and outcome in children with Kawasaki Syndrome compared to other studies.

\section{P-27}

Unexpected results of panel targeted next generation sequencing (NGS) in patients with cardiomyopathies Meliota G. (1), Lombardi M. (1), Varvara D. (2), Resta N. (2), Tagliente M.R. (1), and Vairo U. (1) Cardiologia Pediatrica, Ospedale Pediatrico Giovanni XXIII, Bari, Italy (1); Medical Genetics, Department of Biomedical Sciences and Human Oncology, University of Bari "A. Moro", Bari, Italy (2)

Introduction: Next Generation Sequencing (NGS) panel targeted strategy is increasingly being used to identify mutations associated with cardiomyopathies.

Methods: We carried out NGS-analysis on PGM-Ion Torrent platform in 24 probands with cardiomyopathies, using a panel targeted approach which included 21 disease-causing genes.

Results: We identified at least one pathogenic/likely pathogenic variant in 6 cases, at least one variant of uncertain significance (VUS) in 8 cases, and a co-detection of pathogenic/likely pathogenic variants/VUS in 5 cases. To get insight into the clinical role of the genetic variants detected in the probands, co-segregation analysis was performed in family members.

Case series

Case 1. A 9-year-old boy was diagnosed with Hypertrophic Cardiomyopathy (HCM) when he was 2 year-old. His father was diagnosed with HCM at the age of 20 and suddenly died at the age of 32 years. Molecular testing of the child detected a likely pathogenic variant in TRPM4 (c.1744G > A; p.Gly582Ser) and a VUS in PDLIM3 (c.937C > T; p.Arg313Trp). Despite a negative familiar history and an unremarkable cardiac work-up, the genetic analysis of the mother's proband showed that she carried the variant in TRPM4.

Case 2. A 9-year-old girl was referred to us for acute myocarditis, which later evolved in dilative cardiomiopathy. A previous echocardiogram work-up performed because of a familial history of HCM had turned out unremarkably. Genetic analysis revealed a pathogenetic variant, VCL C.829 C>A; P. Leu277Met. Although her father carried the same genetic variant at genetic screening, he did not suffer of any cardiac disorder. Conversely, her mother was affected by hypertrophic cardiomyopathy, but did not carry her daughter's genetic variant. It has been reported that genetic defects in structural proteins may predispose the myocardium to the induction of inflammation by a pathogenic agent, i.e. the common viral infections of children.

Conclusion: Despite its high diagnostic value, interpretation of NGS-based analysis in patients with cardiomiopathy is often challenging because of unexpected results, such as multiple variants in the same individual or VUS. For a better evaluation of the clinical significance of genetic variants obtained by NGS-based strategy in the clinical practice, pooled data from collaborative studies are required.

\section{P-28 \\ First results from a prospective clinical trial measuring effects of hypothermia for organprotection during cardiac surgery - A new biomarker: Cold response protein RBM3 is upregulated after mild-to-moderate hypothermia during cardiopulmonary bypass \\ Rosenthal L.M. (1,2), Tong G. (1) Wowro S. (1) Pfitzer C. $(1,2,3)$} Berger F. $(1,3,4)$, Schmitt K.R. $(1,3)$ 
(1) Department of Congenital Heart Disease and Paediatric Cardiology, Deutsches Herzzentrum Berlin, Germany. (2) Berlin Institute of Health (BIH), Berlin, Germany. (3) DHZK (German Centre for Cardiovascular Research), partner site Berlin, Germany. (4) Dpartment of Paediatric Cardiology, Charité - Universitaetsmedizin Berlin, Germany

Introduction: Therapeutic hypothermia during cardiac surgery has been widely used for neuroprotection and to attenuate the systemic inflammatory response to cardiopulmonary bypass (CPB). Whereas global protein synthesis is repressed when the body temperature is decreased, a small group of RNA-binding proteins such as RNA-binding motif 3 (RBM3) is induced in response to hypothermia. Experimental data suggests that RBM3 plays a key role in hypothermia-induced neuroprotection. To date all research on RBM3 was performed in vitro or in animal models. Detection and regulation of RBM3 expression in human blood has not been investigated until now. The aim of this study was to investigate RBM3-regulation and cytokine dynamics in patient's blood in response to $\mathrm{CPB}$ and hypothermia.

Methods: A single-center prospective trial with 23 patients undergoing cardiac surgery at German Heart Institute Berlin, Germany was performed. Blood samples were collected from the central venous line during the induction of anesthesia, after weaning from $\mathrm{CPB}$ and 24 hours later. RBM3 protein was quantified in blood samples of patients and from 14 healthy individuals employing an Enzyme-linked Immunosorbant Assay (ELISA). Cytokine levels were analysed via Dry Blood Spot Sample collection (DBS) and enzyme immunoassay (EIA).

Results: RBM3 protein was detectable in human blood of healthy individuals and patient's with congenital heart disease (CHD). Hereby, RBM3 was significantly upregulated in response to CPB and application of mild-to-moderate hypothermia $\left(32-34{ }^{\circ} \mathrm{C}\right)$ after weaning from $\mathrm{CPB}$. A complex immune reaction with significant induction of proinflammatory cytokines and molecules (IL-1 beta, IL-6, IL-8, IL-12p40, IL-16, IL-18, MCP-1, CCL3, CCL4, ICAM-1) in response to CPB and activation of neutrophil cells was detected. Additionally significant elevated VEGF-levels reflecting ischemia-reperfusion-injury and significant reduced coagulation factors due to consumption coagulopathy were observed 24 hours after weaning of CPB.

Conclusions: Despite many technological improvements cardiac surgery with application of CPB remains associated with severe inflammatory response. Cold response protein RBM3 protein can be measured in human blood and is up-regulated after application of mild-to-moderate hypothermia during cardiac surgery employing CPB. RBM3 is therefore a promising new biomarker for hypothermia treatment and a possible new therapeutic target for interventional strategies during $\mathrm{CPB}$.

\section{P-30}

Prevalence of Type D personality in obese adolescents and associated cardiovascular risk

Bruyndonckx L. (1,2), Marchau F. (1), Verhulst S. (1,2),

De Guchtenaere A. (3), Vrints C. (4), Denollet J. $(4,5)$

Department of Paediatrics, Antwerp University Hospital, Belgium (1); Laboratory of Experimental Medicine and Paediatrics, University of Antwerp, Belgium (2); Zeepreventorium, De Haan Belgium (3); Department of Cardiology, Antwerp University Hospital, Belgium (4); CoRPS - Center of research on Psychology in Somatic diseases, Tilburg University, The Netherlands (5)

Introduction: Type D personality refers to the joint tendency to experience negative emotions (negative affectivity) and to inhibit self-expression in social interaction (social inhibition. Type D has been associated with increased cardiovascular risk at adult age, as well as increased levels of depression and anxiety, and more unhealthy behaviours such as a sedentary lifestyle. Little is known about Type D personality and cardiovascular risk at adolescent age. Obese adolescents already display increased cardiovascular risk, including vascular stiffness, endothelial dysfunction, and reduced levels of endothelial progenitor cells (EPC) and endothelial microparticles (EMP). We examined whether Type D personality also contributes to cardiovascular risk among severely obese adolescents.

Methods: Fasting blood samples were taken from obese and healthy children. Peripheral arterial tonometry was used to measure endothelial function. EMP (CD31 +/CD42b- particles) and EPC (CD34 +/KDR +/CD45dim/- cells) were quantified using flow cytometry. Established psychological questionnaires were filled in by the participants.

Results: Obese and normal weight adolescents were comparable for age and sex distribution. Obese adolescents demonstrated worse cardiovascular risk profile with lower HDL concentration, higher CRP, higher systolic blood pressure and pulse wave velocity, endothelial dysfunction, lower EPCs and more EMPs (Table) Type D personality was more prevalent in obese adolescents than in normal weight adolescents $(38 \%$ vs. $17 \% \mathrm{p}=.04)$, as well as symptoms of depression $(43 \%$ vs. $10 \% \mathrm{p}=.002)$.

In obese adolescents, a significant positive correlation was found between negative affectivity and vascular stiffness $(\mathrm{r}=0.28$; $\mathrm{p}=.04$ ), and a negative correlation between social inhibition and numbers of EPC $(r=-.39 ; p=0.04)$. The correlation between social inhibition and reduced EPC numbers was independent of BMI; i.e., social inhibition correlated with a lower BMI $(r=-.27$; $\mathrm{p}=.04)$. Depressive symptoms were not associated with vascular stiffness or EPCs in obese adolescents, and these correlations were not present in normal weight adolescents.

Conclusions: Characteristics of Type D personality are more prevalent in obese adolescents than in their normal weight counterparts. In addition, social inhibition and negative affectivity are associated with cardiovascular risk factors, even at very young age.

Table.

\begin{tabular}{|c|c|c|c|}
\hline & $\begin{array}{l}\text { Normal Weight } \\
\text { Children }(n=30)\end{array}$ & $\begin{array}{l}\text { Obese Children } \\
(n=57)\end{array}$ & $\begin{array}{l}p \\
\text { value }\end{array}$ \\
\hline Female/Male (n) & $21 / 9$ & $42 / 15$ & 0.715 \\
\hline Age (years) & $15.4 \pm 1.5$ & $15.2 \pm 1.4$ & 0.585 \\
\hline $\begin{array}{l}\text { Type D personality } \\
(\%)\end{array}$ & $17 \%$ & $38 \%$ & 0.040 \\
\hline $\begin{array}{l}\text { Depressive } \\
\text { symptoms }\end{array}$ & $10 \%$ & $43 \%$ & 0.002 \\
\hline BMI $\left(\mathrm{kg} / \mathrm{m}^{2}\right)$ & $19.17(17.58 ; 20.65)$ & $\begin{array}{l}35.21(32.05 ; \\
\quad 38.94)\end{array}$ & $<0.001$ \\
\hline \multicolumn{4}{|l|}{$\begin{array}{l}\text { Laboratory } \\
\text { parameters }\end{array}$} \\
\hline $\begin{array}{l}\text { HDL Cholesterol } \\
(\mathrm{mg} / \mathrm{dl})\end{array}$ & $56.9(49.8 ; 65.2)$ & $42.0(36.0 ; 49.0)$ & $<0.001$ \\
\hline $\begin{array}{l}\text { LDL Cholesterol } \\
(\mathrm{mg} / \mathrm{dl})\end{array}$ & $78.1(69.4 ; 88.1)$ & $83.1(73.7 ; 100.1)$ & 0.127 \\
\hline hsCRP (mg/dl) & $0.04(0.02 ; 0.11)$ & $0.24(0.15 ; 0.50)$ & $<0.001$ \\
\hline \multicolumn{4}{|l|}{ Vascular parameters } \\
\hline $\begin{array}{l}\text { Systolic BP } \\
(\mathrm{mm} \mathrm{Hg})\end{array}$ & $111 \pm 8$ & $120 \pm 10$ & $<0.001$ \\
\hline $\begin{array}{l}\text { Diastolic BP } \\
\text { (mm Hg) }\end{array}$ & $64 \pm 5$ & $64 \pm 7$ & 0.956 \\
\hline $\mathrm{PWV}(\mathrm{m} / \mathrm{s})$ & $5.2(4.9 ; 5.7)$ & $5.9(5.4 ; 6.7)$ & $<0.001$ \\
\hline Peak Response (AU) & $2.15(1.37 ; 2.97)$ & $1.50(1.24 ; 1.95)$ & 0.005 \\
\hline $\mathrm{EPC}\left(\# / 10^{6} \mathrm{MNC}\right)$ & $27.1(22.0 ; 42.9)$ & $16.8(12.3 ; 25.2)$ & 0.001 \\
\hline $\operatorname{EMP}(\# / \mu \mathrm{l})$ & $215.7(149.6 ; 255.6)$ & $\begin{array}{c}269.0(195.3 \\
\quad 350.0)\end{array}$ & 0.005 \\
\hline
\end{tabular}




\section{P-31}

Assessment of aortic dilatation and dissection in children with Marfan syndrome

Wozniak-Mielczarek L. (1), Sabiniewicz R. (1),Drezek-Nojowicz M. (2), Nowak R. (3), Gilis-Malinowska N. (4), Wierzba J. (5)

Department of Pediatric Cardiology and Congenital Heart Diseases, 1. Medical University of Gdansk, Gdansk, Poland; 2. Department of Ophthalmology, Medical University of Gdansk, Gdansk, Poland; 3. Department of Cardiosurgery, Medical University of Gdansk, Gdansk, Poland; 4. 1st Department of Cardiology, Medical University of Gdansk, Gdansk, Poland; 5. Department of Pediatrics, Hematology, Oncology, Medical University of Gdansk, Gdansk, Poland

Introduction: Marfan syndrome (MFS) is a connective tissue disorder characterized by a broad range of clinical manifestations. Cardiovascular involvement is the most serious life-threatening aspect of the syndrome. Although it is well studied in adults, there is paucity of data in children. The aim of the study was to evaluate the rate of aortic dilatation and dissection in children with MFS. Methods: Study population included 133 children, who were referred with suspicion of MFS. All patients underwent clinical evaluation (physical, ophthalmological, orthopedic, genetic and cardiac examination). Aortic diameter was measured with transthoracic echocardiography at eight different levels using two techniques (leadingedge in end-diastole and inner-edge in mid-systole). The modified Ghent criteria were used to identify patients with MFS.

Results: MFS was recognized in 40 children (5 days - 17 years, mean 9 years). The rest were categorized as Ehlers-Danlos syndrome $(n=20)$, Loeys-Dietz syndrome $(n=6)$, marfanoid $(n=57)$ and other $(\mathrm{n}=10)$. Among patients with MFS aortic root dilatation $(\mathrm{z}$-score $\geq 2)$ at the level of sinus of Valsalva was found in $32(80 \%)$, while aortic diameter was at the upper limit of normal range $(z$-score $=1,9-1,99)$ in $4(10 \%)$. Surprisingly aortic root dilation was similarly frequent in different age groups: $0-7$ years $(86,67 \%), 8-13$ years $(66,67 \%), 14-17$ years $(84,6 \%)$. Aortic root dilatation ranged from $+2,14$ to $+6,30$, mean $+3,29$ (z-score). The degree of dilatation doesn't depend on patient's sex, age and type of mutation (de novo or familiar). The other segments of ascending aorta were dilated less frequently: valve annulus $(10 \%)$, sinotubular junction (25\%), ascending aorta $(22,5 \%)$. Only in 1 child $(2,5 \%)$ entire aorta was dilated. Within 2-year follow-up in 1 patient $(2.5 \%)$ aortic dissection occured and 1 patient $(2,5 \%)$ underwent elective David procedure.

Conclusions: Aortic dilatation at the level of sinus of Valsalva occurs in most children with MFS and is present since early childhood. There is no linear correlation between the dilation of the aortic root and the patient's age. Serious aortic dilatation can be found in infant, however, in children aortic dissection occurs infrequently and aortic surgery is rarely needed.

\section{P-32}

\section{Comparison of cardiovascular system status between} children and adults with Marfan syndrome

Wozniak-Mielczarek L. (1), Sabiniewicz R. (1),Drezek-Nojowicz M. (2), Nowak R. (3), Gilis-Malinowska N. (4), Wierzba J. (5)

1. Department of Pediatric Cardiology and Congenital Heart Diseases, Medical University of Gdansk, Gdansk, Poland; 2. Department of Ophthalmology, Medical University of Gdansk, Gdansk, Poland; 3. Department of Cardiosurgery, Medical University of Gdansk, Gdansk Poland; 4. First Department of Cardiology, Medical University of Gdansk, Gdansk, Poland; 5. Department of Pediatrics, Hematology, Oncology, Medical University of Gdansk, Gdansk, Poland

Introduction: Marfan syndrome (MFS) is connective tissue genetic disorder caused by mutations in FBN1 gene. MFS affects mainly the cardiovascular, skeletal and ocular system. Cardiovascular complications are the most serious life-threatening aspect of the syndrome. It is still unclear whether cardiovascular manifestation of MFS differs significantly between children and adults.

Methods: The study population included 230 patients (133 children and 97 adults), who were referred with suspicion of MFS. All patients underwent complete clinical evaluation including cardiac examination (ECG, Holter ECG, and transthoracic echocardiography), ophthalmological, orthopedic and genetic consultations. The modified Ghent criteria were used to identify patients with MFS.

Results: MFS was recognized in 105 patients (40 children and 65 adults). The rest were categorized as Ehlers-Danlos syndrome $(n=25)$, Loeys-Dietz syndrome $(n=7)$, marfanoid $(n=79)$ and other $(\mathrm{n}=14)$. Among MFS patients the most common cardiovascular abnormality was aortic root dilation, which was similarly frequent in adults and children $(86,05 \%$ vs $82,14 \%, \mathrm{p}=0,742)$. It was found that dilation of the aortic root progresses with age (mean z-score in children: $+3,18 \pm 0,97$, in adults: $+4,56 \pm 1,92)$, however without linear correlation. In contrast to aortic root dilation, aneurysms of aortic arch and descending aorta (both thoracic and abdominal) were much more common in adults than in children ( $20 \%$ vs $2,5 \%)$. Similarly, aortic dissections occur more often in adults than in children (12,5\% vs $2,5 \%)$. There was no significant difference between children and adults with regard to aortic regurgitation (AR; 37,04\% vs 37,93\%, p =1,000), mitral regurgitation (MR; $57,14 \%$ vs $78,05 \%, \quad p=0,109)$ and mitral valve prolapse (MVP; 71,43\% vs 60\%, $\mathrm{p}=0,441$ ). Suprisingly, pulmonary artery dilatation (PAd) was much more common in adults than in children $(44,19 \%$ vs $25 \%$, $\mathrm{p}=0,035)$.

Conclusions: Both adults and children have similar high rates of aortic root dilatation, but the degree of dilatation is much higher in adults. Dilation of aortic arch and descending aorta, the same as aortic dissection occur more common in adults. The other cardiac abnormalities (AR, MR, MVP) don't differ between the groups. The only exeption is PAd which is much more frequent in adults.

P-33

Investigation of the effect of aortic stiffening and stenosis on ventriculo-arterial interaction in a porcine model of Coarctation repair

Panzer J., De Wolf D., De Somer F., Roossens A., Segers P., Bové T. University Hospital Gent - Gent - Belgium

Introduction: Systemic hypertension is commonly seen despite successful coarctation repair, regardless whether the treatment consisted of surgery or percutaneous stenting. This study aimed to investigate the effect of short- versus long-segment aortic stiffness and stenosis as sequelae of aortic coarctation repair on ventriculoarterial interaction in an animal model

Methods: Short- and long-segment aortic stiffening was created by surgical transection/suture (SUTURE, $n=6$ ) and stent implantation (STENT, $n=5)$ of the proximal descending aorta. Short and long aortic stenosis was realized by wrapping a prosthetic graft around the aorta to reduce the circumference with $1 / 3$, over respectively $1 \mathrm{~cm}$ (SUTURESTENOSIS, $\mathrm{n}=5$ ) and $5 \mathrm{~cm}$ (STENTSTENOSIS, $\mathrm{n}=6$ ). A CONTROL-group comprised 5 animals. After 3 months, measurements consisted of aortic pressure- and flow-recordings proximally and distally from the lesion, and aortic distensibility by intravascular ultrasound. LV-performance was based on PV-loops. Baseline measurements were compared to measurements during dobutamine stimulation.

Results: Data are presented in table. An effective stenosis increases significantly the proximal aortic impedance, resulting in an increased 
backward-to-forward pressure-wave ratio contribution. This phenomenon is accentuated by dobutamine administration. Proximal aortic distensibility is decreased specifically in long-segment stenosis. The increased afterload impairs the ventriculo-arterial coupling in both groups with stenosis, with blunted contractile response during dobutamine (CONTROL $+140 \%$, SUTURE $+103 \%$, SUTURE STENOSIS $+77 \%$, STENT $+116 \%$, STENT STENOSIS $+57 \%$, $\mathrm{p}<0.001)$ and increased myocardial stiffness in long aortic stenosis. Histology showed increased myocardial fibrosis in the group SUTURE STENOSIS and STENT STENOSIS.

Conclusion: This animal study, in which sequelae of aortic coarctation repair were reproduced, demonstrated that short- or longsegment aortic stiffening without stenosis had no significant effect on aortic pressure-flow characteristics. However, the negative effect of stenosis on aortic hemodynamics - especially for a longer segment - leads to rapid impairment of the ventriculo-arterial interaction, which is accentuated during inotropic stimulation. Therefore, therapeutical management needs to focus on improving aortic remodeling after coarctation repair, preferably by minimizing residual stenosis, even if it is at the cost of inducing aortic stiffness.

Table.

\begin{tabular}{|c|c|c|c|}
\hline & CONTROL & SUTURE & $\begin{array}{l}\text { SUTURE } \\
\text { STENOSIS }\end{array}$ \\
\hline \multicolumn{4}{|c|}{ Impedance $\mathrm{Zc}(\mathrm{mmHG} / \mathrm{ml} / \mathrm{s})$} \\
\hline Baseline & $0.18(0.02)$ & $0.15(0.14)$ & $0.27(0.19)$ \\
\hline Dobutamine & $0.14(0.02)$ & $0.14(0.02)$ & $0.31(0.03)$ \\
\hline \multicolumn{4}{|c|}{ Forward P-wave (mmHg) } \\
\hline Baseline & $31.4(2.9)$ & $32.9(2.6)$ & $39.4(3.9)$ \\
\hline Dobutamine & $44.9(2.6)$ & $44.6(4.5)$ & $56.9(5.1)$ \\
\hline \multicolumn{4}{|c|}{ Backward P-wave (mmHg) } \\
\hline Baseline & $13.8(1.0)$ & $8.7(1.2)$ & $10.7(0.6)$ \\
\hline Dobutamine & $14.3(2.9)$ & $10.7(1.1)$ & $17.3(07)$ \\
\hline \multicolumn{4}{|c|}{ Aortic distensibility(\%) } \\
\hline Baseline & 27.1 & 24.4 & 12.7 \\
\hline Dobutamine & 32.1 & 26.6 & 14.8 \\
\hline \multicolumn{4}{|c|}{ Contractility (PRSW-Mw/s) } \\
\hline Baseline & $47.2(1.4)$ & $49.3(3.4)$ & $58.5(1.7)$ \\
\hline Dobutamine & $113.0(3.2)$ & $99.3(5.0)$ & $103.3(1.9)$ \\
\hline \multicolumn{4}{|c|}{ Myocardial stiffness $(\beta)$} \\
\hline Baseline & $0.17(0.02)$ & $0.18(0.02)$ & $0.22(0.01)$ \\
\hline \multirow[t]{2}{*}{ Dobutamine } & $0.12(0.01)$ & $0.13(0.02)$ & $0.19(0.01)$ \\
\hline & STENT & $\begin{array}{l}\text { STENT } \\
\text { STENOSIS }\end{array}$ & Anova-p \\
\hline \multicolumn{4}{|c|}{ Impedance $\mathrm{Zc}(\mathrm{mmHG} / \mathrm{ml} / \mathrm{s})$} \\
\hline Baseline & $0.18(0.03)$ & $0.27(0.04)$ & 0.009 \\
\hline Dobutamine & $0.15(0.03)$ & $0.39(0.07)$ & $<0.001$ \\
\hline \multicolumn{4}{|c|}{ Forward P-wave (mmHg) } \\
\hline Baseline & $32.6(3.3)$ & $41.3(2.3)$ & 0.089 \\
\hline Dobutamine & $41.4(4.9)$ & $63.3(3.0)$ & 0.004 \\
\hline \multicolumn{4}{|c|}{ Backward P-wave (mmHg) } \\
\hline Baseline & $10.1(2.2)$ & $17.2(1.0)$ & 0.001 \\
\hline Dobutamine & $11.5(2.3)$ & $31.7(2.6)$ & $<0.001$ \\
\hline \multicolumn{4}{|c|}{ Aortic distensibility(\%) } \\
\hline Baseline & 17.9 & 8.9 & $<0.001$ \\
\hline Dobutamine & 21.1 & 10.7 & $<0.001$ \\
\hline \multicolumn{4}{|c|}{ Contractility (PRSW-Mw/s) } \\
\hline Baseline & $50.4(1.9)$ & $46.1(5.5)$ & 0.142 \\
\hline Dobutamine & $108.5(4.5)$ & $71.6(8.1)$ & $<0.001$ \\
\hline \multicolumn{4}{|c|}{ Myocardial stiffness $(\beta)$} \\
\hline Baseline & $0.26(0.05)$ & $0.17(0.02)$ & 0.120 \\
\hline Dobutamine & $0.20(0.04)$ & $0.21(0.03)$ & 0.118 \\
\hline
\end{tabular}

\section{P-34}

Decreased exercise tolerance in children after aortic coarctation repair: relationship with changes in cerebral and muscle oxygenation

Vandekerckhove K. (1), Coomans I. (1), Moerman A. (2), Panzer J. (1), De Groote K. (1), De Wilde H. (1), Bove T. (3), François K. (3), De Wolf D. (1), Boone J. (4)

Department of Pediatric Cardiology, Ghent University Hospital, Ghent, Belgium (1); Department of Anesthesiology, Ghent University Hospital,
Ghent, Belgium (2); Department of Cardiac Surgery, Ghent University Hospital, Ghent, Belgium (3); Department of Movement and Sports Sciences, Ghent University, Ghent, Belgium (4)

Introduction: Children after coarctation repair have lower exercise performance compared to their healthy peers. It is unknown whether there are differences in oxygen transportation and extraction at the local cerebral and muscular level during exercise in this patient group.

Methods: 16 children after coarctation repair were compared with 20 healthy peers. Near infrared spectroscopy was used to assess oxygenation at the left $\mathrm{M}$. Vastus medialis and at the prefrontal lobe. Changes of $\mathrm{HHb}$ and $\mathrm{O}_{2} \mathrm{HB}$ in relation to load were compared in both groups; correlation between residual continuous wave Doppler gradient and local oxygenation was evaluated.

Results: Age, length and weight was similar in both groups. Patients with aortic coarctation had lower \%Ppeak $(72.3 \pm 20.2 \%$ vs. $\quad 106 \pm 18.7 \%, \quad \mathrm{P}<0.001), \quad \mathrm{VO}_{2} / \mathrm{kg} \quad(37.3 \pm 9.1 \quad$ vs. $44.2 \pm 7.6 \mathrm{ml} / \mathrm{kg}, \mathrm{P}=0.019)$ and $\% \mathrm{VO}_{2} / \mathrm{kg}(85.7 \pm 21.9$ vs. $112.1 \pm 15.5, \mathrm{P}<0.001)$. Cerebral $\mathrm{O} 2 \mathrm{Hb}$ and $\mathrm{HHb}$ had a higher increase in both curves during exercise, with significant differences from $60 \%\left(\mathrm{O}_{2} \mathrm{Hb}\right)$ and $80 \%(\mathrm{HHb})$ of the peak exercise compared to controls. Muscle $\mathrm{O}_{2} \mathrm{Hb}$ was not different between both groups, but muscle $\mathrm{HHb}$ had a significantly steeper increase, with $\Delta$ muscle $\mathrm{HHb} / \Delta \mathrm{P}$ significantly different from $10 \%$ of the exercise onwards. There was a weak but significant correlation between residual Doppler gradient measured by echocardiography and changes in muscle $\mathrm{HHb}$ at $10-20 \%$ and $20-30 \%$ of peak exercise $(\mathrm{r}=0.37, \mathrm{P}=0.42$ and $\mathrm{r}=0.38, \mathrm{P}=0.40)$

Conclusion: Children after coarctation repair have different patterns of oxygenated and deoxygenated hemoglobin at the level of the brains and a different pattern of deoxygenated hemoglobin at the muscular level. The increased muscular deoxygenation is more pronounced in children with higher residual coarctation gradient on echocardiography.

\section{P-35}

Using patient-derived induced pluripotent stem cells to model left ventricular outflow obstruction defects

Helle E. (1,2), Ampuja M. (1), Balboa D. (3), Eurola S. (3), Ojala T. (2), Otonkoski T. (3), Kivelä R. (1)

Research Programs Unit, Translational Cancer Biology Program, Faculty of Medicine, University of Helsinki, and Wihuri Research Institute, Finland (1); Helsinki University Children's Hospital, University of Helsinki, Helsinki, Finland (2); Research Programs Unit, Molecular Neurology and Biomedicum Stem Cell Centre, Faculty of Medicine, University of Helsinki, Finland (3)

Introduction: Left ventricular outflow tract obstruction (LVOTO) is a subtype of congenital heart disease affecting one or more structures on the left side of the heart - left ventricle, aortic valve and thoracic aorta. At its most severe, LVOTO defects manifest as hypoplastic left heart syndrome (HLHS), in which the left ventricle is underdeveloped, and the systemic circulation depends on the persistence of fetal circulatory physiology. LVOTO defects are at least partially genetic in origin, as they are found to cluster in families.

We have sequenced the exomes of a cohort of 49 LVOTO patients and their family members. We have identified a very rare gene variant that segregates with disease in two unrelated families with multiple affected members with LVOTO defects. This gene has been shown to be important for heart development in mice and zebrafish, but not yet in humans. 
We are studying the impact of this gene variant and the gene itself in cardiomyocyte (CM) and endothelial cell (EC) development and function using patient-derived induced pluripotent stem cells (iPSCs). We hypothesize that the gene is important in cardiac development, affecting either CMs or ECs or both, as ECs have been shown to contribute to CM growth and maturation.

Methods: We obtained iPSCs from a HLHS patient with the rare gene variant and his unaffected mother, and control iPSCs from a healthy donor. We made knockout and heterozygous cell lines for the target gene from the control iPSCs by using CRISPR/Cas9. We have differentiated iPSCs to CMs and ECs, and studied their differentiation by immunohistochemistry and qPCR.

Results: Our results indicate that the patient cells are able to differentiate to both CMs and ECs, however, there are differences in cardiac gene expression patterns during differentiation. Currently, we are analyzing the knockout and heterozygous cell lines in comparison with the patient and control cell lines.

Conclusions: Our results indicate that disease modeling in patientderived iPSCs is a promising method to evaluate the pathogenicity of candidate genes in congenital heart disease.

\section{P-36}

Fetal Echocardiography as a "genetic screening" tool? Germanakis I. , Burianova Bagaki A., Mataliotakis I., Makrigianakis A. Faculty of Medicine, University of Crete, Heraklion, Greece

Introduction: Amniocentesis is routinely recommended in pregnancies with increased risk for fetal karyotype abnormalities (fKA), as assessed by maternal age combined with biochemical and sonographic indexes. Fetal Echocardiography (fEcho), performed upon established indications (EI) allows for fetal congenital heart disease (fCHD) detection, often independently associated with fKA. We evaluated whether the detection of fCHD increases the risk for fCA in pregnancies with and without EI for fEcho.

Methods: Retrospective study of 2,202 fEcho records (years 200816) from a tertiary referral centre, regarding 1 ) referral indications, classified as established (EI) when based on recent recommendations (AHA 2014) or non-established (NEI), in remaining cases 2) diagnosis of fCHD (excluding ventricular or great artery disproportion, inflow valve regurgitation, pericardial fluid, PDA constriction, arrhythmias), 3) results of fetal karyotype (prior or following fEcho).

The prevalence of abnormal karyotype in the presence/absence of fCHD was compared, in subgroups of EI $(+)$ and EI (-) fEcho cases (Pearson Chi-square test, Odds Ratios-O.R)

Results: Included were 258 studies with complete referral data, fEcho diagnoses and available fetal karyotype results.

The prevalence of fetal karyotype abnormalities, in presence vs absence of fCHD respectively, was: A) $15 \%$ vs $10.6 \%$ in the whole group (O.R: 1.5, 95\% C.I:0.7-3.1, p=0.282, n.s) B) $18.2 \%$ vs $14 \%$ for EI $(+)$ cases (O.R: 1.4, 95\% C.I:0.6-2.9, p = 0.429, n.s), C) 4\% vs $2.4 \%$, for EI (-) cases (O.R:1.6, 95\%C.I:0.1-27, p = 0.72, n.s).

Cases having established indications for $\mathrm{fEcho}(\mathrm{EI}+)$ had a significant association with the presence of fKA (16.6\%) compared to cases where fECHO was "routinely" performed (3\%), O.R: 6.3, 95\% C.I:1.4-7.3, $\mathrm{p}=0.005$ )

Conclusions: The detection of fCHD during fetal echocardiography is associated with an increased trend ( 1.5 fold) for fetal karyotype abnormalities. However, the strongest predictor (6-fold increased risk) is the presence of an established indication for fetal echocardiography itself. Although fetal echocardiography should not be used as a means of fetal karyotype "screening", still careful obstetric history talking might identify pregnancies that could benefit from both fetal echocardiography and fetal karyotyping.
Table.

\begin{tabular}{lccc}
\hline $\begin{array}{l}\text { Indication-based } \\
\text { fECHO }\end{array}$ & $\begin{array}{c}\text { Abnormal } \\
\text { fCHD }\end{array}$ & $\begin{array}{l}\text { Normal } \\
\text { Karyotype }\end{array}$ \\
\hline \multirow{2}{*}{ yes } & yes & 18 & 81 \\
& no & 13 & 80 \\
no & yes & 1 & 24 \\
& no & 1 & 40 \\
\hline
\end{tabular}

\section{P-37}

First Report of Autosomal Recessive Catecholaminergic Polymorphic Ventricular Tachycardia (CPVT1), Caused by Homozygous Mutation in the RYR2 Gene

Ta-Shma A., Shauer A., Perles Z., Elitzur Y., Ta-Shma A., Elpeleg O., Luria D.

Hadassah, Hebrew University Medical Center, Jerusalem, Israel

Introduction: Catecholaminergic Polymorphic Ventricular Tachycardia (CPVT) is a rare tachyarrhythmia typically presenting with bidirectional ventricular tachycardia, appearing during physical or emotional stress. Mutations in the Ryanodine receptor gene, RYR2, are known to cause an autosomal dominant type of the disease (CPVT1). As part of our effort to elucidate the molecular basis of cardiac anomalies in consanguineous families, we studied the genetic basis of CPVT in a consanguineous family with healthy parents and two siblings who presented with cardiac arrest and had a diagnosis of CPVT.

Methods: We obtained thorough family history and complete patients' phenotype including: ECG, stress tests, echocardiography. We did comprehensive genotyping with the use of Whole Exome Sequencing analysis and verification was done with Sanger sequencing.

Results: A nineteen years old asymptomatic male presented with cardiac arrest during physical stress. While visiting him in the intensive care unit, his thirteen years old sister experienced major emotional stress, and suffered from cardiac arrest as well. Thorough family workup including ECG, stress test and echocardiography, revealed asymptomatic parents and siblings, one of which was found to have multifocal premature ventricular contractions (PVCs) during stress test. Echocardiography for all family members revealed normal cardiac anatomy. The three affected members underwent implantable cardiac defibrillator (ICD) implantation and received metoprolol therapy. During nine years of follow up, the female had an episode of multiple ventricular fibrillation and shocks. The Metoprolol dose was increased and no further episodes occurred.

Whole Exome Sequencing identified homozygous Gly3118Arg missense mutation in the RYR2 gene. The mutation segregated with the phenotype in the family: The affected members were homozygous for the mutation whereas the non-affected parents and siblings were heterozygous. The mutation is located in the cytosolic component of the RYR 2 protein.

Conclusion: This is a first report of autosomal recessive CPVT1 caused by homozygous RYR2 mutation.

\section{P-38}

CAPZB - A novel player in heart development

Radloff A. (1), Breckpot J. (2), Hoff K. (1), Kramer H.-H. (1), Panakova D. (3), Mercks A.M. (3), Devriendt K. (2), Gewillig M. (4), Sealy I. (5), Collins J. (5), Busch-Nentwich E. (5), Kahlert A.-K. (1,6), Hitz M.-P. $(1,5)$

Department of Congenital Heart Disease and Pediatric Cardiology, Universitätsklinikum Schleswig-Holstein Kiel, Kiel, Germany and 
German Center for Cardiovascular Research (DZHK), Kiel, Germany (1); Center for Human Genetics, University Hospitals Leuven, Catholic University Leuven, Leuven, Belgium (2); Max-Delbrueck-Center for Molecular Medicine in the Helmholtz Association, Electrochemical Signaling in Development and Disease, Berlin, Germany and German Center for Cardiovascular Research (DZHK), Berlin, Germany (3); Department of Fetal and Pediatric Cardiology, University Hospitals Leuven, Leuven, Belgium (4); Wellcome Trust Sanger Institute, Wellcome Genome Campus, Hinxton, United Kingdom (5); Institute for Clinical Genetics, Faculty of Medicine Carl Gustav Carus, TU Dresden, Dresden, Germany (6)

Objectives: Congenital heart defects (CHD) are the most common birth defects in humans with an incidence of 8 per 1000 live births. In $25 \%$ of the cases, the CHD is life-threatening and needs intervention directly after birth or in the first year of life. The aetiology of CHD is still poorly understood. Here we present a new candidate gene for CHD - CAPZB -, which was identified in a patient with hypoplastic left heart syndrome (HLHS). CAPZB encodes the beta subunit of the fast growing-end actin binding protein, which belongs to the F-actin capping protein family. It regulates the assembly and disassembly of actin filaments, modulates the cytoskeleton as well as tethers actin filaments to the Z-line of the sarcomere and is therefore essential for muscle development.

Methods: Using exome sequencing, we identified CAPZB in a patient with HLHS. To understand the functional relevance of the mutation we used a mutant zebrafish. We performed cardiac output measurements on zebrafish larvae at different developmental stages. Additionally, to identify physiological and morphological defects, we performed immunofluorescence staining, electron microscopy and optical mapping. To determine differentially regulated genes influenced by CAPZB mutation we performed RNA-Seq on 48 hours (hpf) old zebrafish larvae.

Results: Zebrafish larvae being homozygous for the mutation (capzb-/-) died after 100 hours hpf. Heterozygous zebrafish (capzb $+/-)$ survived until adulthood and were used for husbandry. Mutant capzb-/- zebrafish larvae developed microcephaly, short stature, hypoplastic jaw and an elongated, unlooped heart at $96 \mathrm{hpf}$ compared to their heterozygous capzb $+/-$ and wildtype siblings. Cardiac output measurements showed an impaired contractility in capzb-/- compared to capzb +/- and wildtype larvae at $72 \mathrm{hpf}$, which was significantly reduced after 96 hpf. Moreover, capzb-/larvae showed physiological and morphological defects after $72 \mathrm{hpf}$ compared to capzb +/- and wildtype siblings. RNA-Seq revealed differentially expressed actin binding genes in capzb-/- larvae.

Conclusion: Loss of CAPZB in zebrafish results in physiological and morphological defects, which phenocopy part of the observed human HLHS phenotype of the identified patient. These results highlight the importance of CAPZB as a new candidate gene for CHD and show its role during early cardiac development.

\section{P-39}

Inflammation, growth, fibrosis and apoptosis in right atrial myocardium of infants with atrial septal defect

Rouatbi H. (1), Gerard A. (2), Heying R. (3), Vazquez-Jimenez J. (4), Seghay M-C. (1-5)

Departments of Pediatric Cardiology, University Hospital Liège, Belgium (1); GIGA Neurosciences, University of Liège, Belgium (2); Department of Pediatric Cardiology, University Hospital Leuven, Belgium (3); Department of Pediatric Cardiac Surgery, University Hospital Aachen, Germany (4); Department of Pediatrics, University Hospital Liège, Belgium (5)

Introduction: Atrial septal defect (ASD) is a relatively common congenital heart disease with an estimated prevalence 2 per 100 live births. Changes in atrial tissue properties, including interstitial fibrosis, increased myocyte size and alterations in ultracellular structure have been described in these patients. The exact mechanisms underlying this remodeling are not yet understood. The aim of this study was to explore mRNA expression of genes coding for inflammation (IL1 $\beta$, IL6, TNF $\alpha$ ), angiogenesis (HIF1 $\alpha$, VEGF), hypertrophy (CT-1, IGF1,), and apoptosis (Fas, BAK, Bcl-xL) in right atrial myocardium of children with ASD.

Patients and Methods: 12 infants with ASD were investigated with a mean age of 64,5 months. Myocardial Samples taken before institution of cardiopulmonary bypass were analyzed by Real time PCR to study the expression of genes involved in myocardial growth, inflammation, hypertrophy and apoptosis.

Results: All patients showed preoperative myocardial inflammation with expression of mRNA coding for IL $1 \beta$, IL 6 , and TNF $\alpha$. Myocardial remodeling led to hypertrophy, angiogenesis, and myocardial fibrosis with expression of mRNA coding for CT-1, HIF-1 $\alpha$, TGF $\beta$ and VEGF. Gene expression of extracellular proapoptotic factor FasL, intracellular apoptosis regulating factors Bak and $\mathrm{Bcl}-\mathrm{xL}$ were also detected.

There was a strong positive correlation between expression of IL1 $\beta$ and TGF $\beta$, respectively (rs: $0,91, \mathrm{p}<0,05)$. There was also an association between myocardial hypertrophy (CT-1) and the expression of the vascular stimulating factor HIF-1 $\alpha$ and the angiogenesis growth factor VEGF, respectively: Expression of CT-1 correlated with that of VEGF ( $r s=0.964 ; p<0.001$ ), and expression of HIF- $1 \alpha$ correlated with that of VEGF ( $\mathrm{rs}=1.0$, $\mathrm{p}=0.003$ )

Conclusion: Myocardial remodeling is a multi-factorial process involving inflammatory- and intracellular signaling pathways, which may have synergistic or antagonistic effects. At a stage of the disease, volume overload activates pro-apoptotic factors that will initiate transition from compensated hypertrophy to decompensated heart failure. This processes, which is highly regulated, has a primordial role in the pathophysiology of heart failure. Our results suggest that IL1 $\beta$ may regulate TGF $\beta$ expression as a feedback mechanism to limit extra cellular matrix degradation in response to injury.

\section{P-40}

The yield of genetic testing in paediatric hypertrophic cardiomyopathy

Field E. (1,2), Norrish G. (1,2), Cervi E. (1), Lord E. (1), Walsh H. (1), Fell H. (1), Jager J. (1,2), Cardenas I. (3), Cicerchia M. (3), Ochoa J.P. (3), Salazar J. (3), Kaski J.P. (1,2)

Centre for Inherited Cardiovascular Disease, Great Ormond Street Hospital, London, UK (1); University College London, London, UK (2); Cardiology Department, Health in Code, A Coruna, Spain (3)

Introduction: Genetic testing of patients with hypertrophic cardiomyopathy (HCM) is increasingly used to enable cascade screening of relatives and to shed light on underlying mechanisms of disease development and progression. We reviewed results of genetic panel testing in paediatric patients at our centre over a three-year period to establish the yield of testing and its diagnostic value.

Methods: Results of all panel tests undertaken in HCM patients between 2015 and 2017 were recorded in a database. Fifteen patients underwent basic panel tests (16-21 genes) and eighteen underwent extended panels (90-104 genes). Relevant clinical details were sourced from patient records.

Results: Thirty-three paediatric patients with HCM (aged 3 months to 17 years) underwent testing. Maximal left ventricular wall thickness in the group ranged from $8 \mathrm{~mm}$ to $30 \mathrm{~mm}$. Three patients had a history of VF arrest, thirteen had an ICD in situ, one 
had undergone surgical myectomy and one underwent cardiac transplantation during the data collection period. Twenty-one of these patients were the family proband.

In twenty-four patients a pathogenic mutation was detected (a positive yield of $72.7 \%$ ). In five patients, only variants of uncertain significance were detected, and in four cases no relevant mutations or variants were identified. Eleven patients with a pathogenic mutation had an additional variant of unknown significance in other panel genes.

Eleven patients had a pathogenic mutation in MYH7, six had a single mutation in MYBPC3 and three had two mutations in MYBPC3. Two patients had a TPM1 mutation and one had a TNNI3 mutation. One patient was found to have a mutation in FHL1, in keeping with his clinical phenotype. One patient with a severe cardiac phenotype and a previously identified DMD mutation was found to carry an additional MYH7 mutation. Conclusions: Genetic panel testing in paediatric HCM has a high positive yield and is of use in establishing underlying mechanisms of disease. It may be helpful in elucidating diagnoses when more than one disease process is contributing towards the HCM phenotype. All pathogenic mutations were found in the genes of the basic panel, however the additional variants detected may play a role as genetic modifiers, which warrants further investigation.

\section{P-41 \\ Clinical presentation and outcomes in paediatric hypertrophic cardiomyopathy patients with compound sarcomeric gene mutations or additional genetic variants Field E. (1,2), Norrish G. (1,2), Cervi E. (1), Lord E. (1), Walsh H. (1), Fell H. (1), Jager J. (1,2), Cardenas I. (3), Cicerchia M. (3), Ochoa J.P. (3), Salazar J. (3), Kaski J.P. (1,2) Centre for Inherited Cardiovascular Disease, Great Ormond Street Hospital, London, UK (1); University College London, London, UK (2);Cardiology Department, Health in Code, A Coruna, Spain (3)}

Introduction: Genetic panel testing of paediatric patients with hypertrophic cardiomyopathy (HCM) enables better understanding of causes of disease in individuals presenting with the condition at an early age. We aimed to examine clinical features of patients carrying multiple pathogenic mutations or with pathogenic mutations in combination with genetic variants of uncertain significance (VUS), in order to explore the significance of these combined genetic factors.

Methods: Between 2015 and 2017, thirty-three paediatric patients with a diagnosis of HCM underwent genetic panel testing at our centre. All genetic tests were processed and reported by the Health-in-Code laboratory in Spain. Test results and clinical records of patients were reviewed and information relating to cardiac phenotype was collated.

Results: Three proband children were found to carry two mutations in MYBPC3. In two of these, cascade screening revealed that one mutation was inherited from each (clinically unaffected) parent. All three had severe hypertrophy, and two had significant history of arrhythmia: one presenting with an out-of-hospital VF arrest aged three years and the other with repeated episodes of non-sustained VT on ambulatory monitoring.

Eleven patients had potentially relevant VUSs in addition to a pathogenic mutation. Variants were found in ten genes: MYBPC3, MYH7, ANKRD1, CSRP3, DES, DSP, FHOD3, FLNC, MYL3 and PKP2. Five of these children were family probands with severe disease. Their phenotypes included two with VF arrests, one requiring cardiac transplantation aged twelve and one presenting at seven years old with complete heart block requiring pacemaker implantation. In six patients with an additional VUS there was family history of HCM; one of these had a VF arrest aged fourteen. Maximal LV wall thickness and diastolic function appeared similar in patients with single mutations compared to those with additional VUSs.

Conclusions: In three cases, patients carried multiple MYBPC3 mutations, which appeared to correlate with a severe phenotype associated with increased risk of ventricular arrhythmia. In paediatric probands with a VUS detected in addition to a pathogenic mutation, disease also appeared more aggressive with an increased risk of ventricular arrhythmia, although detailed comparison to outcomes in single mutation carriers without additional variants will improve understanding of this apparent correlation.

\section{P-42}

Association of vasoactive peptides with the presence and severity of congenital heart disease in children

Papachristou P. (1), Grigoriadou G. (1), Papadopoulos G. (1), Georgakopoulos D. (1), Boutsikou M. (2,3)

Pediatric Cardiology Department, "PEA. Kyriakou" Childrens' Hospital, Athens, Greece (1). Adult Congenital Heart Disease and Pulmonary Hypertension Unit, Royal Brompton Hospital, London, UK (2). MRI Unit, Royal Brompton Hospital, London, UK (3)

Introduction: The course and outcome of congenital heart disease is highly dependent on early diagnosis and accurate assessment of severity. Vasoactive peptides may be useful in the initial evaluation of congenital heart disease, in the follow-up assessment and in the evaluation of the effectiveness of treatment.

Purpose: To compare brain natriuretic peptide (BNP), adrenomedullin (ADM) and urotensin II (UII) plasma levels in patients presenting with non-cyanotic heart disease, and to examine their potential relationship with the presence, the severity and the type of the disease.

Material-Methods: 78 consecutive subjects fulfilling the inclusion criteria [median age 11.56 (1.58-83.75) months], 39 with congenital heart disease - atrial septal defect (ASD), ventricular septal defect (VSD), patent ductus arteriosus (PDA)- and 39 age- and gender matched controls, were prospectively studied. Demographic clinical and echocardiographic data, as well as family, prenatal and medical history, were collected. Disease severity was assessed based on the size of the defect, the significance of shunt, the presence of concomitant anomalies, biventricular size and function as well as the presence of pulmonary hypertension. Plasma BNP, ADM and UII levels were measured using ELISA method.

Results: BNP levels (median (IQR) were significantly elevated in patients as compared to controls $(0.68(0.31-1.15)$ vs $0.41(0.14-$ $0.76), p=0.006)$. ADM and UII levels were higher in the patient group, however these differences were not statistically significant. Increased BNP levels were associated with the presence of congenital heart disease (OR: 1.826, CI95\% 1.159-5.064, p=0.042). On the contrary, history of breast lactation was negatively associated with the presence of congenital heart disease (OR 0.854 , CI95\% 0.735-0.933, $\mathrm{p}=0.040$ ). UII levels were shown to be significantly associated with the complexity of congenital lesions (OR 2.456, CI95\% 1.047-5.758, $\mathrm{p}=0.042$ ). Moreover, higher incidence of failure to thrive ( $(28,2 \%$ vs $2,6 \%, p=0,002)$ and underweight $(23,7 \% \mathrm{vs} 0,0 \%, \mathrm{p}=0,001)$ were observed in patients compared to healthy controls.

Conclusion: The vasoactive peptides' plasma levels are differentially associated with the presence and the severity of non-cyanotic heart disease. BNP and, to a lesser extent, UII levels may be useful as diagnostic markers in the presence of congenital heart disease. 


\section{P-43}

Audit Of A Structured Approach In Paediatric Patients With Dilated Cardiomyopathy

Sri A. (1), Voges I. (2), Prasad S. (2), Daubeney P. (2)

Imperial College London, London, United Kingdom (1); Royal

Brompton \& Harefield Hospital, London, United Kingdom (2)

Introduction: Paediatric dilated cardiomyopathy (DCM) is the most common form of paediatric cardiomyopathy, with a diverse range of aetiologies. However, many patients are still labelled as idiopathic DCM without appropriate investigations. Recently, genetic testing has gained prominence in the diagnostic workup. This study tested the hypothesis that molecular genetic analysis provides incremental value in the identification of the aetiology of paediatric DCM.

Methods: Clinical data of 75 children with DCM were retrospectively analysed, to determine whether their initial diagnosis had changed due to genetic testing or other diagnostic tests. Furthermore, aetiology groups were characterised and a clinical profile of DCM patients in a tertiary referral centre was created.

Results: Idiopathic DCM was diagnosed in 28 (37.3\%) cases, myocarditis and familial DCM were found in 18 (24\%) cases each, inborn errors of metabolism (IEOM) in 10 (13.3\%) and cardiomyopathy due to vitamin $\mathrm{D}$ deficiency in one patient (1.3\%). 15 out of 39 patients who had genetic testing were genotype-positive, resulting in more patients with either familial DCM or IEOM. As a result, a paediatric DCM diagnostic guideline was created to develop a personalised workup strategy for the Royal Brompton Hospital.

Conclusion: Genetic testing provided the clinical breakthrough needed to correctly identify the aetiology in paediatric DCM and thereby decreased the prevalence of idiopathic cases. Increasing the number of specific causes for paediatric DCM may lead to management changes and reduced morbidity and mortality.

\section{P-44 \\ Impaired mood reduces sympathetic overactivity indexed by electrodermal activity in hypertensive adolescents \\ Mestanik M. (1,2), Jurko A. (3), Jurko T. (4), Mestanikova A. $(2,1)$, Micieta $V .(2,1)$; Tonhajzerova I. $(2,1)$ \\ Biomedical Center Martin, Comenius University in Bratislava, Jessenius Faculty of Medicine in Martin (JFM CU), Martin, Slovak Republic (1); Department of Physiology, JFM CU, Martin, Slovak Republic (2); Pediatric Cardiology, Martin, Slovak Republic (3); Clinic of Neonatology, JFM CU, University Hospital Martin, Martin, Slovak Republic (4)}

Introduction: Exaggerated activity of the sympathetic nervous system plays a key role in the pathomechanisms of essential hypertension. Several studies found increased cardiovascular sympathetic stimulation in hypertensive adolescents, however, there is lack of knowledge about the changes of central sympathetic regulation in hypertensive children. Recently, elevated blood pressure was found to be associated with mood disorders in adult population. Therefore, we aimed to study potential relationships between impaired mood and sympathetic regulation in hypertensive adolescents using electrodermal activity (EDA) as a noninvasive index of central cortical and subcortical sympathetic regulation reflecting the effects of various psychophysiological processes.

Methods: Studied population included 40 adolescent boys aged 1316 years with excluded effect under/overweight. EDA was continuously recorded using ProComp Infinity (Thought Technology Ltd., Canada) during 5 minutes of supine rest. Depressive mood was assessed using Children's Depression Inventory (CDI). Afterwards, participants were divided into four groups: normotensives with normal mood (CDI: $2.9 \pm 0.6, \mathrm{n}=10$ ), normotensives with impaired mood (CDI: $12.0 \pm 0.5, \mathrm{n}=10)$, hypertensives with normal mood (CDI: $3.3 \pm 0.6, \mathrm{n}=10$ ), hypertensives with impaired mood (CDI: $12.0 \pm 0.6, \mathrm{n}=10$ ).

Results: Normotensive adolescents with impaired mood had significantly reduced EDA compared to normotensive adolescents with normal mood $(\mathrm{p}<0.05)$. EDA in hypertensive adolescents with normal mood was significantly higher compared to normotensives with normal mood $(p<0.05)$. EDA in hypertensive patients with impaired mood was significantly lower compared to hypertensive as well as normotensive adolescents with normal $\operatorname{mood}(\mathrm{p}<0.001, \mathrm{p}<0.05$, respectively).

Conclusions: Our results revealed increased sympathetic activity indexed by EDA in hypertensive adolescents compared to their healthy peers. In contrast, the impaired mood significantly reduced sympathetic over-activation in adolescent hypertension potentially masking the effects of hypertension-related autonomic dysregulation, which is considered to be an important risk factor for later cardiovascular complications. With respect to recently found increased prevalence of depressive symptoms in patients with hypertension, attention should be paid to potential effects of impaired mood on autonomic regulatory integrity in the evaluation of cardiovascular risk in adolescent hypertension.

Support: VEGA 1/0044/18.

\section{P-45}

\section{Atypical Cardiac Features in Patients with Noonan} Syndrome

Calcagni G. (1), Gagliostro G. (2), Baban A. (1), Albanese S. (1), Trezzi M. (1), Tarani L. (2), Digilio M.C. (1), Tartaglia M. (1), Marino B. (2), Versacci P. (2)

Department of Pediatric Cardiology and Cardiac Surgery, Bambino Gesù Children's Hospital, IRCCS, Rome, Italy (1). Division of Pediatric Cardiology, Department of Pediatrics- "Sapienza" University of Rome, Rome, Italy (2)

Background: Noonan Syndrome (NS) is a relatively common autosomal dominant disorder with typical congenital heart defects (CHDs) such as pulmonary valve stenosis, hypertrophic cardiomyopathy and septal defects. Atypical cardiac features (ACF) are also rarely described in literature. Aim of this study was to report the prevalence and the outcome of these ACF in our population of patients affected by NS.

Design and Methods: A retrospective, multicentric, observational study was performed. All clinical and surgical data of NS patients followed until April 2017 at Bambino Gesù Children's Hospital and Department of Pediatrics - "Sapienza"- University of Rome, were collected. Molecular analysis was performed in all patients. Results: A total of 208 patients with clinical diagnosis of NS were enrolled: 117 males and 91 females. In 167 patients a mutation of one of the known genes was reported: PTPN11, SOS1, RAF1, RIT1, SHOC2, BRAF, KRAS, NRAS, HRAS, CBL and LZTR1. CHDs were reported in 181 patients: 159 presented typical CHDs while ACF were described in 96 patients (isolated or combined with typical CHDs). Mitral, tricuspid and aortic valve were abnormal in 65, 22 and 35 patients, respectively; ascending and descending aorta were abnormal in 17 patients. Eight patients presented coronary dilatation while 1 patient had coronary stenosis; dilatation of left auricle was described in 3 patients. Tetralogy of Fallot (TOF) was described in 1 patient. Mutations of genes reported in patients with ACF were: PTPN11 $(45,8 \%)$, SOS1 $(12,1 \%)$, RAF1 (5,2\%), BRAF (2,1\%), RIT1 (5,2\%), KRAS (1\%), 
SHOC2 (4,2\%), CBL (1\%), NRAS (1\%), HRAS (1\%), LZTR1 (1\%). Twenty patients with ACF had no known mutations $(20,8 \%)$. Cardiac surgery was performed in 10 patients: 5 end-toend anastomosis, 2 aortic replacement, 1 coronary stenosis dilation, 1 cardiac surgery for TOF and 1 mitral valve treatment. This last patient occurred a second intervention for mitral valve replacement. No patients died in our cohort.

Conclusions: Patients with NS usually present a typical spectrum of CHDs. Our data suggest that also ACF must be carefully identified for the possible impact on the clinical outcome of these patients.

\section{P-46}

Insufficient Medical Primary Health Care of Adults with Congenital Heart Defects associated with Genetic Disorders

Neidenbach R. (1), Diebold I. (5), Pieper L. (2), Oberhoffer R. (1,3), Schelling J. (4), Sanftenberg L. (4), Nagdyman N (1), Klein H.-G. (6), Seidel H. (1,6), Ewert P. (1), Kaemmerer $H(1)$

(1) Department of Paediatric Cardiology and Congenital Heart Defects, German Heart Centre Munich, Technical University Munich, Germany; (2) Department of Behavioural Epidemiology, Technical University of Dresden, Germany; (3) Department of Preventive paediatrics, Department of Sport and Health Sciences, Technical University of Munich, Germany; (4) Institute for General Practice, University Hospital of the Ludwig-Maximilians-University Munich, Germany; (5) Medizinisch Genetisches Zentrum Munich, Germany; (6) Zentrum für Humangenetik und Laboratoriumsdiagnostik, MVZ Martinried, Germany; (7) Institut für Humangenetik, Technical University of Munich, Germany

Introduction: Due to recent medical advances, approximately 280,000 adults with congenital heart defects (ACHD) are currently living in Germany. Although most CHD occur sporadically, a causative genetic component is very likely, including chromosomal, monogenic, multifactorial inheritance disorders or environmental factors.

Most ACHD need experienced medical advice. Their care concept not take into account cardiological issues, and also a genetic counseling by a clinical geneticist experienced in CHD.Recent German data show, that more than 200.000 ACHD are not treated by certified ACHD-specialists. As many general physicians do not have specific knowledge concerning CHD, genetic disorders and related comorbidities, particularly patients with rare hereditary diseases are at risk to be not advised or treated sufficiently.

Aim of the study is to demonstrate "real life" primary care of ACHD and with hereditary diseases / genetic disorders.

Methods: A cross-sectional study, questionnaire-based survey including data of ACHD and general practitioners (GP).

Results: Included were 954 ACHD $(51.7 \%$ female, mean age $36.0 \pm 12.4$ years) and 369 general practitioners (GP). GP treat patients with any type of $\mathrm{CHD}$, including rare hereditary diseases such as Marfan syndrome $(\mathrm{n}=95 ; 25.7 \%)$, Ehlers-DanlosSyndrome $(n=28 ; 7.6 \%)$, Turner syndrome $(n=74 ; 20.1 \%)$ or Morbus Fabry $(n=27 ; 7.3 \%)$. In case of cardiac or non-cardiac problems and questions, more than $70 \%$ of the surveyed patients contact their GP primarily. From the patient's perspective, up to $50 \%$ seek for disease oriented advice, which is often unmet. GP indicate in more than $70 \%$ of the cases to be poorly informed about ACHD specific physicians and centres where also a genetic counselling takes place.

Conclusions: ACHD need optimized, comprehensive medical care and modern, scientifically based care concepts, particularly if a genetic disorder coexists.
Although GP's have to guide ACHD to receive adequate diagnosis, treatment and preventive measures, GP's are insufficiently informed about the existing ACHD facilities.

In the future, ACHD-experts and -centers must become more visible and cooperate stronger with GPs to provide adequate management treatment and therapy concepts, including genetic aspects.

\section{P-47}

The impact of myocardial fiber orientation on the left ventricle diastolic compliance: an in-silico study Castaldi B. (1), Angelini A. (2), De Filippi E. (3), Susin F. (3), Cattapan I. (1), Fedrigo M. (2), Boso D. (3), Peruzzo P. (3), Comunale G. (3), Milanesi O. (1)

(1) University of Padova, Women's and Children's Health, Padua, Italy (2) University of Padova, Department of Thoracic and Cardiovascular Sciences, Padua, Italy (3) University of Padova, Department of Civil Environmental and Structural Engineering, Padua, Italy

Background: Left ventricular myocardium is arranged in a complex three-dimensional network of fibers, which form a counterclockwise helix in the endocardial layer and a clockwise helix in the epicardial layer. This architecture may change in congenital and acquired heart disease. However, few studies evaluated the myocardial development as well as the mechanism promoting these changes.

Purpose of the study: Based on morphometric and functional observations on histological sections of fetal human heart and on newborns, we developed an in-silico model in order to test the influence of different myocardial fibers arrangements on diastolic performance.

Methods: In vivo data were obtained by the echocardiographic evaluation in 72 fetuses and 39 premature babies without cardiac pathologies. For the histological section, we studied 20 hearts without cardiac pathologies from autopsy investigation (range 12-40 weeks of gestation). Based on functional and histological data, three finite element models of the left ventricular myocardium were developed, corresponding to 20, 30 and 40 weeks' fetuses. For the $40 \mathrm{GW}$ model alternative models of fiber orientation were tested.

Results: In silico model showed a mild decrease in diastolic compliance since 20 to 40 weeks of gestation. The multi-layer model warrantees the shape of the left ventricle as known. The early development of the endocardial longitudinal layer since $12 \mathrm{WoG}$ gives a pressure-volume plot similar to the adult type plot, while a paradoxical early development of the epicardial longitudinal layer

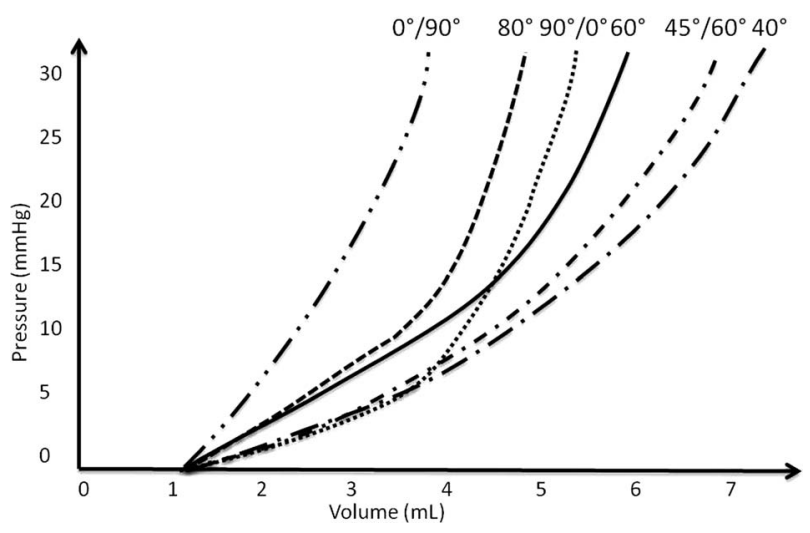

Figure. 
gives higher stiffness of the left ventricle. Finally, a less longitudinal arrangement of the myocardial fibers determinates higher diastolic compliance (see figure).

Conclusions: In silico models showed that a less longitudinal arrangement of the myocardial fibers gives the highest diastolic compliance. The endocardial longitudinal fibers are the main contributors to the preservation of the shape of the left ventricle. Based on these data, the reduction of the fibers' angle in the endocardial longitudinal layer can be a compensatory effect to prevent the increase of left ventricular stiffness. Based on these data, the assessment of the longitudinal strain may be used to improve the diagnostic abilities in left ventricular outflow tract obstruction in fetal life.

\section{P-48}

Aspirin resistance in a paediatric population with congenital heart diseases

Padrini M. (1), Padrini R. (2), Spiezia L. (2), Castaldi B. (1), Milanesi O. (1)

(1) Pediatric cardiology unit, department of woman and child health, university of Padua, Italy (2) Department of Medicine, university of Padua, Italy

Introduction: Aspirin resistance in adults has an incidence of 5\% to $50 \%$ depending on the laboratory test used. Data in paediatric setting are lacking. Our aim was to evaluate the incidence of ASA resistance in a paediatric population with congenital heart diseases. Methods: Included were children (6 months to 18 years old) on maintenance aspirin therapy $(3-5 \mathrm{mg} / \mathrm{kg} / \mathrm{die})$. Venous blood samples were collected 4, 5 and 6 hours after drug administration and plasma concentrations of ASA and salicylic acid were assayed by HPLC. The effect of aspirin on platelet aggregation was assessed using the ASPItest.

Results: Since July 201620 patients were enrolled. There were 11 ASD, 5 univentricular hearts, 1 interrupted aortic arch, 1 TOF, 1 AV canal, and 1 post-actinic cardiomyopathy. The results of ASPItest are available for 17 patients. There were 2 patients (12\%) with high on-treatment platelet reactivity (HPR) (cut off $>39 U$ ). No clinical thrombosis occurred.

The time courses of mean ASA and salicylate concentrations were similar to those outlined in adults (Figure 1 and 2). The ASPI test values were linearly correlated with ASA concentrations $\left(r^{2}=0,36 ; p=0,0113\right)$. The other variables analysed (CBC values, age, weight) were not related to aspirin response. High blood cellularity might play a role in HPR (neutrophil count and MCV reached borderline significance, respectively $\mathrm{p}=0,062$ and $\mathrm{p}=0,075)$. When dichotomous variables were analysed, significance was reached using a lower cut off for the ASPItest suggestive of effective COX-1 inhibition (ASPItest cut off > 30, ASA concentration cut off $>100 \mathrm{ng} / \mathrm{mLxh}, \mathrm{p}=0,005)$.

Conclusions: Aspirin resistance had a non-trivial incidence in our small population. High plasmatic ASA concentration correlates with HPR. Limitations are a low population number and the use of only one platelet reactivity test.

Table.

\begin{tabular}{lccllll}
\hline & \multicolumn{5}{l}{ Pharmacokinetics \& } \\
Demographics & Mean & SD & $\begin{array}{l}\text { pharmacodynamics } \\
\text { phean }\end{array}$ & SD \\
\hline Sex (M/F) & $10 / 10$ & - & Aspirin & AUC (ng/mLxh) & 80 & 83 \\
Age (years) & 8.2 & 4.3 & & Cmax (ng/mL) & 53.7 & 55.3 \\
Weight (Kg) & 28.1 & 15.7 & & Tpeak (h) & 4.80 & 0.77 \\
Height (cm) & 126.8 & 27.0 & Salicylic acid & AUC (ng/mLxh) & 4,528 & 5,004 \\
Dose (mg) & 79.7 & 28.2 & & Cmax (ng/mL) & 3,127 & 3,286 \\
Dose/Kg (mg) & 3.12 & 0.67 & & Tpeak (h) & 4.35 & 0.74 \\
& & & Aspi Test & AUC (U) & 24.9 & 11.4 \\
\hline
\end{tabular}

ASA

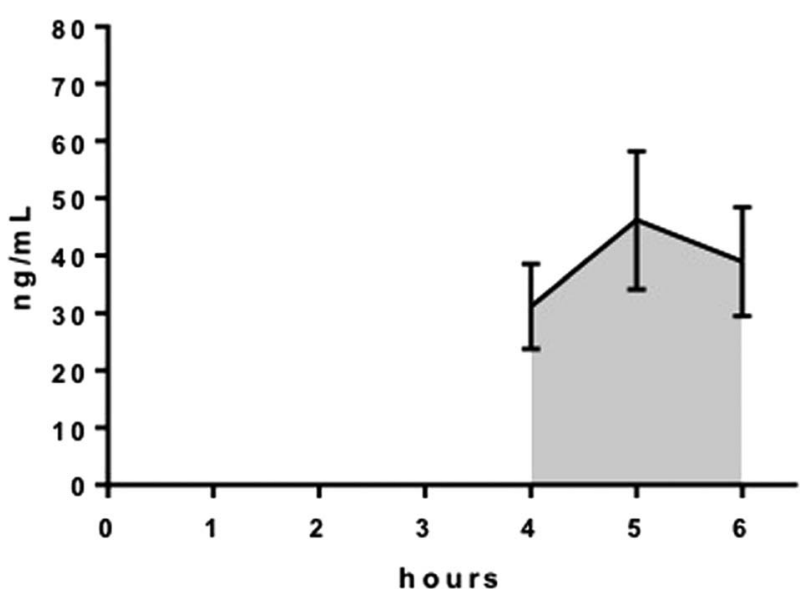

Figure 1.

SA

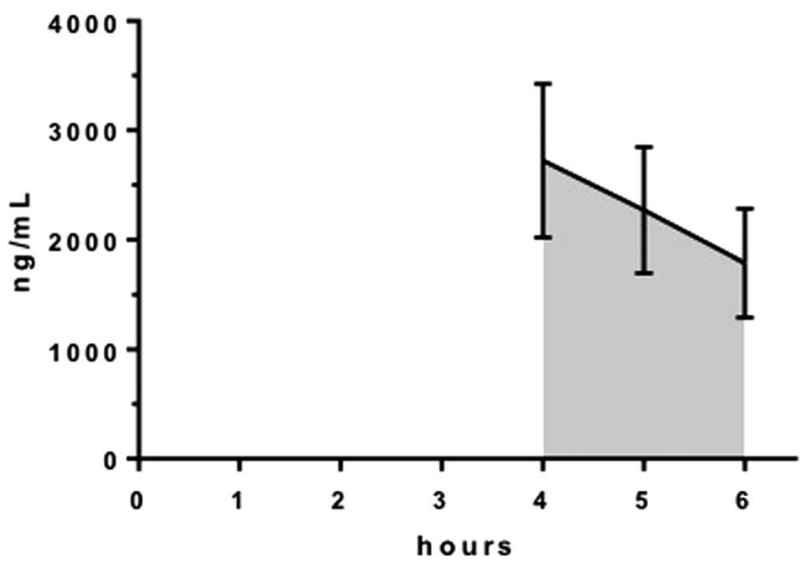

Figure 2.

P-49

Left Pulmonary Artery in 22q11 deletion syndrome: echocardiographic findings, role and expression of Tbx1 in human and knockout mice

Mastromoro G. (1), Calcagni G. (2), Versacci P. (1), Chinali M. (2), Lambiase C. (1), Unolt M. (1), Baban A. (2), Anaclerio S. (1), Bilio M. (3), Digilio M. C. (2), Marino B. (1), Baldini A. (3) Department of Pediatrics, Sapienza - University of Rome, Rome (1); Department of Pediatric Cardiology and Cardiac Surgery, Bambino Gesù Pediatric Hospital, Rome (2); Institute of Genetics and Biophysics "Adriano Buzzati Traverso”, CNR, Naples (3)

Introduction and Hypothesis: Patients with 22q11 deletion syndrome (del22q11.DS) present typical patterns of cardiac defects, with a particular involvement on the ventricular outflow tract and pulmonary arteries (PAs). Mutation of Tbx1 gene is reported in more than $90 \%$ of them. We measured both PAs diameter in patients with and without del22q11.DS during routinary echocardiographic evaluation. Our data were also compared to PAs measurement in Tbx1 mutant mice. Finally, a Call Fate Mapping in Tbx1 was used to understand the expression of this gene mutation in the morphogenesis of PAs. Methods: We evaluated 49 patients with del22q11.DS without cardiac defects. The control group consisted of 49 healthy patients, 
matched for age and sex. All patients underwent a complete transthoracic echocardiography. Subsequently, we crossed Tbx1+/- mice and harvested fetuses, genotyped by PCR. We examined the cardiovascular phenotype of 8 wild type (WT), 37 heterozygous (Tbx1+/-) and 6 null fetuses (Tbx1-/-). Finally, we crossed Tbx1Cre/ + mice with Cre reporter mice R26RmT-mG to reveal Cre recombinase activity. We studied the Fate Map of Tbx1 to evaluate the expression of this gene on the PAs.

Results: Ninety-eight percent of patients with del22q11 showed a smaller left PA (LPA) diameter compared to the right PA (RPA). Seventy-one percent of controls showed the diameter of the LPA smaller than that of the right one. The mean of the LPA/RPA ratio in del22q11 was $0.80 \pm 0.12$ vs $0.97 \pm 0.08$ in the controls with a significant $\mathrm{p}$ value $(\mathrm{p}<0.0001)$.

In WT, heterozygous and null, the average of LPA/RPA ratio were $0.9,0.77,0.6$, respectively. Finally we confirmed the expression of Tbx1 on the pulmonary trunk and particularly of the origin of LPA. Conclusions. Patients with 22q11.DS without cardiac defects show smaller PA compared with healthy patients, and particularly if LPA was compared. The localization of the expression of Tbx1 on LPA, could explain these results considering its significant role on the PAs formation and growth.

\section{P-50}

Change of Voltage-gated Potassium Channel 1.7 and NADPH Oxidase 4 Expressions in Monocrotaline-induced Pulmonary Arterial Hypertension Rat Model

Hong Y.M. (1) , Kim K.C. (2)

Department of Pediatrics (1); Department of Thoracic and Cardiovascular Surgery (2), Ewha Womans University Seoul, Korea,

Introduction: An abnormality of potassium channel expressions influences the function of vessels, including vascular tone and rates of proliferation. Diverse potassium channels, including voltagegated potassium $(\mathrm{Kv})$ channels, have been involved in pathological changes of in pulmonary arterial hypertension (PAH). However, the exact therapeutic importance of Kv1.7 is currently not clear. Therefore, we investigated whether Kv1.7 channel expressions change in the lung tissues of monocrotaline (MCT) -induced PAH rat model and this change is caused by the endothelin (ET)-1 and reactive oxygen species (ROS) pathways.

Methods: The rats were separated into two groups: the control (C) group and the monocrotaline (M) group (MCT $60 \mathrm{mg} / \mathrm{kg}$ ). A hemodynamic study was performed by catheterization into the external jugular vein to estimate right ventricular pressure (RVP) and pathological changes were investigated in the lung tissues. Changes of protein and RNA expression levels were confirmed by western blot and polymerase chain reaction analysis respectively.

Results: MCT caused increased RVP, medial wall thickening of the pulmonary arterioles and increased protein expressions of ET-1, endothelin receptor A (ERA) and NADPH oxidase (NOX) 4. Decreased Kv1.7 channel expression was detected in the lung tissues. Inward rectifier channels 6.1 channels was also increased in the lung tissues. We confirmed ET-1 increased NOX4 protein expression level and decreased glutathione peroxidase- 1 protein expression level in pulmonary artery smooth muscle cells (PASMCs). Thus, ET-1 increased ROS level in PASMCs.

Conclusions: Kv1.7 contributes to MCT-induced PAH and this change might be caused by the ET-1 and ROS pathways.

\section{P-51}

Calculation of the Shunt Flow in Aorto-Pulmonary Collateral Artery model rat with Left Pulmonary Artery ligation under Hypoxia environment

Ito R., Urashima T., Itohisa M., Fujimoto Y., Fujiwara M., Ogawa K., Ida H.
Department of Pediatrics, The Jikei University School of Medicine, Tokyo, Japan

Introduction: Cyanotic congenital heart disease with functional single ventricle often develops aorto-pulmonary collateral arteries (APCA). It is known that APCA can cause the hemodynamically left-to-right shunt and become a burden on the systemic ventricle. It often causes heart failure and pleural effusion, inhibits the growth of pulmonary artery, and affects the remodeling of pulmonary artery. However, detailed study on the mechanisms of APCA development is limited, because the animal models are not reproducible.

Objectives: The purpose of this study is to clarify the timing of APCA expression, and the changes in shunt volume by using animal model.

Methods: Five-week-old Sprague Dawley rat (100-150g) underwent the operation that rats were ligated left pulmonary artery with hilar region under left thoracotomy, and divided into 2 groups: hypoxic (HO) group (10\% of FiO2) and room air (RA) group (21\% of $\mathrm{FiO} 2)$. These rats were measured the blood flow of bilateral ventricular outflow tract using transthoracic ultrasonography and transit time ultrasound probe placed around their aorta and main pulmonary artery, and made up to 10 rats at each timing (3day, 1, 2, 3 , and 4 weeks after operation). In this model, the pulmonary and aorta output was defined as the functional systemic output (Qs) and pulmonary output $(\mathrm{Qp})$. Because the aortic output was the flow that added up the Qp and the quantity of APCA shunt. The Qp/Qs was compared in these groups ( $\mathrm{HO}$ and $\mathrm{RA}$ ).

Results: The Qp/Qs in the HO group was increased more earlier than in RA group significantly, but in RA group caught up with even level since 3 weeks after operation. The Qp/Qs in the HO group was significantly elevated from 1 week after operation compared to the control. The calculation of Qp/Qs by ultrasonography was good correlated with it by transit time ultrasound probe $(\mathrm{R}=0.70)$.

Conclusions: We could create the APCA animal model reproducibly. The quantity of APCA flow in $\mathrm{HO}$ group increased earlier than in RA group. Although the Qp/Qs in RA group caught up with it in $\mathrm{HO}$ group since 3 weeks after operation. In this study, the development of APCA was accelerated from hypoxic environment.

\section{P-52 \\ Modern management of portopulmonary hypertension in children: experience of an expert center \\ Joye R. (1), Wacker J. (2), McLin V. (3), Giostra E. (4), Aggoun Y. (2), Wildhaber B. (5), Lador F. (6) and Beghetti M. (2) Pediatrics (1), Pediatric Cardiology (2), Pediatric Gastroenterology (3), Gatroenterology (4), Pediatric Surgery (5), Pneumology (6), Geneva University Hospitals, Switzerland}

Introduction: Portopulmonary hypertension $(\mathrm{PoPH})$ is a rare but serious complication of portal hypertension or portosystemic shunting. PoPH management may require liver transplantation (LT) but this procedure is contraindicated in patients with severe hemodynamic impairment. There are only few reports describing PoPH management and outcome in children.

Methods: We describe our series of children with PoPH. Diagnostic workup was performed according to the current international guidelines and precapillary pulmonary hypertension was confirmed by right heart catheterization. Patient data was collected via chart review.

Results: 5 patients were identified in our pediatric center from 2013 to 2017 , with a mean age of 15.8 years old (range 14-19). 
Table 1. Patients characteristics, hemodynamic parameters and evolution

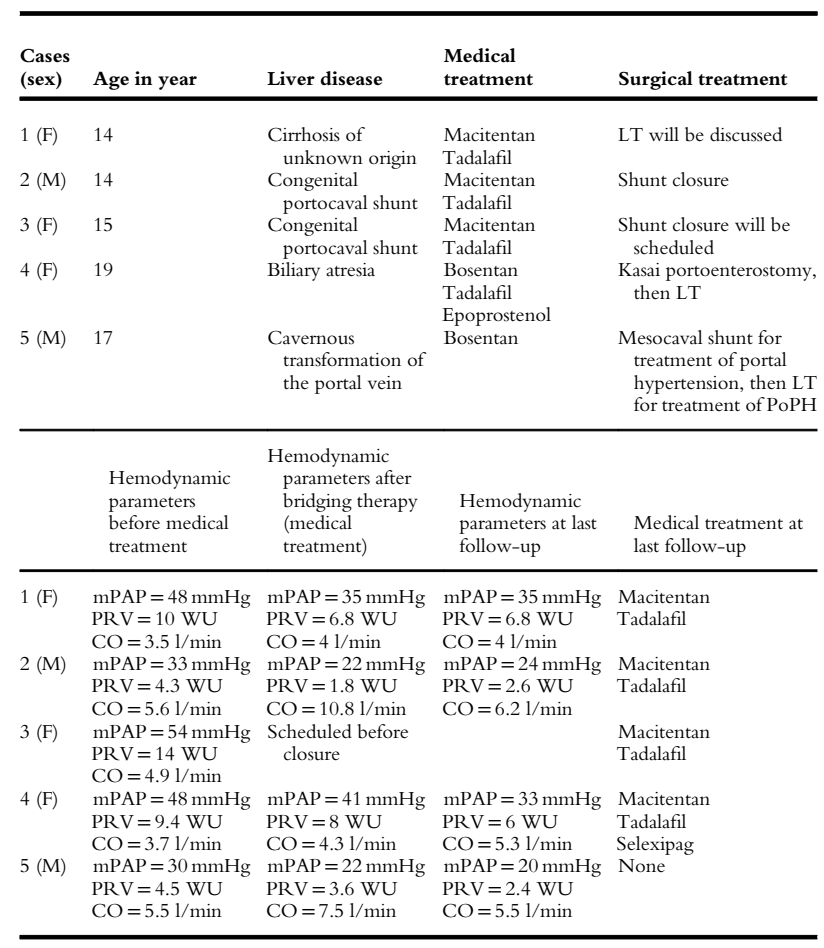

The underlying liver conditions were cirrhosis of unknown origin, 2 portocaval shunts, biliary atresia, and cavernoma of the portal vein. 3 patients suffered with dyspnea and 1 had no cardiopulmonary symptom. The mPAP was $42.6 \mathrm{mmHg}$ (range $30-54$ ) and the indexed mean pulmonary vascular resistance (PVRi) was 8.44 Wood units (range 4.3-14). The 1st was in functionnal class (FC) III and treated by combination therapy consisting of macitentan and tadalafil. After a significant improvement of hemodynamic profiles, LT is discussed. The 2 nd had no cardiopulmonary symptom. PoPH is almost resolved after shunt closure and under treatment of macitentan and tadalafil. The 3rd was in FC III and treated with macitentan and tadalafil. Shunt closure is schedulded. The $4^{\text {th }}$ (biliary atresia) was in FC IV and treated by triple combination therapy with macitentan, tadalafil and IV epoprostenol. PoPH improved dramatically and the patient underwent LT. Epoprostenol was switched to oral selexipag 9 months after LT. $\mathrm{PoPH}$ is now controlled under macitentant, tadalafil and selexipag. The 5 th underwent a surgical mesocaval shunt for a cavernoma of the portal vein. 7 years later, he presented in FC III and was bridged to liver transplantation by bosentan. LT was performed and $\mathrm{PoPH}$ is now resolved. Table 1 shows patients characteristics.

Conclusions: Outcome of pediatric PoPH in the modern era may be largelly improved when managed at an expert center offering $\mathrm{PH}$ and LT multidisciplinary managment. Further studies are needed to better define the pronostic and treatment in this population.

\section{P-53}

Ukrainian Center for Pulmonary Arterial Hypertension in Children: Achievements and Problems

Khanenova V., Dovgaliuk A., Razinkina A., Kalashnikova R.,

Zhovnir V., Rudenko N., Yemets I.

Ukrainian Children's Cardiac Center, Kiev, Ukraine.
Introduction: Pulmonary arterial hypertension $(\mathrm{PAH})$ in children is a hemodynamic and pathophysiological state characterized by increasing of mean pressure in the pulmonary artery above $25 \mathrm{~mm} \mathrm{Hg}$ in patients elder than age of 3 months, and is estimated by the data of the catheterization of the right heart. In 2015 the Ukrainian Center for Pulmonary Arterial Hypertension (UCPAH) in Children was created at the Ukrainian Children's Cardiac Center. The aim of our study was to analyze the demographic and diagnostic spectrum of children in Ukraine with pulmonary hypertension.

Methods: The assessment of our patients included evaluation of clinical symptoms and anamnestic data, roentgenography of the chest, electrocardiography, investigation of the function of the lungs and gases of arterial blood, a 6-minute walk test (6 MW), echocardiography, catheterization of the right heart and a study of vasoreactivity, a high resolution computed tomography, a CT with contrast and pulmonary angiography, a MRI of the heart, blood tests (serology, immunology and genetics), an ultrasonography of the abdominal cavity. Unfortunately, a ventilation perfusion scanning of lungs is not available in Ukraine.

Results: During 2 year 156 patients with $\mathrm{PAH}$ were registrered in UCCC. There were 84 female and 72 male. All patients were with diagnosis of idiopathic PAH (iPAH) - 31 (19,8\%) patients and $\mathrm{PAH}$ - associated with congenital heart disease (PAH-CHD) $125(80,2 \%)$ patients. In group of PAH-CHD 29 (18,5\%) was after total repair of CHD, $52(33,3 \%)$ - after palliative operations, 44 $(28,4 \%)$ - non operative. During our investigation we analyzed their clinical condition, current medications and operability (in case of PAH-CHD). 57 patients with different CHD underwent of total repair, 32 - palliative procedures.

Conclusions: Creation of UCPAH in children helps in creation of a general register of patients with a pediatric group with $\mathrm{PAH}$, carrying out of highly specialized diagnostics of $\mathrm{PAH}$, optimisation of medical therapy, monitoring its effectiveness and assessing the dynamics of patients' clinical condition, providing cardiac surgery, provision of emergency care to patients with complications of $\mathrm{PAH}$ in ICU, iIncrease the level of knowledge about the diagnosis and treatment of children with $\mathrm{PAH}$.

\section{P-54}

\section{Prognostic factors in paediatric restrictive} cardiomyopathy

Abakka S., Malekzadeh-Milani S. Raimondi F., Bonnet D., Khraiche D.

Hôpital Necker-Enfants malades, Paris, France

Introduction: Restrictive cardiomyopathy (RCM) is the rarest and most serious form of heart muscle disease, accounting for $2-5 \%$ of pediatric cardiomyopathies. Identification of prognostic factors is essential to identify high-risk patients that need to be addressed for early heart transplantation.

Methods: We conducted a single-center retrospective study. We enrolled 47 patients that had a diagnosis of RCM between 1996 and 2017. The clinical, ultrasonography, and hemodynamic data were collected at the time of diagnosis, as well as genetic analysis in order to identify early predictors of poor prognosis (transplantation or death). We then compared the evolution according to the type of RCM (pure or hypertrophic cardiomyopathy with restrictive phenotype HRCM), and to the age group ( $<5$ years, 5-11 years, $>11$ years).

Results: 47 patients were included, with a gender ratio of 1.76 (F/M). The mean age at diagnosis was 5.9 years. $57.5 \%$ had a pure RCM and $42.5 \%$ an HRCM. The median follow-up was 
10.2 months. Overall survival at 1, 2 and 5 years was $43 \% 35 \%$ and $15 \%$ respectively. There was no statistically significant difference in terms of survival between RCM and HCMR $(p=0.651)$ and between age groups $(p=0,582)$. Clinical findings at diagnosis were dominated by congestive signs (63.9\%), which were related to a poor outcome $(p=0,041)$. The relationship between a mildly altered left ventricular ejection fraction (LVEF $45-55 \%)$ and mortality was statistically significant $(p=0.01)$. Imaging findings didn't show a late gadolinium enhancement and cavities dilatation were note associated with mortality. Regarding catheterism data, neither cardiac output nor pulmonary vascular resistance nor pulmonary capillary pressure were related to a poor outcome. The rentability of family screening was $60 \%$ : we identified one homozygous and 4 heterozygous mutations on the TNNI3 gene including a neomutation, and 1 mutation on MYH7 gene.

Conclusion: Adverse prognostic factors were dominated by the presence of congestive signs at diagnosis and mild level of left ventricular dysfunction. Patient with these factors should be addressed for Heart transplantation as soon as diagnosed. Autosomic dominant mutation in TNNI3 was the most frequent genetic finding in this population. Family screening and genetic counseling is thus important.

\section{P-55}

Intrauterine diagnosis of coronary artery fistula: Case report

Demir F. (1), Özbarlas N. (1), Erdem S. (1), Akcalı M. (2),

Göcen U. (3), Salih O.K. (3).

Cukurova University Departmant of Pediatric Cardiology Adana-Turkey (1);

Cukurova University Departmant of Neonatology Adana-Turkey (2);

Cukurova University Departmant of Pediatric Cardiovascular Surgery

Adana-Turkey (3).

Coronary artery fistulae represent abnormal communications between a coronary artery and a cardiac chamber, great vessel, or the coronary sinus. Prenatal diagnosis of isolated coronary artery fistulae is extremely rare. We report a case of a very large fistula connecting the circumflex coronary artery to the right ventricle just below the septal leaflet of tricuspid valve posteriorly diagnosed at 21 weeks' gestation. There was right ventricular dilatation at the

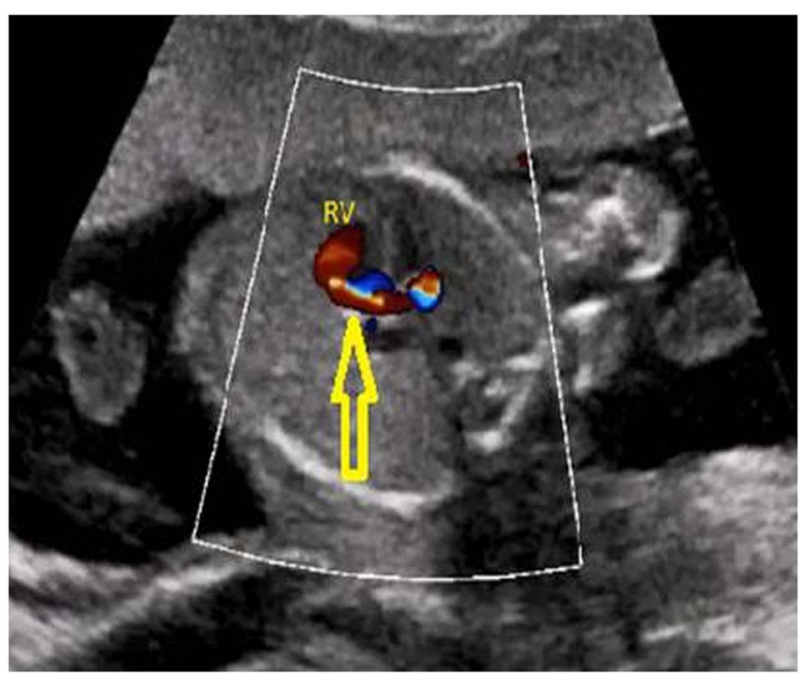

Figure. initial scan. The size of the fistula increased during pregnancy. A postnatal echocardiogram confirmed the diagnosis. Patient developed congestive cardiac failure soon after birth and required surgery for fistula at 17 days after birth. Follow-up echocardiogram following the procedure showed improved forward flow in the descending aorta with decreased RV size. Coronary artery fistulas can be diagnosed accurately during fetal life. Some babies may develop congestive cardiac failure soon after birth requiring early treatment. The correct prenatal diagnosis enabled close perinatal follow-up, prompt clinical evaluation without diagnostic delay and optimal management.

\section{P-56}

Fetal Atrioventricular Block: Management and Outcome from A Single Institution

Duman D. (1), Tunca Sahin G. (2), Babaoglu K. (3), Goynumer G. (4), Beattie B. (5), Conner C. (6),

Uzun O. (7)

Hacettepe University Medicine Faculty Pediatric Cardiology AnkaraTurkey (1), Istanbul Mehmet Akif Ersoy Thoracic and Cardiovascular Surgery Education and Research Hospital Department of Pediatric Cardiology Istanbul Saglik Bilimleri University Istanbul-Turkey (2), Kocaeli University School of Medicine Department of Pediatric Cardiology Kocaeli-Turkey (3), Istanbul Medeniyet University Goztepe Education and Research Hospital Istanbul-Turkey (4), Department of Fetal Medicine University Hospital of Wales Cardiff Wales-United Kingdom (5), Department of Obstetrics and Gynaecology University Hospital of Wales Cardiff- UK (6), Department of Pediatric Cardiology Noah's Ark Children's Hospital for Wales University Hospital of Wales Cardiff Wales- UK (7).

Introduction: Fetal atrioventricular (AV) block carries significant mortality and morbidity. It is related to isoimmune process and may lead to a functional-structural cardiac conduction and myocardial abnormality.

Aim: In this study we aimed to review our 16 years experience of fetuses presented with maternal lupus related cardiac complications.

Methods and Results: 18 fetuses were diagnosed with atrioventricular block from 2001 to 2017 . Of these, only 1 was terminated with hypoplastic left heart syndrome (HLHS) before 20 weeks of gestation. 2 cases had died in fetal period; 1 had hydrops and the other had severe aortic stenosis with atrioventricular septal defect (AVSD). 83,3\% were delivered live. 7/15 (46,6\%) live births were premature deliveries (29 to 35 weeks). 3 of 15 patients died due to prematurity or respiratory causes associated with congenital heart disease (CHD) in the neonatal period. Of 18 patients; $5(\% 27,7)$ were associated with major structural heart defect (1 left atrial isomerism and AVSD; 1 corrected transposition of great arteries; 1 AVSD and HLHS; 1 AVSD + severe aortic stenosis and 1 Ventricular septal defect-VSD). 4/5 fetuses with CHD (except the one with VSD) died ( $2 / 5$ after birth and the others in fetal period). 7 of the remaining fetuses with normal heart structures had maternal anti-Ro antibodies. 7 fetuses $(5 / 7$ with maternal antibodies) had received dexamethasone + salbutamol therapy of which one had heart failure and the other had hydrops and the rest had significant bradycardia. AV block resolved in 1 of these 7 fetuses. In two seronegative patients the fetal heart block resolved without treatment. 10 fetuses with permanent AV block were alive during follow up and 7 of them $(70 \%)$ had been implanted PM (5 were transvenous; 2 ( $<1$ year old) were epicardial). The presence of prematurity, CHD, hydrops are the risk factors for mortality. As the group that treated with steroids were more 
symptomatic; the mortality rate was higher than the cases without treatment $(42,8 \%$ versus $20 \%)$.

Conclusions: This study shows us that fetal AV block causes significant mortality when associated with structural heart disease and prematurity. Immunosuppressive agents or betamimetics do not change the dismal outcome in such cases.

\section{P-57}

Cardiac remodeling and function in aortic coarctation: from the fetus to the newborn

Soveral I. (1), Walter C. (2), Guirado L. (1), García-Cañadilla P. (3), Masoller N. (1), Bennasar M. (1), Sánchez de Toledo J. (2), Martinez JM. (1), Bijens B. (3,4), Gratacós E. (1), Crispi F. (1), Gómez O. (1) Fetal $i+D$ Fetal Medicine Research Center, BCNatal - Barcelona Center for Maternal-Fetal and Neonatal Medicine (Hospital Clínic and Hospital Sant Joan de Déu), Institut Clínic de Ginecologia Obstetricia $i$ Neonatologia, Institut d'Investigacions Biomèdiques August Pi i Sunyer, Universitat de Barcelona, Centre for Biomedical Research on Rare Diseases (CIBER-ER), Barcelona, Spain (1); Pediatric Cardiology, Hospital Sant Joan de Déu, Barcelona, Spain (2); PhySense, DTIC, Universitat Pompeu Fabra, Barcelona, Spain (3); ICREA, Barcelona, Spain (4)

Background: Persistent left ventricular (LV) dysfunction has been described in neonates and adults with coarctation of the aorta (CoA). However, comprehensive assessment of biventricular microstructure, morphology and function in CoA, from fetal to neonatal stage, has not been previously performed. This information might improve prenatal counseling and postnatal management.

Methods: A comprehensive morphometric and functional evaluation was performed in 22 fetuses with confirmed CoA and 44 gestational-age matched controls using 2D, M-Mode, pulsedwave and tissue Doppler imaging (TDI) techniques in the third trimester of pregnancy and in the first $48 \mathrm{~h}$ after birth. Microscopic fiber orientation was assessed in a fetal CoA specimen and compared to a normal heart, using phase-contrast synchrotron tomography. Sphericity index (SphI) was calculated as longitudinal/ mid-transverse ventricular diameter.

Results: Compared to controls, fetuses with CoA showed smaller and more elongated LV (LV SphI: CoA $2.45 \pm 0.84$ vs. controls $1.88 \pm 0.26, p<0.001)$ and larger right ventricles $(\mathrm{RV})$ with less extensive shape changes (RV SphI: CoA $1.46 \pm 0.29$ vs. controls $1.69 \pm 0.23, \mathrm{p}=0.005)$, and no signs of biventricular dysfunction. No signs of ventricular hypertrophy were observed, and synchrotron tomography revealed normal fiber organization. After birth, the LV became more globular (LV SphI: CoA $1.47 \pm 0.22$ vs.controls $1.76 \pm 0.17, \mathrm{p}<0.001)$ and showed signs of diastolic impairment (left E/E' CoA $17.75 \pm 7.6$ vs. controls $9.88 \pm 3.45$ ) with preserved systolic function. The RV had a normalized shape (RV SphI: CoA $1.56 \pm 0.27$ vs. controls $1.67 \pm 0.24, p=0.117$ ) and increased longitudinal function (increased S', E' and A', stroke volume and cardiac output) in the presence of a patent arterial duct.

Conclusions: Fetuses with CoA present biventricular structural changes with preserved fiber organization and function. The prenatal elongated LV's shape suggested a ventricular "modeling" under an unloaded hemodynamic situation, followed by a maladaptive postnatal response, with signs of neonatal modeling and diastolic dysfunction. These results provide new insight into the causes of neonatal LV dysfunction and might help develop new strategies to prevent or ameliorate adverse early ventricular remodeling in CoA.

\section{P-58}

Does timing of surgery predict long term respiratory outcomes in infants with Absent Pulmonary Valve Syndrome? Torok K. (1), Brettle E. (2), Seale A. (1), Miller P. (1) ,Stickley J. (1), Nagakumar P. (2), Desai T. (1)

Department of Cardiology (1), Department of Respiratory Medicine (2) Birmingham Children's Hospital, United Kingdom

Background: Absent pulmonary valve syndrome (APV) is a rare congenital heart defect, usually associated with Tetralogy of Fallot (TOF), characterized by pulmonary insufficiency and marked pulmonary artery dilatation. Some patients develop respiratory failure due to bronchial compression and may require prolonged respiratory support post-surgical repair. We aimed to investigate outcomes of patients with APV and the need for prolonged respiratory support in relation to the timing of the surgery.

Methods: We retrospectively reviewed data of patients diagnosed with APV born or with estimated due date between January 2007 and January 2017. Prolonged ventilation was defined as greater than 3 months of non-invasive or invasive respiratory support.

Results: Thirty patients were identified of which 22 (73\%) were prenatally diagnosed. Pregnancy was discontinued in one, in-utero death in three. One lost to follow-up.

Of the remaining 25 live born, 21 had classical TOF/APV and four were found to be "variants". Variants all had patent ductus arteriosus along with disconnected left pulmonary artery $(n=1)$, no aortic override with ventricular septal defect $(n=1)$ and intact ventricular septum $(n=2)$.

One baby died immediately after birth and two patients had palliative care due to severe airway compression and unable to wean ventilation support. Surgical repair was performed in 20/25 patients $(80 \%)$, two are awaiting surgery.

Of those undergoing surgery, two patients died - one during surgery and a further patient died following worsening bronchomalacia five months post-surgery. Survival from birth of the surgical group at one and five years was 89\% (CI 75 to 100\%).

Six patients who underwent surgery $(30 \%)$ required prolonged respiratory support post-operatively (Table).

Conclusion: The majority of patients with APV are diagnosed antenatally. There is an important pre-operative mortality and $25 \%$ of babies undergoing surgery require prolonged ventilation. Babies having repair in the first month of life are particularly at risk. Further studies are needed to identify prenatal factors that predict postnatal cardio respiratory outcomes so parents can be counseled appropriately.

Table.

\begin{tabular}{lccc}
\hline Timing of & $\begin{array}{l}\text { Number } \\
\text { of patients }\end{array}$ & $\begin{array}{l}\text { Number } \\
\text { alive }\end{array}$ & $\begin{array}{l}\text { Number with } \\
\text { prolonged respiratory } \\
\text { support }\end{array}$ \\
\hline $\begin{array}{l}<\text { month } \\
1 \text { month }-\end{array}$ & 5 & 3 & 4 \\
6 months & 7 & 7 & 2 \\
6 months + & 8 & 8 & None \\
\hline
\end{tabular}

P-59

Initial experience of fetal MRI as adjunct to fetal echocardiography for cardiac tumours tissue characterisation Yeong M., Caldas P., Andronikou S.

Bristol Royal Hospital for Children, Bristol, United Kingdom

Introduction: Fetal echocardiography has been the standard imaging modality for detecting fetal cardiac tumours. The availability of 
ultra-fast MRI sequences has enable visualisation of cardiac structures in-utero. Tissue characterisation of cardiac tumours using cardiac MRI in paediatric population is established however the use of MRI for characterising fetal tumours is not well described.

Methods: Cases of fetal cardiac tumours were referred for MRI. Fetal echocardiography for each case was reviewed. Fetal MRI (figure) was performed on a Siemens $1.5 \mathrm{~T}$ scanner. Following localisers in 3 standard planes, diagnostic imaging was performed using single-shot T2W turbo spin echo (HASTE), T1W fast low-angle shot gradient echo (FLASH) and balanced SSFP (bSSFP) sequences.

Results: 3 fetal cardiac tumour MRI was performed this year, at mean gestation age (GA) of 26 weeks ( $23-29$ weeks). All 3 fetuses had suspected cardiac tumours on fetal echocardiography. The tissue characteristics of the mass were assessed based on T1, T2 and bSSFP signal characteristics. Following the MRI, one pregnancy was terminated and two fetuses were delivered alive.

Conclusions: Our preliminary experience suggests that fetal MRI may be a useful adjunct in tissue characterisation of fetal cardiac tumours however it may not always be possible to be definitive of the diagnosis. Future adjustment of sequences may allow further tissue differentiation of cardiac tumours on MRI.

\section{Table.}

\begin{tabular}{|c|c|c|c|c|c|c|c|}
\hline Case & $\begin{array}{l}\text { GA } \\
\text { (weeks) }\end{array}$ & $\begin{array}{l}\text { Termination } \\
\text { of pregnancy }\end{array}$ & $\begin{array}{l}\text { Fetal MRI } \\
\text { diagnosis }\end{array}$ & T1 & T2 & bSSFP & $\begin{array}{l}\text { Postnatal / } \\
\text { Postmortem } \\
\text { diagnosis }\end{array}$ \\
\hline 1 & 27 & No & Rhabdomyoma & iso & $\begin{array}{c}\text { Mildly } \\
\text { hyper }\end{array}$ & iso & Rhabdomyoma \\
\hline 2 & 23 & No & $\begin{array}{l}\text { Likely } \\
\text { Teratoma }\end{array}$ & Нypo & Hyper & $\begin{array}{c}\text { Mildly } \\
\text { hyper }\end{array}$ & Possible lipoma \\
\hline 3 & 29 & Yes & $\begin{array}{l}\text { Likely } \\
\text { Teratoma }\end{array}$ & Нуро & $\begin{array}{c}\text { Mixed } \\
\text { hypo } \\
\text { and } \\
\text { hyper }\end{array}$ & $\begin{array}{l}\text { Mixed } \\
\text { hypo } \\
\text { and } \\
\text { hyper }\end{array}$ & Teratoma \\
\hline
\end{tabular}

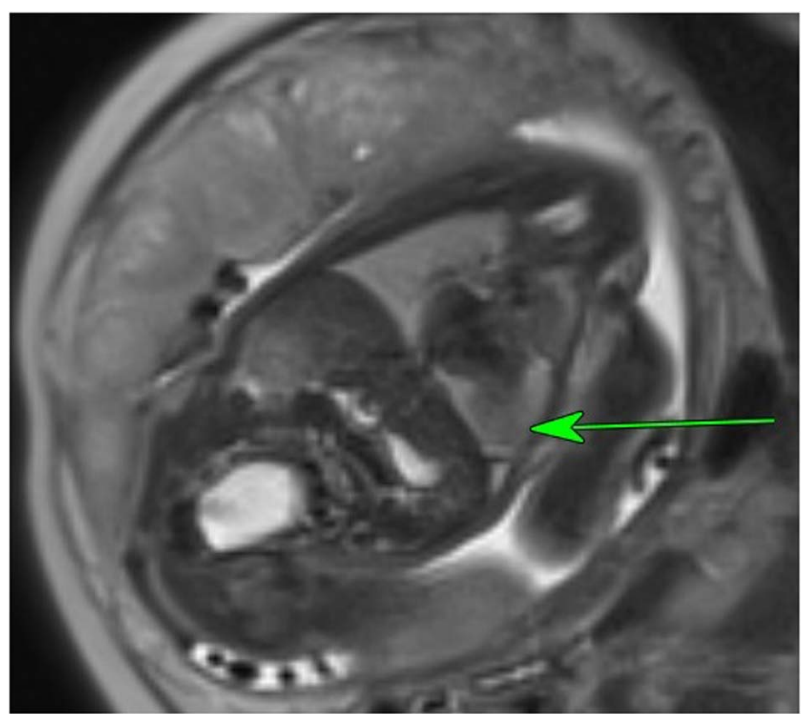

Figure.

\section{P-60}

Fetal Cardiac Interventions: 12-year experience and results in Argentina

Marantz P., Grinenco S., Aiello H., Rivarola M., Trentacoste L., Saenz Tejeira M., Meller C., Otaño L.

Hospital Italiano de Buenos Aires, Buenos Aires, Argentina
Introduction: Fetal Cardiac Interventions (FCI) have been proposed in order to improve outcome in certain types of congenital heart disease that may progress during pregnancy leading to irreversible myocardial or pulmonary damage. The aim of this study is to report our group's experience and results in performing FCI.

Methods: Medical records of patients selected for FCI between January 2005 and November 2017 were reviewed. Measures of central tendency and frequency distributions were calculated.

Results: There were 48 patients selected for FCI, 22 with critical aortic stenosis evolving to hypoplastic left heart syndrome (HLHS); 6 with dysplastic mitral valve, critical aortic stenosis and severely restrictive foramen ovale (FO); 4 with HLHS and severely restrictive $\mathrm{FO} ; 12$ with critical pulmonary stenosis or pulmonary atresia; 4 with severe pericardial effusion. Fetal aortic valvuloplasty was performed in 17 patients, fetal pulmonary valvuloplasty in 3 cases, atrial septum dilatation in 3 fetuses and in 2 of them with stent placement, and pericardiocentesis was performed in 3 fetuses. After 8/26 (30.7\%) procedures there was fetal demise within $48 \mathrm{hs}$ after FCI. There were no associated significant maternal complications.

Conclusions: Fetal cardiac interventions of selected patients in a specialized centre are feasible, with an acceptably low rate of fetal demise and no significant maternal complications.

\section{P-61 \\ Prenatal Detection and Modes of Presentation of Neonatal Congenital Heart Disease in Ireland: a 5-year progress review \\ Lynch A., Ng L, Lawler T, Gardiner F, Lavelle M, Franklin O. National Children's Heart Centre, Our Lady's Children's Hospital Crumlin, Dublin, Ireland}

Aims: This audit aimed to assess the incidence of new cases of severe congenital heart disease in infants in Ireland, and to determine changes in prenatal detection rates by comparing findings to a national audit of prenatal diagnosis in 2009. Furthermore, we aimed to evaluate timing and point of presentation of all infants in Ireland diagnosed postnatally with severe CHD.

Methods: This was a single centre retrospective cohort study. All patients born between May $1^{\text {st }} 2015$ and May $1^{\text {st }} 2016$ and admitted to the National Children's' Heart Centre in Our Lady's Children's Hospital (OLCHC) Crumlin in the first 6 weeks of life with a diagnosis of severe structural CHD were eligible for inclusion. Severe structural congenital heart disease was defined as an abnormality requiring catheter or surgical intervention within the first six months of life.

Results: 184 infants satisfied study criteria. The incidence of severe structural CHD in the study period was 2.8/1000 live births. Within this group the prenatal detection rate was 53\%. This compares to a prenatal detection rate of $22 \%$ in a corresponding period in $2009.27 \%$ of infants with postnatally detected CHD were transferred from maternity hospital in the early neonatal period. The remaining 20\% re-presented from home.

When compared to EUROCAT cases over a similar period, we can see that severe lesions are disproportionately represented in the Irish population with a prevalence of 2.4/1000 live births overall. On comparing prenatally and postnatally diagnosed cohorts, it was evident that those subjects not prenatally diagnosed were more likely to be ventilated for transfer (22/86 postnatally diagnosed, 8/ 98 prenatally diagnosed) and more likely to require admission to ICU on arrival (24/86 postnatally diagnosed, 17/98 prenatally diagnosed).

Conclusion: This study shows a significant improvement in prenatal detection of structural CHD in Ireland. This is likely attributable 
to dedicated training and education of sonographers in fetal detection of CHD. The introduction of pre-discharge oxygen saturations checks in the majority of neonatal units has also improved postnatal detection, reducing the number of children discharged from hospital with severe structural congenital heart disease.

\section{P-63}

Parental Psychological Distress after Prenatal versus Postnatal Diagnosis of Congenital Heart Disease Mellander M. (1), Järvholm S. (2), Ekman-Joelsson B. (1), Johannsmeyer A. (3), Carlsson S.-A. (4), Mattsson L.-A. (2), Bratt E.L. (1,5). Department of Pediatric Cardiology, The Queen Silvia Children's hospital, Sahlgrenska University Hospital, Gothenburg, Sweden (1); Department of Obstetrics and Gynaecology, University of Gothenburg. Sahlgrenska University Hospital, Gothenburg, Sweden (2); Södra Älvsborgs hospital, Department of Obstetrics, Borås, Sweden (3); Norra Älvsborgs hospital, Department of Obstetrics, Trollhättan, Sweden (4); Institute of Health and care sciences, Sahlgrenska Academy, University of Gothenburg, Sweden (5)

Introduction: A diagnosis of congenital heart disease (CHD) in a fetus or in a newborn triggers increased levels of psychological distress in parents. Results of previous studies have been inconsistent regarding the impact of pre- versus postnatal diagnosis in this respect. The purpose of this study was to investigate this and to compare with a group with uncomplicated pregnancies.

Method: Participants were recruited from three hospitals in the western part of Sweden. They were divided into three groups; G1: pregnant women with a fetus diagnosed with CHD and their partners, G2: parents of children with postnatally diagnosed CHD and G3: pregnant women with an uncomplicated pregnancy and their partners. Data was collected during pregnancy within one month of inclusion (T1) and 2-6 months after delivery (T2). The instruments used were questionnaire measures: The Hospital Anxiety and Depression scale (HAD), Sence of Coherence (SOC-13) and Life Satisfaction (LiSat 11).

Results: In total, 28 participants in G1 and 152 in G3 completed the questionnaire at T1: G1 scored lower on Sence of coherence (mean $66.6 \pm 9.6$ ) compared to G3 (mean 70.8 $\pm 9.1 ; \mathrm{p}=0.044$ ). At T2, 15 participants in G1, 30 in G2 and 80 in G3 were included: G1 scored lower on Sence of coherence (mean 59.6 \pm 12.6 ) compared to G2 (mean 72.5 \pm 7.5 ) and G3 (mean 70.4 \pm 2.57 ; G1 vs G2 p $=0.001$; $\mathrm{G} 1$ vs $\mathrm{G} 3 \mathrm{p}=0.001)$. Both $\mathrm{G} 1$ and $\mathrm{G} 2$ had higher levels of anxiety compared to G3 (G1 mean 7.6 $\pm 4.69, \mathrm{G} 2$ mean $8.18 \pm 4.16$ and G3 mean $5.05 \pm 3.15$; G1 vs. G3 p $=0.025$; G2 vs G3 p =0.0003). G1 and G2 scored higher on depression (G1 mean 5.73 \pm 4.18 ; G2 mean $4.37 \pm 3.36$ ) compared to G3 (mean $2.93 \pm 2.57$; G1 vs G3 $\mathrm{p}=0.0018$; $\mathrm{G} 2$ vs $\mathrm{G} 3 \mathrm{p}=0.048)$. Life satisfaction at T2 was lower in G1 (mean 44.9 \pm 9.9 ) compared to G2 (mean 56.5 \pm 5.0 ; $\mathrm{p}=<0.0001$ ) and G3 (mean 52.4 $\pm 6.2 ; \mathrm{p}=0.0004$ ).

Conclusion: A prenatal diagnosis of congenital heart disease was associated with a lower level of sense of coherence and life satisfaction, compared with postnatal diagnosis. This motivates further efforts to improve counseling and support in ongoing pregnancies after a prenatal diagnosis.

\section{P-64}

How to counsel parents after prenatal diagnosis of congenital heart disease? A multidisciplinary approach to develop a questionnaire

Kovacevic A. (1), Simmelbauer A. (1), Starystach S. (2), Elsässer M. (3), Sohn C. (3), Müller A. (1), Bär S. (2), Gorenflo M. (1)
Department of Paediatric and Congenital Cardiology, Heidelberg University Hospital, Heidelberg, Germany (1); Max Weber Institute for Sociology, Ruprecht Karls University Heidelberg, Heidelberg, Germany (2); Department of Gynecology and Obstetrics Heidelberg University Hospital, Heidelberg, Germany (3)

Objectives: To develop a questionnaire suitable to assess parental needs for counselling after prenatal diagnosis of congenital heart disease (CHD) in the fetus.

Methods: The questionnaire was developed by paediatric and fetal cardiologists, maternal fetal medicine specialists and sociologists based on literature research and analysis of daily practice. Likert scaled and open-ended questions are combined with sociodemographical data. The questionnaire ends with an open-ended question asking for feedback and additional remarks. The feasibility of the questionnaire was pre-tested on six individuals. The revised version was pilot-tested on 17 parents.

Results: Median gestational age at fetal diagnosis and first counselling was $28+4$ weeks. At time of data collection all children were alive at a median age of 337 days. Response rate was $89.5 \%$. The pilot-test reliability of the dependent variable's dimensions and the sorrows itemset shows good consistency as shown by high values for the reliability coefficient Cronbach's alpha of $>0.7$ (Table 1). Analysis of the open questions showed that parents evaluated the questionnaire as good and perceived it as a chance to participate in the treatment of their child.

Conclusions: The questionnaire measures reliably and validly parental needs for counselling after prenatal diagnosis of CHD in the fetus. This newly developed tool enables us to measure counselling success not only in the dimension of transfer of medical information but also in the dimensions of trust in medical staff, transparency regarding the treatment process, coping resources, and perceived situational control. A multidisciplinary approach, in particular including social science, seems feasible as theoretical and methodological effort is essential for constructing adequate tests. We plan to carry out factor and cluster analyses to find typical subgroups of parents, which can be distinguished in terms of the characteristic value of the dependent variable effective counselling.

Table. Internal consistency of the questionnaire's subscales (reliability).

\begin{tabular}{ll}
\hline Dimension & $\begin{array}{l}\text { Cronbach's } \\
\text { alpha }\end{array}$ \\
\hline $\begin{array}{l}\text { Transfer of medical knowledge } \\
\text { Trust in health personnel/medical staff }\end{array}$ & 0.857 \\
$\begin{array}{l}\text { Transparency regarding the treatment } \\
\text { process }\end{array}$ & 0.891 \\
$\begin{array}{l}\text { Coping resources } \\
\text { Perceived situational control }\end{array}$ & 0.914 \\
Sorrows (item 4 excluded) & 0.706 \\
\hline
\end{tabular}

\section{P-65}

4D Fetal Echocardiography: STIC vs eSTIC

Germanakis I., Pepes S., Parthenakis F., Makrigianakis A. Faculty of Medicine, University of Crete, Heraklion, Greece

4D fetal echocardiography: STIC vs eSTIC

Introduction: Spatial-Temporal-Image-Correlation (STIC) is a 4D Fetal Echocardiography (FE) technique, based on off-line 4D reconstruction of stored $2 \mathrm{D}$ images. As modern electronic probes 
(eSTIC) could improve 4D FE imaging, we aimed to compare STIC vs eSTIC performance.

Methods: Prospective study, of 33 consecutive routine obstetric scans, median GA:23wk (13-31), (16 early, 40 mid-, 10 late-gestation), performed by an expert sonographer. One pair of each 2D and Color Doppler volumes were acquired, by both STIC and eSTIC, consecutively. Anonymized stored volumes were off-line reconstructed by an expert fetal cardiologist. The success in reconstructing recommended FE views, image quality and diagnostic conclusions by each acquisition were compared.

Results: eSTIC files were larger (63 vs $37 \mathrm{MB})$ and of higher temporal resolution (37 vs $24 \mathrm{fps}$ ), $\mathrm{p}<0.001$. The original (A) plane heart axis angle (189 vs 190 degrees) and the mean reconstruction time (4,96 vs 4,94 min) didn't differ. Motion during acquisition was less in eSTIC vs STIC acquisition (12 vs 20 cases, O.R 7.0, 95\% CI 1.7-27.2, $\mathrm{p}=0.002)$. eSTIC image quality (4 level scale: 1-best to 4-nondiagnostic) was significantly superior on acquisition plane $\mathrm{A}(1.45$ vs $1.67, \mathrm{p}=0.022)$, but also to reconstructed planes (plane B: 2.44 vs $2.79, p=0.008$, plane C: 3.56 vs $3.77, p=0.015)$. Similarly the average image quality (planes A,B,C) was significantly better for eSTIC (1.9 vs 2.2, $\mathrm{p}=0.006)$. Comparing efficiency to obtain all recommended FE views eSTIC was classified as superior in $22(33 \%) / 39$ (59\%) / 21

Table.

\begin{tabular}{lrc} 
& eSTIC normal & abnormal \\
\hline STIC normal & 24 & 4 \\
abnormal & 4 & 16 \\
\hline
\end{tabular}

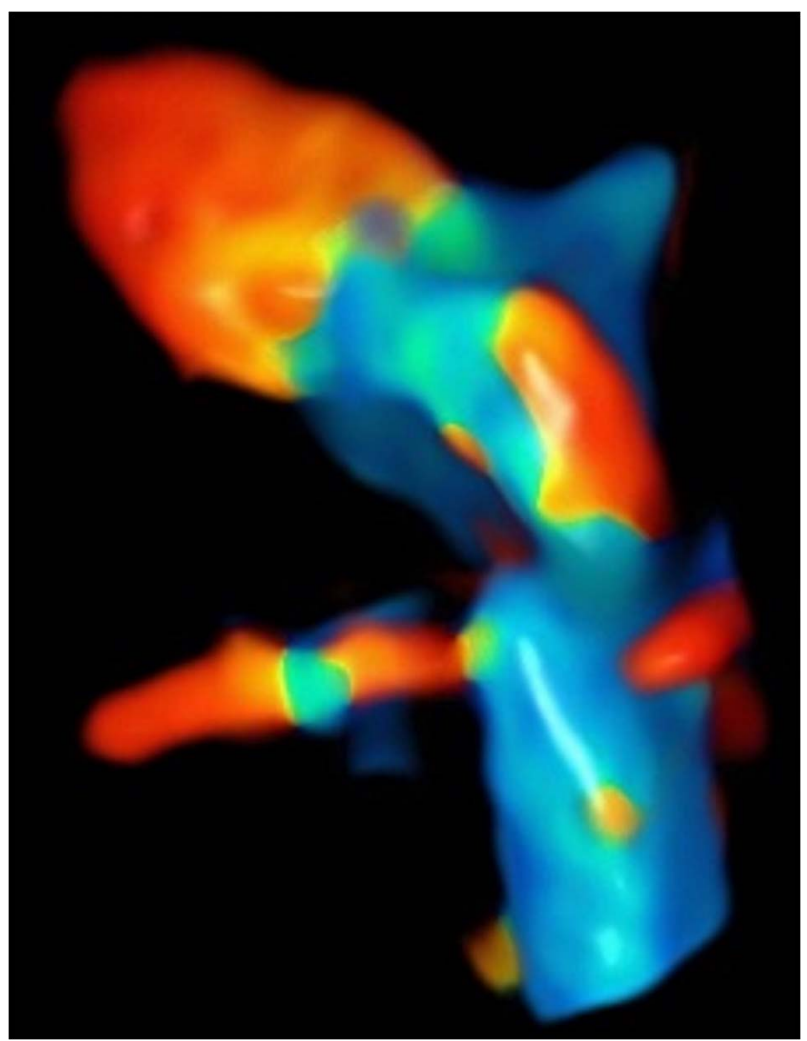

Figure.
(38\%) cases regarding plane A / B / C, respectively, with the remaining cases being classified as of similar performance (out of $<10 \%$ in each plane in favour of STIC).

The diagnostic performance (Table) of the two approaches showed relative good agreement $(\mathrm{kappa}=0,657, \mathrm{p}<0.001)$

Conclusions: Although both STIC and eSTIC require similar offline analysis time, eSTIC being associated with a higher temporal resolution, reduced motion artefacts and a resulting superior image quality in all planes, might offer diagnostic advantages for both offline FE diagnostic purposes. Further study is needed to define potential eSTIC diagnostic advantages.

\section{P-66}

Fetal Echocardiography performance: Indication-based vs "routine" application

Germanakis I. (1), Burianova Bagaki A. (1), Gianias G. (1),

Rogdakis E. (1), Makrigianakis A. (2)

Pediatric Cardiology Unit, Dpt of Pediatrics, University Hospital,

Heraklion, Greece (1); Dpt of Obstetrics and Gynecology, University

Hospital, Heraklion, Greece (2).

Introduction: Established indications (EI) for performing fetal echocardiography (fEcho) are based on documented increased risk for fetal congenital heart disease (fCHD) in the presence of specific indications, over the literature-based baseline fCHD risk in the general population.

Methods: Direct comparison of risk for fCHD (overall and indication specific) relative to the baseline risk for fCHD when fEcho is performed for non-established indications (NEI).

Retrospective study of 2,202 fECHO records (years 2008-16) from a tertiary referral centre, all performed by a single operator under same settings. Referral indications were classified as established (EI) based on recent recommendations (AHA 2014), or non-established (NEI), including: parental wish, "echogenic foci", previous fetus with chromosomal abnormality, NT $<3.5 \mathrm{~mm}$, medications without association with $\mathrm{fCHd}$, maternal heart disease (non-CHD), relatives (non-first degree) with CHD, reduced window in anomaly scan, increased PAPPA.

Odds ratio (O.R, 95\% C.I) for fCHD and critical fCHD (anticipated to require neonatal intervention) were estimated in the presence/absence of EI for fECHO, along with Relative Risk (R.R)

Results: 367 out of 1147 (32\%) of EI cases were associated with fCHD, vs 180 out of 1055 (17\%) of NEI cases (Pearson's Chisquare $65.6, \mathrm{df}=1, \mathrm{p}<0.001)$. O.R for fCHD presence (yes/no): 2.28 (1.86-2.80), R.R for fCHD for EI: 1.42 (1.31-1.53), for NEI: $0.62(0.54-0.7)$

A total of 19 (1.7\%) of EI cases were associated with critical fCHD, compared to $6(0.6 \%)$ of NEI cases $(p=0.016)$, O.R for critical fCHD presence (yes/no): 2.94 (1.17-7.4), and a R.R for critical fCHD for EI: 2.91 (1.16-7.2), for NEI: 0.98 (0.98-0.99)

Among EI cases, the highest probability for fCHD (50-60\%) was documented in cases of suspected fCHD in anomaly scan, polyhydramnion, and fetal chromosomal abnormalities, followed (30-50\%) by increased NT, fetal malformations, with lowest probability $(20-30 \%)$ in $1^{\text {st }}$ degree relatives with CHD, monochorionic twins. The probability for critical fCHD was highest for suspected fCHD during anomaly scan (5\%), increased NT and fetal malformation (1.5-2\%).

Conclusions: Indication-based fetal echocardiography is associated with 2-fold and 3-fold increased diagnostic yield for fetal $\mathrm{CHD}$ and critical fetal CHD, respectively, compared to its performance when applied "routinely" for non-established indications. 


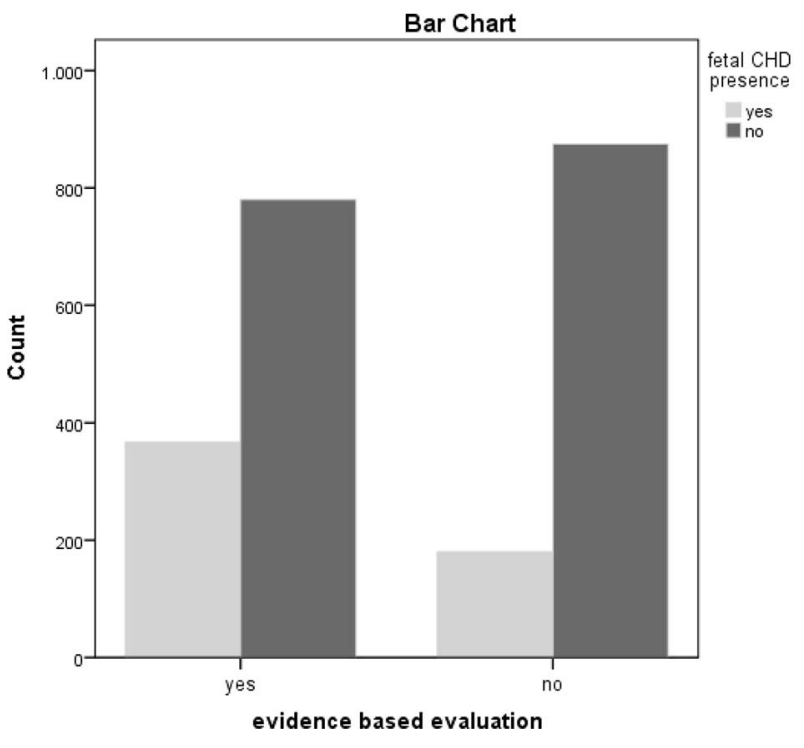

Figure.

\section{P-67}

Two faces of atrial flutter: double tachycardias in the fetus and neonates

Tunca Sahin G. (1), Duman D. (2), Babaoglu K. (3), Goynumer G. (4), Wong A. (5),Beattie B. (6), Conner C. (6), Uzun O. (5) (1)Department of Pediatric Cardiology, Istanbul Saglik Bilimleri University Istanbul Mehmet Akif Ersoy Thoracic and Cardiovascular Surgery Center and Research Hospital, Istanbul, Turkey. (2) Department of Pediatric Cardiology, Faculty of Medicine, Hacettepe University , Ankara, Turkey. (3) Department of Pediatric Cardiology, Faculty of Medicine, Kocaeli University, Kocaeli, Turkey. (4)Department of Obstetrics and Gynecology, Medeniyet University, Istanbul, Turkey. (5) Department of Pediatric Cardiology, Noah's Ark Children's Hospital for Wales, University Hospital of Wales, Cardiff, Wales, UK. (6)Department of Fetal Medicine, University Hospital of Wales, Cardiff, Wales, UK

Introduction: Fetal tachyarrhythmia is an uncommon condition that occurs in $0.4 \%$ to $0.6 \%$ of all pregnancies. Atrial flutter (AFl) accounts for $26 \%$ to $29 \%$ of all fetal tachyarrhythmias. There has been no report specifically studying association of fetal atrial flutter with postnatal atrioventricular tachycardia. We aimed to investigate prenatal and postnatal outcomes of atrial flutter and its role in the development of a second tachycardia following restoration of sinus rhythm in the fetus or newborn.

Methods: This study is a retrospective review of all fetuses presented with atrial flutter between October 2001 and August 2017 at the University Hospital of Wales. Demographical characteristics, type and time of the tachyarryhtmia, echocardiographic findings (structure of heart, hydrops, function), applied medical therapies and outcomes were evaluated.

Results: 70 fetuses were diagnosed with fetal tachycardia of whom 24 cases were intermittant and the rest was persistant tachycardia. 54 had supraventricular tachycardia (SVT) and 16 had atrial flutter. 13 of 16 patients with atrial flutter patient were managed by medical treatment and three were delivered without treatment. Five fetuses with fetal atrial flutter developed AVRT after DC cardioversion immediately at birth and four of them exhibited preexcitation in the ECG. One fetus with long RP tachycardia also developed atrial flutter before birth for which the neonate required cardioversion after birth. Two patients had hydrops, two patients Ebstein's anomaly but the rest of the patients had normal heart. Two of the fetuses presented with atrial flutter was diagnosed in 20 to 21 weeks of gestation and the others were diagnosed at the third trimestr. In two newborns preexcitation became evidend two weeks after discharge from hospital on a Holter recording. One asymptomatic patient with fetal atrial flutter and neonatal preexcitation required accessory pathway ablation.

Conclusion: Fetal AFl in our study showed a strong association with rhythms involving an accessory pathway: atrioventricular reciprocating tachycardia, blocked reentrant premature atrial contractions, and ventricular preexcitation. We suggest that postnatal electrocardiograms after birth should be carefully reviewed in fetuses presenting with atrial flutter and Holter monitoring should be considered as AVRT could emerge in the neonatal period.

\section{P-68 \\ Do prenatal intracardiac echogenic foci affect postnatal cardiac function? \\ Bader R.S. \\ King Abdul Aziz College of Medicine, King Abdul. Aziz University, Jeddah, Saudi Arabia}

Introduction: Echogenic foci in the prenatal hear is not an uncommon finding.

Objective: To determine whether prenatally diagnosed intracardiac echogenic foci are associated with neonatal cardiac dysfunction and persistence.

Methods: Fetuses in which intracardiac echogenic foci were shown on prenatal sonography at 1 perinatal center from (September 2014 to December 2016) underwent postnatal echocardiography at ages 1 month to1 year.

A single pediatric cardiologist assessed cardiac function by measuring the left ventricular shortening fraction and myocardial performance index. The presence of tricuspid valve regurgitation was also sought

Results: Prenatally 60 fetuses had intracardiac echogenic foci mean age \pm SD at diagnosis $(23 \pm 3.1) .53(88.3 \%)$ had left ventricular intracardiac echogenic foci, and 7 (11.6\%) had right ventricular intracardiac echogenic foci.

12 pregnant ladies were lost for follow up ( 2 fetuses of $7(28,5 \%)$ with right ventricular intracardiac echogenic foci., and 10 fetuses of 53 (18.8\%) with LV intracardiac echogenic foci \%)

Post natally, those infants, 32 (66.6\%) males and 16 (33.3\%) females were examined. at a mean age \pm SD of $7.4 \pm 3.1$ months. Prenatally, all infants had a normal left ventricular shortening fraction. The overall mean left ventricular myocardial performance index (reference value, $0.36 \pm 0.06$ ), was normal for both infants with left ventricular intracardiac echogenic foci $(0.32 \pm 0.01)$ and those with right ventricular intracardiac echogenic foci $(0,33 \pm 0.05)$.

Trace tricuspid valve regurgitation were noted in 15 (31\%) of the infants. Left ventricular intracardiac echogenic foci persisted in 15 infants (34.8\%), whereas right ventricular intracardiac echogenic foci persisted in 1 infant (20\%).

Conclusions: Prenatally diagnosed intracardiac echogenic foci can be persistent but is not associated with myocardial dysfunction in the first year of life.

Key words: echocardiography; intracardiac echogenic focus; prenatal diagnosis; sonography.

\section{P-69}

Prenatal diagnosis of vascular rings: single center experience

Pavlova A. (1), Kurkevych A. (1), Mykychak Y. (2), Rudenko N. (1), Raad T. (3), Yemets R. (4) 
Department of Cardiology, Ukrainian Children's Cardiac Center, Kyiv, Ukraine (1); Department of Cardiac Surgery, Ukrainian Children's Cardiac Center, Kyiv, Ukraine (2); Radiology Department, Ukrainian Children's Cardiac Center, Kyiv, Ukraine (3); Department of Anesthesiology and Intensive Care, Ukrainian Children's Cardiac Center, Kyiv, Ukraine (4)

Objectives: In this study we analyzed our experience in the prenatal ultrasound diagnostics vascular rings and the impact of volume growth on its accuracy.

Methods: We analyzed fetal echocardiograms and clinical histories of 42 patients with a diagnosis of vascular ring at our institution from 2004 to 2016 . We evaluated the learning curve of the the prenatal diagnosis of vascular rings.

Results: Between 2004 and 2008 the total number of fetal echocardiographies increased from 27 per year to an average of 1000 per year. Overall, from 2004 to 2016, 6793 pregnant women were examined. Until 2012 there was no vascular ring diagnosis established prenatally. In the period from 2012 to 2016 we diagnosed 42 cases of vascular rings. There were 3 diagnoses of vascular ring established in 2012, 2 in 2013, 11 in 2014, 12 in 2015 and 14 in 2016. The double aortic arch was diagnosed in 5 cases, of which in 1 case there was present a left superior vena cava draining to the coronary sinus. Right aortic arch with left ductus arteriosus and aberrant left subclavian artery was diagnosed in 37 cases. Among the 37 diagnosis the vascular ring was isolated in 28 and associated with other structural congenital heart disease in 9 (1 persisted left superior vena cava draining to the coronary sinus, 7 ventricular septal defects, in of which in 1 case there was present a persistent left superior vena cava draining to the coronary sinus, 1 congenitally corrected transposition of the great arteries with ventricular septal defect and pulmonary atresia).

Conclusions: Prenatal ultrasound diagnostics of vascular rings has high sensitivity and specificity. The 3-vessel and trachea view, the aortic arch long-axis view, the transverse view of the upper abdomen, demonstrating the abdominal aorta are essential for the reliable correct diagnosis.

\section{P-70 \\ Echocardiographic assessment of hemodynamics in fetus with transposition of the great arteries and intact ventricular septum: impact on immediate post natal desaturation \\ Charron M.A., Lachaud M., Peyre M., Mendez Santos A., Carmant L., Birca A., Wu R., Brassard M., Raboisson M.J. \\ Sainte-Justine University Hospital Center, Montreal, Canada}

Introduction: little is known about the physiology and distribution of ventricular flows in fetuses with transposition of the great arteries and intact ventricular septum (TGA-IVS), especially regarding the risk for immediate post natal desaturation. Ductal flow is the result of a complex interaction between left and right ventricular outputs as well as pulmonary and peripheral resistances. Methods: longitudinal hemodynamic assessment of 30 fetuses with TGA-IVS was done 3 times during pregnancy at 18-22, 28-32 and 35-38 weeks of gestation. We measured the size of the inter-atrial defect and calculated left and right ventricular output as well as systolic and diastolic ductal flow. Fetuses were divided into 2 groups depending on their postnatal saturation. Fetuses with postnatal saturation $<65 \%$ at the upper extremities were included in group 1. Echocardiographic parameters were compared between the 2 groups.

Results: 3 fetuses had a restrictive ductus arteriosus with high velocity flow and were excluded from the calculations, they all had postnatal saturation $<65 \%$. Five additional fetuses had a postnatal saturation $<65 \%$. There was no difference in left or right ventricular output between the groups at first evaluation. At 28-32 weeks, the inter-atrial defect was smaller in group $1(3 \pm 0.3$ versus $4 \pm 0.8 \mathrm{~mm}, \mathrm{p}=0.012)$ as well as left ventricular output (221 versus $307 \mathrm{ml} / \mathrm{min}, \mathrm{p}=0.05)$. There was a correlation between left ventricular output and size of the inter-atrial defect $(R 2=0,35$, $\mathrm{p}=0,003)$. Ductal flow was lower in group 1 after 35 weeks (56 versus $130 \mathrm{ml} / \mathrm{min}, \mathrm{p}=0,007)$, mainly due to a decrease in diastolic flow which was retrograde in group $1(-7,1$ versus $22 \mathrm{ml} /$ min, $\mathrm{p}<0.004)$. A total of 12 patients $(4 / 5$ in group 1$)$ had a retrograde diastolic flow in the ductus arteriosus after 35 weeks. Retrograde ductal diastolic flow had a positive predictive value of $40 \%$ and a negative predictive value of $100 \%$ to predict saturation $<65 \%$ at birth.

Conclusion: in third trimester, retrograde diastolic flow in the ductus arteriosus is possibly secondary to low vascular pulmonary resistance but also to decreased left ventricular output in case of small inter-atrial defect. Fetuses with this ductal flow pattern are at risk for postnatal inadequate inter-atrial mixing.

\section{P-71}

Aortic isthmic flow in fetuses with Ebstein's anomaly and/ or tricuspid valve dysplasia

Peyre M. (1), Lachaud M. (1), Mendez Santos A. (1), Berger A. (1), Brisebois S. (1), Thomas Chabaneix J. (2), Raboisson M.J (1).

Sainte-Justine University Hospital Center, Montreal, Canada (1) Hôpital Cardiologique du Haut Lévêque, Pessac, France (2)

Introduction: Ebstein's anomaly and/or tricuspid valve dysplasia (TVD) associated with tricuspid insufficiency has a poor outcome during fetal life. Pre natal echocardiographic parameters based on right chambers size and right ventricular flow are helpful but have varying degrees of sensitivity to predict pre/neonatal outcome. Systolic flow in the aortic isthmus is the result of the balance between left and right ventricular flow and should be affected by lower right ventricular output seen in significant tricuspid regurgitation.

Objective: to evaluate isthmic systolic index (ISI) as a predictor of severity in a population of foetuses with Ebstein's anomaly or TVD and tricuspid regurgitation.

Methods: thirty nine echocardiograms of 21 fetuses (9 Ebstein's anomalies, 12 TVD) diagnosed between January 2010 and June 2017 were reviewed. ISI was calculated on isthmic aortic waveforms as peak systolic velocity/end-systolic velocity and compared to a population of 83 normal fetuses matched for gestational age. Isthmic flow index (IFI = (systolic flow-diastolic flow)/systolic flow) was also calculated as well as right ventricular output. A sub group of 6 fetuses with hydrops, intrauterine or perinatal death were defined as group 1 and compared to the 15 other fetuses (group 2) as well as to the normal population.

Results: two pregnancies were interrupted in group 1 versus 4 in group 2. Overall survival at hospital discharge was $48 \%$. At 21 weeks of gestation ISI was significantly higher for fetuses in group 1 compared to normal population $(0.12 \pm 0.06$ versus $0.18 \pm 0,06, p=0.02)$. At the same gestational age, IFI was increased in group 1 compared to group $2(0.79 \pm 0.36$ versus $0.54 \pm 0.28, p=0.01)$. At 30 weeks of gestation, ISI was higher in fetuses with Ebstein's anomaly/TVD than in normal population $(0.10 \pm 0,40$ versus $0.08 \pm 0.14, p=0.04)$ and right ventricular output was lower in group 1 compared to group $2(78,1 \pm 11$ versus $341.8 \pm 20.3, \mathrm{p}=0.01$ ).

Conclusion: decreased right ventricular output in case of significant tricuspid regurgitation could lead to left ventricular 
preponderance at the level of the aortic isthmus. Isthmic flow indexes could be interesting additional prognosis factors in this at risk situation.

\section{P-72}

An increasing experience in the antenatal diagnosis of right aortic arch

Mantagou L., Forster J., Brown EM., Barwick S.

Leeds General Infirmary, Leeds, UK

Background: The 3VT (3 vessel and trachea) view has been introduced in mid trimester obstetric screening. This images the SVC, transverse aortic arch, ductus arteriosus and trachea. RAA (right aortic arch) can be a variation of normal and 25\% are associated with vascular ring.

Aim: To look at the antenatal detection of a cardiac abnormality where a $3 \mathrm{VT}$ abnormality has been suspected, and to look at the outcome of patients where this is confirmed.

Method: Retrospective Fetal Cardiology database review over 2 years in a Congenital Cardiology centre.

Results: In 2016, RAA was identified in 38\% referred with abnormal 3VT. 5/17 (29\%) children with antenatally diagnosed RAA had surgery to release a vascular ring $(4=$ abnormal $3 \mathrm{VT}$, $1=$ increased NT). All these had RAA and aberrant LSCA and 1 RAA, aberrant LSCA + CoA. The age at operation was 22 days9 months

Cardiac pathology was identified in 53\% referred with abnormal 3VT (15 RAA, 3 bilateral SVCs, 1 TGA, 1 TOF, 1 PS).

In 2017, RAA was identified in 35\% referred with abnormal 3VT. None of the children diagnosed with RAA have had cardiac surgery yet.

Cardiac pathology was identified in $62 \%$ referred with abnormal 3VT ( 26 RAA, 6 bilateral SVCs, 2 single LSVC to CS, 3 TOF, 1 TOF + bilateral SVCs, 2 CoA, 1 simple TGA, 4 complex TGA, 1 Ebstein's)

Conclusion: There is an increasing antenatal detection of RAA due the increasing awareness at mid trimester screening. RAA was previously only diagnosed in a symptomatic postnatal population and therefore remains a grey area as to follow up and management in an asymptomatic well population.

Table 1. Reason for referral to Fetal Cardiology

\begin{tabular}{|c|c|c|}
\hline & 2016 & $\begin{array}{c}2017 \\
\text { (to date) }\end{array}$ \\
\hline Total number of Fetal echoes & 1153 & 1111 \\
\hline $\begin{array}{l}\text { Number referred for suspected fetal cardiac } \\
\text { abnormality }\end{array}$ & 295 & 330 \\
\hline Number referred for abnormal 3VT & 40 & 74 \\
\hline
\end{tabular}

Table 2. Outcome of suspected abnormal 3VT

\begin{tabular}{lll}
\hline & $\mathbf{2 0 1 6}(\mathbf{n}=\mathbf{4 0})$ & $\mathbf{2 0 1 7}(\mathbf{n}=\mathbf{7 4})$ \\
\hline Normal Fetal Echo & 19 & 28 \\
Right aortic arch & 15 & 26 \\
& + 2 RAA referred & + 2 RAA referred \\
Other abnormality & 6 & with inc NT \\
\hline
\end{tabular}

P-73

\section{Abdominal right isomerism with normal intracardiac} anatomy in the fetus

Jowett V. (1,2), Bartsota M. (1,2,3), Carvalho J.S. $(1,4)$

Royal Brompton and Harefield NHS Trust, London, UK (1);

Queen Charlotte's Hospital, London, UK (2); Chelsea and

Westminster Hospital, London, UK (3); St George's Hospital, London, $U K(4)$

Objectives: In the postnatal sequential segmental diagnosis of cardiac abnormalities, it has historically been recognised that atrial situs usually follows that of the abdominal situs. Recognition of abdominal right isomerism (RI) or left isomerism (LI) is often associated with complex congenital heart disease (CHD). However, whilst 'less complex or minor CHD' and 'normal hearts' have been reported with LI, the same has not been previously reported in the setting of abdominal RI. We had identified a number of such cases in our fetal practice and therefore sought to clarify their features.

Methods: Patients were identified from the electronic databases in our tertiary fetal cardiac service comprising three fetal cardiology units. Prenatal echo reports and postnatal reports were reviewed. Results: Six cases of abdominal RI without complex CHD were identified. Gestational age at diagnosis ranged from $18+0$ to $23+1$ weeks gestation. Four cases were livebirths; one pregnancy was terminated and one lost to follow up. The stomach was right sided with levocardia and apex to the left in 5 cases and the stomach left sided with dextrocardia and apex to the right in 1 case. The position of the aorta and inferior vena caca (IVC) is summarised in table 1:

All cases had biventricular atrio-ventricular connections. There was right hand topology in the cases with laevocardia and apex to the left and left hand topology in the case with dextrocardia and apex to the right. Ventriculo-arterial connections were concordant in all cases. In four cases, no cardiac abnormalities were seen. In two cases, abnormalities of systemic venous return were identified: One fetus had a left superior vena cava (SVC) and IVC draining to a dilated coronary sinus; a second fetus had anomalous drainage of the IVC to the left sided atrium, a left SVC draining to the coronary sinus and abnormal drainage of hepatic veins. The presence of a coronary sinus in these 2 cases indicated discordance between abdominal and thoracic situs. One case developed bowel obstruction postnatally secondary to malrotation.

Conclusions: Abdominal RI can occur with normal intracardiac anatomy. This may be explained in some cases by discordance between abdominal and thoracic situs. Detailed sequential segmental analysis of the fetus is important to identify such cases as these fetuses are at risk of the postnatal complications of isomerism such as gut malrotation, splenic abnormalities and primary ciliary dyskinesia.

Table 1. Ultrasound findings of six fetuses with abdominal features of RI

\begin{tabular}{llcccc}
\hline Case & $\begin{array}{l}\text { Position of } \\
\text { stomach }\end{array}$ & $\begin{array}{l}\text { Position of } \\
\text { aorta }\end{array}$ & $\begin{array}{l}\text { Position } \\
\text { of IVC }\end{array}$ & $\begin{array}{l}\text { Position of } \\
\text { heart }\end{array}$ & $\begin{array}{l}\text { Position of } \\
\text { Apex }\end{array}$ \\
\hline 1 & R & Midline & R & L & L \\
2 & R & Midline/R & R & L & L \\
3 & R & Midline/R & R & L & L \\
4 & R & L & L & L & L \\
5 & L & R & R & R & R \\
6 & R & L & L & L & L \\
\hline
\end{tabular}

Key: $R=$ right, $L=$ left; $I V C=$ inferior vena cava 


\section{P-74}

Prevalence of congenital heart disease in fetuses conceived after assisted reproductive technology. Observational study

Fesslova V. (1), Giorgione V. (2), Boschetto C. (1), Brankovic J. (1), Cavoretto P. (2)

(1) Center of Fetal Cardiology, Policlinico San Donato IRCCS, Milan, Italy. (2) Dpt. Obst. Gynecology, San Raffaele Hospital IRCCS, Vita Salute University, Milan, Italy

Background: Assisted reproductive techniques (ART) are nowadays increasingly used in couples with infertility and there is a concern about outcomes of children conceived, both for the preterm delivery and low birth weight and a possible increased risk of congenital anomalies. Data regarding a possibile increase of congenital heart disease (CHD) are coming out and therefore the women pregnant after ART are often referred for fetal echocardiography.

The aim of this study was to analyse the prevalence of CHD in fetuses conceived after ART referred to our center, specifically for an evaluation of the fetal heart

Method: observational prospective study regarding unselected consecutive fetuses conceived after ART referred to our Center between Jan. 2010 - Nov. 2017.

Population: Five hundred seven women (aged 30-55 yrs, median 36) that became pregnant after ART underwent fetal echocardiography at median gestational age 21 week's gestation: 197 pregnancies resulted after in vitro fertilization (IVF), 206 after intracytoplasmatic sperm injection (ICSI) and 104 after heterologous fertilization (egg or embryo-donation ED); 27/197 IVF, 33/206 ICSI and 25/104 ED were twins (one triplet). Cases with known chromosomal anomaly or major extracardiac anomalies were excluded. Suspicious cardiac findings were reported in two cases at the 1st level scan and not confirmed by us.

Results: CHD was found in 23 pregnancies (5 twins of healthy cotwins); 11 cases were product of IVF, 4 of ICSI and 8 of ED. Prevalence of CHD in the whole population was 23/ $507=4.54 \%$, in IVF $11 / 197=5.58 \%$, in ICSI $4 / 206=1.94 \%$ and in $\mathrm{ED} 8 / 104=7.69 \%$. Prevalence in all ART singletons was $18 /$ $425=4.23 \%$, in twins $5 / 85=5.9 \%$. CHD found after IVF were 1 HLH, 2 TGA, 1 PAtr + VSD, 1 DORV, 1 VSD,

1 AVSD, 1 CoA + AS, 1 PLSVC + mild CoA, 2 Ebstein/ NonEbstein; after ICSI 3 HLH, 1 AVSD; after ED 2 PS, 1 TF, 1 PAtr + VSD, 2 CoA, 1 rhadomyoma.

Conclusions: The data of our observational study show an increased prevalence of CHD after all techniques of ART, with respect to references of normal population. Obviously, further research is needed in this topic.

\section{P-75}

Fetal ventricular arrhythmia: the role of superior vena cava-aorta Doppler approach

Mendez A., Mawad W., Peyre M., Lachaud M., Fournier A., Abadir S., Raboisson M.J.

Sainte-Justine University Hospital Center, Montréal, Canada

Introduction: Fetal ventricular arrhythmias are rare, and their diagnosis is often challenging. The M-mode recording technique can be limited and difficult to interpret; the addition of simultaneous superior vena cava-aorta Doppler can improve diagnosis accuracy. Our aim is to illustrate in a didactic approach the main types of ventricular arrhythmias and the findings on superior vena cava (SVC)-aorta (Ao) Doppler images as well as available postnatal ECGs.
Methods: Among our cohort of 23 ventricular arrhythmias diagnosed over a period of 22 years at our institution, we selected 4 examples to illustrate the use of SVC-Ao Doppler recording in rare fetal arrhythmias.

Results: We describe an example of premature ventricular contraction (among 17/24 fetal ventricular arrhythmias) in figure $1 \mathrm{~A}$, followed by an example of ventricular tachycardia (among 4/24 ventricular arrhythmias) in figure 1B. Figures 1C and $1 \mathrm{D}$ illustrate a case of torsade de pointe, and finally figures $1 \mathrm{E}$ and $1 \mathrm{~F}$ display a case of accelerated idioventricular rhythm and in both cases, confirmation of the diagnosis with postnatal ECG tracings.

Conclusions: The SVC-Ao Doppler recording is a valuable tool to improve diagnosis accuracy particularly in rare cases of fetal ventricular arrhythmias.

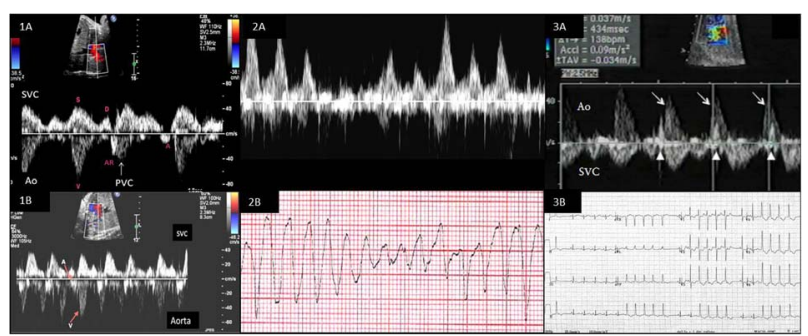

Figure.

1A. Superior vena cava (SVC)-aorta (Ao) Doppler recording demonstrating a premature ventricular contraction $(P V C)$, with the occurrence of a premature aortic ejection with a lower amplitude than those in sinus rhythm with simultaneous retrograde atrial contraction (AR). 1B. SVC-Ao Doppler recording demonstrating an example of sustained ventricular tachycardia with complete atrio-ventricular dissociation and higher ventricular $(V)$ rates compared to atrial contractions $(A) .1 C$ and $1 D$ illustrate a case of torsade de pointe with typical unequal amplitude and irregular fast ventricular ejections (1C) and post natal torsade de pointes in $1 \mathrm{D}$ (lead $\mathrm{II}, 25 \mathrm{~mm} / \mathrm{s}$ speed). $1 \mathrm{E}$ and $1 F$ illustrate a case of accelerated idioventricular rhythm: in $1 E$, $S V C-A o$ Doppler recording shows dissociation between a higher ventricular rate $(V)$ at $170 / \mathrm{min}$ relative to a lower atrial rate $(A)$ at 140/min. 12 lead-post natal ECG illustrated in $1 F$ shows burst of accelerated idioventricular rhythm with a left bundle branch block pattern occurring at a rate about $20 \%$ faster than the sinus rate.

\section{P-76}

Fetal cardiac function in maternal diabetes: a speckle tracking echocardiography study

Miranda J. O. (1,2), Cerqueira R.J. (2,3), Ramaho C. (4), HenriquesCoelho T. (5), Areias J.C. $(1,5)$

Department of Pediatric Cardiology, São João Hospital Centre, Porto, Portugal (1); Department of Surgery and Physiology, Faculty of Medicine, University of Porto, Portugal (2); Department of Cardiothoracic Surgery, São João Hospital Centre, Porto, Portugal (3); Center of Prenatal Diagnosis, Department of Gynecology and Obstetrics, São João Hospital Centre, Porto, Portugal (4); Department of Pediatrics, Faculty of Medicine, University of Porto, Portugal (5)

Introduction: Intrauterine exposure to a diabetic environment is associated with adverse fetal myocardial remodeling. The aim of this study was to assess systolic and diastolic function of fetuses exposed to maternal diabetes when compared with controls, using speckle tracking echocardiography. We hypothesized that fetuses exposed to maternal diabetes present signs of biventricular dysfunction which can be detected by deformation analysis. 
Methods: Cross-sectional study of 129 structurally normal heart fetuses, including 76 fetuses of diabetic mothers and 53 of non-diabetic mothers. Maternal baseline characteristics and echocardiographic data were prospectively collected at 30-33 weeks of gestation.

Results: Fetuses of diabetic mothers had a significantly thicker interventricular septum when compared to controls [median (IQR): 4.25 (3.87-4.50)mm Vs 3.67 (3.40-3.93)mm, p < 0.001], however no effect modification was demonstrated on myocardial deformation. Deformation analysis showed a significantly lower early and late diastolic strain rate for both ventricles in the maternal diabetes group, suggesting a biventricular diastolic impairment. Additionally, a lower global longitudinal strain was observed for the right ventricle in the study group. (Table 1) Multivariate analysis revealed that maternal age is an independent predictor of left and right ventricle global longitudinal strain $(p<0.05)$, with a significant effect only in maternal diabetes after group stratification.

Conclusions: Fetuses of diabetic mothers present signs of biventricular diastolic dysfunction and right ventricle systolic dysfunction by deformation analysis at the third-trimester of pregnancy. They may represent a special indication group for functional cardiac assessment, independently of septal hypertrophy.

Table. Deformation analysis of left and right ventricles

\begin{tabular}{lccc}
\hline & $\begin{array}{c}\text { Control } \\
\text { Group }(\mathbf{n}=\mathbf{5 3})\end{array}$ & $\begin{array}{c}\text { Maternal Diabetes } \\
\text { Group }(\mathbf{n}=\mathbf{7 6})\end{array}$ & p value \\
\hline Frame rate (fps) & $110(96-126)$ & $110(91-124)$ & 0.73 \\
Fetal heart rate (bpm) & $134 \pm 12$ & $137 \pm 12$ & 0.14 \\
LEFT VENTRICLE & & & \\
GLS (\%) & $-16.99 \pm 2.42$ & $-16.01 \pm 3.45$ & 0.076 \\
Systolic SR (1/s) & $-1.73 \pm 0.34$ & $-1.62 \pm 0.39$ & 0.10 \\
Diastolic SR & $2.26 \pm 0.68$ & $1.85 \pm 0.72$ & \\
$\quad$ Early diastolic (1/s) & $1.78 \pm 0.57$ & $1.50 \pm 0.52$ & $\mathbf{0 . 0 0 1}$ \\
$\quad$ Late diastolic (1/s) & $-15.52 \pm 3.86$ & $-13.67 \pm 4.18$ & $\mathbf{0 . 0 0 5}$ \\
RIGHT VENTRICLE & $-1.49 \pm 0.39$ & $-1.42 \pm 0.45$ & 0.012 \\
GLS (\%) & & & \\
Systolic SR (1/s) & $1.97 \pm 0.73$ & $1.57 \pm 0.73$ & $\mathbf{0 . 0 0 2}$ \\
Diastolic SR & Early diastolic (1/s) & $1.68 \pm 0.79$ & $\mathbf{0 . 0 2 1}$ \\
$\quad$ Late diastolic (1/s) & $2.00 \pm 0.77$ & & \\
\hline
\end{tabular}

Data presented as mean \pm standard deviation or median (interquartile range). GLS - Global Longitudinal Strain, SR - Strain Rate.

P-77

A Single-Centre Experience with Antenatally-Diagnosed Atrioventricular Block

Chia W.L.S, Sundararaghavan S., Xu M., Ang W.L., Chen C.K., Tan T.H., Choo J.T.L.

KK Women's and Children's Hospital, Singapore.

Background: Cardiac rhythm abnormalities are found in 1-2\% of fetuses. These may be benign or may be associated with fetal hydrops. Complete atrioventricular (AV) block occurs in approximately 1 in 20,000 live births. 90\% of isolated AV block cases are associated with anti Ro-antibodies which result in direct immunological damage to the AV node. Complete AV block carries a risk of intra-uterine death and the long term risk of dilated cardiomyopathy.

Methods: The Fetal Cardiology database of a tertiary referral center was reviewed retrospectively over a 20 year period from 1997 to 2017. Cases of antenatally-diagnosed AV block were identified and their case histories reviewed.
Results: During the study period, out of $3.1 \%$ of the referrals to Fetal Cardiology, 9 cases of AV block were antenatally diagnosed. Complete AV block made up $27 \%$ of cardiac rhythm disorders in the Cardiac Fetal Database. Detailed case histories and echocardiographic images were available from 2012. Over the last 5 years, 5 cases presented antenatally to our Cardiology centre. All mothers were anti-SSA positive. In 4 pregnancies, the mothers were treated with transplacental oral steroids. Maternal oral salbutamol was used in 2 pregnancies. 1 patient refused steroids and salbutamol. 3 infants required isoprenaline in the neonatal period. 3 infants underwent insertion of permanent pacemaker. None developed cardiomyopathy, and no deaths were noted. Conclusion: The incidence of antenatally diagnosed complete AV block was noted to be higher in our experience in comparison to Western countries. Treatment options include fluorinated steroids and sympathomimetics to increase fetal heart rate. Despite a range of treatments, none of the infants developed cardiomyopathy. Further directions in immune-mediated AV block includes the determination of maternal serum anti-SSA titres.

\section{P-78}

When flecainide is not available... A single center experience in treating fetal supraventricular tachycardia (SVT)

Duliban J. (1), Miszczak-Knecht M. (2), Hamela-Olkowska A. (3), Pręgowska K. (2), Bieganowska K. (2), Dangel J. (3).

Medical University of Warsaw, 2nd Department of Obstetrics and Gynecology (1); Children's Memorial Health Institute, Department of Pediatric Cardiology, Warsaw, Poland (2); Centre of Postgraduate Medical Education, Perinatal Cardiology and Congenital Anomalies Department (3); Warsaw, Poland

The first-line treatment for fetal supraventricular tachycardia (SVT) are digoxin and flecainide. Due to the fact, that flecainide is not available in our country, we decided to use amiodarone in such cases instead.

We reviewed charts of fetuses treated for SVT in our Center between 2002 and 2017. We analyzed the effectiveness of treatment of paroxysmal and permanent SVT. We examined the maximal fetal heart rate (FHR), fetal condition at the introduction of the treatment by Cardiovascular Profile Score (CVPS), drugs applied with special consideration of amiodarone and treatment effectiveness. The time of conversion to sinus rhythm or improvement in CVPS were taken into account.

There were 114 SVT diagnosed cases (47 paroxysmal, 67 permanent). Average gestational age at diagnosis was 28. The VA was long in 59 fetuses, short in 30, 4 had variable time and in 21 VA wasn't determined. Most of the cases were treated (106/114; 93\%). The average maximal FHR among treated group was 246/ min, the average CVPS before treatment was 6 and fetal hydrops was diagnosed in $26,42 \%$ of cases. Digoxin was used in monotherapy in 21 fetal SVT mostly with short VA without hydrops ( $95 \%$ success, average time to conversion to sinus rhythm -8 days). Amiodarone as an only treatment was applied in 38 cases with long VA SVT and/or SVT with hydrops $(97,4 \%$ success, average time to conversion -6 days). A combination of those two drugs was used in 36 patients $(83,3 \%$ success). 10 pts were treated with metoprolol (7 in monotherapy). Other drugs were additionally used in 6 non-responding cases (propranolol - 1, propafenone - 1 , sotalol - 2 or adenosine injected directly to the umbilical vein -3 ). In total, SVT treatment was effective in $90,6 \%$ of cases. In 4 unsuccessful cases, we achieved slowdown of the rhythm and improvement in CVPS. In amiodarone group only mild side effects occurred - TSH rise (8pts, no need of dosage change), 
itchiness/cholestasis/abnormal liver enzymes (3pts), vasculitis (3pts) and rush (2pts).

We proved, that amiodarone is an effective and safe drug for treatment of supraventricular tachycardia in fetuses if flecainide is inaccessible

\section{P-79}

The normal karyotype does not exclude genetic problems in fetuses with congenital heart defects

Szarla K. (1), Duliban J. (2), Grzyb A. (3,4), Nowakowska B.A. (5), Debska M. (6), Wlasienko P. (2,5), Koleśnik A, (3), Dangel J. (3) Medical University of Warsaw, Students' Scientific Club of Perinatal Cardiology (1); Medical University of Warsaw, 2nd Department of Obstetrics and Gynecology (2); Centre of Postgraduate Medical Education, Perinatal Cardiology and Congenital Anomalies Department (3), Children's Memorial Health Institute, Department of Pediatric Cardiology (4); Institute of Mother and Child, Department of Medical Genetics (5), Centre of Postgraduate Medical Education, 2nd Department of Obstetrics and Gynecology (6), Warsaw, Poland

Introduction: Genetic problems which coexisted with congenital heart defects (CHD) significantly changed the prognosis. Classical karyotype had been performed for years, but it was exchanged by chromosomal microarray (CMA). The aim of this study was to determine the type and frequency of genetic abnormalities in fetuses with diagnosis of CHD using CMA.

Methods: 99 fetuses after detailed fetal echocardiography performed between 2015-2017 in referral fetal cardiology center) were referred to invasive genetic testing. Chromosomal aneuploidies and submicroscopic copy number variations (CNVs) were identified in amniocytes DNA samples using high-resolution CMA.

Results: Out of 99 fetuses with CHD 37 (37\%) had genetic problems. There were $15(26 \%)$ out of 58 fetuses with an isolated CHD and 22 (54\%) out of 41 fetuses with CHD and extracardiac malformations. In 16 (43\%) aneuploidy could be diagnosed by classical karyotype, whereas in $67 \%$ only molecular methods gave the final diagnosis.

Conclusions: Classic karyotype could lead to false diagnosis of isolated CHD. CMA should be considered in all cases with prenatally diagnosed CHD.

Table.

\begin{tabular}{|c|c|c|c|c|c|c|}
\hline \multirow[b]{2}{*}{ Category } & & \multirow[b]{2}{*}{$\begin{array}{c}\text { Number } \\
\text { of } \\
\text { fetuses }\end{array}$} & \multirow[b]{2}{*}{$\begin{array}{l}\text { No } \\
\text { CNV } \\
(\%)\end{array}$} & \multicolumn{3}{|c|}{ Pathogenetic variance } \\
\hline & & & & $\underset{(\%)}{\text { Aneuploidy }}$ & $\underset{(\%)}{22 q 11.2}$ & $\begin{array}{l}\text { Other } \\
(\%)\end{array}$ \\
\hline \multirow[t]{7}{*}{ Isolated CHD } & CTD & 21 & $14(66.7)$ & $3(14.3)$ & $3(14.3)$ & $2(9.5)$ \\
\hline & right heart lesions & 5 & $4(80)$ & 0 & 0 & $1(20)$ \\
\hline & left heart lesions & 16 & $16(100)$ & 0 & 0 & \\
\hline & $\begin{array}{l}\text { septal defects/ } \\
\text { AVSD }\end{array}$ & 6 & $3(50)$ & $2(33.3)$ & 0 & $1(16.7)$ \\
\hline & complex CHD & 7 & $4(57.1)$ & 0 & 0 & $3(42.9)$ \\
\hline & $\begin{array}{l}\text { other mild } \\
\text { cardiovascular } \\
\text { abnormality }\end{array}$ & 3 & $2(66.7)$ & 0 & $1(33.3)$ & 0 \\
\hline & total & 58 & $43(74.1)$ & $5(8.7)$ & $4(8.6)$ & $6(10.3)$ \\
\hline \multirow{7}{*}{$\begin{array}{l}\text { CHD with } \\
\text { extra cardiac } \\
\text { defect }\end{array}$} & CTD & 12 & $6(50)$ & $2(16.7)$ & $1(8.3)$ & $3(25)$ \\
\hline & right heart lesions & 2 & $1(50)$ & 0 & 0 & $1(50)$ \\
\hline & left heart lesions & 3 & $2(66.7)$ & 0 & 0 & $1(33.3)$ \\
\hline & $\begin{array}{l}\text { septal defects/ } \\
\text { AVSD }\end{array}$ & 14 & $5(35.7)$ & $6(42.9)$ & 0 & $3(21.4)$ \\
\hline & complex CHD & 2 & 0 & $1(50)$ & 0 & $1(50)$ \\
\hline & $\begin{array}{l}\text { other mild } \\
\text { cardiovascular } \\
\text { abnomality }\end{array}$ & 8 & $5(62.5)$ & $2(25)$ & 0 & $1(12.5)$ \\
\hline & total & 41 & $19(46.3)$ & $11(26.8)$ & $1(2.4)$ & $10(24.4)$ \\
\hline All & & 99 & $62(62.6)$ & $16(16.1)$ & $5(5)$ & $16(16.1)$ \\
\hline
\end{tabular}

CTD conotruncal defect; AVSD atrio-ventricular septal defect
P-80

One gene and different heart defects - How deep should we look?

Wasienko P. (1,2), Gambin T. (3), Nowakowska BA. (2), Dangel J. (1) Centre of Postgraduate Medical Education, Perinatal Cardiology and Congenital Anomalies Department, Warsaw, Poland (1);Institute of Mother and Child, Department of Medical Genetics, Warsaw, Poland (2); Warsaw University of Technology, Institute of Computer Science, Warsaw, Poland (3)

Introduction: Most CHD are isolated cases. However in a few families there is a clear autosomal dominant inheritance. We present a family with GATA6 mutation and the role of Fetal Echocardiography and autopsy examination in establishing the final diagnosis.

Methods: Fetal Echocardiography Examinations were performed in a Tertiary Referral Centre. Sequencing was performed using Illumina Hi-Seq (San Diego, CA) instruments after exome capture with the Sure Select All Human V6 design. Raw sequence data were post-processed using the bc-bio pipeline. Finally, ANNOVAR was used to annotate relevant information about gene names, predicted variant pathogenicity, reference allele frequencies and metadata from external resources.

Results: A mother was operated on for ASD II and PDA during childhood and adolescence with good results. The first son was diagnosed tricuspid atresia in 22 weeks. During pregnancy the karyotyping with detection of 22q11 microdeletion with FISH techniques were performed excluding these aberrations. The cardiac surgery was performed at 9 months of age, however the baby died due to complications.

In next two consecutive pregnancies the spontaneous pregnancy loss occurred about 8 weeks. During 4th pregnancy a common arterial trunk (TAC) with good truncal valve and well developed pulmonary arteries was diagnosed in 20 weeks. After the cordocentesis a routine karyotyping with detection of $22 \mathrm{q} 11$ microdeletion with FISH techniques excluded these aberrations. The baby was born by CS at 35 weeks of gestation due to fetal tachycardia. The baby died unexpectedly at 9 day of life. The diagnosis of TAC was confirmed and a pancreatic aplasia was diagnosed. We have performed WES study the DNA from two born children, from mother and her non-affected parents. New stop mutation in GATA6 gene was identified in mother and both kids. In mother an MRI scan confirmed partial pancreatic aplasia.

Conclusions: The standard G-band chromosome analysis and array analysis are not always sufficient to determine the cause of fetal congenital malformations. WES study allowed a molecular diagnosis in the family and revealed new stop mutation in GATA6 gene. The presence of heart defects and a pancreatic aplasia confirms the clinical phenotype of GATA6 mutation in our patients.

\section{P-81}

Perinatal care after fetal diagnosis of congenital heart defect - Single centre experience

Wójcik-Sęp A. (1), Duliban J. (2), Bokiniec R. (1), Czajkowski K. (2), Dangel J. (3)

Medical University of Warsaw, Department of Neonatology and Neonatal Intensive Care (1); Medical University of Warsaw, 2nd Department of Obstetrics and Gynecology (2); Medical University of Warsaw, 2nd Department of Obstetrics and Gynecology; Centre of Postgraduate Medical Education, Perinatal Cardiology and Congenital Anomalies Department (3); Warsaw, Poland

Introduction: Specialized delivery room planning and early stabilization for neonates with congenital heart disease (CHD) seems to 
be very important to provide a good quality care. Prenatal diagnosis of CHD changed the need for urgent transportation of those babies due to sudden deterioration of their condition. The objective of this study was to determine the impact of fetal echocardiography on way of delivery, immediate postnatal treatment and need of transport to a reference cardiac center from a tertiary neonatal center throughout 15 years of single center experience. Methods: Out of 1095 patients with CHD born in our hospital from 2002 to 2016 we excluded cases of mild defects such as ventricular septal defects, aberrant right subclavian artery, right or double aortic arch. 652 patients with severe and critical CHD were included were included in this retrospective study. The history of perinatal care (way of delivery and indications, pre- and postnatal diagnosis, time of the transport to the cardiac center) were evaluated.

Results: Cesarean section was performed in 241 (37\%) CHD patients. Common causes of instrumental delivery were obstetric indications, risk of asphyxia, mothers' diseases. CHD was the cause only in $4(2,45 \%)$. Before 2009 , the median time from birth to transport to a reference cardiology unit was 4,3 days. From 2009 to 2016 it raised to 6,2 days. From $2009-2012$ only 9 (4,27\%) of babies were discharged directly to home, whereas from 2013 till $2016-60(17,39 \%)$. Mortality rate in the neonatology unit was $2,45 \%$ (16).

Conclusions: Fetal echocardiography enabled much better planning of perinatal care of newborns with CHD in reference centers. Our study revealed that children with CHD don't need to be born by c-section, especially when we do know advantages of a natural labour over c-section and importance of skin to skin contact. Such policy had great benefits for babies and mothers. Neonates got more cangaroo mother care, better stabilization after birth till the time of transportation, better respiratory and cardiovascular adaptation. It also enabled stress reduction and better lactation support for mothers. Such organiztion most probably strengthens motherchild bond from the very first moments and enhanced neurological outcome of children.

\section{P-82}

Fontan Circulation in Adult Patients with Congenital Heart Disease: Results from the CHALLENGE Registry

Apostolopoulou S.C. (1), Ntiloudi D. (2), Giannakoulas G. (2), Vasiliadis K. (3), Frogoudaki A. (4), Ntellos C. (5), Tzifa A. (6), Brili S. (7), Karvounis H. (2), Tsioufis K. (8), Rammos S. (1) on behalf of the CHALLENGE investigators

Onassis Cardiac Surgery Center, Department of Congenital Heart Disease, Athens, Greece (1). AHEPA General Hospital, Thessaloniki, Greece (2). General Hospital G. Papanikolaou, Cardiology Department, Thessaloniki, Greece (3). Attikon University Hospital, Cardiology Department, Athens, Greece (4). Tzaneio General Hospital of Piraeus, Cardiology Department, Athens, Greece (5). Mitera General Hospital, Department of Congenital Heart Disease, Athens, Greece (6).

Hippokration General Hospital, Cardiology Department, Athens, Greece (7). Hellenic Cardiological Society (HCS), Athens, Greece (8)

Introduction: Patients with structurally or functionally univentricular hearts undergo a series of complicated operations ending, if possible, with creation of palliative Fontan physiology, associated with several cardiac and pulmonary sequelae.

Methods: The purpose of this study was to describe the population and clinical characteristics of adult patients with Fontan circulation included in the national registry of adult congenital heart disease (ACHD) in Greece (CHALLENGE registry) from February 2012 until November 2017.
Results: Within the 2320 total patients included in the CHALLENGE registry from 12 centers, we identified 68 patients (3\%) with Fontan type circulation. Age at inclusion in the registry was $26.1 \pm 9.5$ years, $40 \%$ of patients were males, while $45(66 \%)$ had a dominant left and hypoplastic right ventricle. Overall, 30 (44\%) of patients had had 1 and $29(43 \%)>1$ surgeries prior to the Fontan operation, while $34(50 \%)$ had undergone at least one percutaneous intervention. Clinically, $26(38 \%)$ of patients were in WHO class I, $25(37 \%)$ in class II, 14 (21\%) in class III and only $3(4 \%)$ in class IV. The majority (84\%) of patients received medications, $29 \%$ antiarrhythmic, 63\% anticoagulant, 53\% anticongestive and 6\% pulmonary arterial hypertension therapy.

Conclusions: Fontan type circulation patients in our national ACHD registry are in their 2 nd to 5 th decade of life, have had multiple prior operations and percutaneous interventions, are mostly in class I and II, the majority receiving medications. Collection of additional data such as laboratory, echocardiographic, magnetic resonance imaging and exercise stress test data as well as long follow-up will help to further describe this population and possibly identify important factors for the management and prognosis of this population.

\section{P-83}

Late Sequels after Cavopulmonary Anastomosis in Complex Congenital Heart Disease: Thailand Experience

Noiming P., Tanpowpong P., Samankatiwat P., Limsuwan A. Faculty of Medicine Ramathibodi hospital, Mahidol University, Bangkok, Thailand.

Introduction: The survival rate of children with congenital heart disease (CHD) has been improved. Cavopulmonary anastomosis (CPA) has become a standard procedure in patients with complex CHD particularly with single ventricle physiology. These children have been survived with the sequels impact of CPA. The purpose of study is to evaluate late complications in these patients.

Methods: Patients, underwent CPA at least 5 years and had been regularly followed at our institute, were recruited. Medical records had been retrospectively reviewed. Periodic cardiovascular investigations and additional hepatic evaluation had been obtained during Jan-Oct 2017.

Results: Thirty consecutive patients (20 male), were recruited with mean age of 14.8 years ( $7-33$ years). Their median age at operation was 7.6 years old (3-23 years). Three of them (10\%) underwent single-stage total CPA or Fontan versus 21 patients $(70 \%)$ had staged Fontan operation. One fifth of the group (6 patients) underwent palliative inferior CPA or inferior-Glenn (inferior vena cava -to - pulmonary artery) because they presented beyond their infancy period and were not suitable for single- stage Fontan. Of all Fontan patients, 23/24 patients had extra cardiac conduit while 1 patient had lateral tunnel. Fenestration was performed in 23 patients. Median duration of follow up was 7.4 years $(5-17$ years). Majority of the patients (93.3\%) were asymptomatic with NYHA class I or II. Late complications consist of dysrhythmia $16.7 \%$, thromboembolism $16.7 \%$, sinus node dysfunction required permanent pace maker $10 \%$, and protein- losing enteropathy $6.7 \%$. Almost all of the patients had increased hepatic stiffness detected by Fibroscan (which suggested liver fibrosis) while one of the patients had MRI confirmed hepatic nodule. In fenestrated Fontan group, 12 patients (52\%) underwent device closure of fenestrations. There is no statistical significant difference of late complications between patency of fenestration versus device closer groups.

Conclusions: Long term follow up for group of Fontan and inferiorGlenn patients revealed satisfactory result with majority of the 
patients were in functional class I and II. However, there are major concerns regarding systemic venous hypertension and hepatic squeals. Further regularly systematic follow- up is required particularly evaluations for early hepatic fibrosis detection and management.

\section{P-84}

Duplication of Interventricular Septum and a Double Orifice Mitral Valve resulting in a three-ventricular chamber heart: A most rare anomaly

Ariyaratnam J.P., Dua J.S.

Adult Congenital Heart Disease, Manchester Heart Centre, Manchester University Foundation NHS Trust. Manchester, UK

Introduction (or Basis or Objectives): The human heart derives from embryonic mesodermal germ-layer cells that differentiate into mesothelium, endothelium, and myocardium and starts with formation of two endocardial tubes, which merge, loop and septate to form the four chambers. It starts to beat by the 4th week, at the end of which two atrioventricular endocardial cushions appear to form the atrio-ventricular valves. An intra-muscular septum then grows within the ventricle mass consisting of a membranous and a muscular part.

Methods EResults: We assessed a 63-year-old woman with a history of intermittent chest pains, palpitations and shortness of breath on moderate exertion. She had a complex previous history of mild systemic lupus combined with Sjogrens Syndrome, skin angiokeratomas and osteomalacia with Vitamin D deficiency. She also had a dual chamber pacemaker implanted for 2:1 heart block. Initially an echocardiogram demonstrated a thickened interventricular septum with a pattern that followed asymmetric septal hypertrophy and normal left ventricular (LV) systolic function. The septal wall showed marked hypertrabeculation and subsequent cardiovascular magnetic resonance (CMR) imaging demonstrated bulky almost exophytic-looking trabeculae at the septal wall and the global LV mass was 1.4 times normal. Moreover both echocardiography and CMR imaging revealed that the thickened interventricular septum was divided into two muscle bands from the apex to the base giving the appearance of septal duplication and of a three-ventricular chamber heart (Figures 1). Interestingly, duplication of the atrio-ventricular valves was also noted with an appearance of double orifice mitral valve. The most septal valve showed dysplastic leaflets and restricted opening into the rudimentary septal chamber.

Conclusions: We describe this unusual case of congenital heart disease where the first impression was suggestive of hypertrophic

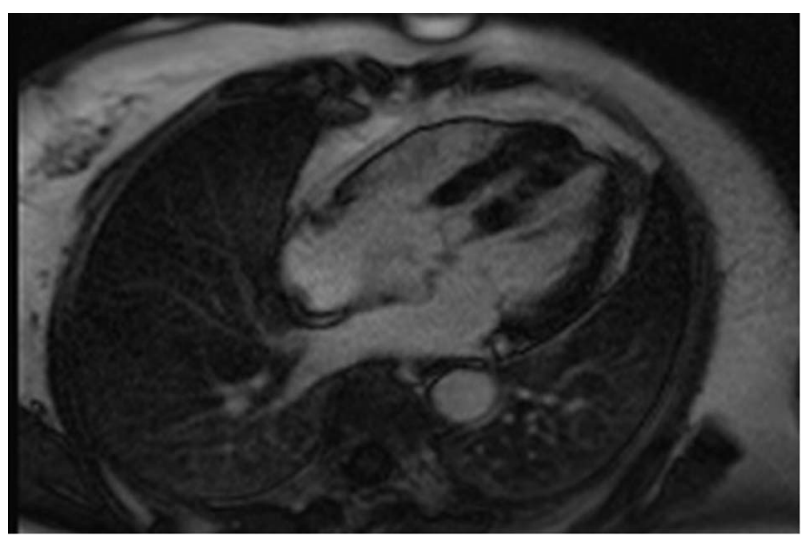

Figure. cardiomyopathy or cardiomyopathy with asymmetric septal hypertrophy and non-compaction appearance. CMR with delayed gadolinium enhancement imaging did not show enhancement compatible with metabolic or infiltrative disorders. Myocardial biopsy unfortunately is not available as the patient did not consent to undergo the procedure. Duplication of the interventricular septum and formation of a third rudimentary ventricular cavity is extremely rare congenital abnormality. To our knowledge this has never been described before.

\section{P-85}

Successful transfer to adult care: A nationwide study in patients with complex congenital heart lesions in Sweden Skogby S. (1,2), Moons P. (1,3), Johansson B. (4), Dellborg M. (5), Christersson C. (6), Nagy E. (7), Winberg P. (8), Thilen U. (9), Hanseus K. (10), Krzynska A. (11,12), Fadl S. (13),

Fernlund E. (14), Kazamia P. (15), Rydberg A. (16), Sunnegårdh J. (2), Goossens E. (17,18), Bratt EL. $(1,2)$.

Institute of Health and Care Sciences, University of Gothenburg, Sweden (1); Department of Pediatric Cardiology, Queen Silvias Childrens Hospital, Gothenburg Sweden (2); KU Leuven Department of Public Health and Primary Care, Leuven, Belgium (3); Heart Center and Department of Public Health and Clinical Medicine, Umeå University, Umeå, Sweden (4); Department of Molecular and Clinical Medicine, Sahlgrenska Academy, University of Gothenburg, Gothenburg, Sweden (5); Department of Clinical Sciences, Uppsala University, Sweden (6); Department of Cardiology, Karolinska University Hospital, Stockholm, Sweden (7); Department of Pediatric Cardiology, Karolinska Hospital, Stockholm, Sweden (8); Department of Cardiology, Lund University Hospital, Lund, Sweden (9); Department of Pediatric Cardiology, Skane University Hospital, Lund, Sweden (10); Division of Cardiovascular Medicine, Department of Medical and Health Sciences, Linköping University, Linköping, Sweden (11);Department of Cardiology, Department of Medical and Health Sciences, Linköping University, Linköping, Sweden (12); Department of Pediatric and Womens Health Care, Orebro University Hospital, Örebro Sweden (13); Department of Pediatrics, Linköping University, Linköping, Sweden (14); Department of Pediatric Cardiology, Uppslala University Sweden (15); Department of Clinical Sciences, Unit of Pediatrics, Umeå University, Umeà, Sweden. (16); University of Leuven, Leuven, Belgium (17); Research Foundation Flanders, Brussels, Belgium (18).

Introduction: Patients with complex congenital heart lesions are in need of life-long medical follow-up. International guidelines advice that patients with complex lesions should be transferred to specialist ACHD care within 12 months after leaving pediatric cardiology. The combination of appropriate care level and appropriate time intervals during transfer to adult care can be considered as successful transfer. Unsuccessful transfer of care in patients with CHD might increase the risk for urgent interventions and long-term complications. The aim of the present study was to determine proportions of successful transfer in patients with complex congenital heart lesions in Sweden.

Methods: Seventy-two patients, born in 1991, 1992, or 1993 and diagnosed with complex congenital heart disease from six university hospitals in Sweden have been enrolled. Predominant diagnosis among the included patients were TGA 42\%, DORV $18 \%$, Truncus arteriosus $9.7 \%$, and $12.5 \%$ Univentricular heart defects. Data were collected from hospital administrative databases, medical files and registries. The time interval between last visit at paediatric cardiology and first visit at ACHD care was calculated. Transfer destinations were categorized as either appropriate (i.e., at ACHD level) or not appropriate (i.e., at nonspecialized adult level). Successful transfer was defined as a transfer 
of care towards the appropriate level and within the recommended time interval.

Results: In total, 68 patients (94\%) were transferred to the appropriate level of care (i.e., ACHD care). Twenty patients (28\%) had their first visit in adult care within 12 months, and 34 patients (47\%) within 18 months (Figure 1). Successful transfer, being transferred to the appropriate care level and within the recommended 12 months, was observed in only 19 patients (26\%). If a more liberal time interval of 18 months would be used, 33 patients (45\%) were considered to be successfully transferred.

Conclusion: The vast majority of patients was transferred to ACHD care. Still, $6 \%$ were not followed up at the appropriate care level. Furthermore, the majority of the patients were not followed up at the recommended time interval, according to guidelines. Indeed, even with a more generous time interval of 18 months, less than half of the patients were successfully transferred.

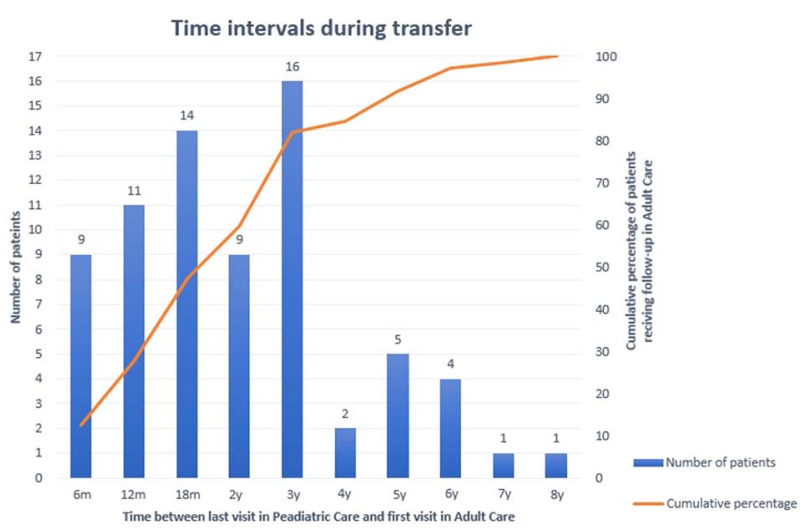

Figure.

\section{P-86 \\ Long-term Result of Right Atrial Isolation for Atrial Fibrillation or Flutter in Adult Congenital Heart Disease Chikada M., Miyairi T., Nishimaki H., Ohno M., Ono H., Chiba K., Ro D., Nagata T., Sakurai Y., Suzuki H., Kita S. \\ Department of Cardiovascular Surgery, St.Marianna University School of Medicine, Kawasaki Japan}

Object: The right atrial isolation procedure was developed by Cox's group at 1988. The method was simple and easy to use for an additional procedure. Some reports presented unfavorable results of right-sided maze procedure for atrial fibrillation with atrial septal defect (ASD). But some presented favorable results of right atrial isolation. In this paper, we present our experience of right atrial isolation and the long-term result with adult congenital heart disease. Materials and Methods: Since 1998 to 2012, 15 patients (6 men, 9 women) underwent right atrial isolation as a concomitant operation. Documented atrial arrhythmias included paroxysmal atrial fibrillation or flutter $(n=9)$ and chronic atrial fibrillation $(n=3)$. Associated cardiac defect included ASD $(n=10)$, ASD with persistent ductus arteriosus $(n=1)$ with pulmonary stenosis $(n=1)$, Ebstein anomaly $(n=1)$, partial $\operatorname{AVSD}(n=1)$ and two chamber of right ventricle $(n=1)$. Diagnoses were based on clinical evaluation and echocardiography. All patients had severe right atrial enlargement revealed on preoperative echocardiography. The procedure included right atriotomy and cryoablation at the level of tricuspid valve annulus. Ages at operation ranged from 25 to 70 years (mean, 54 years). The mean follow-up period was 11.5 years (range, 5 to 19 years).
Results: All but one was free from atrial fibrillation or flutter without medication. One patient underwent a pacemaker implantation with complete atrioventricular block.

Conclusion: Right atrial isolation is a simple and effective procedure that abolishes atrial fibrillation or flutter in adult congenital heart disease.

\section{P-87}

Arrhythmias and heart failure in adults with isolated corrected Transposition of the Great Arteries

Walton C., Martin-Bonnet C., Bozio A., Chakoub S., Galoin

Bertail C., Perouse de Montclos T., Bakloul M., Durousset C., Leconte C., Di Filippo $S$.

Pediatric Cardiology, Cardiovascular Hospital, Lyon, France

The objective of this study was to assess arrhythmias and heart failure occurrence in adults with isolated corrected TGA (ccTGA). Material and Methods: All patients diagnosed with ccTGA at a single-center, were included in the study. Clinical data, echocardiographic parameters and outcomes were collected. All arrhythmic and/or heart failure (HF) events were recorded. Pronostic factors for poor outcome were assessed.

Results: 30 males and 25 females, aged $16.7 \pm 18.4 \mathrm{y}$ at diagnosis of ccTGA and followed-up for $17.9 \pm 12.7 \mathrm{y}$, were included in the study. Mild lesions were associated in 23 cases (small VSD, mild pulmonary valve stenosis, small ASD, mild isthmus stenosis). Complete AV block occurred in 7 cases (13\%). NYHA class I, II, III and IV at onset was respectively: $70 \%, 18 \%, 7 \%$ and $3.6 \%$, and a end-follow-up was respectively: $31 \%, 38 \%, 24 \%$ and $6.7 \%$ at endFU. Systemic right ventricle function at onset FU was normal on $87 \%$ of cases, moderately impaired in $13 \%$ and none had severe RV dysfunction, and changed to respectively $73 \%, 18 \%$ and $9 \%$ at end-FU. Tricuspid regurgitation was grade $0,1,2$ or 3 in $33 \%$, $49 \%, 9 \%$ and $9 \%$ of cases respectively at onset $\mathrm{FU}$ and in $13 \%, 48 \%$, $24 \%$ and $16 \%$ of cases at end-FU respectively. Symptoms of HF occurred in 8 cases $(14.5 \%)$ at the age of $37.5 \pm 13.7 \mathrm{y}$ ( 7 to $61 \mathrm{y}$ ), AVB occurred in $20(36.4 \%)$ at age $31.4 \pm 19.4 \mathrm{y}(5$ to $72 \mathrm{y})$ and arrhythmias in $16(29 \%)$, over FU. RV dysfunction was associated with TR grade $(p=0.025)$, and HF with TR grade $(p=0.015)$ and pacemaker implantation $(p=0.026)$. Freedom for onset of HF was $50 \%$ at mean age of 36years.

Conclusion: Patients with isolated ccTGA experience worsening of $\mathrm{RV}$ function and TR grade, and frequent arrhythmic and HF events over twenty years of follow-up.

\section{P-89}

Factors influencing the participation of adolescents and young adults with congenital heart diseases in a transition education program: a multicenter observational study Werner O., Amedro P., Guillaumont S., Vincenti M., De Lavilleon G., Gavotto A.,Bredy C.

Pediatric cardiology CHU Arnaud de Villeneuve Montpellier-France

Purpose: The improvements of the surgical and medical care of patients with congenital heart disease have led to a significant improvement in their life expectancy. Every year it's 200 more patients (on 100000 birth) that will rich the adulthood. Our next objective now is to improve their quality of life. Therapeutic education can be a very useful tool to reach this goal. But more than $50 \%$ of the patients don't have any medical monitoring after 18 years. The pediatric cardiologist have to prepare their patients to the adulthood to limit the level of "lost of sight", and that is why we created a transition program in our center. The purpose of this 
program is to help the patients between 13 and 25 years to get every skills that will help him in his "patient" life. The goal of this study was to highlight the factors that convince the cardiologist to include his patient in this program.

Methods: Transversal observational multicenter study lead from November 2015 and January 2017.We included all patients that participate to the transition program as the transition group. We selected at random in consultation, patients that wasn't included in the program as the control group. They had to complete 3 questionnaires (Knowledge, quality of life and physical activity).

Results: 123 patients included, 57 in the transition group and 66 in the control group. Mean age 19,7 years. The factors that influence the inclusion in the transition program in univariate and multivariate analysis were: the severity of the disease (OR:4,1, $p=0,03)$, the presence of piercing (OR: 5,5, p =0,007), a great level of knowledge (OR 0,29; $\mathrm{p}=0,02)$ and a young age (OR 0,289; $\mathrm{p}=0,025)$.

Conclusion: this study highlight the factors that influence the cardiologist when they include a patient in a specific transition program. It's a population of patients with more severe diseases, with a lack of knowledge and with behaviors at risk of complication. Our next study will try to assess the effectiveness of the transition education on the quality of life of patients with a congenital heart disease. (Clinical Trials \#NCT03005626)

\section{P-90 \\ Heart Rate Variability and Maximum Oxygen Uptake in adolescent and adult Patients with different Types of congenital Heart Disease \\ Fritz C. (1), Hock J. (1), Oberhoffer R. (1,2), Ewert P. (1), Hager A. (1), Müller J. $(1,2)$ \\ German Heart Centre Munich, Technical University of Munich, Munich, Germany (1); Institute of Preventive Pediatrics, Technical University of Munich, Munich, Germany (2)}

Introduction: The heart rate variability (HRV) is a measure to quantify the autonomous nervous system (ANS) pathways' activities. According to current research there is an indication of an impaired vagal activity considering the Root Mean Square of Successive Differences (RMSSD) in patients with congenital heart disease (CHD). This study evaluates the vagal activity of patients with CHD and their association to exercise capacity.

Methods: In the period from July 2016 until December 2017 a total of 199 patients with different types of CHD (43\% female; $29.3 \pm 10.6$ years) received a breath controlled HRV measurement subsequent to a 3 minutes resting phase in supine position. Based on 130 adjacent heartbeats the RMSSD, a measurement of vagal activity, was assessed. RMSSD values are displayed in median and interquartile [IQR 25; 75]. Additionally all patients underwent a cardiopulmonary exercise test until exhaustion on the bicycle ergometer. For comparison a control group (CG) of 44 (59\% female; $36.9 \pm 13.4$ years) healthy volunteers was recruited. Results: Patients with CHD have impaired vagal activity by means of a reduced RMSSD in comparison to healthy controls (CHD: 33.9 [19.8; 60.8] ms versus CG: 42.4 [29.8; 85.4] ms; $p=0.008)$. Especially patients who underwent palliative Fontan operation $(\mathrm{FP} ; \mathrm{n}=39)$ showed impaired vagal activity (FP: 23.9 [11.7; 38.2] ms; $\mathrm{p}=0.003$ ).

Peak oxygen uptake (VO2peak) of the patients was on average $75.6 \pm 19.8 \%$ of the predicted reference value. In patients with CHD lower vagal activity was associated with a reduction in peak oxygen uptake $(r=0.200 ; p=0.005)$.

Conclusion: The impaired vagal activity and the association with a reduced peak oxygen uptake in patients with CHD suggest a dysfunction of the ANS in this cohort. For improved medical care advanced studies should investigate these impairments in diagnostic subgroups.

\section{P-91 \\ Age-related 10-year risk of lethal Cardiovascular Disease in Adult Patients with Congenital Heart Disease \\ Häcker A.-L. (1,2), Müller J. (1,2), Hager A . (1), Ewert P. (1), Oberhoffer R. $(1,2)$ \\ Department of Pediatric Cardiology and Congenital Heart Disease, Deutsches Herzzentrum München, Technische Universität München, Germany (1); Institute of Preventive Pediatrics, Technische Universität München, Germany (2)}

Background: Cardiovascular diseases are a relevant risk for adults with congenital heart diseases (ACHD), since life expectancy has increased in these patients. While past research studied just single risk factors, this study examines the age-related risk for cardiovascular disease (CVD) based on multiple factors.

Methods: A compound risk factor established to assess the risk of CVD for the next ten years was calculated for 190 ACHD aged 30 years or older ( $45.6 \pm 9.4$ years, $49.7 \%$ female), examined between January 2017 and November 2017. The 10-year CVD risk was estimated with the Prospective Cardiovascular Munster Study (PROCAM) score, including the factors age, sex, systolic blood pressure, LDL cholesterol, HDL cholesterol, triglyceride, diabetes status (yes/no), smoking status (yes/no), and family history of cardiovascular events.

Results: ACHD had significantly lower PROCAM values compared to the national reference (ACHD: $1.7 \pm 3.0 \%$, reference: $4.4 \pm 5.5 \%, \mathrm{p}<.001)$, resulting in a calculated $61 \%$ lower relative risk. In total, only $10.7 \%$ of the ACDH had a reduced HDL cholesterol lower than $40 \mathrm{mg} / \mathrm{dl}, 20.9 \%$ had triglyceride higher than $150 \mathrm{mg} / \mathrm{dl}$ and $2.7 \%$ had increased LDL higher than $190 \mathrm{mg} /$ dl. Diabetes mellitus was prevalent in $4.8 \%$ of the ACHD. Another $14.2 \%$ had a major cardiovascular event in their family before the age of 60 .

Conclusions: Based on the PROCAM score the 10-year CVD risk is lower in ACHD compared to the healthy reference. It has to be further clarified if the lower prevalence of risk factors in LDL cholesterol, triglyceride and diabetes mellitus is a result of a healthy lifestyle and awareness for their disease.

\section{P-92}

Combined Use of Peak Oxygen Uptake and NT-ProBNP to Predict Survival in Adults Congenital Heart Disease Müller J. (1,2), Hock J. (2), Fritz C. (2), Häcker A.-L. (1,2), Oberhoffer R. (1,2), Ewert P. (2), Hager A. (2)

Institute of Preventive Pediatrics, Technische Universität München, Munich, Germany (1); Department of Pediatric Cardiology and Congenital Heart Disease, Deutsches Herzzentrum München, Technische Universität München, Munich, Germany (2)

Introduction: Peak oxygen uptake is a functional outcome measure that proved significance in the prediction of survival in patients with congenital heart disease (CHD). Similar predictive values is also proven for NT-proBNP, a biomarker of myocardial stress. This study evaluates if the combined use of both parameters improves prediction of survival.

Patients and Methods: From 281 (47\% female, $30.4 \pm 10.8$ years) regular outpatient visits that include cardiopulmonary exercise testing and blood sampling in the period from 2008-2016, followup information's were abstracted from the medical records. 
Results: During a follow up of $4.6 \pm 2.9$ years 37 patients died. After correction for sex and age in a multivariable Cox regression, a higher peak oxygen uptake was associated with better survival (HR: 0.984, CI: $0.921-0.976 ; p<.001)$, and increased NTproBNP levels with a worse survival (HR: 2.938, CI: $1.250-$ 6.444; $p=.013)$. The combined use of peak oxygen uptake and NT-proBNP level was higher (AUC: 0.870) than using both parameters alone (AUC peakVO2: 0.846, AUC NT-proBNP: $0.830)$.

Cut-off values for highest sensitivity and specificity from ROC analysis for the two risk factors were a peak oxygen uptake of $60 \%$ predicted and a NT-proBNP level of $300 \mathrm{ng} / \mathrm{ml}$. In comparison to patients with no risk factor (peakVO2 $>60 \%$ and NT-proBNP $<$ $300 \mathrm{ng} / \mathrm{ml}$ ), patients with one risk factor had a 5-fold increased risk for death $(\mathrm{p}=.049)$ and patients with both risk factors a 33fold increase $(\mathrm{p}<.001)$.

Conclusion: The combined use of peak oxygen uptake and NTproBNP level provide important additional information to improved medical care in the clinical management of adult patients with CHD

\section{P-93 \\ Does congenital heart disease affect cognitive function? - A pilot study in adults with congenital heart disease Remmele J. (1,2), Pringsheim M. (2), Nagdyman N. (2), Tutarel O. (2), Kaemmerer H. (2), Oberhoffer R. (1), Ewert P. (2) Chair of Preventive Pediatrics, Technical University of Munich, Munich, Germany (1); Department of Pediatric Cardiology and Congenital Heart Disease, German Heart Center Munich, Technical University of Munich, Munich, Germany (2)}

Objective: It is often assumed, that adults with congenital heart disease (ACHD) have an impairment regarding their cognitive function. In particular it seems reasonable to assume that cyanosis as well as medical and surgical therapies could have potential impact on cognitive function in adulthood. However, currently there is no data regarding cognitive function formally assessed by intelligence tests in ACHD patients. Therefore, the aim of this study was to analyze the cognitive function and its association with cyanotic conditions in ACHD patients.

Methods: Forty-five ACHD patients (female $\mathrm{n}=20$ (44.4\%), mean age at assessment $34.3 \pm 13.9$ years) underwent the Wechsler Intelligence Scale for adults (fourth edition) - a well-established assessment of cognitive function in adults - as inpatients between March-December 2017. In this test impairment is defined as an intelligence quotient achieved which is below more than one standard deviation of the norm.

Results: The mean overall intelligence quotient (oIQ) achieved by the patients was $94.9 \pm 12.9$ points. Regarding the subgroups, the patients achieved $97.7 \pm 13.1$ IQ points (IQp) in verbal comprehension, $94.7 \pm 13.0 \mathrm{IQp}$ in working memory, $94.7 \pm 14.1 \mathrm{IQp}$ in logical thinking, and $96.7 \pm 12.1 \mathrm{IQp}$ in processing performance. Therefore, compared to the norm standard (Ø100 IQp) no impairment of cognitive function was present in the study cohort. Patients with acyanotic CHD $(n=15)$ achieved a mean oIQ of $95.2 \pm 12.1$ points, $96.8 \pm 12.4$ oIQ points in patients with corrected cyanotic CHD $(n=24)$ and a mean oIQ of $86.5 \pm 16.1$ points in patients who were still cyanotic $(n=6)$. The difference between the groups was statistically not significant $(\mathrm{p}=0.277)$.

Conclusions: Contrary to the general assumption, ACHD patients showed no significant impairment in cognitive function compared to the norm. Nevertheless, a trend was shown that cyanotic patients had a lower overall IQ. Further studies in larger cohorts are needed to assess the impact of cyanosis and other possible confounding factors on cognitive function in this growing patient population. The aim would be to identify patients at high risk to allow targeted preventive strategies.

\section{P-94}

Lack of Specific Disease Related Medical Health Care in Congenital Heart Disease: A Call for Reaction? The General Practitioners View

Neidenbach R. (1), Pieper L. (3), Sanftenberg L. (4), Schelling J. (4), Freilinger S. (1), Schwarz M. (1), Krauß A. (1), Seidel L. (1), Nebel K. (1), Oberhoffer R. (1,2), Nagdyman N. (1), Ewert P. (1), Kaemmerer H. (1)

Department of Paediatric Cardiology and Congenital Heart Defects, German Heart Centre Munich, Technical University Munich, Germany. (1) Department of Preventive Paediatrics, Department of Sport and Health Sciences, Technical University of Munich, Germany. (2) Department of Behavioural Epidemiology, Technical University of Dresden, Germany. (3) Institute for General Practice, University Hospital of the LudwigMaximilians-University Munich, Germany(4)

Objective: Mortality in children with congenital heart disease (CHD) has decreased significantly in the last decades. Nowadays, more than $90 \%$ survive into adulthood. According to estimates, there are currently more than 280.000 adults with congenital heart disease (ACHD) living in Germany. ACHD need specific followup, due to a high incidence of cardiac and non-cardiac problems in the long-term course. In Germany, medical follow-up for ACHD is organized in basic treatment through general practitioners (GP) and specific care by ACHD specialized and certified cardiologists and institutions offering maximum ACHD supply. Highly actual data show, that the majority of ACHD (>200.000) is NOT tied to specialized and ACHD-certified physicians and institutions. This study aims to generate "real-life" data regarding the care of ACHD provided by general practitioners.

Methods-Results: A questionnaire-based survey of 541 GP's in Germany was analyzed. According to study results, GP's deal with all types and with any severity grade of CHD, in $58.2 \%$ actually with moderate and severe forms. Even severe problems like heart failure, pulmonary hypertension, arrhythmias or endocarditis are managed by GP. More than $93 \%$ of the surveyed treat ACHD older than 35 years. $69.1 \%$ of GP's are also taking care of rare hereditary disease. ACHD specific physicians are consulted in only $23.6 \%$. More than $35 \%$ of the GP's are not aware of the existing certified clinics/ centers of ACHD. $75.2 \%$ of the GP are not informed about the existence of patient initiatives.

Conclusions: ACHD centers and specialists are inadequately perceived or consulted, despite the increasing rate of cardiac or noncardiac complications of CHD during adult life. Although GP's have to guide ACHD to provide adequate diagnosis, treatment and preventive measures, they are insufficiently informed about the certificated ACHD-specialists or -facilities. A better awareness of the problems concerning follow-up in ACHD should be created, to optimize their care and, as a result, to reduce morbidity and mortality.

\section{P-95}

Medical Rehabilitation in Adults with Congenital Heart Disease (ACHD)

Neidenbach R. (1), Andric D. (1) Einwang H. P. (2), Kodolitsch Y. (3), Bongarth C. (2), Oberhoffer R. (4), Hischke S. (5), Hofbeck M. (6), Ewert P. (1), Kaemmerer H. (1)

(1) Department of Paediatric Cardiology and Congenital Heart Disease, German Heart Centre Munich. (2) Deutsche Rentenversicherung Bayern 
Süd Klinik Höhenried, Germany. (3) University Heart Center, Hamburg, Germany. (4) Department of Preventive Pediatrics Uptown Munich-Campus D, Germany. (5) Department of Medical Biometry and Epidemiology, University Medical Center Hamburg-Eppendorf, Germany. (6) Paediatric Cardiology, Pulmonology and Intensive Care Medicine Tübingen University Hospital, Germany

Introduction: The number of adults with congenital heart disease (ACHD) is steadily rising. In the long-term course, many of them require medical rehabilitation after interventional or surgical treatment of residua, sequela or complications of their congenital heart defect (CHD). However, up to now only scarce data exist about indication, performance and outcomes of cardiac rehabilitation measures in ACHD.

Methods: In ACHD, course and outcome of rehabilitation measures, after previous interventional or surgical treatment, is analyzed. Results: Included were 205 adults (54\% female; mean age $34 \pm 12$ years (range $16-68$ years), with mild $(23.9 \%)$, moderate $(35.1 \%)$ or severe CHD $(41,0)$. 32\% had complex CHD, 21\% rightventricular outflow tract obstructions, $20 \%$ pre-tricuspid shunts, $18 \%$ left-ventricular outflow tract obstructions, $9 \%$ post-tricuspid shunts, $2 \%$ other cardiac anomalies. Main indication for rehabilitation was surgical $(92 \%)$ or interventional $(3 \%)$ treatment of the underlying CHD immediately before rehabilitation.

No severe complications occurred during rehabilitation. The number of patients in function class I/II increased from 189 to 200 , and decreased from 16 to 5 in class III/IV. With the exception of ACE-inhibitors, cardiac medication could be reduced or stopped during rehabilitation in 194 patients. There was an improvement of cardiovascular risk factors.

During a follow-up of 28 months the exercise capacity in 125 ACHD increased from 94 Watt (median $100 \mathrm{Watt}$, range $50-225$ Watt) to 139 Watt (median 135 Watt, range 27- 292 Watt).

While before the medical treatment only 77\% (157) patients were capable of working, the number increased to $82 \%(168)$ at the end of rehabilitation and to $84 \%$ (172) during follow-up. Throughout follow-up $9.3 \%$ (19) of patients had to undergo further cardiac interventions.

Conclusions: The current study provides for the first-time extensive data about the course of rehabilitation in a large cohort of ACHD after surgical or interventional treatment. The over-all outcome of ACHD after rehabilitation appears uneventful and favorable. Further analysis is required to assess the clinical long-term outcome, the impact of rehabilitation on health-related quality of life, disease coping and employment. The results of this study can serve as a benchmark for the development of specific rehabilitation programs in ACHD.

\section{P-96}

Trends in the utilization of outcome measures for proteinlosing enteropathy in contemporary Fontan studies: a systematic review

Udink ten Cate F.E.A. (1,2), ten Dam K. (1), Germund I. (3),

Haustein M. (3), Duijnhouwer A.L. (4), Khalil M. (5), Nazzal R. (3), Apitz C. (6), Helbing W.A. (1,2), Brockmeier K. (3),

Tanke R.B. (1,2), Sreeram N. (3); for the Fontan Care Network. Amalia Children's Hospital, Radboud University Medical Center, Nijmegen, Netherlands (1); Sophia Children's Hospital, Erasmus Medical Center, Rotterdam, Netherlands (2); Heart Center Cologne, University Hospital Cologne, Cologne, Germany (3); Department of Cardiology, Radboud University Medical Center, Nijmegen, Netherlands (4); Pediatric Heart Center, Justus-Liebig University, Giessen, Germany (5); University Children's Hospital Ulm, Ulm, Germany (6).
Objectives. Appropriate selection and standardization of outcome measures are important for advancing Fontan care and research. No study has assessed the use of outcome measures in Fontan studies on protein-losing enteropathy (PLE). The present study determined whether outcome measures of PLE are routinely reported, and to identify the types of outcome measures used in order to classify them in subsets of clinically meaningful endpoints. Methods. A systematic review was conducted in adherence to the PRISMA recommendations. A PubMed search was done on papers published between January 2007 - December 2016 (10 years) using the keywords Fontan AND protein-losing enteropathy. All studies reporting outcome of Fontan patients with PLE were included. We defined an outcome measure as any measurable variable used to describe clinical outcome or effect of a medical intervention. Only variables specific for PLE outcome were selected. Where patient outcome was descriptive (case reports and case series), information was retracted from the text and quantitatively analysed using a lateral thinking tool in which outcome measures were fractionated into objective and constituent pieces of information. Outcome measures were then categorized into appropriate outcome domains.

Results. We identified 162 papers. In the final analysis, data from 77 published articles were extracted, including the outcome of 716 Fontan patients with PLE. A total number of 414 outcome measures were reported, including 115 surrogate outcome measures $(27.8 \%)$. The number of unique outcome measures ranged from 1 to 17 per study. The five most common outcome domains were PLE relapse/resolution (57 [74.0\%] studies), survival (51 [66.2\%]), PLE symptoms (30 [38.9\%]), edema (21 [27.3\%]), and ascites (21 [27.3\%]). Importantly, most studies did not define PLE relapse or resolution. A biochemical assessment of disease activity (serum albumin or protein level) was documented in 40 (51.9\%) reports, whereas enteric protein loss was quantified in only $12(15.6 \%)$ studies.

Conclusions. The outcomes reported in contemporary Fontan-PLE studies are extremely heterogeneous. Thus, there is a great need to develop validated outcome measures to objectively assess disease severity and to quantify the true effectiveness of any therapeutic intervention in Fontan patients with PLE.

\section{P-97}

Liver stiffness as a useful tool in the longitudial follow-up of patients with a Fontan circulation

Jalal Z. (1,2), Iriart X. (1), De Deledinghen V. (3), Foucher J. (3), Thomas J. (1), Seguela P.E. (1), Thambo J.B. $(1,2)$

Congenital Heart disease department. University Hospital of Bordeaux FRANCE. 2. IHU Liryc, Electrophysiology and Heart Modeling Institute, fondation Bordeaux Université, F-33600 Pessac- Bordeaux, France. 3. Hepatology department. University Hospital of Bordeaux FRANCE

Introduction: Liver stiffness (LS) assessed by Transient elastography (TE) is known to be elevated in patients with a Fontan circulation and is considered as a promising tool to evaluate hepatopathy in these patients. However the longitudinal evolution of this parameter has never been studied.

Methods: We conducted a prospective ongoing study since 2007 including all patients with a Fontan circulation who underwent an annual TE assessment in addition to their systematic work-up and/ or in case of a clinical complication.

Results: In this ongoing study, 20 patients were screened with TE and $17 \mathrm{had}$ at least two LS evaluations. Median age at the first TE was 21 years [range 4-32], median duration between the Fontan surgery and the first TE was 4 years [range 1-12], and 6,6 years 
[range 3-15] at the last TE screening. The median LS at the first evaluation was $12.8 \mathrm{kPA}$ [range 6-47] and $12.4 \mathrm{kPA}$ [range 6-27] at the last evaluation. No significant modification of LS values was observed in our cohort, except for 3 who developped a failing Fontan and are currently waiting for cardiac transplant.

Conclusions: Liver stiffness assessed by TE is stable over time in Fontan patients but significantly increases in patients with a failing Fontan. This tool might be useful in the longitudinal follow-up of these patients for anticipating the decrease of cardiac status and to evaluate non-invasively the effects of targeted therapies before heart transplant.

\section{P-98}

\section{The relationship of bicuspid aortic valve phenotype and} pattern of aortopathy: a meta-analysis

Jashari H. (1,2), Bejiqi R. (1), Merovci B. (1), Shala T. (1), Mahmuti B. (1), Henein M.Y. (2)

Pediatric Clinic, University Clinical Center of Kosovo, Republic of

Kosovo (1); Department of Public Health and Clinical Medicine, Umea

University, Sweden (2);

Background: Aortopathy in patients with bicuspid aortic valve (BAV) is increasingly recognized to be a heterogeneous disease entity. Likewise, many studies suggest that the bicuspid valve morphology influences aortic dilatation, but the association of the two is controversial. We therefore, compared, in a meta-analysis, the effect of right-left coronary cusp fusion (RL) and right-non coronary cusp fusion (RN) on the pattern of aortic dilatation.

Methods: Up to November 2017, a systematic search was conducted to identify all studies that compared aortic dimensions in patients with BAV. Raw mean differences in millimeters was analyzed using Comprehensive Meta Analysis software, and data was combined using random-effect model.

Results: Twenty studies reported aortic dimensions for 3854 patients with RL and 1735 patients with RN BAV (with or without raphe). Indexed and non-indexed values were compared at the level of aortic annulus, sinuses of Valsalva, sinotubular junction and ascending aorta. Using indexed values showed only the sinuses of the Valsalva wider in RL BAV patients (mean difference 1.84 (95\% CI: 0.46-3.23), $\mathrm{p}<0.009, \mathrm{I} 2=90.4 \%)$. Using non-indexed values showed the annulus (mean difference 1.38 (95\% CI: 0.62-2.13), $\mathrm{p}<0.000, \mathrm{I} 2=84 \%)$, sinuses of Valsalva (mean difference $2.94(95 \%$ CI: $1.75-4.12), \mathrm{p}<0.000, \mathrm{I} 2=88.2 \%)$, and sinotubular junction (mean difference 1.28 (95\% CI: 0.27-2.29), $\mathrm{p}<0.013$, I2 =76.8\%) wider in RL BAV patients but similar ascending aorta diameter (mean difference 0.14 (95\% CI: $-1.16-1.44$ ), $\mathrm{p}<0.83$, I2 = 87.4\%). Conclusions: The meta-analysis found an association between BAV morphology and the pattern of aortic dilatation, suggesting a contributing effect of the transvalvular flow direction and its relationship with the wall. This does not necessarily exclude differences of development style and tissue composition. Thus, categorizing BAV subtypes may serve as a clinical tool for optimum follow-up strategies in order to provide optimum evidence based management.

\section{P-99}

Positive trajectory of exercise capacity during childhood predicts better adult Fontan physiology

Ohuchi H., Negishi J., Iwasa T., Miike H., Fukuyama M., Kurosaki K., Ichikawa $H$.

National Cerebral and Cardiovascular Center, Osaka, Japan

Introduction: Exercise training is recommended for favorable effects on Fontan pathophysiology. However, long-term benefits of exercise training remain unclear.
Objective: This study was to elucidate an impact of trajectory of exercise capacity during childhood, which mimics effect of exercise training, on the adult Fontan pathophysiology.

Method and Results: Since 1990, consecutive 131 Fontan patients had undergone two serial cardiopulmonary exercise testing (CPX1 and CPX2) during childhood (ages of $8 \pm 2$ and $14 \pm 2$ ) and in their adulthood (CPX3, $23 \pm 5$ years). The change in peak oxygen uptake (PVO2: \% of normal) from CPX1 to CPX2 (PdPVO2) and from CPX2 to CPX3 (AdPVO2) were calculated, then our patients were divided into 4 subgroups according to the dPVO2: groups of II (PdPVO2 $\geq 0 \%+$ AdPVO2 $\geq 0 \%, n=51$ ), ID $(\mathrm{PdPVO} 2 \geq 0 \%+\mathrm{AdPVO} 2<0 \%, \mathrm{n}=46)$, DI $(\mathrm{PdPVO} 2<$ $0 \%+$ AdPVO $2 \geq 0 \%, \quad \mathrm{n}=18), \quad$ and $\quad \mathrm{DD} \quad(\mathrm{PdPVO} 2<0 \%+$ AdPVO $2<0 \%, \mathrm{n}=16)$. When compared with groups of DI and DD, adulthood plasma brain natriuretic peptide levels and renal resistive index were lower and plasma levels of albumin, cholinesterase and PVO2 were higher in the groups of II and ID $(\mathrm{p}<0.05$ to 0.0001$)$. In addition, adulthood body non fat percentage was higher in the groups of II and ID $(\mathrm{p}<0.05)$. After CPX3, 20 unexpected clinical events, including 5 deaths, occurred. Risk of the events was $72 \%$ lower in the groups of II and ID than in the groups of DI and DD $(\mathrm{p}<0.01)$. In particular, the risk reduction was $88 \%$ in the group of II when compared with that in group DD ( $\mathrm{p}<0.001)$.

Conclusions: Positive trajectory of exercise capacity in childhood predicts better adult Fontan pathophysiology, including better prognosis. Thus, prescription of exercise should be a part of postoperative management strategy in pediatric Fontan patients.

\section{P-100 \\ The adult congenital heart disease in Greece: Six years CHALLENGE registry}

Giannakoulas G. (1), Vasiliadis K. (2), Frogoudaki A. (3),

Ntellos C. (4), Tzifa A. (5), Brili S. (6), Manginas A. (7),

Apostolopoulou S. (8), Kolios M. (9), Pitsis A. (10), Ntiloudi D. (1), Karvounis H. (1), Tsioufis K. (6), Rammos S. (8)

Cardiology Department, AHEPA University Hospital, Thessaloniki, Greece (1); Cardiology Department, G. Papanikolaou Hospital, Exohi, Thessaloniki, Greece (2); Cardiology Department, Attikon University Hospital, Athens, Greece (3); Cardiology Department, Tzaneio General Hospital of Piraeus, Greece (4); Department of Congenital Heart Disease, Mitera Children's Hospital, Athens, Greece (5); Cardiology Department, Hippokrateion University Hospital, Athens, Greece (6); Cardiology Department, Mediterraneo Hospital, Athens, Greece (7); Department of Paediatric Cardiology, Onassis Cardiac Surgery Centre, Athens, Greece (8); Cardiology Department, University Hospital of Ioannina, Ioannina, Greece (9); Department of Cardiothoracic Surgery, Saint Luke's Clinic, Thessaloniki, Greece (10)

Introduction: The life expectancy of patients with congenital heart disease (CHD) is impressively improved, however long-term complications do exist. We aimed to study the basic demographic and clinical characteristics of adult patients with congenital heart disease (ACHD) in Greece.

Methods: In January 2012, a registry named CHALLENGE (Adult Congenital Heart Disease Registry. A registry from Hellenic Cardiology Society) was initiated. Enrollment of patients with structural CHD older than 16 years old was performed by 16 specialized centers nationwide. For the severity of CHD, Bethesda classification was utilized.

Results: Of 2304 patients with ACHD (mean age 38 years (SD 16), $51 \%$ women), $50 \%$ suffered from mild, $36 \%$ from moderate and $14 \%$ from severe ACHD. Atrial septal defect (ASD) was the most prevalent diagnosis (31\%). Half of patients had undergone at least 
one open-heart surgery (51\%), while 144 patients (6.3\%) underwent 3 or more surgeries. Among the latter group (mean age 32, SD 11), the majority suffered from tetralogy of Fallot ( $n=62,19 \%$ of tetralogy of Fallot patients), followed by patients with single ventricle ( $n=30,44 \%$ of single ventricle patients) and atrioventricular/ventriculoarterial abnormal connections $(n=20,14 \%$ of patients with atrioventricular/ventriculoarterial abnormal connections). The vast majority of ACHD patients (93\%) was asymptomatic or mildly symptomatic (NYHA class I/II). Elderly patients ( $>60$ years old) accounted for $12 \%$ of the ACHD population. In total, 38\% were under cardiac pharmacotherapy (15\% under antiarrhythmic drugs, 15\% under anticoagulants, $15 \%$ under heart failure medications and $4 \%$ under targeted therapy for pulmonary arterial hypertension).

Conclusions: Patients with ACHD grow older, facing the longterm complications. More than one third of our cohort was receiving cardiac medication. Prospective national registries are of high importance to identify the ongoing needs of these patients and match them with the appropriate resource allocation.

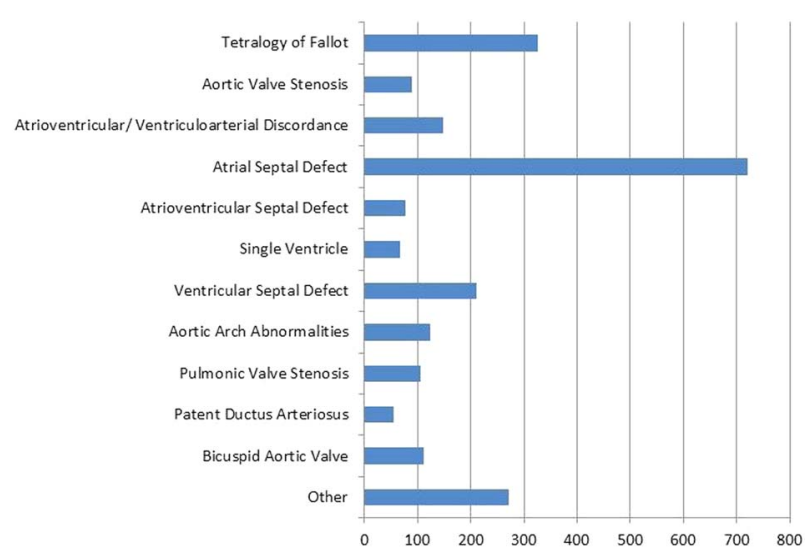

Figure.

\section{P-101}

Decline of estimated glomerular filtration-rate are mainly related to low cardiac output and combination use of spironolactone in adult Fontan patients

Kenji S., Hamamichi Y., Komiya E., Nukaga S., Ishii T., Kishiki.

K., Inage A., Ueda T., Yazaki S., Yoshikawa T.

Sakakibara Heart Institute Tokyo Japan

Background: Criterion values of creatinine (Cre) are different according sex and ages, which makes difficult for us to evaluate renal function by raw Cre value. Japanese Society of Nephrology provides formula of estimated glomerular filtration-rate (eGFR), by which we can estimate renal function in Japanese adults. We assessed reduction of kidney functions by means of eGFR in Fontan adults and sought backgrounds of reduced eGFR.

Methods: The medical records of 63 Fontan adults were reviewed aged from 19 years to 46 years. They underwent cardiac catheterization and routine blood test between 2010 and 2016. We calculated eGFR by formula which was composed of power function for Cre values and ages. Patients with eGFR $80 \mathrm{~mL} / \mathrm{min} /$ $\mathrm{m} 2$ under $(\mathrm{n}=17)$ were defined as eGFR- depression. First, we performed monovariate analysis for eGFR-depression by using the area under a receiving operating characteristics curve (AUROC) and square test. Second, we performed multiple logistic regession model to identify independent predictors for eGFR-depression.
Results: We gained AUROCs for eGFR-depression by 5 indexes; ages (0.683: $p=0.027)$, cardiac output (0.295: $p=0.017)$, end-systolic ventricular volume $(0.310: \mathrm{p}=0.041)$, end-diastolic ventricular volume (0.332: $\mathrm{p}=0.059)$, and ages at Fontan (0.647: $\mathrm{p}=0.075)$. No significant AUROCs were obtained by cardiac performances, such as central venous pressure, ventricular pressure on end-systole and end-diastole, and ventricular ejection fraction. As for internal remedy more patients with eGFR-depression took Spironolactone $(73 \%$ vs. $31 \%, p=0.0042)$. The rates of patients with other drugs were not different between two groups. After multivariate analysis, eGFR-depression was independently associated with cardiac output $1.8 \mathrm{~L} / \mathrm{min} / \mathrm{m} 2$ or under (odds ratio 28 ), diastolic ventricular volume $70 \%$ of Normal or under (odds ratio 19), and internal use of Spironolactone (odds ratio 14). The explanatory coefficient was high $(\mathrm{R}$-square $=0.64)$.

Conclusion: In adult Fontan patients eGFR-depression was intensely related to low cardiac output and small ventricle. Pressure loads, such as high central venous-pressure, and low end-systolic ventricular-pressure, were not connected with eGFR-depression. Shortage of renal blood-flow may be the main cause of deteriorating renal function in adult Fontan patients. Besides, eGFRdepression was associated with Spironolactone. We should to estimate eGFR in adult Fontan patients with these risk factors.

\section{P-102}

Evaluation of Right Ventricular Myocardial Deformation and Tricuspid Valve Displacement in Repaired Tetralogy of Fallot using Feature Tracking Magnetic Resonance Strain

Inage A., Mizuno N.

Sakakibara Heart Institute, Tokyo, Japan

Introduction: The objective of this study was to investigate into correlation between right ventricular (RV) myocardial deformation and tricuspid valve (TV) displacement, and functional parameters in repaired tetralogy of Fallot (TOF) compared to normal subjects using new MR tracking program.

Hypothesis: RV myocardial deformation and TV displacement may provide insight into RV reaction to volume overload in repaired TOF.

Methods: 50 repaired TOF patients $(26.4+/-8.1$ years $)$ and 15 normal subjects $(30.1+/-15.2$ years) underwent a cardiovascular MRI (CMR). Developing CMR software applying a semiautomatic segmentation program of the endo and epicardium was used to perform average longitudinal and circumferential strain, strain rate (LS, LSR and CS, CSR) in mid-systole (MS) and enddiastole (ED). As well, anterior and septal TV displacement relative to the apex were measured at end-systole (ES) as the shortest distance. The displacement velocities at basal lateral and septal segment in MS and early ED were calculated.

Results: $92 \%$ of cases have evident pulmonary regurgitation with $34+/-18 \%$ of pulmonary regurgitant fraction (PRF). LS was reduced in repaired TOF compared to controls while CS was preserved. LS and CS decreased with increasing RV end-diastolic (EDV) and -systolic (ESV) volumes (LS \& ESV r $=0.32$; CS \& EDV $\mathrm{r}=0.41$; CS \& ESV $\mathrm{r}=0.52$ ) and decreasing RV ejection fraction (EF) (LS \& EF r $=-0.55$; CS \& EF $r=-0.52$ ). No relationship was found between strain value and PRF. Increased anterior and septal TV displacement in ES as decreased shortening correlated positively RV volumes $(r=0.66$ and 0.63$)$, negatively $\operatorname{RVEF}(\mathrm{r}=-0.44$ and -0.36$)$ and LS $(r=0.34$ and 0.31$)$. Decreased basal lateral displacement velocities in MS and ED as greater shortening were associated with improved LS, LSR in MS ( $\mathrm{r}=$ 0.57 and -0.48$)$ and LSR in ED $(r=-0.45)$; however, there were 
no correlations between basal septal displacement velocity and RV functional parameters.

Conclusions: The correlations associated LS and CS with RV volumes and function without PRF suggest that preservation of CS is important in maintaining RV function. Greater lateral displacement velocity is associated with improved RV contractility.

\section{P-103 \\ Differences between echocardiographic features of newborns large for gestational age, newborns of mothers with gestational diabetes and newborns appropriate for gestational age}

Erolu E. (1), Akalin F. (2), Karacan M. (1), Sahin O. (1), Bilgin L. (1) Umraniye Education and Research Hospital Istanbul, Turkey (1) Marmara University Istanbul, Turkey (2)

Introduction: We studied echocardiographic features of asymptomatic newborns of mothers with gestational diabetes (GDM), newborns large for gestational age (LGA) and newborns appropriate for gestational age (AGA).

Methods: The study involved 60 singleton full-term newborn babies on whom echocardiogram were perfomed within the $24^{\text {th }}$ $72^{\text {th }}$ hours after delivery. None of the patients had perinatal asphyxia. None of patients had congenital heart disease or metabolic disturbance. There were 20 LGA, 20 newborns of mothers with GDM and 20 AGA. Five of the diabetic mothers used insulin during pregnancy. MMod measurements of left ventricle, left atrium and systolic and diastolic diameters of ascending aorta (Asc Ao) were obtained with 2D Echocardiography (Philips Medical Systems Affiniti 50) equipped with Neonatal Sector S12-4; systolic functions were calculated from MMod. Mitral inflow parameters were measured by Pulse Wave Doppler echocardiography for assesment of left ventricular diastolic functions. Tissue doppler echocardiography was performed, MPI index was calculated.

Results: Left ventricular diastolic diameter (LVDd), left ventricular posterior wall diameter (LVPWd) and left atrial diameter (LAD) were higher in LGA group than AGA group $(p=0.017, p=0.020$; respectively). Aortic diameter and diameter of ascending aorta at diastole were higher in LGA than AGA group $(p=0.001, p=0.039$; respectively). In comparison of AGA and newborn of GDM, no statistical difference was obtained between echocardiographic parameters of two groups. LAD was larger in newborns of GDM in comparison of AGA and newborn of diabetic mothers groups $(p=0.0008)$. There was no correlation between body surface area, height, weight and left atrial and left ventricular dimensions in LGA group.

Conclusions: Fetal macrosomia is associated with cardiac hypertrophy and fetal hypoxia, even intrauterine death. Cardiac biomarkers were found to be increased in LGA newborns compared to AGA newborns in a previous study. Enlargement of left heart chambers in LGA in our study might be a consequence of this finding. Although we did not study elasticity parameters of aorta, the difference in diameters in aorta and Asc Ao might be related to aortic elasticity properties of these infants. Low insulin usage might explain the indifference in myocardial functions in GDM group.

\section{P-104}

The Evaluation of Patients with Corrected Transposition of Great Arteries with Situs Inversus

Kasar T., Ayylldiz P., Ergul Y., Ozturk E., Guzeltas A.

Sağlık Bilimleri University, Mehmet Akif Ersoy Thoracic and

Cardiovascular Surgery Center, Istanbul, TURKEY

Introduction: The aim of the study is to evaluate a rare group of cardiac malformations followed with the diagnosis of congenitally corrected transposition the great arteries (ccTGA) and situs inversus (SI). Here we present the features and follow-up data of this rare group of patients.

Methods: Records of the 110 patients with ccTGA were retrospectively evaluated. Age, gender, associated cardiac defects, surgical history, heart block, arrhythmias, systemic AV valve regurgitation of the ccTGA patients with SI were recorded.

Results: The median age of the 14 patients with SI was 43.3 months (4 days-18 years). 12 patients $(85,7 \%)$ have hemodynamically significant associated lesions where 2 patients were isolated SI-ccTGA. Hemodynamically 9 of the patients were planned to be directed to biventricular correction where univentricular circulation was planned in 5 patients. During the follow-up period [median 43 months (9-84 months)]; 5 patients with associated lesions were operated. 2 patients died, one preoperatively, one after an aortopulmonary shunt procedure. Complete AV block was developed in a patient after surgical VSD closure and an immediate pacemaker implanted. Cardiac Resynchronisation Therapy was performed due to ventricular dysfunction during the postoperative follow-up to the same patient. There was no detected spontaneous AV block or arrhythmia except the supraventricular tachycardia detected in a patient that was ablated successfully.

Conclusion: The clinical presentation and survival are in fact dependant on the the associated defect and conduction abnormalities in this patients. The risk of AV block in ccTGA patients with SI seems to be lower than situs solitus ones due to near normal conduction pathways in SI patients.

Table.

\begin{tabular}{lc}
\hline Feature & Patient \\
\hline Cardiac Apex & \\
$\quad$ Dextrocardia & $7(50 \%)$ \\
Levocardia & $4(25 \%)$ \\
Mezocardia & $4(25 \%)$ \\
Associated lesions & $\mathbf{1 2 ( 8 5 , 7 \% )}$ \\
Ventricular Septal Defect (large) & $10(71,4 \%)$ \\
PS (significant) & $8(57 \%)$ \\
P. Atresia & $2(14,2)$ \\
SVI (significant) & $2(14,2)$ \\
Congestive Heart Failure (Yes/No) & $1 / 14$ \\
Right Ventricular Dysfunction & $1 / 14$ \\
Left Ventricular Dysfunction & $1 / 14$ \\
\hline
\end{tabular}

\section{P-105}

A Case of Atypical Kawasaki Disease with Abducens Nerve Palsy and Sialadenitis

Yilmazer M.M. (1), Meşe T. (1), An H.F. (1), Güven B. (2) Department of Pediatric Cardiology, Izmir Dr.Behçet Uz Children's Hospital, İzmir, Turkey (1); Department of Pediatric Cardiology, Tepecik Training and Research Hospital, İzmir, Turkey (2)

Case: A 5-year-old boy presented with 6 days of fever $>39.0$. he had bilateral non-purulent conjunctivitis and unilateral cervical lymphadenopathy on the left side. He had normal cardiovascular, neurological and gastrointestinal examination. Two dimensional echocardiography was performed, which showed fusiform dilatation in the left and right coronary arteries. The diameter of the left coronary artery (LCA) was measured to be $5.1 \mathrm{~mm}$ (Z-score: $+6.22)$ and the right coronary artery (RCA) was measured to be $6 \mathrm{~mm}$ (Z-score: +11.20$)$. The patient diagnosed with incomplete 
Kawasaki disease according to the clinical, laboratory and echocardiographic data. Intravenous immunoglobulin (IVIG) $(2 \mathrm{gr} / \mathrm{kg}$ ) and high-dose acetylsalicylate (ASA) $(80 \mathrm{mg} / \mathrm{kg})$ were given. Fever recovered on the sixth hour of IVIG administration. He was discharged from the hospital with low-dose ASA on the 10th day of IVIG treatment. The patient was re-admitted to our outpatient clinic due to significant submandibular swelling 17 days after the IVIG treatment. He had not fever since he was discharged from the hospital. Cervical USG showed a lymph node which was spherical, $25 \times 21 \mathrm{~mm}$ in size. In the follow-up swelling continued in the left submandibular region and was characterized as hard by palpation. Biopsy material taken from the submandibular swelling showed that the salivary gland morphology characterized by acini and ductus structures forming the lobules. Eventually eventually the patient was diagnosed with sialadenitis. Meanwhile, $35^{\text {th }}$ day of the illness the patient developed total abducens paralysis in the right eye (Figure 1). Dexamethasone treatment was initiated twice a day at $0.5 \mathrm{mg} / \mathrm{kg} /$ dose. Then dexamethasone treatment gradually reduced day 10 and was cut off on day 16 . Right abducens palsy did not improved completely but coronary dilatations decreased significantly in the echocardiography. The LCA was measured to be $3.1 \mathrm{~mm}$ (Z-score: +1.37), and the RCA was measured to be $2.9 \mathrm{~mm}$ (Z-score: +2.49$)$. Three months after the hospital discharge right abducens paralysis and submandibulary swelling was regressed and the eye movements completely improved.

Conclusions: It should be kept in mind that Kawasaki disease is a multisystemic vasculitis that can effect different organs such as central nervous system, salivary glands and others.

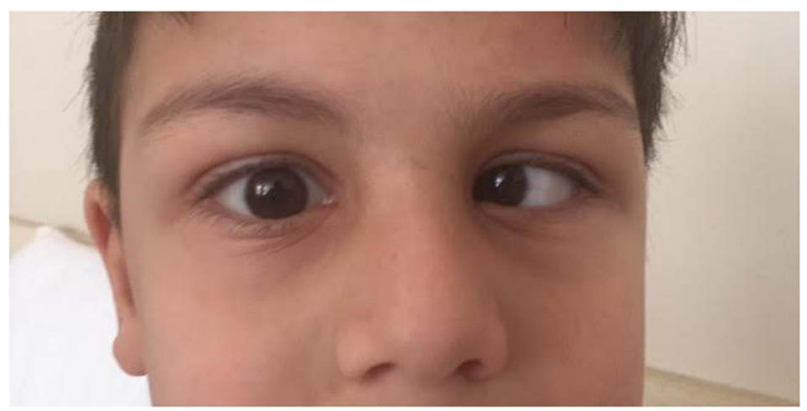

Figure 1.

Outward sight limitation in the right eye, right abducens paralysis

\section{P-106}

World Health Organization and World Heart Federation Echocardiographic Criteria for the Diagnosis of Rheumatic Subclinical Carditis: a Comparative Study

Zaher A.Z., Hamza H.S., Mosaad N.A.

Cairo University Specialized Pediatric Hospitals, Cardiology

Department, Cairo, Egypt

Background: The World Health Organization (WHO) 2004 developed criteria for diagnosing rheumatic subclinical carditis depending only on Doppler echocardiographic criteria of the mitral and aortic valves. In 2012, the World Heart Federation (WHF) developed other criteria depending on both Doppler and morphological features of the same previously mentioned valves. This study aims at comparing the criteria for the diagnosis of subclinical carditis according to the WHO and WHF criteria in patients documented with acute rheumatic fever according to modified Jones criteria.
Methods: This is a retrospective study that included 152 Egyptian patients 4-18 years presenting with acute rheumatic fever and diagnosed as having subclinical carditis according to the 2004 WHO criteria over an 18 months duration from January 2014 to June 2015. Echocardiography was performed by pediatric echocardiographers experienced in rheumatic heart disease in a specialized rheumatic fever clinic. Data were retrieved from the computer system and the WHF criteria for the diagnosis of subclinical carditis were applied on them.

Results: By applying WHF criteria, 3 (2\%) had definite RHD, 23 (15\%) patients had borderline RHD, whereas a total of $126(83 \%)$ patients were considered to have normal hearts.

Conclusion: There is a great discrepancy in the diagnosis of rheumatic subclinical carditis between the WHO and WHF echocardiographic criteria. Given the fact that these patients are already diagnosed as acute rheumatic fever, a dilemma is created that needs further studies to establish a basis for a reliable diagnosis of subclinical carditis where patients in high risk areas might be missed.

\section{P-107}

The influence of dipper pattern on ventricular functions with tissue Doppler, vascular functions with arterial stiffness and heart rate variability in newly diagnosed arterial hypertension in children

Cilsal E.

Adana City Hospital, Turkey

Objectives: This study is designed to investigate the differences of pulsatile hemodynamic, echocardiographic, 24-hour Holter monitorization and heart rate variability parameters in newly diagnosed arterial hypertension $(\mathrm{AH})$ children and also evaluate these parameters among dipper patterns.

Methods: The study group included 30 children with newly diagnosed essential $\mathrm{AH}$ and 30 healthy controls. Children with $\mathrm{AH}$ were also divided into two groups, dippers, and non-dippers. Physical examinations, 24-hour ambulatory blood pressure monitoring, 24hour Holter monitorization, 24-h heart rate variability (HRV), conventional 2-dimensional and Doppler echocardiography, and tissue Doppler imaging (TDI) were performed. Pulse wave analysis using an oscillometric device was performed to measure augmentation index (AI) and pulse wave velocity (PWV).

Results: A total of 60 subjects were included in our study for analysis from November 2016-November 2017. PWV and heart rate values were significantly higher in children with $\mathrm{AH}$. AI value was significantly higher in the non-dipper group. There were significant differences of M-mode and tissue Doppler echocardiographic parameters between $\mathrm{AH}$ and control groups while these parameters were similar according to dipping pattern in $\mathrm{AH}$ group. Time domain values, the standard deviation of all RR intervals (SDNN), which reflect parasympathetic activity, were significantly less in AH group. Also, SDNN values were significantly lower in the non-dipper group compared to dipper group. In frequency domain measures, LF (ms2) measured in daytime value was significantly lower in $\mathrm{AH}$ group compared to control. According to dipper pattern, LF (ms2) measured in nighttime values were also significantly lower in the non-dipper group.

Conclusion: Our study suggests that with the use of vascular function parameters, echocardiographic measurements, and HRV parameters there are significantly lower values observed in children with newly diagnosed $\mathrm{AH}$ compared to controls. While tissue Doppler parameters were similar, HRV parameters related to parasympathetic tone shows significant variations among dipping patterns. Elevated PWV and AI values were found in AH group and non-dipper group respectively. 


\section{P-108}

The Serial changes in Myocardial Functions after Pediatric Hematopoietic Stem Cell Transplantation: from baseline to the 12th months

Ekici F. (1), Uslu Tamburacı Z. D. (1), Yalan K. (2), Dönmez L. (3), Güler Balcı E. (2), Küpesiz A. (2), Kardelen F. (1)

Departments of Pediatric Cardiology (1); Pediatric Hematology and Oncology (2); and Public Health (3); Akdeniz University Faculty of Medicine. Antalya. Turkey

Objectives: To evaluate myocardial functions by tissue Doppler echocardiography (TDE) and serum N terminale Pro-brain natriuretic peptid (ProBNP) assay in children undergoing Hematopoietic stem cell transplantation (HSCT).

Methods: 39 children were evaluated by echocardiography at baseline, the 1st, 3rd, 6th and 12th month after HSCT. Baseline echocardiographic data of the patients were compared with those of 39 age-matched healthy children, as well as patient data after HSCT. Serum ProBNP was measured in patient group at baseline and the first month after HSCT. Our study was designed retrospectively and approved by the ethics committee of the hospital. Transplant types;Matched sibling donor (43\%), Matched unrelated donor (42\%), Autologous (10.2\%) and Haploid HSCT (5\%).

Results: All patient had normal the left ventricular ejection fraction (LVEF) before and after HSCT. Compared to control group, patient group had lower the tricuspid annular-plane systolic excursion rate and the early diastolic velocities (E') for both ventricles and greater the mean pulmonary artery pressure before HSCT. After HSCT, the E/A ratio and E' velocities for both ventricles had decreased for 3 months. The left ventricular diastolic functions returned to baseline level at the 6 months. However, the reductions in the systolic and diastolic myocardial velocities for tricuspid lateral annulus still persisted at the first year after HSCT. ProBNP values were elevated $(>120 \mathrm{pg} / \mathrm{mL})$ in $35.9 \%$ and $33.3 \%$ of patient at baseline and the first month after HSCT, respectively $(\mathrm{p}>0.05)$. Eight patient had positive history of anthracycline exposure, and they had higher baseline ProBNP values $(p>0.05)$. Although in normal limits, they had lower baseline LVEF and E' velocity for septum than patient without history of anthracycline exposure $(p=0.024, p=0.007)$. Ten patient died after HSCT due to non cardiac complication. They had relatively lower baseline LVEF and septal E' velocity than patient who survived $(p=0.012$, $p=0.009)$. There was no significant correlation between baseline ProBNP and mortality $(\mathrm{p}>0.05)$.

Conclusion: HSCT procedures may result subclinical deterioration in myocardial functions during the first year of HSCT. Myocardial function should be monitored by TDE in HSCT recipients.

\section{P-109 \\ Cardiovascular magnetic resonance reveals pathophysiologic background in paediatric patients with ventricular tachycardia and normal echocardiographic findings Giannakopoulou A. (1), Mavrogeni S. (2), Karanasios E. (1), Loukopoulou S. (1), Andreou N. (1), Papadopoulos G. (3) Cardiology Departmen,t Childrens Hospital Aghia Sophia Children's Hospital, Athens, Greece (1), Onassis Cardiac Surgery Center, Athens, Greece (2), First Dept of Paediatrics, National and Kapodistrian University of Athens, Aghia Sophia Children's Hospital, Athens, Greece (3)}

Introduction: To evaluate the pathophysiologic background of ventricular tachycardia (VT) in paediatric patients with normal echocardiographic findings using cardiovascular magnetic resonance (CMR).
Methods: Between 2000-2016, 110 patients (60 male/50 female), aged 10 -18 years, with recent history of ventricular tachycardia VT and normal echocardiographic findings, were referred in our tertiary center for CMR evaluation.

CMR was performed using a $1.5 \mathrm{~T}$ magnet and included functional evaluation in short and long axis, oedema assessment using T2w imaging and fibrosis evaluation using late gadolinium enhancement (LGE). Results: Right and left ventricular function was normal in all paediatric patients. However, the CMR tissue characterization revealed evidence of acute myocarditis in 35/110 and various types of cardiomyopathies in 55/110 (noncompaction cardiomyopathy, arrhythogenic right ventricular cardiomyopathy, hypertrophic, restrictive and dilated cardiomyopathy). In 20/110 no morphologic or functional abnormalities were identified by CMR .

The T2 ratio was $3.4 \pm 0.03$ and $1.5 \pm 0.04$ in myocarditis and cardiomyopathy group, respectively $(\mathrm{p}<0.05)$. In contrary the LGE was $5 \pm 2 \%$ and $15 \pm 3 \%$ in myocarditis and cardiomyopathy group, respectively $(\mathrm{p}<0.05)$.

Conclusion: After CMR evaluation of a paediatric population with history of recent VT and normal echocardiographic findings, evidence of myocarditis and cardiomyopathies was identified. However, in 18\% of them no functional or anatomical abnormality was identified

\section{P-110}

Performance of cardiac MRI for congenital heart disease in a High Field Open (HFO) 1.0Tesla MRI scanner

Tzifa A., Papaioannou G., Patris K., Kritikos N., Loggitsi D.

Mitera Hospital, Athens, Greece

Background and aims: Combined cardiac catheterization and magnetic resonance imaging (XMR) is a well-established approach in some centers, particularly for accurate assessment of pulmonary hypertension (PHT) and its reversibility. Its wider application however, is hampered by limited availability of hybrid XMR suites. We advocate that the procedure can still be performed with minimal changes to the MRI suite's infrastructure with important benefits for the management of borderline inoperable patients due to PHT. The aim of the current study was twofold: First, to test the feasibility of performing such studies in the catheterization laboratory and moving the patient to the MRI suite and secondly, to assess patients' outcomes based on XMR findings, as well as the importance of XMR on the decision planning.

Methods: We reviewed the data of 12 patients who underwent an XMR catheterization because of echocardiographic suspicion of PHT. 1/12 patient had a solely MRI-guided cardiac catheterization procedure.

Results: Twelve patients with median age of 16yrs (range 2-56yrs) underwent an XMR procedure to assess pulmonary vascular resistance (PVR) and guide further management. Median baseline PVR was 5.1 WU.m2 (range 3.1-12WU.m2), which improved to 4.2 WU.m2 (range 2.3-8.5WU.m2) after administration of pulmonary vasodilators. Of the 12 patients, eight were operated and one had percutaneous closure of an ASD at a median time of 5 months (range 0.5-18 months) after the procedure. After administration of pulmonary vasodilators, elevated PVR was found in 3/9 patients (3.5-8.5WU.m2), who therefore had fenestrated closure of their septal defect. The remaining $6 / 9$ patients had complete closure of their defects without fenestration. Two patients had changes in their medical management and one patient has been referred for a heart-lung transplant.

Conclusions: The performance of MRI-assisted or guided catheterization procedures can take place outside the setting of a hybrid XMR suite. XMR procedures are extremely important for the management of patients with CHD and pulmonary hypertension. 
Acquisition of accurate flow data from MRI combined with simultaneous pressure measurement provides a robust assessment of the patient's operability status and type of operation, such as fenestrated defect closure, whilst also guiding the postoperative management in the intensive care unit.

\section{P-111}

Detection of regional wall motion abnormalities by three dimensional speckle-tracking in young patients after $\mathbf{H T x}$ Racolta A. (1), zu Knyphausen E. (1), Klusmeier E. (1), Körperich H. (2), Kececioglu D. (1), Laser K.T. (1)

Heart and Diabetes Center NRW, Pediatric Heart Center and Center for Congenital Heart Defects, Bad Oeynhausen, Germany (1). Heart and Diabetes Center NRW, Institute for Radiology, Nuclear Medicine and Molecular Imaging, Bad Oeynhausen, Germany (2)

Objective: Three dimensional speckle-tracking (3DST) is a new echocardiographic method to investigate left ventricular cardiac deformation. In children the technique has not been widely used. The follow-up of patients after HTx is a potential target as cardiac performance can be negatively influenced by acute or chronic rejections. This study aims to evaluate the potential of the method to detect regional fibrosis in comparison to cardiac magnetic resonance (CMR) imaging, to find correlations with functional parameters of cardiac performance as well as to assess reproducibility in pediatric patients post HTx.

Method: 11 consecutive patients $(20.7 \pm 3.8,14.6-28$ ys, 6 female $)$ who presented for routine outpatient consultation $14.1 \pm 8.2,1.5-22$ ys after HTx were investigated with echocardiography and CMR. In addition to routine diagnostic workup, 3DST (IE 33, X5-1 transducer, calculation with LV-Analysis 3.1[Tomtec]) and estimation of extracellular volume (ECV, 3T TX Achieva with dStream technology, R5.3, Philips, calculation with HDZ-NRW, Bad Oeynhausen) was performed. Regional wall motion abnormalities were calculated as $>20 \%$ segmental deviation from mean global strain values in all directions measured. The same method was applied to T1-Mapping analyses. Reproducibility was assessed by Bland Altman statistics.

Results: All patients were in sinus rhythm, 7 with RBBB, 1 with LBBB. Acute rejection was suspected in none. Ejection Fraction $(\mathrm{EF}, 61 \pm 2.9)$ was normal, no echocardiographic signs for diastolic dysfunction by E/E' $(6.6 \pm 1.8)$. 3DST analysis calculated normal global values for $3 \mathrm{D}$-strain $(-34.9 \pm 4.6 \%)$, circumferential strain $(-28.9 \pm 3.2 \%)$ and longitudinal strain $(-19.7 \pm 3.0 \%)$, reduced values for radial Strain $(40.9 \pm 4.8 \%)$. Segmental wall motion abnormalities were detected in 9 patients distributed on 15 septal segments (AHA 2,3,8). In comparison to CMR these segments did not have elevated regional ECV-values, that did not correlate with the strain values $(r<0.5)$. Intraobserver-variability of mean average segmental strain values was $0.2 \pm 22 \%$ (LOA - 43.5-44\%). Conclusions: 3DST permits identification of septal regional wall motion abnormalities in otherwise normal working hearts post HTx. Most probably this is based on electrical dyssynchrony, as regional fibrosis could not be detected by T1-Mapping. Reproducibility of 3DST results was acceptable. This method may help in the follow-up of pediatric patients post HTx in combination with CMR-ECV measurements.

\section{P-112}

Right Ventricular Evaluation by Echocardiographic Score in Repaired Tetralogy of Fallot

Limsuwan A., Trisauvapak T., Khowsathit P.

Division of Pediatric Cardiology, Faculty of medicine Ramathibodi

Hospital, Mahidol University, Bangkok, Thailand
Background: Cardiac magnetic resonance (CMR) has become a standard tool to evaluate the right ventricle $(\mathrm{RV})$. In repaired tetralogy of Fallot (rTOF), the right ventricular volume load is considered to be one of the key criterions for the pulmonary valve replacement. The objective of this study is to develop echocardiographic score as a parameter to define the right ventricular dilatation in correlation with the CMR measurement. Methods: Patients with rTOF underwent CMR and echocardiogram. The echocardiographic measurement of cardiac chambers including right and left ventricle (LV) was compared and correlated with the RV volume parameter obtained from CMR. The sensitivity and specificity of the echocardigraphic threshold value predict the RV volume were determined. And a scoring system was established.

Results: A total of 91 patients (mean age 14 years, range 12-18, 64 male) were enrolled. The ratio of pulmonary regurgitation (PR) jet width and pulmonary valve annulus diameter (PR/PV) $\geq 0.6$ had $92.9 \%$ sensitivity and $88.2 \%$ specificity to predict pulmonary valve regurgitation fraction $(\mathrm{PRF}) \geq 25 \%$. The echocardiographic measurement of RV end diastolic diameter index (RVEDdi), right ventricular outflow tract diameter index (RVOTdi) plus $\mathrm{RVEDdi}, \mathrm{RV} / \mathrm{LV}$ dimension ratio and the right atrium area index (RAi) were significantly correlated with the RV end diastolic volume index (RVEDVi) obtained by CMR. With multivariate analysis, the $\mathrm{RV} / \mathrm{LV}$ ratio $\geq 0.55$ and the $\mathrm{RAi} \geq 10 \mathrm{ml} / \mathrm{m} 2$ were correlated with the RVEDVi $\geq 150 \mathrm{~mL} / \mathrm{m}^{2}$ by CMR. Therefore, the scoring system was established base on the PR/PV, $\mathrm{RV} / \mathrm{LV}$ ratio and $\mathrm{RA}$ dimension. The $\mathrm{PR} / \mathrm{PV}$ ratio $\geq 0.6$ and echocardiographic score $\geq 2$ would determine significant $P R$ and RV dilation with the sensitivity $79 \%$ and the specificity of $68 \%$. Conclusion: The rTOF score could be potentially use to assess the severity of the PR and RV dilation and predict the RVEDVi by CMR during the routine cardiology follow- up.

\section{P-113}

Myocardial strain using speckle tracking echocardiography in pediatric Duchenne Muscular Dystrophy patients Cesar S. (1), Ortez C. (2), Nascimento A. (2), Bijnens B. (3), Sarquella-Brugada G. (1)

Pediatric cardiology department. Hospital Sant Joan de Déu. Barcelona, Spain (1); Pediatric neurology department. Hospital Sant Joan de Déu. Barcelona, Spain. (2); Pompeu Fabra University. Barcelona, Spain (3).

Introduction: Duchenne muscular dystrophy (DMD) is an inherited X-linked disorder, with an incidence of 1:3500 male births. Cardiomyopathy is the leading cause of death. Cardiac MRI (CMR) with late gadolinium enhancement (LGE) is an important tool to analyze myocardial fibrosis, but early chages could be detected analyzing myocardial strain by echocardiography.

The aim of this study is to analyze the myocardial strain pattern in our pediatric DMD population and to correlate these parameters with global LV function and CMR data.

Methods: We analyzed 17 patients diagnosed with DMD. We obtained epidemiological, clinical, CMR and echocardiography data (LVEF) including speckle tracking offline analysis with QLAB $^{\circledR}$, Philips.

Results. We collected data from 17 pediatric DMD patients. Mean age 13,3y (median 14, IQR 3). All of them received treatment with oral corticoids. Except in the younger case (5yo), the other patients were not still capable of autonomous deambulation. Concerning global LV function analysis 7 patients showed LV systolic dysfunction (LVEF <55\%). Three of these patients showed LGE in the CMR (inferolateral regions). All 7 patients showed regional impairment in the circumferential strain. 
Longitudinal strain was altered only in 2 patients, with LVEF less than $40 \%$.

The other 10 patients showed normal LVEF values. We detected LGE in the CMR in 3 patients (same inferolateral regions and anteroseptal regions). In 6 of these 10 patients, we detected an abnormal behaviour of myocardial deformation in the speckle tracking analysis: abnormal deformation in basal and mid inferolateral regions (two of these 6 patients, only mid inferolateral region abnormalities). Two of the 3 patients with LGE showed impairment of these regional abnormalities.

Conclusion: Circumferential strain is altered in DMD patients, including in those with normal LVEF values. The majority of patients with preserved LVEF and LGE in the CMR showed abnormalities in the regional circumferential strain, specially inferolateral regions. Circumferential strain by speckle tracking echocardiography in pediatric DMD patients could be a marker of myocardial fibrosis.

\section{P-114}

The role of the hepatic elastography in the follow up of patients after Fontan surgery

Meliota G., Lombardi M., Tagliente M.R., Scalzo G., Vairo U. Cardiologia e Cardiochirurgia Pediatrica, Ospedale Pediatrico Giovanni XXIII, Bari, Italy

Introduction: Fontan circulation causes liver congestion with hypoxic stress and local inflammation, that leads to chronic liver damage and fibrosis. Hepatic elastography can non-invasively estimate the level of organ fibrosis, measuring the liver stiffness (LS).

Methods: In order to evaluate usefulness of hepatic elastography in the follow-up of patients with Fontan circulation, we prospectively examined patients with complex congenital heart disease (CHD) who underwent Fontan surgery or Kawashima procedure in our centre between 2012 and 2017. Hepatic elastography was performed at 3,6,12 months after surgery and yearly thereafter. Echocardiographic and blood test data were also collected.

Results: 16 patients were enrolled $(\mathrm{M}: \mathrm{F}=1)$, with mean age at surgery of 6.46 (range 4.0-10.0) years. Native CHD are listed in Table 1. 14 patients underwent extracardiac Fontan surgery (fenestrated in 3 cases), while 2 of them Kawashima procedure. No patient had any preexisting hepatic illness.

Mean age at last follow-up was 12.8 (5-29) years. Echocardiography showed good flow in the Fontan circuit in all patients and a normal systolic ventricular function at follow-up. No one suffered from heart failure nor "protein loosing enteropathy" during follow-up.

Mean LS value was12.35 kPa (range 2.8-22.3). LS was higher in patients who underwent Fontan surgery than Kawashima procedure. Moreover LS was higher at third month after surgery and decreased at 6 and 12 months. It increased again at 5 years. No relevant variations of LS were detected thereafter. Native CHD seemed to be a determinant of the stiffness at 5 years, instead, as right single-ventricle patients showed the highest LS.

Increased LS was linked to hepatomegaly diagnosed by physical examination, but no relevant alteration was found in liver function tests. INR was prolonged in two patients on warfarin and otherwise normal.

Conclusion: Hepatic Elastography is a potential tool to monitor the onset and progression of congestive hepatopathy and fibrosis. Patients who underwent Fontan surgery showed an increase of LS, likely dependent on native CHD, without a relevant alteration of hepatic function. Lacking data regarding liver biopsy, it is not possible to establish whether the increased LS is due to hepatic congestion or fibrosis.
Table.

\begin{tabular}{ll}
\hline Native Congenital Heart Disease & $\begin{array}{l}\text { Number } \\
\mathbf{( \% )}\end{array}$ \\
\hline Tricuspid atresia & $5(31)$ \\
DORV, PS/PA & $3(19)$ \\
ccTGA, PA & $3(19)$ \\
VSD with overriding and straddling tricuspid valve & $2(13)$ \\
TGA, VSD, CoAo, overriding and straddling & $1(6)$ \\
tricuspid valve & $1(6)$ \\
Left isomerism, complete AVCD & $1(6)$ \\
Right isomerism, AVCD with RV dominance & \\
\hline
\end{tabular}

AVCD: atrioventricular canal defect; ccTGA: congenitally corrected trasposition of great arteries; CoAo: coarctation of the aorta; DORV: double-outlet right ventricle; PA: pulmonary atresia; PS: pulmonary stenosis; TGA: trasposition of great arteries; VSD: ventricular septal defect

\section{P-115}

\section{Follow-up of Coarctation of the Aorta: a single-centre experience}

Meliota G. (1), Lombardi M. (1), Tagliente M.R. (1), Scalzo G. (1), Spagnoletti G. (2), Vairo U. (1)

Cardiologia e Cardiochirurgia Pediatrica, Ospedale Pediatrico Giovanni XXIII, Bari, Italy (1), Medical Statistics, University of Foggia, Foggia, Italy (2)

Introduction: Coarctation of the aorta (CoA) is an important treatable cause of secondary arterial hypertension. Left ventricular hypertrophy $(\mathrm{LVH})$ is a marker of organ damage induced by CoA and is associated with a poorer prognosis.

Methods: To identify clinical and instrumental predictors of hypertension and LVH, we retrospectively evaluated 147 patients with CoA, examined for a starting or already on-going follow-up between 2010 and 2017. Medical records were reviewed regarding patient profiles, concomitant congenital heart defects, surgical and percutaneous procedures, echocardiographic evaluations and exercise treadmill testing. R statistical software was used for analysis. Since numeric variables were not normally distributed, nonparametric tests were performed.

Results: Median follow-up period was 5 years (IQR: 2-7 years) and median age at the last follow-up was 12 (IQR: 4-18) years. There was a higher prevalence of male patients (63\%). $21.4 \%$ of patients presented aortic arch hypoplasia and $68.3 \%$ of them had associated congenital heart defects. Total study population showed two clusters of patients according to the age of presentation of CoA: within the first year of life (117 patients, $78.6 \%)$ or $>1$ year (30 patients, 21.4\%). Baseline characteristics and followup data are summarized in the table below. Late hypertension developed independently from recurrent $\operatorname{CoA}\left(\chi^{2}\right.$ test, $\left.p=n s\right)$. Peak trans-isthmic aortic Doppler gradient $\left(\mathrm{PG}_{\mathrm{Ao}}\right)$ at follow-up was significantly related to hypertension and LVH (Mann-Whitney test, $p=0.02$ and $p=0.01$ respectively). $\mathrm{PG}_{\mathrm{Ao}}$ showed a trend of correlation with systolic blood pressure (SysBP) in total population (Spearman's correlation, $\mathrm{p}=0.09$ ) and a significant correlation with SysBP in patients on anti-hypertensive medical treatment (Spearman's correlation, $\mathrm{p}=0.04)$. $\mathrm{PG}_{\mathrm{Ao}}$ was a predictor of pulse pressure in total study population $(\beta=0.034$; $p=0.02$; Figure). Compared with other surgical techniques, patients treated with patch aortoplasty had higher rate of recurrent CoA $\left(\chi^{2}\right.$ test, $\left.p=0.002\right)$ and hypertension $\left(\chi^{2}\right.$ test, $\left.p=0.02\right)$ at follow-up. Hypertensive response to exercise treadmill testing 
Table.

\begin{tabular}{|c|c|c|c|c|c|}
\hline & & $\begin{array}{l}\text { Total } \\
(n=147)\end{array}$ & $\begin{array}{l}\text { I cluster } \\
(n=117)\end{array}$ & $\begin{array}{l}\text { II cluster } \\
(\mathrm{n}=30)\end{array}$ & $\begin{array}{l}P \\
\text { value }\end{array}$ \\
\hline \multicolumn{2}{|c|}{ Age at diagnosis } & $\begin{array}{l}29.9(6.7- \\
11.8)\end{array}$ & $14(4-30) d$ & $\begin{array}{l}6.5(3.25- \\
10.25) \mathrm{y}\end{array}$ & $<0.001$ \\
\hline \multicolumn{6}{|c|}{ Treatment } \\
\hline & Percutaneous & $12 \%$ & $5.0 \%$ & $40 \%$ & $<0.001$ \\
\hline & None & $5 \%$ & $3.0 \%$ & $13.3 \%$ & $<0.01$ \\
\hline \multicolumn{2}{|c|}{ First treatment age } & $\begin{array}{l}29.9(14.4- \\
172.4) \mathrm{d}\end{array}$ & $\begin{array}{l}29.9(10.8- \\
59.9) \mathrm{d}\end{array}$ & $7(3-13) y$ & $<0.001$ \\
\hline \multicolumn{2}{|c|}{ Recurrent coarctation } & $28.3 \%$ & $30.4 \%$ & $24 \%$ & 0.08 \\
\hline \multicolumn{2}{|l|}{ Hypertension } & $55 . \%$ & $53.2 \%$ & $65.5 \%$ & 0.06 \\
\hline \multicolumn{2}{|l|}{ LVH } & $44.7 \%$ & $43.9 \%$ & $46.6 \%$ & 0.08 \\
\hline
\end{tabular}

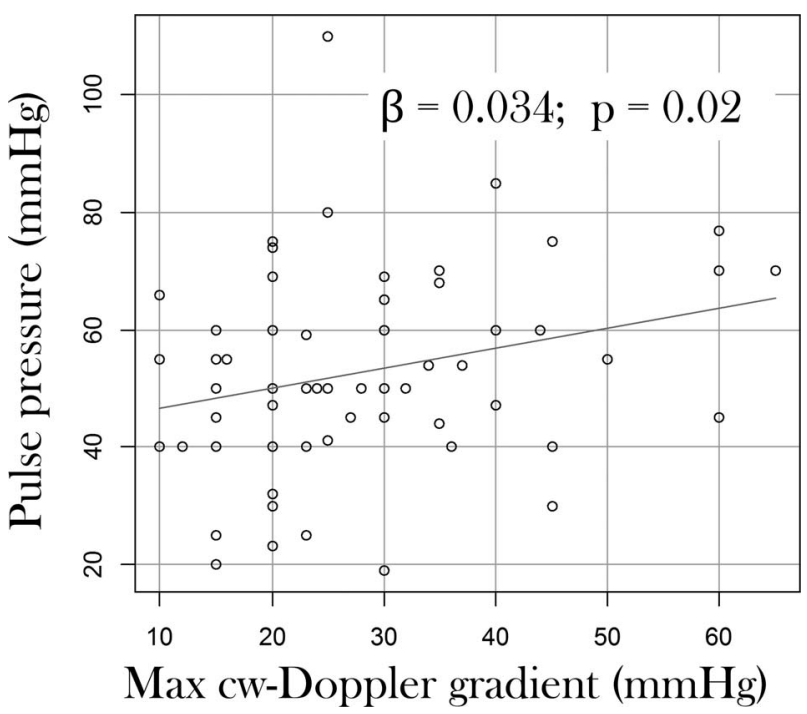

Figure.

showed association with the onset of late hypertension $\left(\chi^{2}\right.$ test, $\mathrm{p}=0.05)$.

Conclusions: Peak trans-isthmic Doppler gradient at follow-up was strongly related to hypertension and $\mathrm{LVH}$ and can have a prognostic value. Hypertensive response to exercise treadmill testing was associated with development of LVH.

\section{P-116}

Parameters of biventricular dyssynchrony in patients with repaired and unrepaired Ebstein's anomaly - Assessment by tissue tracking cardiovascular magnetic resonance Marcora S.A. (1), Weber R. (2,3), Oxenius A. $(2,3)$, Attenhofer Jost C. (4), Valsangiacomo Buechel E.R $(2,3)$ ASST Papa Giovanni XXIII, Bergamo, Italy (1), Pediatric Heart Center, University Children's Hospital, Zurich (2), Children's Research Center, University Children's Hospital, Zurich (3), Klinik im Park, Zurich (4)

Introduction: Different factors, such as left ventricular (LV) noncompaction (LVNC) and atrialization of right ventricle (RV) may influence synchrony in patients with Ebstein anomaly (EA). We studied parameters of ventricular synchrony by using feature tracking (FT) CMR.

Methods: Biventricular function was prospectively assessed by cine CMR using the SSFP sequence in 20 patients with EA ( 6 after TV repair). Median age was 44.5 (17-64) years. Mean TV displacement was $41 \pm 36 \mathrm{~mm}$; LVNC present in 12 patients (60\%). Image temporal resolution was $<25 \mathrm{msec}$. Short-axis (SAX) and longaxis 4 chamber (LAX) images were analyzed by FT (Medis Version 3.3) to assess global and regional time to peak (TTP) in both ventricles. Wall delay (WD) was defined as the TTP difference between the septum and the lateral segments (free wall) on SAX and LAX images.

Results: LV SAX showed a significant WD in basal $(69 \pm 84 \mathrm{~ms}$; $\mathrm{p}<0.01)$ and mid-ventricular segments $(42 \pm 48 \mathrm{~ms} ; \mathrm{p}<0.01)$ but not at the apex $(-4 \pm 57 \mathrm{~ms} ; \mathrm{n} . \mathrm{s})$. Mean septal TTP decreased from basis to apex $(370 \pm 108 \mathrm{~ms}$ vs $334 \pm 60 \mathrm{~ms} ; \mathrm{p} 0.04$ vs $293 \pm 69 \mathrm{~ms}$; $\mathrm{p}<0.01)$. Global and free wall TTP did not differ among segments.

LV LAX had similar TTP values in the septum and free wall $(326 \pm 86 \mathrm{~ms}$ vs $312 \pm 66 \mathrm{~ms})$. RV free wall TTP was significantly delayed compared to the septum ( $383 \pm 114 \mathrm{~ms}$ vs $338 \pm 123 \mathrm{~ms}$; p 0.04; ) and LV free wall $(312 \pm 67 \mathrm{~ms} ; \mathrm{p}<0.01)$.

Mean RV EF\% was $39 \pm 10$ and LV EF\% $53 \pm 10$.TV displacement correlated with WD in the LV mid-ventricular segments (r 0.57, p < 0.01), but not in other biventricular segments. LV EF\% correlated with basal LV SAX WD ( $\mathrm{r}-0.53, \mathrm{p} 0.014)$, but not with global or regional TTP. RV EF\% correlated weakly only with global RV TTP (r - 0.46, p 0.04). LVNC did not affect synchrony parameters.

Conclusions: Significant global and segmental differences in TTP are present in both ventricles of EA patients. LV shows intraventricular dyssynchrony in septal basal and mid-ventricular segments that correlates with the severity of TV displacement. WD is greater in RV free wall than septum and LV free wall, indicating interventricular dyssynchrony.

\section{P-117}

Parameters of biventricular deformation in patients with repaired and unrepaired Ebstein's anomaly - Assessment by tissue tracking cardiovascular magnetic resonance Marcora S.A. (1), Weber R. (2,3), Oxenius A. $(2,3)$, Attenhofer Jost C. (4), Valsangiacomo Buechel E.R $(2,3)$ ASST Papa Giovanni XXIII, Bergamo, Italy (1), Pediatric Heart Center, University Children's Hospital, Zurich (2), Children's Research Center, University Children's Hospital, Zurich (3), Klinik im Park, Zurich (4)

Introduction: In Ebstein anomaly (EA) other factors than the tricuspid valve (TV) malformation, including left myocardial noncompaction (LVNC) and right ventricle (RV) atrialization may influence myocardial function even after TV repair.

We assessed biventricular myocardial deformation in repaired and unrepaired EA by using feature tracking (FT) CMR and tested features potentially influencing myocardial deformation.

Methods: Biventricular function was prospectively assessed by cine CMR using the SSFP sequence in 20 patients with EA. Global circumferential (GCS) and longitudinal strain (GLS) of both ventricles were analysed by FT (Medis Version 3.3). Image temporal resolution was $<25 \mathrm{msec}$.

Patients median age was 44.5 (17-64). Six patients underwent TV repair and twelve (60\%) had LVNC. Correlation with LVNC, degree of TV displacement, ventricular volumes and EF \% was tested.

Results: Mean RVEDV was $137 \pm 34 \mathrm{ml} / \mathrm{m} 2$; RVESV $86 \pm 33 \mathrm{ml} / \mathrm{m} 2$, RV EF\% $39 \pm 10$. RV dilatation (> $110 \mathrm{ml} / \mathrm{m} 2$ ) was measured in 18/20 patients, independently from valve repair. Mean LVEDV was $87 \pm 26 \mathrm{ml} / \mathrm{m} 2$; LVESV $42 \pm 16 \mathrm{ml} / \mathrm{m} 2 ;$ LVEF $\% 53 \pm 10$.

LV GCS was normal $(-26.3 \pm 8 \%)$. GLS was decreased in the LV and RV compared to normal subjects (LV GLS $-16.6 \pm 4 \%$ vs 
$21.3 \pm 4.8 ; \mathrm{p}<0.01$ and RV GLS $-12 \pm 6 \%$ vs $18.9 \pm 4.6$; $\mathrm{p}<0.001)$. GLS of RV septum was inferior to GLS of RV free wall $(12.2 \pm 6 \%$ vs $22.8 \pm 7.3$; p 0.0002). LVNC, previous TV repair, degree of TV displacement did not influence $\mathrm{EF} \%$, nor global strain values of both ventricles.

LV GCS correlated with LV GLS (r 0.749, p < 0.001), LV ESV and LV EF\% (r 0.7128; $\mathrm{p}<0.001)$. A weak correlation was found between LV GCS and RV GLS (r 0.55; p 0.01). LV GLS correlated with LVESV (r-0.52; p 0.01), LV EF\% (r 0.7128; p < 0.001), RV GLS (r 0.49; p 0.029), but not with RV EF\%.

RV GLS significantly correlated with RV ESV ( $\mathrm{r}-0.5 ; \mathrm{p} 0.02)$ and RV EF \% (r 0.54;p 0.01). No correlation was found between LV and RV EF\%.

Conclusions: Patients with EA have a dilated RV with decreased RVGLS and EF\% independently from the severity of TV displacement or RV repair. RV septum deformation is more affected than free wall. LV GCS is normal, while GLS decreased.

\section{P-118}

Midwall fibrosis in children and adolescents with primary inherited dilated cardiomyopathy

Al-Wakeel-Marquard N. (1), Degener F. (1,2), Nordmeyer S. (1,2), Schmitt B. (1,2), Kühne T. (1,2,3,4), Klaassen S. $(2,3,5)$, Berger F. $(1,2,3)$, Messroghli D. $(2,6,7)$

Deutsches Herzzentrum Berlin, Klinik für Angeborene Herzfehler Kinderkardiologie, Berlin, Germany (1); DZHK (German Centre for Cardiovascular Research), partner site Berlin, Berlin, Germany (2); Charité - Universitätsmedizin Berlin, Klinik für Pädiatrie mit Schwerpunkt Kardiologie, Berlin, Germany (3); Charité Universitätsmedizin Berlin, Institut für kardiovaskuläre Computerassistierte Medizin, Berlin, Germany (4); Experimental and Clinical Research Center, a joint cooperation between the Charité Medical Faculty and the Max-Delbrück-Center for Molecular Medicine, Berlin, Germany (5); Deutsches Herzzentrum Berlin, Klinik für Innere Medizin Kardiologie, Berlin, Germany (6); Charité - Universitätsmedizin Berlin, Medizinische Klinik mit Schwerpunkt Kardiologie, Berlin, Germany (7)

Objectives: Midwall fibrosis as detected by late gadolinium enhancement (LGE) cardiovascular magnetic resonance (CMR) is a common finding predicting adverse outcome in adults with idiopathic dilated cardiomyopathy (DCM). So far, its relevance in pediatric patient is relatively unknown. The present study aimed to evaluate focal myocardial fibrosis by LGE in children and adolescents with primary inherited DCM.

Methods: Patients $\leq 18$ years with primary inherited DCM were prospectively enrolled into the study. Standardized CMR including volumetric analysis and LGE for assessment of focal myocardial fibrosis was conducted in all participants. LGE images were acquired 8 to 10 minutes after bolus administration of gadolinium-DOTA at a dose of $0.2 \mathrm{mmol} / \mathrm{kg}$, covering the entire left ventricle (LV) in short axis orientation, and in 2-, 3- and 4 chamber view.

Results: In total, 13 patients (median age (range) 11.6 (7.5-15.0) years, 7 female) were included. Median LV EF was 33 (14-53) \% and LV EDV indexed to body surface area was 124.1 (107.1$225.6) \mathrm{ml} / \mathrm{m}^{2}$. LGE was present in 8 of the 13 patients (62\%). In all 8, LGE was characterized as midwall fibrosis located in the basal and/or midventricular septum. Of the 8 patients with midwall fibrosis, 4 underwent cardiac transplantation (HTx). Prior mechanical circulatory support (MCS) was performed in 3, and additional implantation of an implantable cardioverter defibrillator for secondary prevention of sudden cardiac death in 1 of the 4 HTx patients. Of the 5 LGE negative patients, 1 received MCS and HTx.
Conclusions: Our data suggest that midwall fibrosis appears frequently in children and adolescents with primary inherited DCM and may be associated with unfavorable clinical outcome in these patients. Further longitudinal studies are necessary to assess the incidence and prognostic role of midwall fibrosis in pediatric DCM.

\section{P-119}

Lung ultrasound in the pediatric patient with postoperative congenital heart disease. Towards the new frontier

Hasan T. (1), Bonetti S. (1), Bulgarelli A. (1), Gioachin A. (2), Ragni L (1), Prandstraller D (1), Gagriulo GD (1), Bonvicini M (1), Donti A. (1)

(1) Pediatric and congenital adult cardiology and cardiac surgery unit - S. Orsola - Malpighi Hospital, Bologna, Italy (2) Specialization school of pediatrics - Ferrara, Italy

Introduction: Post-operative recovery of children with heart disease is still encumbered by pulmonary complications like pneumothorax (PNX), pleural effusion (PLE), interstitial edema and pulmonary consolidation (PC). The gold standard for diagnosis of those complications is chest CT which is not feasible in routine clinical care thus chest $\mathrm{x}$-ray is routinely preferred. Recently lung ultrasound (LUS) has become an important diagnostic tool for evaluation of pulmonary diseases in the pediatric context, especially in the emergency and intensive care setting. LUS is accurate in differentiating pleural and parenchymal diseases, it allows for dynamic imaging and is useful in the unstable patient being a bedside examination. Few data in literature support the use in the congenital heart disease pediatric patient, as the method has been developed for the study of pulmonary disease.

Objectives: To evaluate LUS accuracy in the identification of PNX, PLE and PC in a pediatric population of patients with congenital heart disease who underwent heart surgery

Methods: 3 patients aged 0-17 years who underwent cardiac surgery were evaluated in the postoperative period by chest $\mathrm{x}$-ray and LUS at the same time. The exams were executed by two examiners unknown of the other result. The methods where compared for recognition of PNX, PLE and PC. Datas were analyzed for agreement with Cohen's K. Relative sensitivity was then compared with McNemar test.Results: LUS shows good agreement for PNX and moderate agreement for both PLE and PC. LUS shows also a significantly superior relative sensitivity than chest $\mathrm{x}$-ray for PC and a significantly inferior relative sensitivity for PNX. It also shows a tendency to a better relative sensibility (just over the limits for statistical significance). Complete data analysis in table

Table.

\begin{tabular}{|c|c|c|c|}
\hline & $\begin{array}{l}\text { Pleural } \\
\text { effusion }\end{array}$ & Pneumothorax & $\begin{array}{l}\text { Pulmonary } \\
\text { consolidation }\end{array}$ \\
\hline $\begin{array}{l}\text { Chest x-ray } \\
\text { diagnosis }\end{array}$ & 14 & 7 & 21 \\
\hline $\begin{array}{l}\text { Lung } \\
\text { ultrasound } \\
\text { diagnosis }\end{array}$ & 20 & 5 & 28 \\
\hline Agreement & $\begin{array}{l}\text { K } 0,40 \\
\text { (moderate) }\end{array}$ & K 0,63 (good) & $\begin{array}{l}\text { K } 0,51 \\
\text { (moderate) }\end{array}$ \\
\hline $\begin{array}{l}\text { Relative } \\
\text { sensitivity }\end{array}$ & $\begin{array}{l}83 \% \text { (eco) vs } \\
58 \% \text { (x-ray) p } \\
0,060\end{array}$ & $\begin{array}{l}62 \% \text { (eco) vs } \\
87 \% \text { (x-ray) p } \\
0,003\end{array}$ & $\begin{array}{l}90 \% \text { (eco) vs } \\
68 \% \text { (x-ray) p } \\
0,048\end{array}$ \\
\hline
\end{tabular}


Conclusions: This study confirms that LUS has a sufficient agreement rate with the current clinical standard (chest $\mathrm{x}$-ray). The test shows a poor result only in recognition of PNX but the unrecognized PNXs were all minimal (less than $5 \mathrm{~mm}$ ). Non inferiority of LUS in the diagnosis associated with the practicality of bedside performing and absence of radiation recommend its routine use in our population

\section{P-120}

Assessment of pressure-volume-relations in univentricular hearts - comparison of obtainment by $3 \mathrm{D}$-real-time echocardiography and mini pressure-wire with conductance technology

Linden K., Winkler C., Breuer J., Herberg U.

Abteilung Kinderkardiologie, Universitätsklinikum Bonn, Germany

Objectives: Pressure-volume relations (PVR) of the heart allow assessment of systolic as well as diastolic function which may be of particular importance in single ventricle physiology. The gold standard to acquire PVR, the conductance technology (PVRCond), is rarely used in children and restricted to older age due to its catheter size and invasiveness. PVR can also be obtained by $3 \mathrm{D}$-echocardiography (3DE) volume data in combination with simultaneously measured pressure data by a mini pressure-wire (PVR3DE). We sought to compare both methods in patients with univentricular hearts.

Methods: We studied 19 patients (age 2-29 years). PVR3DE (Philips iE33; TomTec; pressure-wire: Radi,St. Jude Medical) and PVRCond (CD Leycom) were assessed under baseline conditions followed by positive inotropic stimulation with dobutamine $(10 \mu \mathrm{g} / \mathrm{kg} / \mathrm{min})$.

Results: Both methods showed that the PVR-specific parameter End-systolic elastance (Ees) as a measure for contractility increased significantly under stimulation with dobutamine. The isovolumic relaxation time constant Tau, reflecting the early active relaxation process decreased as an expression for a positive lusitropic effect of dobutamine (fig.1). Bland-Altman-analyses (bias \pm standard deviation): Ees: $1.6 \pm 2.9 \mathrm{mmHg} / \mathrm{ml}$, Tau: $-4.6 \pm 7.8 \mathrm{~ms}$. While obtaining PVR3DE was successful in all patients, correct placement and signal quality of the conductance catheter could be achieved in only 15 patients under baseline conditions (79\%) and under dobutamine in 12 patients (63\%).

Conclusion: The changes of PVR-specific parameters under dobutamine stimulation were reflected in the same way by both methods. The absolute values for these parameters could vary between the methods, though. So methods are not interchange-
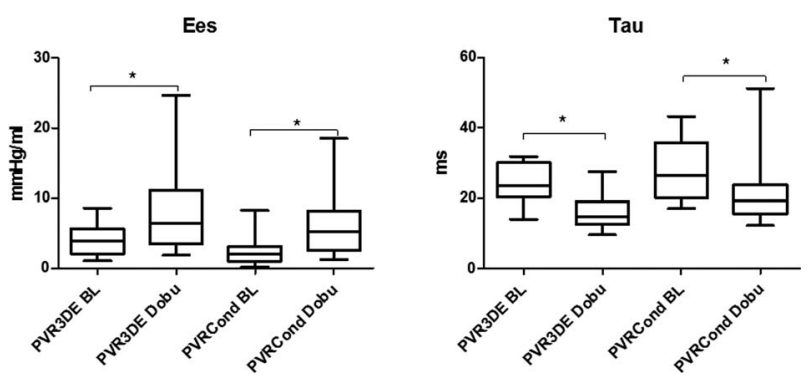

Figure.

End-systolic elastance (Ees) und isovolumic relaxation time constant (Tau) under baseline conditions $(B L)$ and stimulation with dobutamine (Dobu). PVR3DE $=3$ D-echocardiography combined with mini pressure-wire; $P V R$ Cond $=$ Conductance technology; ${ }^{*}=p<0.05 B L$ compared to Dobu. able. We experienced that obtaining PVR3DE in a single ventricle was easier and more often successful than achieving correct placement and signal quality of the conductance catheter for PVRCond. Consequently, PVR obtained by 3DE and mini pressure-wire can serve as a promising and needed alternative to the conductance technology for the assessment of cardiac function in univentricular hearts.

Supported by Fördergemeinschaft Deutsche Kinderherzzentren e.V.

\section{P-121}

Left Ventricular Deformation in $\boldsymbol{\beta}$-thalassemias

Raouf W. A. (1), Hamza H.S. (2), El-Beshlawy A.M. (2)

Center for Social and Preventive Medicine. Cairo University. Cairo.

Egypt (1); Department of pediatrics. Cairo University. Cairo. Egypt (2)

Aims: To assess the value of speckle tracking echocardiography in detecting early left ventricular deformation abnormalities in both $\beta$-thalassemia intermedia and major who were compliant to treatment since infancy, with serum ferritin $<1500 \mathrm{ng} / \mathrm{ml}$, myocardial T2* higher than $20 \mathrm{~ms}$ and normal left-ventricular dimensions and ejection fraction; and to illustrate the deformation pattern in each of the two $\beta$-thalassemia phenotypes and its possible difference from normal.

Methods: The study population comprised 3 groups: group-1 (26 $\beta$-thalassemia major patients), group-2 (24 thalassemia intermedia patients), and group-3 (21 age-matched normal individuals). All subjects had arterial pressure measured, serum ferritin, conventional echocardiography, and speckle tracking echocardiography. Only patients with thalassemia major had myocardial T2^ measurement.

Results: Diastolic blood pressure was lower in both thalassemia groups than in normal ( $\mathrm{P}<0.001)$. All enrolled individuals, including both thalassemia groups, had normal ejection fraction. All groups showed no difference in left-ventricular dimensions, indexed volumes, or global longitudinal strain. The global radial and circumferential strain were lower in $\beta$-thalassemia groups than in the control group $(\mathrm{P}<0.001)$, with no significant difference between both thalassemia groups. Peak twist and peak systolic apical rotation were lower in $\beta$-thalassemia groups $(\mathrm{P}<0.001$ for both parameters), with no significant difference between the two thalassemia groups. Serum ferritin was negatively correlated with diastolic pressure, global radial strain, global circumferential strain, peak apical rotation and peak twist.

Conclusion: Young patients with both $\beta$-thalassemia intermedia and well-treated $\beta$-thalassemia major have lower left-ventricular global radial, circumferential and basal longitudinal (but not global longitudinal) strain values than normal, as well as different rotational pattern characterized by decreased apical systolic rotation and peak twist.

\section{P-122}

Left and Right Ventricular Functions may be Impaired in Children Diagnosed with Subclinical Hypothyroidism Akın A. (1), Unal E. (2), Yildırm R. (3), Ture M. (1), Balı H. (1), Haspolat Y.K. (2)

(1) Dicle University Faculty of Medicine, Department of Pediatric Cardiology, Diyarbakur, TURKEY. (2) Dicle University Faculty of Medicine, Department of Pediatric Endocrinology, Diyarbakır, TURKEY. (3) Diyarbakur Children's Hospital, Department of Pediatric Endocrinology, Diyarbakır, TURKEY

Introduction: Subclinical hypothyroidism may influence left and right ventricular functions. Tissue Doppler Imaging (TDI) is a 
useful method used for the assessment of the effect of subclinical hypothyroidism on cardiac functions.

Methods: We compared left ventricular mass index (LVMI) and TDI parameters of patients with subclinical hypothyroidism (SH) and children with euthyroidism. SH was diagnosed when thyroid stimulating hormone (TSH) level was higher than the reference value of the laboratory $(>4.2 \mathrm{mIU} / \mathrm{L}$ ) and free thyroxine (fT4) level was in normal range.

Results: The study included a group of 35 patients with $\mathrm{SH}$ and a control group of 38 healthy children with euthyroidism (mean age was $7.6 \pm 3.5$ years and $9.0 \pm 2.4$ years, respectievely). LVMI was significantly higher in the patient group $(p=0.005)$. TDI parameters including mitral septal ejection time (ET) was lower $(p=0.003)$ and mitral septal myocardial performance index (MPI) was higher $(p=0.009)$ in the patient group. Right ventricular TDI revealed that tricuspid lateral E/Ea and tricuspid septal E/Ea were higher $(p=0.015$ and $p=0.024$, respectively) and tricuspid septal $\mathrm{Ea} / \mathrm{Aa}$ and ET were lower $(\mathrm{p}=0.018$ and $\mathrm{p}=0.017$, respectively) in the patient group. Intraventricular septal Aa was higher $(p=0.022)$ and $\mathrm{Ea} / \mathrm{Aa}$ was lower $(\mathrm{p}=0.032)$ in the patient group. Conclusions: Subclinical hypothyroidism may lead to increase LVMI. Left ventricular systolic and diastolic TDI parameters (lower mitral septal ET, higher mitral septal MPI) as well as right ventricular systolic (lower tricuspid septal ET) and diastolic (higher tricuspid septal and lateral E/Ea, lower tricuspid septal Ea/Ea) functions may be also impaired in children with $\mathrm{SH}$.

\section{P-123}

Myocardial Deformation and Rotational Mechanics in infants with Down Syndrome in the Early Neonatal Period Breatnach C.R. (1), Bussman N. (2), Smith A. (2), Duignan S. (1), Levy P.T. (3), McCallion N. (2), Franklin O. (1), EL-Khuffash A. (2).

Our Lady's Children's Hospital, Dublin, Ireland (1); The Rotunda Hospital, Dublin, Ireland (2), Washington University School of Medicine, St Louis, United States (3).

Introduction: The impact of Down syndrome on myocardial performance measured using deformation imaging and pulmonary hypertension $(\mathrm{PH})$ in the neonatal period requires exploration. We aimed to assess $\mathrm{PH}$ and measure left (LV) and right (RV) ventricular function using deformation imaging in neonates with DS (without structural heart disease) over 5 days of age.

Methods: Echocardiograms were performed on Days 1, 2 and 5 to measure LV and RV dimensions, LV basal longitudinal strain (LV BLS) and systolic strain rate (LV SR), RV free wall basal longitudinal strain (RV BLS) and systolic SR (RV SR), and LV rotational mechanics. $\mathrm{PH}$ was assessed by examining ductal (PDA's) shunting and tricuspid regurgitation (to estimate RV systolic pressure, RVSp). Values were compared with healthy controls.

Results: 17 infants with DS with a mean \pm SD gestation \& birthweight of $38.4 \pm 2.1$ weeks and $3.1 \pm 0.5 \mathrm{Kg}$ respectively were compared with 16 controls $(38.3 \pm 2.0$ weeks $\& 3.3 \pm 0.5 \mathrm{Kg})$. On Day 1, infants with DS had a higher proportion of bidirectional PDAs $(100 \%$ vs. $20 \%$, p < 0.01 ), a higher RVSp (35vs. $18 \mathrm{mmHg}$, $\mathrm{p}=0.03$ ). Infants with DS had lower RV BLS and RV SR over the three time points. Infants with DS had impaired LV basal rotation on Day 1, 2 and 5 resulting in significantly lower LV twist. Conclusion: Infants with DS demonstrate elevated pulmonary pressures during the early neonatal period translating into lower RV function measured using deformation imaging. They also demonstrate impaired twist driven by reduced basal rotation. The clinical implications warrant further study.

\section{P-124}

D-Transposition of the Great Arteries and Intact

Ventricular Septum: morphological and functional echocardiographic pattern in newborns before the surgical correction

Walter C (1), Gómez O. (2), Bartrons J. (1), Carretero J. (1),

Sánchez-De-Toledo J. (1)

Hospital Sant Joan de Déu, Barcelona, Spain (1); Hospital Clínico de

Barcelona - Sede Maternidad, Barcelona, Spain (2)

Objectives: To describe morphological and functional pattern in newborn with D-Transposition of the Great Arteries and intact ventricular septum (IVS/D-TGA) before surgery, and to analyze the differences in the group with pulmonary hypertension (PHT). Methods: Anthropometric, clinical and echocardiographic data were obtained in 17 newborns with IVS/D-TGA. 40 bodysurface matched-controls were selected. Patients were divided in two groups, PHT (2) and no-PHT (15). Cardiac morphologic and biventricular function were assessed after 48 hours of life (before the correction surgery) by $2 \mathrm{D}$-Echo, M-mode,pulse-wave and Tissue-Doppler-Imaging techniques.

Results: PHT patients (2) had desaturation that required oxygen therapy with conventional-mechanical-ventilation and nitricoxide. The other 15 had pulmonary overcirculation, requiring diuretics and high-flow nasal cannula. Morphometric evaluation in IVS/D-TGA revealed heart enlargement predominantly in right cavities and systemic arteries (Right-Left-mid ratio 1.2 vs 0.8 , $\mathrm{p}=0.001$; Pulmonary-Aortic ratio 0.9 vs $1.4, \mathrm{p}=0.002)$. This enlargement wasn't seen in PHT compared with the non-PHTgroup (RightVentricle end-diastolic mid-diameter 11.2 vs $15.2 \mathrm{~mm}, \mathrm{p}=0.04$; RVdiastolic area 1.8 vs $3.1 \mathrm{~cm} 2, \mathrm{p}=0.04$; RVsystolic area 1.1 vs $1.8 \mathrm{~cm} 2, \mathrm{p}=0.02$ ).

Biventricular functionality was increased in IVS/D-TGA [RVsystolic (s'7.8 vs $5.3 \mathrm{~cm} / \mathrm{s}, \mathrm{p}=0,001$; ICT 36.9 vs $45.0 \mathrm{~ms}$, $\mathrm{p}=0.03$ ) and diastolic function (E 90.7 vs $50.8 \mathrm{~cm} / \mathrm{s}, \mathrm{p}=0.001$; e'13.1 vs $7.3 \mathrm{~cm} / \mathrm{s}, \mathrm{p}=0,001$; a'11.1 vs $8.2 \mathrm{~cm} / \mathrm{s}, \mathrm{p}=0,002$; E/A 1.3 vs $0.9, \mathrm{p}=0.002$ ), LeftVentricle systolic (Teichholz EjectionFraction 75.4 vs $69.9 \%, p=0.04$; MAPSE 6.5 vs $5.6 \mathrm{~mm}, \mathrm{p}=0.03$; s'6.2 vs $4,4 \mathrm{~cm} / \mathrm{s}, \mathrm{p}=0,001$; VFC 0.26 vs $0.24, \mathrm{p}=0.02$ ) and diastolic function (E 96.5 vs $59.5 \mathrm{~cm} / \mathrm{s}, \mathrm{p}=0,001$; A 71.4 vs $52.6 \mathrm{~cm} / \mathrm{s}, \quad \mathrm{p}=0,002$; e'8.7 vs $6.4 \mathrm{~cm} / \mathrm{s}, \mathrm{p}=0.002$; a' 8.4 vs $6.3 \mathrm{~cm} / \mathrm{s}, \mathrm{p}=0.01 ; \mathrm{E} / \mathrm{A} 1.5$ vs $1.2, \mathrm{p}=0.03)]$.

In PHT group, right systolic (TAPSE 8.7 vs $9.4 \mathrm{~mm}$, s'5.6 vs $79 \mathrm{~cm} / \mathrm{s}$ ), diastolic (E $43 \mathrm{vs} 97 \mathrm{~cm} / \mathrm{s}$, A 54.8 vs $71 \mathrm{~cm} / \mathrm{s}$, e' 5.5 vs $13.3 \mathrm{~cm} / \mathrm{s}$, a' 7.3 vs $11 \mathrm{~cm} / \mathrm{s}$ ), and left systolic (MAPSE 5.1 vs $6.5 \mathrm{~mm}$, SAPSE 3.8 vs $6.7 \mathrm{~mm}$, mitral s' 5 vs $6.3 \mathrm{~cm} / \mathrm{s}$, septal s' 4.8 vs $5.5 \mathrm{~cm} / \mathrm{s}$ ) and diastolic function (E 86.3 vs $98.9 \mathrm{~cm} / \mathrm{s}$, A 58 vs $71 \mathrm{~cm} / \mathrm{s}$, mitral e' 5.9 vs $9.1 \mathrm{~cm} / \mathrm{s}$, mitral a' 4.3 vs $8.8 \mathrm{~cm} / \mathrm{s}$, septal e'4.9 vs $7 \mathrm{~cm} / \mathrm{s}$, septal a' 4.6 vs $7.8 \mathrm{~cm} / \mathrm{s}$ ) are decreased.

Conclusions: Non-corrected IVS/D-TGA have an increase in global size and biventricular function. PHT produces the opposite effect, with a global morphological and functional decrease. Larger series should be needed to identifiy whereas PHT IVS/D-TGA patients have a different preoperative funciotnal pattern compared with noPHT patients.

\section{P-125}

3D printed heart models are highly anticipated innovation in medical education

Meyer-Szary J. (1), Sabiniewicz R. (1), Kwiatkowska J. (1),

Fijałkowska J. (2), Dorniak K. (3)

Department of Paediatric Cardiology and Congenital Heart Defects (1); 2nd Department of Radiology (2); Department of Noninvasive Cardiac Diagnostics (3) Medical University of Gdansk 
Introduction: The parallel advances in medical imaging, image processing, and 3D printing techniques have led to greater availability of this innovation in medicine. Recognising the difficulties of comprehending complex anatomy of congenital heart defects and the educational potential of the 3D printed anatomical models we have assessed their reception by the medical students.

Methods: Using available software and Zortrax M200 desktop printer we have prepared 18 models of varied congenital heart diseases (ToF, CAT, PA, CoA, IAA, HAA, DAA, VR) pre- and post-operatively. They were introduced to the curriculum of the course for medical students in our department. Each model played a double role - first as a riddle to engage the students and see if they can give the correct diagnosis. Secondly as an illustration to the detailed case description each model represents. At the end, we circulated a questionnaire to assess the participants opinions.

Results: We have assessed a group of 45 students, $57 \%$ males, median age 24 (22-29) years, 66\% fifth and 33\% sixth year of the studies. All fully agreed that $3 \mathrm{D}$ printed models are helpful educational aid.

Almost all (98\%) fully, and the rest rather agreed that they help understand complex anatomy of congenital heart diseases. Similarly, 93\% fully, and 7\% rather agreed that they help understand the principles of cardiosurgical corrections of the defects. Regarding aiding understanding physiology of the defects $76 \%$ fully agreed, and $24 \%$ rather agreed. Students would also like to interact with the models if they allowed simulated interventional procedures $-71 \%$ definitely, $24 \%$ rather and only $4 \%$ had no opinion. Interestingly, $80 \%$ (definitely and rather in equal proportions) would like to participate in extracurricular course on the technical aspects of 3D printing in medicine, living only $18 \%$ undecided and $2 \%$ rather not interested. Ninety eight percent of students claimed that such models should also be used in other classes naming 17 specialties in an opened question. Finally, 98\% would definitely, and $2 \%$ would rather recommend other students to attend classes featuring such models.

Conclusion: The results unequivocally display that $3 \mathrm{D}$ printed heart models are highly anticipated innovation in medical education.

\section{P-126}

Interference of BMI with accuracy and validity of 3D-STE data

AbdelMassih A.F. (1), Agha H.M. (1), AbdelRahman M.Y. (1), Milanesi O. (4), Castaldi B. (4), Geranio G. (5) Putti M.C. (5), Kharabish A. (3), Esmail R. (1), El-Kamah G. (7), Hamdy M. (2), ElBaz H. (6), El-Behary N. (3), Hanna C. (8), El-Tagy H. (11), Mishriky M.A. (10), Mamdouh M. (10), Livio A. (5),

Ramadan T. (12), Afdal P. (12)

Pediatric Cardiology Unit, Pediatrics' Department, Specialized Children Hospital, Faculty of Medicine, Cairo University, Cairo, Egypt (1); Pediatric Hematology Unit, Pediatrics' Department, Specialized Children Hospital, Faculty of Medicine, Cairo University, Cairo, Egypt (2); Cardiac MRI unit, Radiology Department, Faculty of Medicine, Cairo University, Cairo, Egypt (3); Pediatric Cardiology unit, Department of Woman and Child health, Faculty of Medicine, Padova University, Padova, Italy (4); Pediatric Hematology unit, Department of Woman and Child health, Faculty of Medicine, Padova University, Padova, Italy (5); Clinical Pathology Department, Faculty of Medicine, Cairo University, Cairo, Egypt (6); Pediatric Hematology Department, National Research Center, Cairo, Egypt (7); Computer Science and software development department, American University of Cairo, Cairo, Egypt (8); Pediatric Residency Program, Faculty of Medicine, Cairo University, Cairo, Egypt (9); Cardiology Residency Program, Faculty of Medicine, Cairo University, Cairo, Egypt (10); Orthopedics' Residency Program, Faculty of Medicine, Cairo University, Cairo, Egypt (11); Medical Students, Faculty of Medicine, Cairo University (12)
Introduction and Objectives: 3D echocardiography is recently the gold standard for volumetric assessment of LV as well as EF (EAE guidelines), deformation imaging alternatively called speckle tracking is undergoing constant development; one of those most revolutionary advances is the development of 3D derived STE. Compared to 2D STE, 3D STE not only allows assessment of the 3 components of strain simultaneously but also markedly shortens the duration of processing. However many known limitations exist to 3D STE, one of them is the occurrence of stitch artifact in the absence of breath holding which makes it difficult to use in infants. To our knowledge no or few studies have shown the effect of increasing BMI on the accuracy and validity of 3D echocardiographic data. The aim of this study is to show the pattern of variation of a single parameter: Global longitudinal strain (GLS) using 2D and 3D approach.

Methods: Twenty seven normal persons were recruited from outpatient clinics in Padova/Cairo universities. 2D and 3D-STE were initially performed to all candidates to study the pattern of variation of GLS with varying BMI. Candidates were divided into two groups Group I with BMI (Body mass index) between 15-19 and Group II with BMI 20-25, in each group 2D and 3D GLS was determined and the coefficient of variation between both was determined.

Results: There was no statistically significant difference between $2 \mathrm{D}$ derived GLS $(-16.47 \% \pm 0.93)$ and 3D derived GLS $(-16.45 \% \pm 0.81)$ in Group I while in Group II 2D derived GLS $(-19.36 \% \pm 2.59)$ was significantly higher from 3D derived GLS $(-16.69 \% \pm 0.88)$ with a P-value of 0.002 . The coefficient of variation between $2 \mathrm{D}$ derived and 3D derived GLS in Group II was $11.2 \% \pm 7.2$ compared to $1.12 \% \pm 0.84$ in Group I with a P-value $<0.005$.

Conclusion: We conclude that statistically significant differences exist between 2D and 3D derived GLS in higher BMI with tendency of 3D STE to underestimate GLS with higher body weights. This may reflect the interference of fat with acoustic windows and full volume acquisition in $3 \mathrm{D}$ echocardiography.

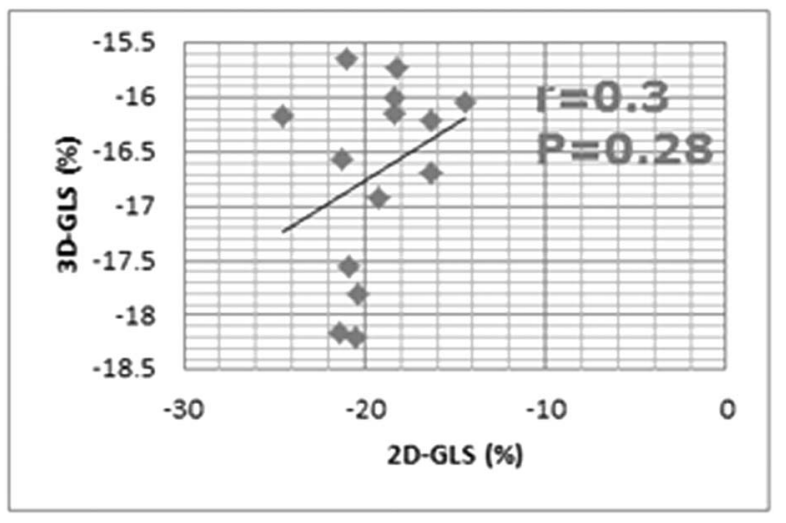

Figure.

Correlation graph between $2 \mathrm{D}$ and $3 D$ GLS in the group II with BMI between 20-25.

\section{P-127}

First Presentation of Rheumatic Fever in Preschool Egyptian Children Younger Than 5 Years

Marzouk P.A., Hamza H.S., Mosaad N.A., Emam S.M., Fattouh A.M. Cardiology department, Cairo University Specialized Pediatric Hospitals, Cairo, Egypt.

Objectives: Rheumatic fever usually occurs in children between 5 to 15 years, less frequently in younger age. The aim of this study 
was to detect the frequency of first presentation rheumatic fever in ages less than 5 years and determining the pattern of presentation in this age group.

Methods: This retrospective study was conducted on Egyptian children presenting to a specialized rheumatic fever clinic from March 2014 to March 2016.The data of children younger than 5 years presenting with proven rheumatic fever according to the modified jones criteria were recorded. These data included demographic data, clinical presentations, laboratory findings and echocardiographic findings.

Results: Out of 621 patients diagnosed with Proven rheumatic fever; $47(7.5 \%)$ were younger than 5 years. Twenty six $(55.3 \%)$ were females and twenty one $(44.7 \%)$ were males. Positive family history was encountered in 13 patients $(27.7 \%)$. Their most common presentations were arthritis in 36 (76.6\%), carditis in 13 $(27.7 \%)$ and chorea in only 1 patient (2.1\%). Subclinical carditis was detected by echocardiography in 4 patients $(8.5 \%)$. The most common valvular lesion encountered was mitral regurge in 16 (34\%) followed by aortic regurge in 7 patients (14.9\%). Both mitral and aortic regurge were present in 6 patients $(12.8 \%)$. Rheumatic morphological changes in the mitral valve were encountered in 9 patients in the form of thickened mitral valve leaflets, thick subvalvular apparatus, restricted posterior mitral valve leaflets, lack of systolic coaptation and mitral valve prolapse where 8 out of 9 had 2 or more rheumatic mitral morphological changes while 1 patient had only 1 rheumatic mitral morphological change. Thickened aortic valve leaflets were encountered in 3 patients.

Conclusion: Acute rheumatic fever can occur in children younger than 5 years, thus the possibility of rheumatic fever should be adequately investigated in these young patients presenting with arthritis, carditis or chorea especially in developing countries like Egypt. Echocardiography is an essential tool to diagnose subclinical carditis which can influence the management strategy. Long-term follow-up is mandatory to determine the outcome for young children with subclinical echocardiographic evidence of carditis.

\section{P-128}

Hypertrophic cardiomyopathy in childhood show difference in degree of hypercontractility between MYH7 mutations and MYBPC3 mutations

Allahyari P. (1), Östman-Smith I. (2)

Department of Clinical Physiology, Sahlgrenska University Hospital, Gothenburg, Sweden (1). Department of Pediatric Cardiology, Queen Silvia Childrens Hospital, Sahlgrenska Academy, Gothenburg, Sweden (2)

Background: MYH7- and MYBPC3-mutations are the most common causes of HCM in Northern Europe. HCM due to MYH7-mutations has been reported to have earlier penetrance of overt disease than MYBPC3-mutations. It thus seemed interesting to study if there were any systematic differences in cardiac function measures on cardiac ultrasound that might be utilized for early detection of mutation carriers.

Methods: Among consecutive patients attending the Queen Silvia Childrens Hospital in Gothenburg with HCM caused by pathogenic mutations, and with clearly pathological hypertrophy $<19$ yrs of age, we identified 11 patients with MYH7-mutations, and 13 with MYBPC3-mutations. Patients with compound heterozygosity were excluded.

Results: Median age at presentation with pathological hypertrophy within this pediatric cohort were 11.0yr for MYH7 and 13.6yr for MYBPC3 $(p=0.49)$. There were no significant differences in degree of septal or posterior wall hypertrophy with median
Detroit Z-score for septal thickness 2.68 for MYH7 and 2.60 for MBPC3 ( $p=0.23$ ), and for posterior wall 1.87 versus 1.80 . Circumferential contractility as measured by fractional shortening was notably higher in MYH7, median 53\% [IQR 44-60\%] versus $42 \%$ [40-46\%] in MYBPC3, $\mathrm{p}=0.008$. Related to that systolic wall-to-cavity ratio was also higher in MYH7: median 1.00 [0.72$1.34]$ versus 0.63 [0.58-0.85], $\mathrm{p}=0.010$. There were no significant differences in left atrial-to aortic ratio, median 1.39 and 1.34 respectively, but nevertheless there were clear differences in diastolic function with trans-mitral E:A ratio 1.28 [1.11-1.52] in MYH7 versus 1.80 [1.63-2.21; $\mathrm{p}=0.005]$ in MYBPC3. TissueDoppler also differed with E:e ratio 12.8 [10.6-14.3] and 8.2 [7.25-10.40; $\mathrm{p}=0.006]$, and e:a ratio $1.30[0.80-1.86]$ and 2.10 [1.70-2.40; $\mathrm{p}=0.034]$ respectively. Pulmonary venous SD-ratio tended to be higher in MYH7: 1.3 [0.9-1.9] versus $0.8[0.7-1,2 ; p=0.051]$. There was also a trend for a lower LV end-diastolic volume measured with 3-D ultrasound, but with some missing values it did not reach significance: $28.3 \mathrm{ml} / \mathrm{m} 2 \mathrm{BSA}$ [24.2-39.9 ml] for MYH7 versus $44.3 \mathrm{ml} / \mathrm{m} 2$ BSA [28.9-45.8, $\mathrm{p}=0.07]$ in MYBPC3.

Conclusions: It may be possible to diagnose mutation carriers with MYH7-mutations early in situations where genetic testing cannot be offered or is declined by looking for high fractional shortening, unusually small cavity and for age-inappropriate values in diastolic function.

\section{P-129}

Morpho-functional myocardial status in preterm infants with hemodynamically significant patent ductus arteriosus Gonchar M., Boichenko A., Kondratova I., Matsiyevska N., Onikiienko O.

Kharkiv National Medical University, Kharkiv, Ukraine

Purpose: to improve the diagnosis of hemodynamically significant patent ductus arteriosus (HSDA) in prematurely born infants.

Methods: The study involved examination of 165 newborns at gestational age (GA) of 24-37 weeks. The study implied determination of indices of systolic, diastolic ventricular functions. Doppler imaging was conducted in the first day of life and in 24-48 hours, in newborns with HSDA daily until stabilization of the patient's condition or surgical correction. The newborns were divided into the groups: Group $1(\mathrm{n}=34)$ included infants with an extremely low body weight, GA 24-29 weeks. Group $2(\mathrm{n}=37)$ involved newborns with very low birth weight, GA 30-34 weeks, Group $3(\mathrm{n}=36)$ comprised patients with low body GA 31-35 weeks; Group $4(\mathrm{n}=58)$ included infants at GA 35-37 weeks.

Results: Diagnosis of HSDA was established according to the criteria of Sehgal A, McNamara PJ. (2009) and additional criteria: IR ACA and/or MCA >0.8, «diastolic steal» syndrome in the renal and/or mesenteric arteries or IR renal and/or mesenteric arteries $>0.85$ and/or reverse blood flow in the abdominal aorta, an increase in the linear size of the left ventricle (LV) and/or atrium by $10 \%$ or more from the initial size with hypertrophy of the interventricular septum and posterior LV wall; cardiothoracal index $>60 \%, \quad \mathrm{FiO} 2 \geq 40 \%$. HSDA was established in $52.9 \%$ $(p<0.05)$ of Group 1 infants, all of them revealed disturbance of diastolic function of LV and RV. Tissue Doppler imaging (TDI) showed a decrease in the velocity of fibrous rings, $\mathrm{cm} / \mathrm{sec}$ (LV lateral TDI $\mathrm{S}=5.74 \pm 0.87 \quad(\mathrm{p} 1 / 4 \leq 0.05), \quad \mathrm{E}^{\prime}=6.32 \pm 1.14$, $\mathrm{A}^{\prime}=6.89 \pm 1.15 \quad(\mathrm{p} 1 / 4 \leq 0.05) ; \quad$ septal $\quad$ TDI $\mathrm{S}=4.97 \pm 0.55$ $(\mathrm{p} 1 / 4 \leq 0.05), \mathrm{E}^{\prime}=6,06 \pm 1.13, \mathrm{~A}^{\prime}=6.42 \pm 1.06 \quad(\mathrm{p} 1 / 4 \leq 0.05)$, RV TDI $\mathrm{S}=6.70 \pm 1.15 \quad(\mathrm{p} 1 / 4 \leq 0.05), \quad \mathrm{E}^{\prime}=7.01 \pm 1.12$, $\mathrm{A}^{\prime}=7.14 \pm 1.20(\mathrm{p} 1 / 4 \leq 0.05)$. Newborns in severe condition with HSDA of Group 1 have demonstrated hypokinetic type of 
central hemodynamics (systolic index $1.8 \pm 0.61 / \mathrm{min} \times \mathrm{m} 2$, $(\mathrm{p} \leq 0.01))$, Tei index $\mathrm{LV}=0.36 \pm 0.10$ and $\mathrm{RV}=0.34 \pm 0.10$ $(\mathrm{p} \leq 0.05)$ and SS genotype polymorphism of SOD2 T58C $(\mathrm{x} 2=6.258, \mathrm{p}=0.044), \mathrm{GG}$ and $\mathrm{AG}$ genotypes of polymorphism ADRB1 Ser49Gly $(x 2=6.627, p=0.036)$.

Conclusion. The use of target Doppler imaging in preterm infants with HSDA is useful in assessing the global function of the myocardium. We consider it expedient to conduct further research to confirm our results and determine their clinical significance.

\section{P-130}

The improvement of Echo Criteria to determine the hemodynamic significance of PDA among premature neonates

Kulikova D.A., Buchneva O.V., Safonova I., Pisklova Y.V., Krylova O.S., Shafer Y.V.

Institute of General and Urgent Surgery, Department of Cardiac surgery, Kharkiv, Ukraine

Introduction: Annually the number of premature labors grows, causing urgent need to improve diagnostics and treatment of premature infants in a neonatal ICU. The PDA is one of the most common conditions among such children. It is diagnosed in more than $40 \%$ of infants with weight less than 1500 gr. The PDA considerably aggravates patient's condition, increases risk of complications in the bronchopulmonary system, CNS, GIT and limits life expectancy. In many countries the method of choice for treatment is a surgical ligation of PDA. The uniform diagnostic criteria and indications for this procedure among premature infants are nowadays a pending issue. We introduced new ECHO's diagnostic criteria to determine the PDA's hemodynamic significance.

Methods: Between 2010 and 2017 - 187 patients were examined with the preliminary diagnosis of hemodynamically significant PDA. In addition to the standardized ECHO criteria we developed a new method: the percentage of blood flow in PA which originated in PDA in the ratio of pulmonary trunk area (PTA). Patients were distributed by the following groups: hemodynamically insignificant PDA - the area of blood flow - $10 \%$, the average hemodynamic importance from $10-30 \%$ of PTA and the obvious hemodynamic significance - the area of blood flow from PDA was more than $30 \%$ of the PTA.

Results: All 187 patients were distributed in the following groups: hemodynamically insignificant PDA $-9(4,81 \%)$ patients, the average hemodynamic importance - 101 (54,01\%), the obvious hemodynamic significance -77 (41,18\%). $8(4,28 \%)$ patients from the second group were reevaluated and transferred to the third group. On the basis of these criteria, PDA was ligated in 85 $(45,45 \%)$ premature newborns. The gestational age of children varied from 24 to 33 weeks $(28,00 \pm 0,54$ weeks), the weight from $600 \mathrm{gr}$ to $2000 \mathrm{gr}(1218,67 \pm 63,42)$. All children were intubated since their birth, had pneumonia and affected CNS in perinatal period, IRDS. The surgery was done on the 10-34 day of life in neonatal ICU.

Conclusions: The study optimized echocardiographic indications for surgical ligation of PDA. The new criterion developed and applied by us showed good results. The method's sensitivity is $90,59 \%$.

\section{P-131}

Prevalence of Fontan associated liver disease using standard investigations

Hansen J.H. (1), Moritz J.D. (2), Senkpiehl I. (2), Fischer G. (1), Logoteta J. (1), Rinne K. (1), Attmann T. (3), Scheewe J. (3), Kramer H. - H. $(1,4)$
Department of Congenital Heart Disease and Paediatric

Cardiology, University Hospital of Schleswig-Holstein, Campus Kiel, Germany (1); Department of Radiology and Neuroradiology,

University Hospital of Schleswig-Holstein, Campus Kiel, Germany (2); Department of Cardiovascular Surgery, University Hospital of SchleswigHolstein, Campus Kiel, Germany (3); DZHK (German Center for Cardiovascular Research), partner site Hamburg/Kiel/Lübeck, Germany (4).

Introduction: Liver fibrosis is increasingly recognized as a potentially serious morbidity associated with Fontan circulation (Fontan associated liver disease, FALD). The purpose of this cross-sectional study was to assess the prevalence of liver abnormalities using standard investigations.

Methods: Fontan patients were screened for liver abnormalities by abdominal ultrasound and routine laboratory tests. Patients were divided into three groups based on follow-up since Fontan surgery ( $<5$ years, 5 to 10 years, $>10$ years). The relation to hemodynamic data from cardiac catheterization and echocardiography was analyzed.

Results: 249 Fontan patients who had routine follow-up between January 2014 and November 2017 were included. Median age was 11 (7-16) years, the median follow-up since Fontan surgery 8 (413) years. Hepatic ultrasound was performed in 171 (68.7\%) patients; laboratory assessment was completed in 247 (99.2\%) cases; 211 (84.7\%) patients underwent cardiac catheterization, echocardiography studies were available in all cases. Sonographic signs of fibrotic changes included heterogeneous parenchyma, surface nodularity or hyper-echoic lesions in 80 (46.8\%) patients. The prevalence of fibrotic changes increased with longer follow-up since Fontan completion ( $<5$ years: 25.6\%; 5-10 years: $45.5 \%$; $>10$ years: $58.4 \%$; $p=0.004)$. Elevated gamma-glutamyltranspeptidase (gGT) levels were observed in $165(66.8 \%)$ patients. Glutamic-oxaloacetic-transaminase (GOT) levels above normal range and thrombocytopenia were more frequently observed in cases with more than 10 years follow-up since Fontan surgery (26/92 vs. $15 / 150, \mathrm{p}<0.001$ and $26 / 94$ vs. $5 / 151, \mathrm{p}<0.001$ ). Hypoproteinemia and reduced prothrombin activity were found in $5.1 \%$ and $27.8 \%$, respectively. The frequency was not different between age groups. Among laboratory parameters, only gGT levels were higher in cases with suspected fibrotic changes $(70 \pm 48$ $\mathrm{U} / 1$ vs. $50 \pm 32 \mathrm{U} / 1, \mathrm{p}=0.003)$. The number of patients with impaired ventricular function or more than mild atrioventricular valve regurgitation was not different between cases with or without suspected fibrosis. Systemic venous pressures were not different $(11 \pm 3$ vs. $11 \pm 2 \mathrm{mmHg}, \mathrm{p}=0.765)$.

Conclusions: The prevalence of ultrasound and laboratory abnormalities suggestive of FALD increases with time since Fontan surgery. However, ultrasound abnormalities were not necessary reflected by laboratory abnormalities and vice versa. Further studies are needed to guide surveillance and therapeutic strategies in the management of FALD.

\section{P-132}

Abnormal blood flow dynamics are associated with anatomical torsion of the aortic arch and eccentric geometry of the RV in Patients with Hypoplastic Left Heart Syndrome after three-stage palliation

Trotz P. (1), Gabbert D.D. (1), Wadle F. (1), Kheradvar A. (2), Kis E. (1), Scheewe J. (1,3), Kramer H.-H. (1), Rickers C. (1) University Hospital Schleswig-Holstein, Campus Kiel, Department for Congenital Heart Disease and Paediatric Cardiology, Kiel, Germany (1); University of California, Irvine, The Edwards Lifesciences Center for Advanced Cardiovascular Technology, Irvine, United States (2); 
University Hospital Schleswig-Holstein, Campus Kiel, Department of Cardiovascular Surgery, Kiel, Germany (3)

Objectives: The reconstructed aortic arch of patients with Hypoplastic Left Heart Syndrome (HLHS) has an important impact for long-term prognosis. 4D Flow MRI allows determining a variety of novel fluid-dynamic parameters (e.g. helicity, circulation, vorticity in defined volumes of the vessel). Therefore, we thought to test the hypothesis that aortic arch geometry effects blood flow dynamics and may effect right ventricular function in HLHS.

Methods: Twenty-four HLHS patients (median age: 4.4; 2-17 years; all NYHA I) underwent a comprehensive MRI examinations including 4D-flow acquisitions 1.8 (0.4-14.1) years after completion of the Fontan circulation. Volumetric data were used to estimate right ventricular (RV) function. With an in-house analysis software $(\mathrm{C}++$ based), novel parameters of blood flow dynamics were calculated within the cardiac cycle along the entire thoracic aorta from the neo-aortic valve towards diaphragm and were correlated with geometric parameters (such as geometric torsion which describes the twist of a curvature out of the plane) and parameters of the RV.

Results: Peak effective torsion (geometric torsion multiplied with curvature) of the neo-aortic root correlated significantly with peak helicity density of the blood flow $(\mathrm{p}<0.01$, adj. $\mathrm{R} 2=0.33$, rho $=0.59$ ). The peak relative helicity density correlated with the variation (difference between maximum and minimum) of the aortic diameters $(p=0.03$, adj. $\mathrm{R} 2=0.18$, rho $=0.46)$ as well as with the maximum diameter of the aorta $(p=0.04$, adj. $\mathrm{R} 2=0.15$, rho $=0.44) . \mathrm{RV}$ function $(\mathrm{EF} 57 \% \pm 10 \%)$, volumes and mass did not correlate with any of the flow parameters. The mass-to-volume ratio, a parameter of eccentric geometric remodeling of the RV, correlated inversely with the peak helicity density $(p=0.05$, adj. $\mathrm{R} 2=0.14$, rho $=-0.43$ ).

Conclusion: Our data show that abnormal blood flow patterns in the thoracic aorta in HLHS after three-stage palliation are strongly associated with geometric torsion of the aortic arch, variations of the aortic diameters and eccentric RV geometry. Our findings may trigger modifications of surgical reconstruction to optimize fluid-dynamic conditions in the future.

\section{P-133}

\section{Comparison of Echocardiography Indexes and Heart Failure Plasma Biomarkers in Young Adults with Single Ventricle after the Total Cavopulmonary Connection Pozniak Y., Lebid I., Salamanyna A., Bairamov E., Rudenko N., Khanenova $V$., Iershova $Y$., Klymyshyn $Y$. \\ Ukrainian Children's Cardiac Center, Kyiv, Ukraine}

Objectives: We aimed to clarify correlation between echocardiography volumetric and hemodynamic indexes, myocardial function by tissue doppler imaging and level of Heart Failure (HF) Plasma Biomarkers (N-Terminal Pro-Brain Natriuretic Peptide, Galectin-3) in young adults with Fontan circulation.

Methods: From 01.Jan.2005 to 31.Dec.2016 132 patients underwent Fontan, TCPC, external conduit. Eighteen young adults (>18 y.) were included (10 males, 8 female, mean age $21.3 \pm 1.9 \mathrm{y}$, 18-23 years). EDV, ESV, EDI, EF (Simpson's method) estimated by Echo and MRI. Peak S, E, A, ratio E/A, E/E', Myocardial Performance Index (MPI) estimated by Echo (TDI).

Results: The peak S $(9.2 \pm 1.8 \mathrm{~mm})$ by TDI and EF $(51 \pm 5 \%)$ had direct significant correlation NT-proBNP $-134 \mathrm{pg} / \mathrm{mL}$, range 78 to $284 \mathrm{pg} / \mathrm{mL}(\mathrm{r}=+0.6, \mathrm{p}<0.05)$. MPI by TDI $(0.51 \pm 0.03)$ and $\mathrm{EF}$ $(51 \pm 5 \%)$ had direct significant correlation Galectin-3 $-9.5 \mathrm{ng} / \mathrm{mL}$, interquartile range 9.9 to $15.0 \mathrm{ng} / \mathrm{mL}(\mathrm{r}=+0.3, \mathrm{p}<0.05)$.
Conclusions: HF Plasma Biomarkers (NT-proBNP, Galectin-3) are reliable tests for estimation HF in young adults with Fontan circulation, specifically with right ventricular morphology. Echo indexes (peak S, MPI) could be used for assessment of SV myocardial function with good correlation with NT-proBNP, Galectin-3. HF Plasma Biomarkers may be an extremely useful, quick, and simple prognostic tests for identification high risk patients after TCPC.

\section{P-134 \\ Importance of coronary CT angiography after arterial switch operation in transposition of the great arteries patients \\ Abreu S., António M., Pinto F. \\ Hospital Santa Marta, Serviço de cardiologia pediátrica, Lisboa, Portugal}

Introduction: Mortality and morbidity in patients with transposition of the great arteries (TGA) submitted to arterial switch operation (ASO) depends mainly on the state of coronary perfusion, being that coronary abnormalities are an important late cause of death in these patients. CT angiography provides accurate information on coronary anatomy, very useful for postoperative management. It has also proven to be a safe exam in the paediatric population. The aim of this study is to describe our institutional experience with CT angiography in TGA patients, and it's role on postoperative management of these patients.

Methods: retrospective analysis of clinical processes of TGA patients who performed coronary CT angiography CT between January 2013 and September 2017.

Results: Between January 2013 and September 2017, 18 TGA patients performed coronary CT angiography, with a medium age of 14 years. $66 \%$ (12) performed the exam due to suspicion of ischemia, $22 \%$ (4) to evaluate coronary anatomy and the remaining to evaluate the relationship between coronary arteries and surrounding structures.

Among patients with suspicion of ischemia, 83\% were symptomatic (chest pain/ fatigue). The remaining performed the exam due to altered previous exams and one due to iatrogenic coronary lesion during surgery.

$60 \%$ (6) of symptomatic patients had significant lesions identified in coronary angiography CT (kinking: 3, occlusion:1, filiform coronary:2), with modification of action in 3 patients, two of them being submitted to coronary surgery afterwards.

Only $16 \%$ of all patients needed to perform complementary exams to clarify their clinical status (mainly cardiac MRI to evaluate perfusion). $55 \%$ previously performed conventional angiography, and in half of the patients a coronary abnormality was identified, but all patients needed CT angiography to clarify the anatomy or the significance of the abnormalities found.

None of the patients presented complications after CT angiography, and only $22 \%$ (4) needed general anaesthesia. The medium doses of radiation were $2,4 \mathrm{mSv}$.

Conclusion: CT angiography proved to be safe and useful in paediatric patients. It is a very fast technique that provides detailed information on coronary anatomy, crucial for the postoperative management and selection of high-risk patients after ASO.

\section{P-135}

Neoaortic root dilatation and its impact on neoaortic regurgitation in adolescents and young adults with transposition of the great arteries after an arterial switch operation

Michalak K.W. (1), Moll M. (2), Sobczak - Budlewska K. (1), Moll J.A. (1), Szymczyk K. (3), Dryżek P. (1), Moszura T. (1), Moll J.J. (2) 
Department of Cardiology, Polish Mother's Memorial Hospital, Lodz, Poland (1); Department of Cardiac Surgery, Polish Mother's Memorial Hospital, Lodz, Poland (2); Department of Diagnostic Imaging, Medical University, Lodz, Poland (3)

Introduction: Neoaortic regurgitation (NeoAR) is a common sequel in patients with the transposition of the great arteries (TGA) after an arterial switch operation (ASO). Aside the native pulmonary valve functioning in the systemic circulation, neoaortic root abnormalities are proved to be related to this feature.

The aim of this study was to assess the frequency of neoaortic root dilatation and its impact on neoaortic valve function in patients with TGA after ASO.

Patients and methods: Among 750 patients with TGA who had ASO between years 1991 and 2016, we qualified for this study the initial cohort of 53 consecutive patients older than 17 years, who had complete follow-up visit according to our institutional protocol with echocardiographic (TTE), coronary computed tomography angiography (CCTA) and magnetic resonance imaging (MRI) examinations. The presence and grade of NeoAR obtained in echo was compared with MRI findings. Differences in calculated Z-scores between two modalities (CT and TEE) and between patients who developed significant NeoAR (over $10 \%$ neoaortic regurgitant fraction in MR) were calculated.

Results: On the basis of TTE, neoaortic regurgitation was a common finding (39/53 patients; $73.6 \%)$. In most of the cases it was trace or mild (12/53 patients; $22.6 \%$ and $21 / 53$ patients $39.6 \%$ respectively) and had no clinical significance. Moderate stenosis was present in 4 cases $(7.5 \%)$, severe in two $(3.8 \%)$. TTE and MR results correlate well $(\mathrm{R}=0,62 ; \mathrm{p}<0.001)$, however there is significant difference between them in qualitative assessment $(p<0.001)$.

Neoaortic valve $Z$-score was significantly bigger in those patients, who developed significant NeoAR (mean Z-score 3.45 vs 2.02; $\mathrm{p}=0.001)$. Results from TTE and CT correlate well $(\mathrm{r}=0.68$; $\mathrm{p}<0.001)$ but differences were statistically significant $(\mathrm{p}<0.001)$. Neoaortic sinus was also significantly bigger in patients with significant NeoAR (mean z-score 2.98 vs $1.72 ; \mathrm{p}=0.006$ ), and results obtained from CT despite their good correlation with TTE $(\mathrm{R}=0.79 ; \mathrm{p}<0.001)$ were significantly bigger $(\mathrm{p}<0.001)$. Sinotubular junction diameter was similar in patients with and without significant NeoAR $(p=0.34)$, but the difference between CT and ECHO measurements was still significant $(p=0.003)$.

Conclusions: TTE has a tendency to overestimate the grade of NeoAR in relation to the MRI results. Diameters of neoaortic valve and sinus are bigger in patients with significant NeoAR, however in most patients with abnormal $\mathrm{Z}$ - scores neoaortic valve remain competent.

\section{P-136}

Cardiovascular Assessment Of Children With Alagille Syndrome Prior To Liver Transplantation: Should Hybrid MRI-Catheter Have The Last Word

Bordin G. (1,2), Valverde I. (1,3,4), Cernat E. (5), Baker A. (5), Miller O. (1)

Department of Congenital Heart Disease, Evelina London Children's Hospital,London, UK (1); Paediatric Cardiology Unit, Department of Women's and Children's health, University of Padua, Italy (2); Division of Imaging Sciences, Kings College London, UK (3); Cardiovascular Pathology Unit, Institute of Biomedicine of Seville, Spain (4); Department of Child Health and Institute for Liver Studies, King's College Hospital, London, UK (5)

Objectives: Alagille syndrome is associated with congenital heart disease, leading to a higher mortality rate after liver transplantation
(LT). Combined MRI-Catheterisation (XMR) is a "one-stop shop" investigation to assess anatomy, Cardiac Output (CO) reserve by Stress MRI and risk stratification. Patients who are unable to increase CO after dobutamine stress above a $40 \%$ threshold, have been traditionally considered unsuitable for LT, even though the final decision is influenced by individual case discussion, as previously described by Razavi et al. We present our updated experience with a new era cohort and re-visit the conventional XMR criteria for LT.

Methods: This is a retrospective single centre study. Eighteen children (5.6 \pm 4.3 years) with Alagille syndrome underwent $\mathrm{XMR}$ at rest and during two-stages dobutamine stress (10 and 20 $\mathrm{mcg} / \mathrm{kg} / \mathrm{min}$ ) between August 2008 and February 2016 in our institution.

Results: Twenty-two XMR were completed successfully, 4 patients had 2 repeated XMR procedures and one had 3 XMR procedures. The $18 \mathrm{XMR}$ performed as pre LT assessment, showed a significant increase in heart rate and $\mathrm{CO}$ from baseline to maximal dobutamine stress $(\mathrm{p}<0.001)$. Three patients with significant pulmonary valve or arterial stenosis required interventions prior to transplant consideration.

Fifteen patients were able to increase their CO more than $40 \%$ and judged suitable for LT: 12 patients were transplanted uneventfully, while 3 patients had stable liver condition.

Three patients did not increase their $\mathrm{CO}$ above the threshold of $40 \%$. All had a CO at rest in the normal range for BMI and gender. Two of them were judged unsuitable for liver transplant and died from end stage liver disease: the first patient had significant heart comorbidity, whereas the second had a normal cardiac anatomy. However, the third patient was listed as high risk transplant candidate, interestingly he was successfully transplanted.

Conclusions: Dobutamine-stress XMR provides accurate information of $\mathrm{CO}$ response to stress, and hemodynamic and anatomic detail allowing planning of any required cardiac interventions prior to LT. It also helps to identify high risk patients, but a clear threshold cannot be confirmed from this study, and final decision still needs a case by case multidisciplinary discussion.

\section{P-137}

The Impact of Acute Preload Changes on Left Ventricular Cardiac Mechanics in Children who Underwent Percutaneous Closure of Patent Ductus Arteriosus

Prota C., Sabatino J., Smith B., Paredes J., Josen M., Michielon G., Fraisse A., Di Salvo G.

Royal Brompton Hospital, PaediatricCardiology, London, United Kingdom

Introduction: Patent ductus arteriosus (PDA) is one of the most common congenital heart defects, representing $5 \%$ to $10 \%$ of all congenital heart cases. Usually PDA spontaneously resolve but those that lead to clinical instability require invasive management; among closure technique, the transcatheter percutaneous one is safe and effective. However, PDA closure is always associated with abrupt hemodynamic changes. For this reason, the evaluation of children undergoing percutaneous PDA closure could be a unique clinical model to assess the impact of acute preload changes on cardiac mechanics.

Aim of our study was to evaluate the left ventricular mechanics before and within 24 hours after transcatheter percutaneous PDA closure.

Methods: twenty-one children (age 57.4 \pm 55.1 months, age range 1-186 months) diagnosed with hemodynamically significant PDA underwent percutaneous PDA closure.

Complete echocardiographic evaluation, including standard and advanced speckle-tracking echocardiography (STE) with 
acquisition of left ventricular ejection fraction (LVEF), left ventricular global longitudinal (LS), radial (RS) and circumferential strain (CS), was performed at pre-closure and within 24 hours post-closure.

Results: Mean PDA diameter was $2.1 \pm 0.9 \mathrm{~mm}$. At baseline evaluation, all patients presented normal $\operatorname{LVEF}(62.7 \pm 4.0 \%)$. The baseline strain values were: LV LS $-22.1 \pm 2.1 \%$; LV CS $-25.3 \pm 2.5 \%$; LV RS $39.1 \pm 13.1 \%$. At the post-closure evaluation there was a significant reduction in LV volumes (baseline: LV end diastolic volume $=40.4 \pm 24.4 \mathrm{ml}$ vs. $34.5 \pm 24.4 \mathrm{ml}, \mathrm{p}=0.001)$, a significant drop in LV EF $(57.1 \pm 7 \%, \mathrm{p}=0.0016)$ and also a significant reduction in LV LS $(-18.00 \pm 3.1 \%, p<0.001)$, LV CS $(-17.7 \pm 5 \%, \mathrm{p}<0.01)$, and LV RS $(28.85 \pm 10.4 \%, \mathrm{p}<0.001)$ In $6(28.6 \%)$ patients, LV EF dropped below $55 \%(49 \pm 4 \%$, p vs baseline $=0.001)$ at the 24 hour assessment. These 6 patients were comparable with the remaining 15 patients for baseline age, LV end-diastolic volume, PDA size and LVEF $(p=0.09)$. Only LV LS was significantly $(p=0.04)$ reduced in the 6 patients who presented a LVEF $<55 \%$ after percutaneous PDA closure.

Conclusions: Preload changes after transcatheter PDA closure cause a significant decrease in left ventricular systolic performance indexes. Of note, evaluation of pre-closure LS can help to identify the subgroup of patients who may experience an immediate drop in $\mathrm{LVEF}<55 \%$ after the procedure.

\section{P-138}

Kawasaki disease: cardiac mechanics analysis by speckle tracking echocardiography in children and young adults

Prota C. (1), Sabatino J. (1), Josen M. (1), Paredes J. (1), Johansen

Vidia V. (1), Pernia M. (1), Herberg J. (2), Fraisse A. (1), Di Salvo G. (1) (1) Royal Brompton Hospital, Paediatric Cardiology, London, United Kingdom; (2) Imperial College Healthcare NHS Trust, London, United Kingdom

Introduction: Kawasaki disease (KD) is the most common acquired children's heart disease in developed countries. Despite optimal medical treatment, coronary artery abnormalities may still occur in $2-4 \%$ of the patients, making myocardial dysfunction the most important complication during long-term follow-up. Aim of our study was to assess cardiac mechanics in children and young patients with a history of KD and persistent coronary aneurysm (s) by using speckle tracking echocardiography.

Methods: Forty-five patients ( 22 males, mean age of $10.5 \pm 7.9$ yy), all with history of $\mathrm{KD}$, persistent coronary aneurysms and normal left ventricular ejection fraction (LVEF), were included in the study. The 2D datasets were acquired for quantification of LV longitudinal, radial and circumferential strain, global and regional, and LV twist; moreover, also the assessment of the right ventricular $(\mathrm{RV})$ global longitudinal function was obtained. The results were compared with those of 30 age-matched controls. Biventricular volumetric data and EF were obtained by cardiac magnetic resonance (CMR).

Results: Of the overall population, 17 patients had left, 8 right, and 20 both left and right coronary aneurysms. Patients and controls showed the same mean LVEF (respectively $65 \pm 5 \%$ vs $65 \pm 5 \%$, $\mathrm{p}=\mathrm{NS}$ ) as assessed by biplane Simpson. LV end-diastolic and endsystolic volumes and EF correlated well between 2D echocardiography and CMR. However, KD patients had significantly lower LV systolic global strain on the longitudinal axis $(-19.3 \pm 2.4 \%$ vs. $-22.4 \pm 2.4 \%, \mathrm{P}<0.05)$, radial axis $(28.4 \pm 9.3$ vs $45 \pm 5.5 \%$, $\mathrm{p}<0.0001)$, and circumferential axis $(-16.4 \pm 5.4 \%$ vs. $-25.1 \pm 4.1 \%, \mathrm{p}<0.0001)$. Also LV twist was significantly reduced in $\mathrm{KD}$ patients $\left(7.2 \pm 1.4^{\circ}\right.$ vs. $\left.13.1 \pm 5.3^{\circ}, \mathrm{P}<0.001\right)$. Regarding
RV function, patients showed standard echocardiographic indexes of RV function comparable to controls (respectively TAPSE: $19.5 \pm 3.1 \mathrm{~mm}$ vs $19.8 \pm 3.4 \mathrm{~mm}, \mathrm{p}=\mathrm{NS}$; $\mathrm{FAC}=42.2 \pm$ $9 \%$ vs $43.5 \pm 8 \%, \mathrm{P}=\mathrm{NS})$. RVEF assessed by CMR in KD patients was normal,too. Of note, RV global systolic longitudinal strain was significantly impaired $(-22.2 \pm 4.14 \%$ vs. $-33 \pm 9 \%$, $\mathrm{p}<0.0001)$.

Conclusions: In young patients with history of $\mathrm{KD}$ and persistent coronary aneurysm (s), impairment of cardiac mechanics occurs regardless normal LVEF and $2 \mathrm{D}$ echo indexes of RV systolic function. Our data suggest to include speckle tracking echocardiography during the follow-up of these patients.

\section{P-139 \\ Cardiac mechanics in children with left ventricular non compaction \\ Sabatino J., Prota C., Paredes J., UY Pernia M., Josen M., Karagodova E., Krupickova S., Daubeney P., Di Salvo G. \\ Royal Brompton Hospital, Paediatric Cardiology, London, United \\ Kingdom}

Introduction: Left ventricular non-compaction (LVNC) is typically characterized by prominent trabeculations and deep intertrabecular recesses within the left ventricular (LV) wall. Despite multiple diagnostic indices have been proposed, no definitive methods have been established to recognize real LVNC from borderline hypertrabeculated hearts yet, particularly in young patients.

Therefore, the aim of the present research was to evaluate the LV mechanics and to explore its diagnostic value in children and young patients with real LVNC or borderline prominent LV hypertrabeculations (LV-HTr).

Methods: Study population (overall age $9.2 \pm 5$ years) included 37 patients (12 LVNC children, 9 LV-HTr children not meeting standard LVNC diagnostic criteria, and 16 CTRL). Biplane LV ejection fraction (LVEF) was assessed in the whole population. The $2 \mathrm{D}$ echo datasets were acquired for quantification of LV global longitudinal (GLS), radial (RS) and circumferential strain (GCS). Furthermore, biventricular volumetric data including $\mathrm{EF}$ were measured by cardiac magnetic resonance (CMR).

Results: LVNC, LV-HTr and CTRL showed a similar LVEF as assessed by CMR (LVNC: $64.1 \pm 8.9 \%$ vs. LV-HTr $68.9 \pm 4.7 \%$ vs CTRL $65.9 \pm 5.2 \%, \mathrm{p}=\mathrm{NS})$. On the contrary, LVEF assessed by echo was significantly lower in LVNC compared to LV-HTr and CTRL groups $(54 \pm 10.3 \%$ vs $61 \pm 5.5 \%$ vs $65.2 \pm 5.5 \%$, $\mathrm{p}<0.05)$. GLS, GCS and RS were all significantly reduced in children with LVNC compared to CTRL (GLS: $-16.1 \pm 3.9 \%$ vs $-22.8 \pm 2.4 \%$; GCS: $-16.8 \pm 4.7 \%$ vs $-25.9 \pm .4 .1 \%$, p < 0.05$)$. Moreover, children with LV-Tr showed a significant reduction of longitudinal, circumferential and radial strains compared to controls (GLS: $-17.7 \pm 3.0 \%$ vs $-22.8 \pm 2.4 \%$; GCS: $-19.6 \pm 2.9 \%$ vs $-25.9 \pm .4 .1 \%, \mathrm{p}<0.05)$, but their values were not significantly better compared to those of LVNC (GLS: $-17.7 \pm 3.0 \%$ vs $-16.1 \pm 3.9 \%$; GCS: $-19.6 \pm 2.9 \%$ vs $-16.8 \pm 4.7 \%, p=N S)$. Of note, apical CS was significantly reduced either in LVNC group compared to LV-HTr and CTRL groups, and in LV-HTr compared to CTRL (apCS: $-14.9 .1 \pm 5.1 \%$ vs $-19.1 \pm 3.5 \%$ vs $-28.9 \pm 8.2 \%$ ).

Conclusions: In conclusion, impairment of cardiac mechanics was observed both in children with LVNC and with LV-HTr regardless normal CMR LVEF. In particular, apical CS might help to differentiate children with LVNC from borderline prominent LV hypertrabeculations. 
P-140

Peripheral Arterial Structure And Function In Young Survivors Of Childhood Cancer

Broberg O. (1), Maxedius A. (1), Öra I. (2), Liuba P. (1)

Pediatric Cardiology Lund Sweden (1), Pediatric Oncology Lund Sweden (2)

Introduction: Survivors of childhood cancer are at risk for cardiovascular complications earlier in life than the normal population. Besides cardiomyocyte damage it has been shown that chemotherapy also negatively affects the endothelial function. Peripheral arterial tonometry (PAT) is a measurement of endothelial dysfunction and a surrogate marker for atherosclerosis. Carotid dimensions and intima media thickness (CIMT) can be used to calculate arterial elastic properties such as distensibility and stiffness. These indices are linked to increased risk of future cardiovascular events.

Methods: 58 survivors of childhood cancer of different diagnoses and treatments aged 20 to 30 years without any previous cardiac event or chronic disease and 53 healthy age matched controls were evaluated with PAT using the ENDOPAT-2000 (Itamar Medical). Finger plethysmographic probes were used to record pulsatile flow before and after 5 minutes of occlusion with a blood pressure cuff. The contralateral arm served as a control. A reactive hyperemia index (RHI) was yielded and the result was log transformed (lnRHI). Both left and right main carotid were examined with the Philips Epiq 7 ultrasonographic machine using the linear array L15-7io transducer. Maximum diastolic (DD) and systolic diameters (SD) were measured as well as CIMT. Blood pressure measurements were taken at the time of carotid ultrasound. Statistical analyses were done using SPSS.

Results: Baseline demographic data are seen in table 1. Diastolic blood pressure was significantly higher in patients. All parameters (stiffness index, strain and distensibility index) of carotid elasticty were highly different from the control group. The reactive

Table 1.

\begin{tabular}{|c|c|c|c|c|c|}
\hline & Group & $\mathbf{N}$ & Mean & Std. Dev. & $\mathbf{P}$ \\
\hline \multirow[t]{2}{*}{ Age (years) } & Patient & 58 & 25,76 & 2,50 & ns \\
\hline & Control & 53 & 24,46 & 2,65 & \\
\hline \multirow[t]{2}{*}{ Sex (male) } & Patient & $58(37)$ & & & \\
\hline & Control & $53(35)$ & & & \\
\hline \multirow{2}{*}{$\begin{array}{l}\text { Body Mass Index } \\
\left.\text { (kg/height }^{2}\right)\end{array}$} & Patient & 58 & 24,56 & 3,82 & ns \\
\hline & Control & 53 & 25,32 & 3,65 & \\
\hline \multirow[t]{2}{*}{$\mathrm{SBP} \mathrm{mmHg}$} & Patient & 58 & 119,43 & 12,21 & ns \\
\hline & Control & 53 & 115,93 & 13,65 & \\
\hline \multirow[t]{2}{*}{$\mathrm{DBP} \mathrm{mmHg}$} & Patient & 58 & 77,30 & 9,17 & 0,016 \\
\hline & Control & 53 & 73,59 & 6,49 & \\
\hline \multirow[t]{2}{*}{ CIMT (mm) } & Patient & 55 & 0,46 & 0,06 & ns \\
\hline & Control & 53 & 0,45 & 0,05 & \\
\hline \multirow[t]{2}{*}{$\mathrm{SD}(\mathrm{cm})$} & Patient & 55 & 6,59 & 0,54 & 0,04 \\
\hline & Control & 52 & 6,80 & 0,51 & \\
\hline \multirow[t]{2}{*}{$\mathrm{DD}(\mathrm{cm})$} & Patient & 55 & 6,03 & 0,53 & ns \\
\hline & Control & 52 & 5,91 & 0,47 & \\
\hline \multirow{2}{*}{$\begin{array}{c}\text { Stiffness Index }(\beta) \\
(\ln (\mathrm{SBP} / \mathrm{DBP}) / \\
(\mathrm{SD}-\mathrm{DD} / \mathrm{DD})\end{array}$} & Patient & 55 & 4,84 & 1,41 & $<0,001$ \\
\hline & Control & 52 & 3,18 & 0,99 & \\
\hline \multirow{2}{*}{$\begin{array}{l}\text { Distensibility Index } \\
\left(1 / \beta^{\star} \text { CIMT }\right)\end{array}$} & Patient & 55 & 0,10 & 0,03 & $<0,001$ \\
\hline & Control & 52 & 0,16 & 0,09 & \\
\hline \multirow[t]{2}{*}{ LnRHI } & Patient & 53 & 0,67 & 0,29 & 0,001 \\
\hline & Control & 47 & 0,85 & 0,22 & \\
\hline
\end{tabular}

SD main carotid systolic diameter, DD main carotid diastolic diameter, SBP systolic blood pressure, DBP diastolic blood pressure, LnRHI reactive hyperemia index log transformed, CIMT carotid intima media thickness hyperemic response was blunted compared to controls. CIMT did not differ.

Conclusions: These young childhood cancer survivors have decreased arterial elastic properties and endothelial dysfunction compared to healthy controls indicating increased risk for future cardiovascular disease.

\section{P-141}

Circulating Biomarker for Myocardial Angiogenesis in Children and Adolescents with Severe Hypertrophic Cardiomyopathy

Fernlund E. (1,6), Gyllenhammar T. (2), Larsson A. (3), Ärnlöv J. (4,5), Carlsson M. (2), Liuba P. (6)

(1) Dept. of Pediatrics, Linköping University Hospital, Linköping University, Sweden; (2) Dept. of Clinical Physiology, Skane University Hospital, Lund University, Sweden; (3) Dept. of Medical Sciences, Uppsala University, Sweden; (4) Dept. of Medical Sciences, Cardiovascular Epidemiology, Uppsala University, Sweden; (5) School of Health and Social Studies, Dalarna University, Falun, Sweden; (6) Pediatric Cardiology, Skane University Hospital and Lund University, Lund, Sweden

Introduction: Hypertrophic cardiomyopathy (HCM) is characterized by gradual thickening of the myocardium with disruption of coordinated cardiac hypertrophy and angiogenesis resulting in impaired coronary collateralization. Endostatin, an important angiogenesis inhibitor, is a $20-\mathrm{kD}$ a carboxy-terminal proteolytic fragment from the first non-collagenous domain of collagen XVIII, which is a central component of the extracellular matrix in the cardiovascular system. Objective: To assess in young patients with familial HCM whether plasma endostatin relates to the severity of HCM as defined by the presence of intracardiac cardioverter-defibrillator (ICD).

Methods: Children and adolescents with family history and echocardiographic signs of HCM ( $\mathrm{n}=14$; median age 15 years, range $7-27$ years) as well as healthy controls $(n=21)$ underwent echocardiography with tissue Doppler imaging, cardiac magnetic resonance (CMR) and peripheral venous blood sampling for endostatin analysis. Half of the HCM patients ( $\mathrm{n}=7$; "ICDHCM") had earlier received ICD either for primary or secondary prevention for sudden cardiac death (SCD). The remaining HCM patients ("HCM") were age-matched with ICD-HCM patients. Results: The left ventricular (LV) thickness and mass, and the mitral septal and lateral E/e' were significantly higher in the ICD-HCM group than in the other 2 groups $(\mathrm{p}<0.01)$. Plasma endostatin was higher in the ICD-HCM group ( $p=0.09$ vs. controls) and correlated with septal $\mathrm{E} / \mathrm{e}^{\prime}(\mathrm{p}=0.006, \mathrm{r}=0.5)$ and with $\mathrm{LV}$ septal hypertrophy $(\mathrm{p}=0.04, \mathrm{r}=0.4)$. In the subgroup of HCM patients who underwent CMR, there was an inverse correlation between endostatin and myocardial perfusion response to adenosine on CMR ( $\mathrm{r}=-0.45, \mathrm{p}=0.08)$.

Conclusion: In patients with HCM, the degree of septal hypertrophy, diastolic dysfunction and myocardial perfusion response to adenosine correlate with plasma levels of endostatin, which is elevated in the ICD-HCM group. The findings suggest endostatin as additional biomarker for more severe adverse changes in the myocardium of these patients.

\section{P-142}

Prediction of the heart function in patients with single ventricle physiology by using a simulation model Hakacova N. (1), Enocksson M. (1), Winkler K. (1), Broome M. (2) Childrens Heart Centre, Lund, Sweden (1); Astrid Lindgren Children's Hospital, Karolinska University Hospital, Stockholm, Sweden (2) 
Introduction: In patients with single ventricle physiology, atrioventricular (AV) valve regurgitation leads to volume overload of the single ventricle and eventually may lead to heart failure. On the other hand, heart failure may lead to valve regurgitation. Symptoms and hemodynamic findings that result from primary heart failure and heart failure secondary to the AV valve regurgitation overlap. It is necessary to distinguish two pathophysiological features for guidance of the appropriate treatment. Operation of the regurgitating valve, where the regurgitation is primarily a result of ventricular dysfunction can lead to decreased cardiac output due to decreased preload and thus mortality and morbidity after operation.

Aim: The aim is to predict the ventricular function and cardiac output after surgical repair of the atrioventricular valve by using simulation model. We hypothesize that simulated and patient specific hemodynamic data agree.

Methods: Hemodynamic data of patinet with HLHS and tricuspid valve regurgitation were assessed preoperatively, simulated and followed prospectivelly. Ventricular and valvular function was assessed before operation, one day, one week, one month and six months after operation. Simulation program APLYSIA was used to predict ventricular function after changes in valve regurgitation over time. Predicted hemodynamic data by simulation program were compared with patinets follow up data.

Results: The simulated and patient specific ejection fraction and cardiac output agreed by using Aplysia as simulation program. We could simulate that first week after surgery, in $80 \%$ of patients, the ejection fraction decreaced and cardiac output increased after the resolving of the AV-valve reggurgitation. After one moth and six months, the ventricular and atrial dimensions decreased, however, the cardiac output remained higher.

Conclusions: Simulation model Aplysia could predict the ventricular function after surgery, based on preoperative anatomical and functional variables.

\section{P-143}

Cardia Magnetic Resonance in young patients with Hypertophic Cardiomiopathy

Ait Ali L. (1), Sandrine C. (2), Todiere G. (3), Barison A. (3), Aquaro D.G. (3), Khraiche D. (2), Legendre A. (2), Boddaert N. (2), Festa P. (3), Bonnet D. (3), Raimondi F. (2)

(1) Institute of clinical physiologie CNR, Italy; (2) Hôpital Universitaire Necker Enfants malades, Assistance Publique-Hôpitaux de Paris, France; (3) Fondazione G. Monasterio, CNR-Regiorne Toscana, Pisa Italy

Background: although the role of cardiac magnetic resonance (CMR) in adult with hypertrophic cardiomiopathy (HCM) has been wildly validated, limited data existed on the role of CMR young patients with HCM. We sought to assess hypertrophy extension of HCM as well as late gadolinium enhancement (LGE) in a young population by CMR and evaluate their impact on event or procedure at follow-up.

Methods: we restrospectively evaluated 75 HCM or HCM-like (associated to a genetic syndrome) young patients (aged 10.4 \pm 5.2 years, 49 males) who underwent CMR in 2 tertiary pediatric cardiac centers. Left ventricle volume, mass and function, wall thickness and LGE were assessed. According to the extension of the hypertrophy we divided our population in 3 groups: Focal: less than 3 ventricular hypertrophic segments; Intermediate: from 3 to 8 hypertrophic segments and Diffuse: more than 8 hypertrophic segments. Clinical history was abstracted from medical record. During a median follow-up of 2 years, one patient died, 2 underwent surgical procedure associated with ICD implant in one; ICD was implanted in 2 others patients, one of them was subsequently transplanted.
Results: thirty-two patients (43\%) have a family history of HCM. In 22 Patients HCM was associated to a genetic syndrome. A gene mutation was present in 14 patients, in 4 patients any mutation has been found and genetic testing was unknown in the remaining patients. $55 \%$ of the whole population presented intermediate hypertrophy extension, $27 \%$ a diffuse one. The maximum wall thickness was $14.7 \pm 6 \mathrm{~mm}$. LGE was present in $22 \%$, mainly in the interventricular septum. Patients with LGE had a greater wall thickness and left ventricular mass index than those without LGE (respectively: $20 \pm 6.8 \mathrm{~g} / \mathrm{m} 2$ vs. $13 \pm 4.9 \mathrm{~g} / \mathrm{m} 2, \mathrm{p}=0.001$ and $97 \pm 29 \mathrm{~g} / \mathrm{m} 2$ vs. $76 \pm 26 \mathrm{~g} / \mathrm{m} 2, \mathrm{P}=.02)$. Moreover, the presence of LGE was associated with subsequent surgical/ICD procedure at follow-up (Chi2:6.1, $\mathrm{P}=.01)$.

Conclusions: In our population the intermediate pattern of HCM is the most frequent. LGE is less prevalent in young population than in adult HCM patients but it is associated with subsequent surgi$\mathrm{cal} / \mathrm{ICD}$ procedure. Further longitudinal studies are needed to evaluate the progressive development of LGE and its prognostic significance.

\section{P-144}

Young Fontan patients with strong regurgitation of atrioventricular valve possess cardiac overloads and also hepatic disorders

Horimoto Y., Hamamichi Y., Wakamiya T., Mastui T., Kuwata S., Kobayashi T., Saito M., Ishii T., Inage A., Ueda T., Yazaki S.,

Yoshikawa T.

Sakakibara Heart Institite, Tokyo, Japan

Backgrounds. Regurgitation of atrio-ventricular valve (AVVR) potentially depresses cardiac functions in young Fontan patients. We also predicted strong AVVR would impair hepatic functions. The purpose of this study was to investigate cardiac and hepatic functions in young Fontan patients with AVVR.

Methods. The medical records of 174 Fontan patients were reviewed aged from 2 to 18 years. They underwent cardiac catheterization between 2010 and 2015. We divided the whole into two groups, such as patients with AVVR mild-to-moderate or over (Strong AVVR: $n=27$ ) and all remaining patients $(n=147)$. First, we compared cardiac and hepatic performances between groups with and without Strong AVVR. Second, we excluded patients with Strong AVVR from 174 patients, using rest of whom we compared these indexes between patients with AVVR (Mild AVVR: $n=65$ ) and without AVVR (No AVVR: $\mathrm{n}=82$ ).

Results. Fontan patients with Strong AVVR possessed larger ventricular volume on end-systole (66\% vs. $49 \%: p=0.0043)$ and on end-diastole (132\% vs. $102 \%$ : $p=0.0023)$. Ejection fraction was almost same between two groups. Strong AVVR group had higher ventricular pressure on end-diastole (10.5 vs. $8.3 \mathrm{mmHg}$, $\mathrm{p}=0.016$ ); higher pressure of pulmonary capillary wedge ( 9.4 vs. $6.8 \mathrm{mmHg}: \mathrm{p}=0.00033)$; higher pressure of inferior vena cava (13.7 vs. $12.1 \mathrm{mmHg}: \mathrm{p}=0.0019)$. Levels of NT-proBNP were much higher in Strong AVVR group (1156 vs. $241 \mathrm{pg} / \mathrm{ml}$, $\mathrm{p}=0.0014)$; albumin levels were higher (4.2 vs. $4.4 \mathrm{~g} / \mathrm{dl}$, $\mathrm{p}=0.046$ ); gamma - glutamyl peptidase levels were higher (136 vs. 66 IU/1: $p=0.00011)$. Between patients with Mild AVVR and with No AVVR, all cardiac indexes and hepatic indexes mentioned above were not significantly different.

Conclusion. Young Fonatn patients with Strong AVVR possessed hepatic disorder as well as cardiac overloads. In case young Fontan patients had only Mild AVVR, they preserved as same cardiac and hepatic functions as those with No AVVR. We should perform Fontan with fenestration, when we predict Strong AVVR would 
leave after procedure. Otherwise we should attempt to repair Strong AVVR proactively, even if we found their ejection fraction did not fall.

\section{P-145 \\ We should note that lower pressure of pulmonary capillary wedge than we expected lifts up central venous pressure in Fontan circulation \\ Horimoto Y., Hamamichi Y., Wakamiya T., Mastui T., Kuwata S., Kobayashi T., Saito M., Ishii T., Inage A., Ueda T., Yazaki S., Yoshikawa $T$. \\ Sakakibara Heart Institute, Tokyo,Japan}

Background: In patients with chronic heart failure high pressure of pulmonary capillary wedge (PCWP), which is affected by decreased left-cardiac functions, provoked pulmonary hypertension. We predicted that increased PCWP in Fontan heart also induced high central-venous pressure (high CVP). We investigated minimum PCWP which caused high CVP in Fontan patients. Methods: The medical records of 174 Fontan patients were reviewed from 2 yrs to 18 yrs. They underwent cardiac catheterizations between 2010 and 2015. We defined CVP $16 \mathrm{mmHg}$ or over as high CVP (CVP $\geq 16 \mathrm{mmHg}: \mathrm{n}=27)$. First, we examined whether the area under a receiving operating characteristics curve (AUROC) was calculated to determine the best discriminating PCWP for predicting high CVP. Second, we sought minimum PCWP which was connected with high CVP. Third, AUROCs were calculated about each cardiac performance for predicting minimum PCWP. Results: We gained quite a positive AUROC for predicting high CVP by PCWP (0.824: 95\% C.I.0.713 0.934). The rate of patients with $\mathrm{PCWP} \geq 12 \mathrm{mmHg}$ in high CVP group was higher than that in non-high CVP group (48\% vs. $1 \%$ : p < 0.00001). Similarly, significant patient-rate differences between high CVP and non-high CVP were obtained by PCWP $\geq 11 \mathrm{mmHg}(59 \%$ vs. $4 \%$ : $\mathrm{p}<0.00001)$, PCWP $\geq$ $10 \mathrm{mmHg}(66 \%$ vs. $8 \%$ : $\mathrm{p}<0.00001)$, and by lower pressures. Significant differences were not obtained by PCWP $\geq 6 \mathrm{mmHg}$ (85\% vs. $65 \%$ : $p=0.069)$, and by lower pressures. Minimum value of PCWP was $7 \mathrm{mmHg}$ which was potentially connected with high CVP (85\% vs. 43\%: $p=0.00016)$. We obtained significant AUROCs for predicting this minimum values of PCWP $(\geq 7 \mathrm{mmHg})$ by end-diastolic ventricular pressure $(0.832,95 \%$ C.I. $=0.772-0.889)$, by end-diastolic ventricular volume $(0.587$, 95\%C.I.: $0.502 \sim 0.672$ ), and by end-systolic ventricular volume (0.599, 95\%C.I.: 0.515 0.683) . Conclusion: In Fontan patients $\mathrm{PCWP} \geq 7 \mathrm{mmHg}$ was potentially connected with high CVP. This PCWP value was not so higher than we expected. However, it was related to ventricular overloads, particularly end-diastolic ventricular pressure. We should employ the strategy avoiding high PCWP in Fontan candidate patients to acquire adequate CVP after procedure.

\section{P-146 \\ Assessment of right ventricular diastolic by tricuspid annular plane systolic excursion (TAPSE) and its prognostic value in patients with pulmonary arterial hypertension \\ Bobhate P., Colaco S., Karande T., Kasar P., Kulkarni S. \\ Childrens' heart centre, Kokilaben Dhirubai Ambani Hospital, Mumbai, India}

Background: Complete assessment of right heart function includes assessment of the right atrial and ventricular function. Right ventricular diastolic function is difficult to assess especially in pediatric patients. TAPSE has an upstropke and a downstroke. The displacement in the upstroke is used to measure the RV systolic function. The downstroke could be utilised for assessing the right ventricular diastolic function. In this study we aimed to quantify the right ventricular diastolic function using TAPSE and establish its prognostic significance.

Materials and methods: We studied patients of $\mathrm{PAH}$ without shunt lesions referred to our centre. All patients underwent a detailed clinical examination, echocardiogram, Cardiac catheterization, NT pro-BNP and six minute walk test whenever feasible. Clinical deterioration was defined as death, creation of POTTS shunt or addition of prostacyclin analogues. Total right heart function was measured using TAPSE. Down-stroke in TAPSE was broken down into two components; excursion occurring from the peak of the TAPSE to the beginning of $p$ wave on ECG (TAPSE $\mathrm{Rv}_{v}$ ) and from the beginning of the $\mathrm{p}$ wave to the trough of TAPSE $\left(\right.$ TAPSE $\left._{R A}\right)$. The percentage of TAPSE RA $\left(\%\right.$ TAPSE $\left._{R A}\right)$ in relation to the entire TAPSE was calculated.

Results: 48 children (17 F), median age 3 yrs (range 0.3-17), median BSA $0.56 \mathrm{~m}^{2}(0.2-1.8)$. Echocardiogram was done on all patients at the time of initial presentation to the PAH clinic. 8 Patients underwent Potts shunt, 1 was started on iloprost and there were 6 deaths. One and 3 yr event free survival were 86 and $58 \%$ respectively. \% TAPSE RA was significantly higher in patients with clinical deterioration (72 \pm 6 vs $34 \pm 8$ ), $p=<0.001$ and correlated with higher NT-proBNP and lower 6 mins walk distance. \%TAPSE RA of $>60 \%$ and RVFAC of $<25 \%$ were independently associated with event free survival ay 3 years $(p=0.02$ and $p=0.03)$.

Conclusion: Diastolic dysfunction precedes systolic dysfunction in patients with PAH. \%TAPSE RA can be used as a marker of reliance on atrial contribution in maintaining cardiac output. Loss of this contribution can lead to clinical deterioration. Regular monitoring of can identify patients who are prone to clinical deterioration and escalation of therapy.

\section{P-147}

Evaluation of left ventricle systo-diastolic function, epicardial adipose tissue and carotid intima-media thickness in HIV children and young adults

Marsico F., Lo Vecchio A., Paolillo S., D'Andrea C., Bruzzese E., Guarino A., Trimarco B., Perrone Filardi P.

AOU Policlinico Federico II, Naples, Italy

Introduction: Human immunodeficiency virus (HIV) infection is a major cause of morbidity and mortality worldwide. In developed countries, life expectancy has increased considerably as a result of antiretroviral therapy, and cardiovascular (CV) disease has emerged as an important late concern. Today is known the effect of HIV infection on systolic function, but few data are present in literature regarding the association between HIV, diastolic function, and some emerging CV risk factors. The aim of this study is to evaluate the left ventricle systo-diastolic function, the epicardial adipose tissue (EAT) and the intima-media thickness (IMT) in HIV children and young adults.

Methods: We enrolled in the study 29 HIV young patients affected by vertical transmission, under highly active antiretroviral therapy (HAART) (48\% male; median age 11,86 $\pm 5,34)$, and 26 agematched controls. Two groups underwent in the same day to echocardiographic evaluation, for the study of the systo-diastolic function and for the measurement of the EAT, and to carotid ultrasound study for the evaluation of the IMT.

Results: Our analysis showed a statistical significant increase of EAT and IMT in HIV patients, compared to healthy control 
subjects (EAT: 3,16 $\pm 1,05 \mathrm{~mm}$ vs 1,24 $\pm 0,61 \mathrm{~mm} ; \mathrm{p}<0,0001$. IMT: $0,77 \pm 0,15 \mathrm{~mm}$ vs $0,51 \pm 0,11 \mathrm{~mm} ; \mathrm{p}<0,0001)$, and a statistical significant reduction of systolic function, evaluated with biplane Simpson method, in HIV patients, compared to healthy control subjects, although absolute value were normal in both populations $(52,3 \pm 17,49$ vs $66 \pm 4,24 ; p=0,029)$. Regarding diastolic function, there were no difference between HIV patients and control subjects (E/A: 1,62 $\pm 0,48$ vs $2 \pm 0 ; p=0,076$. E/E': $3,75 \pm 0,96$ vs $4,75 \pm 1,5 ; \mathrm{p}=0,076)$.

Conclusion: In our study, HIV children and young adults showed an increased CV risk, concerning in an increased EAT and IMT. On the other hand, our patients showed also a worst systolic function compared with healthy subjects. However, it isn't known if these results are correlated to the infection or to the HAART. Nevertheless, more large multicentric study are needed to confirm our results.

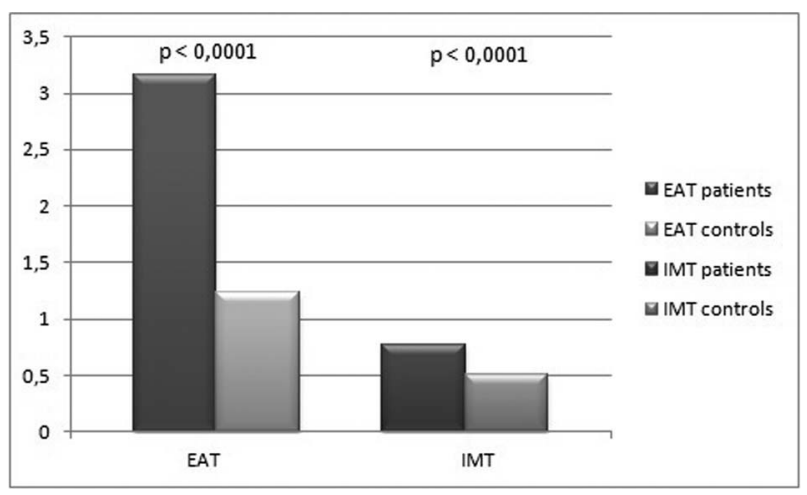

Figure.

\section{P-148}

Evaluation of Revised Jones Criteria for Diagnosing Acute Rheumatic Fever in the Setting of a Tertiary Cardiac Centre in India

Saxena A., Narang R., Ramakrishnan S., Gupta S.K., Juneja R., Kothari S.S.

All India Institute of Medical Sciences, New Delhi, India

Introduction and Objective: There is no gold standard or diagnostic test for diagnosis of acute rheumatic fever (ARF). The diagnosis is guided by Jones criteria which were last updated in 2015. The revised criteria are population specific, being different for moderate and high risk populations. We evaluated the diagnostic yield of these criteria for diagnosing ARF in a tertiary referral centre with facilities for cardiac valve surgery.

Methods: Data of patients with ARF (diagnosed by physician judgment and not strictly by criteria) recruited in a prospective, hospital based, pediatric rheumatic heart disease (RHD) registry was analyzed to see the fulfillment of Jones criteria. There were a total of 124 children with ARF seen over a 4 year period. All underwent echocardiography.

Results: The age ranged from 5 to 15 years (mean $10.4 \pm 2.2$ years) and $60 \%$ were males. $31 \%$ presented in NYHA class III or IV, only $15 \%$ were in NYHA class I. Of the total 124 cases, $62 \%$ were already diagnosed with RHD and had recurrence of ARF. Only $18 \%$ fulfilled revised Jones criteria. The commonest reason for diagnosing ARF in these children was worsening of functional class, congestive heart failure and increase in valvular lesion severity (48\%). ASO titer was elevated ( $>333 \mathrm{IU})$ in $34 \%$ and $\operatorname{CRP}(>3 \mathrm{mg} / \mathrm{dl})$ in $70 \%$. ESR of $>30 \mathrm{~mm} / \mathrm{h}$ was present in $37 \%$. One patient presented with syncope secondary to complete atrio-ventricular block requiring temporary pacemaker, which resolved in 3 days. Echocardiography revealed significant (moderate or severe) mitral regurgitation in $97 \%$ and aortic regurgitation in $23 \%$. Nodules were seen on mitral valve in $43 \%$ and on aortic valve, in $8 \% .19 \%$ had mild pericardial effusion and flail mitral valve was seen in $5 \%$ of patients. One patient died during ARF due to progressive heart failure and one underwent valve replacement surgery during acute phase.

Conclusion: In the setting of a tertiary cardiac referral centre, most patients with ARF present in an advanced stage of the disease and may not fulfill the conventional Jones criteria. The diagnosis should be considered in those demonstrating recent worsening of their symptoms and valvular regurgitation.

\section{P-149}

Blood pressure measurements in infants; who does what? A Nordic survey

Granlund P.A. (1), Ødegaard J.S. (1), Skjerven H.O. (2), Carlsen K.C.L. (1,2), Hanseus K. (3), Rognvaldsson I. (4), Sunnegård J. (5), Turanlahti M. (6), Holmstrom H. $(1,2)$

1. Institute of Clinical Medicine, University of Oslo, Oslo, Norway;

2. Division of Paediatric and Adolescent Medicine, Oslo University

Hospital, Oslo, Norway; 3. Department of Paediatric Cardiology, Skane University Hospital, Lund, Sweden; 4. Division of Paediatric Cardiology, University Hospital of Iceland, Reykjavik, Iceland; 5. Institute of Clinical Sciences, Gothenburg University, Gothenburg, Sweden; 6. Division of Tertiary Pediatrics, Helsinki University Hospital, Helsinki, Finland

Introduction: Despite frequent use of non-invasive blood pressure (NIBP) measurements in infants, generally accepted measurement guidelines are lacking. The primary aim of the present study was to determine the use of standardised procedures for infant NIBP measurements in the Nordic countries. Secondarily we aimed to identify factors included in the standardisation and interpretation of NIBP measurements in infants.

Methods: A cross-sectional electronic questionnaire survey was completed by physicians in all university hospitals in Sweden, Norway, Denmark, Finland and Iceland, targeted through national coordinators.

The survey contained four parts: 1) respondent characteristics, 2) presence and description of standardized procedures for BP measurements, 3) actual daily practice of BP measurements, and 4) the respondent's methodological considerations and interpretation of BP measurements in a healthy 6-monthold baby.

Results: The overall response rate of 55/84 physicians (91\% paediatricians) included all Nordic university hospitals in the sub-fields of cardiology $(n=22)$, general paediatrics $(n=16)$, nephrology $(\mathrm{n}=14)$ and other fields $(\mathrm{n}=3)$, but lacked responses from the 18 physicians working within intensive care units. Age-specific NIBP procedures for infants were available in 13 units $(23.6 \%)$ only. The respondents referred to 19 different sources of information. Factors most commonly assessed for interpretation were age (100\%), arousal state (78.2 \%) and cuff size $(76.4 \%)$. Interpretation of the highest- and lowest assumed normal systolic and diastolic BP overlapped substantially.

Conclusion: We conclude that the majority of university hospital units treating children lack age-specific written procedures for measuring and interpreting infant BP. There is a strong need for common guidelines and updated reference values. 


\section{P-150}

Myocardial deformation imaging identifies early phenotypic changes in children with HCM-causing sarcomere protein gene mutations

Argiolas A. (1), Norrish G. (1,2), Green D. (2), Marek J. (1,2), Kaski J. $(1,2)$.

Great Ormond Street Hospital London UK (1); University College London UK (2)

Background: Hypertrophic cardiomyopathy (HCM) is caused by mutations in the cardiac sarcomere protein genes inherited as an autosomal dominant trait with age-related penetrance. We sought to analyse myocardial mechanics in mutation-positive children without overt left ventricular hypertrophy, using 2D speckle tracking echocardiography.

Methods: Offline 2D speckle tracking analysis was performed in 18 paediatric sarcomeric gene mutation-carriers $(G)$ and compared with 30 phenotypically affected children with sarcomeric mutations (HCM) and 24 healthy controls (C).

Results: Global longitudinal strain (GLS\%) was lower in hypertrophic cardiomyopathy $(-15.8 \%)$ than controls $(-24.8 \%)$ and mutation-carriers $(-22.5 \%)(\mathrm{p}<0.0005$ and 0.002 , respectively). In addition, significant $(p<0,0005)$ segmental changes were observed for HCM in peak longitudinal strain, predominantly at basal septum BS (HCM $=-5,9 \% ; C=-20,2 \% ; G=-17,4 \%)$, mid septum MS (HCM $=-7,9 \% ; C=22,1 \% ; G=-20,8 \%)$, mid inferior segment $\mathrm{MI}(\mathrm{HCM}=-9,7 \% ; \mathrm{C}=-22,6 \% ; \mathrm{G}=-21,2 \%)$, basal anterior septum BAS (HCM $=-4,8 \% ; \mathrm{C}=-18,4 \% ; \mathrm{G}=-22,5 \%)$. Strain rate was significantly $(\mathrm{p}<0,0005)$ reduced at $\mathrm{BS}(\mathrm{HCM}=$ $-0,5 / \mathrm{s} ; \mathrm{C}=-1,5 / \mathrm{s})$, BAS $(\mathrm{HCM}=-0,4 / \mathrm{s} ; \mathrm{C}=-1,3 / \mathrm{s} ; \mathrm{G}=-1,1 / \mathrm{s})$, mid anterior septum $(\mathrm{HCM}=-0,9 / \mathrm{s} ; \mathrm{C}=-1,7 / \mathrm{s})$, MI $(\mathrm{HCM}=$ $-0,7 / \mathrm{s} ; \quad \mathrm{C}=-1,6 / \mathrm{s}), \quad \mathrm{MS} \quad(\mathrm{HCM}=-0,6 / \mathrm{s} ; \mathrm{C}=-1,5 / \mathrm{s}-\mathrm{C} ; \mathrm{G}=$ $-1,2 / \mathrm{s})$, apical inferior segment $(\mathrm{HCM}=-1,5 / \mathrm{s} ; \mathrm{C}=-2,1 / \mathrm{s})$.

Post-systolic shortening was significantly $(\mathrm{p}<0,0005)$ increased at BS $(\mathrm{HCM}=8,4 \% ; \quad \mathrm{IQR} 1,6-24,5 ; \quad$ vs $\mathrm{C}=0 \% \mathrm{IQR} 0-1,2 \%)$, BAS $(\mathrm{HCM}=6,9 \% \mathrm{IQR} 0,2-36,9$ vs $\mathrm{C}=0 \% \mathrm{IQR} 0-0)$, and $\mathrm{MS}(\mathrm{HCM}=$ $4,7 \% \mathrm{IQR} 0,4-22,8$ vs $\mathrm{C}=0 \% \mathrm{IQR} 0-0)$. Moreover, mutation-carriers had slower strain rate than controls at $B S(C=-1,5 / \mathrm{s}$ vs $G=-1,1 /$ s, $\mathrm{p}=0,015)$ and $\mathrm{MI}(\mathrm{C}=-1,6$ vs $\mathrm{G}=-1,1 / \mathrm{s}, \mathrm{p}=0,036)$.

Mechanical dispersion was prolonged in hypertrophic cardiomyopathy (HCM = 67,7 IQR54,1-101 $\mathrm{ms}$ vs C =27,8 IQR22,733,2 $\mathrm{ms}$ and $\mathrm{G}=31,6 \mathrm{IQR} 27,3-47,2 \mathrm{~ms}, \mathrm{p}<0,0005)$. Regression showed a $7,87 \mathrm{~ms}$ increase in mutation-carriers as compared to controls, after adjusting for maximal wall thickness, LVOT gradient and history of VT.

Conclusions: Global and segmental systolic strain is significantly impaired in children with sarcomeric hypertrophic cardiomyopathy. Importantly, phenotype-negative mutation carriers have reduced global and regional strain parameters as well as mechanical dispersion as compared to controls, suggesting that impairment in myocardial mechanics could be an early phenotypic expression of sarcomeric disease.

\section{P-151}

Relationship between ventricular function and exercise performances in adult patients with Fontan circulation Ladouceur, M. (1), Laurito M. (1,2), Pontnau F. (1), Karsenty C. (1), Iserin L. (1), Legendre A (1).

(1) Hôpital Européen Georges Pompidou and Necker, AP-HP ; Paris Descartes university, Paris, France; (2) A Gemelli Hospital-Catholic University of Sacred Heart, Rome Italy

Background: In adult patients with Fontan circulation, exercise performances are reduced and have a prognostic relevance.
Skeletal, pulmonary and cardiac factors contribute to a progressive decline in exercise capacity. We have recently shown that chronotropic incompetence, increased pulmonary vascular resistance, and lack of adequate preload limit exercise performances in Fontan circulation. However cardiac intrinsic mechanisms involved in this exercise limitation have not been specifically studied.

Objectives: To evaluate the relationship between single ventricle function and exercise capacity in adult patients with Fontan circulation.

Methods: Patients with Fontan circulation who underwent 2dimensional echocardiography with speckle tracking analysis and cardiopulmonary exercise testing (CPET) were included. We analyzed the associations between echocardiographic systolic and diastolic parameters, and exercise capacity measurements evaluated with CPET.

Results: 30 patients with Fontan circulation were retrospectively studied, $25(83 \%)$ patients had adequate ventricular echo images allowing strain measurements. The ventricle longitudinal peak systolic strain was significantly correlated with exercise peak VO2 $(\mathrm{R}=0,62 ; \mathrm{p}<0.01)$ and percentage of predicted peak $\mathrm{VO} 2$ $(\mathrm{R}=0,47 ; \mathrm{p}=0,01)$. There was no significant correlation between ventricular ejection fraction and exercise measurements. Finally a significant correlation between percentage of predicted peak VO2 and $\mathrm{E} / \mathrm{A}(\mathrm{R}=-0,43, ; \mathrm{p}=0,03)$ demonstrated a role of diastolic dysfunction in exercise limitation.

Conclusion: In Fontan patients, decrease in global longitudinal strain and diastolic dysfunction of the single ventricle is significantly associated with exercise limitation. Like peak VO2, these parameter may have a prognostic value in Fontan circulation and could be useful to identify patients at risk for failing Fontan who should require a closer follow-up.

\section{P-152}

Cardiac index is not related to Ventricular Ejection Fraction in different types of Total Cavo Pulmonary Connection

Ciliberti P. (1), Valenti V. (2), Ciancarella P. (2), Napolitano C. (2), Curione D. (2), Santangelo T. (2), Leonardi B. (1), Drago F. (1), Iorio F.S. (1), Giannico S. (1), Carotti A. (1), Secinaro A. (1).

(1) Pediatric Cardiology and Cardiac Surgery Department, Bambino Gesù Children's Hospital IRCSS, Rome, Italy; (2) Department of Imaging, Bambino Gesù Children's Hospital IRCSS, Rome, Italy.

Introduction: Cardiac Magnetic Resonance (CMR) is nowadays the "gold standard" diagnostic technique for patients underwent Total Cavo Pulmonary Connection (TCPC) completion. Little is known about MRI findings after different types of Fontan palliations.

Methods: We retrospectively reviewed CMR performed between 2008 and 2017 in patients underwent TCPC completion with Extracardiac conduit or Classic Fontan in a single center. All the studies comprehended ventricular function assessment and a complete set of cardiac flow allowing calculation of cardiac index and effective cardiac index (measured from the sum of SVC and IVC net flow). Atrio ventricle valve regurgitation was calculated by a combined analysis of volumes and cardiac flows. Medical, surgical and clinical data were collected from hospital records.

Results: One hundred thirteen (113) patients were included. Mean age at MRI was $19.7 \pm 6.7$ years, mean age at TCPC was $5.7 \pm 4.5$ years. Extracardiac tunnel was the most common type of palliation (105 pts; 93\%), whilst Classic Fontan was performed in 8 pts (7\%). Main CMR findings between the two subgroups are summarized in Table 1. 
Comparing the two subgroups of different type of palliation there were no differences in term of EF $(p=0.75)$, and of presence of at least moderate AV valve regurgitation $(p=0.9)$. No differences $(p=0.62)$ were found in term of MACE (death, heart transplant, and listing for heart transplant due to failing).

Despite the comparable findings a significant difference was found in term both of cardiac index and effective cardiac index $(\mathrm{p}<0.001$ and $\mathrm{P}=0.02$ respectively) between the Extracardiac conduit and the Classic Fontan subgroups.

Conclusions: Despite comparable value of ventricle function, AV valve regurgitation and percentage of systemic to pulmonary collaterals, cardiac indexes measured by CMR are higher in patients with Extracardiac Tunnel Fontan compared to the ones underwent Classic Fontan. This finding suggests a better efficiency of the Fontan system in this subgroup, and confirms that cardiac output in Fontan patients is not only related to ventricle function but is dependent on the combination of many different factors.

Table 1.

\begin{tabular}{lccc}
\hline & $\begin{array}{c}\text { Classic } \\
\text { Fontan } \\
\mathbf{N = 8}\end{array}$ & $\begin{array}{c}\text { Extra- } \\
\text { cardiac }\end{array}$ & \\
& $\mathbf{N = 1 0 5}$ & $\mathbf{p}$ \\
\hline Male sex; n (\%) & $4(50 \%)$ & $62(59 \%)$ & 0.61 \\
Age at MRI; (yrs) & $29.8 \pm 7.9$ & $18,9 \pm 6$ & $<0.001$ \\
Age at TCPC; (yrs) & $4.9 \pm 2.4$ & $5.8 \pm 4.7$ & 0.581 \\
Time MRI from Fontan; (yrs) & $25.5 \pm 5.7$ & $13.5 \pm 5.8$ & $<0.001$ \\
Saturation at rest (\%) & $93 \pm 5$ & $95 \pm 3$ & 0.186 \\
Heterotaxy; n (\%) & $0(0 \%)$ & $15(14 \%)$ & 0.25 \\
Main ventricle type: & $6(75 \%)$ & $62(59 \%)$ & 0.374 \\
-Left; (\%) & $2(25 \%)$ & $27(26 \%)$ & 0.964 \\
-Right; n (\%) & $0(0 \%)$ & $16(15 \%)$ & 0.228 \\
-BIventricular; n (\%) & $69.3 \pm 19.3$ & $98.4 \pm 27.1$ & 0.004 \\
Indexed EDVV (ml/m2) & $51 \pm 11$ & $50 \pm 9$ & 0.749 \\
EF (\%) & $3(38 \%)$ & $24(23 \%)$ & 0.349 \\
EF <45\%; n (\%) & $2.2 \pm 0.6$ & $3.1 \pm 0.6$ & $<0.001$ \\
CI (1(min/m2) & $2 \pm 0.5$ & $2.6 \pm 0.6$ & 0.02 \\
Effective CI (1/min/M2) & $1.1 \pm 0.3$ & $1.7 \pm 3.4$ & 0.621 \\
RPA/LPA net flow ratio & $16 \pm 12$ & $19 \pm 10$ & 0.2 \\
SP collateral flow; (\%) & $1(12.5 \%)$ & $13(12 \%)$ & 0.9 \\
AVVR $\geq$ moderate; n (\%) & $1(12.5 \%)$ & $8(8 \%)$ & 0.62 \\
MACE; n (\%) & & & \\
\hline
\end{tabular}

\section{P-153}

Joint reference values for cardiac MR imaging derived ventricular size and function for children aged $0-18$ years van der Ven J.P.G. (1), Sadighy Z. (1), Valsangiacomo-Buechel E. (2), Sarikouch S. (3), Kellenberger C.J. (4), Kaiser T. (2), Beerbaum P. (5), Boersma H. (6), Helbing W.A. $(1,7)$

Department of Paediatrics, division of Cardiology, Erasmus university Medical Center-Sophia Children's Hospital, Rotterdam, the Netherlands (1); Department of Paediatrics, division of Cardiology, University Children's Hospital, Zurich, Switzerland (2); Department of Heart, Thoracic, Transplantation and Vascular Surgery, Hannover Medical School, Hannover, Germany (3); Department of Diagnostic Imaging, University Children's Hospital, Zurich, Switzerland (4); Department of Pediatric Cardiology and Intensive Care, Hannover Medical School, Hannover, Germany (5); Department of Cardiology, Erasmus university Medical Center, Rotterdam, the Netherlands (6); Department of Radiology, Erasmus university Medical Center-Sophia Children's Hospital, Rotterdam, the Netherlands (7)

Introduction: Cardiac magnetic resonance imaging (CMR) is an important tool in the assessment of congenital heart disease (CHD). Although widely used in the management of CHD, available normative data is considered suboptimal, considering the relatively small sample sizes. We sought to create more comprehensive reference values for ventricular size and ejection fraction. Methods: We combined data of 141 healthy children (68 male), aged 0 to 18 years, from previously published Caucasian cohorts from 3 european centers. CMR images were obtained using a standard cine-SSFP sequence. Pre-analysis consensus was obtained for the contouring method. All CMR images were manually contoured in the short-axis orientation under supervision of one experienced expert according to the established guidelines. Biventricular volumes and myocardial masses were derived. Reference curves of volumes and masses, indexed for BSA, in relation to age, ranging from 0 to 18 years, were constructed using the LMS method.

Results: We report normative values stratified per gender and age group representing developmental stages. Age-related reference curves showed non-linear growth patterns. As an example, normal right ventricular end-diastolic volume (RVEDV) in boys was approximately $55 \pm 9 \mathrm{~mL} / \mathrm{m} 2$ at age 3 , increasing to $75 \pm 10$ at age 9 and $95 \pm 10$ at age 18 . The reference curve is shown in fig 1 . In this curve, a rapid growth is initially observed and growth decelerates past age 9 years.

During puberty, a significant difference between girls and boys in biventricular volumes and mass develops (e.g. RVEDV $76 \pm 11$ in girls vs $89 \pm 12 \mathrm{~mL} / \mathrm{m} 2$ in boys $>12$ years, $\mathrm{p}<0.01$ ). No differences between genders could be observed in children under 6 years.

Our data was generally in concordance with previously published normative data. Interobserver agreement (coefficient of variation $(\mathrm{CoV})-9$ to $11 \%$ for right ventricular parameters) and intraobserver variability (CoV -6 to $0 \%$ for right ventricular parameters) were comparable to previous studies.

Conclusions: This study provides normal reference ranges for biventricular volumes and masses in a large group of healthy children. These data can be used as reference for the interpretation of CMR studies in patients with CHD.

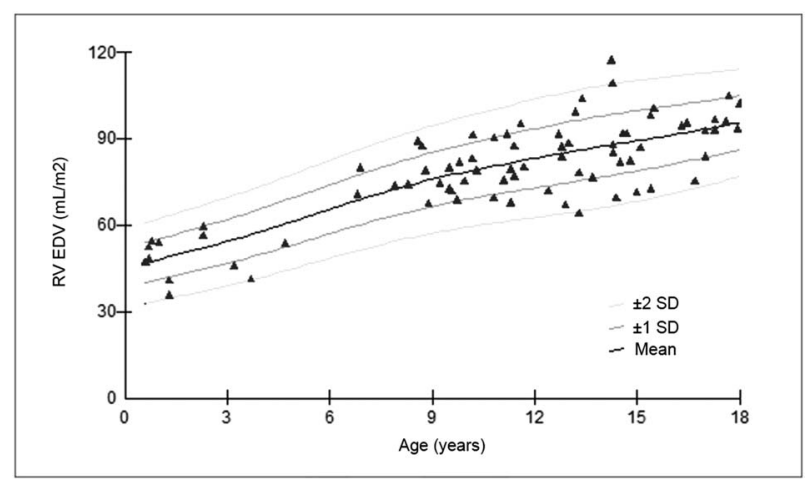

Figure 1.

\section{P-154}

Echocardiographic study in children suffering from Familial Mediterranean fever. A preliminary study of a cohort of patients from Northern Greece

Vampertzi O. (1), Papadopoulou-Legbelou K. (1), Triantafyllou A. (2), Douma S. (2), Papadopoulou-Alataki E. (1)

4th Department of Pediatrics Papageorgiou Hospital Aristotele University of Thessaloniki, Thessaloniki, Greece (1); 3rd Department of Internal Medicine Papageorgion Hospital Aristotele University of Thessaloniki, Thessaloniki, Greece (2) 
Objectives: Familial Mediterranean Fever (FMF) is anautoinflammatory periodic disease, inherited in an autosomal recessive manner. It is characterized by recurrent febrile episodes and sterile serosal inflammation attacks.Echocardiographic abnormalities (systolic or diastolic dysfunction) have been described in a few studies, which were related with sustained subclinical inflammation. The aim of the present study was to assess cardiac function in children diagnosed with FMF as well as the correlation of echocardiographic parameters with the disease duration,inflammatory markers [Erythrocyte Sedimentation Rate (ESR), CRP] and the serum amyloid levels.

Methods: 24 patients with FMF (12 males,12 females,mean age: $8.64 \pm 3.55$ years) during attack-free period and 18 healthy controls (7 males, 11 females, mean age:9.51 \pm 2.78 years) were enrolled in this study, excluding children with obesity, hypertension and dyslipidemia. All FMF patients fulfilled the diagnostic Tel Hashomer criteria and were under colchicine treatment. The disease was confirmed by genetic analysis. A single experienced paediatric cardiologist recorded all echocardiographic measurements using conventional echocardiography.

Results: Mean disease duration of the study population was $5.88 \pm 2.97$ years. Serum amyloid levels in FMF patients ranged between 0.7 and $128 \mathrm{mg} / \mathrm{L}$ (normal values $<6.4 \mathrm{mg} / \mathrm{L}$ ). Although ESR and CRP values where within normal limits, mean values of ESR were statistically significant increased in patients compared to controls $(11.39 \pm 5.74$ vs $6.72 \pm 2.56, p=0.003)$. The echocardiographic study did not yield statistically significant differences in the parameters of systolic and diastolic function (table). Furthermore, no statistical significant correlations were found between echocardiographic parameters, inflammation markers and amyloid levels.

Conclusions: Children suffering from FMF that are under colchicine treatment do not display early echochardiographic abnormalities. This could be related to the young age, the early diagnosis along with the short duration of the disease. Moreover, colchicine probably protects patients from the harmful effects of autoinflammation on myocardial tissue. However, FMF children should be followed with echocardiography as they are considered to have an increased risk of cardiac function abnormalities.

Table.

\begin{tabular}{lccc}
\hline & FMF & Controls & p \\
\hline Age (years) & $8.63 \pm 3.54$ & $9.5 \pm 2.78$ & 0.392 \\
ESR (mm) & $11.39 \pm 5.74$ & $6.72 \pm 2.56$ & 0.003 \\
CRP(mg/dl) & $0.49 \pm 0.65$ & $0.21 \pm 0.19$ & 0.088 \\
LVIDd (mm) & $39.1 \pm 6.4$ & $41.8 \pm 6.6$ & 0.189 \\
LVIDs (mm) & $24.6 \pm 0.41$ & $26.1 \pm 4.1$ & 0.232 \\
LVPWd (mm) & $6.6 \pm 1$ & $6.7 \pm 1.1$ & 0.762 \\
IVSd (mm) & $6.5 \pm 1$ & $6.5 \pm 1$ & 0.918 \\
EF\% & $67.4 \pm 5.05$ & $67.7 \pm 5.67$ & 0.835 \\
FS\% & $37 \pm 3.97$ & $37.44 \pm 4.46$ & 0.736 \\
LAD (mm) & $25.6 \pm 3$ & $26.2 \pm 2.6$ & 0.529 \\
LA/Ao & $1.26 \pm 0.21$ & $1.24 \pm 0.1$ & 0.681 \\
E wave MV (m/sec) & $0.85 \pm 0.11$ & $0.81 \pm 0.07$ & 0.165 \\
A wave MV(m/sec) & $0.45 \pm 0.11$ & $0.45 \pm 0.07$ & 0.913 \\
dt MV (ms) & $131 \pm 35.16$ & $133.77 \pm 21.78$ & 0.770 \\
E/A MV & $1.96 \pm 0.43$ & $1.84 \pm 0.27$ & 0.305 \\
E wave TR (m/sec) & $0.72 \pm 0.1$ & $0.74 \pm 0.1$ & 0.421 \\
A wave TR (m/sec) & $0.45 \pm 0.05$ & $0.45 \pm 0.1$ & 0.974 \\
\hline
\end{tabular}

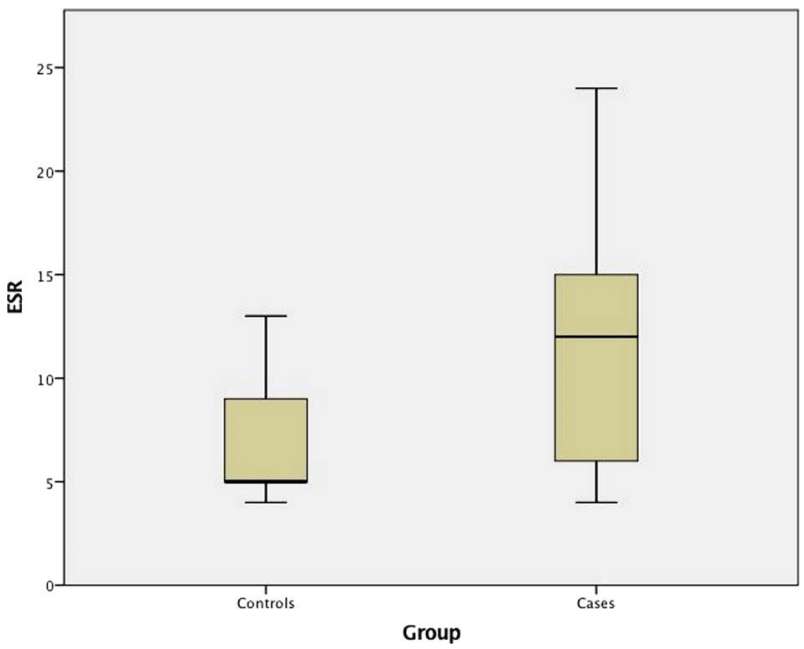

Figure.

ESR by group. Significantly higher for Cases.

\section{P-155}

Paracetamol for the Treatment of Pre-term Patent Ductus Arteriosus - A Systematic Review and Meta-analysis Gibb J., Tulloh R.

Department of Congenital Heart Disease, University Hospitals Bristol NHS Foundation Trust, Bristol, UK

Introduction: Ibuprofen is commonly used to close arterial ducts (PDA) in preterm neonates. In order to help determine if Paracetamol offered similar efficacy, a systematic review and meta analysis was performed to compare outcomes in significant PDA in neonates $<37$ weeks gestation. Primary outcome was PDA closure after one or two courses ( 3 or 6 days) of medication.

Methods: Medline, Scopus, Embase and Google scholar were searched. Manual screening of results revealed six completed trials. Data regarding the risk of selection, detection, attrition and reporting bias were collected and studies were evaluated using the Cochrane collaboration tools for their risk of bias. Using a predesigned data extraction form, the PDA closure rates, as well as rates of complications were extracted, including gastrointestinal bleeding; oliguria; necrotising enterocolitis; Brochopulmonary dysplasia; and mortality. The mean post treatment serum creatinine; aspartate transaminase; and alanine aminotransferase level were also extracted. Data from studies were entered into Stat version 13.0 and compared using the Metan functions.

Results: 733 neonates from six studies were randomised for PDA closure after one course of study medication (371 to Paracetamol, 362 to Ibuprofen). 646 from five studies were randomised for PDA closure after two courses (327 to Paracetamol, 319 to Ibuprofen). The relative risk of PDA closure with Paracetamol compared to Ibuprofen was 1.07 (95\% CI 0.98-1.17, $\mathrm{p}=0.124)$ and $1.05(95 \%$ CI $0.99-1.11, \mathrm{p}=0.129)$ after one and two courses respectively. The relative risk of gastrointestinal bleeding was $0.27(95 \% \mathrm{CI}=$ $0.11-0.67, \mathrm{p}=0.005)$ compared to those treated with Ibuprofen. There was a non significant trend towards lower rates of oliguria with Paracetamol (relative risk $=0.46$ 95\% CI 0.2-1.1, p = 0.083). Conclusion: The effectiveness of Paracetamol for PDA closure in pre-term neonates appears to be similar to that of ibuprofen. It confers a lower risk of gastrointestinal bleeding and may reduce the incidence on oliguria. Paracetamol should be considered as a first line medication for haemodynamically or clinically significant PDA closure in pre-term neonates. 


\section{P-156}

Chronological change of the interventricular septal thickness in hypertrophic cardiomyopathy associated with inborn errors of metabolism

Nakano K., Hayashi T., Ono H., Kato H.

National Center for Child Health and Development, Tokyo, Japan

Introduction: It has been demonstrated that hypertrophic cardiomyopathy (HCM) associated with inborn errors of metabolism has poor outcome with the mortality rate exceeding $50 \%$ at 2 years. However, the clinical characteristics of patients with HCM and inborn errors of metabolism who survive their infancy are not well described.

Methods: The study included 54 patients with inborn errors of metabolism who underwent echocardiography at our institution from January 2009 to August 2017. The end-diastolic interventricular septal thickness at the latest examination was retrospectively collected and standardized using z-scores based on body surface area. The diagnosis of HCM was made if the septal thickness z-score exceeded +2. For patients with HCM, the chronological changes of the septal thickness were also evaluated. Results: Of 54 patients, 45 patients were diagnosed with mucopolysaccharidosis, 5 with mucolipidosis (including 2 with mucolipidosis type II), and 4 with glycogen storage diseases. Patients were 13.7 (range, 0.3 to 52.3) years at the latest examination. Overall, HCM was present in $18(33 \%)$ patients. The frequency of HCM was $26 \%$ in mucopolysaccharidosis, $80 \%$ in mucolipidosis, and $50 \%$ in glycogen storage diseases. Chronological changes of the septal thickness z-scores were available in 10 patients with mucopolysaccharidosis, 3 with mucolipidosis, and 2 with glycogen storage diseases, which were presented in Figure. The annual increase rates of septal thickness z-scores were 0.3 (range, -0.2 to 0.7 ) in mucopolysaccharidosis, 1.4 (range, 1.3 to 1.7 ) in mucolipidosis, and 0 (range, -0.1 to 0.1 ) in glycogen storage diseases ( $\mathrm{p}<0.05$ by Kruskal-Wallis test). Of note, rapidly progressive HCM was observed in both patients with mucolipidosis type II.

Conclusions: Left ventricular hypertrophy was prominent and progressed rapidly in patients with mucolipidosis type II. In other forms of inborn errors of metabolism, left ventricular hypertrophy progressed more slowly. The study demonstrates that there are distinct differences in terms of frequency and severity of HCM among patients with inborn errors of metabolism. The plan for follow-up echocardiography would need to be optimized according to the diagnosis.

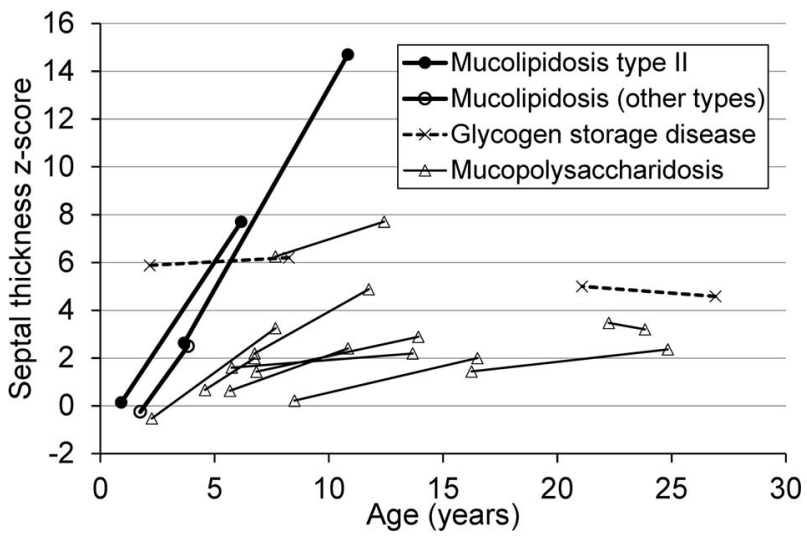

Figure.

P-157

Feasibility and reproducibility of new 3D automatic software for left heart chamber quantification in children Hadeed K., Amadieu R.; Dulac Y.; Karsenty C.; Ratsimandresy M.; Acar P. Department of Pediatric Cardiology, Children's Hospital, Toulouse, France.
Introduction: New 3D automatic software (Heart model (HM), Philips Healthcare, Andover, Massachusetts) is now available to quantify left heart chamber size and function. Its feasibility and accuracy in children have not been reported. The aim of this study was to assess feasibility and reproducibility of HM in healthy children, and to compare LV indices obtained by this technique with classic 2D biplane method.

Methods: 60 consecutive healthy children aged more than 5 years were included (mean age 6.5 years, mean weight $20.8 \mathrm{~kg}$ ). All infants underwent TTE (Epic 7, X5-1, Philips Healthcare, Andover, Massachusetts). Left ventricle (LV) end-systolic and enddiastolic volumes, LV ejection fraction (EF) and left atrium (LA) end-systolic volume were obtained by biplane Simpson method. 3D dataset was obtained from apical 4 chamber view and analyzed using HM software. LV indices obtained by the two methods were compared.

Results: Feasibility of HM was $80 \%$, in 30 cases (62.5\%) with contour adjustment and 18 cases $(37.5 \%)$ without contour adjustment. Intraobserver variability was $(0 \%, 4 \%$ and $8 \%$ for HM without contour adjustment, HM with contour adjustment, and biplane method respectively). Interobserver variability was (6\% and $12 \%$ for HM with contour adjustment and biplane method respectively). Test- retest variability for $\mathrm{HM}$ was $6.5 \%$. Correlation between HM and biplane measurements were strong ( $r=0.85$ to 0.94). LVEF obtained by HM was lower than that obtained by biplane method (bias -8\%). However LV volumes and LA endsystolic volume were larger (bias +12 and +8 respectively).

Conclusion: HM is a promising software for assessment of left heart chamber volume and function. Its feasibility in infants aged more than 5 years is good with excellent reproducibility. Working is going on to validate this technique in children with congenital heart disease and to compare it with MRI measurement as the gold standard.

\section{P-158}

Pericardium-covered stent implantation in a complicated giant coronary artery aneurysm in an adolescent boy Meşe T. (1), Yilmazer M.M. (1), Nazlı C. (2), Varı̧ E. (2), Güven B. (3) Department of Pediatric Cardiology, İzmir Dr.Behçet uz Children's Hospital, Izmir, Turkey (1); Department of Cardiology, Atatürk Training and Research Hospital, İmir, Turkey (2); Department of Pediatric Cardiology, Tepecik Training and Research Hospital, Izmir, Turkey (3)

Case: A 16-year-old boy Syrian refugee re-admitted with the complaint of chest pain 8 months after the first admission. During the initial hospitalization, a giant coronary artery aneurysm was detected, but the patient and the family refused the therapeuric option and they left the hospital without permission. On this admission electrocardiogram demonstrated ST-T wave changes in precordial leads. Transthoracic echocardiography showed a mild dilatation of left heart chambers with mild systolic dysfunction. Echocardiography also demonstrated giant coronary aneurysm previously detected and huge thoracic mass compressing the heart and left lung. The patient was transferred immediately to the intensive care unit (ICU). A multi-slice CT was performed in order to evaluate coronary arteries and the mediastinal mass. Multislice CT showed a 20x15 cm mediastinal mass arising from the pericardial space with bulging into the distal lobe of left lung. Sudden cardiac arrest occurred on the first day of ICU admission, and responded to cardiopulmonary resuscitation. We discussed the patient with cardiac surgeons and radiology department. Blood leaking from coronary aneurysm was considered as the source of 
pericardial hematoma. Therefore, we decided to use covered stent for the exclusion of giant aneurysm. Selective right coronary angiography showed a giant saccular aneurysm at the distal part of RCA and another small saccular aneurysm which was located just before the crux (Figure 1A). A $3.0 \mathrm{~mm} \times 27 \mathrm{~mm}$ equine pericardium covered stent (Aneugraft PCS, ITGI Medical, Or Akiva, Israel) was implanted to exclude both aneurysms (figure 1B). However, we noted the persistence of stenosis at the proximal site (figure 1B). Thereafter, we implanted an another $3.0 \mathrm{~mm} \times 27 \mathrm{~mm}$ equine pericardium covered stent at the proximal site so that which resulted that stents overlapped each other. The final angiogram showed an improvement of blood flow and the disappearance of both aneurysms without residuel stenosis. (Figure 1C).

Conclusions: Up to our knowledge, this is the first patient who had a successful transcatheter pericardium- covered stent implantation to exclude partially ruptured giant aneurysm with huge pericardial hematoma. With percutaneous coronary stenting, improvement of the stagnant coronary blood flow in a giant aneurysm and dilatation of the stenotic segment were successfully achieved.

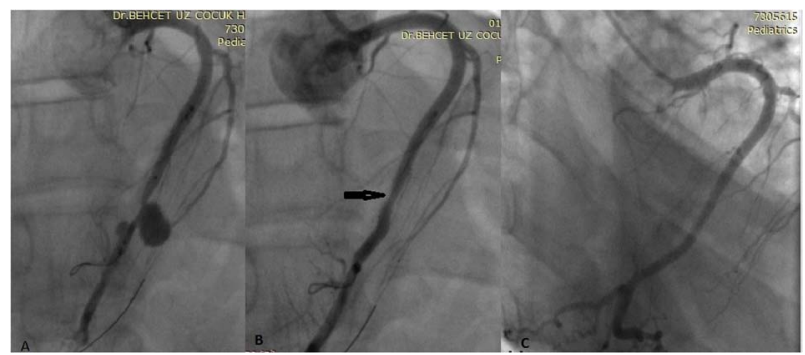

Figure 1.

A: Caudal coronary angiographic view showing an implantation of the pericardium-covered stent to exclude both aneurysm. B: Caudal coronary angiographic view immediately after the pericardium- covered stent implantation showed no aneurysm at the RCA with a localized stenosis on the proximal side of the stent (arrow). C: Coronary angiogram (RAO caudal view) immediately after the second pericardium covered stent implantation on the proximal side of the first implanted stent showed the resolution of the stenosis and the improvement of the coronary blood flow

\section{P-159}

Successful Melody valve implantation in a stenotic conduit with large RVOT aneurysm

Abu Haweleh A., Fraisse A.

Queen Alia heart institute, Amman,Jordan

A 21 year old male with aortic valve stenosis, status post balloon aortic valvuloplasty at the age of 5 years, status post Ross operation with a 22 Contegra in the right ventricle outflow tract (RVOT) presented with left pulmonary artery stenosis and stenosis of the calcified Contegra with a huge RVOT aneurysm at the proximal part of the Contegra.

On the 30th of May 2016, he had successful LPA stenting using $36 / 12 \mathrm{~mm}$ intra stent ev3, Contegra and RVOT stenting using a $45 \mathrm{~mm}$ covered Numed CP stent to close the ostia of the aneurysm, with mild residual shunt. Before coronary artery testing was accomplished with aortic root angiogram while a $20 \mathrm{~mm}$ diameter balloon was inflated in the RVOT conduit. This was followed by $22 \mathrm{~mm}$ Melody valve implantation that completely abolished the shunt through the aneurysm.
Immediately post procedure the pressure gradient across the RVOT dropped from $85 \mathrm{mmHg}$ to $20 \mathrm{mmHg}$ with a systolic RV pressure of $40 \mathrm{mmHg}$.

During follow up, he remained stable and last RVOT gradient by echo was $30 \mathrm{mmHg}$.

In summary, we present the successful treatment of a RVOT stenosis and aneurysm with covered stent and Melody valve implantation.

\section{P-160}

Persistent Left Superior Vena Cava Closure In a Patient With Unroofed Coronary Sinus

Dogan M.T., Tutar E., Ucar T., Atalay S.

Department of Pediatric Cardiology, Ankara University Medical School, Ankara, Turkey.

Introduction: Unroofed coronary sinus (URCS) is a rare congenital cardiac anomaly and has been almost always associated with a persistent left superior vena cava (PLSVC). PLSVC draining into the left atrium is responsible for a right-to left shunt that can be a source of oxygen desaturation. Systemic thromboembolism and brain abscesses are possible complications of this condition. In order to prevent recurrent cerebral emboli and abscesses, correction of this anomaly has been recommended. Although transcatheter based treatment therapies are used to correct this condition in adults, there was a little experience in children.

Case Report: A 6 year-old girl was admitted with cough and fever. Physical examination revealed slight cyanosis and clubbing of the fingers. Transthoracic echocardiographic evaluation with agitated saline injection from the left arm revealed a PLSVC draining into

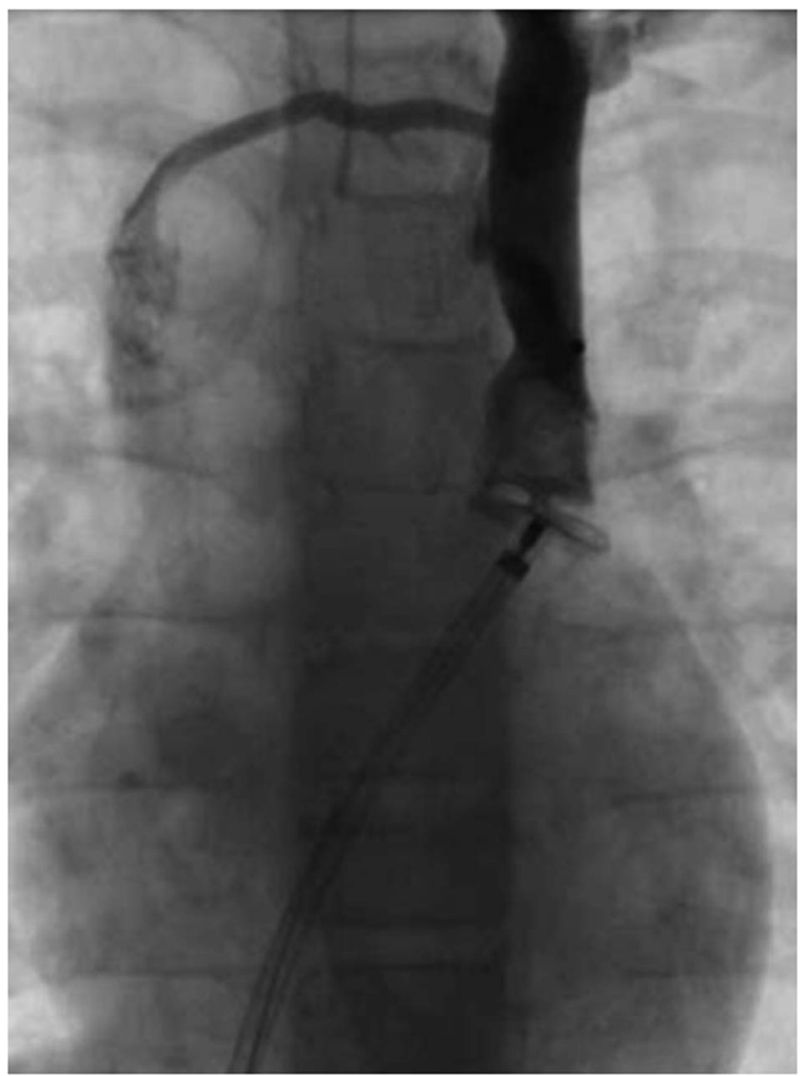

Figure. 
the left atrium suggesting URCS. Diagnosis was confirmed by CT. Under general anesthesia, a catheterisation was performed with left jugular venous and right femoral venous access. An angiocardiogram through the left jugular vein confirmed the LSVC draining directly into the left atrium. There was no significant pressure rise in LSVC during test occlusion of the distal part of the LSVC. Occlusion angiography showed sufficient drainage to the right SVC via bridging vein. Sheat advanced through the coronary sinus to the LSVC. Distal part of the LSVC was occluded with 16/12 mm Amplatzer Vascular Plug II (below the drainage of the accessory hemiazygos vein). Control angiography demonstrated that there was no residual shunt (Figure). After the procedure, arterial oxygen saturation in room air immediately increased from $91 \%$ to $98 \%$. Patient was discharged the day after the procedure without any complication.

Conclusion: If URCS left untreated it can cause complications such as systemic thromboembolism, infective endocarditis, and brain abscess. Early diagnosis and treatment are very important. Although surgery has good results, in selected cases, transcatheter closure appears to be a safe and effective alternative to surgical treatment.

\section{P-161}

Trans-septal Septostomy And Balloon Septoplasty Of The Atrial Septum For Decompression Of The Left Atrium In Patients Supported With Extracorporeal Membrane Oxygenation

Dogan M.T. (1), Ucar T. (1), Tutar E. (1), Odek C. (2),

Ozcınar E. (3), Cakıcı M. (3), Kendirli T. (2), Eyileten Z. (3),

Akar R. (3), Atalay S. (1)

Department of Pediatric Cardiology, Ankara University Medical School, Ankara, Turkey (1); Department of Pediatric Intensive Care, Ankara University Medical School, Ankara, Turkey (2); Department of Cardiovascular Surgery, Ankara University Medical School, Ankara, Turkey (3)

Introduction: Venoarterial extracorporeal membrane oxygenation (VA-ECMO) has been used in myocardial failure to provide immediate cardiac and respiratory support. Rarely left heart hypertension leds to pulmonary edema can detected due to veno arterial ECMO support in patients. Left atrial (LA) decompression in this setting is very important. The experience in pediatric age group is limited. We aimed to evaluate the feasibility of percutaneous trans-septal LA decompression in pediatric patients. We described that the use of trans-septal septostomy and balloon septoplasty of the atrial septum with transesophageal echocardiographic guidance has effectively decompressed of pulmonary venous hypertension.

Methods: We retrospectively analyzed 5 VA-ECMO patients with 6 months to 17 years age children have pulmonary edema from April 2017 to November 2017. Mean left ventricular ejection fraction of the patients was $16 \%$. Cardiogenic shocks were due to dilated cardiomyopathy in 3 of the patient and myocarditis in 2 of the patient .The procedures were performed 1-5 days after ECMO. Trans-septal atrial septostomy and balloon atrial septoplasty were successfully performed in 5 patients on veno-arterial ECMO. Average procedure time was $45 \mathrm{~min}$ (range, 15-75 min). There were no procedure-related complications. After 12 hours from the procedure, it is seen that pulmonary edema showed improvement radiologically. (Figure) and it was also seen that patient's respiratory support requirenments decreased. Two patients were successfully weaned from mechanical ventilator and removed from ECMO.Heart transplantation was required only in one patient.
Table.

\begin{tabular}{llllllll}
\hline & $\begin{array}{l}\text { Weight } \\
\text { (Kg) }\end{array}$ & $\begin{array}{l}\text { Age } \\
\text { (Year) }\end{array}$ & $\begin{array}{l}\text { Gender } \\
\text { (M/F) }\end{array}$ & $\begin{array}{l}\text { Balloon } \\
\text { Size } \\
(\mathbf{m m})\end{array}$ & $\begin{array}{l}\text { Procedur } \\
\text { Time } \\
\text { (Minute) }\end{array}$ & $\begin{array}{l}\text { EF } \\
\text { (\%) }\end{array}$ & $\begin{array}{l}\text { LA } \\
\text { Pressure } \\
(\mathbf{m m H g})\end{array}$ \\
\hline Patient 1 & 6 & $1 / 2$ & $\mathrm{M}$ & 13 & 25 & 12 & $44-21$ \\
Patient 2 & 15 & 5 & $\mathrm{M}$ & 16 & 65 & 15 & $40-14$ \\
Patient 3 & 38 & 13 & $\mathrm{M}$ & 18 & 75 & 20 & $28-20$ \\
Patient 4 & 50 & 16 & $\mathrm{~F}$ & 16 & 45 & 13 & $22-13$ \\
Patient 5 & 60 & 16 & $\mathrm{~F}$ & 16 & 15 & 20 & $9-2$ \\
\hline
\end{tabular}

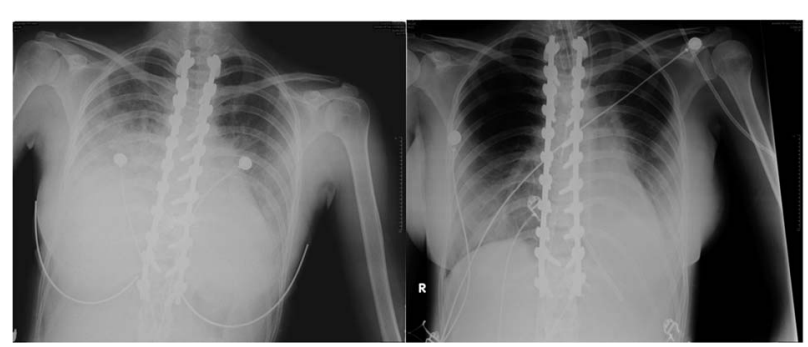

Figure.

Conclusions: Transseptal atrial septostomy and balloon atrial septoplasty can be an effective and safe method for decompression of the LA pressure in the children with ECMO. LA decompression procedure, is a time-saving way for patients in VA-ECMO and it allows heart transplantation and insertion of the left ventricular support device.

\section{P-162}

Cardiac catheterization in infants $<2500$ grams

Anagnostopoulou A., Eleftherakis N.,Andreou N.,Loukopoulou S., Giannakopoulou A., Papafylaktou M., Karanasios E.

Cardiology Department, Aghia Sophia Childrens Hospital Athens, Greece

Introduction: Low birth weight infants are born with a weight $<2500$ grams. Very low birth weight infants weigh $<1500$ grams and extremely low birth weight infants weigh $<1000$ grams.

Since low birth weight infants have higher morbidity and mortality and face more challenges in their care, our goal was to examine the complications during cardiac catheterizations performed for diagnostic or interventional purposes.

Methods: We searched our catheter laboratory retrospectively for all catheterizations between 01/01/2007 and 31/12/2016 covering a 10 year period. We selected all neonates and infants whose weight at the time of catheterization was $<2500$ grams. Subsequently we discovered the causes for the catheterization, whether the cathetererization was diagnostic or interventional and any complications that may have arisen.

Results: 54 children who had undergone catheterization were identified. $29(54 \%)$ were boys and 25 (46\%) were girls. They were from 1 to 171 days old at the time of catheterization. Their weight ranged from 980 to 2500 grams. One baby was extremely low weight at 980 grams and one was very low weight at 1200 grams.14 (26\%) of the catheterizations were interventional. The indications for the interventional catheterization were : 1) DTGA -Balloon atrial septostomy (5/14), 2) DORV + TGA - Balloon atrial septostomy (1/14), 3) Pulmonary Valve atresia- Balloon atrial septostomy $(1 / 14), 4)$ Valvuloplasty for pulmonary stenosis $(3 / 14), 5)$ Valvuloplasty for Aortic stenosis (1/14) 6) Valvuloplasty 
for Coarctation of the aorta (2/14) and 7) Removal of retained central venous catheter $(1 / 14)$. The most common indications for diagnostic catheterization was Coarctation of Aorta (9/40), followed by Tetralogy Of Fallot (5/40), Double Outlet Right Ventricle (3/40), Pulmonary Atresia (3/40), Ventricular Septal Defect $(3 / 40)$ and Univentricular heart $(3 / 40)$.

The children on the interventional group were younger (mean 12.5 days vs 30.95 days) and less heavy (mean 2109 grams vs 2142 grams) than the non interventional group.

None of the children died during the postperative period, but one child in the interventional group had recurrent SVT during valvuloplasty managed successfully by pacing.

Conclusions: Low birth weight infants tolerated the procedures, well even the interventional catheterization. Our experience shows that the indications have been expanding through the years.

\section{P-163}

Prevention of the complete heart block complication after transcatheter perimembranous ventricular septal defect correction

Sideris E., Macuil B., Zanzani S., Sokolov A., Varvarenko V., Pursanov M., Toumanides $S$.

Athenian Institute of Pediatric Cardiology, Athens, Greece

Surgery is currently indicated for the majority of perimembranous ventricular septal defects (PVSDs), since transcatheter correction by Amplatz type devices was associated with an incidence of complete heart block (CHB) up to $20 \%$. The justification for this choice will be examined by retrospective review of the results of other interventional devices including the buttoned device, the transcatheter patch and the PFM coil. The Buttoned Device has two disks buttoned together and is not interferring with the defect itself. Sixty cases were performed in children more than 2 years. Defect size was 3-6 mm, subaortic rim more than $2 \mathrm{~mm}$. No case of $\mathrm{CHB}$ was noticed. However, three cases were operated because of aortic insufficiency.

The transcatheter patch (TP) is a wireless, soft, absorbable device. Thirty cases were performed in children over $8 \mathrm{~kg}$ or older than 1 year with defect size $5-16 \mathrm{~mm}$. Four malalignment defects (Fallot Tet) and 5 cases of Down Syndrome were included. There was no subaortic rim limitation. There was no CHB.There was one device embolization and no long term complications.

The PFM coil has been used in more than 300 defects in clinical trials. The subaortic rim was more than $3 \mathrm{~mm}$ and patient age more than 2 years. There was no CHB. There were good long term results.

In conclusion, there are several devices not causing $\mathrm{CHB}$, avoiding compression of the conduction system. They have good acute and long term results. The wireless TP has wider application, although some metal devices can have good results in selective defects. Larger clinical trials are necessary prior to changing the current treatment choice.

\section{P-164}

Catheter Closure of Patent Ductus Arteriosus in Adult Patents Using an Outpatient Protocol. A randomized multicentre study

Thanopoulos B.D. (1,4), Dardas P. (1), Ninios V. (1), Giannakoulas G. (2), Deleanou D. (3), Lancovici S. (3)

"Agios” Lukas Klinic, Thessaloniki, Greece (1); Ahepa"University

Hospital, Thessaloniki, Greece (2); AresCardilogy Center, Bucharest,

Romania (3); Iatrikon Medical Center, Athens, Greece (4)

Introduction: Transcatheter closure is the treatment of choice for the majority of patients with a patent ductus arteriosus (PDA).
However, the standard technique of this procedure uses an arterial access and requires immobilization of the patients for 24 hours and may be associated with arterial complications. The aim of this study was to report experience with catheter closure of PDA in 104 consecutive adult patients with the Cocoon PDA occluder on outpatient basis using an exclusive venous approach.

Methods: The age of the patients ranged from 16-72 years (median 36 years. In group 2 the procedure was guided using hand injections of contrast media through the delivery sheath and 2D and color Doppler echocardiography from suprasternal and parasternal long and short axis, respectively.

Results: Group 1: The PDA diameter range from 3 to $10 \mathrm{~mm}$. The device diameter ranged from 4 to $12 \mathrm{~mm}$. The PDA occluders were permanently implanted in all patients. Two and 4 patients had absent and reduced arterial pulses, respectively, that were restored with intravenous infusion of heparin and rtPA, were the main complications of the procedure. . The hospital stay of the patients ranged from 24-48 hours.Group 2. The mean PDA diameter ranged from $2-9 \mathrm{~mm}$. The device diameter ranged from $4-10 \mathrm{~mm}$. The PDA occluders were permanently implanted in all but 2 patients. There were no arterial complications. .. The hospital stay of the patients ranged from $6-8$ hours. There was a reduced cost that ranged from $300-500 €$. Complete echocardiographic closure of the ductus at 1-month follow-up was observed in all 102 patients (100\%).

Conclusions: Exclusive transvenous PDA occlusion using combined angiographic and echocardiographic guidance is an effective and safe method that prevents the arterial complications of the standard approach. In addition, in adult patients, this technique, may be used on outpatient basis resulting in early patient mobilization and reduce hospital cost.

\section{P-165}

Definition of Institutional Diagnostic Reference Levels in Pediatric Interventional Cardiology Procedures

Tsapaki V. (1), Kollaros N. (2), Tsoutsinos A. (2), Plemmenos C. (2), Mastorakou I. (2), Apostolopoulou S.C. (2)

Konstantopoulio General Hospital, Athens, Greece (1); Onassis Cardiac Surgery Center, Athens, Greece (2)

Introduction: Diagnostic and interventional procedures in congenital heart disease is a rapidly developing field and transcatheter therapy of congenital lesions, where applicable, is the standard of care. At the same time, the lack of pediatric Institutional Diagnostic Reference Levels (DRLs) prohibits the identification of good practices.

Methods: This study aimed to evaluate pediatric radiation doses in a dedicated cardiology center, with the objective of characterizing patterns in dose variation. The ultimate purpose was to define DRLs for different types of paediatric cardiac interventional procedures, according to patient age.

Results: A total of 477 interventional procedures were performed during 3 consecutive years, by operators with $>15$ years of experience. The median values obtained for Fluoroscopy Time (FT), Number of Frames $(\mathrm{N})$, and Kerma Area Product (PKa) were 3.1-15.8 min, 579-1779 and 1.0-20.8 Gy. $\mathrm{cm}^{2}$ respectively. Median FT, N and PKa values analyzed by age range are depicted in the Table. Conclusions: A large range of patient dose data is observed during pediatric cardiac interventional procedures, depending greatly on procedure type and patient weight. Consequently, the definition of DRLs for pediatric cardiac interventional procedures presents challenges due to multiple clinical and technical factors affecting the outcome. A consensus is needed on interventional procedures nomenclature and grouping in order to allow common assessment and comparison of doses. 
Table.

\begin{tabular}{llll}
\hline Age at cath & $\begin{array}{l}\text { Fluoroscopy } \\
\text { Time (FT) }\end{array}$ & $\begin{array}{l}\text { Number of } \\
\text { Frames (N) }\end{array}$ & $\begin{array}{l}\text { Kerma Area } \\
\text { Product } \\
(\mathbf{P K a})\end{array}$ \\
\hline$<1$ year old & $5.8 \mathrm{~min}$ & 1322 & $2.0 \mathrm{~Gy} \cdot \mathrm{cm}^{2}$ \\
1-5 year old & $6.5 \mathrm{~min}$ & 1403 & $3.0 \mathrm{Gy.cm}$ \\
5-10 year old & $5.9 \mathrm{~min}$ & 950 & $7.0 \mathrm{Gy.cm}$ \\
$10-16$ year old & $5.7 \mathrm{~min}$ & 940 & $14.0 \mathrm{~Gy} \cdot \mathrm{cm}^{2}$ \\
\hline
\end{tabular}

\section{P-166}

Results of MRI guided or assisted procedures in Greece

Tzifa A., Patris K., Apostolidis Ch., Avramidis D.,Loggitsi D,

Kalangos $A$.

Mitera Hospital, Athens, Greece

Background and aims: Combined cardiac catheterization and magnetic resonance imaging (XMR) is a well-established approach in some centers, particularly for accurate assessment of pulmonary hypertension (PHT) and its reversibility. Its wider application however, is hampered by limited availability of hybrid XMR suites. We advocate that the procedure can still be performed with minimal changes to the MRI suite's infrastructure with important benefits for the management of borderline inoperable patients due to PHT. The aim of the current study was twofold: First, to test the feasibility of performing such studies in the catheterization laboratory and moving the patient to the MRI suite and secondly, to assess patients' outcomes based on XMR findings, as well as the importance of XMR on the decision planning.

Methods: We reviewed the data of 12 patients who underwent an XMR catheterization because of echocardiographic suspicion of PHT. 1/12 patient had a solely MRI-guided cardiac catheterization procedure.

Results: Twelve patients with median age of 16yrs (range 2-56yrs) underwent an XMR procedure to assess pulmonary vascular resistance (PVR) and guide further management. Median baseline PVR was 5.1 WU.m2 (range 3.1-12WU.m2), which improved to 4.2 WU.m2 (range 2.3-8.5WU.m2) after administration of pulmonary vasodilators. Of the 12 patients, eight were operated and one had percutaneous closure of an ASD at a median time of 5 months (range 0.5-18 months) after the procedure. After administration of pulmonary vasodilators, elevated PVR was found in $3 / 9$ patients (3.5-8.5WU.m2), who therefore had fenestrated closure of their septal defect. The remaining 6/9 patients had complete closure of their defects without fenestration. Two patients had changes in their medical management and one patient has been referred for a heart-lung transplant.

Conclusions: The performance of MRI-assisted or guided catheterization procedures can take place outside the setting of a hybrid XMR suite. XMR procedures are extremely important for the management of patients with CHD and pulmonary hypertension. Acquisition of accurate flow data from MRI combined with simultaneous pressure measurement provides a robust assessment of the patient's operability status and type of operation, such as fenestrated defect closure, whilst also guiding the postoperative management in the intensive care unit.

\section{P-167}

Thrombus Aspiration in Acute Myocardial Infarction in Children with Intra-cardiac Mass

Waithayawongsakul P., Limsuwan A., Jongviriyavong P.,

Meemook K., Jirotjananukul T., Samankatiwat P., Siripornpitak S., Wattanatranon D.

Ramathibodi Hospital, Mahidol University, Bangkok, Thailand
Introduction: Acute ST-segment elevation myocardial infarction (STEMI) is rarely present in children. The primary goal of management is to reperfuse the ischemic myocardium. Primary percutaneous coronary intervention (PCI) has become standard of care. We presented the PCT in management of STEMI in a male adolescent with left atrial (LA) mass.

Patient and Methods: A 13 year-old male known protein S deficiency and LA mass after being evaluated for stroke in the young. He received low molecular weight heparin for intracardiac mass located at LA which appeared to adhere to the anterior mitral valve leaflet. Echocardiogram evaluation revealed a hyperechogenic mass, (diameter $3 \times 3 \mathrm{~cm}$ ), located adjacent to the lower part of the LA septum and moved along with anterior mitral valve leaflet. With the result of protein S deficiency, the LA mass was presumed to be the thrombus in origin. After 20 months of medical treatment, he presented with sudden onset of chest pain. Result: The patient was referred to our service due to STEMI within 2 hours of onset. The thrombolytic agent was not given because the patient had been taken warfarin. He was transport to the cath labs. After femoral artery sheath was inserted, the 0.014 Pilot50 wire and the 6FJL3 catheter were advanced into the left main coronary. Selective left coronary angiograms were obtained and revealed the total occlusion of the distal left circumflex artery. Decision was made to perform manual thromobectomy using Thrombuster aspiration catheter. The small thrombus like tissue $(3 \mathrm{~mm} \times 5 \mathrm{~mm})$ was obtained. Another left coronary angiogram was obtained which revealed the unobstructed flow to the left circumflex artery.

Cardiac MRI evaluation showed the heterogenic density of the LA mass which was well defined to attach to the LA septum. The primary impression was LA myxoma. Therefore, the patient underwent an elective surgery to remove the mass with the pathological confirm diagnosis of myxoma.

Conclusion: This case revealed the role of PCI and thrombus aspiration in a child with LA mass presented with STEMI. Thrombuster has a beneficial role in management of tumor emboli to the coronary artery.

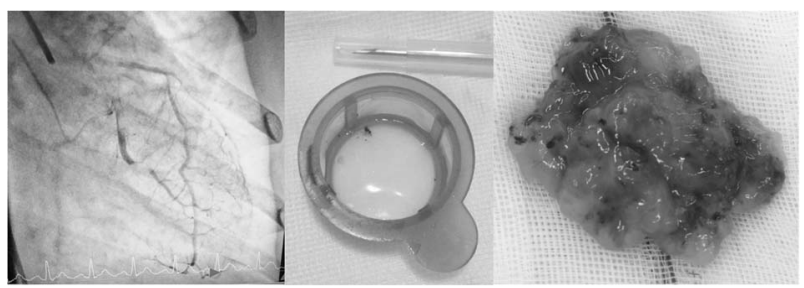

Figure.

\section{P-168}

Is transhepatic vascular access a feasible and safe alternative for cardiac catheterization in children with congenital heart disease?

Jakob D.-M., Knirsch W., Kretschmar O.

Departement of Cardiology. University Children's Hospital Zurich, Switzerland

Introduction: Diagnostic and therapeutic cardiac catheterization is performed in children with congenital heart disease using femoral vascular access. In some cases, this access may be unavailable due to thrombosis from previous catheterization, indwelling central lines or patient's small size. In the last few years the transhepatic approach has been described as "viable" and safe alternative to the femoral access. 
Methods: We analyzed safety and complications of transhepatic access in children performed in our center from 2005 to 2016. Results: During this 11 year period, we performed 8 catheters in 6 children via transhepatic access. Median age was 18.5 months (range 2-174 months), median weight $10 \mathrm{~kg}$ (range 4.6-18 kg). Sheath size ranged from 4-11Fr. Transhepatic puncture was always ultrasound guided. 7 catheters were interventional and 1 only diagnostic. Interventions included stenting of main pulmonary artery, pulmonary side branches and superior caval vein, balloon dilatation of pulmonary vein, closure of pulmonary artery, pulmonary side branches, veno-venous collaterals and atrial septal defect.

After catheter in 3 patients we closed the punctured hepatic vein using coils or amplatzer devices. The day after, all patients had abdominal ultrasound, echocardiography and ECG. There were no complications in all the patients.

Conclusion: Data on transhepatic catheterization in children is limited. Our data show that a broad spectrum of different cardiac interventions can be performed via transhepatic vascular access. It seems a feasible and safe alternative for cardiac catheterization if routine femoral access is not possible.

\section{P-169}

Transcatheter Closure of Atrial Septal Defects and Patent Ductus Arteriosus using Cocoon Devices - single pediatric center experience

Mahmoud H. (1,2), Nicolescu A. (1), Filip C. (1), Bogdan A. (1), Nicolae G. (1), Duica G. (1), Cinteza E. (1,3)

"Marie Curie" Emergency Children's Hospital, Bucharest, Romania (1); University of Damascus, Damascus, Syria (2); "Carol Davila" University of Medicine and Pharmacy, Bucharest, Romania (3)

Introduction: Starting a new paediatric interventional cardiology program is not an easy task to accomplish. The tremendous need for treating children in order to reduce the morbidity and mortality in pediatric population with congenital heart disease was the main purpose for this project.

Methods: Between June 2015 and October 2017, more than 150 cases of congenital heart diseases were diagnosed and treated in our department after initiating this new activity. For more than half of the cases we used Cocoon devices.

Results: A total number of 83 patients were included in the studied group, 27 for atrial septal defect (ASD) closure and 56 cases for patent ductus arteriosus (PDA) closure. The median age for the ASD was 8.5 years (range $3-25$ years) and median weight was $25 \mathrm{~kg}$ (range $11.5-63 \mathrm{~kg}$ ). The mean follow-up was $15.4 \pm 6.7$ months (range 1-24 months). The mean ASD diameter by transesophageal echocardiography was $15.2 \pm 4.1 \mathrm{~mm}$ (range $8-26 \mathrm{~mm}$ ), and by balloon sizing $20.5 \pm 5.2 \mathrm{~mm}$ (range $13.5-32 \mathrm{~mm}$ ). The mean device diameter was $17.3 \pm 5.6 \mathrm{~mm}$ (range $8-32 \mathrm{~mm}$ ). The median age for the PDA was 36 months (range 4-192 months) and median weight was $14 \mathrm{~kg}$ (range $5-58 \mathrm{~kg}$ ). The mean follow-up was $13 \pm 8$ months (range 1-26 months). The mean PDA minimum diameter was $2.5 \pm 0.8 \mathrm{~mm}$ (range $1-5 \mathrm{~mm}$ ). $12.6 \%$ were performed in cases associated with pulmonary hypertension. The success implantation rate was $98.9 \%$. The complication rate was $2.3 \%$ (including two ASD device embolization in patients with less than $5 \mathrm{~mm}$ deficient posterior and inferior rim). The closure rate for ASD was 96.3\% in the first 24 hours and $100 \%$ at 1 -month follow-up. For PDA, the closure rate in the first 24 hours was $98.2 \%$ and $100 \%$ at 1 -month follow-up. On short and intermediate follow-up (1-26 months) no device-related complications were noted.

Conclusions: After this series of cases, the early and intermediate results are very encouraging. The Cocoon devices are safe for transcatheter closure of both ASD and PDA, also as initial experience in our developing centre.

\section{P-170}

Transcatheter closure of atrial septal defects in children and adolescents: a single center experience with the occlutech figulla septal occluder

Bilici M., Akin A., Demir F., Ture M., Balik H., Yilmaz K., Киуитси M.

Dicle University Medical Faculty, Department of Pediatric Cardiology, Sur, Diyarbakir, Turkey

Background: The aim of this study was to evaluate the efficacy and reliability of the Occlutech Figulla Septal Occluder (OFSO) device in children and adolescents with isolated secundum atrial septal defect (ASD).

Methods: We reviewed the clinical records of 70 patients who underwent percutanous closure of ASD with an Occlutech OFSO betwen May 2012 and May 2017.

Results: OFSO was applied to 70 patients during the study period and the results were analysed in this study. Forthy seven of the patients were girl and 23 of them were boy. The median age of the cases was 7 year (range; 4-16 year) and the median weight was $21 \mathrm{~kg}$ (range, $12 \mathrm{~kg}-75 \mathrm{~kg}$ ). 69 patients (98.5\%) underwent successful ASD closure with the OFSO. Transoesophageal echocardiography showed complete closure in 68 patients $(98.5 \%)$ following the procedure, minimal residual shunt in 1 patients (1.5\%)). Color flow Doppler echocardiogpaphy at 12 month after postimplantation showed that complete closure was achieved in this patient. All patients (100\%) had complete closure at 12th months of follow-up. No cardiac rupture, device embolisation, thromboemboli or procedure-related deaths were observed.

Conclusion: The results of this study showed that transcatheter ASD closure with OFSO is a safe and effective method. Although the total closure and complication rates with OFSO are similar to those of ASO, the procedure time is shorter and the radiation exposure is less.

\section{P-171}

Percutaneous closure of paravalvar leak after Cone repair for Ebstein's anomaly

Alderweireldt A., Bové T., De Groote K., De Wilde H., François K., Minne A., Panzer J., Vandekerckhove K., Zaqout M., De Wolf D.

Ghent University Hospital, Ghent, Belgium

Ebstein's Anomaly is a rare congenital heart disease. The Conerepair is used to repair the tricuspid valve. As in valve replacements, paravalvar leaks can affect the outcome.

A 5 year old boy with severe Ebstein's anomaly underwent a successful Cone-repair. At follow-up, his right ventricle function was reasonable, but he developed a paravalvar leak. The leak increased progressively and echocardiography at 1 year post repair showed right atrial dilatation, decreased RV function and important flow over the oval paravalvar defect of $8 \times 5 \mathrm{~mm}$ in postero-lateral position. Because of the hemodynamic importance of the leakage, percutaneous closure was scheduled. Transoesophagal echocardiography confirmed the defect in infero-lateral position, but failed to provide accurate visualisation of the margins of the defect. As we failed to see the native valve under fluoroscopy, we monitored the procedure with transthoracic echocardiography which allowed perfect visualisation of the defect. An oval amplatzer vascular plug III type size $10 / 5 \mathrm{~mm}$ was 
positioned in the defect. The immediate limited residual leak was completely abolished after a few weeks, with recovery of RV function.

The "cone-repair" involves delamination and clockwise rotation of the anterosuperior leaflet, and use of the remnants of the septal and inferior leaflets to create a cone, subsequently the atrialized portion of the right ventricle is plicated. This is comparable to a valve replacement, re-inserting the own tricuspid valve instead of a (bio)prosthetic valve.

As in any other type of valve replacement, a paravalvar leak can compromise cardiac function, in which case closure of the leak is mandatory. Percutaneous closure is an option and is done under fluoroscopic guidance. If a bioprosthesis is inserted, fluoroscopic visualisation of the tricuspid annulus is often lacking and TEE is used in addition to guide the closure procedure. In our case, fluoroscopy and TEE failed to delineate the tricuspid annulus and the defect, but fortunately transthoracic echo provided accurate visualisation of the anatomic structures, which allowed us to close this paravalvar leak.

Conclusion: Paravalvar leak can compromise the results of Cone-repair for Ebstein's disease. Percutaneous closure is possible, taking into account the limitations of guiding the procedure by fluoroscopy or even TEE.

\section{P-172}

Endovascular pulmonic 'Bentall procedure' as tailored treatment strategy in a ruptured and dilated pulmonic homograft of a patient with congenital heart disease following Ross operation

Mueller G. (1), Christoph Sinning C. (2), Kozlik-Feldmann R. (1), Biermann D. (3), Koelbel T. (4), Schaefer U. (2)

Department for Paediatric Cardiology, University Heart Centre Hamburg (1), Department for Cardiology, University Heart Centre Hamburg (2), Department for Cardiac surgery of congenital heart defects, University Heart Centre Hamburg (3), Department for vascular medicine, University Heart Centre Hamburg, Germany (4)

Introduction: Due to signs of severe right heart failure a 31-year old female patient was hospitalized. The patient had known for Shone complex after ROSS and mitral valve replacement with a history of 5 open-heart surgeries. Computertomography revealed a ruptured and severely dilated, 21-years old pulmonic homograft with substernal and sternal bleeding. Due to the ruptured homograft with substernal bleeding an alternative strategy for the dilated homograft has do be developed to prevent high risk surgery.

Methods: In order to cover the perforation a self-expanding stentgraft in the enlarged right-ventricular outflow tract and ruptured homograft was performed. As no stent-grafts with the desired diameter and length are commercially available, $34 \mathrm{~mm}$ wide and $77 \mathrm{~mm}$ long covered Zenith TBE was modified on a side-table during the procedure. The stent-graft was partially deployed through the Captor-valve out of the peel-away sheath. The fabric was shortened using electrocautery between the mid- and the distal stent, thereby shortening the stent-graft to a length of $52 \mathrm{~mm}$. The distal fixation of the stent-graft was replaced using $25 / 0$ monofilament sutures that were attached to stainless steel triggerwire controlling the distal fixation. The surgeon-modified covered stent-graft was then reloaded and implanted with full coverage of the $15 \mathrm{~mm}$ perforation. Finally an Edwards Sapien 3 valve was inserted via a $16 \mathrm{Fr}$ e-sheat and anchored within the Zenith TBE.

Results and Conclusions: Tailored modifying of a self-expandable aortic stent graft opens an alternative for treatment of severely enlarged and perforated homograft to prevent high risk surgery.

\section{P-173}

Closure of the iatrogenic cleft in mitral valve in two years old child

Saygil A. (1), Yildiz P (1), Oktay A. (1), Yalcmbas Y.K. (2),

Yuksek A. (3)

Aclbadem University, School of Medecine, Department of Pediatric

Cardiology, Istanbul, Turkey (1) ;Acibadem University, Department of Cardiovascular Surgery, Istanbul,Turkey (2);Acıbadem Bakırköy

Hospital, Department of Anesthesiology, Istanbul, Turkey (3)

Objective: Iatrogenic cleft or hole may occur as a rare complication after surgery in the mitral valve. Surgery is the approved treatment modality. However, interventional methods also provide solutions for the treatment of paravalvular leakage which develop during valve replacement in adult cases. There is no literature study on the interventional closing of mitral valve cleft in children. We present a case of a two-year-old who underwent an interventional closure of iatrogenic mitral valve cleft.

Results: At the age of 2 days during the neonatal period, the patient was scheduled for cardiology follow-up following detection of a heart murmur due to aortic outflow tract stenosis, mild stenosis in the subaortic ridge membrane and isthmic aorta, and mild mitral insufficiency diagnosed during echocardiographic examination. She was operated on at the age of 16 months following detection of a subaortic maximal gradient of $63 \mathrm{mmHg}$ and a mean gradient of $34 \mathrm{mmHg}$. Membrane resection was performed in the patient who was diagnosed with a subaortic fibromuscular ring during surgery. She was discharged on postoperative 12th day following a problemfree postoperative period. Postoperative echocardiography of the patient revealed anterior mitral valve insufficiency with a cleft of 3-4 $\mathrm{mm}$ in diameter. She was scheduled for follow-up with ACE inhibitor therapy. However, there was a significant increase in the left atrial diameter $(32 \times 30 \mathrm{~mm})$ and an increase in the diameter of the mitral anterior cleft and of the insufficiency. Patient was taken in for catheterization under general anesthesia, with prophylaxis and thrombophylaxis for endocarditis. The $5 \mathrm{~mm}$ cleft in the mitral valve was safely closed with Amplatzer Ductal Occluder II (Figure 1). No mitral insufficiency flow was detected in transesophageal echocardiography. The patient was discharged with oral thrombophylaxis therapy. Follow-up results demonstrated a decrease in left atrial diameter $(22 \times 20 \mathrm{~mm})$ and no valvular insufficiency apart from the pre-existing central minimal insufficiency in the mitral valve.

Conclusion: It is possible to perform interventional closure of clefts in the mitral valve on the right patient and under appropriate positions. This is the first case in which the mitral cleft is closed with ductal occluder in children.

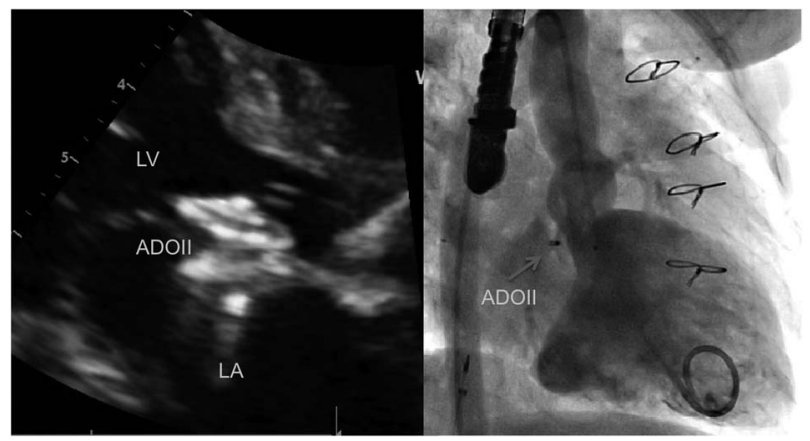

Figure 1. 


\section{P-174}

Intra-cardiac Echocardiography guided trans-catheter closure of Patent Ductus Arteriosus without contrast angiography

Suda K. (1), Maeda Y. (1), Kuwaraha H. (1), Kagiyama Y. (2), Takase R. (1), Kotada Y. (1), Kudo Y. (2), Iemura M. (2)

Kurume University School of Medicine (1); St. Mary's Hospital (2), Kurume City, Japan

Introduction: Though contrast angiography is the standard guidance of trans-catheter closure of patent ductus arteriosus (TC-PDA), it is contra-indicated in patients with severe renal disease that often seen in senile patients. We have developed intra-cardiac echocardiography (ICE) guided TC-PDA (Cathet Cardiovasc Intervent 2015). We report sequential 6 cases that successfully underwent TC-PDA without contrast angiography.

Methods: Subjects were 6 patients with PDA and median age of 54.4 (35.4 - 66.1) years old. The median size of PDA was 4.0 (3.2 11.7) $\mathrm{mm}$ with median Qp/Qs of 1.8 (1.4 and 2.4), respectively. The oldest patient suffered from renal dysfunction and 2 patients had pulmonary hypertension. Prior to the TC-PDA, all patients underwent contrast X-ray computed tomography to clarify the anatomy. ICE catheter was inserted through $2^{\text {nd }}$ sheath at femoral vein and placed at main or left pulmonary artery. During the TC-PDA, we primarily used ICE to guide the procedure.

Results: We could successfully place Amplatzer Duct Occluders in 5 and Amplatzer Septal Occluder in 1 without contrast angiography. ICE at main or left pulmonary artery has allowed us to determine the diameter and length of PDA, to monitor the device placement, and to determine the residual shunts. ICE did not increase the risk of complication except for transient arrhythmia, though new operator needs some learning time to understand orientation of ICE.

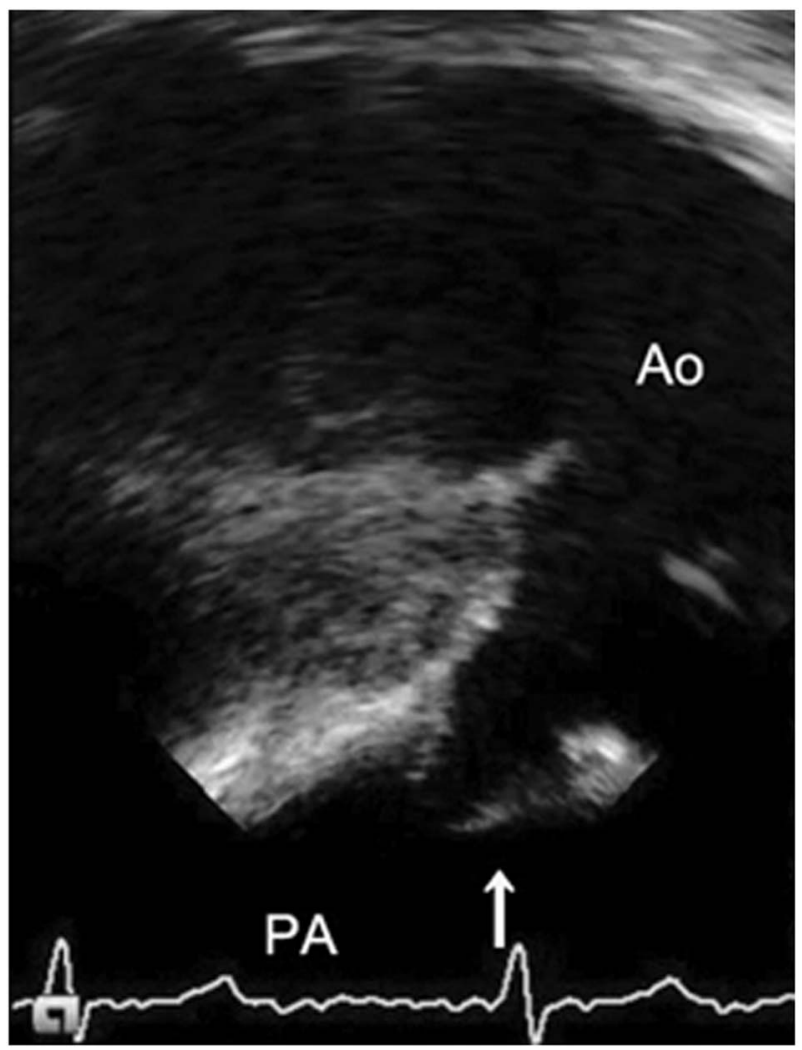

Figure.
Conclusions: ICE-guided TC-PDA without contrast angiography is feasible and can be the standard treatment for adult patients, especially complicated by renal dysfunction.

\section{P-175}

Difficulties and possible solutions in establishing a paediatric cardiac program in a developing country Sadiq M., Khan A.

The Children's Hospital and Institute of Child Health Lahore Pakistan

Background: Establishment of pediatric cardiac services in developing countries is a major challenge. Once established, it is even bigger a challenge to cater for the needs of very large populations. In Pakistan with population of $>200$ millions, over 15000 babies require some form of heart surgery or intervention each year. The challenges include paucity of centers and personnel, limited resources, overcrowding, illiteracy, and infection control.

Objectives: To analyze difficulties and propose solutions, in establishing a pediatric cardiac program.

Methods: The Children's Hospital Lahore is a 1100-beded public sector multidisciplinary hospital, with all the support facilities. We established a congenital cardiac program where neonatal interventions and open-heart surgeries are being performed at all ages. Results: Pediatric cardiac program started in 1999. The major problems faced include recruitment of trained staff and equipment and lack of resources for ongoing financial needs. So far we have performed surgery on $>6000$ children, with an overall mortality of $7 \%$ in the year 2015. The cost for an open-heart surgery is about US $\$ 2000$ to $\$ 4000$ and for a closed heart surgery is US $\$ 1500$ to $\$ 2000$. This is still too much for a poor patient and support by the government and philanthropic organizations ensures no patient is denied treatment for lack of funds. The current waiting list for routine open heart surgery is over 5 years. The interventional program was developed with the help of international experts visiting the unit on regular basis. There is no waiting time for the interventions.

Conclusions: Despite improved infrastructure and skills, the logistics, affordability, staffing, late presentation, nutritional status, infection, and unfavorable economics continue to negatively influence the overall results. Continued determination and governmental as well as philanthropic support, is key to the establishment of pediatric cardiac program.

\section{P-176}

When portal blood bypasses the liver: presentation and management of congenital Abernethy extra-hepatic shunts Roggen M., Eyskens B., Maleux G., Witters P., Cools B., Heying R., Suys B., Boshoff D., Gewillig M. Leuven University, Belgium

Introduction: Congenital porto-systemic shunts are rare and hard to diagnose. Clinical manifestation can vary from asymptomatic to extreme forms of cyanosis, pulmonary hypertension, liver insufficiency and mental retardation. Treatment options, depending on the type of abnormality and timing of detection, vary from (stepwise) occlusion to liver transplant. We report our experience.

Methods: Test balloon occlusion was performed to confirm portal pressure $<18 \mathrm{mmHg}$ or portal-caval gradient $<8 \mathrm{mmHg}$. The shunt was then either closed or partially obstructed.

Results: 5 patients presented between 2006 and 2017. Presenting symptoms were very heterogeneous: antenatal screening (2), cyanosis 75\% (1), PMR \& pulmonary hypertension by torrential arterio-arterial collaterals (1), and fortuitous in scimitar 
syndrome (1). The shunts were reached percutaneously either via the jugular or femoral vein (4) or by direct hepatic puncture (1). Four shunts were closed with a device in a one-step-procedure with Amplatzer Vascular or mVSD plugs (3) or a coil (1), depending on the size of the shunt. One patient failed the test occlusion; a very short landing site was determined (12 $\mathrm{mm}$ long, $10 \mathrm{~mm}$ stretched diameter); flow-reductor implanted through an 8 F sheath using a $6 \mathrm{~mm}$ Occlutech Muscular VSD occluder (disks $13 \mathrm{~mm}$ ) preperforated 5/16 mm Bentley covered stent (image).

In 2 patients the shunt was completely closed with no residual flow. In one patient the largest shunt was closed but three collaterals became evident at closure requiring later closure; another patient had mini collaterals which remain monitored. In the patient with the flow-reductor, echo Doppler one month later, showed small portal veins appearing in the liver allowing later complete closure.

Conclusions: Porto-caval shunt can be managed percutaneously. Early diagnosis allows early and complete closure; this stresses importance of fetal screening. Treatment at late presentation with hypoplastic portal veins may require stepwise occlusion with a flow-reductor, hoping to avoid liver transplantation.

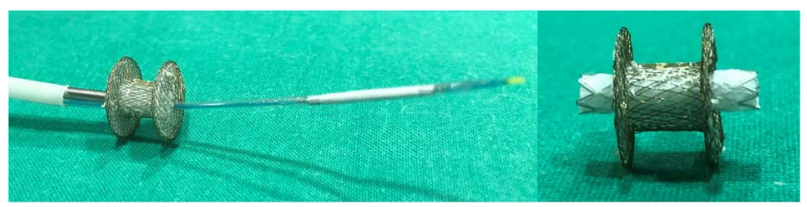

Figure.

\section{P-177}

\section{Coronary Artery Fistula in Infant}

Culqui Levano E. (1), Mariño Vigo C. (1), Salinas Mondragón C. (1) Instituto Nacional De Salud Del NiñoLima-Perú

Introduction: Defined as an abnormal connection between a coronary artery and a cardiac chamber or vessel, represents $0.2-0.4 \%$ of congenital heart anomalies, is the most common coronary anomaly, occurs in $60 \%$ of the right coronary and $40 \%$ of the left coronary. They can drain to the right ventricle (40\%), right atrium $(25 \%)$, the pulmonary artery $(15 \%)$, the coronary sinus $(7 \%)$, the left atrium (5\%) or the left ventricle (3\%) . Only $10 \%$ of $20 \%$ of pediatric patients are symptomatic. It is usually congenital, it can be acquired after thoracic trauma, cardiac surgery or surgery. Case Report: Infant of 3 months, P: 3.320, Down syndrome, hospitalized three times due to heart failure and respiratory symptoms presenting cough, rhinorrhea, interrupted lactation, profuse sweating and respiratory difficulty at admission. CF: 154 FR : 58 T: 36.8 SAT: $99 \%$, snoring, expiratory wheezing, sub costal tirage, ejection systolic murmur II / VI in left sternal border pulmonary focus and continuous murmur at level 3 to 4 EII line for right sternal, hepatomegaly. Normal CPK and CPKMB, chest $\mathrm{x}$-ray with cardiomegaly. Normal electrocardiogram Echocardiography: Intrauricular communication OS of $17 \mathrm{~mm}$. Tricuspid insufficiency of moderate degree. Ao deficiency of slight degree. Mild pulmonary insufficiency. Dilation of right coronary artery of severe degree of $6 \mathrm{~mm}$. Dilation of right cardiac chambers of moderate degree. Left ventricular systolic function preserved. Angiotem: interatrial communication. Dilation of right coronary artery. Catheterization Coronary fistula from right coronary to right atrium $(6 \mathrm{~mm})$ Mild pulmonary hypertension (PAPS: $39 \mathrm{mmhg}$ ). Stationary evolution awaiting surgical treatment, closure was attempted by catheterization but due to the tortuosity of the fistula, closure was not achieved.
Conclusions: Surgical closure or interventional catheterization is the treatment for patients with symptoms. Only $10 \%$ of $20 \%$ of pediatric patients are symptomatic. Some authors recommend leaving the coronary fistula without treatment in pediatric patients if the shunt is small, in the case presented the coronary fistula produced symptoms early and hemodynamic compromise so the indication is the surgical closure when presenting the fistula a complex anatomy.

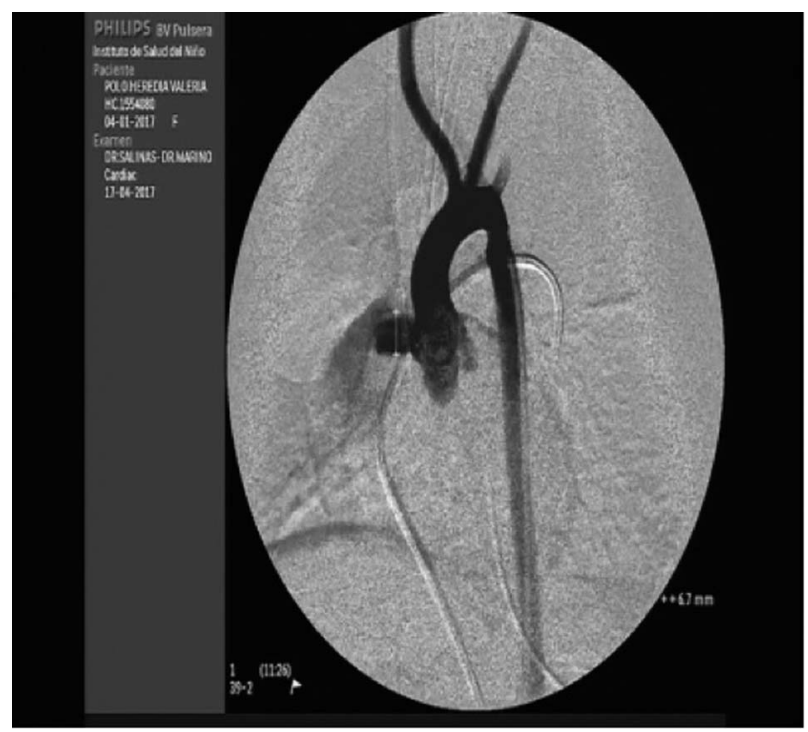

Figure.

\section{P-178}

Exercise Doppler gradients in patients with effectively treated aortic coarctation

Rato J. (1), Martins D. (1), Congo K. (2), Mendes S.C. (1), Anjos R. (1)

1 - Hospital Santa Cruz, Lisboa, Portugal; 2 - Hospital do Espírito Santo, Evora, Portugal

Introduction: Aortic coarctation causes abnormal responses to exercise such as induced hypertension and increased gradients in the descending aorta and these may persist despite correction. Different options of treatment may have distinct patterns of cardiovascular response to exercise, and its knowledge may influence management of these patients.

Methods: We performed exercise testing and transthoracic echocardiography before and up to one minute after maximum exercise on patients with effectively treated aortic coarctation (with corrected gradients $<20 \mathrm{mmHg}$ ) by surgery or stent implantation. Peak and corrected Doppler gradients at the isthmus were recorded at rest and up to one minute after maximum exercise.

Results: Thirty four patients were included, 28 male (82\%), median age 29 years (range 13-53). The last intervention for coarctation treatment was surgery in 11 patients $(32 \%)$ and stent placement in $23(68 \%)$. Median age at the last procedure was 23 years (1-47). Median time after treatment was 2 years $(0.5-22)$. Twenty one patients had hypertension (62\%) and 16 of these were on antihypertensives. Exercise tests had a median duration of 10 minutes (7-15). There were 11 exercise induced hypertensive responses (32\%). Median peak gradients were $18 \mathrm{mmHg}(5-40)$ at rest and $38 \mathrm{mmHg}(8-74)$ after exercise. Median corrected gradients were $12 \mathrm{mmHg}(5-20)$ and $25 \mathrm{mmHg}(5-70)$ respectively. There was no 
statistically significant difference between surgical or stent groups in terms of hypertensive response or measured Doppler gradients either at rest or after exercise. The group of patients who were treated before 20 years of age had higher gradients before treatment and a significantly higher gradient at rest and after exercise $(\mathrm{p}=0.003$ and $\mathrm{p}=0.001$, respectively).

Conclusion: These results suggest that response to exercise in patients with effectively treated aortic coarctation by surgery or stent implantation is similar, specifically in terms of exercise induced hypertension and Doppler gradients. There were significantly higher peak gradients at rest and after exercise on patients treated at a younger age, presumably because of higher gradients before treatment.

\section{P-179}

Early postoperative cardiac catheterization in patients with functionally univentricular hearts

Loureiro P. (1), Tanase D. (2), Eicken A. (2), Ewert P. (2),

Cleuziou J. (3), Georgiev S. (2)

(1) Paediatric Cardiology Department, Hospital de Santa Marta, Centro Hospitalar Lisboa Central - EPE, Lisbon - Portugal; (2) Paediatric Cardiology and Congenital Heart Defects Department, German Heart Center Munich, Munich - Germany; (3) Department of Cardiovascular Surgery, German Heart Center Munich, Munich - Germany

Introduction: Patients with univentricular hearts undergo palliative operations that require complex surgical techniques. Cardiac catheterization (CC) may be useful, in the early postoperative period, to clarify and manage residual lesions, however there have been concerns about its safety.

Methods: We retrospectively analyzed all consecutive patients with functionally univentricular hearts who underwent CC in the immediate post-operative period, between 2005 and 2016 .

Results: Over 11 years, 118 patients underwent 166 CC in the first 30 days after a surgery. 62 patients $(53 \%)$ were male, the median age was $1.5 \pm 4.6$ years $(\min 0.01, \max 31.6)$ and CC was performed on average $9.8 \pm 7.8$ days (min 1 , max 29) after surgery. 98 CC (59\%) were diagnostic and 68 (41\%) interventional. During the 68 interventional $\mathrm{CC}$ a total of 87 interventions were performed, $54(62 \%)$ of which on a recent surgical anastomosis. One, two and three similar or distinct procedures, were performed on 50 (73.5\%), $17(25 \%)$ and $1(1.5 \%)$ interventional CC respectively. There were 14 intraprocedural complications, 4 (29\%) classified as minor, $6(43 \%)$ as moderate, $3(21 \%)$ as major and $1(7 \%)$ as catastrophic. There was no association between the incidence of CC complications and the presence of mechanical ventilation, inotropic support, ECMO, open chest, type of CC performed (diagnostic versus interventional) $(\mathrm{p}=0.198)$, or intervention on a recent surgical anastomosis versus intervention elsewhere $(\mathrm{p}=0.151)$.

Twenty eight surgical reinterventions were performed on average $1.9 \pm 1.8$ days $(\min 0.5 ; \max 7)$ after a CC: $20(71 \%)$ after a diagnostic CC and 8 (29\%) after an interventional CC.

Sixteen patients $(14 \%)$ died in the first 30 days after a CHS. Those that underwent an interventional CC had a better survival than those who underwent only a diagnostic study $(p=0.015)$. No difference was noticed between the patients who had an intervention on a recent surgical anastomosis and those who had an intervention elsewhere $(\mathrm{p}=0.178)$.

Conclusions: Early postoperative CC is a safe procedure in the treatment of patients with functionally univentricular hearts. It not only allows an attempted diagnosis of residual lesions and a timely surgical repair, but also the management of some abnormalities percutaneously.

\section{P-180}

Long term outcomes of balloon aortic valvuloplasty in neonates, infants and children

Quyam S., Bellsham-Revell H., Rosenthal E., Simpson J.M.,

Hewett S., Patel J., Qureshi S.A.

Evelina London Children's Hospital, London, UK

Introduction: Percutaneous balloon aortic valvuloplasty (BAV) is an established and efficacious treatment for the relief of aortic stenosis (AS). We aimed to describe its long term outcomes in children.

Methods: Patients $>16$ years who underwent primary BAV for AS at our institution between 2004 and 2017 were included. Exclusion criteria were; intended single ventricle palliation; predominant sub/supra-valvar AS; and predominant aortic regurgitation (AR). All patients were followed up until death or end of study period.

Results: Study Population: 73 patients with median age 0.2 years (range 1 day -15.2 years) underwent BAV. Patient demographics and echocardiography-derived parameters are summarised in Table 1. 39 (53\%) were neonates, $11(15 \%)$ were infants and $23(32 \%)$ were older children. 16 (22\%) had critical AS. Patients were growth restricted with a mean weight $\mathrm{z}$-score of $-0.7 \pm 1.6$. The peak Doppler derived gradient was $71 \pm 29 \mathrm{mmHg}$ and mean was $38 \pm 17 \mathrm{mmHg}$. There was no difference in severity between age groups, but neonates were more likely to have impairment of ventricular function. Prior to intervention, no patient had more than mild AR which was present in $6(8 \%)$. Morphology was bicuspid in 39 (55\%). Immediate Results: Echocardiographic Doppler derived gradient was reduced from 71 to $36 \mathrm{mmHg}$ (P $<0.001), 14(19 \%)$ patients had a residual gradient of $>50 \mathrm{mmHg}$. AR increased in $50(68 \%)$ patients, to moderate in $14(20 \%)$ and severe in $2(3 \%)$. There was no procedural mortality. Follow up results: Median follow up was 5.3 years. Three late deaths occurred (3.8\%), all in neonates. Patients showed catch up growth with an increase in mean weight $\mathrm{z}$ score from $-0.7 \pm 1.6$ to $-0.4 \pm 1.7$ (P $0.36)$ a median of 1.5 years post intervention.

$26(36 \%)$ patients required re-intervention, most commonly Ross procedure $(\mathrm{n}=11,14 \%)$.

10 -year Freedom from re-intervention was $66 \% \pm 7 \%$ (95\% CI 61 $86 \%$ ) and median time to re-intervention was 9.3 years. Reintervention was more commonly associated with critical AS (P $0.05)$ and lower aortic annular $\mathrm{z}$ scores.

Conclusions: BAV is effective and associated with low procedural mortality in children of all ages with a lengthy freedom from reintervention. Re-intervention is more common in neonates with critical AS and impaired LV function.

Table 1. Patient demographics and echocardiography-derived parameters

\begin{tabular}{lccccc}
\hline & All & Neonates & Infants & Child & $P$ \\
\hline $\mathrm{N}(\%)$ & $73(100)$ & $39(53)$ & $11(15)$ & $23(32)$ & \\
Age (y) & $0.2(1 \mathrm{~d}-15.2 \mathrm{y})$ & $0.08(1-30 \mathrm{~d})$ & $0.5(0.3-0.7)$ & $7.2(1-15.2)$ & - \\
Female $\mathrm{n}(\%)$ & $26(36)$ & $11(28)$ & $3(27)$ & $12(52)$ & 0.17 \\
Weight (Kg) & $11.5 \pm 14.3$ & $3.9 \pm 1.2$ & $6.1 \pm 2.9$ & $28 \pm 17.3$ & - \\
Weight z-score & $-0.7 \pm 1.6$ & $-1.0 \pm 1.3$ & $-1.4 \pm 2.1$ & $0.1 \pm 1.5$ & 0.01 \\
Annulus z-score & $-0.3 \pm 1.6$ & $-1.0 \pm 1.6$ & $0.4 \pm 1.8$ & $0.1 \pm 1.5$ & 0.28 \\
Bicuspid n (\%) & $39(55)$ & $22(52)$ & $6(55)$ & $28(53)$ & 0.89 \\
Peak gradient (mmHg) Pre & $71 \pm 29$ & $72 \pm 34$ & $66 \pm 31$ & $71 \pm 17$ & 0.84 \\
AR none/trivial/mild Pre & $54 / 11 / 6$ & $35 / 2 / 2$ & $5 / 4 / 1$ & $14 / 5 / 3$ & 0.01 \\
FS (\%) Pre & $34 \pm 12$ & $28 \pm 12$ & $40 \pm 6$ & $41 \pm 9$ & $<\mathbf{0 . 0 0 1}$ \\
Normal LV function n (\%) & $50(70)$ & $20(51)$ & $8(80)$ & $22(100)$ & $\mathbf{0 . 0 0 5}$ \\
Re-intervention & $26(36)$ & $14(36)$ & $3(27)$ & $9(39)$ & 0.89 \\
Peak gradient (mmHg) & $38 \pm 17$ & $36 \pm 16$ & $38 \pm 15$ & $38 \pm 20$ & 0.35 \\
$\quad$ Post & & & & & \\
AR none/trivial/mild n & $11 / 21 / 23$ & $3 / 11 / 16$ & $2 / 5 / 2$ & $6 / 5 / 5 / 7$ & 0.19 \\
AR mod/severe n & $14 / 2$ & $6 / 2$ & $1 / 0$ & $7 / 0$ & - \\
\hline
\end{tabular}




\section{P-181}

Use of Transcatheter Flow Reducer in Complex Fontan Circulation Based on Computation Fluid Dynamics Simulation

Liuba P. (1), Frieberg P. (2), Strand D. (1), Maxedius A. (1), Forslid A. (3), Carlsson M. (2)

Pediatric Cardiology (1) and Clinical Physiology (2), Skåne University Hospital and Lund University (3), Lund, Sweden

Background: Patients with single ventricle (SV) and heterotaxy syndrome are at increased risk for pulmonary arteriovenous malformations (PAVM) after Fontan completion. We report a complex case in whom Computation Fluid Dynamics (CFD) simulation was initially employed to evaluate the potential use of a new transcatheter flow reducer (TFR) to improve flow distribution to the AVM-affected lung and preliminary results from an animal study aimed to test the efficacy of this device and its percutaneous retrieval several weeks after device implantation.

Methods and Results: A 13-year old patient with functionally SV and absent hepatic segment of the IVC with hemyazygos continuation to the left SVC, with previous Kawashima followed by surgical incorporation of the hepatic veins to the central PA, was referred to our institution due to significant desaturation. Cardiac catheterization revealed normal pressures, diffuse AVM in the right lung and hypoplastic central PA. CMR showed no hepatic flow to the lung with AVM. Based on CFD simulation, most optimal hepatic flow distribution to the lungs was achieved with enlargement of central PA combined with reduction of IVC flow. A prototype flow reducer (Occlutech GMBH,Germany; $20 \times 30 \mathrm{~mm}$ with $10 \mathrm{~mm}$ fenestration) made of nitinol mesh with polyurethane covering, was then implanted percutaneously via a 12 Fr Occlutech delivery sheath in the intrahepatic segment of the IVC in 3 domestic swine followed by re-catheterization 2 weeks later. During this period, all animals received warfarin o.d. The device implantation and the follow-up were uneventful. Two weeks after implantation, the device was patent in 2 animals and found embolized in the 3rd animal most likely due to significantly larger IVC in this animal. Although there was no significant gradient across the device, collaterals were noted on angiography with approximately $10 \%$ increase in SVC/IVC flow on CMR. In 1 animal, the device was easily retrieved percutaneously. Conclusion: Transcatheter reduction of systemic venous flow in complex univentricular circulation with PAVM might be useful in optimizing hepatic flow distribution. Additional studies at our institution are under way to further assess the utility of CFD simulation in interventional and surgical treatment of patients with Fontan circulation.

\section{P-182}

The outcome of percutaneous pulmonary valvuloplasty in infants. A single center experience

Sodini C., Lunardini A., Giusti S., Spadoni I.

Pediatric Cardiology and GUCH Unit, 'G. Monasterio' Tuscan

Foundation, Massa (Italy)

Objectives: we aimed to evaluate 30 years' experience in pulmonary valvuloplasty (PVP) performed in children with pulmonary valve stenosis (PVS), within 12 months of age, in our Institution. A particular focus was paid to incidence and risk factors of recurrent PVS and progressive pulmonary insufficiency (PI).

Methods: retrospective review of paper-based and digital archives from June 1988 to February 2017 was undertaken. Statistical significance was set as P-value $<0,05$. Success of PVP was defined as systolic pressure gradient $(\mathrm{SPG})<36 \mathrm{mmHg}$.
Results: ninety-two patients (critical PVS 34, severe PVS 58) underwent 104 PVP, in the first year of life, of which 12 (11,5\%) were repeated PVP in early follow-up. Immediate success rate was $88 \%$, need of surgical RVOT reconstruction $5 \%$, complication rate $6,8 \%$, absent mortality. Seventy-one patients were followed for $>1$ year ( $9,6 \pm 6,6$, range $1,1-27,9$ years). Long-term success rate was $87 \%$, freedom from surgery $94 \%, 94 \%$ and $84 \%$ at 5,10 and 15 years respectively. Three patients needed RVOT reconstruction, and 1 PVP. In 65 patients Doppler peak SPG 21,2 $\pm 10,5$, mean $12,9 \pm 5,6 \mathrm{mmHg}$. Prevalence of moderate/severe PI was $13 \%$; 3 patients (4\%) required pulmonary valve replacement, after 15,4 $\pm 4,6$ years. Balloon to pulmonary valve annulus ratio $(\mathrm{B} / \mathrm{A})$ was $1,18 \pm 0,16$ in the first PVP, without variation along the study period, and $1,29 \pm 0,11$ in the second $(p=0,005)$, annulus $Z$-score was $<2$ in $50 \%$ patients with repeated PVP. Predictive factors of recurrent stenosis were age $<11$ days and annulus Z-score $<-1,21$ at first PVP. BSA at first PVP $<0,22 \mathrm{~m} 2$ was a risk factor for significant PI; no correlation was found with $\mathrm{B} / \mathrm{A}$ nor with period of intervention.

Conclusions: overall critical stenosis had a worse outcome. Prevalence of progressive PI in our series is lower than that found in literature: choosing a low B/A since the beginning of our experience $(<1,25)$ could have protected by this serious complication. Our results confirm the effectiveness of PVP as first-choice therapy for PVS in pediatric patients. Multicenter studies are warranted to further evaluate risk factors for residual defects in larger populations.

\section{P-183}

Percutaneous closure of traumatic atrial septal defect, where the problem lies?

Sirico D., Annoni G., Mauri L., Moreo A., Giannattasio C., Vignati G. Ospedale Niguarda Cà Granda, Milano (Italy)

Introduction: We report the unique case of a man, who sustained violent blunt chest trauma resulting in cardiac septal defect (ASD), followed by paradoxical cerebral fat embolism. We also describe the challenging percutaneous closure of traumatic ASD.

Case report: A 21-year-old male was involved in a high-speed motor vehicle accident. Vital signs were: BP 115/90 mmHg; HR120 bpm; PO2 84\%. X-ray: ribs and sternum fractures, and a displaced right femoral shaft fracture. Lab tests: leucocytosis $(30540 / \mathrm{mm} 3)$, CPK $871 \mathrm{U} / \mathrm{L}, \mathrm{CK}-\mathrm{MB} 8.7 \mathrm{mcg} / \mathrm{L}$, Hs Troponin T $107 \mathrm{ng} / \mathrm{L}$. EKG: sinusal tachycardia. Three hours later, the patient underwent right femoral fracture nailing. On 2nd day, he showed psychomotor agitation and spread cutaneous petechiae. Brain CT scan revealed multiple bilateral hypodense spots of the white matter. Fat embolism syndrome (FES) with paradoxical cerebral involvement was suspected. 2D TEE showed the presence of $8 \mathrm{~mm}$ large atrial septal defect (ASD), with left-to-right shunt, of probably traumatic origin. The patient underwent urgent ASD percutaneous closure with $13 \mathrm{~mm}$ occluder device. The next day, routine 2D-TTE revealed device embolization and 2D-TEE showed a larger ASD (diameter shifted from 8 to $18 \mathrm{~mm}$ ) with oedematous borders and significant left-to-right shunt. CT scan revealed device migration at infradiaphragmatic aorta. Through left groin access, the device was successfully removed using a Goose-neck catheter and inferior vena cava filter was positioned. On 7th day from admission, new ASD percutaneous closure was performed, using a balloon sizing catheter, a $22 \mathrm{~mm}$ occluder device was deployed without any residual shunt. The patient was discharged on day $23 \mathrm{rd}$.

Discussion: Although uncommon, cardiac septal defects are known complications of blunt chest trauma. In our patient FES, due to femoral shaft nail fixation, unmasked a new born ASD previously excluded. Urgent percutaneous ASD closure resulted in device 
embolization due to progressive ASD enlargement maybe because traumatic ASD is surrounded by necrotic and friable tissue that may not hold an occluder device. From these data, we conclude that percutaneous closure of traumatic ASD is a feasible procedure, but it should be avoided in the acute phase after trauma due to the high risk of device embolization.
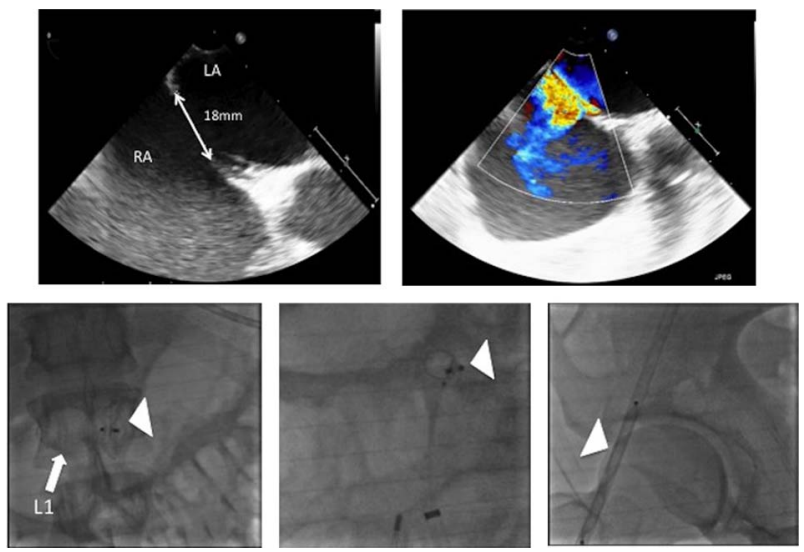

Figure.

\section{P-184}

Single center experience in usage of the ultrahigh pressure balloons in dilatation of rigid vessel stenosis in patients with congenital heart disease

Pursanov M.G., Chiaurelly M.R., Sobolev A.V., Atajanov U.U. A.N. Bakoulev Scientific Center for Cardiovascular Surgery, Moscow, Russian Federation

Objective. to exam our experience in usage of UHPB in patients with CHD and rigid vessel stenosis in various vascular systems. Materials and methods. During the period from April 2015 until January 2017 PTBA of 58 various vessel segments was performed in 47 pts. Patients age varied from 6 months to 32 years $(10,34+5,57$ years $)$ and weight from $4,6 \mathrm{~kg}$ to $96 \mathrm{~kg}$ $(39,4+22,6 \mathrm{~kg})$. In $30(63,8 \%)$ patients were previously performed ineffective repeated PTA (12-16 atm).

Balloon dilatation using UHPB were performed in 37 pts with pulmonary artery stenosis (48 segments); in 4 pts with aortic isthmus coarctation; 1 patient with stenosis of right ventriclepulmonary artery conduit; in 1 patient with superior vena cava (SVC) stenosis. Pulmonary artery PTBA were performed after right ventricle outflow tract reconstruction in 21 patients due to tetralogy of Fallot, in 14 after radical surgery in complex CHD, in 4 after bidirectional cavopulmonary anastomosis.

Results. Usage of the UHPB was examined according to a full balloon expansion. In 98,3\% of all cases (57 of 58 segments) of PTBA full expansion to the nominal volume were achieved. Only in 1 case $(1,7 \%)$ we were unable to achieve full expansion of the UHP due to fracture of previously implanted pulmonary artery stent. We've registered no complications associated with usage of UHPB. Conclusion. Usage of UHPB is an effective and safe method of managing rigid stenosis of various vascular systems, allowing to eliminate stenosis in $98,3 \%$ of cases.

\section{P-185}

Hybrid stenting of hypoplastic pulmonary arteries in surgical repair of complex CHD

Pursanov M.G., Shatalov K.V., Chiaureli M.R., Berishvili D.O., Kim A.I., Sobolev A.V., Arnautova I.V.,Baveyan A.O.
A.N. Bakoulev Scientific Center for Cardiovascular Surgery, Moscow, Russian Federation

Objective: to determine results and efficiency of hybrid stenting of the pulmonary artery in patients with various CHD with the obstructive pathology of the pulmonary artery (PA).

Methods: hybrid stenting of the PA was performed in 24 patients (26 lesions) with various CHD. Patient age was from 10 month to 19 years (median 4,4+4,6) and weight from 5,3 to $77 \mathrm{~kg}$ (median $18,6+18,7)$. Hybrid stenting was performed in 14 patients $(58,3 \%)$ under 3 years old (y.o.), in $4(16,7 \%)$ - from 3 to 6 y.o. and in $6(25 \%)$ - older than 6 y.o. Hybrid stenting was performed in operation suit using mobile angiographic C-arm GE 9900. In $7(29,2 \%)$ patients PA stenting was performed during hemodynamic correction of CHD, in $5(20,8 \%)$ - during primal surgical repair of $\mathrm{CHD}$, in $6(25 \%)$ - in patients with complications after surgical repair of CHD and in $6(25 \%)$ - in patients with hypoplastic left heart syndrome. In general 6 operations were performed as an emergency procedure and in 18 - as a scheduled surgery. Stenting of left PA was performed in 18 cases, of right $\mathrm{PA}$ - in 7 cases and in 1 case we performed a stenting of the pulmonary trunk.

Results: in $23(95,8 \%)$ of 24 patients hybrid stenting was effective. In 1 case $(4,2 \%)$ after balloon expansion, stent migrated to the proximal part of the left LA which resulted in surgical removal and repair of the left PA ostium. After stent implantation in all cases, we achieved a complete management of the PA obstruction. One patient (after primal repair of the PA atresia) died due to increasing polyorganic insufficiency in short follow-up period, remaining 23 patients were discharged from a hospital with significant improvement.

Conclusion: intraoperative hybrid stenting is effective and save procedure. Vascular access should be determined individually, according to patient anatomy and morphological specifics of CHD. Hybrid procedure allows more effective and safe hemodynamic and primal surgical repair. In cases of complications, they can be managed with direct surgical intervention.

\section{P-186}

Infective Endocarditis after percutaneous pulmonary valve implantation - Long-term single centre experience Tanase D. (1), Ewert P. (1), Hager A. (1), Georgiev S. (1), Cleuziou J. (2), Hess J. (1), Eicken A. (1) 1 German Heart Centre Munich, Department of Paediatric Cardiology and Congenital Heart Defects, Munich, Germany; 2 German Heart Centre Munich, Department of Cardiovascular Surgery, Munich, Germany

Aims: This study sought to investigate the incidence of infective endocarditis (IE) after percutaneous pulmonary valve implantation (PPVI). Recently, there is some concern, that increased rates of IE occur after PPVI with the Melody valve. It is imaginable that procedural steps prior to implantation, like crimping of valved stents or mechanical forces during dilation might favor the onset of IE in percutaneously implanted valves.

Methods and Result: The purpose of this study was to assess the prevalence and the incidence of IE after PPVI in a single centre long-term experience. The cumulative follow up time comprised 883.4 patient years for 226 valved stents implanted in the pulmonic position. The annualized incidence of IE for all patients receiving valved stents in the RVOT was $1.9 \%$.

Conclusion: The incidence for IE after PPVI is acceptable and comparable to surgically implanted biological valves. Despite some events of IE, freedom from reoperation is high, showing a good performance of the valve in the long time follow up. 


\section{P-187}

Transcatheter Closure of Arterial Duct in Infants < $6 \mathrm{~kg}$ : Amplatzer Duct Occluder type I vs. Amplatzer Duct Occluder II Additional Sizes.

Giordano M. (1), Santoro G. (2), Gaio G. (1), Palladino M.T. (1), Iacono C. (1), Capozzi G. (1), Carrozza M. (1), Russo M. G. (1) Paediatric Cardiology, A.O.R.N. "Ospedali dei Colli", University of Campania "Luigi Vanvitelli", Naples, ITALY (1); Paediatric Cardiology and GUCH unit, Ospedale del cuore "G.Pasquinucci", Massa, ITALY (2)

Objectives. Transcatheter closure of AD remains challenging in low-weight patients and using Amplatzer Duct Occluder devices is still considered off-label in infants less than $6 \mathrm{~kg}$. This study aimed to report a large, single-center experience of percutaneous arterial duct (AD) closure in infants $<6 \mathrm{~kg}$ as well as to compare the most frequently used devices, Amplatzer Duct Occluder type I (ADO I) and Amplatzer Duct Occluder II Additional Sizes devices (ADO II-AS) (St. Jude Medical Corp, St. Paul, MN, USA).

Methods. From November 2002 to December 2016, among the 764 patients submitted to percutaneous closure of $\mathrm{AD}$ at our Institution, 31 were infants $<6 \mathrm{~kg}$ (mean age $4.8 \pm 2.11$ months, range 0.03-10; mean weight $5.1 \pm 0.94 \mathrm{~kg}$, range 3-6). 14 patients (45\%) underwent ADO I (Group I) and 17 pts ADO II-AS (Group II) implantation. Results. AD diameter was $2.61 \pm 0.72$ (range 1.5-4.0) mm resulting in $\mathrm{QP} / \mathrm{QS}$ of $2.57 \pm 0.95$ (range 1.5-4.5). Mean pulmonary artery $(\mathrm{PA})$ pressure and $\mathrm{PA} /$ aortic pressure ratio were $25.58 \pm 6.41 \mathrm{mmHg}$ (range 14-38) and $0.50 \pm 0.19$ (range 0.230.91 ), respectively. Successful device deployment was achieved in all patients without procedural morbidity or mortality. Procedural and fluoroscopy times were not significantly different between groups. However, total absorbed $x$-ray was significantly lower in the Group II $(120.75 \pm 69.16$ vs $27.64 \pm 16.06 \mathrm{mGy}, \mathrm{p}<0.01)$. Immediate, $24 \mathrm{~h}$ and mid-term $(12 \pm 1 \mathrm{mos})$ complete occlusion was recorded in $71 \%, 90 \%$, and $97 \%$ of patients, respectively. Midterm occlusion rate did not significantly differ between the groups. Conclusions. Trans-catheter closure of AD with Amplatzer Duct Occluder devices is feasible, safe and effective also in infants less than $6 \mathrm{~kg}$, without significant difference between the most commonly used devices, ADO I and ADO II-AS.

\section{P-188}

Off-label use of duct occluder devices in low-income countries to close hemodynamically relevant perimembranous ventricular septal defects: a multicenter experience.

Sreeram N. (1), Udink ten Cate F.E.A. (1,2), Sobhy R. (3),

Kalantre A. (4), Sachdev S. (5), Subramanian A. (6), Koneti N.R. (5), Hamza H. (3), Jayaranganath M. (6), Kumar R.K. (4)

Heart Center Cologne, University Hospital of Cologne, Cologne,

Germany (1); Amalia Children's Hospital, Radboud University Medical Center, Nijmegen, the Netherlands (2); Cairo University Children's

Hospital, Cairo, Egypt (3); Amrita Institute of Medical Sciences, Kochi, India (4); CARE Hospital, Hyderabad, India (5); Sri Jayadeva Institute of Cardiovascular Sciences and Research, Bengaluru, India (6)

Objectives. The off-label use of duct occluder devices to close perimembranous ventricular septal defect (pmVSD) is of interest in low-income countries. Limited data exist on this technique. We sought to evaluate the feasibility, technical aspects, and outcome of transcatheter pmVSDs closure using duct occluder devices with a single retention disc.

Methods. From 2010 to 2016, 222 patients (female 47.7\%) were identified from databases of 5 participating institutions in whom
pmVSD closure was attempted using an Amplatzer Duct Occluder I or Lifetech duct occluder device.

Results. Patients ranged in age from 0.7 to 52 years (median, 7.0 years; $15.8 \%$ were 2 years of age or younger). Weight range was 4.3 to $70 \mathrm{~kg}$ (median, $18 \mathrm{~kg} ; 61.1 \%$ were less than $20 \mathrm{~kg}$ ). The mean size of the VSD was $6.2 \pm 1.5 \mathrm{~mm}$ (median $6.0 \mathrm{~mm}$ ). A large defect $(>6 \mathrm{~mm})$ was present in 137 patients $(61.7 \%)$. Device closure was successful in 218 patients $(98.2 \%)$. The $10 / 8 \mathrm{~mm}$ device was used in most patients $(\mathrm{n}=85,38.3 \%)$, and the vascular approach was from the right ventricle in 169 patients (76.1\%). There were 18 complications in $17 / 218$ patients $(7.8 \%)$. Three patients $(1.4 \%)$ developed complete heart block (transient $n=2$; requiring permanent pacing $\mathrm{n}=1)$. Median follow-up was 6 months (6 months -6 years). A mild residual shunt without clinical significance was seen in 10 patients at 6 months follow-up. Conclusions. The immediate results of transcatheter pmVSD closure using a duct occluder device with a single retention disc are promising. It is an effective and straightforward technique with a low rate of complications.

\section{P-189}

Cerebral oxygenation normalization is delayed following balloon atrial septostomy in neonates with transposition of the great arteries compared to preductal peripheral saturation

Puska V. (1), Pitkänen O. (2), Rautiainen P. (2), Kaskinen A. (2), Boldt T. (2), Pihkala J. (2), Andersson S. (2), Rahkonen O. (2) University Hospital of Oulu and University of Oulu, Oulu, Finland (1); University Hospital of Helsinki and University of Helsinki, Helsinki, Finland (2)

Introduction: Fetal hypoxia has been implicated in the abnormal brain development seen in newborns with congenital heart disease. Following a balloon atrial septostomy (BAS) in patients with transposition of the great arteries (TGA) cerebral oxygenation increases rapidly but very little is known about the direct effect of BAS on the neonatal brain and on cerebral oxygenation. It is yet unclear how BAS affects cerebral oxygenation and if rapid increase of oxygen delivery results in brain reperfusion injury in neonates with TGA.

Methods: We performed a prospective single center study to evaluate changes in the cerebral oxygenation before and for a period of 48 hours following BAS in 12 neonates with TGA. Data collected included peripheral oxygen saturation, mixed venous saturation, regional cerebral tissue oxygen saturation $\left(\mathrm{r}_{\mathrm{c}} \mathrm{SO}_{2}\right)$ measured by using near infrared spectroscopy, heart rate, blood pressure and hemoglobin prior and following BAS. Differences in the course of NIRS, preductal oxygen saturation, and calculated cerebral oxygen consumption between the two groups at different time points were analyzed. We also analyzed metabolic status of the patients including acidosis, lactate, and ischemia markers e.g. S100 before and following BAS.

Results: $9(75 \%)$ patients underwent BAS at the age $4.6( \pm 2.7)$ hours. Six $(50 \%)$ patients had prenatal diagnosis. There were $1(1 / 12)$ early death prior to the septostomy. Lowest preductal peripheral oxygen saturation at admission was median of 64.5 (range 39.0 - 92.0), before BAS 85.6 (range 62.0 - 90.6), 5 minutes following BAS 86.8 (range $70.6-91.4$ ) and $24 \mathrm{~h}$ after BAS90.0 (range 85.2-93.6). Lowest $\left(\mathrm{r}_{\mathrm{c}} \mathrm{SO}_{2}\right)$ at same time points were 50.0 (range $35.0-70.0$ ), 52.8 (range $36.4-72.5$ ), 54.5 (range $37.2-$ 73.8 ) and 69.2 (range $58.8-80.8$ ).

Conclusions: $\left(\mathrm{r}_{\mathrm{c}} \mathrm{SO}_{2}\right)$ levels rise at a slower rate than peripheral oxygen saturation values achieving stable level in 48 hours after BAS. Our data suggests that normalization of the cerebral 


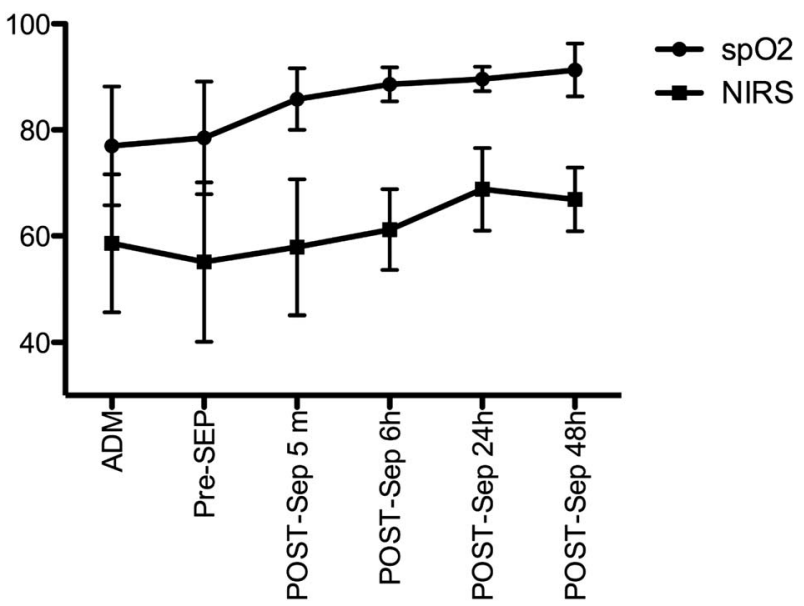

Figure.

oxygenation is delayed following the atrial septostomy. This may be due to brain reperfusion injury following the balloon atrial septostomy.

\section{P-190}

Ductus arteriosus stenting in patients with ductaldependent pulmonary blood flow- single center experience Gendera K., Ewert P., Georgiev S., Tanase D., Genz T., Hosny Fayed M., Eicken A.

Department of Pediatric Cardiology and Congenital Heart Disease, German Heart Center Munich, Technische Universität München, Germany

Background: Ductus arteriosus stenting has become an alternative, less invasive management option to Blalock-Taussig (BT) shunt implantation in selected patients with ductal-dependent pulmonary blood flow. The aim of this study was to assess safety, feasibility, short- and mid-term follow up and complications associated with this intervention.

Methods: Between August 2013 and July 201734 patients (one patient twice) with ductal-dependent pulmonary blood flow underwent cardiac catheterization for ductus arteriosus stenting as a first step management. Diagnoses: pulmonary atresia (PA) with a ventricular septal defect $(n=5)$, PA with an intact ventricular septum $(n=7)$, critical pulmonary stenosis (PS) with a doubleoutlet right ventricle $(n=5)$, critical PS $(n=3)$, Tetralogy of Fallot $(n=2)$, transposition of the great arteries $(n=1)$ and complex heart defect with ductal-dependent pulmonary circulation $(\mathrm{n}=11)$. The median age of the patients was 10 days (min.1; max.1840) and median weight was $3 \mathrm{~kg}$ (min.1.7; $\max .13,6)$. Premounted coronary stents CoroFlex Blue (diameter:3-4 mm; $\mathrm{n}=33$ ) and Formula 414 stents (diameter: $6 \mathrm{~mm} ; \mathrm{n}=1$ ) were used to cover whole length of the ductus arteriosus. In 17 cases the intervention was performed from the femoral access (femoral artery $=15$,femoral vein $=2$ ), in 18 from the axillary access.

Results: Access and stenting for the ductus arteriosus were successful in 29 patients (85\%). In follow up, ductus restenting was required in one patient. Complications occurred in 6 interventions $(17 \%)$ (stent dislocation $n=2$; not covering whole ductus length $\mathrm{n}=2$; stent dislocation over balloon $\mathrm{n}=1$, arteriovenous fistula $\mathrm{n}=1$ ) and 5 of these patients needed surgical BT shunt placement. After successful ductus stenting the median time between intervention and next-step surgery was 86 days $(n=18$; $\min .48$; max.431). In re-catheterization (usually performed before surgery) median Z-score value of the LPA and RPA diameter $(\mathrm{n}=22)$ increased from $-0,87$ (min.-2,86; max.1,81) and -0,28 (min.-1,69; $\max .1,39)$ to 1.37 (min.-3.14;max.3.55; $\mathrm{p}<0.001$ ) and 0.95 (min.-0.91;max.3.67; $\mathrm{p}<0.001$ ), respectively.

Conclusion: Stenting of the ductus arteriosus is an appropriate alternative to surgical shunt interposition in many patients with ductal-dependent circulation. It provides satisfactionary pulmonary arterial growth and enables to postpone surgical treatment. However in some patients procedural complications may occur and surgical shunt placement is needed.

\section{P-191}

Percutaneous catheter interventions via Glidesheath Slender in small children

Gendera K., Ewert P., Georgiev S., Tanase D., Genz T., Hosny Fayed M., Eicken $A$.

Department of Pediatric Cardiology and Congenital Heart Disease, German Heart Center Munich, Technische Universität München, Germany

Objectives: In small children the diameter of the vessels comprises a significant limitation for catheter interventions. Femoral arterial occlusion is a serious complication that can cause limb length shortening. Subclavian arterial occlusion may lead to subclavian steal syndrome or growth disorders of the upper extremity if distal. Bilateral vessel occlusion can preclude further catheter interventions. The Glidesheath Slender is an innovative sheath with a thinner wall and hydrophilic coating. The inner diameter is compatible with $5 \mathrm{~F}$ or $6 \mathrm{~F}$ guiding catheters, depending on the sheath being used, whereas the outer diameter is similar to that of a regular $1 \mathrm{~F}-\mathrm{less}$ sheath. To reduce risk for scarring and occluding the vessels, interventions can be performed via Glidesheath Slender sheath.

Methods: We present a group of 22 small children (median age: 60 days (min.3; max.790), median weight: $4.1 \mathrm{~kg}$ (min.1,4; max.10.3)) in whom percutaneous interventions were performed via Glidesheath Slender. The type of intervention: VSD occlusion $(n=6)$, re-coarctation management with stent implantation $(n=5)$, stenting of the ductus arteriosus $(n=4)$, LPA angioplasty $(n=3)$, stenting of the Sano shunt $(n=1)$, Rashkind procedure $(n=1)$ and coil embolization of MAPCAs $(n=1)$. In 20 children the intervention was performed from femoral access (artery $n=15$, vein $n=5$ ) and in 2 from the subclavian arterial access. $5 \mathrm{~F}(\mathrm{n}=20)$ and $6 \mathrm{~F}(\mathrm{n}=2)$ Glidesheath Slender were used in our study group. In all patients vessel access was obtained under ultrasound guidance.

Results: No serious complications associated with vessel cannulation occurred. None of the children presented symptoms of the vessel narrowing or occlusion. In all patients in whom the procedure was performed from the arterial access the pulse on the peripheral arteries (posterior tibial artery or radial artery) was palpable afterwards. No signs of vein occlusion was noticed in patients in whom venous access was used.

Conclusion: The Glidesheath Slender enables to perform life-saving interventions in very small children with 1 F-less outer sheath diameter. The risk of vessel narrowing/occlusion probably is lower with this sheath. The use of Glidesheath Slender, which was developed for coronary interventions with radial artery access, in small children is promising.

\section{P-192}

Successful percutaneous recanalization of the RPA origin after surgical ligation in patient with Ebstein anomaly and Glenn procedure

Gendera K., Ewert P., Georgiev S., Tanase D., Genz T., Hosny Fayed M., Eicken A. 
Department of Pediatric Cardiology and Congenital Heart Disease, German Heart Center Munich, Technische Universität München, Germany

Objectives: Absence of a hypothetical hepatic factor passing through the pulmonary circulation is supposed to be responsible for pulmonary arteriovenous fistulas (AVF) development what can lead to severe cyanosis in patients after partial cavo-pulmonary anasthomosis (PCPA). Redirection of hepatic blood flow into the pulmonary artery may cause fistulas to close, what significantly improves the clinical state of the patient. In some patients a transcutaneous approach for blood redirection of hepatic blood supply into the pulmonary circulation is possible and repeated open-heart surgery can be avoided.

Methods: A 12-year-old girl with Ebstein anomaly, who at the age of 3 years was managed with PCPA and surgical ligation of the right pulmonary artery (RPA) origin, was scheduled for cardiac catheterization due to severe central cyanosis ( $\mathrm{SaO} 2$ 80-85\%). The right jugular vein and the right femoral vein were cannulated both with $6 \mathrm{~F}$ sheaths. Angiography showed multiple intrapulmonary fistulas in the right lung. The mean blood pressure RPA: $13 \mathrm{mmHg}$, LPA: $35 \mathrm{mmHg}$. Simultaneous injection into SVC and LPA showed the ligated RPA origin with the length of about $5 \mathrm{~mm}$. A radiofrequency wire (Baylis) was advanced through a left Judkins guiding catheter from the jugular access. Repeated energy (up to 15 Watts) applications (under fluoroscopy control) caused slow advancement of the radiofrequency guidewire through the ligated segment. After entering the LPA-lumen the guidewire was snared from the femoral site. A $6 \mathrm{~F}$ long sheath was introduced from the femoral vein and a $6 \times 16 \mathrm{~mm}$ covered LifeStream stent was advanced and implanted into the recanalized segment.

Results: The narrowest segment of the implanted stent was assessed with 2-3 mm what was found optimal due to the increased LPA blood pressure. Control angiography showed no contrast extravasation. After the procedure the clinical state of the patient improved significantly. Three months after stent implantation oxygen saturation was measured with $90 \%$.

Conclusion: Redirection of hepatic blood into the pulmonary artery in patients after PCPA with AVF and severe cyanosis can cause fistulas to close and significantly improve the clinical state of the patient. If possible an interventional approach can be considered in selected patients.

\section{P-193}

\section{Complex decision making in the paediatric catheterisation} laboratory

Duignan S. (1), Ryan A. (1), Burns B. (2, 3), Kenny D. (1), McMahon C.J. (1)

Paediatric Cardiology Department, Our Lady's Children's Hospital, Crumlin, Dublin, Ireland (1); Greater Sydney Area Helicopter

Emergency Medical Service, NSW Ambulance, Australia (2); Sydney

Medical School, Sydney University, Australia (3)

Optimal outcomes are as much influenced by critical decision making pathways as by the technical skill of the operator. The complexity and potential cognitive traps underlying critical decision making has long been recognised in the aviation and business communities, however remains a largely subconscious, unexamined discipline amongst congenital cardiac interventionalists. Challenges to making good decisions in the catheterization laboratory include heuristics, biases and cognitive traps. In this paper we discuss some of the more common decision challenges encountered and we address potential solutions to such decision making with particular focus towards standardization. We use the example of balloon aortic valvuloplasty to demonstrate potential pitfalls in our current decision making processes and ways in which these could be improved upon by standardization.

\section{P-195}

Dilating side struts of open cell stents - An in vitro study

Krasemann T., Kruit M.N., van der Mark A.E., Zeggelaar M., Dalinghausb M., van Beynum I.M.

Sophia Kinderziekenhuis, Erasmus MC Rotterdam Department of

Pediatrics, Division of Pediatric Cardiology Rotterdam/Netherlands

Background: Open cell stents are frequently used in interventional therapy of congenital heart disease. Overstenting of vessel branches may lead to the need of stent strut dilation.

Methods: In vitro we tested the strut size achievable in Bard Valeo and Cook Formula stents, and the pressure necessary to achieve strut rupture. Balloon position was either perpendicular to the stent or through the lumen through one side strut only.

Results: With the original balloon at nominal pressure, in Valeo stents the side struts could be dilated to ca. $90 \%$ of the stent diameter, in Formula stents to ca. $80 \%$. With bigger high pressure woven balloons, strut size increased to ca. $125 \%$ of the stent diameter in Valeos, and to ca. 105\% in the Formulas. Strut rupture can connect two adjoining struts leading to even bigger diameters. These are dependent on the size of the balloon utilized. Pressures were depended on the balloon utilized.

Discussion: Dilation and overdilation of side struts in open cell stents can be achieved. Dependent on the clinical context, the original balloon used to place the premounted stent can be used to achieve strut dilation, but woven high pressure balloons offer higher security. Should a larger diameter be required, these high pressure woven balloons can achieve bigger diameters and even strut rupture.

\section{P-196}

Interventions and strategies in patients with pulmonary atresia and multifocal lung perfusion

Zartner P., Hart C., Suchowerskyj P., Asfour B., Schneider, M.B.

German Paediatric Heart Centre, Sankt Augustin, Germany

Introduction: Patients with pulmonary atresia (PAtr) and ventricular septal defect (VSD) occur in 7 of 100000 births and account for $1 \%$ of the patients with a congenital heart disease (CHD). They usually present with multifocal lung perfusion in form of multiple aorto-pulmonary collaterals (MAPCA).

Methods: Between 2003 and 2017 we collected 60 patients with PAtr or critical pulmonary valve stenosis (cPS) and VSD, age ranged from 0.17. to 42.5 years (mean 5.1 years). All had MAPCA and the first aim was to differentiate who had native pulmonary arteries (nPA). These were selected as the later main branch pulmonary arteries and all strategies applied targeted to promote growth of these vessels, by closing collaterals doubly suppling lung areas and stenting of stenotic arteries. As a next step these vessels were connected to the right ventricle either by intervention or surgery and unifocalisation of all available MAPCA was performed. Patients without recognizable nPA were set on a palliative approach with optimisation of the lung perfusion and later VSD closure only if the right ventricular (RV) pressure was to expect lower than the systemic pressure.

Results: Follow-up time is 1.1 to 11.8 years (mean 4.7 years). Patients underwent 0 to 4 surgical operations (mean 2.1) and 0 to 14 catheterisations (mean 6). Two patients died during follow up. 15 patients $(25 \%)$ had no native pulmonary artery (PA). 45 patients 
had a native pulmonary artery and followed our biventricular approach, while 11 of these patients still await VSD closure with or without fenestration. The RV pressure compared to the left ventricular (LV) pressure was significantly higher in the patients without $\mathrm{nPA}$, in mean 94\% (standard deviation 31\%), than in mean $73 \%$ (standard deviation $21 \%$ ) in the group with $\mathrm{nPA}$, after the VSD was closed.

Conclusions: The use of MAPCA as pulmonary arteries increases the afterload of the RV after "corrective" surgery like unifocalisation and VSD closure, so the recruitment of nPA, even if hypoplastic, improves the prognosis in many patients. They should be recognised and their growth pursued consequently by increasing forward blood flow through these vessels.

\section{P-197}

Transcatheter embolectomy with thrombus fragmentation and aspiration in adolescent pulmonary embolism

Levunlieva E., Kaneva A., Dimitrov L., Nenova K., Alexandrov A. Department of Pediatric Cardiology and Cardiac Surgery, National Heart Hospital, Sofia, Bulgaria

Pulmonary embolism is rare in pediatric patients, but the incidence of the disease is increasing in past decades. It may be lifethreatening and requires prompt and active treatment.

We present two cases of pulmonary embolism in adolescent patients with successful transcatheter embolectomy with thrombus fragmentation and aspiration.

The first case is 15 year-old girl. At the age of 10 years she was operated on for astrocytoma. The patient was admitted in severe condition, with dyspnea, tachycardia, and hypoxemia. Echocardiography showed right ventricular (RV) dilation (RV/LV ratio -0.85$)$ and pulmonary hypertension. CT showed massive thrombi with subtotal occlusion of the two pulmonary arteries. Transcatheter treatment was performed with thrombus fragmentation with rotation of a pigtail catheter and suction embolectomy. $\mathrm{RV}$ pressure dropped from $75 \mathrm{mmHg}$ before the procedure to $55 \mathrm{mmHg}$ after the procedure.

The second case is 17 year-old female one month after delivery of a healthy baby. Two weeks after the delivery she presented with shortness of breath, chest pain, tachycardia, and hypoxemia. CT showed subtotal left pulmonary artery (LPA) occlusion and partial RPA occlusion. In this patient thrombus fragmentation, suction embolectomy and PTA of the LPA were performed. Pulmonary blood flow was improved, but RV pressure showed no significant change.

In the two patient local fibrinolysis with Alteplase was performed. Standard anticoagulant treatment with heparin followed by acenocumarol was carried-out.

In both patients six months after the procedure CT examination showed no residual thrombi in the lung vessels.

Transcatheter treatment with thrombus fragmentation, embolectomy, and local fibrinolysis is safe and effective approach in pulmonary embolism in adolescent pediatric patients.

\section{P-198}

Occlusion of Complex Veno-atrial Collaterals in a Fontan Circulation

Rehman R., Elarabi A., Alwi M.

Institut Jantung Negara (National Heart Institute), Kuala Lumpur. Malaysia

Introduction: An 8-year-old boy with Double outlet right ventricle and hypoplastic left ventricle underwent Fontan completion by 4 years of age. He developed progressive cyanosis with resting Oxygen saturations of $65 \%$. Absence of a fenestration made a venous collateral decompressing the Fontan circuit likely. We proceeded to cardiac catheter to assess his fontan circulation. Case: Mean pressure in the Fontan was $13 \mathrm{mmHg}$ and pathways unobstructed. IVC angiogram showed multiple complex systemic venous collateral channels from the IVC and hepatic veins draining to a common site in the left atrium.

Engaging the collateral from femoral approach was difficult due to the angle and tortuous course. A jugular vein approach was adopted. Multiple stenoses resulted in inability to advance the sheath to the desired position, the LA.

We occluded the major individual channels using a $10 \mathrm{~mm}$ (a) and $12 \mathrm{~mm}$ (b) Amplatzer Vascular plug II and a $6 \times 6 \mathrm{~mm}$ (c) Amplatzer ductal occluder II. Angiogram confirmed reduced flow through the collaterals and stable device position. Saturations improved to $86 \%$.

Discussion: Systemic venous collaterals can decompress the higherpressure Fontan circuit to the lower pressure atrium or pulmonary vein, resulting in symptomatic cyanosis. After bidirectional cavopulmonary anastomosis incidence of systemic venous collaterals was $17 \%$ in a series by Gross et al and $31 \%$ by Magee et al. Weber detected significant systemic venous collaterals in $18 \%$ post Fontan.

Risk factors for collateral development include: gradient between the superior vena cava and right atrium, increased transpulmonary gradient, heterotaxia and high mean PA pressure. Sugiyama et al found 68 systemic venous collaterals in 50 cyanosed patients post Fontan. These were supracardiac in $36(53 \%)$, cardiac in $12(18 \%)$, and infracardiac in $20(29 \%)$. The brachiocephalic vein was the most common site with 30 of the 68 collaterals (44\%).

Mehta et al have described accessing the pulmonary venous atrium from the PA floor, a potential route for us to place a device at the LA exit site.

Decompressing systemic venous collaterals continue to be problematic in the Fontan circuit. Longer term data is required to assess the impact of collaterals in patients who have and have not undergone occlusion.

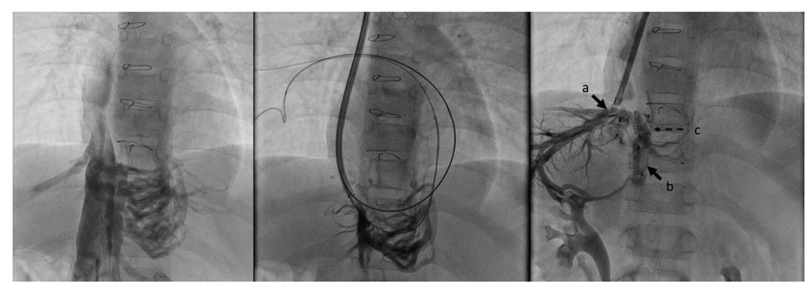

Figure.

\section{P-199}

Outcomes of Ductal Stenting with Pre-existing Branch Pulmonary Artery Stenosis - Revisiting the American Heart Association Guidelines

Rehman R., Marhisham C.M., Samion H., Alwi M.

Institut Jantung Negara (National Heart Institute), Kuala Lumpur. Malaysia

Introduction: Patent Ductal Arteriosus (PDA) stenting in the setting of pre-existing branch pulmonary artery (PA) stenosis is controversial. Current American Heart Association guidance is to avoid ductal stenting in this setting.

Over the recent years we have undergone a learning curve in complex ductal stenting. CT imaging enabled better understanding 
of complex ducts, aided patient selection, planning and consideration of access routes. Improved procedural success, long surgical waiting lists as well as the mortality and morbidity of the mBTS in the current era led us to cautiously revisit our position with regards to the AHA guidelines.

Methods: Prospective review of patients ( $<3$ months, $>2.5 \mathrm{~kg}$ ) undergoing PDA stenting with ductus related branch PA stenosis between Jan 2014 - Dec 2015. The growth of the jailed stenotic PA branch with the contralateral PA was compared angiographically 6-12 months later.

Results: 46 patients underwent ductal stenting. 37\% had ToF-PA (17/46).

4 patients required early modified Blalock Taussig Shunt (mBTS) and were further excluded.

The unaffected PA showed good growth (mean Z score from -0.6 to +2.2 ). The jailed PA also showed proportionate growth (mean $\mathrm{Z}$ score from -1.4 to +1.1$)$ in $93 \%(37 / 40) .3$ patients showed poor PA growth (2 underwent restenting, $1 \mathrm{mBTS}$ to affected side). There was no in-hospital mortality and 3 deaths occurred. Conclusions: In an era where neonatal mBTS continues to have significant morbidity and mortality, we feel that ductal stenting in complex PDA's with pre-existing PA stenosis is a feasible alternative. The stenosed, jailed PA still has potential for proportionate growth. Patient selection, antiplatelet therapy and close follow up is vital. Stent removal with surgical repair within 1 year of age is our current recommendation.

\section{P-200}

Accuracy of imaging fusion between echocardiography and fluoroscopy in percutaneous atrial septal defect closure in children

Hadeed K., Hascoet S. (1,2), Ratsimandresy M. (1), Karsenty C. (1), Alacoque X. (1), Heitz F. (1), Fraisse A. (1,3), Dulac Y. (1),

Acar P. (1).

(1) Departement of Paediatric and Congenital Cardiology, Children's Hospital, Toulouse, France. (2) Department of Paediatric and Congenital Cardiac Surgery, Marie-Lannelongue Hospital, Paris, France. (3) Department of Pediatric Cardiology, Royal Brompton Hospital, London, United Kingdom.

Introduction: EchoNavigator ${ }^{\mathbb{B}}$ allows imaging fusion between $3 \mathrm{D}$ echocardiography and fluoroscopy in the catheterization laboratory. We aim to test the accuracy of the imaging fusion in a pediatric population.

Methods: During percutaneous closure of atrial septal defect in 31 patients (26 kgs [21-37]), occluder devices, visualized on echo and fluoroscopy, were used as a reference tool. A first distance was measured between a marker positioned on echo and the thread pitch on fluoroscopy. A second distance was measured between the thread pitch on the 3D echo view and on fluoroscopy. The 2 distances were measured on 4 orthogonal views in systole and diastole.

Results: Fusion and marker positioning were feasible in real-time in all cases. In 5 cases (16.1\%), fusion was instable during the procedure with transient loss of the automatic tracking of the probe. Quality of the fusion imaging was rated good in all cases. On the fusion view, minimal and maximal first distances during the cardiac cycle were $0.5[0.3-1]$ and $2 \mathrm{~mm}$ [1.5-2.5] $(\mathrm{p}<0.0001)$. The marker positioned on echo was fixed and did not follow the movement of the thread pitch. Minimal and maximal second distances were $0.5[0-0.5]$ and $2 \mathrm{~mm}$ [1.5-2.5] $(\mathrm{p}<0.0001)$.

Conclusion: Feasibility and accuracy of imaging fusion between echocardiography and fluoroscopy using the EchoNavigator ${ }^{\circledR}$ software is high in children above $20 \mathrm{kgs}$. This software offers a new real-time imaging guiding modality in the catheterization laboratory with potential interest in complex procedures as well as for fellow training.

\section{P-201}

Hybrid-implantation of a pulmonary valve avoiding cardiopulmonary bypass

Wiegand G. (1), Schlensak C. (2), Mustafi M. (2), Schneider W. (2), Sieverding L. (1), Hofbeck M. (1)

University Children's Hospital Tuebingen, Department of Pediatric Cardiology, Tuebingen, Germany (1); University Hospital Tuebingen, Department of Thoracic and Cardiovascular Surgery, Tuebingen, Germany (2)

Objectives: Percutaneous pulmonary valve replacement with the Melody valve is well established in patients with a weight of $>25 \mathrm{~kg}$. In children $<20 \mathrm{~kg}$ implantation becomes difficult due to small vessel size. In selected cases problems of access and cardiac anatomy can be solved by a hybrid approach.

Case report: We report a patient who was referred at the age of 4 years with dextrocardia, double discordance, 1-transposition of the great arteries, VSD, subvalvular and valvular pulmonary stenosis. She underwent VSD-patch closure and implantation of a valveless $14 \mathrm{~mm}$ Gore-Tex LV-PA conduit. Postoperatively she suffered from chronic effusions as well as renal and hepatic failure. Since percutaneous interventional implantation of a pulmonary valve was impossible due to the complex ventricular anatomy and small size of the patient $(18.1 \mathrm{~kg})$, we opted for a hybrid procedure. The chest was reopened via a median sternotomy. To avoid extracorporeal circulation the distal LV-PA conduit was incised and anastomosed to a $6 \mathrm{~mm}$ Gore-Tex tube. An 18Fr sheath was introduced into this tube and advanced into the conduit via a 0.035 " wire. The $18 \mathrm{~mm}$ Melody valve was crimped on a $14 \mathrm{~mm}$ BIB-balloon and introduced into the $18 \mathrm{Fr}$ sheath, following removal of its check-flow valve. The balloon was advanced into the LV-PA conduit and the position was controlled under TEE guidance. After confirmation of the correct position just distal to the ventricular end of the conduit the valve was expanded with the balloon. The balloon and sheath were removed and the $6 \mathrm{~mm}$ Gore-Tex tube was oversewn at its entry into the LV-PA conduit. The patient made an uneventful recovery with disappearance of the effusions. 12 months later the valve is well functioning within the conduit with a systolic peak velocity of $3 \mathrm{~m} / \mathrm{sec}$.

Conclusion: In order to avoid the use of cardiopulmonary bypass the hybrid approach may offer interesting solutions for pulmonary valve replacement in children with inadequate size of venous vessels and/or complex anatomy. In the presence of a nonvalved PTFE-conduit hybrid placement of the Melody valve is facilitated by introduction via a temporary distal graft connecting to this conduit.

\section{P-202}

Pulmonary atresia with intact ventricular septum: medium term follow-up

Bacca A., Donti A., Mariucci E.M., Egidy Assenza G., Bonvicini M. Cardiologia Pediatrica e dell'Età Evolutiva, Bologna, Italy

Introduction: Pulmonary atresia with intact ventricular septum (PAIVS) is a rare and complex congenital heart disease characterized by imperforate pulmonary valve and intact ventricular septum. Treatments have been improved over time: cardiosurgical techniques have become safer during the neonatal period and 
percutaneous intervention has been developed as an alternative. This resulted in a reduction mortality over time, but clinical trend, final cardiac physiology (biventricular, one-and-a-half, univentricular), long term complications and therapeutic history can be very complex and currently not well known. Purpose of this study is to clarify this topic by analyzing a surviving popolation affected by PAIVS in retrospect.

Methods: 59 PAIVS patients and older than 6-year were selected in our Institution. Neonatal heart anatomy (tricuspid valve $\mathrm{z}$-score, right ventricular morphology), therapeutic history, final cardiac physiology, clinical outcome and complications of these patients were evaluated in a median follow-up of 16 years.

Results: Patients with more unfavorable neonatal charateristics were immediately directed to univentricular pallation (9). In the other patients percutaneous valve perforation (PVP) was performed to less diminutive right ventricle and was effective in $64 \%$. Almost all patients (90\%) underwent further surgery/percutaneous intervention during follow-up and each patient underwent an average of 3,1乃1,2 interventions.

Final biventricular phisiology was reached by $78 \%$ of candidates of biventricular repair. They had higher tricuspid z-score $(-1,04$ vs $-2,89, p<0,05)$, severe tricuspid regurgitation ( $97 \%$ vs $64 \%$, $\mathrm{p}<0,05)$, a more represented right ventricle (bi/tri-parted $100 \%$ vs $55 \%, \mathrm{p}<0,05)$, no difference in surgical/percutaneous approach.

Half patients with biventricular repair has severe pulmonary regurgitation and 12 patients underwent pulmonary valve replacement for right ventricle enlargement $(141319 \mathrm{ml} / \mathrm{mq}$ vs non-valve replacement $99338 \mathrm{ml} / \mathrm{mq}, \mathrm{p}<0,05)$. Those who developed dilatation of the right ventricle enlargement had higher neonatal tricuspid $\mathrm{z}$-score $(-0,37$ vs $-1,29, \mathrm{p}<0,05)$ but there wasn't difference about surgical/percutaneous treatment.

Most patients have NYHA I-II regardless final phisiology. Only $10 \%$ has clinical arrhythmias (all supraventricular).

Conclusions: Most of APSI patients reached biventricular phisiology, they had higher tricuspid z-score, more tricuspid regurgitation, more represented right ventricle. A significant number of these patients underwent pulmonary valve replacement. There were few clinical arrhythmias.

\section{P-203 \\ Three-dimensional rotational angiography in newborns and infants with hypoplastic left heart syndrome \\ Koleśnik A., Zubrzycka M., Rewers B., Migdał A., Brzezińska- Rajszys G. \\ Cardiovascular Interventions Laboratory, The Children's Memorial Health Institute, Warsaw, Poland}

Introduction: Recently 3D rotational angiography (3DRA) became a standard imaging method. It allows to create three-dimensional images, three-dimensional roadmaps for navigation during diagnostic catheterization and interventional procedures as well as to obtain tomographic images similar to those created with standard computed tomography. In newborns and infants, however, the volume of injected contrast medium and radiation dose should be strictly considered. We present our experience with 3DRA in infants after first stage palliation for hypoplastic left heart syndrome.

Methods: Twelve 3DRAs were performed in 12 patients with hypoplastic left heart syndrome using Philips Allura Clarity system. The age of patients was 1-10 months (avg. 6 months). In all cases undiluted contrast medium was injected into the right ventricle without rapid pacing. Rotation time was $4.1 \mathrm{~s}$, the injection delay $2 \mathrm{~s}$, injection time $5 \mathrm{~s}$, contrast medium dose maximum $2 \mathrm{ml} / \mathrm{kg}$, contrast medium flow $1-3 \mathrm{ml} / \mathrm{s}$.
Results: 3DRA was done during 9 interstage diagnostic catheterisations, 2 implantations of stents into the Sano conduit and one duct stenting following bilateral pulmonary artery banding. In all cases the quality of 3DRA images was satisfactory for 3D reconstruction and roadmaping. Quality of cone-beam computed tomography images (XperCT, Philips) allowed for accurate visualisation and measurements of anatomical structures. Moreover, the details impossible to depict with two-dimensional angiography, e.g. relationship of vascular structures to airway, could be assessed. Contrast volume ranged from 5 to $18 \mathrm{ml}$. Radiation dose per acquisition was 2.09-5.59 mGy (Air Kerma, median 3.06 mGy) and 153-527 mGycm2 (Dose Area Product, median 323.5).

Conclusions: In newborns and infants with hypoplastic left heart syndrome 3DRA can be safely used to obtain diagnostic images and support interventions with low volume of contrast medium and reasonable radiation dose.

\section{P-204}

Coronary artery size and event free survival after transcatheter closure of coronary fistulas

Radtke W.A.K. (1), Rubinstein C. (2), Tsuda T. (1)

Nemours Cardiac Center, Wilmington, DE, USA (1); MUSC

Children's Hospital, Charleston, SC, USA (2)

Introduction: During followup after transcatheter closure of coronary fistulas, ischemic events appear to occur more frequently after fistula closure at older age. It has been inferred that earlier closure may ameliorate the risk of such late complications. Supporting evidence for an underlying mechanism is lacking.

Methods: We performed a retrospective review of our experience with transcatheter closure of coronary fistulas during infancy and childhood with emphasis on event-free survival and size change of the feeding coronary artery. Fourteen patients underwent transcatheter closure of coronary fistulas at age 10 days to 18 years (weight $3.6-64.8 \mathrm{~kg}$ ). The fistula originated from the right coronary in 5 , from the left in 7, and from both in 2. There were no major procedural complications. Followup ranged from 1 to 19 years.

Results: There were no ischemic events during followup. Recanalization of the fistula occurred in 1 patient and was successfully occluded in a second procedure. At the time of the procedure, the feeding coronary artery measured $3.2-9.0 \mathrm{~mm}$ in diameter by angiography and $3.6-8.3 \mathrm{~mm}$ by echocardiography. At latest followup, the echocardiographic diameter of the former feeding coronary measured $3.3-8.2 \mathrm{~mm}$, suggesting only minimal regression in the size of the feeding vessel. The patient with recanalization had an increase in the size of the feeding coronary from 7.0 to $8.5 \mathrm{~mm}$ over 3 years. Our findings suggest that there is little regression in the size of the feeding coronary during followup but closure of the coronary fistula appears to prevent a size increase.

Conclusions: The benefit of coronary fistula closure at early age may be prevention of further coronary dilatation and allowing patients to grow into the size of their feeding coronary vessel thus decreasing the risk of thrombotic events.

\section{P-205}

Our experience with SHSMA (Hyperion) occluders for different intra- and extarcardiac shunts closure Galeczka M., Fiszer R., Litwin L., Smerdzinski S., Szkutnik M., Karwot B., Bialkowski J.

Department of Congenital Heart Defects and Pediatric Cardiology, Silesian Center for Heart Diseases, SMDZ, Medical University of Silesia, Zabrze, Poland 
Introduction: Transcatheter closure with nitinol wire mesh occluders has become the treatment of choice for many congenital heart defects. Comed BV (The Netherlands) / Lepu MT Company (China) have recently introduced the SHSMA (Hyperion) occluders, similar to Amplatzer devices. Lower nickel ion release and improved biocompatibility due to oxidization differentiate the device. We present our preliminary experience with Hyperion Atrial Septal Defect (ASD), Patent Foramen Ovale (PFO), Patent Ductus Arteriosus (PDA) and Ventricular Septal Defect (VSD) occluders.

Methods: Retrospective analysis of 248 patients (pts; 172 females), 0,25-77 years old (yo) with secundum ASD (125 pts: 17 double/ multiple), PFO (90 pts), PDA (29 pts) or VSD (4 pts: 1 muscular, 3 perimembranous) who had transcatheter shunt closure with Hyperion device between March 2014 and November 2017 in a single tertiary center. Median age of ASD pts was 26 yo (4-77), PFO - 40 yo (18-68), PDA - 2 yo (0,3-73) and VSD - 2,6 yo $(0,25$ -31). Patients were treated with appropriate type and size of Hyperion device (n-249) without any preliminary patients' selection. The implantation techniques were the same as previously described for Amplatzer occluders. Two infants with VSD had hybrid procedure performed. Perimembranous symmetrical (3 pts) or asymmetrical (1 pt) and muscular (1 pt) VSD occluders were applied. In 3 pts with multiple ASDs the PFO occluder was used.

Results: All procedures but 1 were performed successfully. In one patient with perimembranous VSD a stable position of occluder was achieved with neither symmetrical nor asymmetrical VSD occluder. The mean follow-up was 1,5 years $(0,2-3,7 y)$. Except for transient pulmonary hypertensive crisis after hybrid muscular VSD closure, there were no procedure-related complications. Complete shunt closure was achieved in all except 7 pts with double/multiple ASDs, 6 pts with PFO and 2 pts after hybrid VSD closure - insignificant residual leaks persisted with no need for reintervention (no recurrent neurological events in PFO pts; aspirin treatment maintained).

Conclusions: Our preliminary experience with the clinical application of Hyperion occluders in the transcatheter closure of ASD, PFO, PDA and VSD has confirmed their utility and safety in short- and mid-term observation.

\section{P-206}

The Amplatzer Duct Occluder II Additional Sizes - Our experience with different intra- and extracardiac shunts closure in paediatric and adolescent population

Galeczka M., Litwin L., Fiszer R., Smerdzinski S., Szkutnik M., Bialkowski J.

Department of Congenital Heart Defects and Pediatric Cardiology, Silesian Center for Heart Diseases, SMDZ, Medical University of Silesia, Zabrze, Poland

Introduction: Amplatzer Duct Occluder II Additional Sizes (ADO II AS), introduced in 2012, was designed to address percutaneously medium to large patent arterial ducts (PDA) in small children. We report label and innovative off-label applications of ADO II AS. Methods: Retrospective analysis included 128 elective patients (pts; 81 females) with median age of 3,7 years (y; range $0,3-24 y, 11,7 \%$ pts older than 10y), who had interventional shunt closure with ADO II AS between July 2014 and November 2017 in a single tertiary center. We closed 120 PDA (mainly complex and elongated type; median narrowest PDA diameter 1,6 mm, 1,2-4 mm; median PDA length $7 \mathrm{~mm}, 2,5-15 \mathrm{~mm}), 4$ perimembranous ventricular septal defects, 2 postsurgical ventricular septal defects in pts with Tetralogy of Fallot (including 1 Gerbode type), one aortopulmonary window (APW; 4 months, $6 \mathrm{~kg}$ ) and one major aortopulmonary collateral artery (MAPCA; 5 months, $8 \mathrm{~kg}$ ). The implantation techniques were routine. Venous delivery site was applied in less than $7 \mathrm{~kg}$ pts ( $3 \mathrm{PDA}, 1 \mathrm{APW})$ and arterio-venous loop was used in 2 pts with perimembranous ventricular septal defect and in $1 \mathrm{pt}$ with Gerbode type defect. Median fluoroscopy time was 3 minutes $(1,5-36)$.

Results: All procedures but one were successful. In one infant with huge A-type PDA a stable position of occluder was achieved with neither ADO II AS nor ADO I (successful surgery 1 month later). There were transient access site complications in 3 pts (pulse loss; alteplase administration in $1 \mathrm{pt}$ ) and transient bifascicular block in $1 \mathrm{pt}$ after perimembranous VSD closure observed in periprocedural period. The median follow-up was 12 months (1-40 months; $121 \mathrm{pts}$ in follow-up) with no procedure-related complications. Insignificant residual shunt was observed in 2 pts with postsurgical ventricular septal defect with no need for reintervention.

Conclusions: ADO II AS is an effective and safe occluder, which could be used not only to close different types of PDA in infants and small children, but also in adolescents and adults, replacing coils. Furthermore, off label ADO II AS application in properly selected ventricular septal defect or extracardiac shunts is possible.

\section{P-207}

Safety and efficiency in transcatheter closure of atrial septal defect (ASD) using Cocoon Septal Occluder

Knop M., Fiszer R., Szkutnik M., Smerdzinski S., Bialkowski J.

Department of Congenital Heart Diseases and Pediatric Cardiology

Silesian Center for Heart Diseases in Zabrze, Poland

Introduction: Transcatheter closure of secundum type of atrial septal defect (ASD) is currently considered the first-choice therapeutic option. Various Amplatzer modifications are currently available on the market. The aim of the study was to evaluate feasibility, safety and efficiency of percutaneous ASD closure using Cocoon Septal Occluder (Sanare Company, Philippine) in our institution during the short term follow-up. It is made of nitinol wires covered with platinum using nano fusion technology to prevent nickel release from it.

Methods: There were $33(28 \mathrm{~F})$ patients in whom transcatheter closure of ASD was made from 26.06.2017-27.11.2017 in Silesian Centre for Heart Diseases in Zabrze, Poland. The indications for atrial septal defect closure were: right ventricle enlargement and hemodynamically significant left-right shunt. Mean weight of patients who underwent transcatheter closure was $33,9 \pm 25,3 \mathrm{~kg}$ (range $8-92 \mathrm{~kg}$ ) and age $13,85 \pm 17,7$ years (range 1,3-65 years) respectively. Transcatheter closure of ASD was conducted in all patients using Cocoon Septal Occluders (CSO).

Results: The devices were implanted successfully in all patients. Mean ASD diameter in patients, who underwent transcatheter closure in TEE was $12,14 \pm 4,1 \mathrm{~mm}$ (single ASD). There were 8 patients with multiple ASD. Mean implant size was $14,7 \pm 4,5 \mathrm{~mm}$ (range $8-26$ ). Balloon calibration were made in 14 patients. Mean stretch diameter of ASD in these cases was $16,6 \pm 4,25 \mathrm{~mm}$ (range $10-24$ ). Mean fluoroscopy time was $5,6 \pm 2,6 \mathrm{~min}$ (range 2-12). In 3 patients minor complications occurred: atrial fibrillation $(n=1)$, arteriovenous fistula $(n=1)$, short term fever after procedure $(n=1)$. Mean follow-up time was 5 months. During this time no complications were observed. Conclusions: Percutaneous closure of ASD using Cocoon Septal Occluder is safe and effective procedure, however long-term follow-up is needed. 


\section{P-208}

Bare-metal stenting in recurrent coarctation of the aorta below $12 \mathrm{~kg}$ of weight

Grootendorst R.J., Krings G., Molenschot M., Breur J.M.P.J.

Wilhelmina Children's Hospital, Utrecht, the Netherlands

Introduction: Recurrent coarction of the aorta (CoA) occurs relatively often after surgical correction. Surgical and interventional treatment for recurrent $\mathrm{CoA}$ in infants below $12 \mathrm{~kg}$ of weight has specific limitations. Stenting is a promising alternative and increasingly accepted in older children. Technical stent properties continue to improve. This study investigates bare-metal stenting as a treatment modality in recurrent CoA in small children.

Methods: In vitro study of expandability and breakage characteristics of the Cook Formula $418(8 \mathrm{~mm})$ and $535(8$ and $10 \mathrm{~mm})$ stents was followed by clinical use in 14 patients. All patients below $12 \mathrm{~kg}$ undergoing treatment of native or recurrent CoA with a Cook Formula stent between November 2012 and October 2016 were included in this study. Patient and procedural characteristics were obtained as well as procedural success, complications and follow up. Results: In vitro testing of the 8 and $10 \mathrm{~mm}$ Cook Formula stents showed low profile when being downcrimped on smaller balloons and reached a wide diameter with favorable breaking characteristics and limited foreshortening. 14 patients underwent implantation of a Cook Formula stent during the study period. Median time interval between surgery and intervention was 4.2 months. Median age was 0.5 years and median weight $5.6 \mathrm{~kg}$. Arterial sheath size ranged from 5 to $7 \mathrm{Fr}$. Stent diameters of 4.4 to $9.1 \mathrm{~mm}$ were obtained. A median residual gradient of $0 \mathrm{~mm} \mathrm{Hg}$ was achieved. Intima stripping in 1 patient was adequately treated with no permanent damage. 2 redilatations due to somatic growth were performed after a median interval of 38 months. An "in stent" breaking procedure as proposed as the next step in this optimized workflow was not necessary yet.

Conclusions: This study is the first to report mid-term follow-up of stenting therapy in this population. This study is limited by its case study design, but does indicate that bare-metal stenting with Cook Formula stents is effective with minimal complication rates and might be used in children below $12 \mathrm{~kg}$ with recurrent CoA.

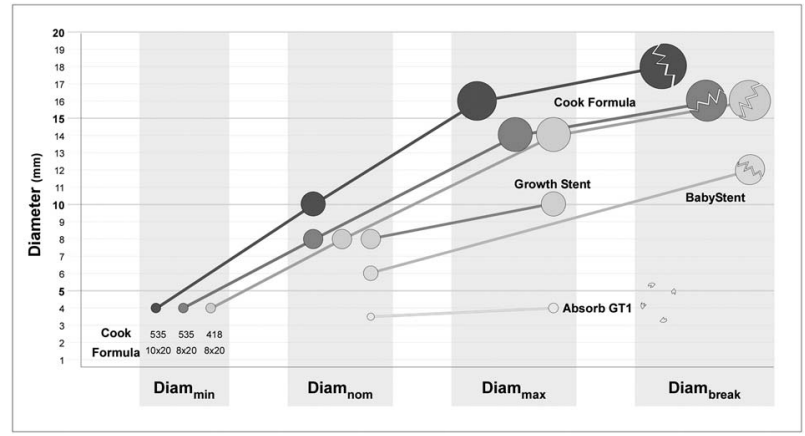

Figure.

P-209

Suspected protamine-induced lethal myocardial ischemia after congenital heart surgery in an infant with congenital disorder of glycosylation

Panagiotou E. (1), Ostermayer S.H.U. (1), Gruszka A. (1), Koester S. (1), Hanten J. (1), Maizza A.F. (2), Vazquez J. (2), Buding B. (3), Kerst G. (1)

Department of Pediatric Cardiology, RWTH Aachen University

Hospital, Germany (1); Department of Pediatric and Congenital Heart
Surgery, RWTH Aachen University Hospital, Germany (2); Department of Anesthesiology, RWTH University Hospital Aachen, Germany (3)

Introduction: Congenital heart disease (CHD) can be associated with the heterogenous group of congenital disorders of glycosylation (CDG). Affected children are exposed to risks of both thrombosis and bleeding. We report on lethal myocardial ischemia associated with heparin neutralization by protamine after cardiopulmonary bypass (CPB) for biventricular repair in an infant with CDG and Double-Outlet Right Ventricle (DORV).

Methods: A 10 month old infant with CDG type Ia and DORV with a severely hypoplastic pulmonary valve and hypoplastic pulmonary arteries was admitted for surgery. Previously, a 2,5 mm modified BT-Shunt had been implanted at 6 weeks of age because of increasing cyanosis. To further promote pulmonary artery growth, balloon angioplasty of the shunt and the hypoplastic pulmonary valve had been performed at 6 months of age. After shunt implantation the infant received acetylsalicylic acid at $3 \mathrm{mg} /$ $\mathrm{kg} / \mathrm{d}$ despite congenital thrombocytopenia. Coagulation tests were normal. There never was evidence for bleeding or thrombosis. Biventricular repair consisted of VSD-closure, transannular pulmonary patch-plasty and implantation of a monocusp contegra conduit. Transesophageal echocardiography (TOE) showed a good biventricular function with no residual defects and no pulmonary regurgitation. Weaning from $\mathrm{CPB}$ was uneventful. Protamine was given for heparin neutralization, immediately followed by cardiopulmonary reanimation an intensified catecholamine support. TOE showed new-onset massive cardiac edema with severely impaired biventricular function accompanied by ST-elevations on ECG. We decided to put the patient on extracorporeal membrane oxygenation (ECMO) using heparin for anticoagulation. Neither excessive bleeding nor thrombosis occurred. The myocardial edema initially regressed only to recur upon reduction of ECMO-flow. Given the severe neurodevelopmental delay and the limited prognosis in CDG, parental consent was given to discontinue ECMO-support on the 6th postoperative day. The patient died 24 hours later in catecholamine-resistant heart failure.

Results: Administration of protamine in an infant with CHD and CDG resulted in massive myocardial edema leading to severely impaired biventricular function and death.

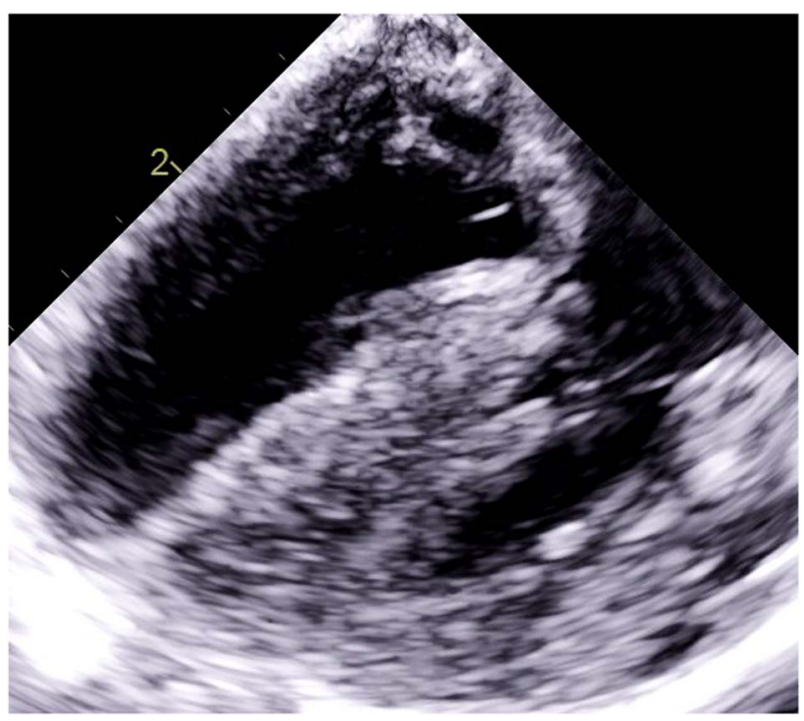

Figure. 
Conclusions: Protamine as a polycationic protein might interact with abnormally glycosylated membranes in CDG and lead to thrombotic myocardial microangiopathy, myocardial ischemia and terminal heart failure. It deems pruden to avoid protamine during $\mathrm{CPB}$ in patients with $\mathrm{CHD}$ and CDG.

\section{P-210}

Effect of Modified Ultrafiltration of Blood on the Immune Response of Body in Children

Baizhigitov N.B. (1,2), Kuatbekov K.N. (1,2), Abzaliev K.B. (1), Mullen A.K. (1,2), Sepbayeva A.D. (1)

Center for Perinatology and Pediatric Cardiosurgery, Almaty, Kazakhstan (1); Kazakh National Medical University named after S.D. Asfendiyarov, Almaty, Kazakhstan (2)

Objectives: This paper deals with the scientific analysis of the immune response during cardiopulmonary bypass. Methods of ultrafiltration (UF) and modified ultrafiltration (MUF) have found application in the practice of surgical interventions with the use of artificial circulation (IC) more than 20 years ago and remain relevant at the present time.

Methods: Authors conducted a retrospective analysis of the cases of children born with congenital heart disease from 2011 to 2016.

Results: Before the operation, the plasma levels of TNF in the Group 1 was within physiological limits and was $13.4 \pm 2.24 \mathrm{pg} / \mathrm{ml}$. In Group 2, at this stage, the figure was $11.5 \pm 0.02 \mathrm{pg} / \mathrm{ml}$. In Phase 2 of the study, during the $\mathrm{CPB}$, no significant changes in the levels of TNF in the Group 1 were observed and its scale was $16.4 \pm 2.61 \mathrm{pg} / \mathrm{ml}$. In Group 2, at this stage, the figure also did not change significantly and amounted to $14.9 \pm 0.28 \mathrm{pg} / \mathrm{ml}$. In Phase 3 after UF in Group 1, there was a significant change of the levels of TNF, which have dropped to $9.9 \pm 0.84 \mathrm{pg} / \mathrm{ml}$. In Group 2, on the contrary, was a significant increase up to $18.94 \pm 2.15 \mathrm{pg} / \mathrm{ml}$. In Phase 4 of the study, 3 hours after the operation, there was a speaker in relation to the plasma concentrations of TNF - its value was significantly decreased to $7.1 \pm 5.26 \mathrm{pg} / \mathrm{ml}$.

Conclusions: These data suggest that ultrafiltration of blood can effectively eliminate unrelated cell receptors and plasma protein cytokines. The use of UF and MUF methods allows controlled removal of excess fluid from the bloodstream, reducing the likelihood of edema development. Removal of inflammatory mediators from the bloodstream reduces the emergence of the syndrome of a systemic inflammatory response.

\section{P-211}

Early and Late Outcomes of Total Correction of Tetralogy of Fallot; A single center experience

Surucu M. (1), Erdogan I. (2), Tokel K. (2), Varan B. (2),

Ozkan M. (3), Kllic M. (2), Aslamaci S. (3)

Gazi Yasargil Education and Research Hospital, Diyarbakir-Turkey (1); Department of Pediatric Cardiology Faculty of Medicine, University of Başkent, Ankara-Turkey (2); Department of Cardiovascular Surgery, Faculty of Medicine, Basskent University, Ankara-Turkey (3)

Introduction: Tetralogy of Fallot (TOF) is the most common form of cyanotic congenital heart disease. It carries a very high mortality if left untreated. The 10 -year survival in patients untreated is $24 \%$ only. The purpose of this study was to analyze the factors affecting morbidity and mortality and follow-up results in postoperative period in TOF patients.

Methods: We retrospectively investigated 476 consecutive patients who were diagnosed with tetralogy of Fallot (TOF) and underwent a surgical intervention from 2002 to 2017. Data were obtained from hospital records, clinical records, echocardiographic, operative and catheterization reports and referring physician notes. We investigated the following clinical characteristics before and after surgery: gender; age at the time of surgery; follow-up period.

Results: Mean age at the time of diagnosis was $4.9 \pm 28$ months and $26.1 \pm 34.3$ months at the time of operation. Mean weight at the time of operation $11.2 \pm 5.6 \mathrm{~kg}(4.6-70 \mathrm{~kg})$. The most common presenting symptoms were physical examination of the murmur in 317 patients, cyanosis in 139 patients. Sixty one patients had initial palliative operations. A transannular patch was inserted in 258 (60\%) patients. Mean postoperative intensive care unit stay was $4.5 \pm 6.6$ days, mean hospital stay was $9.6 \pm 8.2$ days. The mean follow-up period was $4.4 \pm 4.4$ years ( 1 months -17 years). On postoperative echocardiography 72 patients had severe pulmonary regurgitation, pulmonary gradient was $19.1 \pm 10 \mathrm{mmHg}$ $(2-65 \mathrm{mmHg})$. Postoperative pulmonary valve insufficiency and residual pulmonary stenosis grade did not affect intensive care unit and hospital stay. Use of a permenant pacemaker was required in $8(1.7 \%)$ patients. Fifteen patients died in the early period and the operative mortality was $3 \%$. In the follow-up period, pulmonary valve replacement was performed in 33 patients and aortic valve replacement was performed in 3 patients. Late death from cardiac causes occurred one patient.

Conclusion: Total correction of TOF can be performed with a very low mortality rate. However, the postoperative re-operation or re-intervention rates remain relatively high. So it is important for patients to follow them for many years.

\section{P-212}

Resolution of Pulmonary Arterial Hypertension after Successful Repair of a Large Aortopulmonary Window (APW) in an Adult Patient

Hakim I.N., Zografos M.P., Protopapas M.E., Zavaropoulos P., Tsaousis G., Alexopoulos C., Sarris E.G.

Iaso Children's Hospital, Athens, Greece

Introduction: Aortopulmonary window (APW), if unrepaired early in life, leads to fixed pulmonary hypertension and Eisenmenger's Syndrome. We report the case of a 23 year old man with a large type I APW who was judged to remarkably still have reversible pulmonary hypertension, which indeed resolved after successful surgical repair. Methods: Auscultation of a cardiac murmur in an asymptomatic 23 year old $70 \mathrm{~kg}$ army recruit led to the diagnosis of a large, $4 \mathrm{~cm}$ (by ECHO), aorto-pulmonary window. Cardiac catheterization at another institution revealed pulmonary artery (PA) pressure at systemic level, pulmonary to systemic flow ratio (Qp/Qs) greater than $4: 1$, and pulmonary vascular resistance (PVR) approximately $1 / 10$ of systemic. Oxygen challenge resulted in further increase of Qp/Qs and PVR decreased. Lung biopsy via left thoracotomy showed no evidence of irreversible pulmonary hypertension (PAH), and the patient was referred to our center for further management. Surgical repair was performed through a median sternotomy, cardiopulmonary bypass with aortic and bicaval cannulation, and cardioplegic arrest. The aorta and pulmonary artery were separated at the level of the window, and the resulting defects in the great vessels were closed with a Dacron Hemashield patch to the aortic side, and a separate autologous pericardial patch to the pulmonic side.

Results: Immediately postoperatively, under anesthesia, PAP pressure was reduced to approximately one quarter of systemic. The postoperative period was uneventful, and the patient was discharged in 7 days on prophylactic sildenafil therapy, at which time estimated PA pressure was half systemic. At follow-up one year later, the patient remains clinically well, at NYHA class I, and with echocardiographically estimated PA pressure remaining at the same level. 
Conclusion: Although previously unrepaired APW in adults is typically inoperable due to the establishment, early in life, of irreversible pulmonary arterial vascular disease and hypertension, nonetheless, if careful evaluation demonstrates reversibility of PA hypertension, surgical repair should be offered, as it can achieve restoration of normal anatomy, abolishment of shunting, and resolution of pulmonary hypertension. Long term close follow-up is recommended to monitor PA pressure, in the hope of eventual complete discontinuation of sildenafil therapy.

\section{P-213}

Neonatal aortic coarctation repair: early and midterm results in a low volume Paediatric Cardiac Centre Kalavrouziotis G., Kallikourdis A., Giannakopoulou E., Tzalavra S., Prapa M., Agrogianni X., Kouna N., Garini E., Eleftherakis N., Paraschou D., Barbaressou Ch., Kourtesis A., Paphitis Ch. "Aghia Sophia" Children's Hospital, Athens, Greece

Objective: Repair of aortic coarctation (CoA) in neonates (1-30 days old) is associated with a higher mortality and an increased incidence of restenosis compared to older children. We present our early and mid-term results in neonatal CoA repair.

Methods: From June 2001 to June 2016, 99 neonates with a mean age of 16.8 (range, 4-30) days and a mean body weight of 2.79 (range, 1.40-4.56) kg, underwent CoA repair at our institution. Concomitant cardiac defects (besides PDA) were: VSD, 31; ASD / PFO, 16; hypoplastic aortic arch (HAA), 11; aortic stenosisbicuspid aortic valve, 7; mitral stenosis, 3; AVSD, 1; smallish left ventricle, 2. Surgical techniques for CoA repair included: resection and end-to-end anastomosis (ETE), 61; extended ETE anastomosis (EETE), 12; radically extended ETE (REETE), 4; subclavian flap (SF), 4; synthetic patch (SP), 4; SP + ETE, 2; SF + ETE, 1; SF + SP, 1. Pulmonary artery banding was performed in 21 cases with unrestrictive VSD.

Results: Mortality was 7\% (7/99). Causes of death were intractable cardiac $(n=4)$ and/or respiratory failure $(n=3)$. Follow-up of 87 patients (94\% of the survivors) for 6 to 178 (mean: 88) months, revealed no late death; 15 patients $(17 \%)$ developed recoarctation 2 to 5 months postoperatively, and were submitted to 17 percutaneous balloon dilatations. Three of these patients (all with HAA) had reoperation for aortic arch reconstruction $(n=2)$, or post-balloon aneurysm formation $(\mathrm{n}=1) ; 21$ patients ( $23 \%$ of the survivors) are under antihypertensive therapy. Age, weight, and surgical technique were not identified as risk factors for mortality or recoarctation. Conclusions: CoA repair in neonates can be performed with low early and mid-term mortality; recoarctation is relatively high, especially in smallish / hypoplastic aortic arch, but is irrelevant to age, body weight and surgical technique.

\section{P-214}

Vascular Rings - Aortic Arch Abnormalities. Single Center 31 years of Experience

Kallikourdis A., Kalavrouziotis G., Antonopoulos N., Schoretsanitis P., Garini E., Eleftherakis N., Giannakopoulou E., Andreou N., Karanasios E., Kourtesis A.

"Aghia Sofia" Children's Hospital. Athens, Greece

Objective: To retrospectively analyze our center's experience in the diagnosis and management of this rare cardiac malformation ( $<1 \%$ of congenital heart diseases) and it's complications.

Methods: Between January 1986 and December 2017, 34 congenital vascular rings were submitted to our Department for surgical correction. There were 26 infants (age: 12 days-12 months, median: 4.5 months; weight: $1.9-8.9 \mathrm{~kg}$, median: $7.4 \mathrm{~kg}$ ), and 8 children (age: 1-6 years, median: 3.5 years; weight: 7.5$24 \mathrm{~kg}$, median: $15.5 \mathrm{~kg}$ ). Patients were divided into five groups: 1) Double aortic arch with both arches patent and left ligamentum arteriosum (12 cases), 2) Double aortic arch with atresia mainly in the distal part of the left arch and left ligamentum arteriosum (5 cases), 3) Right aortic arch with left ligamentum arteriosum (11 cases), 4) Left aortic arch with aberrant right subclavian artery in combination with CoA and/or PDA (4 cases), and 5) Pulmonary artery sling (2 cases). Main diagnostic tools were 1) cardiac echo 2) oesophagography 3) bronchoscopy and lately 4) CT angiogram with 3D reconstructions and 5) MRI angiogram

Results: We used the left posterolateral thoracotomy for the majority of the cases except 2 cases with pulmonary artery sling where median sternotomy was performed. In all cases we did extended mobilization of the compressed structures from surrounding soft fibrous bands. We also had to perform tracheal plasty reconstruction to one infant with pulmonary artery sling due to severe tracheomalachia. We had no intraoperative mortality but in the postoperative period, 1 neonate died of respiratory failure and sepsis. The children have been followed up every 6 months for the first three years and then once every year. All are clinically well and mostly asymptomatic.

Conclusions: High suspicion, early diagnosis and prompt surgical treatment are necessary to relief symptoms and avoid future severe complications, especially tracheomalachia.

\section{P-215}

Re-operations For Tricuspid Valve Regurgitation Following Tetralogy Of Fallot Repair

Ntalarizou E., Sfyridis P., Tzifa A., Mitropoulos F., Azariadis P., Mponou P., Anderson D., Kalangos A.

Mitera Hospital, Athens, Greece

Background: Significant tricuspid valve regurgitation (TVR) is an occasional finding after repair of Tetralogy of Fallot (ToF) that should be dealt with at the time of reoperation for replacement of a regurgitant pulmonary valve (PVR).

Methods: Single-center retrospective series of patients referred to our practice for re-do PVR after total correction of ToF at a younger age between 2013-2017. Initial ToF correction was performed in another center. We report on the frequency and causes of TVR in this cohort.

Results: 7/82 patients referred for PVR and 1 isolated ToF patient (total 10\%) had significant TVR and underwent tricuspid valvuloplasty. Median age at the time of reoperation was 16 (range 10-27) years; median time between initial correction and reoperation was 13 years (range 10-27yrs). The functional class was NYHA class I in $2 / 8$, class II in $4 / 8$ and class III in 2/8. Median TV regurgitant fraction on cardiac MRI prior to surgery was $25 \%$ (range $22-33 \%$ ). Median RV enddiastolic volume and RV ejection fraction were $170 \mathrm{ml} / \mathrm{m}^{2}$ and $45 \%$, respectively. We noticed two responsible mechanisms for the TR. Dense retraction of the AS commissure with restricted motion of the adjacent anterior and septal leaflet to the AS commissure due to the extension of the fibrotic reaction to the pledgeted sutures used for the VSD closure. The second mechanism was retraction of the AS commissure with rupture of its primary chordae. Annuloplasty ring was placed in 6 patients. Tricuspid valve repair with a septal leaflet sliding plasty and creation of a neo-commissure between the anterior and the septal leaflet was performed in 7 cases. Closure of the AS commissure with a bovine patch performed in one case. Mortality was $0 \%$ with no major postoperative complications. One patient had moderate TR/TS. Conclusion: Iatrogenic regurgitation of the tricuspid valve post-ToF repair due to the placement of pledgeted sutures for the VSD closure 
is surgically challenging. Fibrotic reaction induced by these sutures is a progressive phenomenon. The extent of the fibrosis to the adjacent leaflets will be a determinant factor for the fate of the TV repair.

\section{P-216}

Long Term Results after Arterial Switch Operation (ASO) for Transposition of the Great Arteries: Complications necessitating re-intervention

Protopapas E. (1,2), Zografos P. (1,2), Hakim N. (1,2),

Zavaropoulos P. (1,2), Alexopoulos C. (3), Sarris G. $(1,2)$

Athens Heart Surgery Institute (1); Department of Congenital Cardiac

Surgery at Iaso Children's Hospital (2); Pediatric Cardiac Intensive Care

Unit at Iaso Children's Hospital (3), Athens, Greece

Background/Purpose: Although the Arterial Switch Operation (ASO) is long established as the procedure of choice for Transposition of the Great Arteries (TGA), follow up has frequently revealed development of complications which may necessitate late re-intervention (percutaneous and/or re-operative). This study aims to review the long-term results of the ASO in our experience, with emphasis on the need for re-intervention. Methods: The medical records of all sixty survivors of an ASO for TGA at our institution between 1997 and 2017 were retrospectively reviewed. Median age and weight at ASO were 14 days (3d-3y) and $3.5 \mathrm{~kg}(2.35+11.8)$, respectively. The indications for the ASO included TGA with intact ventricular septum (IVS) $(n=43)$, TGA with ventricular septal defect (VSD) $(\mathrm{n}=15), \mathrm{TGA} / \mathrm{VSD}$ and aortic coarctation $(n=2)$. Seven patients had a two-stage repair.

Results: One late death occurred due to pulmonary infection. Six patients underwent reoperation, five had a catheter intervention, and two patients had reoperation after prior re-intervention (balloon dilation and stenting). Median time for re-interventions (percutaneous or surgical) was 6.5 years after ASO (range 1y-16y). Freedom from reoperation at 20 years was $86,6 \%$ and from re-intervention (percutaneous or surgical) was $78.5 \%(n=13)$. The cause for re-intervention was RVOTO obstruction, mainly in PA branches $(n=12)$, TV regurgitation $(n=1)$, and none for neoaortic valve regurgitation or coronary artery stenosis. There was no mortality for re-intervention. At follow up (median time $=13$ years) all patients are in NYHA I.

Conclusions: Following the ASO for TGA, compared to atrial level repairs, complications requiring intervention have shifted from the inflow to the outflow of the ventricles. Our experience confirms that the most common complication is pulmonary artery stenosis, which can be treated effectively by catheter procedures and /or surgery, the latter also providing effective treatment after failed percutaneous interventions. Most late reoperations are needed within the first 10 years after an ASO. Larger series and possibly longer follow-up may be necessary to detect more rare complications, such as aortic valve insufficiency and coronary stenosis, which have not been encountered in our patients. Finally, survival and functional outcomes of the ASO were excellent in the long term and seem unaffected by the need for re-intervention.

\section{P-217}

Consistent improvement of right ventricular (RV) size and function after late pulmonary valve replacement following complete repair of Tetralogy Fallot (TOF): A 20-year experience

Zografos M.P., Protopapas M.E, Hakim I.N., Zavaropoulos P., Alexopoulos C., Kyrvasillis G., Sarris E.G.

Iaso Children's Hospital, Athens, Greece
Introduction: Pulmonary valve insufficiency (to various degrees) is unavoidable for most patients after TOF repair, but can be well tolerated for several years. However, longstanding pulmonary valve insufficiency can lead to progressively severe RV dilatation and eventual dysfunction, which may predispose to heart failure, arrhythmias, and sudden death. Pulmonary valve replacement (PVR) is advocated in an increasing over time proportion of patients with prior TOF repair, aiming to prevent (and hopefully reverse) further excessive RV enlargement and dysfunction. We report our experience with PVR late after compete repair of TOF. Methods: Between 1/9/1997 and 1/11/2017, 60 patients (45 male - 15 female), median age 12 years (range 5 - 46) underwent PVR late after compete TOF repair. Most patients $(n=36)$ had undergone total TOF repair at other institutions, while twenty four were among the more than 300 TOF repairs performed by our group over the same time period. Median time interval between repair and PVR was 10.5 (range 1-43) years. Over the study period, we have used uniform indications for PVR, including symptoms, increase in RV end-diastolic-volume-index (RVEDVI) to $160 \mathrm{ml} / \mathrm{m}^{2}$ or more, and/or reduction of $\mathrm{RV}$ ejection fraction (RVEF) below 50\%, as measured by MRI. Heterologous valved conduits (ContegraR, diameter 18-22 mm), were used in 19 patients, 2 patients received HancockR conduits (16 and $22 \mathrm{~mm}$ ), and all 39 remaining patients had bioprosthetic valves (Edwards-MagnaR, 19-29 mm). Concomitant residual lesions (tricuspid or aortic valve insufficiency, residual VSD, RVOTO or pulmonary artery stenosis) were repaired in 23 patients.

Results: There was no early or late mortality. All symptomatic patients improved their NYHA functional class from median II to I $(p<0.001)$. At follow up (median 8 years, range 1 to 20), RVEDVI improved from $165 \mathrm{ml} / \mathrm{m}^{2} \pm 15$ to $132 \mathrm{ml} / \mathrm{m}^{2} \pm 16$ $(p=0.003)$, and RVEF from $42 \% \pm 7$ to $49 \% \pm 7$ ( $p=0.007)$. No patient has required redo PVR.

Conclusions: Late PVR after prior compete TOF repair can be performed safely, and results not only in stabilization but actually in improved patient functional status, reduced RV size and also in improved RV function.

\section{P-218}

Functional results (midterm)after double switch operation in congenitally corrected trasnposition of great arteries Vazaios C. (1), Kántorová A. (1), Valentík P. (1), Śagát M. (1), Nagi A.S. (1), Sabateen F. (1), Nosal'M. (1)

Children's Cardiac center of Bratislava, Department of Cardiac Surgery, Bratislava Slovakia

Introduction: Surgical therapy of CCTGA currently aims at re-establishing anatomically favourable hemodynamic by Senning-Rastelli (SR) or Senning-Switch (SS) procedure. In our study we summarise the midterm results of patients who underwent anatomical correction of CCTGA.

Methods: We retrospectively analysed our results with patients who underwent SS or SR operation from 1991 until 2017 with a median follow up of 6 years. Post-operative follow up echocardiographic data were collected and evaluated for every patient along with 10-year survival and 10 year freedom from reoperation. Results: Out of 11 patients who underwent final surgery for CCTGA, 8 patients underwent anatomic correction in our centre (3 underwent single ventricle palliation). Five patients had S,L,L and 3 I,D,D segmental anatomy. Ventricular septal defects were present in 7 patients. Pulmonary atresia was present in 4 patients, 1 patient had valvar pulmonary stenosis. Left ventricular retraining using pulmonary artery banding was used in 3 patients. Median 
time of LV retraining was 625 days (1,7years). Six patients underwent Senning-Rastelli procedure and 2 patients Senning-switch. The survival at 10 -years is $100 \%$. There were 4 reoperations, 2 conduit replacements and 2 for other cause. Overall freedom from redo is $45 \%$ at ten years. At the latest echocardiography follow up all patients have normal left ventricular function. There was no neoartic insufficiency in both patients after arterial switch. Tricuspid insufficiency of second degree is found in 2 patients. Atrial baffles are unobstructed in all patients. Post-operative arrhythmias were diagnosed in 2 patients with one of them diagnosed with supraventricular tachycardia and AV-block in the otehr patient. Three patients presented with spontaneous AV-block. All patients are in NYHA I functionall class.

Conclusions: Midterm results after anatomical correction of CCTGA are excellent. Half of our patients required reoperation within 10 years, which were mostly conduit replacements after SR procedure. All patients are in good clinical condition without any major anatomical residua. The incidence of complete AV blok is mostly spontaneous within the expected range.

\section{P-219}

Pulmonary artery banding (PAB): Fez's experience Atmani S. (1), Elkouach M. (1), Labib S. (2), Adnan B. (3)

University hospital center Hassan II Fez Morocco

Introduction: Pulmonary artery banding itself has not been an entirely benign procedure. Hence, the exact place of the pulmonary banding should be well defined.

Material and methods: A retrospective study was done with 33 patients undergoing a PAB procedure between January 2011 and July 2015. Criteria for banding were not closely defined; patients presented with severe cardiac insufficiency with failure to thrive. The charts of all patients undergoing PA banding operations were analyzed retrospectively.

Results: The mean age of PAB was around 16 month. The mean weight was $7 \mathrm{~kg}$. Six patients have Down's syndrome. The diseases are; ventricular septal defect (II b) (8 patients), Swiss cheese ventricular septal defect (2patients), double-outlet right ventricle ( 2 patients), atrio-ventricular canal (5 patients), Transposition of the greats arteries with VSD (2 patients), single ventricle with tricuspid atresia (6 patients), single ventricle (5) and coarctation with VSD (3 patients). The pulmonary artery systolic pressure prior to banding was 60 to $100 \mathrm{~mm} \mathrm{Hg}$.

The same surgical technique was used for all patients (Toronto formula). The re-interventions that were done following the application of the band were as follows: the PAB was moved and replaced since there was left coronary artery compression by the band in one case. Pericardial effusions were drained in two patients. A pneumo -mediastinum in one case spontaneously improved.

The mean hospital stay was $10 \pm 3$ days. Four patients died by nosocomial infection and severe pulmonary hypertension with a hospital mortality of (4/33). An additional 6 patients died while they were awaiting definitive repair, total mortality is $30 \%$.

Conclusion: PAB still useful under in our area, can relieve heart failure symptom. But the mortality in our study is high, significant factor of death are advanced disease with high pulmonary hypertension.

\section{P-221}

Sildenafil Plasma Concentrations during Routine Treatment of Children with Pulmonary Arterial Hypertension

Ziesenitz V. (1,2), Grommes S. (1), Burhenne J. (3), Uhl S. (1), Mikus G. (3), Haefeli W.E. (3), Gorenflo M. (1)
Paediatric \& Congenital Cardiology University of Heidelberg, Germany (1); Pediatric Pharmacology \& Pharmacometrics, University of Basel Children's Hospital, Basel, Switzerland (2); Clinical Pharmacology \& Pharmacoepidemiology, University of Heidelberg, Germany (3)

Introduction: Sildenafil, a substrate of CYP3A, is a treatment standard of children with pulmonary arterial hypertension $(\mathrm{PAH})$. The current dosing recommendation for sildenafil in children is $0.5-2.0 \mathrm{mg} / \mathrm{kg}$ thrice daily. The objective of this study was to generate a dataset of sildenafil plasma concentrations during routine in-patient treatment in infants and children with PAH using opportunistic blood sampling for pharmacokinetic analysis. Methods: After obtaining informed consent, sparse remnant blood samples from routine blood analyses were analyzed if drawn within $8 \mathrm{~h}$ after the last oral sildenafil administration using a validated LC/MS/MS assay. In addition, patient characteristics, diagnoses, and comedications in order to screen for drug interactions were extracted from the medical records. Obtained plasma concentrations were compared with those of traditional studies in children using serial sampling.

Results: Twenty-two patients (11 males) with a median age of 7.5 months (range 4 days- 9.5 months), median weight of $6.54 \mathrm{~kg}$ $(3.05-26.7 \mathrm{~kg})$ were included. Twenty-two patients had pulmonary hypertension associated with congenital heart disease and two had other causes. Mean pulmonary artery pressure was $29 \pm 14 \mathrm{mmHg}$ during therapy. Seven patients were classified as Rosenthal class B and 15 were class C. In total, 271 blood samples were collected (3-38 samples per patient). Maximum sildenafil concentration ranged from 2.80 to $645 \mathrm{ng} / \mathrm{ml}$ (median Cmax $87.4 \mathrm{ng} / \mathrm{ml}$ ), time of maximum plasma concentration (Tmax) ranged from 0.1 to $5 \mathrm{~h}$ after dosing (median $2 \mathrm{~h}$ ). Median administered dose was $0.86 \mathrm{mg} / \mathrm{kg}(0.45-1.95 \mathrm{mg} / \mathrm{kg})$ three or four times a day. Cmax normalized to dose was $0.99-215 \mathrm{ng} / \mathrm{ml} / \mathrm{mg}$. Eleven patients were co-treated with the CYP3A inducer phenobarbital.

Conclusions: The mean sildenafil dose matches the recommended dose range of $0.5-2.0 \mathrm{mg} / \mathrm{kg}$. Plasma concentrations using remnant samples were comparable to those of previously conducted pediatric studies, indicating rapid elimination in children. Sildenafil plasma concentrations showed substantial inter-patient variability; the correlation between plasma and effect site concentrations and the predictive value of plasma concentration measurements, however, still have to be investigated in children, as well as the extent of CYP3A induction by phenobarbital in children.

\section{P-222}

Early Postoperative Low Cardiac Output Syndrome Due to Restrictive Right Ventricle Physiology After Surgical Repair of Tetralogy Fallot

Cevik A., Tuncel Z.,Balci S.T.,Karaci A.R., Yalcin Y.

Istanbul Bilim University Florence Nightingale Hospital Pediatric Cardiac Intensive Care Unit, Istanbul, Turkey

Objectives: The restrictive right ventricle physiology ( $\mathrm{RVVF}$ ) after surgical repair of tetralogy of Fallot (TOF) is common. Clinically significant rRVF leading to high morbidity and mortality is seen due to low cardiac output syndrome (LCOS) in early postoperative period. We investigated the role of echocardiography to determine the risk of clinically significant rRVF after surgical repair of TOF.

Methods: 46 patients who had surgical repair of TOF between May 2014 and June 2017 were taken to this study. Group 1 ( $\mathrm{n}=10$, 
median age $14,0 \pm 2,3$ months) was found echocardiographic evidence of RVF with LCOS; group $2(n=12$, median age $8,1 \pm 3,0$ months) was found $\mathrm{rRVF}$ without LCOS; while group 3 ( $n=24$ patients, median age 9,6 $\pm 2,9$ months) showed neither LCOS nor rRVF. Presence of end diastolic antegrade forward flow greater than $30 \mathrm{~cm} / \mathrm{sec}$ during atrial systole using pulse wave Doppler measurements of at least three different consecutive cycles was accepted as echocardiographic evidence of rRVF. The right ventricle and left ventricle diastolic functions were also assessed by analyzing mitral and tricuspid inflow patterns and Doppler tissue imaging (DTI). Right ventricle myocardial performance index (RVMPI), and left ventricle myocardial performance index (LVMPI) values were evaluated.

Results: The end diastolic antegrade pulmonary flow velocity was significantly higher in group $1(\mathrm{p}<0.001)$. RVMPI in group 1 was higher compared to group $3(\mathrm{p}<0.001)$. The concurrent use of end diastolic antegrade pulmonary flow velocity (cut-off value $>99 \mathrm{~cm} / \mathrm{sec}$ ) and DTI values showed 100\% sensitivity and $90 \%$ specificity.

Conclusion: In patients who had surgical repair of TOF, the LCOS development risk can be determined by using DTI and end diastolic antegrade pulmonary flow velocity measurements in the early postoperative period.

\section{P-223 \\ Bivalvation Procedure with Plicated Interatrial Septum Remnant for Common Atrioventricular Valve Regurgitation in Neonate with Asplenia Syndrome Imoto Y., Matsuba T., Ogata Y., Yamamoto H., Matsumoto K., Arata K., Imagama I., Kanda H., Toyokawa K., Tateishi N., Nagatomi S. Cardiovascular and Gastroenterological Surgery, Kagoshima University Graduate School of Medical and Dental Sciences, Kagoshima, Japan}

Background: Regurgitation of common atrioventricular valve (CAVV) with moderate grade or more in a neonate with asplenia syndrome is a formidable problem, especially when complicated with TAPVC with pulmonary venous obstruction. Morphological understanding is very difficult in neonatal CAVV, and repair technique has not been established.

Case: The patient was a 7-day-old boy with asplenia syndrome complicated with single ventricle with CAVV, infracardiac type TAPVC, pulmonary atresia and PDA. Echocardiography showed severe CAVV regurgitation from the central portion. He presented with failure to thrive. Operation was performed with conventional cardiopulmonary bypass with aortic and bicaval venous cannulae. Performed procedures were as follows; PDA ligation, Blalock-Taussig shunt (EPTFE $3.5 \mathrm{~mm}$ ), patch augmentation of the pulmonary bifurcation, anastomosis of the common $\mathrm{PV}$ to the atrium, ligation of the vertical vein, and CAVV repair. Saline test in CAVV revealed significant regurgitation, but responsible valve lesions were unclear. Meticulous techniques for valve repair as are used in older patients seemed unrealistic, and we performed plication of the interatrial septum remnant (IASR) expecting the bridge of IASR would improve coaptation of the central portion of the CAVV and also protect against valve prolapse. Postoperative echocardiography showed mild CAVV regurgitation. He underwent bidirectional Glenn procedure at the age of one year, and total cavopulmonary connection (TCPC) at 3 years old. Eight years have passed after TCPC, but his CAVV regurgitation remains mild and no significant stenosis is noted.

Conclusion: Bivalvation procedure with plicated IASR is a useful technique for CAVV regurgitation in neonates with asplenia syndrome.

\section{P-224}

Left internal Jugular aneurysm in a 15 month old Boy Loggos S. (1), Kannangara R. (1), Chetcuti K. (2), Pelella G. (1), Lotto A. (1), Dhannapuneni R. (1), Guerrero R. (1) (1)Department of Congenital Cardiac Surgery, Alder Hey Children's Hospital, Liverpool, UK; (2)Department of Radiology, Alder Hey Children's Hospital, Liverpool, UK

Introduction: Jugular vein aneurysm are extremely rare in the paediatric population, especially lesions that were not exhibiting clinical symptoms. We present a 15 month old boy, with a mass protruding from the left side of the neck. There was no history of injury or puncture and no history of infectious diseases. From the clinical examination. there was a palpable mass in the left aspect of the neck, with no sings of compression of surrounding structures, and no signs of distension in the rest of the venous system. Laboratory tests did not reveal any abnormal findings. Initially he had an US of the neck, which highlighted the presence of an internal Jugular Aneurysm. A CT of his Head, neck, chest, abdomen and pelvis followed using contrast, which revealed no further vascular abnormalities. Finally he had a cardiac ECHO as part of the Pre-op assessment.

Method and Presentation: The Patient was presented in out MDT meeting, after an extensive literature search of this extremely rare lesion. Very few case reports have been found. Some did not advocate any intervention. However in some cases intervention has been performed. It was also reported in children and operations were performed in a 3 month old and a 1 year old. Cardiology and interventional radiology teams were not able to offer interventional procedures, due to technical limitations. We decided to offer surgery and excision of the aneurysm. The aneurysm was doubly ligated above and below the junction with the left subclavian and was completely excised and sent to histopathology which confirmed diagnosis.

Comment: Jugular aneurysm are extremely rare conditions, especially in the paediatric population, with few reports, but also in the adult population. They are usually presented as enlarging masses, neurological symptoms but most of the time are asymptomatic. The treatment options include, conservative management and follow ups, interventional procedures, surgery, and Hybrid procedures. There has been reports of increased incidence of thrombosis and resulting thromboembolic phenomena and also bleeding.

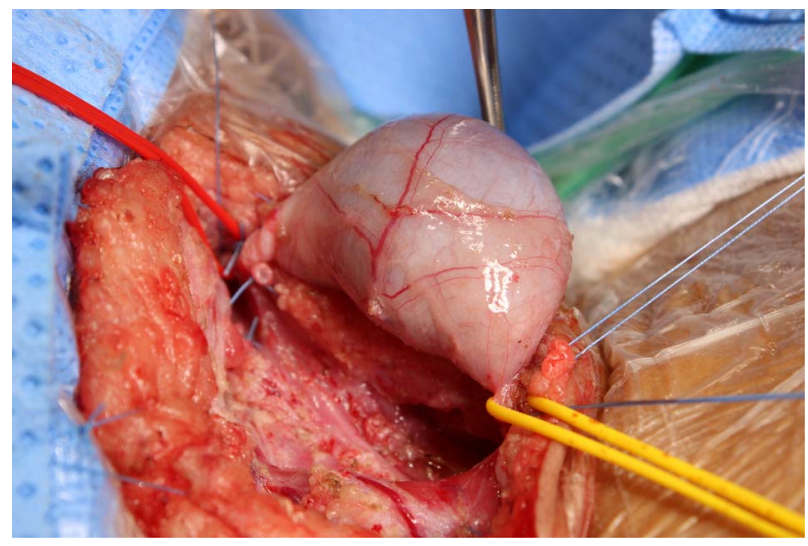

Figure.

P-225

Outcomes following general anaesthesia in Paediatric Hypertrophic Cardiomyopathy

Norrish G. (1,2), Forshaw N. (1), Field E. (1), Kaski J.P. (1,2)

Great Ormond Street Hospital, London UK (1); Institute of

Cardiovascular Sciences University College London, UK (2) 
Introduction (or Basis or Objectives): Hypertrophic cardiomyopathy (HCM) is the second most common cause of cardiomyopathy in childhood. These patients have historically been considered to be high risk candidates for general anaesthesia, however there is currently a paucity of evidence regarding the safety of anaesthesia and peri-operative outcomes in this population.

Methods: A retrospective single centre cohort study of all paediatric patients ( $<18$ years) with hypertrophic cardiomyopathy undergoing general anaesthesia (GA) between 2000-2016 was performed to assess peri-anaesthetic outcomes. Patients undergoing cardiac surgery were excluded. Primary outcome was peri-operative mortality or need for re-hospitalisation within 30 days of anaesthesia.

Results: 86 patients aged 0-18 years (median 12.4 years) with HCM underwent 164 separate general anaesthetic procedures over the study period. General anaesthetic was most commonly required for a non-surgical cardiac procedure $(n=87,53 \%)$, followed by a general surgical, radiology or orthopaedic procedure ( $\mathrm{n}=17, \mathrm{n}=16, \mathrm{n}=12$ respectively). The underlying aetiology was heterogenous and included sarcomeric/idiopathic disease $(56 \%)$, a malformation syndrome $(26 \%)$, inborn error of metabolism (12\%) or neuromuscular disease (4\%). Maximal wall thickness (MWT) ranged from $4-49 \mathrm{~mm} .23$ patients $(1 \%)$ had severe left ventricular hypertrophy (MWT $>30 \mathrm{~mm}$ or Z score $>+6$ ) and 47 patients $(30.5 \%)$ had left ventricular outflow tract obstruction at rest. The majority of patients $(n=143,77 \%)$ had no complications. Length of stay ranged from $0-21$ days although two thirds of patients $(n=104,65 \%)$ were performed as day-cases. 20 patients $(12 \%)$ had minor peri-anaesthetic complications which included intra-operative bradycardia or hypotension requiring treatment. 1 patient $(0.6 \%$ of GA procedures) experienced a cardiac arrest during anaesthetic induction which required extra-corporeal membrane oxygenation (ECMO) supported cardiopulmonary resuscitation. The presence of left ventricular systolic impairment was the only clinical feature associated with a peri-anaesthetic complication.

Conclusions: We report the largest series of paediatric HCM patients undergoing general anaesthesia to date with a low incidence of minor complications $(12 \%)$ and a mortality rate of $0.6 \%$. In an experienced centre, paediatric patients with HCM can be anaesthetised with a low risk of adverse events. Future studies are required to identify clinical features that may predict anaesthetic risk.

\section{P-226 \\ Improving Use of Peri-operative Therpeutic Heparin Infusions on a Paediatric Cardiology Ward \\ Searle J., Bentley S., Rowlinson G. \\ Royal Brompton Hospital, London, UK}

Introduction: Heparin infusions are commonly used to minimise the risk of thromboembolic events in paediatric cardiology patients. Evidence for infusion rates and target ranges for monitoring are however largely extrapolated from adult data. Given differences in drug clearance and clotting factor expression, the paediatric population is very different. Where paediatric-specific evidence exists, it is largely from a non-cardiology PICU population. Furthermore, evidence arbitrarily distinguishes patients above and below one year old. These age ranges persist within current ACCP guidelines.

Methods: Retrospective observational study of heparin infusion dosing in patients on a general paediatric cardiology ward, at a surgical centre, over a one year period. All inpatients between 0-16 years old, who received a heparin infusion, were included. The patient cohort was generated from an electronic prescribing system, on which timings of dose changes were also recorded. Correlation was made against pathology results for both APTT and anti-Xa levels. Demographic data was collected from patient records. Statistics were analysed using STATA.

Results: Thirty-six patients had 243 blood tests to monitor heparin infusions. Current guidelines were followed in all cases. No bleeding, thromboembolic events, or adverse reactions were recorded. The most common indication was post-operative prophylaxis for fenestrated-TCPC $(n=7)$. Median delay till achieving therapeutic dosing was 26 hours (range $0-115$ hours). This was longer for patients following PICU step-down, at 32 hours (0-115 hours). Inverse correlation was demonstrated between age and therapeutic infusion rate $(\mathrm{r}=-0.59)$. Significant differences were seen between under-1, 1 to 6 and over- 6 year age groups $(\mathrm{p}=0.02$, figure).Increasing initial heparin dose for a ' 1 to 6 year' group from 20 to 24 units/kilogram/hour would reduce time till therapeutic dosing by $10(0-30.5)$ hours per patient.

Conclusions: Evidence underlying heparin dosing is very limited in paediatric cardiology ward patients. Demographic-based dosing relies upon data using arbitrary age boundaries. We demonstrate that patients currently experience significant delays before achieving accepted therapeutic targets. This may increase risk of thromboembolic events, and certainly increases the burden of blood tests. Modifying the current guideline, to increase initial infusion rates for an intermediate age group, may reduce this risk exposure.

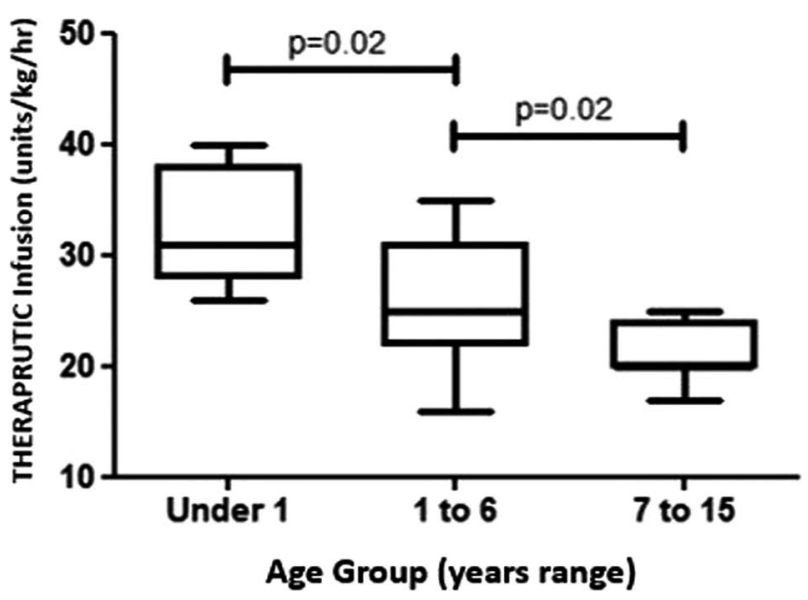

Figure.

\author{
P-227 \\ Risk Factors for Pericardial Effusion After Cardiac Surgery \\ in Children - A 17-year Retrospective Survey \\ Alsafi Z. (1), Maxedius A. (1), Tran P.K. (2), Johansson Ramgren J. (2), \\ Liuba P. (1) \\ Pediatric Cardiology (1) and Cardiac Surgery (2), Pediatric Heart Center, \\ Skane University Hospital and Lund University, Lund, Sweden
}

Background: Although postoperative pericardial effusion (postopPE) is a well-recognized complication after cardiac surgery in children, there is a very limited number of studies assessing its prevalence and risk factors.

Methods: A retrospective single-centre survey of all children who underwent surgery for CHD between January 2001-October 2017 and who required PE drainage within the first 30 days after surgery was conducted. Demographics, cardiac and surgical data, as well as PE drainage data including time from surgery to PE drain insertion, duration of postopPE and eventual relapse were 
obtained from the hospital's digital database. Right atrial (RA) and left ventricular diameters and areas were measured from echocardiographic digital scans obtained before the cardiac surgery and upon drain removal in all biventricular patients with surgery during 2008-2017. Data is expressed as median and range.

Results: Of nearly 5500 pediatric cardiac surgeries over the last 17 years, 126 patients $(0.02 \%$; age $0.7(0-16)$ years; weight $6(1-80)$ $\mathrm{kg} ; 69$ (55\%) girls) needed pericardial drainage at 10 (0-29) days after surgery with a drainage time of $2.5(1-24)$ days. The vast majority $(n=114$ (90\%); age: $0.9(0-16)$ years; 61 (54\%) girls) underwent cardiopulmonary bypass $(\mathrm{CPB})$ surgery. Of these, 70 patients $(61 \%$; age $1.1(0-16)$ years; 33 (46\%) girls) had surgery for non-shunt lesion as primary lesion, whereas VSD or complete AVSD was the primary lesion in 30 of 44 patients (68\%) with shunt surgery. Among CPB patients, there was no significant difference in surgical age, gender, time between surgery and postopPE drainage and drainage duration between the non-shunt and the shunt groups $(p>0.1)$. Longer need of pericardial drain ( $\geq 7$ days) was encountered in $15(13 \%) \mathrm{CPB}$ patients and was associated with male gender $(p=0.04)$ and elevated CRP on days 1 and 2 postoperatively ( $p=0.02$ for both). Later onset of postopPE ( $\geq 14$ days) was encountered in $22(19 \%)$ CPB patients and was associated with decrease in RA area $(\mathrm{p}=0.02)$.

Conclusion: PostopPE after pediatric cardiac surgery is a rare complication occurring mostly in young patients after $\mathrm{CPB}$ surgery with later onset associated with postoperative decrease in RA size, and longer need for drainage associated with male gender and early postoperative inflammatory response.

\section{P-228}

Factors Influencing Need for Late ASD Closure after Neonatal Repair of Severe Pulmonary Valve Obstruction and Intact Ventricular Septum

Björk J. (1), Odermarsky M. (1), Steiner K. (2), Jarring J. (1), Sjöberg G. (2), Johansson Ramgren J. (1), Liuba P. (1) Pediatric Cardiology and Cardiac Surgery, Skane University Hospital and Lund University, Lund (1); Karolinska University Hospital, Stockholm (2), Sweden

Background: In neonates with critical pulmonary stenosis (CPS) or pulmonary atresia with intact ventricular septum (PAIVS), a nonrestrictive atrial septal defect (ASD) has been speculated to improve the initial clinical course after pulmonary valvotomy (PV) but some concerns exist in terms of its potentially longterm adverse effect on the right ventricle's (RV) growth and persistent desaturation due to right-to-left (R-L) shunt.

Objective: To assess the relationship between the size of ASD and the need for post-valvotomy reinterventions.

Method: Patients with PAIVS and CPS treated at our center during 2001- 2015 were reviewed. Exclusion criteria were associated cardiac malformations and hypoplastic RV deemed unsuitable for biventricular circulation. Clinical and echocardiographic data were retrieved from the hospital's databases.

Results: In total, 48 patients (18 with PAIVS and 30 CPS) were included. The median follow-up was 5 and 8 years, respectively. One patient with PAIVS died on day 3 after surgical valvotomy and Blalock-Taussig shunt (BTs). The majority (89\%) of patients with PAIVS had surgical valvotomy whereas transcatheter valvotomy was used in the majority (87\%) of patients with CPS. Palliation with BTs or PDA stenting was used in 13 (72\%) patients with PAIVS and in 4 (13\%) patients with CPS. Reintervention within 1 month after initial repair was needed in $4(22 \%)$ patients with PAIVS and in $4(13 \%)$ patients with CPS. Later reinterventions were performed in in $11(61 \%)$ patients with PAIVS and in $10(33 \%)$ patients with CPS. Of these, 7 (39\%) patients with PAIVS and 5 (17\%) with CPS underwent ASD closure due to persistent resting desaturation. The latter did not correlate with ASD size after valvotomy $(p>0.1)$. Initial palliation with BTs was the only variable associated with ASD device closure $(p=0.04)$. No patient required univentricular conversion.

Conclusion: Neonatal biventricular repair for severe pulmonary valve obstruction has low mortality but significant need for late reinterventions, mostly consisting of ASD closure due to clinically significant desaturation secondary to R-L shunt. The use of aortopulmonary shunt, probably illustrating a more severe form of RV hypoplasia, but not the size of the ASD, predicts the need for later ASD closure due to desaturation.

\section{P-229}

Early Electrocardiographic Changes in Patients with Tetralogy of Fallot After Surgical Repair with Monocusp Pulmonary Valve

Ljung L. (1), Jeremiasen I. (1), Rosenkvist C.J. (1), Maxedius A. (1), Sjöberg G. (2), Hanseus K. (1), Liuba P. (1)

1) Cardiology, Pediatric Heart Center, Skåne University Hospital, Lund, Sweden; 2) Pediatric Cardiology, Karolinska University Hospital

Stockholm, Sweden

Background: Surgical repair of Tetralogy of Fallot (TOF) with right ventricular outflow tract (RVOT) enlargement with transannular patch (TAP) results in pulmonary regurgitation (PR) with subsequent increasing RV volume load and need for later pulmonary valve replacement. These changes are typically associated with progression of QRS and QTc duration, which are predictive for long-term adverse events. RVOT reconstruction with monocusp pulmonary valve has been increasingly used during the past decade in the attempt to improve early postoperative recovery and delay PR particularly during the first years after surgery.

Objective: To assess whether repair of TOF with monocusp pulmonary valve influences the progression of QRS and QTc duration during the first two years after surgical repair of TOF.

Methods: We performed a retrospective survey of all patients with TOF who underwent surgical repair between 1998-2014, who had conventional electrocardiograms available (A) before surgery, (B) upon postoperative discharge and (C) and at 18-24 months after surgery, and who did not require reoperation during this period. Demographic, clinical, surgical and electrocardiographic data were obtained. Data are median and range.

Results: In total, 93 patients who fulfilled the above criteria were included. There was no mortality. Of these, 34 patients (median age/weight at repair: 4.8 months $/ 6.9 \mathrm{~kg}$ ) underwent pulmonary valve-sparing repair repair, 30 patients (median age/ weight:5.2 months $/ 6.7 \mathrm{~kg}$ ) underwent TAP repair, and 29 patients (median age/weight:5.3 months $/ 6.5 \mathrm{~kg}$ ) had repair with TAP and monocusp There were no differences between the groups in QRS and QTc durations preoperatively or upon discharge after repair ( $p>0.15)$. At 18-24 months, both QRS and QTc durations were greater in the monocusp group $(\mathrm{p}<0.05$ for QRS and $\mathrm{p}<0.01$ for $\mathrm{QTc})$. The percentage change in QRS and QTc durations from the early postoperative time were increased in the monocusp group as compared to the other groups ( $\mathrm{p}<0.05$ for both). Conclusion: Surgical repair of TOF with TAP and monocusp pulmonary valve appears to be associated with adverse changes in QRS and QTc durations during the first years after repair.

\section{P-230}

Levosimendan therapy in children with chronic/acute cardiomyopathy compared to congenital heart disease Galoin-Bertail C., Mulatier C., Perouse de Montclos T., Bakloul M., Walton C., Durousset C., Leconte C., Di Filippo $S$.

Pediatric Cardiology, Cardiovascular Hospital, Lyon, France 
The objective of this study was to compare levosimendan therapy administered to hemoynamically compromised pediatric patients with either myocardial dysfunction (CMP) or congenital heart disease (CHD).

Methods: This is a retrospective study of patients aged $<18$ years, who received one or more levosimendan infusions, for failure to wean off inotrope and/or ECMO support. Demographic data, clinical, biological, and echocardiographic parameters (LVSF, subaortic VTI and TAPSE) were collected at onset of LEVO (T0), at 2 days (T2), 10days (T10), 30days (T30) and 6months (M6) after LEVO termination. Patients were divided into CHD and CMP groups. Patients were LEVO responder if weaned off inotrope and/or ECMO support after LEVO termination.

Results: CMP group included 34 patients (53\% females) with myocardial dysfunction ( 5 acute myocarditis) who received 65 LEVO and CHD included 37 cases (29.7\% females) who received 47 LEVO. Mean number of levosimendan infusions was 1.27 $(\max 3)$ in $\operatorname{CHD}$ and $1.9(\max 6)$ in $\operatorname{CMP}(p=0.03)$. Mean age at LEVO was 3.8 versus 2.3years in CMP and CHD $(p=0.04)$. Twelve percent of CMP and $51 \%$ of CHD were dependent on ECMO support $(\mathrm{p}<0.0001)$. ECMO was discontinued in $6 \%$ of CMP and $36 \%$ in CHD ( $<<0.0001$ ), $21.6 \%$ of CMP and $36 \%$ of CHD could be weaned off inotrope support $(p=0.08)$, i.e. $26 \%$ of CMP versus $66 \%$ of CHD were responders $(p<0.0001)$. Failure (heart transplantation and/or death) occurred in $76 \%$ o CMP versus $43 \%$ in CHD $(p=0.0039)$. Shortening fraction (SF) was constantly lower in CMP compared to CHD at each time point from $\mathrm{T} 0$ to $\mathrm{T} 30$, and increased in both group from $\mathrm{T} 0$ to $\mathrm{T} 30$. LVSF did not differ between chronic and acute CMP at onset (T0) and increased in acute myocarditis (AM) but not in chronic CMP, while none of AM was LEVO responder. Survival at 5-year follow-up were $60 \%$ in CHD versus $20 \%$ in CMP $(p=0.0002)$. Conclusion: Levosimendan therapy can be safely administered in children and seems to be effective in CHD compared to CMP patients.

\section{P-231 \\ Prognostic value of a new lung ultrasound score in pediatric cardiac surgery \\ Ait Ali L. (1), Gargagni L. (1), Scalese M. (1), Franchi E. (2), Giordano R. (2), Haxiademi D. (2), Moschetti R. (2), Festa P. (2), Murzi B. (2), Santora G, Cantinotti M. (2) \\ (1) Institute of Clinical Physiology, National Research Council, Massa-Pisa, Italy; (2) Fondazione Regione Toscana G. Monasterio, Massa, Pisa, Italy}

Background: Lung ultrasound (LUS) is gaining consensus for the diagnosis and prognosis of pulmonary disease. However, although pulmonary complications are common in the post-operative (post-op) of pediatric cardiac-surgery, the use of LUS is still limited. We sought to evaluate if LUS evaluation of lung congestion after post-op extubation could predict intensive care unit length of stay (ICU-LOS) and if it's reflect conventional biomarkers in this setting.

Methods: LUS was performed in the 24 hours $(13 \pm 6 \mathrm{~h})$ after extubation in 132 patients who underwent pediatric cardiac surgery (median age:0.9 year, IQ: $0.22 ; 4.4$ ). For each hemithorax, 3 areas (anterior/lateral/posterior) have been evaluated in the upper and lower halves of the chest (for a total of 6 scanning sites per side). Pulmonary congestion score was assessed as percentage of B-lines and atelectasis. For each site a score was calculated as follow: score: 0 normal aeration, absence of b-lines: Score1 (mild congestion): 10-30\% B-lines; Score 2 (moderate congestion): 40-60\% B-lines; Score 3 (severe congestion): 70-100\% B-lines;
Score 4 (complete loss of aeration): lung consolidation. Total LUS-score was calculated as a sum of all sites score. Demographic, surgical data and Biomarkers (brain natriuretic peptide: BNP- and cystatin-C) were recorded.

Results: The total LUS-score $(13 \pm 6)$ correlates inversely with age: $\mathrm{r}:-0.38, \mathrm{p}<0.001$ and positively with both $\mathrm{BNP}$ and Cystacin-C (respectively r:0.29, $\mathrm{p}=0.01$ and $\mathrm{r}: 0.38, \mathrm{p}<0.001$ ) as well as with a surgical complexity score ( Arisotle score) (r:0.22, $\mathrm{p}=0.01)$ and by-pass time ( $\mathrm{r}: 0.24, \mathrm{p}<0.001)$. Moreover there was a good correlation also with ICU-LOS: (r: 0.4, $\mathrm{p}<0.001)$. At multivariate analysis: the LUS-Score was identified as an independent variables for $\operatorname{lnICU}$ LOS (beta: .22, 95\% CI: 0.01-0.058, $\mathrm{p}=0.005$ ) together with Cystacin (beta: .34, 95\% CI: $0.52-1.265, \mathrm{p}<0.01$ ), age (beta: $-, 14,95 \% \mathrm{CI}:-.086--0.01$, $\mathrm{p}=0.047$ ) and Aristotle score (beta: $0.13,95 \%$ CI: $0.073-0.205$, $\mathrm{p}<0.01)$.

Conclusions: We demonstrated the prognostic values of LUS-score as an independent associated factor with ICU-LOS after pediatric cardiac surgery. LUS score increases with the complexity of surgical procedure, and its trend reflects that of other prognostic biomarkers such as BNP and Cystatin-C. Further studies are required to validate and reinforce these data.

\section{P-232}

Could 3D-printed models improve pre and peroperative management of anomalous origin of coronay artery?

Batteux C. (1), Piéchaud J.F (1), Belli E. (1), Zoghbi J. (1), Van Aerschot I. (1), Bouzguenda I. (2)

Hôpital Privé Jacques Cartier, Massy, France (1); Hôpital Privé La Louvière, Lille, France (2)

Introduction: Anomalous Origin of Coronary Artery (AOCA) is the second most frequent cardiovascular etiology of sudden death during exercice among children and its pre- and per-operative management remain discussed.

Methods: We retrospectively analysed patients who underwent surgery for AOCA in our hospital. We evaluated their 3D-printed models to describe their anatomy and predict the most efficient surgical gesture and its potential difficulties.

Results: between 2010 and 2017, 19 patients were operated on (Median age 15 years old, 37\% asymptomatic). Preoperative functionnal or invasive tests (stress test, coronarography) didn't

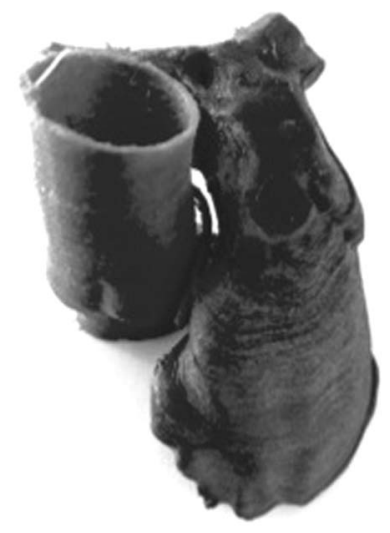

Photo: Anomalous Origin of Right Coronary Artery (from left sinus) : printed model

Figure. 
improve the management of AOCA. Coronary unroofing was the most frequent surgical gesture performed $(\mathrm{n}: 16 ; 84 \%)$, associated in $50 \%$ to a complementary gesture (pulmonary trunk translation, Lecompte's manoeuvre, sino-tubular jonction's resection). There were no postoperative adverse events except pericardial effusion (n : $7 ; 36 \%$ ) and no mortality. Median follow-up was 27 months. All patients are now asymptomatic. Based on CT-scan, we modelled, 3D-printed (photo) and presented 17 patients to a pluridisciplinary group. Due to a bad CT-scan quality, 2 models weren't 3D-printed. Referring to per-operative anatomy, our models improved the preoperative anatomy description in $47 \%$ of cases $(\mathrm{n}: 8)$. Main surgical gesture decided on 3D printed models was similar to surgical choice in $82 \%$ of cases $(n: 14)$. Furthermore, complementary or alternative gesture to unroofing have been predicted in 7/11 cases $(63 \%)$.

Conclusions: Unroofing in AOCA surgical management shows good and safe results but preoperative management remain variable. With $3 \mathrm{D}$ printed models, we showed a real benefit on $3 \mathrm{D}$ anatomy description, and a good matching in the prediction of associated/alternative gesture to coronary unroofing.

\section{P-233}

Incidence of congenital heart disease in a neonatal unit of a tertiary care hospital in Tunisia

Chioukh F.Z. (1), Ben Ameur K. (1), Khemis T. (1), Ajmi H. (2), Hamdi S. (3), Maatoug F. (3), Kortas C. (4), Ben Hamida H. (1), Bizid M. (1), Zaydi T. (1), Monastiri K. (1)

Maternity Center, Department of Neonatal Intensive Care Unit Monastir-Tunisia (1); Teaching Sahloul Hospital Department of Pediatrics Sousse-Tunisia (2); Teaching Fattouma Bourguiba hospitalTunisia, Department of Cardiology B Monastir-Tunisia (3); Teaching Sahloul Hospital Department of cardiovascular Surgery Sousse-Tunisia (4)

Objectives: To determine the incidence and pattern of various congenital heart diseases (CHD) in a neonatal unit of a tertiary care hospital.

Methods: The prospective study was carried out in a neonatal unit of a tertiary care hospital from 2015 to 2016 . All neonates admitted with gestational age of $>28$ weeks irrespective of birth weight were included in the study. Neonatologist carried out the neonatal examination during the first 12 hours of life. Neonates suspected of having congenital heart disease were further evaluated by pulse oxymetry, X-ray chest and neonatologist performed echocardiography. The findings of echocardiography were confirmed by pediatric cardiologist in case of structural and/or functional cardiac abnormalities.

Results: Of the 13406 neonates born during the period of the study, 123 were found to have congenital heart disease with an incidence of 9.2/1000. 91\% of newborns were inborn. Prenatal screening was done in $11 \%$ of cases. Median gestational age at diagnosis was 36 weeks. Most common lesion was atrial septal defect 35 (28.5\%), followed by ventricular septal defect 29 (23.6\%) and patent ductus arteriosus 25 (20.3\%). The other CHD were essentially tetralogy of fallot $04(3.2 \%)$, transposition of great arteries $04(3.2 \%)$, troncus arteriosus $04(3.2 \%)$ and interruption of arch artery $04(3.2 \%)$, double- outlet right ventricule $03(2.4 \%)$ and atrioventricular canal defects 03 (2.4\%).

Conclusion: Congenital heart diseases are a common congenital anomaly which must be diagnosed as earlier as possible. Its incidence varies from centre to centre due essentially to method of detection and early examination by a neonatologist. Promote early diagnosis is important to consider in order to improve management of CHD in our country.

\section{P-234}

Mortality of congenital heart diseases in a neonatal intensive care unit

Chioukh F.Z. (1), Ben Ameur K. (1), Khemis T. (1), Ajmi H. (2), Hamdi S. (3), Maatoug F. (3), Kortas C. (4), Ben Hamida H. (1), Bizid M. (1), Zaydi T. (1), Monastiri K. (1)

Maternity Center, Department of Neonatal Intensive Care Unit Monastir-Tunisia (1); Teaching Sahloul Hospital Department of Pediatrics Sousse-Tunisia (2); Teaching Fattouma Bourguiba hospitalTunisia, Department of Cardiology B Monastir-Tunisia (3); Teaching Sahloul Hospital Department of cardiovascular Surgery -Sousse-Tunisia (4)

Objectives: To determine the mortality of congenital heart disease (CHD) in a neonatal unit of a tertiary care hospital.

Methods: The prospective study was carried out in a neonatal unit from april 2015 to marsh 2017. All confirmed CHD admitted with gestational age of $>28$ weeks irrespective of birthweight were included in the study. Atrial defects and patent ductus arteriosus were excluded from the study. Data was collected on a predesigned proforma containing information regarding consanguinity, gender, gestational age, and weight at birth, family history, and associated malformations.

Results: Fifty five newborns were included in the study. Sex ratio was 1.03. Antenatal diagnosis was noted in $11 \%$ of cases. Parental consanguinity was present in $22 \%$ of cases. $38 \%$ of cases were syndromic especially trisomy 21 . Mean age at echocardiographic diagnosis was 2 days and the most frequent indications for this exam were the presence of a murmur followed by respiratory distress and cyanosis. Most frequent anomalies were ventricular septal defect 29 (52.7\%), followed by tetralogy of fallot $4(7.2 \%)$, transposition of great arteries $04(7.2 \%)$, troncus arteriosus $4(7.2 \%)$, interruption of arch artery 4 $(7.2 \%)$ and atrioventricular canal defects $3(5.5 \%)$. Mecanical ventilation and prostaglandines infusion were respectively indicated in $45 \%$ and $30 \%$ of cases. $18 \%$ of babies were operated. Mortality rate was $40 \%$ and the letality by CHD was $68 \%$.

Conclusion: Despite advances in neonatal diagnosis and resuscitation of CHD, their management is limited in Tunisia because of an insufficient technical platform and human resources especially in cardiovascular surgery departments. Furthermore, the lack of insurance coverage for all types of severe CHD fails to provide surgery in developed countries for all patients.

\section{P-235 \\ Degenerative alterations in RVOT samples - what to learn from histopathological analysis? \\ Peivandi A.D. (1), Seiler M. (1), Asfour B. (2), Sindermann J. (1), Martens S. (1), Osada N. (1), Malec E. (3), Lueck S. (3) Dept. of Cardiothoracic Surgery, Division of Cardiac Surgery, University Hospital of Muenster, Muenster, Germany (1); German Pediatric Heart Center, Sankt Augustin, Germany (2); Dept. of Cardiothoracic Surgery, Division of Pediatric Cardiac Surgery, University Hospital of Muenster, Muenster, Germany (3)}

Objectives: Many children with reconstructive surgery of the RVOT during childhood need re-operation mostly due to re-obstruction. Focusing on histological correlation of degenerative alterations, we examined the calcification degree, number of nuclei per $\mathrm{mm} 2$ as well as the expression of tissue inhibitors of metalloproteinases (TIMPs) and matrix metalloproteinases (MMPs) in explanted RVOT samples. Methods: RVOT samples from 38 patients (male: $n=24,63 \%$; female: $n=14,37 \%$ ), who underwent re-operation of the RVOT at a median age of 16 years [2-60 years] (median 14.2 years [1.8-49 years] after primary surgery), were gathered. After standardized specimen preparation, staining was performed with alizarin-red 
dye to visualise calcification. Nuclei were identified using nuclear fast red staining. Additionally, immunohistochemistry was performed focusing on TIMP1 and MMP9. Calcification intensity was quantified in a standardized fashion. Furthermore, present nuclei per mm2 as well as TIMP and MMP expression were analysed microscopically.

Results: Indications for primary surgery were Tetralogy of Fallot $(\mathrm{n}=21 ; 55.3 \%)$, double outlet right ventricle $(\mathrm{n}=3 ; 7.8 \%)$, truncus arteriosus communis $(\mathrm{n}=2 ; 5.3 \%)$ and other malformations of the RVOT and pulmonary arteries $(n=11 ; 28.9 \%)$. During re-operation either allografts $(n=3)$, autologous $(n=6)$ and bovine $(n=16)$ tissue or synthetic material $(n=5)$ that was previously used for a transannular patch repair $(n=22)$ and reconstruction of the RVOT $(\mathrm{n}=16)$ was explanted. 18 specimens $(52.6 \%)$ showed severe calcifications macroscopically. Bovine explants showed a higher degree of calcification than autologous tissue $(\mathrm{p}=0.004)$ but were less calcified than synthetic materials such as GORETEX ${ }^{B}$ or DACRON ${ }^{B}(p=0.001)$. Amount of nuclei per $\mathrm{mm}^{2}$ did not differ significantly between the 3 groups (human: $11.31 / \mathrm{mm}^{2}$; bovine: $10.20 / \mathrm{mm}^{2}$; synthetic: $10.25 / \mathrm{mm}^{2}$ ). TIMP1 and MMP9 showed positive results in all samples.

Conclusions: Bovine samples seem to be more susceptible to calcification than autologous tissue. If transannular patch repair is needed, we recommend to use autologous tissue at primary repair. Nuclei per $\mathrm{mm}^{2}$ were equal in all groups. The presence of MMP9 indicates processes of angiogenesis and neovascularisation, while TIMP1 expression is important for the integrity of connective tissue. To further classify degenerative alterations, complementary examination will be performed.

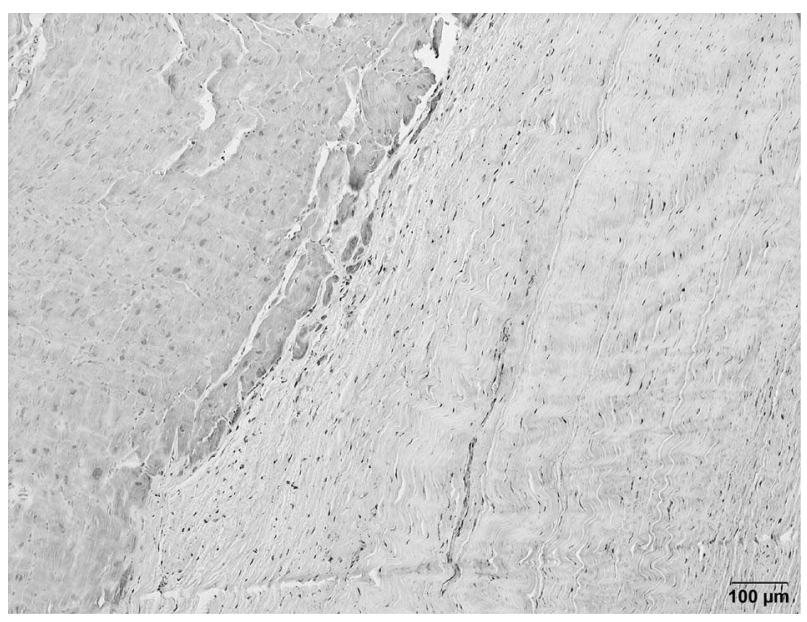

Figure.

\section{P-236}

Cardiac function and heart failure treatment in Fontan patients - Impact of right and left ventricular physiology

Kampf K. (1), Lehner A. (2), Dalla-Pozza R. (2), Hagl C. (1), Haas NA. (2), Ulrich S. (2), Hakami L. (1)

(1) Hospital of the Ludwig-Maximilians-University (LMU) Munich, Heart Surgery, Munich, Germany, (2) Hospital of the Ludwig-MaximiliansUniversity (LMU) Munich, Pediatric Cardiology, Munich, Germany

Objectives: The impact of the ventricular morphology in patients who underwent Fontan procedure on midterm and late outcomes are discussed controversially. We compared the postoperative outcomes in single left ventricle and single right ventricle morphology regarding the ventricular function and the need of susequent chronic heart failure therapy.

Methods: Fontan palliation was performed in 168 patients with a single ventricle morphology (median age 2.6 years, median weight $12.4 \mathrm{~kg}$, female $\mathrm{n}=68,40.2 \%$; male $\mathrm{n}=101,59.8 \%$ ) between 2003 and 2012. Group I: n=116 with hypoplastic left heart syndrome (HLHS) s/p Blalock-Taussig-Shunt (BT-Shunt) $\mathrm{n}=39$, RV-PA-Conduit $n=67$ or hybrid procedure $n=10$. Group II: $\mathrm{n}=35$ with hypoplastic right ventricle were $\mathrm{s} / \mathrm{p}$ pulmonary artery banding $(\mathrm{PAB}, \mathrm{n}=8)$, Damus-Kaye-Stansel anastomosis $(\mathrm{n}=3)$ and $\mathrm{s} / \mathrm{p}$ BT-Shunt $(\mathrm{n}=24)$. Cardiac function and heart failure treatment was assessed 6, 12, 60, 120 and 160 month post Fontan palliation. Results: Patients with right ventricular morphology had a significant higher need of medical heart failure therapy pre-Fontan $(\mathrm{p}=0)$ and after six $(\mathrm{p}<0.02)$, twelve $(\mathrm{p}<0.04)$ and sixty months $(\mathrm{p}<0.004)$. After 10 and 13 years we could not find a significant difference. Echocardiographic evaluation of ventricular function showed no significantly higher occurrence of decreased ventricular function in the groups.

Conclusion: The midterm and late outcomes of patients with single left or single right ventricle at our hospital are similar with international results.

\section{P-237}

Impact of age on early postoperative outcome in infants after primary repair of tetralogy of Fallot with a transannular patch - Single Center Experience Schnizer S. (1), Dalla-Pozza R. (2), Hagl C. (1), Kur F. (1), Pichlmaier M. (1), Brenner P. (1), Sadoni S. (1), Haas NA. (2), Lehner A. (2), Ulrich S. (2), Hakami L. (1)

(1)Hospital of the Ludwig-Maximilians-University (LMU) Munich, Heart Surgery, Munich, Germany, (2) Hospital of the Ludwig-MaximiliansUniversity (LMU) Munich, Pediatric Cardiology, Munich, Germany

Objectives: The optimal timing for surgical repair of tetralogy of Fallot (TOF) remains controversial. The aim of this study was to analyse the impact of age on early postoperative outcome.

Methods: From 2002 to 2013, in total 69 patients (median age 4.5 months, range 5 days -42 months, median weight $5.9 \mathrm{~kg}$ (range $2.5-13.0 \mathrm{~kg}$ ) with diagnosis of TOF underwent a transannular patch procedure at our center. $11(16 \%)$ patients had previous palliation. Methods: Multivariate analysis was used for evaluation of ventilator time, length of ÍCU stay and mortality till 30 days postoperatively in three different patient groups (age: 0-3 months, 4-6 months, 6 months and older).

Results: Patients at the age of $0-3$ months had a significantly $(\mathrm{p}<0.05)$ longer ICU-stay (median 9.1d, range $0-68.9 \mathrm{~d})$ and time on ventilator (median $3.5 \mathrm{~d}$, range $0-21.3 \mathrm{~d}$ ) compared to older patients.

Age of 3-6 months: Median ICU 6.1d (range 0 - 12.2d); median time on ventilator $2.0 \mathrm{~d}$ (range $0-8.1 \mathrm{~d}$ ).

Age 6 months and older: Median ICU 3.0d (range 0 - 31.4d); median time on ventilator $1.0 \mathrm{~d}$ (range $0-9.0 \mathrm{~d}$ ).

In the group 6 months and older one patient died due to cerebral infarction perioperatively and one re-do surgery was necessary. Mortality was $0 \%$ in the other two groups. In all three groups were no sign of clinically relevant pulmonary valve regurgitation or stenosis in echocardiographic controls. All three groups showed good left and right ventricular functions on 30 days postoperatively.

Conclusion: This data promotes the fact that a younger patient age is associated with longer time on ventilator and longer ICU stay. 30 day mortality was not influenced. 


\section{P-238}

Long-term outcomes of the arterial switch operation in a population-based follow-up

Puska V. (1), Kallio M. (1), Ojala T. (2), Rahkonen O. (2),

Mattila I. (2), Pihkala J. (2)

University Hospital of Oulu/University of Oulu, Oulu, Finland (1); University Hospital of Helsinki/University of Helsinki, Helsinki, Finland (2)

Objectives: To evaluate long-term outcomes of the arterial switch operation (ASO) for transposition of great arteries (TGA) in Finnish nationwide follow-up.

Methods: All patients who underwent ASO in Finland between years 2004 and 2014 were included in this retrospective study. Overall 148 were operated, 81 (55\%) with simple TGA, 50 (34\%) TGA and ventricular septal defect (VSD) and 17 (11\%) Taussig-Bing anomaly. 129 (88 \%) patients underwent balloon atrial septostomy prior to ASO.

Results: Total of $35(24 \%)$ patients had prenatal diagnosis. The mean age at the time of the ASO was $10.1 \pm 8.6$ days. Thirty-one patients $(20.9 \%)$ underwent 60 reinterventions (19 reoperations and 41 cardiac catheterizations) during the follow-up, most commonly due to pulmonary artery stenosis (53\%), and coarctation of the aorta (13\%). One patient underwent heart transplantation at 10 months of age due to severe heart failure and left ventricular dilatation. Longer perfusion time $(\mathrm{p}=.008)$, and abnormal coronary anatomy $(\mathrm{n}=34, \mathrm{p}=.000)$ were associated with increased risk for reintervention. Overall survival rate after ASO was $96.6 \%$. The early mortality (within 30 days or before hospital discharge) was $2.7 \%(n=4)$ and late $0.7 \%(\mathrm{n}=1)$.

Conclusions: Long-term survival after ASO is excellent. The most common reason for reintervention after ASO is pulmonary artery stenosis.

\section{P-239}

Surgery of single ventricles: is the humanitarian action justified?

Gouton M. (1,2), Bical O. (1), Lucet V. (1), Leca F. (1)

(1) Mécénat-Chirurgie Cardiaque - Paris-France; (2) Institut Mutualiste Montsouris - Paris - France

Objectives: Children with a single ventricle usually require several surgeries before eventually benefiting from a total cavopulmonary connection. The aim of this study is to know if such support is possible for children from developing countries, managed by an humanitarian association, taking in account the follow-up and the survival improvement, and if so, for what types of single ventricles.

Methods: We studied retrospectively all single ventricle patients managed by our association Mécénat-Chirurgie Cardiaque (MCC) since its creation in 1996. After their return to their country, the children were followed up in collaboration with the corresponding doctors in the countries of origin.

Results: From 1996 to 2017, 138 children (90 boys, 48 girls) with single ventricle were managed by MCC at a mean age of 4 years. $33 \%$ are from sub-Saharan Africa, 25\% from North Africa, 24\% from the Middle East and 15\% from Eastern Europe. 19 children were withdrawn from surgery because of pulmonary hypertension or overly complex heart disease. 119 were operated on, with a total of 165 procedures: 41 had only palliative surgery (systemicpulmonary anastomosis or banding), 47 a partial cavo-pulmonary connection (PCPC), and 31 a total cavo-pulmonary connection (TCPC). The mean age at the TCPC was 8.5 years. The overall operative mortality was 10/160 (6.2\%). After a mean follow-up of 5.5 years, 18 children (13\%) were lost to follow-up. Survival rate of operated children was $82 \%$ and $79 \%$ at 5 and 10 years, compared with $39 \%$ and $29 \%$ for non-operated children. The prognosis is better for tricuspid atresia (90 and 86\%) and double inlet ventricles (87 and $83 \%$ ) than for DORV or atrio-ventricular canal (64\% and $68 \%$ at $5 y$ ).

After palliative surgery, survival rate was $72 \%$ at $5 y$ and $63 \%$ at 10 , whereas after PCPC it was $81 \%$ and $77 \%$ respectively, and $97 \% 10$ years after TCPC.

Conclusion: Surgical management of children with single ventricles from developing countries by a humanitarian association is possible and legitimate, with a very satisfactory long-term follow-up and survival rate. Favourable forms are tricuspid atresia or double inlet single ventricles. The management should aim towards a TCPC as soon as possible.

\section{P-240}

1996-2017: a humanitarian action for 3000 children with congenital heart diseases from developing countries Gouton M. (1,2), Saint-Picq M. (1), Lucet V. (1), Bical O. (1), Leca F. (1) (1) Mécénat-Chirurgie Cardiaque - Paris-France; (2) Institut Mutualiste Montsouris - Paris - France

Objectives: Mécénat-Chirurgie Cardiaque is a humanitarian association whose mission is to enable children suffering from cardiac malformations to come to France to access the care that is not available in their native countries. Since its creation in 1996, near 3000 children were managed by MCC. The purpose of this study is to analyse the follow-up of this cohort.

Methods: We reviewed all patients managed by our humanitarian organisation since 1996, and analysed long-term survival.

Results: 2984 children were entrusted, from 66 different countries, mainly from sub-Saharan Africa: 70\%, but also from North Africa: 11\%, the Middle East 8\%, South-East Asia: 8\%, Eastern Europe: $5 \%$. Mean age at arrival in France was 7 years old. The diagnoses, made by the cardiologists in the countries of origin of the children, had to be confirmed and sometimes corrected at the arrival in France. The encountered heart diseases were tetralogy of Fallot $25 \%$, VSD $17 \%$, acquired valvular diseases $16 \%$, complex congenital heart disease: single ventricle, pulmonary atresia with VSD, DORV, D-TGV 5\% each, truncus arteriosus 2\%. 11 children died before surgery. 223 children were withdrawn for surgery, mainly because of pulmonary hypertension or overly complex heart disease, or because of venial disease. 2750 children underwent surgery or intervention. Post-operative mortality has been $2.3 \%$. After a mean follow-up of 4.4 years, 401 children were lost to follow-up. At last follow-up, 288 children were dead at a mean age of 10.5 years old. Mean delay until death was 2.3 years. Survival rate at 5 and 10 years was $89 \%$ and $84 \%$ in operated children, and $74 \%$ and $62 \%$ in non-operated children respectively. Survival rates at 5 and 10 years were: Fallot 95.4\%-94.9\%; VSD 94.9\%-91.1\%; acquired valvular diseases $77.9 \%-68.5 \%$; single ventricle $78.2 \%-71.7 \%$.

Conclusion: With low operative mortality for often very severe heart disease, and good long-term follow-up (87\%), the management of children from countries without cardiac surgery is justified by a similar survival to that of Western cohorts - except for acquired valvulopathies, presumably because of the difficulty of anticoagulant treatment. Thus our humanitarian action must continue, and improve for an optimal management of the disinherited populations. 


\section{P-241}

Early and long-term results of biventricular repair of Tetralogy of Fallot with or without pulmonary atresia Mostefa-Kara M., Villemain O., Meot M., Raisky O., Bonnet D. M3C-Necker Enfants malades, AP-HP, Université Paris Descartes, Sorbonne Paris Cité, Paris, France

Objective: We sought to determine the results of biventricular repair of patients with Tetralogy of Fallot without pulmonary atresia (TOF) and Tetralogy of Fallot with pulmonary atresia (TOF-PA) to clarify variables affecting reintervention and mortality.

Methods: We performed a retrospective study on patients with a diagnostic of TOF and TOF-PA who underwent a biventricular surgical strategy between 1995 and 2016, in our center.

Result: We reviewed 1091 patients, divided into three anatomical groups: TOF $(\mathrm{n}=857,79 \%)$, and patients with a TOF-PA where separated into those who had a pulmonary trunk $(\mathrm{T}+)$ or not (T-): TOF-PA/T + ( $\mathrm{n}=81,7 \%)$, and TOF-PA/T- $(n=153,14 \%)$. Overall patient mortality was $30(2.7 \%)$, six of them occured before discharge $(0.5 \%)$. The survival at 10 years after the biventricular repair was $98.8 \%$ for TOF and $92 \%$ for TOF-PA ( $p<0.001)$. In multivariate analysis, the absence of pulmonary trunk (HR 11.5 IC95\%[2.8-47.5], $\mathrm{p}<0.001$ ) and presence of MAPCAs (HR 8.5 IC95\%[2.1-34.2], $\mathrm{p}=0.002)$ were associated with a higher mortality. The median follow-up was 8.9 years after the biventricular repair. 227 patients underwent reintervention after biventricular repair $(20.8 \%)$, but it was not associated with additional mortality $(p=0.40)$. The freedom from reintervention at 10 years after the biventricular repair was $89.5 \%, 69 \%$, and $47.8 \%$ for TOF, TOF-PA/T + and TOF-PA/T- respectively $(\mathrm{p}<0.001)$. In multivariate analysis, a post-natal diagnosis (HR 1.3 IC95\%[1.03-1.8], p < 0.028), the absence of pulmonary trunk (HR 2.5 IC95\%[1.6-3.7], $\mathrm{p}<0.001)$, pulmonary artery branches stenosis (HR 2.5 IC95\%[1.6-3.7], $\mathrm{p}=0.011$ ), and prior palliative surgery (HR 1.7 IC95\%[1.2-2.5], $\mathrm{p}=0.008$ ) were reintervention risk factors.

Conclusion: The absence of the main pulmonary artery seems to be a major prognostic factor in ToF. The surgical options aiming to reduce the rate of reoperation on right ventricular outflow tract is the main source of progress in this defect.

\section{P-242}

The risk of recoarctation of aortic arch reconstruction varies among different type of patch material van Beynum I.M. (2), Kurul S. (1,2), Krasemann T (2)., Dalinghaus M. (2), Etnel J.R (1)., Bogers A.J.J.C. (1) Erasmus Medical Centre, Rotterdam, the Netherlands. Department of Cardiothoracic Surgery (1); Pediatric Cardiology (2)

Background: Recoarctation after aortic arch reconstruction is a known complication in neonates and infants. Homograft is the most commonly used patch material for aortic arch reconstructions in our centre. Since 2014, tissue engineered bovine pericardium (CardioCel $^{\circledR}$, Admedus Regen Pty Ltd, Perth, WA, Australia), was used as an alternative. The aim of our study was to determine if the material used for arch reconstruction had an influence on the development of recoarctation.

Methods: Data of all neonates and infants who underwent aortic arch reconstruction with use of any patch material between 2005 and 2016, in either univentricular or biventricular repair, was reviewed. Recoarctation was defined by the need of reintervention, either percutaneous or surgical. 41 patients were included. The aortic arch was reconstructed with a homograft $(\mathrm{n}=30) \operatorname{or~CardioCel}^{\mathbb{R}}(\mathrm{n}=11)$, at a median age of $2(1-49)$ weeks and $2(2-30)$ weeks, respectively. The median weight at the procedure was $3.5(2.4-9.7) \mathrm{kg}$ and $3.4(2.8-7.6)$ $\mathrm{kg}$ for the homograft and $\mathrm{CardioCe}^{(\mathbb{R})}$ group, respectively.
Results: Recoarctation was documented in 15 patients; 7 patients $(23 \%)$ in the homograft group and 8 patients $(73 \%)$ in the Cardio$\mathrm{Cel}^{\mathbb{B}}$ group. Freedom from reintervention estimated $77 \%$ and $17 \%$ at 1 year, for homograft and CardioCel ${ }^{\circledR}$ group, respectively. The difference was highly significant $(p=0.000)$. The median time at the first intervention for recoarction was 23 (14-461) weeks and 14 (7-10) weeks for the homograft group and Cardi$\mathrm{oCel}^{\circledR}$, respectively. The time difference did not reach the level of significance $(P=0.07)$. Among patients with recoarctations, the number of subsequent reinterventions needed to treat the recoarctations was higher among the CardioCel $^{\circledR}$ (mean 1.9 reinterventions per patient) compared to the homograft group (mean 1.3/patient) $(\mathrm{P}=0.057)$. Treatment for recoarctation was percutaneous balloon angioplasty in 11 patients, stent implantation in 2 , and operative enlargement in 2.

Conclusion: The patch material used for aortic arch reconstruction in neonates influences the rate of recoarctation and the number of reinterventions needed to treat them. Based on our results $\mathrm{CardioCel}^{\circledR}$, a bovine pericardial patch, cannot be recommended for reconstruction of the aortic arch in neonates.

\section{P-243}

Pulmonary Vein Obstruction After Repair of Isolated Total Anomalous Pulmonary Venous Return

Ko J. K., Jun H. O., Baek J. S.

Department of Pediatric Cardiology, Ulsan University College of

Medicine, Seoul, Korea

Introduction: Pulmonary venous obstruction (PVO) after surgical repair for total anomalous pulmonary venous return (TAPVR) remains the most significant complication, with high morbidity and mortality. The location of PVO is mainly at the anastomotic site after standard repair, probably owing to the combination of inflammation, fibrosis, and direct purse string effect. However, obstruction in the individual pulmonary veins (PV) could happen through retrograde extension of the fibrotic process of anastomotic site obstruction or other unknown reasons. In this study we studied the characteristics of PV obstruction after TAPVR repair. Methods: Records of 100 consecutive patients having undergone surgery for isolated TAPVR without other combined cardiac anomalies were reviewed focusing specifically on the development of PV obstruction after repair.

Results: 13 patients developed PVO after repair. Among these 13 patients, PV obstruction was found in 9 patients and in 3 patients both anastomotic site stenosis and PV obstruction had occurred. Preoperatively PVO at various site was noted in 38 patients, however individual PV obstruction was not present. Among these 38 patients, 4 developed PV obstruction after repair. $\mathrm{PV}$ obstruction was more frequently seen in the patients with PVO before repair than in the patients without PVO $(p<0.05)$. In 5 patients both side PV were involved and right and left PV obstruction were in one and 3 patients respectively. The age or body weight at TAPVR repair was not different between the patients with and without late PV obstruction $(p>0.05)$. The difference in the type of TAPVR between 2 groups couldn't be evaluated due to small number of PV obstruction. The interval between TAPVR repair and PV repair was from 2 months to 17 months (median, 8 months). All PV repair were done through sutureless technique with one early surgical mortality and in 4 patients stenosis progressed again at the same PV or another PV. Conclusions: PVO is still significant residual problem after surgical repair for TAPVR and especially individual PV obstruction is troublesome, its risk factor is unknown and it remains high morbidity even after recent sutureless technique. 


\section{P-244}

Ibuprofen for the treatment of patent ductus arteriosus in preterm and/or low birth weight infants-our expirience Krstevska Blazhevska S.

Clinical hospital Acibadem Sistina, Skopje, FYROM

Background: Ibuprofen is cyclo-oxygenase inhibitor, is use in closer of patern ductus arteriosus in preterm end/or low birth weight neonates.

Objectives: To determine the efficacy and safety of ibuprofen for closing a PDA in preterm and/or low birth weight infants in our hospital.

Results: We use ibuprofen intravenous for closure of PDA in 8 preterm and low birth neonates. Three of them had hyperglycemia during administration period of ibuprofen, in 5 neonates PDA was succeed closed, one neonates had surgical closure of PDA because of failed closure by ibuprofen and one baby die because of intracranial hemorrhagia.

Conclusions: Ibuprofen is as effective in closing a PDA and reduces the risk of NEC and transient renal insufficiency. Given the reduction in NEC ibuprofen currently appears to be the drug of choice. . Studies are needed to evaluate the hyperglycemia as side effect during administration of ibuprofen.

\section{P-245}

Recovery in the early postoperative period after Fontan operation - 9 years experience of a single institution in favor of early extubation strategy

Simeonov L., Shishkov S., Pechilkov D., Kaneva A.

National Heart Hospital-Sofia, Bulgaria

Background: Fontan circulation is strongly dependent on low cavopulmonary pressures and favors negative pressure ventilation. Early postoperative extubation of such patients should have a strong positive effect on stabilizing the hemodynamics and lowering the inotropic support needs. The aim of this study is to establish the differences among the patients that were successfully extubated early after operation and those who failed to do so.

Methods: Medical files of patients after Fontan operations for nine years period (2009-2017) were retrospectively analyzed. Patients with missing data from the medical files were excluded from the study. We divided the patients in two groups: first group of early extubated patients - extubated up to 12 hours after the operation, second group of later extubated patiens. Preoperative and serially measured postoperative data in the first 48 hours were collected. Data were presented as medians with range or as means \pm standard deviation. A non-parametric Mann-Whitney $U$ test integrated in the statistical software SPSS 19.0 was used. A value of $\mathrm{P}<0.05$ was considered significant.

Results: For a period of 9 years 73 patients were operated. 64 patients were included in the study. The median age was 3.5 years (range 1.8-9). In 62 patients (96.9\%) an extracardiac conduit was used and in $2(3,1 \%)$ a lateral tunnel was done. In 43 patients $(64.1 \%)$ a fenestrated Fontan was performed and 21 (35.9\%) were non-fenestrated. 20 patients $(31.3 \%)$ were included in the early extubated group. Compared to later extubated group, early extubated group had higher pre-operative ejection fractions $66 \%$ $(51-87)$ vs. $59.5 \%(45-86) \mathrm{p}=0.004$, lower initial cavo-pulmonary pressures $13 \mathrm{mmHg}(8-17)$ vs. $14 \mathrm{mmHg}(9-21) \mathrm{p}=0.04$, lower initial inotropic scores 11.89 (5-24) vs. $23.6(3-57) \mathrm{p}=0.0001$ and shorter ICU stay 5 days $(2-11)$ vs. 6 days $(2-47) \mathrm{p}=0.01$. Within the early extubated group there was a lowering in the inotropic score in pre- and postextubation values 11.9 (5-24) vs. 7.4 (2.312.9) $\mathrm{p}=0.0001$.
Conclusion: In the early postoperative period after Fontan operation patients that can be extubated in the first 12 hours had higher pre-operative ejection fraction, lower cavo-pulmonary pressures and lower inotropic scores after the operation. After establishing a spontaneous ventilation patients need much less inotropic support to sustain a stable hemodynamics.

\section{P-246}

Survival rate drops substantially in patients with hypoplasic left heart syndrome who possess abnormality in pulmonary vein or left atrium

Hamamichi Y., Komiya E., Nukaga S., Sonoda K., Ishii T., Kishiki K., Inage A., Ueda T., Yazaki S., Yoshikawa T.

Sakakibara Heart Institute, Tokyo, Japan

Background: Hypoplastic left heart syndrome and its variant (HLHS) are poor prognostic disease group, even now when operative procedures against them have been adjusted better. We predicted that innate properties and pre-operative conditions would make HLHS prognosis worse. We investigated adverse prognostic factors related to HLHS.

Methods: The medical records of 108 HLHS patients (variant 45) were reviewed who were born between 2004 and 2014. All patients underwent Norwood procedure with shunting to pulmonary artery (PA) at a stage prior to Glenn procedure. First, we sought following factors by Kaplan-Meier methods: survival rate, Glenn achievement rate, and Fontan achievement rate. Log rank analysis was performed dividing the whole into two groups by each prognostic factor. Second, we reckoned hazard ratio by Cox proportional-hazards model using prognosis related factors which had significant differences with log rank.

Results: Whole survival rate was $58 \%$ in patients who underwent Norwood procedure: Glenn achievement rate was 58\%; Fontan achievement rate was $41 \%$. Of 45 dead patients, $82 \%$ did not attain to Glenn procedure. Log rank analysis showed that survival rates were decreased by existence of following adverse prognosisfactors; anomalous pulmonary-vein connection or abnormal partition wall in left atrium (abnormal PV/LA: 8\% vs. 64\%, $\mathrm{p}<0.0001$ ); aortic atresia or mitral atresia (AA/MA: $43 \%$ vs. $72 \%$, $\mathrm{p}=0.0007)$; birth until the year $2010(48 \%$ vs. $79 \%: \mathrm{p}=0.0082)$. Glenn achievement rate was decreased only by abnormal PV/LA (17\% vs. $63 \%$ : $\mathrm{p}<0.0001)$. After Cox proportional-hazards model, we obtained hazard ratio of death-rate increase by following factors: 4.9 for abnormal PV/LA (p<0.001), 3.2 for AA/MA $(p<0.001)$, and 3.2 for birth until $2010(p=0.001)$. We also obtained hazard ratio of un-accomplishment of Glenn only by abnormal PV/LA (5.5, $\mathrm{p}=0.019)$. Of 8 patients who died after Glenn procedure, 7 patients possessed abnormal PV/LA, or AA/MA ( $87 \%$ vs. $38 \%$ : $\mathrm{p}=0.027)$.

Discussion: Most of HLHS patients with dead did not achieve Glenn procedure yet. Survival rates were decreased in HLHS patients who owned abnormal PV/LA, or AA/MA. Even after Glenn procedure, patients who owned either of abnormal PV/LA or AA/MA died much more than those without these 2 factors.

\section{P-247}

Decellularized fresh aortic homografts for aortic valve replacement in children: Initial experience with the ARISE valve

Schmiady M. (1,3,6), Dave H. (1,3), Valsangiacomo E. (2,3), Greutmann M. (4), Santos Lopes B. (4), Kuen H.P. (5),

Kretschmar O. (2,3), Hübler M. (1,3) 
1 Division of Congenital Cardiovascular Surgery, University Children's Hospital Zurich, Switzerland; 2 Division of Paediatric Cardiology, University Children's Hospital Zurich, Switzerland; 3 Children's Research Centre, University Children's Hospital Zürich, Switzerland; 4 Division of Grown Up Congenital Heart Diseases, University Hospital Zürich, Switzerland; 5 Centre for Congenital Heart Disease, Pediatric and Adult Cardiology, Luzerner Kantonsspital, Lucerne, Switzerland; 6 Department of Cardiovascular Surgery, University Hospital, Zurich, Switzerland

Introduction: Valve replacement remains the last therapeutic option for patients with severe aortic valve dysfunction not amenable for valve repair. Ross Procedure has been the preferred procedure for aortic valve dysfunction, owing to its excellent haemodynamic profile, no need for anticoagulation and a belief that it adapts with growth. Despite this, long-term results reporting significant incidence of aortic root dilatation, not so insignificant neo-aortic valve dysfunction and the newly acquired pulmonary valve disease have fueled development of alternative concepts. Decellularized fresh aortic homograft (ARISE) have been proposed as an alternative. Here, we report our preliminary results of aortic valve replacement (AVR) using ARISE valved conduits in children.

Methods: After obtaining informed consent (Sep 2016 - June 2017), 9 patients (5 male) with a median age of 8.7 years (3.1 - 18.3) underwent aortic valve replacement using ARISE valves. Median ARISE Z-value at implantation was 1.4 (-0.3 3.4). In three patients concomitant other procedures were performed. Echocardiography and magnetic resonance Imaging were used for pre-and postoperative evaluation. The implantation involved root replacement using proximal and distal continuous sutures and coronary button reimplantation.

Results: The handling and haemostatic properties of the ARISE valves were satisfactory. All patients survived the operation and are alive at a median follow-up of 8.1 (3.5-15) months. The valves were well accepted by all recipients without clinical signs of haemolysis. All patients received acetyl salicylic acid postoperatively for one year. Peak postoperative gradient remained stable with a median of 3.5 (2-9) $\mathrm{mmHg}$ at last follow-up. While 3 patients had mild neo-aortic regurgitation (AR), the remaining valves had none/trivial AR. The $\mathrm{Z}$ value of the ARISE valve remained stable at follow-up. Freedom from reoperation and reintervention was $100 \%$ at a median follow-up of 8.1 (3.5-15) months.

Conclusion: Initial experience with the use of the ARISE valve is promising. It has raised hopes of a viable alternative to the Ross Procedure in children. Whether this concept leads to alleviation of the early risks and long-term morbidity associated with the Ross Procedure and actually demonstrates favorable remodeling with somatic growth of the young patient is what only time will tell.

\section{P-248 \\ Effect of Mechanical Circulatory Support Bridging to Pediatric Heart Transplant \\ Kocabeyoglu S.S. (1), Pac M. (1), Pac A. (2), Kervan U. (1), \\ Sert D.E. (1), Karahan M. (1), Eris D. (2) \\ (1) Cardiovascular Surgery Yuksek Ihtisas Hospital, Ankara, Turkey; \\ (2) Pediatric Cardiology Yuksek Ihtisas Hospital, Ankara, Turkey}

Introduction: In recent times, the number of heart transplants in the pediatric patient population has been growing increasingly. Many centers have started their pediatric cardiac transplant programs every passing day. The purpose of this study was to introduce the results of our pediatric heart transplants and to analyze our outcomes.
Methods: Twenty-four pediatric patients who underwent heart transplantation between 2001 and 2017 were included in this study. Twelve patients were male and nine of the patients were under 10 years of age. (One was less than 1 year, two were between $1-5$ years, six were between 6-10 years, fifteen were between 11-18 years). Dilated cardiomyopathy was the most frequent cause of heart failure (Ten patients with dilated cardiomyopathy, seven with noncompaction cardiomyopathy, four with restrictive cardiomyopathy, one with hypertrophic cardiomyopathy and one has arrhythmogenic right ventricular cardiomyopathy).

Results: Nine patients were bridged to transplant with adult longterm left ventricular assist device (Seven with HVAD, two with HVAD as an LVAD + ECMO as an RVAD), one patient was bridged with Berlin Heart LVAD and one other patient was bridged with ECMO to the heart transplant. No early mortality was encountered in all these bridged patients. There were four inhospital mortality and late mortality was observed in four patients. The remaining 16 patients out of 24 were able to followed-up uneventfully till April 2017.

Conclusion: In pediatric patient population, heart transplant can be performed as safely as the adult heart transplants with similar mortality and morbidity rates. Additionally, bridging with durable adult left ventricular assist device to heart transplant can be done safely in the pediatric population.

\section{P-249}

Epidemiologic Data, Prognosis and Biopsy Guided Therapy in Children with Myocarditis and Cardiomyopathy

Kourelis G., Vagenakis G., Rallis D., Apostolopoulou S.C., Kyriakoulis K., Kakava F., Ntegiannis D., Kaklamanis L., Rammos S. Onassis Cardiac Surgery Center, Athens, Greece

Introduction: Myocarditis and cardiomyopathies are acquired causes of heart failure with a wide range of aetiology, pathophysiology and prognosis. In Greece there is a paucity of data regarding this group of diseases in the paediatric population. This study reports epidemiologic, management and prognosis data of children with myocarditis and cardiomyopathy, referred to a tertiary center because of cardiac failure.

Methods: We performed retrospective data collection for paediatric patients (0-18 years old) diagnosed with myocarditis / cardiomyopathy and hospitalised from 2004 to 2017 . Analysis of data was performed on the basis of demographics, diagnosis, biopsy results, management and prognosis.

Results: 80 patients were analysed (44 boys; 36 girls), with the following diagnoses: 37 cardiomyopathy (26 dilated; 4 hypertrophic; 5 restrictive); 2 arrhythmogenic right ventricular dysplasia and 43 myocarditis. We defined the following age groups for age distribution: $0-1$ year old $(n=13)$; $1-5$ y.o. $(n=25)$; $5-16$ y.o. $(n=37)$ and $>16$ y.o. $(n=5)$. Diagnosis was based on echocardiography findings, myocardiac enzyme levels and biopsy findings [active inflammation; necrosis; hypertrophy; fibrosis; (near) normal; other]. All patients but 3 underwent cardiac biopsy. Overall, 27 cardiac histological specimens (23 out of the 'myocarditis' group and 4 out of the rest) were positive for viral or bacterial genetic material through polymerase chain reaction (PCR) testing. Of the positive patients, 21 received targeted therapy (antiviral or antibiotic agents). No specific therapy was available for the remaining patients at the time of diagnosis. Based both on biopsy findings (active inflammation or not) and on PCR in blood/cardiac specimen, 20 patients in total received intravenous immunoglobulin and 4 systemic corticosteroids. During follow up, 11 children (15\%) died; 9 (11\%) were transplanted or are in the transplant list; $9(11 \%)$ required prolonged 
medical support for heart failure; the rest (63\%) improved or had full recovery.

Conclusions: Acquired heart failure in the paediatric population is mostly due to myocarditis and dilated cardiomyopathy, while the majority of affected patients survive over long follow up. Cardiac biopsy and PCR testing in blood and cardiac specimen are very useful tools for diagnosis as well as in guiding specific treatment, especially in patients with myocarditis.

\section{P-250}

Treatment of pediatric patients with acute myocarditis from Parvovirus B19 with the off label use of Telvibudine Vagenakis G.A., Apostolopoulou S., Laskari C., Tsoutsinos A., Kakava F., Kyriakoulis K., Kourelis G., Rammos S. Onasis Cardiothorasic Center, Athens, Greece

Introduction: Myocarditis is an inflammatory disease of the myocardium caused from various causes. One of the most common is viral infection from parvovirus B19. Children with this infection often do not fully recover and may develop heart failure, or worse, need heart transplantation or die. It is our intension to share our experience with the off label use of telvibudine, a drug used for the treatment of hepatitis B, in the treatment of parvovirus B19 myocarditis.

Methods: In the course of 4 years (2013-2017) five children (age 2 to 16 years) were diagnosed in our institution with myocarditis due to parvovirus B19 after performing myocardial biopsy. PCR identification of the causative agent and viral load (number of copies) was performed. With admission four out of five needed intravenous inotropes and one child mechanical ventilation. Mean ejection fraction was $25 \%$ and the $\mathrm{z}$ score of the LVEDD was 2.4. All had ECG findings, elevated troponin levels and NT-proBNP levels $>4000 \mathrm{pg} / \mathrm{ml}$. Myocardial biopsy was performed 24-48 hours from admission. They were all treated with oral telvibudine (dose $15-20 \mathrm{mg} / \mathrm{kg} /$ day) for duration of three to six months, after authorization from the National Drug Organization of Greece.

Results: All five patients experienced a full recovery of their myocardial function. Mean ejection fraction is $60 \%$ and the $\mathrm{z}$ score of the LVEDD was 1.3. NT-proBNP levels dropped $<100 \mathrm{pg} / \mathrm{ml}$ and till now there has been no deterioration of their myocardial function. They have been followed up in our department by cardiac ultrasound every 6 months and MRI.

Conclusion: Patients with the suspicion of myocarditis, symptoms and signs of heart failure should undergo myocardial biopsy immediately and specialized treatment, if possible, should be commenced. Treatment of patients with acute myocarditis, from parvovirus B19, with telvibudine might become a helpful tool in the prevention of serious sequels of the inflammatory process like heart failure, need for heart transplantation or even death and may even help in the full recovery of such patients.

\section{P-252 \\ Multi-biomarker approach using ANP, BNP, NT-Pro BNP and hs-TnT improve prediction of adverse cardiac events in pediatric heart failure with congenital heart disease \\ Mori Y., Nakashima Y., Murakami T., Inoue N., Kaneko S. Division of Pediatric Cardiology, Seirei Hamamatsu General Hospital, Hamamatsu, Japan}

Introduction: Few reports describe the prognostic values of measuring multiple biomarkers in pediatric patients with congenital heart disease (CHD). We aimed to determine whether atrial natriuretic peptide (ANP), B-type natriuretic peptide (BNP), $\mathrm{N}$-terminal pro BNP (NT-proBNP) and high sensitivity troponin $\mathrm{T}$ (hs- $\mathrm{TnT}$ ) predicted the adverse events, and whether a combination with use of these biomarkers impoved prediction of events in pediatric patients with CHD.

Methods: Pre-and post operative 259 patients with CHD who were measured 4 biomarkers at the same time were included in the study. Adverse events were defined as cardiac death, arrest and acutely decompensated heart failure requiring inotropic agents. The receiver operating characteristic (ROC) curve was performed to determine high-risk levels of 4 biomarkers for predicting adverse events. Patients were divided into 5 groups based on number of high-risk levels (group 1: No biomarkers elevated, group 2: one biomarker elevated, group 3: 2 biomarkers elevated, group 4; 3 biomarkers elevated, group 5: all biomarkers elevated). Usefulness of combinations of biomarkers was investigated by comparing 5 groups.

Results: The median age at entry was 6.1 years [IQR $1.8 \sim 10.2$ years]. There were 10 adverse events during a median follow-up of 3.9 years. The C-statistic of all biomarkers was over 0.8 , and patients with levels of ANP $>77.6 \mathrm{pg}$, BNP $>61 \mathrm{pg} / \mathrm{ml}$, NT-pro $\mathrm{BNP}>1090 \mathrm{pg} / \mathrm{ml}$ and $\mathrm{hs}-\mathrm{TnT}>0.016 \mathrm{ng} / \mathrm{ml}$ were high-risk for predicting adverse events by ROC analysis. There were 174 patients in group 1, 45 in group 2, 20 in group 3, 4 in group 4 and 16 in group 5 . There were significant differences among 5 groups $(\log -\operatorname{rank} \mathrm{p}<.0001)$. Also significant differences were found between group 1 and 3 or 4 (Log-rank $\mathrm{p}<0.005)$.

Conclusions: The mesurements of ANP, BNP, N-T pro BNP and hs-TnT provide prognostic imformation in pediatric patients with $\mathrm{CHD}$. When use together, these biomarkers improve prediction of adverse events.

\section{P-253}

\section{Cardiomyopathy and acquired long QT syndrome in propionic acidemia}

Kovacevic A. (1), Garbade S.F. (2), Kölker S. (2), Hoffmann G.F. (2), Gorenflo M. (1), Staufner C. (2)

Department of Pediatric and Congenital Cardiology, Heidelberg University Hospital, Heidelberg, Germany (1); Department of General Pediatrics, Division of Neuropediatrics and Metabolic Medicine, Heidelberg University Hospital, Heidelberg, Germany (2)

Objectives: Propionic acidemia (PA) is an inborn error of metabolism caused by a deficiency of propionyl-CoA carboxylase. Cardiomyopathy (CMP) and QT prolongation are known to be potentially critical complications, but systematic analyses of cardiac phenotypes in PA patients are lacking.

Methods: 18 patients diagnosed with PA were enrolled in a retrospective single center study (data from 1989-2017). Echocardiography parameters (left ventricular end-diastolic-diameter LVEDD, systolic LV function - fractional shortening FS, mitral valve Doppler inflow patterns - E/A ratio) and 12-leadelectrocardiogram recordings (corrected QT interval QTc, according to Bazett's formula) were analyzed. Symptomatic patients were dichotomized to the group "neonatal onset" (symptoms within 28 days of life) and "late onset" (symptoms after 28 days). Besides descriptive analysis, correlations between cardiac function, LVEDD, QTc and clinical parameters (age at onset, beta-blocker or ACE-inhibitor therapy) were calculated.

Results: 18 patients with PA were enrolled, 17 of them were symptomatic ( $\mathrm{n}=1$ asymptomatic) with a median age at diagnosis of 6.0 days. 15/17 (88\%) were early onset and 2/17 (12\%) late onset. CMP was diagnosed in 7/18 (39\%) patients at a median age of 14.4 
years; all of those were early onset patients. Mean QTc of all cases was $445 \mathrm{~ms}(+/-18.11 \mathrm{SD})$; however individual range was up to $564 \mathrm{~ms}$. Longer QTc is associated with higher LVEDD z-scores. Beta-blocker therapy leads to decreased QTc in all patients treated $(n=4)$. However, decreased QTc intervals were observed also in untreated patients. ACE-I therapy did not improve FS. 11/18 (61\%) individuals show pathological mitral valve inflow patterns (E/A ratio) demonstrating LV diastolic dysfunction.

Conclusions: We report a 39\% CMP rate in our cohort, all of those early onset PA patients. Two thirds of those show signs of diastolic LV dysfunction. Mean QTc intervals are prolonged and directly correlated to higher LVEDD z-scores. Beta-blocker therapy may be considered effective for prolonged QTc in PA. In this cohort, ACE-I therapy did not lead to an improvement of systolic LV function. Prospective studies are warranted to further investigate cardiac phenotypes in PA. A multicenter approach is desirable due to limited case numbers per center for this rare disease.

\section{P-254 \\ Risk factors for RV failure in paediatric patients requiring LVAD implantation due to heart failure secondary to cardiomyopathy}

Rico-Armada A., Crossland D., Simpson E., O'Sullivan J., Reinhardt Z. Freeman Hospital, Newcastle upon Tyne, UK

Introduction: The assessment of the right ventricular (RV) function in patients with a failing left ventricle is important and difficult. We hypothesise that echocardiographic imaging is insufficient to evaluate the degree of dysfunction and the symptoms of RV failure are not always apparent on admission. Our objective was to evaluate the presence of clinical and biochemical risk factors suggesting RV failure in patients undergoing left ventricular assist device (LVAD) insertion.

Methods: We conducted a retrospective study of paediatric patients with end-stage heart failure secondary to cardiomyopathy who required biventricular assist device (BIVAD) between January 2014 and January 2016.

Results: During the 5 year period 25 patients required BIVAD support. Twelve (48\%) patients had simultaneous BIVAD insertion, $9(36 \%)$ had RVAD inserted after LVAD during the same operation and $4(16 \%)$ had RVAD at a later stage. The mean age was 4.3 years ( 2 months-14 years), $68 \%$ patients had symptoms of RV failure pre-operatively, $48 \%$ renal, $54 \%$ liver impairment, $40 \%$ had arrhythmias. Echocardiogram In most patients showed severe RV function, in 8/25 'moderate' and in 1 satisfactory. Post VAD insertion, 6 patients had mesenteric hypoperfusion, 5 requiring intestinal resection; 2 developed liver dysfunction, 3 had acute kidney injury requiring dialysis, 7 patients had thromboembolic complications and 5 had cerebral haemorrhage. The outcome for $68 \%$ was transplantation, $24 \%$ died and $8 \%$ recovered.

Conclusions: Patients undergoing BIVAD support have a high mortality rate $(24 \%)$ hence the major importance of an early detection of the RV failure. In half of the cases the severity of the $\mathrm{RV}$ failure was not detected prior to LVAD insertion. The classical signs of right heart failure (liver and renal failure) were present in most of our patients. Moreover, this cohort showed increased incidence of arrhythmias, both atrial and ventricular. Whilst echocardiography and clinical risk factors alone may not accurately predict the need for RVAD, they may serve as a warning that such support may be required and all efforts should be made to medically optimise the functional performance of the RV before LVAD implantation with agents such as milrinone, diuretics and pulmonary vasodilators.
P-255

Predictors of outcomes in paediatric dilated cardiomyopathy

Belhadjer Z., Iserin F., Khraiche D., Raisky O., Bonnet D.

M3C-Necker, University Paris Descartes, Hospital Necker Enfants

malades, Paris, France

Background: Dilated cardiomyopathies (DCM) is a severe disease and remains the leading cause of heart transplantation in children. Objective: To determine predictors of outcome during sequential follow-up of a cohort of pediatric DCM.

Methods: The study was retrospective (2000-2016) monocentric and included children with DCM. Clinical, biological, echocardiographic parameters and treatment information were collected throughout the follow-up.

Results: We included 110 patients. Mean follow-up was 4 years. 75\% had no events during the first year of follow-up. 39\% of patients died or underwent cardiac transplantation. In $27 \%$ of cases, the left ventricular function fully recovered. Predictors of events at baseline (death and transplantation) were age at diagnosis $>5$ years $(p=0.017)$, and cardiogenic shock as a presenting symptom $(\mathrm{p}=0.04)$. During follow-up, hospitalization for acute cardiac failure ( $p<0.0001$ ), and the need for loop diuretics during follow-up $(p=0.01)$ predicted events. In multivariate analysis, recurrent hospitalizations for heart failure and persistence of mitral insufficiency were risk factors of death $(p=0.02$ and $p<0.001$ respectively). Conclusion: We identified number of hospitalizations for heart failure and persisting congestive status requiring diuretics as predictors of outcome in DCM. In addition, we identified mitral insufficiency as a string predictor of events suggesting that progressive dilatation of the left ventricle should be considered as a risk factor for subsequent events and that reducing mitral regurgitation potentially with pulmonary banding could be considered in these patients.

\section{P-256 \\ Cardiac involvement and therapeutic outcomes in pediatric patients with Duchene Muscular Dystrophy in the pediatric cardiology department of Ahepa in Thessaloniki during the period 2014-2015: a retrospective study \\ Papadopoulou A., Keivanidou A., Papazisi D., Giannopoulos A. Department of Pediatric Cardiology, 2nd Pediatric Clinic, School of Medicine, Aristotle University of Thessaloniki, University General Hospital AHEPA, Thessaloniki, Greece}

Introduction: Duchene muscular dystrophy (DMD) represents the most common and severe form of muscular dystrophy and it is due to mutations in the dystrophin gene on chromosome Xp21.1. Typical presentation of cardiac involvement are dilated cardiomyopathy affecting the left ventricle, chronic heart failure and heart rhythm disorders.

Materials and Methods: It is a retrospective survey that took place during the period 01/01/14-01/01/15 in Ahepa pediatric cardiology department. Children with muscular dystrophy diagnosed by muscular biopsy were enrolled in the study. The statistical methods that were used are the following: a) descriptive analysis of demographic data and cross-tabulation matrices, b) Pearson Chi -Sqared Test. The statistical analysis was carried out with the use of statistical parcel IBM SPSS Statistics 22.

Results: Of the total of 99 children with muscular dystrophies, 48 had DMD. The mean age of diagnosis of DMD was 5 years and 2 months. Cardiac involvement was seen in 14 children (29, 2\%). All of them had an LV ejection fraction (EF) $<70 \%$ measured with M-Mode. Drug treatment in the means of angiotensin- converting enzyme inhibitors (ACE) and beta blockers (BBs), received 
8 patients. No improvement of EF (>70\%) were seen after the treatment. ECG changes were noticed in $71 \%$ of the patients with cardiac involvement and $\mathrm{EF}<70 \%$. Muscular biopsy was positive in 10 out of 14 DMD patients with cardiac involvement and no significant correlation was found between these two parameters. Conclusions: The current recommendations of the DMD Considerations Working Group identified ACE as first line therapy in patients with DMD with LV dysfunction. On the other hand, the use of BBs is controversial and limited in small-sized studies. According to our study, the use of drug treatment preserved rather than improved the LV function.

\section{P-257}

Diastolic dysfunction whose etiologies were mostly obscure in Glenn patients frequently causes increased pressure of superior vena cava

Hamamichi Y., Nukaga S., Komiya E., Sonoda K., Ishii T., Kishiki K., Inage A., Ueda T., Yazaki S., Yoshikawa T. Sakakibara Heart Institut, Tokyo, Japan

Introduction: It is unknown what factors induce diastolic dysfunction of ventricle (DDSV) in Glenn patients. Furthermore, it is unclear what harms are provoked by DDSV. The purpose of this study was to investigate the related factors to DDSV and the adverse effects by DDSV in Glenn patients.

Methods: The medical records of 146 Glenn patients were reviewed aged from 9 months to 24 years. They underwent cardiac catheterization between 2010 and 2017. We defined DDSV as end-diastolic pressure of ventricle $13 \mathrm{mmHg}$ or over $(\mathrm{n}=34)$. First, indexes were determined which were connected with DDSV. Second, we compared pulmonary circulation factors between Glenn patients with and without DDSV.

Results: In monovariae analysis DDSV in Glenn patients was associated with expanded ventricular volume on end-systole and on end-diastole, increased ventricular pressure on end-systole. Following factors were not related to DDSV; study ages, 1st strategies, additional flow to pulmonary arteries, strong regurgitation of atrioventricular valve, and ventricular ejection fraction. After multivariate analysis, DDSV in Glenn patients was independently associated with odds ratio of 8.1 for ventricular pressure on end-systole $(\geq 97 \mathrm{mmHg}$ ), and 4.4 for ventricular volume on end-systole $(\geq 66 \%)$. The explanatory coefficient was low (R-square $=0.22$ ). As for pulmonary circulation factors, pressure of pulmonary capillary wedge or left atrium was higher in Glenn patients with DDSV (11.7 vs. $7.6 \mathrm{mmHg}$ : $\mathrm{p}<0.0001)$; pressure of superior vena cava was higher 16.3 vs. $12.1 \mathrm{mmHg}$; $<0.0001$ ). The ratio of patients with pressure of superior vena cava $16 \mathrm{mmHg}$ or over was more in DDSV patients (48\% vs. $14 \%$ : p < 0.0001).

Conclusion: Our study showed that half Glenn patients with DDSV possessed so high pressure of superior vena cava that indicated risk factor for Fontan. Our study also showed DDSV in Glenn patients was connected with ventricular volume on end-systole and on enddiastole, and systolic pressure of ventricle. However, eight out of ten patients did not possess obvious risk factors for DDSV in Glenn circulation. We might tighten medical management for Glenn patients not to fall into DDSV, if we could not catch risk factors.

\section{P-258}

Pulmonary-circulation disturbance arises in Glenn patients with high values of $\mathbf{n}$-terminal pro-brain natriuretic peptide Nukaga S., Hamamichi Y., Komiya E., Sonoda K., Ishii T., Kishiki. K., Inage A., Ueda T., Yazaki S., Yoshikawa T. Sakakibara Heart Institute, Tokyo, Japan
Backgrounds: We generally consider that values of $\mathrm{n}$-terminal pro-brain natriuretic peptide (NT-proBNP) would be decreased in Glenn circulation. However, high values of NT-proBNP (high NT-proBNP) subsist in Glenn patients. We predict Glenn patients with high NT-proBNP would possess not only cardiac depression but also pulmonary circulation disturbance.

Objective: We investigated factors related to high NT-proBNP and the feature of pulmonary circulation in high NT-proBNP group. Methods: The medical records of 125 Glenn patients were reviewed aged from 10 months to 23 years. We defined high NT-proBNP as NT-proBNP $800 \mathrm{pg} / \mathrm{ml}$ or over (by Japan heart failure society, $n=27$ ). First, indexes were determined which were connected with high NT-proBNP. Second, we compared pulmonary circulation factors between Glenn patients with and without high NT-proBNP.

Results: In monovariate analysis high NT-proBNP was associated with following 6 factors: histories of conduit from right ventricle to pulmonary arteries, ventricular volume on end-systole $(\geq 80 \%)$ and on end-diastole ( $\geq 178 \%$ ), ventricular pressure on end-diastole ( $\geq 12 \mathrm{mmHg}$ ), ventricular ejection fraction $(\leq 35 \%)$, and strong valve regurgitation of atrio-ventricle. After multivariate analysis high NT-proBNP was independently associated with odds ratio of 4.4 for expanded ventricular volume on end-systole, 3.9 for history of right ventricle conduit, and elevated ventricular pressure on end-diastole. Explanatory coefficient was 0.39. As for pulmonary circulation, pressure of superior vena cava was elevated in high NT-proBNP group (15 vs. $12 \mathrm{mmHg}$ : $\mathrm{p}=0.00048$ ); pressure of pulmonary capillary wedge (or left atrium) was elevated (9.6 vs. $8.0 \mathrm{mmHg}: \mathrm{p}=0.041$ ); the ratio of patients with pulmonary resistance $2.4 \mathrm{U} \cdot \mathrm{m}^{2}$ over was higher $(30 \%$ vs. $9 \%$ : $\mathrm{p}=0.013)$

Conclusion: High NT-proBNP values in Glenn patients were connected with myocardial stretch, which has been said in Fontan patients. Our study also showed history of conduit from right ventricle was independently related to high NT-proBNP in Glenn patients. As for pulmonary circulation, pressures of pulmonary capillary wedge and superior vena cava were increased in Glenn patients with high NT-proBNP. Depressed cardiac functions would build up afterload on pulmonary arteries. Heart failure therapy might be useful for lowering pressure of pulmonary artery in Glenn patients.

\section{P-259}

Evaluation of reverse remodeling in Right Ventricle Hypertrophy using PA debanding model rat

Urashima T., Itohisa M., Baba S., Ito R., Ijima M., Mori T., Kawachi F., Minamisawa $S$.

Jikei University School of Medicine, Tokyo, Japan

Introduction: The investigation for right ventricular reverse remodeling is critical for maintaining of the long-term right heart function, and deciding the right timing to release the pulmonary artery stenosis. To clarify this problem, we create a PA debanding model rat to evaluate the reverse remodeling in RV by repetitive hemodynamic evaluation, and electron micrography.

Design/Methods: PA debanding model rats were surgically generated by pulmonary artery banding using SD rats. UCG was performed at 2 weeks' post-operative period. Then rats with an RV-PA PG over $80 \mathrm{mmHg}$, and either a flat interventricular septum underwent re-thoracotomy and removed the band. UCG measurements were determined again at 4 weeks' post-operative period. The rats with evidence of RV-PA PG under $60 \mathrm{mmHg}$ were included to estimate more further studies. The level of LC3, and p62 were measured by Western blotting. Thus, we examined 
the morphology of the mitochondria by electron microscopy, which revealed the presence of damaged mitochondria with degraded cristae and mitochondrial membranes, whereas damaged mitochondria appeared to be partially restored. The comparison performed between PA debanded ( $\mathrm{G}-\mathrm{db} ; \mathrm{n}=18)$, just PA banding for $2(\mathrm{G}-2 \mathrm{w} ; \mathrm{n}=12)$ or 4 weeks $(\mathrm{G}-4 \mathrm{w} ; \mathrm{n}=18)$.

Results: There were significant increases in RV cardiac output derived from UCG in the G-db. Median survival time in G-db was significantly longer than PA banding $(29.8 \pm 5.1$ days vs $17.1 \pm 2.9$ days; $\mathrm{P}<0.01)$. RV weigh,and \%fibrosis of $\mathrm{G}-\mathrm{db}$ was significantly inhibited at 4wks compared with G-4w, but no significant difference with G-2w. Both LC3A/B, and p62 expressions were reduced in the G-db compared with G-4w. The rate of fibrosis in RV was significantly lower in the G-db compared with G-4w, but without significance compared with G-2w. Damaged mitochondria ratio was reduced in G-db compared with G-4w. Conclusions: A release of RV obstructive lesion is important to inhibit progress of fibrosis. The progress of fibrosis, and mitochondrial dysfunction were inhibited by the amelioration of the RV hypertrophy, but developed fibrosis was not restored. The myocardial damage estimated from the LC3, and p62 accumulation improved following reverse remodeling which is amelioration of the right ventricular hypertrophy.

\section{P-260}

Anxiety, Depression and Behavioral Rating Scales in Children with Non-Cardiac Chest Pain

Yoldaş T. (1), Kenar A. (2), Örün U.A. (1), Kayalı Ş. (1), Bodur Ş. (3), Özgür S. (1), Doğan V. (1), Karademir S. (1). Department of Pediatric Cardiology, Dr. Sami Ulus Maternity, Children's Health and Diseases Training and Research Hospital, Ankara, Turkey (1); Department of Pediatrics, Dr. Sami Ulus Maternity, Children's Health and Diseases Training and Research Hospital, Ankara, Turkey (2); Department of Child and Adolescent Psychiatry, Dr. Sami Ulus Maternity, Children's Health and Diseases Training and Research Hospital, Ankara, Turkey (3)

Objectives: Chest pain is the second most common reason for referral to pediatric cardiologists after benign heart murmurs. Etiology frequently depends on non-cardiac reasons. Non-cardiac etiologies of chest pain can include skeletal-muscle system disorders, gastrointestinal abnormalities and psychological symptoms. In addition, individuals may experience non-cardiac chest pain (NCCP) which is idiopathic, or of unknown origin. The aim of this study is to examine psychological symptoms in children and adolescents with medically unexplained chest pain.

Methods: 76 patients (ages 8-18 years) were included in the study who referred to the pediatric cardiology department with the complaint of chest pain but did not have any detected cardiac etiology or any other organic causes of chest pain. The control group was composed of 51 healthy volunteers in the same age group. Selfevaluation scales were given to both groups which included Beck Anxiety İnventory and Children's Depression Inventory. Also parents of both groups completed the Conner's Parent Rating Scale for assessment of Attention-deficit/hyperactivity disorder.

Results: Anxiety scores of the non-cardiac chest pain group were significantly higher compared to controls. No significant differences were found between patients and controls in terms of attention-deficit/hyperactivity disorder and depression scores. In patient group, patterns were similar for boys and girls and for children and adolescents; except girls scored significantly higher than boys in children's depression inventory.

Conclusions: In children and adolescents, non-cardiac chest pain is associated with increased levels of anxiety. These results show the importance of psychiatric evaluation in non-cardiac chest pain patients. Larger controlled studies are needed to determine the prevalence and impact of attention-deficit/hyperactivity disorder and depression in children and adolescents with NCCP.

\section{P-261}

Outcomes of a multidisciplinary approach for paediatric cardiac patients yield high satisfaction but complete preparedness is unattainable

Garratt V., King G., Ellison N., Cutler H., Hughes J., Stoica S., Caputo M., Parry A.

University Hospital NHS Foundation Trust Bristol Bristol, United Kingdom

Background: The patient-doctor relationship has moved far from the paternalistic approach to a partnership. With direct patient input we sought to modify our surgical pathway to improve patient experience, ensuring the information given to families met their needs for effective surgical preparation. At an open forum we sought patients' experiences and learnt that despite best efforts many felt unprepared for hospital admission. We therefore changed our surgical pathway to provide more information and support in a sequential and reinforcing manner involving the whole multidisciplinary team.

Methods: Following initial written information, a face-to-face surgical clinic meeting (SC) is held at which the intervention is discussed and the patients meet with the primary surgeon, Paediatric Clinical Nurse Specialists (PCNS) and Cardiac Clinical Psychologists (CCP). This is followed-up with further written information. Those identified as requiring additional psychology support are seen in the interim. At pre-admission clinic (PAC) patients see PCNS, CCP and play specialists for child preparation. Results: 79 patients were followed through their surgical pathway from February 2016-July 2016, with questionnaires at SC and PAC and telephone questionnaires post-operatively. $69.4 \%$ of patients considered initial written information prepared them for SC and 100\% reported the surgeon, PCNS and CCP clearly explained all aspects of the forthcoming surgery. Following PAC $66.7 \%$ of patients were satisfied and felt prepared for surgery. However, after surgery fewer patients reported that leaflets, surgeons, PCNS and CCP had been helpful in preparing for surgery than at PAC. Overall, only $29.6 \%$ reported that in retrospect they felt they had been completely prepared for their child's surgery.

Conclusions: Although at the time patients report high satisfaction and feel prepared for surgery using this multidisciplinary approach, after the event only $29.6 \%$ felt completely prepared, suggesting complete preparedness is unattainable. Ongoing work for improvements will involve previous patients in pre-intervention preparation.

\section{P-262}

Neurological development of Congenital Heart Disease with Very Low Birth Weight Infants -Multicenter Study in Japan

Nakashima Y. (1), Mori Y. (1), Toyoshima K. (2), Masutani S. (3), Yoda H. (4), Tanaka Y. (5)

Seirei Hamamatsu General Hospital, Hamamatsu, Japan (1); Kanagawa Children's Medical Center, Yokohama, Japan (2); Saitama Medical Center, Kawagoe, Japan (3); Toho University Omori Medical center, Tokyo, Japan (4); Shizuoka Children's Hospital, Shizuoka, Japan (5)

Objectives: The purpose of the study was to investigate neurological outcome in 6-year survivors of very low birth weight (VLBWI) with congenital heart disease (CHD). 
Methods: We sent a questionnaire to newborn intensive care units (NICU) in Japan. We analyzed the Developmental Quotient (DQ) evaluated at 3 years old and full scale IQ at 6 years old in VLBWI born from 2000 to 2006 . We compared the clinical and developmental data in patients with CHD (CHD group) to 229 VLBWIs without CHD admitted in our NICU (no-CHD group). Results: We obtained a questionnaire from 12 NICU. There were 45 VLBWIs with CHD (CHD group). The most common lesions were ventricular septal defect (44\%), tetralogy of Fallot (16\%) and pulmonary valvular stenosis (9\%). Of 45 patients, $9(20 \%)$ had chromosomal abnormality, 21 patients (47\%) performed surgical repair or catheter interventions. Median periods of hospital stay and intubation were 80 days $(43 \sim 215$ days $)$ and 7 days ( $0 \sim 138$ days), respectively. There were no significant differences in the periods of hospital stay, intubation periods, and the frequency of severe complications such as chronic lung disease, necrotizing enterocolitis and brain hemorrhage between CHD and no CHD group. In CHD group, the Cognitive-Adaptive (C-A) DQ, Language-Social (L-S) DQ, Postural-Motor (P-M) DQ, and overall DQ were 83 (14 117), 81 (16 114), 77 $(12 \sim 124), 81$ (19 114), respectively. All DQ scores were below reported normal range. When compared to scores in the no-CHD group, all scores were also lower in the CHD group. However, there were no significant differences of all DQ scores between in CHD (median of overall DQ: 87, P-M DQ: 180, C-A DQ: 87, L-S DQ: 84), and no-CHD patients (median of overall DQ: 86, C-A DQ: 86, L-S DQ: 86, P-M DQ 100, when patients with chromosome anomaly were excluded (All scores, $\mathrm{p}>0.05$ ). Full scale IQ of patients excluded chromosomal anomaly was not statistically deferent between CHD and no-CHD groups (88 (63$103)$ vs. 92 (45-129).

Conclusions: The degree of neurocognitive deficits of survivors of VLBWI with CHD is similar to that of no-CHD survivors of VLBWI at the age of 6 years.

\section{P-263}

Correlation between cognitive impairment and prognostic parameters in adult patients with congenital heart disease Kourkoveli P., Apostolopoulou S., Moutsou A., Rammos S. Department of Pediatric Cardiology and Adult Congenital Heart Disease, Onassis Cardiac Surgery Centre, Athens, Greece

Introduction: Cognitive deficits have been observed in patients with congenital heart disease (CHD). This impairment could lead to difficulties in the management of these patients with regards to the understanding of their disease or in adhering to pharmacological treatment and medical advice. Aim of this study is to assess the cognitive and psychological function in adult patients with moderate and great complexity CHD and to attempt to correlate the results with prognostic parameters.

Methods: Thirty consecutive clinical stable patients, mean age $28.1 \pm 11.7$ years old, $50 \%$ male, with moderate and great complexity congenital heart defects were recruited from a tertiary center. Patients cognitive capacity, psychological and neurohormonal status was assessed with a mini mental examination test (MMSE), Beck depression inventory and Zung depression scale questionnaires and plasma B-type brain natriuretic peptide (BNP) respectively.

Results: All patients were symptomatic (NYHA $\geq \mathrm{II})$. Mean plasma BNP concentration was $210 \pm 300 \mathrm{pg} / \mathrm{ml}$. In $26.6 \%$ of cases a pathological MMSE score emerged. $33.3 \%$ were characterized as having depressive symptoms. A positive correlation between MMSE and age $(p=0.01), \operatorname{BNP}(p=0.02)$ and the presence of depressive symptoms $(p=0.02)$ was observed but not between MMSE and NYHA class.

Conclusions: MMSE and BNP plasma levels could be considered simple tests for identifying CHD patients who need special attention and systematic neuropsychological testing and who require a strong doctor/patient interaction for patient's adherence to therapy.

\section{P-264}

Psychoemotional status of families of preterm newborns with patent ductus arteriosus in the industrial region of a country with a developing economy

Onikiienko O.L. (1), Kulikova D.O. (2), Gonchar M.O. (1), Buchneva O.V. (2), Matsiyevska N.K. (1)

Kharkiv National Medical University (1). State Institute of General and Urgent Surgery named after Zaitsev V.T. of National Academy of Medical Sciences (2)

Introduction: The birth of a very preterm child in the family is a great stress, which is associated with the presence of a multiple congenital abnormalities, including congenital heart disease. Successful nursing management of such children significantly increased the number of congenital heart defects, in particular, patent ductus arteriosus (PDA). PDA in developing countries usually requires surgical treatment due to absence of parenteral forms of non-steroids, which in turn is an additional stress factor. Methods: We interviewed 112 families of preterm infants. 47 children (group 1) have had surgery of PDA. In 65 families (group 2) the situation did not require surgical treatment in the neonatal period. The survey was conducted using adapted Symptom Checklist-90-revised questionnaires, Family Environmental Scale.

Results: The average score for clusters of questionnaires among mothers was: somatization $1.29 \pm 0.46$; anxiety $2.06 \pm 0.52$; depression $1.30 \pm 0.53$, an index of general severity $1.56 \pm 0.49$. The same parameters for fathers were $0.60 \pm 0.33,0.82 \pm 0.35,1.21 \pm 0.40$, $0.74 \pm 0.44$, respectively, and were significantly lower than that of mothers. In group 1, the average index of total severity in the parents was $1.95 \pm 0.62$, in the second $1.36 \pm 0.43(p<0.01)$.

Conclusions: The presence of the PDA which required surgical treatment was the main stress factor. The level of anxiety was higher in mothers than in fathers. The level of fathers' distress affected on the relationship in the family significantly more often in group 1. All parents would prefer the availability of professional psychological assistance. Certification of intravenous NSAIDs would reduce surgical management of PDA.

\section{P-265}

Parental anxiety and comprehension before cardiac intervention in a population of children with congenital heart disease (CHD): contributing factors and consequences

Werner O., Ovaert C., El Louali F.

Pediatric cardiology, CHU La Timone, Marseille - France

Introduction: The medical information (diagnosis and treatment) around congenital heart disease can induce major stress to the parents. Visual analog scales have been validated to assess anxiety in the adult population. The aim of this study was to analyze parental anxiety using a Visual analog scales and to explore the influencing factors, in particular the level of understanding of the disease and its support.

Methods: Prospective transversal study lead from January 2017 to May 2017 in the pediatric cardiology and intensive care unit of -La 
Timone Enfants' University Hospital (Marseille, France). All available parents (mother and/or father) of children admitted for surgical or transcatheter cardiac intervention were offered to participate. The parents were asked to complete 2 Visual analog scales in order to assess their level of anxiety and comprehension, before intervention and after complete information given by the cardiologist, the surgeon and the anesthetists.

Results: We included 73 patients, mean age 3 years, median 11 months (range 7 days-13 years), 42 requiring surgery and 31 transcatheter intervention. The mean score of maternal anxiety was significantly higher than the paternal anxiety (8.2 vs 6.3 , $\mathrm{p}<0.0001)$. The factors that influenced the maternal anxiety were: the paternal anxiety $(p=0.015)$, children's comorbidity $(p=0.013)$, the distance home-surgical center $(p=0.036)$ and the surgical risk scale (RACHS) $(p=0.025)$. In multivariate analysis, maternal anxiety was still influenced by the paternal anxiety $(\mathrm{OR}=4.995 \% \mathrm{CI} 1.05-19.42)$ and the RACHS (OR $=11.495 \%$ CI 1.19-116.2). No relation was found between the mean score of comprehension and anxiety. In the neonatal group, there was no difference in parental anxiety between prenatal and postnatal diagnosis.

Conclusion: This study highlights several factors that influence the parental anxiety. Identifying the parents at risk of high stress can be useful to set up psychological support during hospitalization.

\section{P-266}

Transition program for children with congenital heart disease: the importance of patient engagement and partnership

Fournier A. (1), Badr J. (2), Dore A. (3), Pomey M.P. (2)

CHU Sainte-Justine (1), Public Health School, University of Montreal

(2), Montreal Heart Institute (3); Montreal, Quebec, Canada

Introduction: Most children with congenital heart disease (CHD) reach adulthood. In Canada, $>50 \%$ of adults with $\mathrm{CDH}$ don't benefit from a close follow up by healthcare providers and $>60 \%$ of children with CHD in Quebec discontinue follow up at adulthood.

Methods: This study aims to build a transition program for children with $\mathrm{CDH}$ in partnership with patients and healthcare professionals. Our hypothesis is that patient engagement through this process and peer support between adult patients who went through the transition and adolescents with $\mathrm{CDH}$ will help them acquire skills for self-management and lead to better follow up in adulthood. The study includes quantitative and qualitative data collected through focus groups, individual interviews and questionnaires with healthcare professionals, adult with CHD, adolescents with CHD and their parents. The study assesses the potential benefit of peers in supporting patients through their transition, by implementing a co-constructed program in partnership with patients and their families.

Results: Healthcare professionals $(\mathrm{n}=15)$ consider patient engagement in transition program to be important. Even if most feel comfortable with engaging patients as partners in transition programs, they have questions regarding their professional/legal responsibilities in such programs and worry about the burden of increased workload. The transition for adult patients $(n=6)$ was smooth even though the majority didn't have any information or discussion with the medical team about the process. Surgical interventions and changes in treatment after transfer were described as more traumatic that the transition itself. Parents of adolescents $(n=7)$ where much more affected by the transition that the adolescents themselves. In this study, the average age of adolescent was 17.8 [17-18 yrs, $n=6] ; 83 \%$ of the parents mentioned that they had not discussed the transition with healthcare professionals and $100 \%$ said they didn't have any information about it. Parents felt helpless and needed support.

Conclusions: Transition is an important issue to address, and implementing a program based on peer support and partnership with patients and their family is a promising path to respond to the needs of children with CHD and their parents and improve the quality of healthcare services.

\section{P-267}

Eye Movement Desensitization and Reprocessing in Children and Adolescents with Congenital Heart Disease (ConHD) and Medically Related Subthreshold PTSD: a Randomized Controlled Trial

Utens E.M.W.J. (1,3,5), Meentken M.G. (1), van Beynum I.M. (2), Aendekerk E.W.C. (1), Legerstee J.S. (1), El Marroun H. (1), van der Ende J. (1), Lindauer R.J.L. (3), Hillegers M.H.J. (1), Moll H.A. (4), Helbing W.A. (2)

(1) ErasmusMC-Sophia, dept. of Child and Adolescent Psychiatry/ Psychology; (2) ErasmusMC-Sophia, dept. of Pediatric Cardiology; (3) Academic Center for Child psychiatry the Bascule/Dept. Child and Adolescent Psychiatry, Academic Medical Center, Amsterdam; (4) ErasmusMC-Sophia, Dept. General Paediatrics; (5) Research Institute of Child Development and Education, University of Amsterdam

Introduction: About 3 in every 10 children/ adolescents admitted to a hospital or undergoing medical treatment, develop elevated symptoms of posttraumatic stress disorder (PTSD). If untreated, subthreshold PTSD seriously affect quality of life, psychosocial functioning and long-term psychiatric comorbidity. Research into subthreshold PTSD due to pediatric medical treatment and/or hospitalization is scarce. Even less is known about the treatment of subthreshold PTSD in youth with ConHD. Eye Movement Desensitization and Reprocessing (EMDR) is a fast, innovative and non-invasive psychotherapy for posttraumatic stress symptoms. Effectiveness of EMDR in medically related trauma in children has not previously been systematically investigated.

Methods: Included are children aged 4 to 16 who have undergone a one-time (trauma type I; patients from emergency unit) or repeated (trauma type II) hospitalization/ medical treatment for ConHD up to 5 years ago. Participating children are first screened for PTSD symptoms. Thereafter, children with subthreshold PTSD are randomly assigned to 1) ca. 6 sessions of standardized EMDR or 2) care as usual (CAU = medical care only). Follow-up measurements take place after 2 and 8 months.

Results: Preliminary analysis from the screening assessment at baseline reveals that $68 \%$ of all participating children were symptom-free and $32 \%$ show elevated, subtreshold PTSD symptoms at baseline. Data gathering and screening of patients will be ongoing until February 2018. Outcomes of this assessment and the EMDR intervention as such will be presented during the conference.

Conclusions: Our data show that symptoms of PTSD were experienced by 1 in every 3 pediatric patients. Clinical implications and specific needs for psychosocial care will be discussed. Our results indicate that children with ConHD should be structurally screened and if needed treated for PTSD symptoms.Possibilities to provide psychosocial interventions are discussed.

\section{P-268}

Quality of life and long-term follow-up after stenting of Coarctation of the Aorta

Dobrev D., Lekova R, Kaneva A.

Department of Pediatric Cardiology, National Heart Hospital, Sofia, Bulgaria 
Background: Stenting of coarctation of the aorta (CA) requires lifelong medical follow-up for late complications, hypertension sequelae and treatment (including reinterventions). Lack of adequate care is a frequent problem with Grown-up congenital heart defect $(\mathrm{GUCH})$ patients.

Objective: To evaluate the long-term clinical condition and quality of life (QoL) after stenting for CA in tertiary heart center.

Methods and patients: QoL was assessed with a custom-made questionnaire through direct contact during regular visit or phone call. The main topics include self-assessment, arterial hypertension, medication, symptoms, follow-up and demographics. A comparison with official statistical analysis was performed. Hospital database was used to assess health care accessibility, insurance, specific diagnostics and treatment. 41 patients underwent CA stenting between 2003 and 03.2017. Procedure related complications were observed in 8 cases (19\%). The patients were followed-up for a mean of $3.2 \pm 2.8$ years ( 0.1 to 10.2 years). Six patients were excluded from the study ( 2 urgent operations and 4 foreigners). During follow-up 10 additional interventional procedures were conducted (all of them in children).

Results: There is no regular follow-up for more than 4 years in $52 \%$ of GUCH. By October 2017 nine patients (26.5\%) are children and 26 patients $(74.3 \%)$ are GUCH. 26 responded to the questionnaire; $9(34.6 \%)$ of the adults did not due to inactive phone number/refusal to participate. There is significant difference in follow-up free period - mean 1.86 years for children versus 5.86 years for adults $(p=0.003)$. Educational level corresponds to the general population. Follow-up is performed in secondary $(37.5 \%)$ or a tertiary center $(56.3 \%)$ by cardiologist $(43.8 \%)$ or pediatric cardiologist (50\%). 25\% of GUCH are not followed up at all. $11 \%$ are unemployed with no insurance. All but 5 patients report good general health and $13(50 \%)$ take antihypertensive therapy.

Conclusion: There is clear lapse in the care for GUCH with stented CA. QoL for these patients is not significantly different from the general population. There is a discrepancy between general selfassessment as being "cured" and symptomatic findings. GUCH with stented CA need proactive care with multimodality imaging methods and timely solution of potential complications.

\section{P-269 \\ Newborn Screening for Congenital Heart Disease Using Echocardiography and Follow-up at High Altitude in China \\ Li J.J. (1), Liu Y. (2), Xie S.Y. (1), Zhao G.D. (2), Dai T. (2), Chen H. (2), Mu L.F. (2), Qi H.Y. (2), Li J. (1) \\ Capital Institute of Pediatrics, Peking University Teaching Hospital, Beijing, China (1); Women and Children's Hospital of Qinghai \\ Province, Xining, Qinghai, China (2)}

Introduction: Screening newborns for congenital heart disease (CHD) mostly focuses on critical CHD using pulse oximetry. But this is inapplicable at high altitude due to variedly decreased arterial saturations. Altitude hypoxia induces pulmonary arterial hypertension that is main morbidity in left to right shunt CHD. We aimed to screen newborns for all forms of CHD using echocardiography at high altitude and follow up their outcomes.

Methods: Echocardiography was performed in consecutive asymptomatic 1,002 newborns in 3-5 days after birth (459 girls; 73 (7.3\%) Tibetan, 701 (70.0\%) Hui, Salar, and other ethnics, and 228 (22.7\%) Han; residence altitude range: 1,800-4,223 m, median 2,450 m) born in Xining, Qinghai between Mar 2015 and Aug 2016. Followup echocardiography was performed at $1-3,6$, and 12-18 months. Results: The incidence of CHD was $27.8 \%(279 / 1,002)$ [140 girls; 16 (5.7\%) Tibetan, 179 (64.2\%) Hui, Salar, and other ethnics, and
84 (30.1\%) Han]. Secundum atrial septal defect (ASD)[175 $(62.7 \%)]$ was the most dominant, followed by patent ductus arteriosus (PDA)[61 (21.9\%)], and ventricular septal defect (VSD) [8 (2.9\%)]. At 1-3 months follow-up in 98/279 (35.1\%), ASD, PDA, and VSD closed in $12.7 \%, 82.4$, and $16.7 \%$, respectively. At 6 months in 78/252 (31.0\%), ASD, PDA, and VSD all closed in $50 \%$. At $12-18$ months in 85/208 (40.9\%), ASD, PDA, and VSD closed in $64.2 \%, 72.2 \%$, and $80.0 \%$ respectively. In total, among 173 babies with CHD (62.0\% of 279) at follow-ups, $28.9 \%$ CHD babies remained unclosed. During follow-up period, 2 babies died of pneumonia at 4 and 6 months. The incidence of CHD and spontaneous closure were not correlated with altitudes and ethnics ( $\mathrm{p}>0.1$ for all).

Conclusions: The incidence of CHD is many folds higher than that at low altitude, consisting solely of simple forms with left to right shunt in asymptomatic newborns. By 18 months of age, the incidence of CHD was $10 \%$. Follow-up remains challenging due to the poor socioeconomic conditions, and must be reinforced in order to provide early repair and prevent from significant pulmonary arterial hypertension and even death.

\section{P-270 \\ Incidence and Spectrum of Congenital Heart Disease in Neonatal Intensive Care Unit at High Altitude in China Li J.J. (1), Wang X.R. (2), Qi H.Y. (2), Li J. (1) Capital Institute of Pediatrics, Peking University Teaching Hospital, Beijing, China (1); Women and Children's Hospital of Qinghai Province, Xining, Qinghai, China (2)}

Introduction: Our previous study on echocardiography screening asymptomatic newborns for congenital heart disease (CHD) at high altitude showed a high incidence (30\%) consisting solely of simple forms. This study evaluated the incidence and spectrum of CHD in neonatal intensive care unit (NICU) in order to depict a truer picture of $\mathrm{CHD}$ at high altitude.

Methods: We reviewed charts of 4,214 neonates in the Women and Children's Hospital in Xining (2,260m), Qinghai in 2015-2016. Echocardiography was performed in 1,943 babies (aged 10 minutes to 2 months; 1,220 boys; altitude ranged 1,800 to $4,300 \mathrm{~m}$, median 2,526m) when CHD was suspected based on heart murmur, cyanosis or pneumonia.

Results: These babies were hospitalized because of pneumonia $(62.6 \%)$, asphyxia $(13.2 \%)$, or hyperbilirubinemia $(7.3 \%)$. The incidence of CHD was $26.0 \%(1,096 ; 658$ boys). Mild and moderate CHD accounted for $97.6 \%(1,070)$, including 580 (52.9\%) secundum atrial septal defect, 224 (20.4\%) patent ductus arteriosus, $18(1.6 \%)$ ventricular septal defect, 248 (22.6\%) multiple defects with left to right shunt, $1(0.1 \%)$ bicuspid aortic valve, 7 (0.6\%) pulmonary stenosis, $2(0.2 \%)$ aortic stenosis, $6(0.5 \%)$ atrioventricular septal defect. Critical CHD accounted for $2.4 \%$ (26), including $5(0.5 \%)$ complete transposition of the great arteries (TGA), $6(0.5 \%)$ hypoplastic right heart, $3(0.3 \%)$ hypoplastic left heart, $3(0.3 \%)$ double outlet right ventricle, $3(0.3 \%)$ tetralogy of Fallot, $2(0.2 \%)$ truncus arteriosus, $2(0.2 \%)$ total anomalous pulmonary venous connection, $2(0.2 \%)$ severe aortic stenosis, $2(0.2 \%)$ severe pulmonic stenosis. Among those with CHD, pulmonary arterial hypertension occurred in 2.8\% (54/1,943) (systolic pressure $69 \pm 24 \mathrm{mmHg}$ ). By 2-12 months follow-up in 26 patients with critical CHD, 17 died before cardiac surgery, 1 of the 4 survivors had corrective operation of TGA, and 5 lost track. Conclusions: The incidence of CHD in NICU at high altitude is about 20 folds higher than that at low altitude, with substantially less incidence of critical CHD but with high mortality. Routine 
echocardiography and follow-up should be implemented in all NICU patients to provide early intervention.

\section{P-271}

Isolated left ventricular apical hypoplasia in a 9-year-old girl - A rare differential diagnosis for dilatated cardiomyopathy

Zimmer M. (1), Bergdolt M. (2), Follmann P. (1), Pohler J. (1),

Schumacher B. (2), Kriebel T. (1)

Department of Pediatrics, Westpfalz-Klinikum Kaiserslautern, Germany (1); Department of Cardiology, Westpfalz-Klinikum Kaiserslautern, Germany (2)

Introduction: Isolated left ventricular apical hypoplasia (LVAH) is a very rare primary cardiomyopathy with non-specific symptoms. This entity is probably contributed to an inadequate dilatation of chambers during partitioning.

Methods: In our female patient already prenatal ultrasound showed high echogenicity of the left ventricular myocardium. Postnatal echocardiograms showed a spherical dilated left ventricle with a normal function and no clinical symptoms. The diagnosis was congenital dilatative cardiomyopathy. During follow up echocardiographics enddiastolic diameter increased. Therefore and due to the atypical left ventricular configuration, we performed a catheter study at the age of 9 years. There were no further pathological results apart from a mild elevated left-ventricular enddiastolic pressure (LVEDP) of $12 \mathrm{mmHg}$ and the spheric configuration of the left ventricle. The histological analysis of myocardial biopsies showed no signs for an inflammation but a moderate interstitial myocardial fibrosis, consistent with dilatative cardiomyopathy. Subsequent cardiac magnetic resonance imaging revealed the four typical morphological characteristics of LVAH consisting of a truncated and spherical left ventricular configuration with rightward bulging of the interventricular septum, a fatty replacement of the left ventricular apex, a complex papillary muscle network in the anterior apex, an elongation of the right ventricle wrapping around the deficient left ventricular apex and a left ventricular out-pouching (Figure). Due to the elevated LVEDP we started a treatment with Captopril.

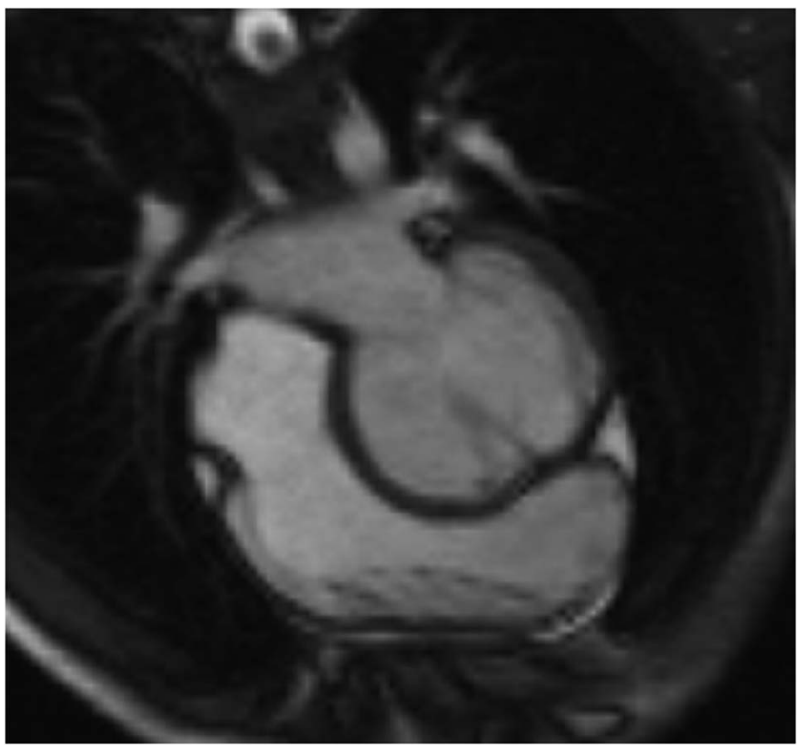

Figure.
Conclusion: To the best of our knowledge this is the first report of LVAH with abnormalities already observed during fetal echocardiography. Therefore this rare congenital heart defect should be considered as a differential diagnosis to dilatative cardiomyopathy in the newborn.

\section{P-272}

AV Channel is very rare in T21 patients at high altitude in Bolivia

Heath A., Medina N., Balanza G., Scherer C., von Alvensleben I.

Kardiozentrum, La Paz, Bolivia

Introduction (or Basis or Objectives): Down Syndrome (DS) children have a major cardiac anomaly, the most common being atrioventricular septal defects (AVSD) in regions below 1500 meters of altitude. Aim: To stablish frequency and distribution of Congenital Heart Disease (CHD) in children with Down Syndrome (DS) at different altitudes above sea level in Bolivia.

Methods: Retrospective, descriptive study of echocardiographic examination of 595 children with DS performed with a standarized protocol. The patients were distributed in two groups, according with the residency altitude. G1 (n: 480): >3000 masl and G2 (n: 115): 1500-3000 masl.

Results: Between 2004 and 2016 we studied 5954 patients with suspicion of CHD, 595 of those were diagnosed with DS (10\%). The mean age at diagnosis was 2,94 years. $390(65,5 \%)$ patients had a CHD. Female patients with DS were more likely to have a cardiac anomaly compared to male patients. The male/female ratio was: 1,05:1. We also analyzed the frequency of CHD of each group: G1: 69,4\% (n:333) and G2: 49,5\% (n:57). The group 1 had higher prevalence of CHD. In all two groups the most frequent CHD were: VSD, PDA und ASD. The frequency of AV channel of each group was: G1: 0,8\% (n:3), G2: 1,5\% (n:6). The hemodynamic significance of CHD of each group at the moment of the study were: G1: $67 \%$ (n: 299), G2: 44,2\% (n: 46). The association of cardiac malformations by altitude were: $\mathrm{G} 1: 33,6 \%$ (n:112) and G2: $19,3 \%$ (n:11). Ductus arteriosus shows an increasing prevalence with altitude.

Conclusions: CHD frequency increases with altitude. AV Channel is not the most frequent CHD at high altitude in patients with DS, but associations of VSD, ASD and ductus are the most common finding. AV Channel seems to increase with lower altitude (G1: $0,8 \%$ vs $G 2: 1,5 \%)$. AV channel has a significant fewer frequency in Bolivian patients. More studies are needed to investigate if this difference with other populations is racial or due to hypoxia.

Table: Comparision of frequency and CHD distribution in our study and in different populations

\begin{tabular}{|c|c|c|c|c|c|c|}
\hline & $\begin{array}{l}\text { Our } \\
\text { Data. } \\
2017\end{array}$ & $\begin{array}{l}\text { Morris } \\
\text { et al. } 2014\end{array}$ & $\begin{array}{l}\text { Bell et al. } \\
2003\end{array}$ & $\begin{array}{l}\text { Freeman } \\
\text { et al. } 1998\end{array}$ & $\begin{array}{l}\text { Stoll et al. } \\
1990\end{array}$ & $\begin{array}{l}\text { Källén } \\
\text { et al. } 1996\end{array}$ \\
\hline Place & Bolivia & Europe & England & Atlanta & France & Europe \\
\hline Study period & 2004-2016 & $2000-2010$ & $1985-1999$ & 1989-1995 & $1979-1987$ & 1976-1993 \\
\hline $\begin{array}{l}\text { Number of cases } \\
\text { with Down } \\
\text { Syndrome }\end{array}$ & 595 & 6151 & 577 & 227 & 139 & 5581 \\
\hline $\begin{array}{l}\text { Percentage with } \\
\text { CHD }\end{array}$ & 65,5 & 43,6 & 39,1 & 44 & 44,6 & 26 \\
\hline AVSD & 2,3 & 23,2 & 43,4 & 45 & 41,9 & 38,5 \\
\hline VSD & 36 & 24,3 & 28,8 & 35 & 29 & 28 \\
\hline ASD OS & 19 & N/A & N/A & 8 & N/A & N/A \\
\hline PDA & 35,6 & 1,6 & 16,8 & 7 & & 3,6 \\
\hline TOF & 0,5 & 2,7 & 3,5 & 4 & 3,2 & 3,5 \\
\hline $\begin{array}{l}\text { Coarctation of } \\
\text { the aorta }\end{array}$ & 0,3 & 1,6 & 4 & N/A & 4,8 & 0,6 \\
\hline Other & 6,3 & 18,5 & 4,7 & 1 & 14,5 & 26,4 \\
\hline
\end{tabular}




\section{P-273}

Asymptomatic interarterial course of the left coronary artery from the right sinus of Valsalva. Which management should be recommended?

Gildein H.P. (1), Brangenberg R. (2), Epp A. (1), Buheitel G. (3), Meierhofer C. (1), Ewert P. (1)

Deutsches Herzzentrum München, Müchen, Germany (1); Klinikum Traunstein, Traunstein, Germany (2); Klinikum Augsburg, Augsburg, Germany (3)

Aberrant course of the left coronary artery (LCA) originating from the right coronary sinus of Valsalva (RCSV) and coursing between the aorta and pulmonary artery is a rare coronary anomaly. On forced exercise there is a risk of compression of the LCA causing ischemia or even sudden cardiac death. In symptomatic patients surgery is recommended. We report 2 cases of asymptomatic adolescents.

Case 1: M.P. complained at the age of 12 years of uncharacteristic chest-pain on exercise but also at rest. Echocardiography showed origin of the LCA from the RCSV taking course to the left between the aorta and the main pulmonary artery which was confirmed on MRI. On exercise test he tolerated a peak strain of $4.1 \mathrm{~W} / \mathrm{kg}$ without any symptoms or electrocardiographic changes. Five minutes after the exercise test he complained of right thoracic pain which was related to respiration. On stress MRI with adenosine there were no perfusion defects nor regional wall motion abnormalities.

Case 2: S.S. is a 14-year-old adolescent competitive ice-hockey player without any complaints on maximal exertion. He had echocardiography for his annual sports evaluation which showed the above mentioned coronary abnormality. On exercise test there were no symptoms or electrocardiographic changes at a peak-strain of $4.7 \mathrm{~W} / \mathrm{kg}$. The systolic blood pressure rose to $227 \mathrm{mmHg}$. Competitive sports were denied. Surgical treatment was asked for by the parents in order to continue participation in training and tournaments.

Besides conservative treatment there are three surgical options, reimplantation, coronary artery bypass and unroofing (if anatomically feasible). We decided in an interdisciplinary discussion with the surgeons not to operate due to the complexity in case 1 and the lack of symptoms in case 2 unless a slitlike orifice could be demonstrated. However, competitive sports were forbidden.

Summary: Aberrant origin of the LCA from the RCSV is well recognized on echocardiography and an intramural course might be identified. In symptomatic patients surgery is indicated with unroofing of the coronary artery as the most elegant method when possible. In asymptomatic patients surgery remains a question of discussion.

\section{P-274}

Isolated left ventricular apical hypoplasia

Manso P. H., Jurca M. C., Trad H.S.

Ribeirao Preto Medical School, department of Pediatrics; Ribeirao Preto- Brazil

An asymptomatic 13-year-old boy was diagnosed with a mild systolic murmur during a routine evaluation. He also had arthrogryposis multiplex congenita with mild spine deviation. ECG showed sinus bradycardia $(\mathrm{HR}=50 \mathrm{bpm})$, and a transthoracic echocardiogram identified a mild left ventricle systolic dysfunction and an abnormal papillary muscle.

One year later, the routine echocardiogram identified a spherical left ventricle with ejection fraction of $51 \%$, a parachute mitral valve with no dysfunction, and a banana-shaped right ventricle. Therefore, a cardiac MRI was performed.

The cardiac MRI identified an isolated left ventricular apical hypoplasia. It showed an abnormal mitral valve apparatus and the diagnosis aspects of the disease: spherical $\mathrm{lv}$ configuration, fatty material at left ventricle apex, elongation of the right ventricle wrapping around the deficient LV apex, and both mitral papillary muscles originating in the flattened anterior apex. After one-year follow-up, two years after the initial diagnosis, the patient is asymptomatic, with sinus rhythm.

Discussion: Isolated left ventricular hypoplasia is a very rare disease, with no more than 25 reports since its first description in 2004. Diagnosis age ranges from three months to 66 years old.

In most patients the condition is discovered incidentally. Wrapping rv, bulging ventricular septum toward the rv, and abnormal papillary muscle aroused the diagnosis suspicion and posterior MRI.

There is not a single pattern regarding the ECG: It may present right or left deviation. Non-sustained ventricular tachycardia and atrial tachycardia have also been reported in these patients. Although our patient presented with sinus bradicardia, he did perform well in a cardiopulmonary test.

Clinical evolution may also vary from asymptomatic to NY class II. Therefore, the patient should be followed up concerning the emergence of heart failure and arrhythmias.

Pathogenesis: So far, a clear cause for this disease has not been established. In most congenital lv heart diseases, the apical component exists as incomplete ventricle.

A previous case report has described association of arthrogryposis with this cardiomyopathy. A mutation in the LMNA gene (p.Arg644Cys) has been described as a potential genetic cause.

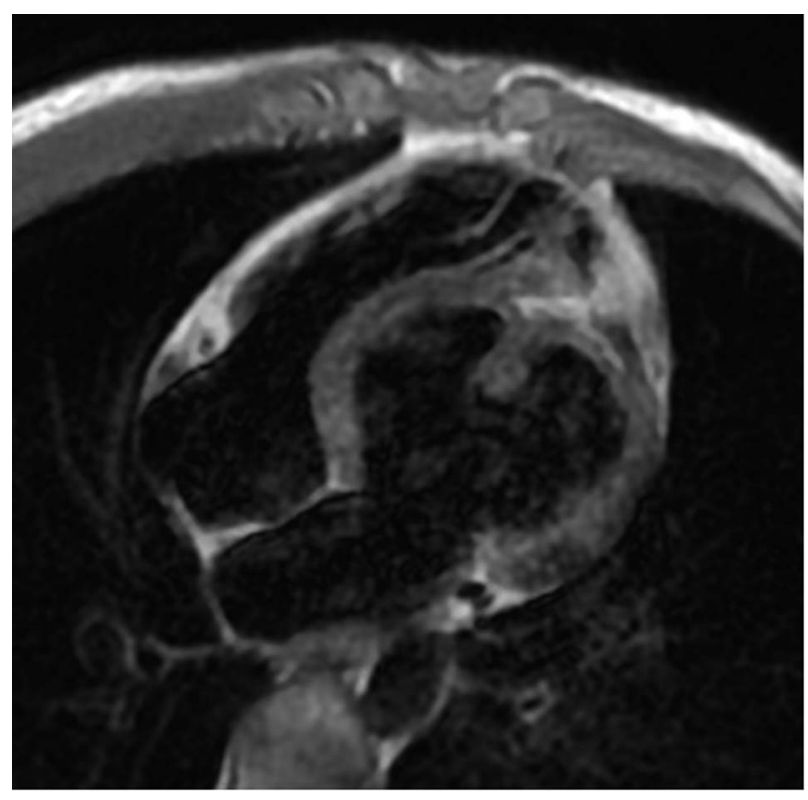

Figure.

\section{P-275}

Left ventricular noncompaction in cardiac explants from paediatric patients and adults with congenital heart disease: prevalence and associated factors

Silva P.G. (1), Azeka E. (2), Jatene M.B. (2,3), Aiello, V.D. (2) Faculdade de Medicina da Bahia - Federal University of Bahia, Salvador, Brazil (1); Instituto do Coração - HCFMUSP, Sao Paulo, Brazil (2); Faculdade de Medicina da Universidade de São Paulo, Sao Paulo, Brazil (3) 
Introduction: Left ventricular noncompaction (LVNC) is a rare anomaly characterized by prominence of the ventricular trabeculations that may be associated with severe heart failure and need for a heart transplant or occur as an associated feature in other cardiomyopathies or congenital heart defects. There are no data concerning the prevalence of LVNC in patients who have undergone heart transplantation nor its associated factors. Our objectives are to assess the prevalence of LVNC phenotype (focal or diffuse) as the main or associated diagnosis in cardiac explants from pediatric patients or adults with congenital heart disease investigating the association between LVNC and sex/age, comparing the gross findings with the data obtained through pre-surgical imaging studies.

Methods: Cardiac explants from paediatric patients and adults with congenital heart defects were selected. Medical records were consulted for the demographic, anathomopathological, echocardiographic and MRI data. Measurement of the compacted and noncompacted portions of the left ventricular wall was performed with a pachymeter at 3 different points: the inflow, the apical, and the outflow walls. The echocardiographic-based criteria of Chin and the Jenni were used to determine whether LVNC was present.

Results: 94 hearts were evaluated, being $51.06 \%$ from male individuals. The average age was $9.81 \pm 6.87$ years. Three $(3.19 \%)$ had LVNC as the main pre-transplant diagnosis. However, $59.57 \%$ of the heart specimens fulfilled any of the criteria for LVNC in at least one ventricular segment. $32.9 \%$ presented focal LVNC at just one point, $17 \%$ presented at 2 different points, and $7.4 \%$ at all 3 points. The apex had the highest prevalence of LVNC $(28.72 \%$ under Jenni and $41.48 \%$ under Chin). Dilated cardiomyopathy was the most prevalent anatomopathological diagnosis (67.02\%) overall and among the hearts with LVNC at gross evaluation (69.09\%). The prevalence of LVNC in hearts with structural and restrictive cardiopathies were $52,4 \%$ and $61,5 \%$, respectively. The average ejection fraction was $34.16 \pm 15.30 \%$, but no difference was found between hearts with or without LVNC.

Conclusion: Focal LVNC is a prevalent feature in cardiac explants from the paediatric population, present mainly at the ventricular apex and frequently associated with the primary diagnosis of dilated cardiomyopathy.

\section{P-276}

How does right and left isomerism look like? A portrait of cardiac anomalies and visceral arrangement

Oreto L. (1,3), Ciliberti P. (2), Del Pasqua A. (2), Santangelo T.P. (4), Napolitano C. (4), Manuri L. (1), De Zorzi A. (1), Gitto P. (1), Saitta M.B. (1), Anderson R.H. (5), Agati S. (1), Carerj S. (3), Secinaro $A$. (4)

(1) Mediterranean Pediatric Cardiology Center, Bambino Gesù Pediatric Hospital, Taormina, Italy; (2) Medical-Surgical Department of Pediatric Cardiology, Bambino Gesù Pediatric Hospital, Rome, Italy; (3) Clinical and Experimental Department of Medicine, University Hospital of Messina, Italy; (4) Department of Imaging, Bambino Gesù Pediatric Hospital, Rome, Italy; (5) Institute of Genetic Medicine, Newcastle University, United Kingdom

Objectives: To describe principal cardiac anomalies, and the arrangement of organs, in patients with right as opposed to left isomerism.

Methods and Results: We retrospectively reviewed data of patients diagnosed with so-called visceral heterotaxy at our Institutions in the last two years, including echocardiography in all patients and computed tomography angiography in the most complex cases. We identified right isomerism (RI) in 24 patients (14 males). All had common atrioventricular junctions (AVSD), except for one with tetralogy of Fallot. AVSD was nearly always (85\%) unbalanced with prevalent right-dominance. Right outflow obstruction was present in 19/24 cases (79\%), and extracardiac anomalous pulmonary venous connections (APVC) in 13/24 (54\%). The heart was right-sided in 10 cases (42\%), midline in 1. The inferior caval vein (ICV) was left-sided in 9 cases (37\%) and interrupted with azygos continuation in 1 case.The spleen was absent in all but 2 patients with a right-sided spleen, and 1 with left-sided multiple spleens, all having right bronchial isomerism. The liver was midline in 18/24 patients (75\%).

We identified left isomerism (LI) in 15 patients (4 males). An AVSD with atrial and ventricular shunting was found in 8 cases (53\%), and with only atrial shunting in 3 cases (20\%). In addition, we observed 2 partial APVC (one associated to subaortic ventricular septal defect), 1 muscular defect and 1 supra-aortic stenosis. Right outflow obstruction was present in only $3 / 15$ cases (20\%). The heart was right-sided in 4 cases (27\%). A right-sided ICV was noted in 1 case, while ICV was interrupted with azygos continuation in $10 / 15$ cases (67\%), and with hemiazygos continuation in 4 cases. Multiple left-sided spleens were found in 11 patients, multiple right-sided spleens in 2, and solitary spleens to the right and left each in 1 patient.All had left bronchial isomerism. The liver was midline in 11 of the patients (73\%).

Conclusions: As expected, the majority of patients with RI had AVSDs guarded by a common valve, often with right outflow obstruction, while AVSD was less frequent in LI. Right-sided hearts were found in both subsets. Splenic morphology was not consistently associated with either form. These unexpected arrangements of the organs can be associated with equally unusual cardiac anomalies. The key is to analyse each system in its own rights.

\section{P-277}

Cardiotoxicity in Acute Lymphoblastic Leukaemia Long-term follow-up in a group of paediatric patients Pires M. (1), Francisco A. (2), Brito J. (3), Pires A. (1, 2)

Faculdade de Medicina, Universidade de Coimbra, Portugal (1); Serviço de Cardiologia Pediátrica, Centro Hospitalar e Universitário de Coimbra, Portugal (2); Serviço de Oncologia Pediátrica, Centro Hospitalar e Universitário de Coimbra, Portugal (3)

Introduction: The use of anthracyclines in the treatment of acute lymphoblastic leukaemia (ALL) is limited by its cardiotoxic effects. Anthracycline-induced cardiotoxicity in long-term survivors of childhood cancer is well known. The aim of this study was to evaluate the effects of anthracycline therapy on cardiac function in a group of children with high risk LLA.

Methods: The authors carried out a retrospective echocardiographic analysis in a group of 40 patients with high risk ALL aged from 1 to 17 years over a period of 10 years (2007-2017) treated with at least $250 \mathrm{mg} / \mathrm{m}^{2}$ of a cumulative anthracycline dose. The parameters used were: fraction shortening (FS), ejection fraction (EF) by the Simpson biplane method and the tricuspid annular plane systolic excursion (TAPSE). The echocardiographic evaluation was carried out at diagnosis, 3, 6 and 12 months after the onset of chemotharapy and then yearly.

Results: Twenty five patients $(62,5 \%)$ were male. The cardiovascular risk factors identified prior to therapy were: arterial hypertension (5); type 1 diabetes mellitus (2); dyslipidaemia (3) and chronic kidney disease (2). None underwent mediastinal radiotherapy. The sample was divided into 3 groups according to their age at diagnosis and the beginning of chemotherapy: $0-5$ years $(n=11), 6-11(n=15)$ and $12-18(n=14)$. The ALL cell lineage was evenly distributed (1:1 ratio between $\mathrm{B}$ and $\mathrm{T}$ cell lineage) and 2 patients had the Philadelphia chromosome. 
The fraction shortening after the first year of treatment was lower in the female group $(n=29 ; \mathrm{p}=0,039)$ as was the ejection fraction in the 6-11 and $12-18$ years age groups when compared to the $0-5$ years age group $(n=32 ; p=0,035)$. We also found that overall the fraction shortening $(n=15 ; \mathrm{p}=0,019)$ and TAPSE $(\mathrm{n}=20$; $\mathrm{p}=0,003$ ) were lower during the first year of treatment.

Conclusion: Based on our results, systolic dysfunction is most likely to occur during the first year of anthracycline treatment and is most prominent in girls and older children. This highlights the importance of a long-term follow-up in paediatric cancer survivors treated with anthracyclines in order to diagnose potential cardiovascular toxicity.

\section{P-278}

\section{The Association Of Birth Weight With Cardiovascular Risk Factors In Early Childhood}

Dogan M.T.

Department of Pediatric Cardiology, Ankara University Medical School, Ankara, Turkey

Introduction: Cardiovascular disorders are one of the leading causes of mortality and morbidity in the modern World. Since cardiovascular disorders are initially subclinical and it takes a long time for the symptoms of the disease to become apparent. Therefore it is important to point out the predisposing factors and early subclinical findings to improve the outcome. In adult studies, mortality due to cardiovascular diseases was high in low and high birth weight. Prevention of cardiovascular risks would be an ideal method for the future well-being of people .The aim of this study was to determine the effect of birth weight on cardiovascular disease and body composition in early childhood.

Methods: This study was carried out prospectively in healthy children admitted to Pediatric Department of Cumhuriyet University between January 2012 and January 2013. The study population comprised of early childhood aged $2-5$ years, that is a term AGA children $(n=22)$, term SGA children $(n=22)$, term macrosomic children $(\mathrm{n}=22)$. Anthropometric measurements including height,weight, skinfold thicknesses, waist circumference, and head circumference. The plasma glucose, insulin levels, serum total

Table .

\begin{tabular}{|c|c|c|c|c|}
\hline & $\begin{array}{l}\text { AGA infants } \\
(\mathrm{n}=22)\end{array}$ & $\begin{array}{l}\text { SGA infants } \\
(\mathbf{n}=22)\end{array}$ & $\begin{array}{l}\text { Macrosomic } \\
\text { infants }(n=22)\end{array}$ & $\mathbf{P}$ \\
\hline Birth weight, kg, median (range) & $3.4(3.2-3.5)$ & $2.3(2.3-2.4)$ & $4.4(4.2-4.6)$ & 0.001 \\
\hline $\begin{array}{l}\text { Length at birth, } \mathrm{cm} \text {, median } \\
\text { (range) }\end{array}$ & $50.2 \pm 1.2$ & $48.3 \pm 2.4$ & $52.2 \pm 1.4$ & 0.04 \\
\hline $\begin{array}{l}\text { Head circumference at birth,cm, } \\
\text { median (range) }\end{array}$ & $34.8 \pm 1.7$ & $31.5 \pm 2.4$ & $35.4 \pm 1.8$ & 0.01 \\
\hline Weight, kg, median (range) & $17(16-18)$ & $16(15-17)$ & $19(18-20)$ & 0.01 \\
\hline Height, $\mathrm{cm}$, median (range) & $106(102-109)$ & $102(98-105)$ & $106(102-110)$ & 0.1 \\
\hline $\begin{array}{l}\text { Head circumference, } \mathrm{cm} \text {, median } \\
\text { (range) }\end{array}$ & $50(49-51)$ & $50(49-51)$ & $50(49-51)$ & 0.6 \\
\hline BMI $\left(\mathrm{kg} / \mathrm{m}^{2}\right)$, median (range) & $16(15-17)$ & $15(15-16)$ & $17(16-18)$ & 0.05 \\
\hline $\begin{array}{l}\text { Triceps skinfold thickness, median } \\
\text { (range) }\end{array}$ & $11(10-13)$ & $11(10-12)$ & $11(10-13)$ & 0.9 \\
\hline $\begin{array}{l}\text { Fasting glucose, mg/dL, median } \\
\text { (range) }\end{array}$ & $80(75-85)$ & $85(80-91)$ & $87(82-92)$ & 0.04 \\
\hline Insulin, $\mathrm{mIU} / \mathrm{mL}$, median (range) & $5.3(3.2-7.3)$ & $7(3.7-10.0)$ & $4.5(3.2-5.9)$ & 0.02 \\
\hline $\begin{array}{l}\text { Total cholesterol, mg/dl, median } \\
\text { (range) }\end{array}$ & $123(106-140)$ & $112(99-125)$ & $110(94-124)$ & 0.6 \\
\hline $\begin{array}{l}\text { Low-density lipoprotein } \\
\text { cholesterol, mg/dl, median } \\
\text { (range) }\end{array}$ & $67(56-78)$ & $43(33-55)$ & $40(33-47)$ & 0.01 \\
\hline $\begin{array}{l}\text { High-density } \\
\text { lipoproteincholesterol, } \\
\text { mg/dl, median (range) }\end{array}$ & $33(29-38)$ & $33.2(29-38)$ & $30(23-36)$ & 0.3 \\
\hline $\begin{array}{l}\text { Triglycerides, mg/dl, median } \\
\text { (range) }\end{array}$ & $82(68-97)$ & $80(55-105)$ & $94(71-117)$ & 0.4 \\
\hline $\begin{array}{l}\text { HOMA-IR index, median } \\
\text { (range) }\end{array}$ & $1.02(0.5-3.1)$ & $1.5(0.7-2.4)$ & $1.01(0.6-5.1)$ & 0.02 \\
\hline
\end{tabular}

cholesterol, LDL, HDL, and triglyceride concentrations were measured.

Results: The children in SGA group had significantly lower weight and body mass index compared with those in AGA group respectively and to those in macrosomic group respectively. The fasting glucose levels were significantly higher in SGA group and macrosomic group respectively than in AGA group. The children in SGA group had significantly higher insulin levels and HOMA-IR index when compared to the children in AGA and macrosomic groups. Total cholesterol, HDL cholesterol, triglycerides, systolic and diastolic blood pressure were comparable among the study groups.

Conclusion: Our findings indicated that although BMI was significantly higher for macrosomic births when compared to term AGA children, anthropometric measurements were not related to insulin resistance and metabolic syndrome. However, children in small for gestational age group were shown to develop insulin resistance in early childhood. Further studies are needed in larger cohorts of children to confirm whether or not there is a relationship between birth weight and anthropometric measurements and the cardiovascular risk.

\section{P-282}

Pilot Study of Pulse Oximetry Screening Test in Azerbaijan

Petropoulos A.C (1,2), Behbudov V. (1), Khudiyeva A. (1), Mustafayeva G. (1), Seyidov N. (3,4)

Merkezi Klinika, Baku Azerbaijan (1); Dep. Of Pediatrics Azerbaijan Medical State University (2); Public Health and Reforms Center of MoH of Azerbaijan (3); Mediclub hospital Baku Azerbaijan (4)

Introduction: Preliminary studies show that Azerbaijan has a higher incidence of Congenital Heart Disease (CHD), then the average world prevalence of $\sim 1 \%$. Pulse oximetry screening (pox.scr.) test has proven its efficiency as cheap, easy applicable, non-invasive screening test in early detection of critical CHD (c-CHD).

Aim: Prospective, double blind study, proves combination of pox.scr, medical history + physical examination, by neonatologists and hyperoxygenation test (when indicated) offers high early detected c-CHD. Population-Method: Ministry of Health, designated two public maternity hospitals in which 1670 term neonates $(1.05 \%$ healthy deliveries/year), 959 boys and 801 girls, born by SVD from June 2016 to May 2017, were studied with Pox.scr., medical history + physical examination by a neonatologist at 37 and 85 hours postdelivery. Simultaneous measurement of pox.scr. using highquality devices, both pre/post DA, were performed. A SatO2 =/ $>95 \%$ and difference of less of $3 \%$ between the measurements was accepted as normal. All failing pox.scr. had echo-2D study, establishing diagnosis. Hyperoxygenation test under indications was done. Blind part involved neonatologists/cardiologists, as they did not know about findings of the other team. All cohort had echo-2D scan on day 12 from birth.

Results: 37 hours post birth, pox.scr, detected17c-CHD. Additionally, 38 failures, were proved suffering from variety of respiratory disease (16) and early onset sepsis (22). Sensitivity of $68.2 \%$ and specificity of $50.2 \%$, was found. When adjusted to clinical assessment and indicated hyperoxygenation test, these, rose to $86.3 \%$ and $82.6 \%$ respectively. Pox.scr. applied at 85 hours of age detected additional 4 c-CHD. Estimated sensitivity was $83.9 \%$ and specificity $90.2 \%$. Adjusted to clinical assessment, indexes rose to $94.5 \%$ and $99.1 \%$ respectively. At the 12th day of life, echo-2D scan was applied to cohort. One more c-CHD as well as 30/1670 detected simple CHD's were found, estimating incidence of CHD of $3.11 \% / 40.38 \%$ c-CHD. 
Conclusions: This pilot study, proved combination of pox.scr. and neonatologist examination after the $84 \mathrm{~h}$ from birth, increasing significant early detection of c-CHD including a large proportion of critical left heart obstructive CHD's. Additional sever pathology was also detected. In a country with limited surgical capacity, mandatory use of this combination is beneficial.

\section{P-283}

Impact of Prenatal and Early Postnatal Diagnosis of Critical Congenital Heart Defects in Romanian Newborns Nicolae G. (1), Nicolescu A. (1), Diaconu A. (2), Cinteza E. (1,2), Duica G. (1), Safta G. (1), Cirstoveanu C. (1,2), Filip C. (1) M.S. Curie Emmergency Children Hospital, Bucharest, Romania (1). Carol Davila University of Medicine and Pharmacy, Bucharest, Romania (2)

Introduction: Congenital heart defects are the most common form of congenital anomaly, with a $0.8 \%$ incidence of all live births. In the case of critical malformations, prognosis and outcomes are significantly influenced by the time of diagnosis and proper postnatal management.

Material and Method: A prospective study was conducted at M.S curie Hospital, Bucharest, between January 2011 and December 2016, on 205 newborns with critical CHD (congenital heart disease). We aimed to identify the impact of prenatal and early postnatal diagnosis in our country's socio-economic context.

Results: Of the 205 newborns, only 61 pts $(29,7 \%)$ had prenatal diagnosis while the other 144 pts $(70,2 \%)$ were diagnosed postnatally. It was found that scheduled births were more frequent in the prenatally diagnosed group (82\%) compared with postnatally diagnosed group (38\%); invasive preoperative respiratory support was needed more frequent in the postnatally diagnosed group $(75 \%)$ versus the prenatally diagnosed group (48\%). A greater number of pts requiring preoperatory antibiotic treatment for confirmed infections was also identified in the postnatally diagnosed group (88\%) versus $(60.6 \%)$ in the prenatally one. The median age at the time of surgery was 14 days for newborns with prenatal diagnosis and 25 days for those with postnatal diagnosis. Overall mortality was significantly lower for the prenatally diagnosed group (7.1\%) compared to the postnatally diagnosed group $(14.7 \%)$. The increased mortality in the second group was due to the higher mortality rate of the late diagnosed patients (at more than 72 hours after birth or even after discharging from maternity) going up to $40 \%$ in those diagnosed after discharge from maternity.

Conclusions: In the socio-economic context of our country, prenatal or early postnatal diagnosis in the first 48-72 hours of life provide similar (comparable) prognosis, while late diagnosis after more than 72 hours leads to a significant increase in mortality. The increase in mortality in this subgroup is due to the increased preoperative morbidity, to the delay of surgical moment secondary to late diagnosis, to the difficulty of finding a transport solution and a specialized center for properly resolution of cases.

\section{P-284}

Pulse oximetry screening in the asymptomatic newborns Chaudhry S.H., Singh Y.

Cambridge University Hospitals, UK

Background: The current screening tools (Fetal anomaly screening and routine neonatal examination) fail to detect up to $39-50 \%$ of critical congenital heart defects (CHDs). However, the addition of pulse oximetry screening (POS) as an adjunct may detect up to $92 \%$ critical CHDs.
Aims and Objective: Retrospective audit with the following two outcomes:

1) to assess the uptake of the pulse oximetry screening on the postnatal ward;

2) to review the efficacy of the pulse oximetry screening in detecting CHDs and other non-cardiac conditions such as sepsis.

Study design and Methods: A retrospective observational study involved all eligible infants for POS and born between September 2015 to April 2016. Infants already admitted to NICU and already symptomatic infants were excluded.

Results: A total of 3,646 eligible infants for POS were identified from the patient records and uptake was $98 \%(3,574$ of 3,646 babies). In around two-third infants screening was performed within 4-8 hours as recommended by the local guideline.

64 infants $(1.7 \%)$ were noted to have abnormal POS results (POS positive results). 3 babies were diagnosed with CHD. The defects were as follows:1) small ASD, 2) Critical pulmonary stenosis with right heart, 3) AVSD with mild TR. 2 babies had minor findings like PFO on echocardiography and 1 was antenatal diagnosis of AVSD. Echo was performed in 9 out of 64 (14\%).

The remaining 33 of 64 (51\%) babies had significant non-cardiac pathologies such as sepsis (11), suspected sepsis (5), RDS (5), feeding issues (8) and jaundice (4). 25 of the 64 (39\%) babies had no significant cardiac or non-cardiac pathologies (false positive). Conclusion: When pulse oximetry screening is performed early it has slightly higher false positive rate as compared to the reported studies. However, it helps in detecting other non-cardiac conditions in otherwise well infants. In our cohort, it did not put any extra burden on echocardiography services.

\section{P-285}

Assessment of cardiac functions, aortic stiffness and heart rate variability parameters in children with Attention Deficit Hyperactivity Disorder

Gürses D. (1), Kızılkaya A. (1), Ünlü G. (2), Büber A. (2)

Pamukkale University Faculty of Medicine, Department of Pediatric

Cardiology (1) and Department of Child and Adolescent Psychiatry (2), Denizli, TURKEY

Introduction: Attention deficit hyperactivity disorder (ADHD) is characterized by decreased attention span, impulsiveness, and hyperactivity. Cardiovascular involvement can be seen secondary to autonomic dysfunction. This study is conducted in order to assess possible cardiac involvement in children with ADHD and whether we investigate differences in ADHD subtypes or not. Methods: In this cross-sectional study, we evaluated 73 children with ADHD (14 with hyperactivity, 25 with inattentive, and 35 with combined type), and 37 healthy children as controls. Cardiac evaluation was made using an electrocardiogram, conventional and tissue Doppler echocardiography and ambulatory holter monitoring. Results: Systolic blood pressure and mean heart rate increased in ADHD patients $(\mathrm{p}<0.05)$. QT and QTc dispersion, which show ventricular repolarization homogeneity were similar in the groups $(\mathrm{p}>0.05)$. Left ventricle Sdm, Edm/Adm and interventricular septum Edivs/Adivs decreased by the measurements made by tissue Doppler echocardiography in ADHD patients $(p<0.05)$. Aortic stiffness parameters were found similar in the two groups $(p>0.05)$. SDNN, rMSSD and pNN50 decreased in the children with ADHD ( $\mathrm{p}<0.01)$. As compared within ADHD subtypes; heart rate was higher, pNN50 and RMSSD were lower in combined subtype than the other subtypes $(\mathrm{p}<0.01)$.

Conclusions: Our findings showed cardiac involvement can exist in ADHD patients as secondary to autonomic dysfunction. This involvement was higher in combined subtype. Children with 
ADHD were at risk for cardiovascular complication. Before treatment, assessing all children with ADHD, especially combined subtype using with ambulatory Holter monitoring and tissue Doppler echocardiography in addition to detailed physical examination and electrocardiography might be helpful to detect cardiac complication in preclinical stage of the cardiac involvement.

\section{P-286 \\ Time Domain Heart Rate Variability Analysis In Familial Mediterranean Fever Patients \\ Gürses D. (1), Yüksel S. (2), Yildırm-Arslan S. (1) \\ Pamukkale University Faculty of Medicine, Department of Pediatric \\ Cardiology (1) and Pediatric Nephrology (2), Denizli, TURKEY}

Introduction: Familial Mediterranean Fever (FMF) is an autosomal recessive autoinflammatory disease, characterized with recurrent fever, abdominal pain, chest pain and joint involvement. Cardiovascular involvement can be seen secondary to chronic inflammation. This severe outcome is a major cause of morbidity and mortality in FMF patients. There are a few studies investigating cardiovascular involvement in FMF patients. Heart rate variability (HRV) is a non-invasive index of neuronal modulation of heart rate. In this study, autonomic functions of heart in preclinical stage of heart disease were evaluated in FMF patients with time domain heart rate variability analysis.

Methods: The study population included 66 patients with FMF during non-attack period and 40 healthy children as controls. Patients and controls underwent 24-hour ambulatory Holter monitoring.

Results: There was no significant differences between the two groups in age, height, sex, body mass index, arterial blood pressure ( $\mathrm{p}>0.05)$. QT and QTc dispersion s, which shows ventricular repolarization homogeneity were similar in the two groups $(\mathrm{p}>0.05)$. Mean RR time, minimum and maximum heart rate, SDNN and SDANN were similar in the two groups $(p>0.05)$. SDNN-i, RMSSD and pNN50 significantly decreased in the FMF patients $(\mathrm{p}<0.05)$. In the FMF patients, SDNN-i, RMSSD and pNN50 were found $85.521 .2 \mathrm{~ms}, 62.828 .3 \mathrm{~ms}$, and 24.8 $12.3 \%$ respectively. In FMF patients with and without M694V mutation, time domain HRV parameters were found as similar $(\mathrm{p}>0.05)$.

Conclusion: Our findings show cardiac involvement can exist in FMF patients even during non-attack period. The analysis of heart rate variability might be helpful to detect cardiac complications in preclinical stage of the cardiac involvement.

\section{P-287 \\ Breastfeeding and vitamin $D$ supplementation reduce the risk of Kawasaki disease in a German population-based case-control study \\ Meyer K. (1), Volkmann A. (2), Hufnagel M. (3), Schachinger E. (1), Horstmann J. (1), Stiller B. (1), Jakob A. (4) \\ Department of Congenital Heart Disease and Pediatric Cardiology, University Heart Center Freiburg, Freiburg, Germany (1); Institute for Medical Information Processing, Biometry and Epidemiology, Ludwig- Maximilians-University of Munich, Munich, Germany (2); Division of Pediatric Infectious Disease and Rheumatology, Center for Pediatrics and Adolescent Medicine, University Medical Center Freiburg, Freiburg, Germany (3); Department of Pediatric Cardiology, Ludwig- Maximilians-University of Munich, Munich, Germany (4)}

Objectives: Kawasaki disease (KD), a vasculitis of unknown etiology, is in some cases complicated by the development of coronary artery aneurysm (CAA). To date, the exact pathomechanism of $\mathrm{KD}$ is unknown, both environmental and genetic factors seem to be associated with the development of the disease.

Methods: Data on $308 \mathrm{KD}$ patients, recruited from the active population-based German Pediatric Surveillance Study in the years 2012 - 2014, were used to evaluate the impact of different perinatal factors on KD. The design was a matched case-control study with regard of age, sex and place of residence $(n=326$ controls). All KD patients were evaluated and fulfilled the international diagnostic KD criteria. A standardized questionnaire was used to review breastfeeding practices, vitamin D supplementation and birth characteristics. Logistic regression analyses were used to obtain odds ratios (OR) for the case-control pairs. Simple dependency analyses were performed to test the impact of the factors on the clinical course.

Results: There was no difference in birth weight or parturition between the KD patients and the controls, but vitamin D supplementation and breastfeeding were independently negatively associated with KD, even when adjusted for age, place of residence and sex. The duration of vitamin $\mathrm{D}$ was significantly less in children with $\mathrm{KD}$ than in children without $\mathrm{KD}(\mathrm{p}=0,039$, $\mathrm{OR}=0,964,95 \%$ CI: $0,931-0,998)$ and the duration of breastfeeding was significantly shorter in patients with KD than in the controls ( $p=0,013, \mathrm{OR}=0,471,95 \% \mathrm{CI}$ : 0,260 - 0,853). However, we were not able to detect any influence of either vitamin D supplementation or breastfeeding on the course of disease. Developing CAA in the acute phase or in long term (one year after disease onset), as well as being refractory to intravenous immunoglobulin treatment and the level of inflammatory lab values, did not differ between KD patients with and without breastfeeding/ vitamin D supplementation.

Conclusion: Our results indicate protective effects of breastfeeding and vitamin D supplementation on the development of KD in our study population, but they do not influence the natural course of the disease. Although the effects were small, it underlines the overall benefit of both interventions.

\section{P-288}

Determinant factors of physical fitness in children with congenital heart disease

Zaqout M. (1,2), Vandekerckhove K. (1), Bové T. (3), François K. (3), De Henauw S. (2), De Wolf D. (1), Michels N. (2)

1. Ghent University Hospital, Pediatric Cardiology Department, Ghent, Belgium; 2. Ghent University, Public Health Department, Ghent, Belgium; 3. Ghent University Hospital, Cardiac Surgery Department, Ghent, Belgium

Introduction: Advances in the treatment of children with congenital heart disease (CHD) have resulted in notable improvements in survival and life expectancy. Thus, attention has shifted from mortality and cardiac morbidity to long-term cardiovascular and psychosocial health, which are best predicted by physical fitness.

Objective: To determine factors associated with physical fitness in children who underwent surgery for CHD.

Methods: Sixty-six children (6-14y) who underwent surgery for ventricular septal defect $(n=19)$, coarctation of aorta $(n=10)$, tetralogy of fallot $(n=15)$ and transposition of great arteries $(\mathrm{n}=22)$ were included. All children performed physical fitness tests: cardio-respiratory fitness, upper and lower-limb muscular strength, balance, flexibility and speed. Cardiac evaluation was done via echocardiography and cardio-pulmonary exercise test. Determinant factors related to child's characteristics (sex, age, body mass index), child's lifestyle (breakfast habit, fruit and vegetable consumption, dietary fat and sugar propensity, screen time, 
physical activity, quality-of-life), physical activity motivators/ barriers and parental factors (socio-economic status, body mass index) were obtained. Multivariate (six fitness components together as outcome) and linear regression analyses were conducted, adjusted for CHD lesion type.

Results: No differences in physical fitness performance nor in any of the tested determinant factors were observed according to CHD lesion type. Age and sex were the only factors associated with physical fitness in children with repaired CHD (partial èta squared $=0.797, p<0.001 ;$ partial èta squared $=0.567, p=0.008$ ) respectively. Older children performed better than younger in muscular strength and balance and less in cardio-respiratory fitness. Sex and motivations showed an association with some physical fitness components. Boys had better cardio-respiratory fitness $(p=0.007)$, whereas girls were better in flexibility $(p=0.017)$ and balance $(\mathrm{p}=0.041)$. Age, sex and motivators together reached an adjusted $\mathrm{R}^{2} 0.444$ for speed, 0.484 for lower muscular strength, 0.707 for upper muscular strength, 0.021 for flexibility, 0.149 for balance and 0.382 for CRF. Adding other possible determinants did not significantly increase the adjusted $\mathrm{R}^{2}$.

Conclusions: Children with different repaired CHD lesions did not differ in physical fitness or activity. Apart from age and sex as non-modifiable determinants, motivation for physical activity seems the only relevant target to increase fitness in this patient group.

\section{P-289}

Vitamin D Deficiency Cardiomyopathy: A National Experience

Ferguson R., Hunter L, McDevitt $H$.

NHS Greater Glasgow \& Clyde, Scotland, UK

Introduction: Dilated cardiomyopathy (DCM) is the leading cause of heart transplantation in children over the age of five years. Idiopathic DCM remains the most common aetiology but Vitamin D deficiency DCM is reported in the literature.

Methods: A retrospective review of consecutive cases of vitamin D deficiency DCM presenting to a national congenital cardiac centre between January 1st 2000 and August 31st 2017. Patient demographics; maternal vitamin D status; echocardiographic parameters; x-rays; serum biochemistry and length of hospital stay were reviewed.

Results: Six patients were identified during the study period (three male), three of whom were Caucasian. Median age at presentation was 210 days (range 2, 302.) All six patients had high serum parathyroid hormone levels (median 45pmol/l, range 27, 120), a sensitive marker of total body calcium deprivation secondary to vitamin D deficiency. All patients demonstrated clinical and echocardiographic improvement following high dose vitamin D treatment. One patient failed to comply with medical management and died five months after initial diagnosis.

Conclusion: Vitamin D supplementation is recommended for pregnant women and children under the age of five years. Although vitamin D deficiency DCM is relatively rare, it is wholly preventable. In Northern Europe children remain at risk of vitamin $\mathrm{D}$ deficiency secondary to inadequate exposure to sunlight, particularly during the winter months. In addition, social deprivation and maternal vitamin D deficiency are important risk factors for vitamin D deficiency DCM in the paediatric population. Prophylactic vitamin D supplementation may obviate future morbidity and mortality and improve clinical cost effectiveness.
P-290

Is infective endocarditis of any concern in pediatric Marfan patients?

Olfe J., Stark V., Kozlik-Feldmann R., Mueller G.C., Pesch J., Mir T.S.

Clinic for Pediatric Cardiology, University Heart Center, Hamburg, Germany

Introduction: In historical data there has been concern about a higher risk of infective endocarditis in Marfan patients, especially in those with mitral valve prolapse (Mark H, 1974). Patients with congenital heart disease are known to have a higher risk for endocarditis. Still, current adult guidelines do not suggest antibiotic prophylaxis in Marfan patients (Habib G, 2015). So far there have been no data concerning the risk of endocarditis in a pediatric Marfan population.

Methods: Since 2008 we investigated 424 patients with suspected MFS. 149 patients were diagnosed with MFS $(9.5 \pm 5.8 \mathrm{y}, 62 \%$ male). We retrospectively analyzed our databank looking at the incidence of infective endocarditis. Subgroup analysis concerning risk factors like mitral valve prolapse, tricuspid valve prolapse, aortic insufficiency and bicuspid aortic valve were analyzed. Patients being operated on the aortic arch or any heart valve were excluded.

Results: In our cohort we have not seen a single case of endocarditis over 978.5 patient years. Risk factors like mitral valve prolapse $(40,9 \%)$, tricuspid valve prolapse $(30,2 \%)$, aortic insufficiency $(6,7 \%)$, bicuspid aortic valve $(12,7 \%)$ had no effect on the incidence of infective endocarditis.

Conclusions: In our cohort of pediatric Marfan patients we have not experienced a single case of infective endocarditis over a time period of 978,5 patient years. Even patients with known risk factors were not affected. These early data from our single centre study suggest that the recommendations to not apply endocarditis prophylaxis in pediatric Marfan patients seem appropriate. Nationwide registry based data could help to elucidate this question.

Table: Demographic data and incidence of infective endocarditis in pediatric Marfan patients

\begin{tabular}{lcrl} 
Demographic data & $\mathbf{n}$ & & \\
\hline Patients & 149 & & \\
Age (years) at first visit & 9,5 & $\mathbf{\pm} .8$ & \\
Total patient years & 978,5 & & \\
\hline & $\mathrm{n}$ & $\%$ & Endocarditis \\
\hline Mitral valve prolapse & 61 & 40,9 & 0 \\
Tricuspid valve prolapse & 45 & 30,2 & 0 \\
Aortic insufficiency & 10 & 6,7 & 0 \\
Bicuspid aortic valve & 19 & 12,7 & 0 \\
Total & & & 0 \\
\hline
\end{tabular}

P-291

Particularities in Diagnosis and Treatment for Infectious Endocarditis at Children in North Eastern of Romania 11 Years Study

Iordache C. (1), Luca A.C. (2)

"Sfanta Maria" Children's Emergency Hospital of Iasi, Romania (1),

"Gr.T.Popa" University of Medicine and Pharmacy, Paediatric

Cardiology Department, Iasi, Romania (2)

Introduction: Infectious endocarditis represents a rare pathology in children, but with lethal potential. The goal of the therapy is fast 
and total eradication of the infection. The purpose of this study is to evaluate the particularities in diagnosis and treatment for infectious endocarditis in children.

Methods: Were taken in study 43 children with infectious endocarditis hospitalized between January 2006 - January 2017 in the Cardiology Department of the „Sfanta Maria” Children Emergency Hospital of Iasi, Romania.

Results: The patients are aged between 23 days and 16 years, the average age being 7.8 years. At approximately $86.04 \%$ of the patients, the endocardial damage appeared in the pre-existent valvular lesions, specially mitral and aortic. As associated congenital malformations, the patients prevailingly presented ventricular septal defect, mitral valve prolapse, arterial canal persistence, aortic stenosis, coarctation of the aorta, aortic prosthesis. Blood cultures were collected and the most frequent identified etiological agents were: Staphylococcus coagulase-positive, Streptococcus mitis, Staphylococcus speciae coagulase-negative, Staphylococcus haemolyticus, Streptococcus bovis, Escherichia coli, for which the antibiogram showed sensitivity for beta-lactam, cephalosporins, glycopeptides, trimethoprim-sulfamethoxazole, rifampicin, quinolone, lincosamides, oxazolidinones, and thus specific treatment was set up according to the antibiogram. The echocardiografic reevaluation as well as the cardiologic consultation in dynamics allowed an adequate and permanent monitoring of the therapeutic behavior. The most frequent complications were: pericarditis $(30.23 \%)$, congestive heart failure $(65.11 \%)$, trombembolism (18.6\%) and stroke syndrome (6.97\%). 34 of the patients evolved favorably, in 6 cases $(13.95 \%$ of the patients) it has been surgically intervened with an as complete as possible resection of the vegetations, restoring of valvular and ventricular function and correction of the risk factor (subjacent cardiopathy), and for 3 of the patients this problem was lethal.

Conclusions: The infectious endocarditis is a severe disease that affects young age too, leading towards exitus in some cases. Diagnostic imaging and early blood cultures are of relevance in order to intervene promptly. The treatment must be targeted and applied as fast as possible.

\section{P-292 \\ Incidence and characteristics of Kawasaki Disease in Northwestern Greece \\ Vlahos A. P., Makis A.C., Tsitsari I., Chaliasos N. \\ Child Health Department, School of Medicine, University of Ioannina, Ioannina, Greece}

Introduction: Kawasaki Disease (KD) is a vasculitis with potentially significant cardiovascular complications. Its incidence in Europe is $5-15 / 100.000 /$ year. The creation of KD registry is important for each country's health system. The aim of the study is the tracing and analysis of KD characteristics in Northwestern Greece.

Methods: A medical record review study was performed in a tertiary University Hospital that consists the referral center for Northwestern Greece.

Results: There were 13 children hospitalized (9 males) with an incidence of 3,6/100.000/year, with $<1$ year: $5,1-5$ years: 7 and $>5$ years: 1 child. All of them had a history of fever of at least 5 days duration, 12 had conjunctivitis, 10 cheilitis, 4 glossitis, 12 cervical lympadenopathy, 12 a rash, 8 edema of the extremities and 8 desquamation of fingers and toes. There was 1 child with incomplete KD. There was leukocytosis $(11.490-40.530 / \mu \mathrm{l})$, anemia $(\mathrm{Hb} 7,7-10,3 \mathrm{~g} / \mathrm{dl})$, thrombocytosis (470.000-1.678.000/ $\mu \mathrm{l})$ and increased inflammation indices in all patients. 4 had hepatic involvement and 5 had aseptic pyuria. 12 had an abnormal echocardiography with ectasias in either right or left coronary artery, 4 of whom in both arteries and 3 had a pericardial effusion. All patients received IVIG and aspirin. 2 patients required more than one IVIG infusion due to persistence of fever and 1 patient received corticosteroids. One infant had severe KD and died despite the addition of anticoagulation treatment. All other patients demonstrated clinical improvement.

Conclusions: The incidence of KD in Northwestern Greece is somewhat lower comparing with the European data recorded. The majority of children were males $<5$ years of age and fulfilled the clinical criteria of KD. Regional registries are as important as national in order to accurately record variations in incidence and clinical characteristics which will have implications in both public health and research.

\section{P-293}

Booster Immunization in children and adolescents with congenital heart disease in Germany

Wacker-Gussmann A. (1,2), Becker R. (1), Unterleitner C. (1),

Remmele J. (1), Reiner B. (1), Hock J. (1), Nagdyman N. 2), Ewert P. (2), Oberhoffer R. (1)

(1) Institute of Preventive Pediatrics, Faculty of Sport and Health Sciences, Munich, Germany. (2) German Heart Centre, Department of Pediatric Cardiology and Congenital Heart Defects, Munich, Germany

The first two authors equally contributed to the manuscript

Introduction: High risk patients like children and adolescents with congenital heart disease benefit most of the reduced infection rate caused by immunization. The question raises, if these patients are sufficiently immunized.

Patient and Methods: Children with congenital heart disease aged between 2 and 18 years were enrolled in a prospective study at the German Heart Center Munich, Germany. Immunization and medical status were received by a specific questionnaire and patient survey. Excel and SPPS. Statistics 20 were used for statistical analysis. Results were referred to the STIKO recommendations.

Results: Out of 657 enrolled children with a mean age of 9.8 (SD +/- 4.8) years, $179(27.2 \%)$ received the recommended basic immunization within the compulsory time frame. Vaccination rates for all recommended basic immunizations were over $80.0 \%$, except for Hepatitis B (75.7\%), meningococci $(74.3 \%)$, varicella (49.9\%) and pneumococci (37.7\%).Regarding booster vaccinations for patients with 16 years or above $(\mathrm{n}=90), 81(90.0 \%)$ were vaccinated against tetanus, $82(91.1 \%)$ against diphteria, $59(55.6 \%)$ against poliomyelitis and $77(85.6 \%)$ against pertussis within the recommended time frame. Out of these, 18 (20.0\%) had completed all recommended booster vaccinations.

Discussion: The immunization rate was moderate in patients with congenital heart disease. The rate of basic pneumococcal immunization was extremely low, as well as the rate of booster immunizations within the recommended time frame. Reasons were low patient advice, need of surgical intervention and concerns about vaccination. In addition, late basic immunizations led to late booster vaccinations.

Conclusion: Low booster and pneumococcal immunization rates within the recommended time frame revealed the essential elucidation concerning the need of vaccinations.

\section{P-294}

\section{Oral Health and Awareness in Adults with Congenital} Heart Disease

Wacker-Gussmann A. (1,2), Hollatz S. (1), Wilberg S. (3),

Folwaczny M. (3), Kaemmerer H. (1), Ewert P. (1), Oberhoffer R. (1) (1) Institute of Preventive Pediatrics, Faculty of Sport and Health Sciences, Munich, Germany; (2) German Heart Centre, Department of Pediatric 
Cardiology and Congenital Heart Defects, Munich, Germany; (3) Department of Conservative Dentistry and Periodontology, LudwigMaximilians-University, Munich, Germany

The first two authors equally contributed to the manuscript

Background: Patients with Congenital Heart Disease (CHD) are at high risk to develop aninfective endocarditis. Bacteria entering from the oral cavity can superinfect endocardial microlesions. Thus, oral health is essential to prevent the potentially lethal infective endocarditis. Data on the prevalence of oral health and patients' awareness of risk factors in adults with CHD is limited and therefore the aim of the study.

Methods: Adults with CHD were enrolled in a prospective observational study at the German Heart Center Munich, Germany. The patients were asked to complete a specific questionnaire. Additionally, a dentist performed an examination and documented the amount of decayed filled and missing teeth and the plaque-index as indicators for caries and quality of dental hygiene. The DMFT-Score (decayed + missing + filled) was calculated and the cardiological profile of the patient noted.

Results: The median age of the 112 participants was 31,5 (range 18-77) years. $38,4 \%$ did not know about the link between oral health and heart disease. $10,7 \%$ did not identify poor oral health as risk factor for cardiac complications.10,7\% felt anxious about dental treatment because of their CHD. In 20,5\% active caries was found. The mean DMFT-Score was 7,91 (+/- 6,54). No association between severity of CHD and DMFT-Score was shown $(p=0.45)$. The DMFT-Score was higher in patients requiring an antibiotic endocarditis prophylaxis $(p=0,001)$. These patients were also older $(\mathrm{p}=.045)$. Age and DMFT-score correlate positively $(\mathrm{p}=.000)$.

Conclusion: Awareness about the importance of an excellent oral health in ACHD must be improved. The effective prevention measures in dentistry can lower the prevalence of poor oral health.

\section{P-295}

Maternal smoking before and during pregnancy and congenital heart defects

Tzimou I. (1), Papadopoulou-Legbelou K. (2), Keivanidou A. (1), Emmanouil-Nikoloussi E.N. (3), Giannopoulos A. (1)

2nd Pediatric Department of Aristotle University of Thessaloniki, AHEPA University Hospital Thessaloniki, Greece (1); 4th Pediatric Department of Aristotle University of Thessalonii, Papageorgiou General Hospital, Thessaloniki, Greece (2); European University Cyprus, Nikosia, Cyprus (3)

Introduction/Aim: Maternal smoking before and during pregnancy has been associated with increased risk for congenital heart defects. The aim of this study was to examine the effect of maternal smoking before and during pregnancy to the frequency of congenital heart defect (CHD) and determine the risk for select CHD phenotypes.

Methods: In a retrospective case-control study, the association between congenital heart defects and maternal smoking was investigated. Data was attained from the archives of a Pediatric Cardiology unit during a 16-year period (2005-2015). An inperson interview was performed to the mothers of children with congenital heart defects and mothers of children with structurally normal heart matched by the year of delivery. Mothers reported smoking behavior during 5 time periods: 4-6 months before conception, 1-3 months before conception, first trimester of pregnancy, second and third trimester of pregnancy. The number of cigarettes was grouped in the following categories: $0,1-10$,
11-20, 21-39, $\geq 40$ cigarettes per day. A logistic regression model was used to control for confounding factors.

Results: Of the 10.537 children examined in the Pediatric Cardiology Unit during this time period, 989 were found with congenital heart defects. The most frequent congenital heart disease was Ventricular Septal Defect (376 cases, 38\%). It was also found a statistically significant positive association between maternal smoking (a month before conception and during the first trimester) with pulmonary valve stenosis (OR 1.34, 95\% CI 1.05-1.73) and atrial septal defects (OR 1.41, 95\% CI 1.04-1.79).

Conclusions: Maternal smoking before and during the first trimester of pregnancy is related to higher risk for atrial septal defects and pulmonary valve stenosis.

\section{P-297}

Characteristics of infective endocarditis in children and adolescents with congenital heart defects in Norway

Jortveit J. (1), Klcovansky J. (2), Eskedal L. (3), Birkeland S. (4), Døhlen G. (2), Holmstrom H. $(2,5)$

1. Department of Cardiology, Sorlandet Hospital, Arendal, Norway; 2. Division of Paediatric and Adolescent Medicine, Oslo University Hospital, Oslo, Norway; 3. Research Department, Serlandet Hospital, Kristiansand, Norway; 4. Department of Cardiothoracic Surgery, Oslo University Hospital, Oslo, Norway; 5. Institute of Clinical Medicine, University of Oslo, Oslo, Norway

Introduction: Norwegian health registers provide a unique opportunity to conduct nationwide population-based studies. The aims of this study were to investigate the occurrence of infective endocarditis (IE) according to the European Society of Cardiology criteria in children and adolescents with congenital heart defects CHDs) and to characterize their heart defects, previous treatments, diagnostic features, and outcomes of IE.

Methods: Data concerning all children with CHD and IE born in Norway between 1994 and 2016 were retrieved from the Oslo University Hospital's Clinical Registry for Congenital Heart Defects. Survivors were followed through 2016, and supplementary information was retrieved from medical records.

Results: The study includes all 1357543 live births in Norway between 1994 and 2016. The incidence of IE according to the European Society of Cardiology criteria was estimated to 2.2 per 10000 person-years $(n=36)$ among children and adolescents (0-18 years old) with CHDs. The mean follow-up time was 12.4 years $( \pm 5.5$ years) and the incidence was stable throughout the period. Twenty five $(75 \%)$ of the patients had severe CHDs and most had undergone open chest cardiac surgery or catheter-based cardiac interventions the last year before IE (fig 1). Thirteen (38\%) patients underwent cardiac surgery as a part of the IE treatment. Mechanical heart valves were implanted in two cases. Three patients died $(8 \%)$ of multiple organ failure related to IE.

Conclusions: In conclusion, the incidence of IE among children and adolescents with CHDs in Norway 1994-2016 was higher than the reported incidence in the general population. Despite the restrictions against antibiotic prophylaxis to the highest-risk patients in 2009, the incidence of IE was stable throughout the period. IE was associated with severe CHDs and recent complex cardiac interventions, and had significant mortality. In our view, it is still essential to underline the importance of adequate prevention, early detection, and rapid treatment of IE in many patients with CHDs. 


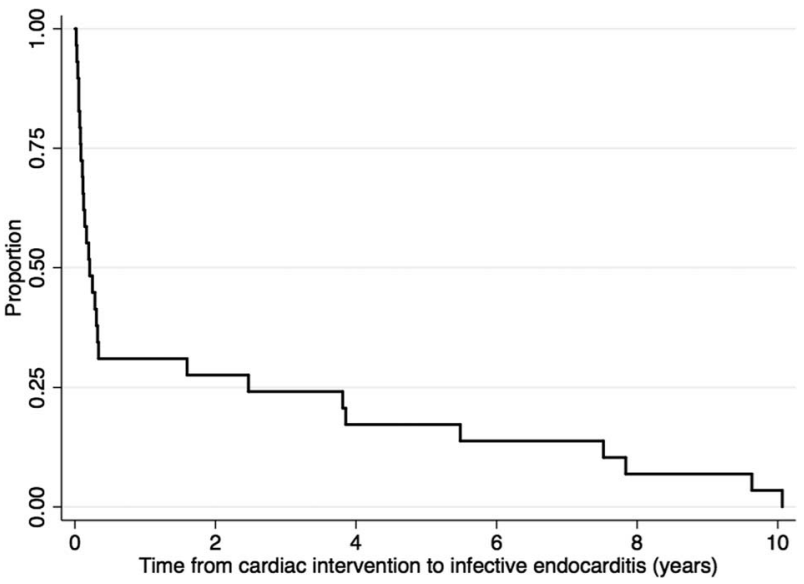

Figure.

\section{P-298}

Initiation of warfarin therapy in children: a new safe and practical clinical algorithm

Bajolle F. (1), Moreau C. (2), Neuraz A. (1), Moreau de Bellaing A. (1), Malekzadeh-Milani S. (1), Lasne D. (1), Bonnet D. (1) (1) Hôpital Necker-Enfants-Malades, Paris, France; (2) CHU Pontchaillou, Rennes, France

Purpose: Managing vitamin-K antagonist therapy is challenging in children. We developed a simple weight-adapted warfarin algorithm (target INR, 2.0-3.0) for starting warfarin therapy in children in order to reduce the frequency of over-anticoagulation and the time required to obtain the maintenance dose. To build this initiation warfarin regimen, we used a derivation cohort from 2009 to 2012. In this cohort, the loading dose was chosen according to the CHEST recommendation $(0.2 \mathrm{mg} / \mathrm{kg}$ whatever the age). The observation of this cohort allowed us to create an empirical regimen in a validation cohort from 2012. In this regimen, the initial fixed warfarin dose was weight-dependent according to three growth periods. The two cohorts are independent.

Methods: Sixty-one children (median age 7.1 years; from 0.1 to 17.9 years) were included in the derivation cohort. In our warfarin regimen, the induction dose was adapted to three weight subgroups: $<20 \mathrm{~kg} ; 20$ to $30 \mathrm{~kg}$ and $>30 \mathrm{~kg}$ with respectively 2,3 and $5 \mathrm{mg} /$ day during 2 consecutive days. The day 3 daily dose was predicted from the INR value obtained the day after the second intake. To validate this algorithm, we used prospectively this regimen in consecutively hospitalized children between 2012 and 2017. This validation cohort included 66 consecutively children (median age 5.6 years; from 0.3 to 18 years) who had a target INR of 2.0 to 3.0 .

Results: The time to achieve a therapeutic INR was 5 days in both cohorts (derivation, [3-8]; range, 3-24 days vs validation [4-6]; range, 3-11 days, $\mathrm{p}$ 0.82) but only 3 patients of the validation cohort had an INR $>5$ versus 7 in the derivation cohort. No major bleeding was recorded in this validation cohort. Finally, the time to achieve the maintenance dose was significantly shorter in the validation cohort than in the derivation cohort (11 days ([8-15]; range, 6-35 days) vs 14 days ([11-30]; range, 7-82 days), p 0.00012).

Conclusion: Our warfarin induction regimen is simple, practical, safe, and accurate in predicting the daily maintenance warfarin dose in children. It reduces the frequency of over-anticoagulation and the time required to achieve the maintenance dose.

\section{P-300}

Assessment of Pulse Oximetry Screening Trends in AEPC Jakab A. (1), Dalla Pozza R. (2), Ehringer-Schetitska D. (3), Fritsch P. (4), Oberhoffer R. (5), Petropoulos A. (6)

University of Szeged, Department of Pediatrics, Szeged, Hungary (1). Department of Pediatric Cardiology, Ludwig Maximilians-University of Munich, Munich, Germany (2). Dept. of Paediatrics, Landesklinikum Wiener Neustadt, Vienna, Austria (3), Dept. of Paediatric Cardiology, University Childrens Hospital, Graz, Austria (4); Institute of Preventive Paediatrics, Technical University of Munich, Munich, Germany (5); Merkezi Klinika, Baku, Azerbaijan (6)

Introduction: Critical congenital heart diseases (CCHD) are conditions in which neonates require surgery intervention in the first weeks of life to survive. Thus, early detection is an urgent clinical priority. Despite prenatal ultrasound examination and postnatal physical examination are performed for screening, a great number of cases remain undetected. Pulse oximetry screening has been adopted in several countries as an adjunct to postnatal examination in order to improve the detection rate and clinical outcomes.

Aim of the study: To assess current practice of screening methods for critical congenital heart diseases in Europe, mainly focusing on pulse oximetry screening trends.

Methods: An internet-based questionnaire was forwarded to the Ordinary and Junior Members of the Association for European Paediatric and Congenital Cardiology. Questions were focused on prenatal echocardiogram, the details of pulse oximetry screening practice and the providers' opinions regarding pulse oximetry implementation.

Results: Total 160 responses were received from 35 different European and 12 non-European countries. Foetal echocardiography is performed in almost all hospitals (90.6\%) mainly on selected foetuses with higher risk of developing congenital heart disease $(86.2 \%)$. More than half of the providers use pulse oximetry screening for CCHD (68.1\%), however, there is no consensus regarding oxygen saturation cut-off level and the timing of the measurement. The majority of responders (91.3\%) are interested in the implementation if a European guideline existed.

Conclusion: In Europe, pulse oximetry screening for CCHD has grown in its acceptance, yet clinical practice in its application may vary among countries and hospitals. Some providers are waiting for the general consensus to adopt pulse oximetry screening for $\mathrm{CCHD}$, which indicates the need for creating a universal European guideline.

\section{P-301}

Estimation of health-related quality of life in children and adolescents with congenital heart disease by subjective and objective method

Batinica M. (1), Dilber D. (2), Božikov J. (3), Matković H. (4), Melsa M. (4), Malčić I. (2)

University Children's Hospital Zagreb, Department of Pediatrics, Cardiology Unit, Zagreb, Croatia (1); University of Zagreb, School of Medicine and University Hospital Centre Zagreb, Department of Pediatric Cardiology, Zagreb, Croatia (2); University of Zagreb, School of Medicine, Andrija Stampar School of Public Health, Zagreb, Croatia (3); University of Zagreb, School of Medicine, Zagreb, Croatia (4)

Objectives: To compare perception of health-related quality of life (HRQOL) between patients with congenital heart disease (CHD) and their parents, and both with cardiopulmonary exercise testing (CPET) parameter - peak oxygen uptake (pVO2) - as the best represent of exercise capacity and cardiopulmonary fitness. 
Methods: A cross-sectional, single-centre, observational study was performed on 38 patients aged 8 to 18 years $(20 \mathrm{M}, 18 \mathrm{~F})$, with various $\mathrm{CHD}$, under routine follow-up. Previous Fontan procedure have had $13 \%$ of patients. Patients and their parents completed a HRQOL questionnaire - PCQLI ${ }^{\circledR}$. We analysed answers on the general health perception item, given at 5-point Likert scale, and subscales: Disease Impact (physical functioning) and Psychosocial Impact (psychological and social functioning). Afterwards, CPET with Bruce treadmill protocol was performed under same conditions, conducted by one of the authors. Descriptive and inferential statistics were used for data analysis, including calculation of Spearman's correlation coefficients between patients' and parents' HRQOL scores and for the comparison of HRQOL scores with pVO2.

Results: To describe the study group, mean $\mathrm{pVO} 2$ were compared with reference values of healthy pediatric population, local and from the literature; by Student's t-test, patients value was significantly lower $(p<0.01)$ for both gender. Excellent matching of self- and parent-estimated health-related quality of life was found.

Peak oxygen uptake correlated significantly with all scores Disease Impact, Psychosocial Impact, Total score - of patients and of their parents $(p<0.05)$. All scores increase linearly with $\mathrm{pVO} 2$ up to $\mathrm{pVO} 2=<40 \mathrm{ml} / \mathrm{kg} / \mathrm{min}$, when they meet their plateau. Conclusions: In general, our patients had reduced exercise capacity. Peak oxygen uptake was in good correlation with the perception of all aspects of health, made by patients and by their parents: better perception of health mainly means better pVO2. So, complementary usage of these two methods should get an comprehensive overview of the health status of young patients with CHD, as it's already established in adult medicine.
P-302

Nocturnal Dipping in Prehypertensive Patients

Herceg-Cavrak V., Svigir A., Tokic Pivac V., Vugrinec Mamic M., Cuk M. Children's Hospital Zagreb, Croatia

Introduction: The nocturnal dipping pattern in prehypertensive children and adolescents has not been studied well. Decreased dipping in nocturnal blood pressure in children and adolescents is related to secondary hypertension, obesity, obstructive sleep apnea, low birth weight, diabetes mellitus type 1 and type 2. Obesity is often associated with primary arterial hypertension and prehypetension in children and adolescents and affects not only average 24-hour BP values, but also decreases drop in nocturnal BP. Prehypertension is defined as blood pressure (BP) $\geq 90$ th percentile, or $\geq 120$ / $80 \mathrm{mmHg}$, but $<95$ th percentile for age, sex and height.

The aim: of our study was to investigate the nocturnal drop in blood pressure in patients with prehypertension.

Patients and methods: We studied 24-hour blood pressure monitoring results in a group of 206 patients (109 males) aged 6-20 years, mean age $14.62 \pm 2,73$ years referred to our cardiology and nephrology clinic as hypertension.

Results: Normal findings had 58 (28\%), prehypertension was present in $89(43 \%)$ and confirmed arterial hypertension (primary and secondary) in $59(29 \%)$ of patients. In the group of prehypertensive patients 53 $(60 \%)$ of them were non-dippers. Obesity played important role in prehypertensive blood pressure changes - among non-dipping patients almost one half were obese (47\%), in comparison with dippers with the significantly lower prevalence of obesity $(39 \%)$ ( $p<0,01)$.

Conclusion: The absence of nocturnal drop in blood pressure is a common finding in prehypertensive patients and is significantly associated with the increased body mass index. 